\title{
Grupos de tranças Brunnianas e grupos de homotopia da esfera $\mathbb{S}^{2}$
}

\author{
Oscar Eduardo Ocampo Uribe
}

\section{TESE APRESENTADA}

$\mathrm{AO}$

Instituto De Matemática e Estatística

DA

Universidade DE SÃo Paulo

PARA

OBTENÇÃO DO TÍTULO

$\mathrm{DE}$

Doutor EM Cî̂NCIAS

\author{
Programa: Matemática \\ Orientador: Prof. Dr. Daciberg Lima Gonçalves \\ Coorientador: Prof. Dr. John Guaschi
}

Durante o desenvolvimento deste trabalho o autor recebeu auxílio financeiro da FAPESP, projeto número 2008/58122-6

São Paulo, julho de 2013 



\title{
Grupos de tranças Brunnianas e grupos de homotopia da esfera $\mathbb{S}^{2}$
}

\begin{abstract}
Esta versão da tese contém as correções e alterações sugeridas pela Comissão Julgadora durante a defesa da versão original do trabalho, realizada em 02/07/2013. Uma cópia da versão original está disponível no Instituto de Matemática e Estatística da Universidade de São Paulo.
\end{abstract}

Comissão Julgadora:

- Prof. Dr. Daciberg Lima Gonçalves (orientador) - IME-USP

- Prof. Dr. John Guaschi - Université de Caen (França)

- Prof. Dr. Daniel Vendrúscolo - UFSCAR

- Prof. Dr. Alcibiades Rigas - UNICAMP

- Prof. Dr. Tomas Edson Barros - UFSCAR 

Douter de tout ou tout croire, ce sont deux solutions également commodes, qui l'une et l'autre nous dispensent de réfléchir. Henri Poincaré

In mathematics, what is intriguing, puzzling, interesting, surprising, boring, tedious, exciting is crucial; they are not incidental, they shape how we think.

Bill Thurston 

Dedico esta tese à minha família; especialmente a meus pais Carlos e Nubia, e à minha namorada Eliane. 



\section{Agradecimentos}

Agradeço, primeiramente a Deus, por me permitir conquistar este sonho.

Não tenho palavras suficientes para agradecer aos Professores Daciberg e John pela orientação, pela incansável disponibilidade, por tudo que generosamente têm me ensinado durante toda minha pósgraduação. Pela participação sempre ativa e presente, pelas inúmeras discussões dos assuntos da tese, tanto no Brasil como à distância. Agradeço pela confiança depositada em mim durante este projeto de pesquisa, eu não teria conseguido alcançar este objetivo sem o constante apoio dos meus orientadores. Estou profundamente agradecido a vocês professores!

À banca julgadora, composta pelos professores Daciberg Lima Gonçalves, John Guaschi, Daniel Vendrúscolo, Alcibiades Rigas e Tomas Edson Barros, agradeço pelas sugestões dadas visando melhorar meu trabalho de tese.

Esta tese foi apoiada pela FAPESP projeto número 2008/58122-6, pela Capes-Cofecub projeto número Ma 733-12 (França) e Cofecub projeto número 1716/2012 (Brasil). O apoio destas agências foi muito importante para o desenvolvimento deste projeto de pesquisa.

Sou muito grato com toda a comunidade do Instituto de Matemática e Estatística, da Universidade de São Paulo, onde passei muitos anos aprimorando minha formação acadêmica e profissional. Quero agradecer aos meus professores do IME, o aprendido durante todo esse tempo tem sido indispensável para completar esta fase da minha carreira acadêmica. Agradeço à equipe de Topologia Algébrica do IME, às Professoras Lucília, Fernanda e aos meus colegas. Para mim, a participação neste grupo tem sido uma experiência fantástica, levarei comigo boas lembranças e ensinamentos, fruto desta interação. Aproveito para agradecer ao Professor Raul e à Professora Rosa, pelas várias conversas acadêmicas, bem como as não acadêmicas, as quais tem me ajudado bastante durante este processo. Também ao meu amigo, Anderson Paião dos Santos, pelas sugestões na redação deste texto.

Gostaria de estender meus agradecimentos aos membros do Laboratoire de Mathématiques Nicolas Oresme, da Université de Caen, pela magnífica recepção e hospitalidade durante meu estágio de pesquisa, no LMNO, entre janeiro e junho de 2012. Parte deste trabalho foi obtido sob a supervisão do Professor John neste estágio. Também sou muito grato com os "Jeunes Chercheurs" Hugues, Mathieu, Willy e Justine e com os outros doutorandos do LMNO, sua companhia e receptividade foi valiosa durante minha estada 
em Caen.

Não posso deixar de agradecer aos meus professores da Universidad Nacional de Colombia, sede Medellin. Uma conquista como esta, é parte de um processo que começou muito tempo atrás, na Colômbia, onde iniciei meus estudos na Matemática. Agradeço à Professora Débora Tejada. Meus primeiros passos na Topologia Algébrica foram guiados por ela e a motivação que tive para continuar estudando esta maravilhosa área da Matemática, na pósgraduação, foi inspirada pelos seus ensinamentos, quando eu estava ainda na graduação.

A toda minha família agradeço enormemente. Especialmente aos meus pais Carlos e Nubia, aos meus irmãos Carlos e Luis e a minha irmã Patricia agradeço pelo apoio, pela confiança e pelo respeito de todos vocês nas minhas decisões, pela comprensão de ficarmos distantes por tanto tempo. Só vocês sabem quanta saudade eu sinto. Muito obrigado pela força e apoio, que tem me dado desde que empreendi meus estudos fora da Colômbia.

A Eliane agradeço profundamente pelo carinho, pela confiança, pelo apoio, pela paciência. Sua ajuda, sua fortaleza, tem sido fundamental para mim durante todo o doutorado. Durante todo este tempo você tem estado comigo comemorando as vitórias e me dando força nos momentos difíceis. Sua participação nesta conquista tem sido muito grande, este trabalho também é dedicado a você.

Agradeço ao Sr. Carlinhos, Dona Lindinalva, Elane, Elma e Gilmar pela fortaleza, pelo apoio, durante estes anos.

Sem dúvida, quero também agradecer aos meus amigos, tanto aos mais distantes quanto aos menos distantes. De fato, no final das contas, sou eu quem fico entretido no meu trabalho, ou seja, o distante sou eu mesmo. Escrever nomes é arriscado, pois posso esquecer algum. Mesmo assim, vou tentar, quero registrar meus agradecimentos a Anderson Paião, Gustavo, Rafael, Sérgio, Vinicius, Anderson Vieira, Adilson, Itailma, Maikel, Glauce, Rosilene, William, Gláucia, Pricila, Cleber, Tatiane, Maysa, Danilo, Héctor, Tiago, João Paulo, Bruno M., Bruno, Naiara, Samir, Cesar, Sabrina, Graciele, Alejandra, Daniel, Luz Marina, Raibel, Rafael A., F. David, Natalia S., Emer, Natalia M., Nacho, Oscar Daniel, Andres, Lenny, Elisa, Vivian, Daniela e Wendy. A todas as pessoas e amigos que de uma forma ou outra colaboraram neste processo quero lhes dizer muito obrigado.

Bom, por último, aos meus orientadores, aos meus professores, a Eliane, a minha família, aos meus amigos, a todos vocês agradeço pela paciência e apoio, durante todo este processo.

Muito obrigado a todos!!!

Oscar. 


\section{Resumo}

OCAMPO URIBE, O.E. Grupos de tranças Brunnianas e grupos de homotopia da esfera $\mathbb{S}^{2}$. 2013. 127 f. Tese (Doutorado) - Instituto de Matemática e Estatística, Universidade de São Paulo, São Paulo, 2013.

A relação entre os grupos de tranças de superfícies e os grupos de homotopia das esferas é atualmente um tópico de bastante interesse. Nos últimos anos tem sido feitos avanços consideráveis no estudo desta relação no caso dos grupos de tranças de Artin com $n$ cordas, denotado por $B_{n}$, da esfera e do plano projetivo. Nessa tese analisamos com detalhes as interações entre a teoria de tranças e a teoria de homotopia, e mostramos novos resultados que estabelecem conexões entre os grupos de homotopia da 2-esfera $\mathbb{S}^{2}$ e os grupos de tranças sobre qualquer superfície. No andamento deste trabalho, descobrimos uma conexão surpreendente dos grupos de tranças com os grupos cristalográficos e de Bieberbach: para $n \geq 3$, o grupo quociente $B_{n} /\left[P_{n}, P_{n}\right]$ é um grupo cristalográfico que contém grupos de Bieberbach como subgrupos, onde $P_{n}$ é o subgrupo de tranças puras de $B_{n}$. Com isto obtivemos uma formulação de um Teorema de Auslander e Kuranishi para 2-grupos finitos e exibimos variedades Riemannianas compactas planas que admitem difeomorfismo de Anosov e cujo grupo de holonomia é $\mathbb{Z}_{2^{k}}$. Além disso, durante esta tese, detectamos e, quando possível, corrigimos algumas imprecisões em dois importantes artigos nessa área de estudo, escritos por J. Berrick, F. R. Cohen, Y. L. Wong e J. Wu (Jour. Amer. Math. Soc - 2006) assim como por J. Y. Li e J. Wu (Proc. London Math. Soc-2009).

Palavras chave: grupos de tranças de superfícies; tranças Brunnianas; grupos simpliciais; grupos de homotopia das esferas; comutadores; grupos cristalográficos; grupos de Bieberbach. 


\section{Abstract}

OCAMPO URIBE, O.E. Brunnian braid groups and homotopy groups of the sphere $\mathbb{S}^{2}$. 2013. 127 f. Tese (Doutorado) - Instituto de Matemática e Estatística, Universidade de São Paulo, São Paulo, 2013.

The relation between surface braid groups and homotopy groups of spheres is currently a subject of great interest. Considerable progress has been made in recent years in the study of these relations in the case of the $n$-string Artin braid groups, denoted by $B_{n}$, the sphere and the projective plane. In this thesis we analyse in detail the interactions between braid theory and homotopy theory, and we present new results that establish connections between the homotopy groups of the 2-sphere $\mathbb{S}^{2}$ and the braid groups of any surface. During the course of this work, we discovered an unexpected connection of braid groups with crystallographic and Bieberbach groups: for $n \geq 3$, the quotient group $B_{n} /\left[P_{n}, P_{n}\right]$ is a crystallographic group that contains Bieberbach groups as subgroups, where $P_{n}$ is the pure braid subgroup of $B_{n}$. This enables us to obtain a formulation of a theorem of Auslander and Kuranishi for finite 2-groups, and to exhibit Riemannian compact flat manifolds that admit Anosov diffeomorphisms and whose holonomy group is $\mathbb{Z}_{2^{k}}$. In addition, during the thesis, we have detected, and where possible, corrected some inaccuracies in two important papers in the area of study, by J. Berrick, F. R. Cohen, Y. L. Wong and J. Wu (Jour. Amer. Math. Soc-2006), and by J. Y. Li and J. Wu (Proc. London Math. Soc - 2009).

Keywords: surface braid groups; Brunnian braids; simplicial groups; homotopy groups of spheres; commutators; crystallographic groups; Bieberbach groups. 


\section{Sumário}

Lista de Símbolos $\quad$ Xv

Lista de Figuras $\quad$ xix

Introdução $\quad$ xxi

Resumo dos resultados principais $\quad$ xxvii

1 Preliminares 1

1.1 Rudimentos da teoria de grupos . . . . . . . . . . . . . . . . 1

1.1 .1 Grupos livres ... . . . . . . . . . . . . . . 3

1.1.2 Subgrupos comutadores em grupos . . . . . . . . . . . . . . . 4

1.1.3 O subgrupo comutador simétrico . . . . . . . . . . . . 6

1.2 Topologia Algébrica e Teoria de Tranças . . . . . . . . . . . . . . . . 9

1.2.1 Grupos de homotopia . . . . . . . . . . . . . . . . 9

1.2.2 Espaços de configuração . . . . . . . . . . . . . . . . . . . . 13

1.2.3 Grupos de Tranças sobre superfícies . . . . . . . . . . . . . . . . . 14

1.2.4 Enlaçamentos e tranças Brunnianas . . . . . . . . . . . . . 20

1.3 Teoria de Homotopia Simplicial . . . . . . . . . . . . . . . . . . . . 25

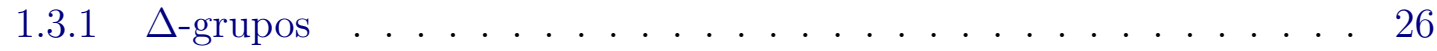

1.3.2 Grupos simpliciais . . . . . . . . . . . . . . 30

2 Grupos de tranças Brunnianas, quase-Brunnianas e grupos de homoto$\begin{array}{ll}\text { pia da esfera } & 37\end{array}$

2.1 Grupos de tranças Brunnianas . . . . . . . . . . . . . . . . . 37

2.2 Grupos de tranças quase-Brunnianas e grupos de homotopia da 2-esfera . . 43

2.3 Tranças puras sobre superfícies e grupos de homotopia da 2-esfera . . . . . 58

2.4 Um monomorfismo entre tranças sobre a esfera e tranças sobre o disco . . . 66

2.4.1 Construindo um homomorfismo de $\operatorname{Brun}_{n+1}\left(\mathbb{S}^{2}\right)$ em $\operatorname{Brun}_{n}\left(\mathbb{D}^{2}\right)$. . . 66

2.4.2 Uma prova diferente do Teorema 1.2 de Cohen et al. [9] . . . . . . . 71

2.4.3 Uma prova diferente do Teorema 1 (1) de Li e Wu em [58] . . . . . 72 
3.1 Grupos de homotopia de $\Delta$-grupos formados por tranças puras . . . . . . . 75

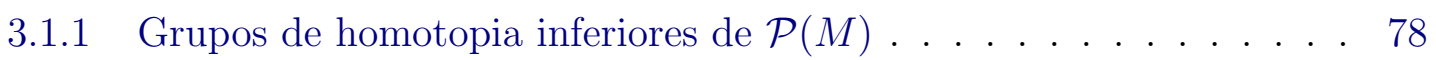

3.1.2 Grupos de homotopia superiores de $\mathcal{P}(M) \ldots \ldots$. . . . . . . 83

3.2 O grupo $T_{k, n}(M)$ para uma superfície $M \ldots \ldots \ldots \ldots$. . . . . 84

3.3 Sequências exatas de grupos de tranças Brunnianas e grupos de homotopia 85

3.4 Uma trança Brunniana com 5 cordas na esfera . . . . . . . . . . . . . . 90

4 Centro de $G_{n+1} / \mathcal{B} d_{n} \mathcal{H}$ e os grupos de homotopia $\pi_{n}(\mathcal{H})$

4.1 O Teorema de Extensão Central e o Teorema de Decomposição . . . . . . . 96

4.2 Construção de grupos simpliciais . . . . . . . . . . . . . . . . . 107

$4.2 .1 \quad$ O grupo simplicial $\mathcal{K}\left(\mathbb{D}^{2}\right) \quad \ldots \ldots \ldots$. . . . . . . . . . . 109

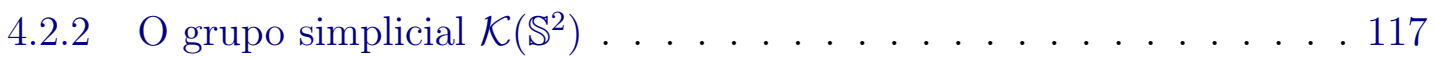

4.3 Aplicações . . . . . . . . . . . . . . . . . . . . . . . . . . 121

4.3.1 Os conjuntos de grupos de tranças $\mathbb{P}$ e $\mathcal{P}\left(\mathbb{S}^{2}\right) \ldots . . . . .121$

4.3.2 Quocientes finitamente apresentados e $\pi_{*}\left(\mathbb{S}^{2}\right) \ldots . . . . . . .125$

5 Grupos de tranças e Grupos cristalográficos 135

5.1 Grupos cristalográficos e de Bieberbach . . . . . . . . . . . . . . 136

5.2 Tranças com 3 cordas sobre o disco e grupos cristalográficos . . . . . . . 140

5.2.1 A representação integral $\Theta: \Sigma_{3} \rightarrow \operatorname{Aut}\left(P_{3} / \operatorname{Brun}_{3}\left(\mathbb{D}^{2}\right)\right) \quad \ldots . . .140$

5.2.2 Elementos de torção em $B_{3} / \operatorname{Brun}_{3}\left(\mathbb{D}^{2}\right) \ldots \ldots$. . . . . . . . . . 144

5.3 Tranças com $n$ cordas sobre o disco e grupos cristalográficos . . . . . . . . 154

5.3.1 O grupo quociente $B_{n} /\left[P_{n}, P_{n}\right]$ é um grupo cristalográfico . . . . . . 155

5.3 .2 Sobre a torção 2 em $B_{n} /\left[P_{n}, P_{n}\right] \ldots$. . . . . . . . . . . . . 159

5.3.3 Elementos de ordem ímpar em $B_{n} /\left[P_{n}, P_{n}\right] \ldots$. . . . . . . . . . 162

5.4 Tranças, variedades planas e existência de difeomorfismos de Anosov . . . . 166

5.4.1 Os grupos de Bieberbach e o Teorema de Auslander e Kuranishi . . 166

5.4.2 Grupos de Bieberbach $\mathcal{B C G}(4, H)$ e com grupo de holonomia $\mathbb{Z}_{2^{k}}$. . 168

5.4.3 Existência de difeomorfismos de Anosov para algumas variedades planas . . . . . . . . . . . . . . . . . . . 174

6 Apêndice $\quad 177$

6.1 O artigo de J. Berrick, F. Cohen, Y.L. Wong e J. Wu . . . . . . . . . . . . 177

6.1.1 Algumas imprecisões detectadas nas Subseções 6.5 e 6.6 de [9] . . 178

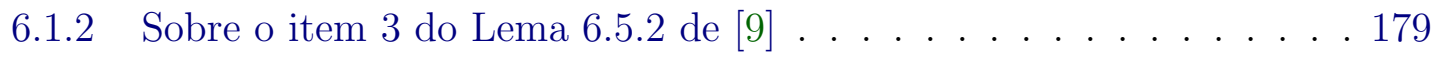

6.1 .3 Sobre o exemplo da Subseção 7.4 de [9] . . . . . . . . . . . . . . . . 181

$6.2 \mathrm{O}$ artigo de J.Y. Li e J. Wu . . . . . . . . . . . . . . . . . . . . . . . 184

6.2.1 Sobre a Seção 2 . . . . . . . . . . . . . . . . . . . . . . . . 184

6.2 .2 Sobre a Seção $3 \ldots$. . . . . . . . . . . . . . . . . . . . 188 
6.2.3 Sobre a Seção 4 . . . . . . . . . . . . . . . . . . . . . . 190

6.3 Algumas provas alternativas . . . . . . . . . . . . . . . . . . 191

6.4 Sobre difeomorfismos de Anosov . . . . . . . . . . . . . . . . . 195

Referências Bibliográficas $\quad 199$

$\begin{array}{ll}\text { Índice Remissivo } & 207\end{array}$ 


\section{Lista de Símbolos}

$[G, G]$

$\left[R_{1}, R_{2}, \ldots, R_{n}\right]_{S}$

$\Gamma_{n+1}(G)$

$\langle a\rangle^{G}$

$B(m, n)$

$F_{n}$

$\Sigma_{n}$

$\mathbb{Z}$

$\mathbb{Z}_{m}$

$D_{n} M$

$F_{n} M$

I

$S^{n}$

$\mathbb{S}^{n}$

$\mathbb{R}^{n}$

$\pi_{n}\left(X, x_{0}\right)$

$B_{n}$

$B_{n}(M)$

$B_{n, k}(M)$

$P_{n}$
Subgrupo comutador de $G$

Subgrupo comutador simétrico dos grupos $R_{1}, \ldots, R_{n}$

$(n+1)$-ésimo termo da série central de $G$

Fecho normal de $a$ em $G$

Grupo de Baumslag-Solitar

Grupo livre de posto $n$

Grupo de permutações em $n$ elementos

Grupo dos inteiros

Grupo cíclico de ordem $m$

$n$-ésimo espaço de configuração não ordenado de $M$

$n$-ésimo espaço de configuração ordenado de $M$

Intervalo unitário $I=[0,1]$

Esfera simplicial de dimensão $n$

Esfera de dimensão $n$

Espaço euclidiano de dimensão $n$

$n$-ésimo grupo de homotopia de $X$ com ponto base $x_{0}$

Grupo de tranças de Artin com $n$ cordas

Grupo de tranças com $n$ cordas sobre $M$

Grupo misto de tranças com $n+k$ cordas sobre $M$

Grupo de tranças puras de Artin com $n$ cordas 
$P_{n}(M)$

$\chi_{\sigma_{k}}: P_{n} \rightarrow P_{n}$

$\operatorname{Brun}_{n}(M)$

$\operatorname{Brun}_{n}^{k}(M)$

$\mathcal{Q}^{k} \operatorname{Brun}_{n}(M)$

$\mathcal{S}^{k} \operatorname{Brun}_{n}(M)$

$A_{i, j}$

$\sigma_{i}$

$C l(\beta)$

$\Delta_{n}$

$\chi: B_{n} \rightarrow B_{n}$

$\bar{\sigma}: \frac{B_{n}}{\left[P_{n}, P_{n}\right]} \rightarrow \Sigma_{n}$

$\sigma: B_{n} \rightarrow \Sigma_{n}$

$\mathbb{P}$

$\widetilde{\Omega P}$

$\mathcal{K}$

$\mathcal{K}\left(\mathbb{D}^{2}\right)$

$\mathcal{K}\left(\mathbb{S}^{2}\right)$

$\mathcal{P}(M)$

$N \mathcal{G}=\left\{\mathcal{N}_{n} \mathcal{G}\right\}_{n \geq 0}$

$\pi_{n}(\mathcal{G})=\mathcal{Z}_{n} \mathcal{G} / \mathcal{B} d_{n} \mathcal{G}$

$\mathcal{B} d_{n} \mathcal{G}$
Grupo de tranças puras com $n$ cordas sobre $M$

Ação por conjugação de $\sigma_{k}$ sobre os elementos de $P_{n}$

Grupo de tranças Brunnianas com $n$ cordas sobre $M$

Grupo de tranças $k$-decomponíveis com $n$ cordas sobre $M$

Grupo de tranças quase-Brunnianas de ordem $k$ com $n$ cordas sobre M

Grupo de tranças semi-Brunnianas de ordem $k$ com $n$ cordas sobre $M$

Gerador de $P_{n}$

Gerador de $B_{n}$

Fecho de uma trança $\beta$

"Full twist" com $n$ cordas

Reflexão espelho

Homomorfismo que envia tranças (módulo o comutador $\left[P_{n}, P_{n}\right]$ ) em permutações

Homomorfismo que envia tranças em permutações

Sequência formada por grupos de tranças de Artin

$\Delta$-grupo formado por subgrupos de tranças de Artin cuja primeira aplicação face é $\partial$

Grupo simplicial $\left\{\pi_{1}\left(\mathbb{D}^{2} \backslash Q_{n}\right)\right\}_{n \geq 0}$

Grupo simplicial tal que $\left(\mathcal{K}\left(\mathbb{D}^{2}\right)\right)_{n}=\operatorname{Ker}\left(d_{n+1}: P_{n+1} \rightarrow P_{n}\right)$ para $n \geq 1$

Grupo simplicial tal que $\left(\mathcal{K}\left(\mathbb{S}^{2}\right)\right)_{n}=\operatorname{Ker}\left(d_{n+2}: P_{n+2}\left(\mathbb{S}^{2}\right) \rightarrow P_{n+1}\left(\mathbb{S}^{2}\right)\right)$ para $n \geq 2$

$\Delta$-grupo formado por grupos de tranças puras sobre $M$

Complexos de Moore de um $\Delta$-grupo $\mathcal{G}$

n-ésimo conjunto de homotopia de Moore de um $\Delta$-grupo $\mathcal{G}$

n-ésimo bordo de Moore de um $\Delta$-grupo $\mathcal{G}$ 
n-ésimo ciclo de Moore de um $\Delta$-grupo $\mathcal{G}$

$G L(n ; \mathbb{R})$

$J_{n}$

$O(n ; \mathbb{R})$

$S O(n ; \mathbb{Z})$

$\mathcal{B C G}(n, H)$

$\operatorname{Aff}\left(\mathbb{R}^{n}\right)$

$\operatorname{Aut}(G)$

$\operatorname{PCM}(n, c)$
Grupo de Lie formado por matrizes invertíveis de tamanho $n \times n$ sobre os reais

Grupo de matrizes unimodulares de tamanho $n \times n$ sobre os inteiros

Grupo de Lie formado por matrizes ortogonais de tamanho $n \times n$ sobre os reais

Grupo matrizes ortogonais de tamanho $n \times n$, com determinante 1 , sobre os inteiros

Grupo cristalográfico de tranças com $n$ cordas com grupo de holonomia $H$

Grupo afim de $\mathbb{R}^{n}$

Grupo dos automorfismos do grupo $G$

Matriz circulante principal de tamanho $n \times n$ e com entrada principal $c$ 


\section{Lista de Figuras}

1.1 Concatenação de tranças . . . . . . . . . . . . . . . . . . . . . . . 14

1.2 Permutação associada às tranças . . . . . . . . . . . . . . . . . . 15

1.3 Tranças com 3 cordas sobre o disco, o cilindro e o pantalon . . . . . . . . . 15

1.4 Geradores $\sigma_{i} \in B_{n}$, para $1 \leq i \leq n-1 \ldots \ldots \ldots \ldots$

1.5 Geradores $A_{i, j} \in P_{n}$, para $1 \leq i<j \leq n \ldots \ldots \ldots \ldots$

1.6 Trança $A_{-1, j} \ldots \ldots \ldots \ldots \ldots \ldots \ldots$

1.7 "Full twist" com 5 cordas . . . . . . . . . . . . . . . . . . . . . . 19

1.8 Nó Trefoil. . . . . . . . . . . . . . . . . . . . . . . . . . . 20

1.9 Enlaçamento de Hopf. . . . . . . . . . . . . . . . . . . . . . . . . . 20

1.10 Enlaçamento de Whitehead. . . . . . . . . . . . . . . . . . 20

1.11 Forma geral do fecho de uma trança . . . . . . . . . . . . . . . . . . 21

1.12 Trança $\sigma_{1}^{3}$ e seu fecho, o nó Trefoil. . . . . . . . . . . . . . . . . . . 21

1.13 Trança $A_{1,2}$ e seu fecho, o enlaçamento de Hopf. . . . . . . . . . . . . . . . 21

1.14 Trança $A_{1,2} \sigma_{2}^{-1} \sigma_{1} \sigma_{2}^{-1}$ e seu fecho, o enlaçamento de Whitehead. . . . . . . 21

1.15 Anéis de Borromeo. . . . . . . . . . . . . . . . . . . . . . . 22

1.16 Esquecendo a segunda corda de uma trança com 7 cordas, que não é pura, e fazendo um re-arranjo do ponto base. . . . . . . . . . . . . . . . 27

1.17 Esquecendo a primeira corda de uma trança com 7 cordas, que não é pura, e fazendo um re-arranjo do ponto base. . . . . . . . . . . . . . . . . . 27

1.18 Esquecendo a quarta corda do "full twist" com 5 cordas e fazendo um re-arranjo do ponto base. . . . . . . . . . . . . . . . . . . . 28

2.1 Trança com 4 cordas sobre o disco que não é Brunniana. . . . . . . . . . . 39

2.2 Trança com 2 cordas no disco (Brunniana) e no anel (não Brunniana). . . . 39

2.3 Esquecendo as duas primeiras cordas de uma trança $\beta \in B_{5} \ldots \ldots$. . . . . 44

2.4 Esquecendo as três últimas cordas de uma trança $\beta \in B_{5} \ldots \ldots$. . . . . . . 44

2.5 Trança quase-Brunniana, de ordem 2, com 5 cordas sobre o disco. . . . . . 45

2.6 Trança semi-Brunniana de ordem 2, com 5 cordas, na esfera. . . . . . . . . 48

2.7 Fecho da trança $\left[A_{1,3}, A_{2,3}\right]$ : Anéis de Borromeo. . . . . . . . . . . . . 66

3.1 Laço $c$ que representa $\gamma \ldots \ldots$. . . . . . . . . . . . . . . . 79

3.2 Geradores livres $w_{0}, w_{1}, w_{2} \ldots \ldots \ldots \ldots \ldots$ 
4.1 Geradores de $\pi_{1}\left(\mathbb{D}^{2} \backslash Q_{n}\right)$ para $n \geq 1 \ldots \ldots$. . . . . . . . . . . . .

5.1 Trança $\gamma_{i, k} \ldots \ldots \ldots \ldots \ldots \ldots \ldots$ 


\section{Introdução}

Os grupos de tranças do plano, denotados por $B_{n}$, foram introduzidos por E. Artin em 1925 [2], de uma forma geométrica e intuitiva, e posteriormente, em 1947, ele os estudou mais rigorosamente de um ponto de vista algébrico [3], [4]. Cada elemento deste grupo tem uma representação geométrica como um trançado de cordas com certas restrições. Estes grupos, também chamados de grupos de tranças de Artin, têm sido generalizados em várias direções, como por exemplo, os grupos de tranças de superfícies, os grupos de ArtinTits, assim como os grupos de tranças virtuais e "welded". Neste trabalho utilizaremos a primeira generalização, em que as tranças são consideradas sobre qualquer superfície $M$ e que são denotados por $B_{n}(M)$. Um aspecto interessante dos grupos de tranças sobre superfícies é que eles podem ser definidos de pontos de vista diferentes, tendo cada uma destas definições diversas vantagens operacionais, dependendo do objetivo de estudo. A noção de grupo de tranças sobre superfícies foi introduzida inicialmente por O. Zariski, e generaliza de forma natural a definição geométrica de Artin [98], [99]. Os grupos de tranças sobre superfícies foram estudados na década de 60 por R. Fox e L. Neuwirth em [31]. Neste trabalho eles mostraram que o grupo de tranças (resp. tranças puras) sobre uma superfície coincide com o grupo fundamental do espaço de configuração não ordenado (resp. ordenado).

Os grupos de tranças são interessantes por si próprios, mas também têm um papel importante em diferentes áreas da matemática, como por exemplo em topologia, geometria, álgebra, sistemas dinâmicos e física teórica. Destacamos o seu uso no estudo de nós e enlaçamentos [64]; na definição de invariantes topológicos como o polinômio de Jones e os invariantes de Vassiliev [51], [64]; dos Mapping Class Groups [13], [94], [30]; da propriedade de Borsuk-Ulam para aplicações em superfícies [39]; e da teoria de homotopia clássica, que começou a ser desenvolvida desde a década de 80 por F. R. Cohen [18]. Também têm aplicações na biologia, robótica e criptografia [8]. Finalmente observamos que a $K$-teoria de grupos de tranças sobre algumas superfícies tem sido estudada, ver [52], [42].

Na teoria dos grupos de tranças sobre superfícies, aqueles grupos de tranças sobre a esfera $\mathbb{S}^{2}$ e o plano projetivo $\mathbb{R} P^{2}$ são de grande importância e são excepcionais na teoria geral, uma vez que estas são as únicas superfícies em que seus espaços de configuração não são espaços de Eilenberg-Mac Lane. O tipo de homotopia destes espaços de configuração, assim como a periodicidade cohomológica dos grupos de tranças da esfera $\mathbb{S}^{2}$ e do plano 
projetivo $\mathbb{R} P^{2}$, podem ser vistos na seção 3.6 de [42] com algumas de suas referências. Para uma leitura inicial da teoria de tranças recomendamos os livros de V.L. Hansen [45], de C. Kassel e V. Turaev [54], e de K. Murasugi e B. Kurpita [75]. Para uma leitura mais avançada e resultados recentes desta teoria recomendamos o capítulo de D. Rolfsen no livro [8]; assim como os textos de J. Birman e T. Brendle [11] e de J. Guaschi e D. Juan-Pineda [42].

Entre os diferentes subgrupos dos grupos de tranças de superfícies, estudaremos aqui os chamados grupos de tranças Brunnianas sobre superfícies. Vejamos como surgiram estes grupos no ambiente da teoria de tranças. O matemático alemão Hermann Brunn (1862-1939) estudou a existência de enlaçamentos com certas propriedades especiais e publicou em 1892 um artigo com os resultados da sua pesquisa [15]. Muitos anos depois, em 1954, no artigo intitulado "Link groups" J. Milnor estudou n-enlaçamentos quasetrivias ("almost trivial links"), que ele definiu como sendo $n$-enlaçamentos com a propriedade que qualquer subenlaçamento próprio é trivial [70, Página 189]. Os $n$-enlaçamentos quase-triviais definidos por J. Milnor, na verdade estavam entre os $n$-enlaçamentos estudados por H. Brunn em [15]. Milnor classificou os enlaçamentos quase-triviais, a menos de homotopia de enlaçamentos, usando os invariantes que hoje em dia são chamados de invariantes de Milnor, ver [70], cuja definição se baseia nos produtos de Massey no complemento do enlaçamento. Estes invariantes de Milnor têm sido muito importantes na teoria dos nós. Posteriormente H. Debrunner, num artigo de 1961 intitulado "Links of Brunnian type", continuou os estudos que H. Brunn começou em [15], onde além de estudar o problema de forma mais rigorosa apresentou vários outros exemplos dos tipos estudados por H. Brunn, ver [22]. Na introdução de [22] Debrunner chamou de enlaçamento Brunniano os $n$-enlaçamentos não triviais, para $n>1$, com a propriedade que todo subenlaçamento próprio é trivial quando qualquer uma das componentes for retirada, argumentando que os primeiros exemplos de tais enlaçamentos foram dados por H. Brunn em [15]. O enlaçamento chamado de Anéis de Borromeo é um dos exemplos mais representativos de um enlaçamento Brunniano.

Motivados pelo estudo dos $n$-enlaçamentos, vamos considerar o conjunto $\operatorname{Brun}_{n}(M)$ das tranças com $n$ cordas sobre uma superfície $M$ com a propriedade que ao removermos qualquer uma de suas cordas obtemos a trança trivial com $n-1$ cordas. O conjunto acima forma um subgrupo de $B_{n}(M)$. Na década de $70 \mathrm{H}$. Levinson [57] chamou de tranças decomponíveis ("decomposable braids") as tranças de $\operatorname{Brun}_{n}\left(\mathbb{D}^{2}\right)$. Neste trabalho H. Levinson também considerou um tipo mais geral de tranças, que ele chamou de $k$-decomponíveis, que são as tranças que têm a propriedade que ao removermos qualquer subconjunto com $k$ cordas obtemos a trança trivial com $n-k$ cordas. Uma questão clássica proposta por G. S. Makanin em 1980, no bem conhecido "Kourovka notebook" [61], era

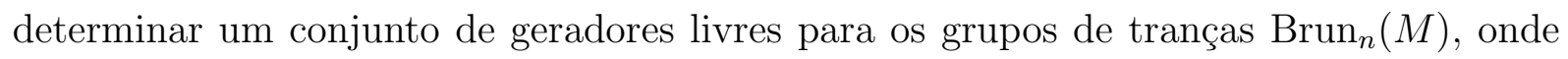
$M$ é o disco, os quais ele chamou de tranças suaves ("smooth braids"). D. L. Johnson [49] 
usou o método conhecido como "Hall commutator collection process" [60] para dar um conjunto de geradores de $\operatorname{Brun}_{n}(M)$. Como mencionado no artigo de Johnson [49], apareceu nas atas de uma conferência um resumo de G. G. Gurzo [43] anunciando a solução do problema proposto por Makanin. Anos mais tarde, em 1999, T. Stanford usou o termo trança Brunniana no artigo intitulado "Brunnian braids and some of their generalizations" [88] para denotar os elementos de $\operatorname{Brun}_{n}\left(\mathbb{D}^{2}\right)$. Até o nosso conhecimento esse é o primeiro lugar onde aparece o termo trança Brunniana. Claramente um enlaçamento obtido pelo fecho de uma trança Brunniana sobre o disco é um enlaçamento Brunniano, mas existem enlaçamentos Brunnianos que não são obtidos pelo fecho de uma trança Brunniana sobre o disco, como por exemplo o enlaçamento de Whitehead.

Observamos que essas primeiras considerações de grupos de tranças Brunnianas foram dadas nos grupos de tranças sobre o disco, e em $2000 \mathrm{~K}$. Whittlesey [94] considerou $\operatorname{Brun}_{n}\left(\mathbb{S}^{2}\right)$, que os chamou grupos de tranças Brunnianas sobre a esfera. A partir de 2006, em vários artigos [9], [17] e [58], [5], foi considerado o estudo de $\operatorname{Brun}_{n}(M)$ para $M$ uma superfície e desde então é usada a notação para este grupo como $\operatorname{Brun}_{n}(M)$. A definição formal e algumas das propriedades dos grupos de tranças Brunnianas aparecem na Subseção 1.2.4 e na Seção 2.1.

Esta tese está fortemente inspirada e ligada ao artigo [9] de 2006, escrito por F. Cohen, J. Berrick, Y. L. Wong e J. Wu, o qual estabeleceu uma conexão entre os grupos de tranças Brunnianas sobre a esfera e os grupos de homotopia da esfera $\pi_{*}\left(\mathbb{S}^{2}\right)$, onde as ferramentas usadas para estabelecer a ponte entre as duas teorias foi a teoria de homotopia simplicial. O Teorema 1.2 de [9], também formulado no Capítulo 1 como Teorema 1.2.13, diz que para $n \geq 5$, a sequência de grupos

$$
1 \longrightarrow \operatorname{Brun}_{n+1}\left(\mathbb{S}^{2}\right) \longrightarrow \operatorname{Brun}_{n}\left(\mathbb{D}^{2}\right) \stackrel{f_{*}}{\longrightarrow} \operatorname{Brun}_{n}\left(\mathbb{S}^{2}\right) \longrightarrow \pi_{n-1}\left(\mathbb{S}^{2}\right) \longrightarrow 1
$$

é exata, onde $f: \mathbb{D}^{2} \hookrightarrow \mathbb{S}^{2}$ é a inclusão natural. Prosseguindo com estes estudos, uma conexão entre os grupos de homotopia da esfera $\mathbb{S}^{2}$ foi exibida por J. Y. Li e J. Wu em [58] no caso dos grupos de tranças de Artin (ver o Teorema 1.3.25) e por V. Bardakov, R. Mikhailov, V. Vershinin e J. Wu em [5] no caso dos grupos de tranças da esfera e do plano projetivo (ver o Teorema 1.2.14). Recentemente, em [10], J. Berrick, L. Hanbury e J. Wu conseguiram outros resultados nesta direção, iniciada em [9].

É bem conhecido que o entendimento dos grupos de homotopia das esferas ajudaria no desenvolvimento de varias áreas da matemática. O objetivo principal deste trabalho consiste em explorar novas conexões entre a teoria de tranças e a teoria de homotopia, em particular mostraremos que pode se estabelecer uma conexão entre os grupos de homotopia da esfera $\mathbb{S}^{2}, \pi_{*}\left(\mathbb{S}^{2}\right)$, e os grupos de tranças sobre qualquer superfície, estendendo os resultados já conhecidos na literatura. Para fazer isto precisaremos introduzir a noção de grupo quase-Brunniano de tranças, de ordem $k$, sobre superfícies. Este conceito estende a 
noção de tranças Brunnianas, na medida que flexibiliza a definição destas tranças, e será de grande ajuda no desenvolvimento desta tese.

Como parte do entendimento dos grupos de tranças Brunnianas sobre superfícies estudamos vários quocientes envolvendo estes grupos, em particular o grupo $B_{3} / \operatorname{Brun}_{3}\left(\mathbb{D}^{2}\right)$. Neste processo descobrimos um resultado inesperado: $B_{3} /\left[P_{3}, P_{3}\right]$ é um grupo cristalográfico de dimensão 3 e com grupo de holonomia sendo o grupo de permutações $\Sigma_{3}$. O Corolário 2.1.4 garante que $\operatorname{Brun}_{3}\left(\mathbb{D}^{2}\right)=\left[P_{3}, P_{3}\right]$, onde $P_{3}$ é o subgrupo de tranças puras de Artin com 3 cordas e $\left[P_{3}, P_{3}\right]$ é seu subgrupo comutador. Assim, motivados pelo resultado encontrado para $B_{3} / \operatorname{Brun}_{3}\left(\mathbb{D}^{2}\right)$, decidimos estudar o caso geral do grupo quociente $B_{n} /\left[P_{n}, P_{n}\right]$, encontrando que ele é um grupo cristalográfico de dimensão $n(n-1) / 2$ e com grupo de holonomia sendo o grupo de permutações $\Sigma_{n}$. Os grupos cristalográficos, assim como os grupos de Bieberbach (grupos cristalográficos livres de torção), têm sido bastante estudados durante muitos anos. Suas propriedades algébricas são muito interessantes e em vista dos Teoremas de Bieberbach eles estão relacionados com variedades Riemannianas compactas planas, e portanto são, por sua vez, de muito interesse na Geometria Diferencial, Topologia Algébrica e Sistemas Dinâmicos. De especial interesse para a Geometria são os grupos de Bieberbach, pois eles correspondem a variedades Riemannianas compactas planas cujo grupo fundamental é o grupo de Bieberbach. Então, estudamos o problema de encontrar os subgrupos de $B_{n} /\left[P_{n}, P_{n}\right]$ que são de Bieberbach e que têm a mesma dimensão que o grupo cristalográfico $B_{n} /\left[P_{n}, P_{n}\right]$. Entre os vários resultados obtidos destacamos aquele que mostra que os grupos cristalográficos $B_{n} /\left[P_{n}, P_{n}\right]$ não tem torção 2, permitindo mostrar que para qualquer 2-grupo finito $H$ existe uma variedade Riemanniana compacta plana com grupo de holonomia $H$. Portanto, uma prova alternativa de um caso de um Teorema de Auslander e Kuranishi pode ser dada. Finalmente, estudaremos a existência de difeomorfismos de Anosov para algumas das variedades Riemannianas compactas planas encontradas.

A seguir, descreveremos em linhas gerais a divisão deste trabalho. Ressaltamos que um resumo, com os enunciados completos dos resultados principais, encontra-se depois desta introdução, na página xxvii. Destacamos ainda a seguinte convenção seguida neste trabalho. Os resultados enunciados nesta tese mas que não foram obtidos por nós sempre serão destacados com parênteses, onde faremos a respectiva referência bibliográfica.

O Capítulo 1, chamado de Preliminares, está dividido em três seções. Na Seção 1.1 encontramos alguns conceitos da teoria de grupos, na Seção 1.2 faremos uma exposição curta de grupos de homotopia e teoria de tranças e na Seção 1.3 descreveremos os aspectos principais da teoria de homotopia simplicial que será usada nesta tese.

No Capítulo 2 começaremos nosso estudo da interação entre a teoria de tranças e a teoria de homotopia. Na Seção 2.1 lembraremos a definição dos grupos de tranças Brunnianas sobre superfícies e provaremos o Lema 2.1.5, que dá uma descrição dos grupos Brunnianos, que será usado nesta tese. Os grupos de tranças quase-Brunnianas, de ordem 
$k$, com $n$ cordas sobre uma superfície $M$, serão definidos na Seção 2.2, e mostraremos que, no caso em que $M$ é a esfera $\mathbb{S}^{2}$ ou o plano projetivo $\mathbb{R} P^{2}$, estes grupos estão relacionados com os grupos de homotopia da esfera $\mathbb{S}^{2}$. Na Seção 2.3 vamos demonstrar que certos subgrupos de tranças sobre qualquer superfície $M$ se projetam nos grupos de homotopia da esfera $\mathbb{S}^{2}$. Relações entre os grupos de homotopia da esfera $\mathbb{S}^{2}$ e os grupos de tranças são conhecidos na literatura apenas no caso dos grupos de tranças sobre a esfera [9], o disco [58], e o plano projetivo [5]. Na última seção deste capítulo, a Seção 2.4, vamos construir um homomorfismo injetor de $\operatorname{Brun}_{n+1}\left(\mathbb{S}^{2}\right)$ em $\operatorname{Brun}_{n}\left(\mathbb{D}^{2}\right)$ e finalizaremos a seção demonstrando de forma alternativa o Teorema 1.2 de [9] e o Teorema 1(1) de [58].

No Capítulo 3 consideraremos os $\Delta$-grupos formados por tranças puras sobre superfícies. Na Seção 3.1 vamos calcular os grupos de homotopia de Moore (usada em teoria de homotopia simplicial) destes $\Delta$-grupos, estendendo assim o Teorema 1.1 de [9], onde foram calculados os grupos de homotopia de Moore do $\Delta$-grupo formado por tranças puras sobre a esfera $\mathbb{S}^{2}$. Nas Seções 3.2 e 3.3 descreveremos sequências exatas envolvendo os grupos de tranças Brunnianas, quase-Brunnianas e os grupos de homotopia da esfera $\mathbb{S}^{2}$. Na Seção 3.4 daremos um exemplo de trança Brunniana com 5 cordas sobre a esfera que se projeta no gerador de $\pi_{4}\left(\mathbb{S}^{2}\right)$.

No Capítulo 4 nos ocuparemos com o estudo de grupos de homotopia de Moore de $\Delta$-grupos e/ou grupos simpliciais como sendo o centro de quocientes de grupos apropriados. Para isto, na Seção 4.1 daremos alguns resultados da teoria de homotopia simplicial, entre os quais se destacam o Teorema de Extensão Central e o Teorema de Decomposição. $\mathrm{Na}$ Seção 4.2 vamos construir dois grupos simpliciais específicos, um deles com tranças sobre o disco e outro com tranças sobre a esfera. Tendo construido estes grupos simpliciais, aplicaremos neles os resultados obtidos na Seção 4.1 e com isto obteremos novos resultados relacionando a teoria de tranças com a teoria de homotopia.

O Capítulo 5 tem por objetivo o estudo da relação entre a teoria de tranças e os grupos cristalográficos. Observamos que não é feito neste capítulo o estudo da interação entre a teoria de homotopia (clássica e simplicial) e a teoria de tranças, como feito nos capítulos anteriores. No entanto, este capítulo surgiu na investigação dos grupos de tranças Brunnianas e a conexão com os grupos de homotopia da esfera $\mathbb{S}^{2}$. Mostraremos que existe uma surpreendente ponte entre a teoria de tranças e a teoria dos grupos cristalográficos. Na Seção 5.1 faremos uma revisão dos conceitos básicos necessários sobre grupos cristalográficos e de Beiberbach. Na Seção 5.2 provaremos que o grupo quociente $B_{3} / \operatorname{Brun}_{3}\left(\mathbb{D}^{2}\right)$ é um grupo cristalográfico, sendo este o ponto de partida para o estudo de grupos cristalográficos via os grupos de tranças. Uma vez analisado o caso motivador de 3 cordas passaremos a estudar, na Seção 5.3, o grupo quociente $B_{n} /\left[P_{n}, P_{n}\right]$. Demonstraremos que o grupo $B_{n} /\left[P_{n}, P_{n}\right]$ é um grupo cristalográfico, estudaremos seus elementos de torção e veremos que ele tem subgrupos de Bieberbach com grupo de holonomia um 2-grupo finito. Na Seção 5.4 vamos estudar alguns subgrupos de $B_{n} /\left[P_{n}, P_{n}\right]$ que têm como grupo 
de holonomia um 2-grupo finito e formularemos um Teorema de Auslander e Kuranishi para 2-grupos finitos. Para finalizar este capítulo, exibiremos uma família de variedades Riemannianas compactas planas, de diferentes dimensões e com diferentes grupos de holonomia cíclica de ordem uma potência de 2, que admitem difeomorfismo de Anosov.

O Capítulo 6 é um apêndice. Na Seção 6.1 vamos registrar algumas imprecisões detectadas no artigo de 2006 de J. Berrick, F. R. Cohen, Y. L. Wong e J. Wu [9], com destaque a imprecisão encontrada no Lema 6.5.2 do mencionado artigo. Em cada caso daremos um argumento formal evidenciando o problema encontrado. O Lema 6.5.2 de [9] foi usado por J. Y. Li e J. Wu num subsequente artigo de 2009 [58]. Na Seção 6.2 faremos uma exposição detalhada dos problemas detectados em [58]. Estas imprecisões são de naturezas diferentes, mas algumas delas provem diretamente daquelas encontradas no Lema 6.5.2 de [9]. Muitos dos resultados de [58], que apresentam algum tipo de problema, foram formulados e provados corretamente nas Seções 4.2 e 4.3 desta tese. Em particular, o Teorema 1 de [58], que é o principal resultado deste artigo, foi formulado como Teorema 4.3.12 e demonstrado na Subseção 4.3.2. Na Seção 6.3 daremos algumas provas alternativas de resultados obtidos nessa tese. Na última seção, a Seção 6.4, definiremos formalmente os chamados difeomorfismos de Anosov e enunciaremos um Teorema de Porteous que resultou ser de grande ajuda, na Subseção 5.4.3, para estudarmos a existência de difeomorfismos de Anosov em variedades planas com grupo de holonomia cíclica. 


\section{Resumo dos resultados principais}

Apresentaremos aqui um resumo que consiste dos principais resultados obtidos durante o doutorado e registrados nessa tese. A exposição será feita capítulo a capítulo, dando algumas definições e eventualmente tecendo alguns comentários sobre sua relevância. Como mencionado na introdução faremos a seguinte convenção neste trabalho. Os enunciados nesta tese que não foram obtidos por nós sempre serão destacados com parênteses, onde faremos a respectiva referência bibliográfica. Porém, de forma excepcional no caso deste resumo de resultados principais, indicaremos na frente de cada enunciado sua numeração dentro deste texto no meio de parênteses.

\section{Capítulo 1}

No Capítulo 1 faremos uma revisão da maior parte dos conceitos que serão usados ao longo do texto. O leitor poderá encontrar nesse capítulo alguns rudimentos da teoria de grupos, dando atenção particular aos grupos livres e à teoria de comutadores; alguns aspectos básicos da teoria de tranças; e também noções básicas de teoria de homotopia simplicial.

\section{Capítulo 2}

Começamos o Capítulo 2 com uma revisão dos grupos de tranças Brunnianas, começando por sua definição. Para cada $i=1, \ldots, n$ existe um homomorfismo natural

$$
\rho_{i *}: P_{n}(M) \longrightarrow P_{n-1}(M)
$$

induzido pela aplicação geométrica que "esquece" a $i$-ésima corda. Definimos o grupo de tranças Brunnianas com $n$ cordas sobre $M$ como sendo o grupo

$$
\operatorname{Brun}_{n}(M)=\bigcap_{i=1}^{n} \operatorname{Ker}\left(\rho_{i *}\right) .
$$

Ou seja, uma trança $\beta$ pertence a $\operatorname{Brun}_{n}(M)$ se, e somente se, esquecendo qualquer uma das cordas de $\beta$ temos que a trança resultante é a trança identidade em $n-1$ cordas. 
O primeiro resultado obtido neste capítulo é uma reformulação do Lema 3.8 de [5], que será usado ao longo desse texto. Em particular a expressão dada em (1) para os grupos de tranças Brunnianas sobre superfícies será de grande utilidade.

Lema 1 (Lema 2.1.5). Suponhamos que $n \geq 5$ se $M=\mathbb{S}^{2}$, que $n \geq 4$ se $M=\mathbb{R} P^{2}$ e que $n \geq 2$ nos outros casos. Então, a intersecção

$$
\operatorname{Ker}\left(d_{j}: P_{n}(M) \rightarrow P_{n-1}(M)\right) \cap \operatorname{Ker}\left(d_{k}: P_{n}(M) \rightarrow P_{n-1}(M)\right)
$$

é tal que

$$
\begin{aligned}
\operatorname{Ker}\left(d_{j}\right) \cap \operatorname{Ker}\left(d_{k}\right) & =\left\langle A_{k, j}\right\rangle^{i_{j_{*}}\left(\pi_{1}\left(M \backslash\left\{q_{1}, \ldots, \widehat{q_{j}}, \ldots, q_{n}\right\}\right)\right)} \\
& =\left\langle A_{k, j}\right\rangle^{i_{k_{*}}\left(\pi_{1}\left(M \backslash\left\{q_{1}, \ldots, \widehat{q_{k}}, \ldots, q_{n}\right\}\right)\right)}=\left\langle A_{k, j}\right\rangle^{P_{n}(M)}
\end{aligned}
$$

para quaisquer $1 \leq k<j \leq n$.

Fixemos $m$, com $1 \leq m \leq n$. Portanto, para $n \geq 5$ se $M=\mathbb{S}^{2}, n \geq 4$ se $M=\mathbb{R} P^{2}$ e $n \geq 3$ nos outros casos, vale que

$$
\operatorname{Brun}_{n}(M)=\left(\bigcap_{1 \leq l<m}\left\langle A_{l, m}\right\rangle^{P_{n}(M)}\right) \bigcap\left(\bigcap_{m<r \leq n}\left\langle A_{m, r}\right\rangle^{P_{n}(M)}\right) .
$$

Uma flexibilidade na definição de tranças Brunnianas dá lugar às tranças chamadas de quase-Brunnianas, as quais irão aparecer bastante nos Capítulos 2 e 3 . Para $0 \leq k \leq m-1$ e $m \geq 3$ o conjunto

$$
\mathcal{Q}_{B}^{k} \operatorname{Brun}_{m}(M)=\left\{\beta \in B_{k, m-k}(M) \mid d_{j}(\beta)=1, \forall j=k+1, \ldots, m\right\}
$$

é um subgrupo do grupo misto $B_{k, m-k}(M)=\sigma^{-1}\left(\Sigma_{k} \times \Sigma_{m-k}\right)$. Com isto temos a seguinte definição.

Definição 2 (Definição 2.2.1). O grupo $\mathcal{Q}_{B}^{k} \operatorname{Brun}_{m}(M)$ será chamado de grupo quaseBrunniano misto de ordem $k$, com $m$ cordas, sobre $M$. Definamos o grupo $\mathcal{Q}_{B}^{k} \operatorname{Brun}_{m}(M)$ restrito ao grupo de tranças puras, $\mathcal{Q}^{k} \operatorname{Brun}_{m}(M)=\mathcal{Q}_{B}^{k} \operatorname{Brun}_{m}(M) \cap P_{m}(M)$, que chamaremos de grupo quase-Brunniano de ordem $k$, com $m$ cordas, sobre $M$.

Depois de termos definido as tranças quase-Brunnianas, demonstramos algumas propriedades que estes grupos têm e depois passamos a definir as chamadas tranças semiBrunnianas.

Definição 3 (Definição 2.2.4). Sejam $m \geq 3$ e $1 \leq k \leq m-1$. Definimos o grupo semiBrunniano de ordem $k$, com $m$ cordas, sobre $M$ denotado por $\mathcal{S}^{k} \operatorname{Brun}_{m}(M)$ como sendo a intersecção do núcleo $\operatorname{Ker}\left(\eta_{k}: B_{k, m-k}(M) \rightarrow B_{m-k}(M)\right)$ com o grupo $\mathcal{Q}_{B}^{k} \operatorname{Brun}_{m}(M)$, onde $\eta_{k}$ é o homomorfismo induzido pela fibração de Fadell-Neuwirth generalizada.

Por convenção diremos que $\mathcal{S}^{0} \operatorname{Brun}_{m}(M)=\operatorname{Brun}_{m}(M)$. 
Em outras palavras uma trança $\beta \in B_{k, m-k}(M)$ é semi-Brunniana de ordem $k$, com $m$ cordas, sobre $M$ se ela torna-se a trança identidade em $B_{m-k}(M)$ quando removemos o conjunto das primeiras $k$ cordas, assim como vira a trança trivial em $B_{k, m-k-1}(M)$ quando retiramos qualquer uma das últimas $m-k$ cordas. Observemos que se $k=1$, então

$$
\mathcal{S}^{1} \operatorname{Brun}_{m}(M)=\operatorname{Brun}_{m}(M)
$$

A seguir temos uma proposição que garante que, em geral, os grupos definidos até agora são grupos livres.

Proposição 4 (Proposição 2.2.7 (2)). Suponhamos que $m \geq 3$. Os grupos de tranças quase-Brunnianas e semi-Brunnianas de ordem $k$, com $m$ cordas, sobre uma superfície $M$ são grupos livres, exceto para $m=3$ e $M=\mathbb{S}^{2}$.

Durante esta parte da tese temos especial interesse nos grupos de tranças quaseBrunnianas, já que estes grupos estão relacionados com os grupos de homotopia da esfera, como veremos posteriormente. No seguinte resultado daremos uma descrição para o grupo de tranças quase-Brunnianas sobre superfícies.

Teorema 5 (Teorema 2.2.12). Seja $M$ uma superfície qualquer, vamos supor que $m \geq 3$ se $M \neq \mathbb{S}^{2}, \mathbb{R} P^{2}$, que $m \geq 4$ se $M=\mathbb{R} P^{2}$ e que $m \geq 5$ se $M=\mathbb{S}^{2}$. Então, para $1 \leq k \leq m-2$, vale que

$$
\mathcal{Q}^{k} \operatorname{Brun}_{m}(M)=\left[\left\langle A_{k+1, m}\right\rangle^{P},\left\langle A_{k+2, m}\right\rangle^{P}, \ldots,\left\langle A_{m-1, m}\right\rangle^{P}\right]_{S} .
$$

No caso $k=m-1$, para $m \geq 4$ se $M=\mathbb{S}^{2}$ e para $m \geq 3$ nos outros casos, temos que

$$
\mathcal{Q}^{m-1} \operatorname{Brun}_{m}(M) \cong \pi_{1}\left(M \backslash\left\{q_{1}, \ldots, q_{m-1}\right\}, q_{m}\right)
$$

O Teorema 5 junto com a Proposição 2.2.10 dão uma descrição completa dos grupos de tranças quase-Brunnianas sobre superfícies.

Na demonstração da Proposição 2.2.2 consideraremos a projeção que esquece as primeiras $k$ cordas, para $1 \leq k \leq m-1, \eta_{k}: B_{k, m-k}(M) \rightarrow B_{m-k}(M)$, e veremos que $\eta_{k}\left(\mathcal{Q}^{k} \operatorname{Brun}_{m}(M)\right) \subseteq \operatorname{Brun}_{m-k}(M)$. Portanto, podemos considerar a seguinte restrição de $\eta_{k}$, que por abuso de notação também denotaremos por

$$
\eta_{k}: \mathcal{Q}^{k} \operatorname{Brun}_{m}(M) \rightarrow \operatorname{Brun}_{m-k}(M)
$$

Com esta notação obtemos o primeiro resultado envolvendo grupos de tranças e grupos de homotopia.

Teorema 6 (Teorema 2.2.13). Sejam $k \geq 1$ e $m \geq 3$ e suponhamos que $k \leq m-5$ se $M=\mathbb{S}^{2}$, que $k \leq m-4$ se $M=\mathbb{R} P^{2}$ e $k \leq m-3$ nos outros casos. 
1. Se $M$ é uma superfície do tipo Eilenberg-Mac Lane, então $\operatorname{coKer}\left(\eta_{k}\right)=\{1\}$.

2. Se $M=\mathbb{R} P^{2}$ então, $\operatorname{coKer}\left(\eta_{k}\right) \cong \pi_{m-k-1}\left(\mathbb{S}^{2}\right)$.

3. Se $M=\mathbb{S}^{2}$ então, $\operatorname{coKer}\left(\eta_{k}\right) \cong \pi_{m-k-1}\left(\mathbb{S}^{2}\right)$.

Segue imediatamente do teorema anterior, usando as definições dos grupos envolvidos, o seguinte corolário.

Corolário 7 (Corolário 2.2.14). Consideremos o homomorfismo $\eta_{k}: B_{k, m-k}(M) \rightarrow B_{m-k}(M)$.

1. Se $1 \leq k \leq m-3$ e $M$ é uma superfície do tipo Eilenberg-Mac Lane, então a seguinte sequência é exata curta

$$
1 \longrightarrow \mathcal{S}^{k} \operatorname{Brun}_{m}(M) \longrightarrow \mathcal{Q}^{k} \operatorname{Brun}_{m}(M) \stackrel{\eta_{k}}{\longrightarrow} \operatorname{Brun}_{m-k}(M) \longrightarrow 1
$$

2. Se $1 \leq k \leq m-4$ e $M=\mathbb{R} P^{2}$, então a seguinte sequência é exata

$$
1 \rightarrow \mathcal{S}^{k} \operatorname{Brun}_{m}\left(\mathbb{R} P^{2}\right) \rightarrow \mathcal{Q}^{k} \operatorname{Brun}_{m}\left(\mathbb{R} P^{2}\right) \stackrel{\eta_{k}}{\rightarrow} \operatorname{Brun}_{m-k}\left(\mathbb{R} P^{2}\right) \rightarrow \pi_{m-k-1}\left(\mathbb{S}^{2}\right) \rightarrow 1
$$

3. Se $1 \leq k \leq m-5$ e $M=\mathbb{S}^{2}$, então a seguinte sequência é exata

$$
1 \longrightarrow \mathcal{S}^{k} \operatorname{Brun}_{m}\left(\mathbb{S}^{2}\right) \longrightarrow \mathcal{Q}^{k} \operatorname{Brun}_{m}\left(\mathbb{S}^{2}\right) \stackrel{\eta_{k}}{\longrightarrow} \operatorname{Brun}_{m-k}\left(\mathbb{S}^{2}\right) \longrightarrow \pi_{m-k-1}\left(\mathbb{S}^{2}\right) \longrightarrow 1
$$

Com a ajuda de um Teorema de J. Wu (Teorema 1.2.3) podemos relacionar tranças sobre qualquer superfície e os grupos de homotopia da esfera. Para isto será necessário uma certa construção, onde aparecem grupos $G_{n+1}(M)$ e $E_{n+1}(M)$, que é explicada na Seção 2.3. Destacamos que, em alguns casos, ainda é possível exibir tranças que se projetam em geradores dos grupos de homotopia baixos da esfera, os quais são livres de torção, ver as Observações 2.3.4.

Teorema 8 (Teorema 2.3.3). Suponhamos que $n \geq 4$ se $M=\mathbb{S}^{2}, n \geq 3$ se $M=\mathbb{R} P^{2}$ e que $n \geq 1$ nos outros casos. As seguintes sequências são exatas curtas.

1. Para $k=0$ :

$$
1 \longrightarrow G_{n+1}(M) \longrightarrow \mathcal{Q}^{1} \operatorname{Brun}_{n+1}(M) \cap E_{n+1}(M) \longrightarrow \pi_{n+\delta(g-1)+2}\left(\mathbb{S}^{2}\right) \longrightarrow 1
$$

2. Para $k \geq 1$ :

$$
1 \longrightarrow G_{n+1}(M) \longrightarrow \operatorname{Brun}_{n+1}(M) \cap E_{n+1}(M) \longrightarrow \pi_{n+\delta(g-1)+k+2}\left(\mathbb{S}^{2}\right) \longrightarrow 1 .
$$


Gostaríamos de destacar que o Teorema 8 estende a qualquer superfície alguns resultados já conhecidos na literatura sobre a conexão entre os grupos de tranças sobre a esfera, o disco e o plano projetivo com os grupos de homotopia da esfera, ver Observação 2.3.4.

No Lema 2.4.1 provaremos que para $n \geq 3$ existe um monomorfismo

$$
\psi_{n+1}: \operatorname{Ker}\left(\delta_{n+1}: P_{n+1}\left(\mathbb{S}^{2}\right) \rightarrow P_{n}\left(\mathbb{S}^{2}\right)\right) \rightarrow P_{n}\left(\mathbb{D}^{2}\right)
$$

e com este monomorfismo provaremos o seguinte teorema.

Teorema 9 (Teorema 2.4.4). Para $n \geq 4$ o homomorfismo

$$
\psi_{n+1}: \operatorname{Brun}_{n+1}\left(\mathbb{S}^{2}\right) \rightarrow \operatorname{Brun}_{n}\left(\mathbb{D}^{2}\right) \cap \operatorname{Ker}\left(f_{*}\right)
$$

é um isomorfismo. Em particular, $\psi_{n+1}:$ Brun $_{n+1}\left(\mathbb{S}^{2}\right) \rightarrow$ Brun $_{n}\left(\mathbb{D}^{2}\right)$ é um homomorfismo injetor, para $n \geq 4$.

Na Subseção 2.4.2 daremos uma prova diferente do Teorema 6.6.5 de [9], usando os Teoremas 8, 9. O Teorema 9 também será usado na Subseção 2.4.3 para dar uma prova diferente do isomorfismo descrito no item (1) do Teorema 1 de Li e Wu em [58], que está formulado no Teorema 1.3.25.

\section{Capítulo 3}

Neste capítulo estamos interessados principalmente em calcular os grupos de homotopia de Moore dos $\Delta$-grupos $\mathcal{P}(M)=\left\{P_{n+1}(M)\right\}_{n \geq 0}$ formados por grupos de tranças puras sobre qualquer superfície. A estrutura simplicial dos grupos de tranças é dada no Exemplo 1.3.2.

Tomando $k=1$ no Teorema 6 , obtemos o seguinte resultado que descreve os grupos de homotopia superiores do $\Delta$-grupo formado por grupos de tranças de superfície.

Teorema 10 (Teorema 3.1.8).

1. Sejam $n \geq 2$ e $M$ uma superfície diferente da esfera $\mathbb{S}^{2}$ e do plano projetivo $\mathbb{R} P^{2}$. Então,

$$
\pi_{n}(\mathcal{P}(M))=\{1\}
$$

2. Suponhamos que $n \geq 4$ se $M=\mathbb{S}^{2}$ e que $n \geq 3$ se $M=\mathbb{R} P^{2}$. Então,

$$
\pi_{n}(\mathcal{P}(M))=\pi_{n}\left(\mathbb{S}^{2}\right)
$$

No Teorema 3.1.6 são calculados os grupos de homotopia inferiores dos $\Delta$-grupos formados por grupos de tranças puras sobre superfícies. Desta forma, o Teorema 3.1.6 junto 
com o Teorema 10 fornecem uma descrição completa para $\pi_{n}(\mathcal{P}(M))$ com $M$ e $n$ quaisquer. É de nosso conhecimento que os Teoremas 3.1.6 e 10 também foram demonstrados por J. Berrick, L. Hanbury e J. Wu, e apareceram num artigo que será publicado em breve [10]. No entanto, as técnicas usadas por eles em [10] são diferentes das nossas.

A seguir mostramos outras sequências exatas curtas de tranças e grupos de homotopia da esfera $\mathbb{S}^{2}$. Um resultado similar foi obtido no Corolário 7 e é usado na prova do Teorema 11.

Teorema 11 (Teorema 3.3.3). Vamos supor que $k \geq 3$ se $M=\mathbb{S}^{2}$, que $k \geq 2$ se $M=\mathbb{R} P^{2}$, e que $k \geq 1$ nos outros casos. Seja $Q_{k}$ um conjunto de $k$ pontos no interior de $M$.

1. Para todo $n \geq 3$ e $M \neq \mathbb{S}^{2}, \mathbb{R} P^{2}$ a seguinte sequência é exata curta

$$
1 \longrightarrow \mathcal{S}^{k} \operatorname{Brun}_{k+n}(M) \longrightarrow \operatorname{Brun}_{n}\left(M \backslash Q_{k}\right) \stackrel{f_{*}}{\longrightarrow} \operatorname{Brun}_{n}(M) \longrightarrow 1
$$

2. Para todo $n \geq 5$ a seguinte sequência é exata

$$
1 \longrightarrow \mathcal{S}^{k} \operatorname{Brun}_{k+n}\left(\mathbb{S}^{2}\right) \longrightarrow \operatorname{Brun}_{n}\left(\mathbb{S}^{2} \backslash Q_{k}\right) \stackrel{f_{*}}{\longrightarrow} \operatorname{Brun}_{n}\left(\mathbb{S}^{2}\right) \longrightarrow \pi_{n-1}\left(\mathbb{S}^{2}\right) \longrightarrow 1
$$

3. Para todo $n \geq 4$ a seguinte sequência é exata

$$
1 \rightarrow \mathcal{S}^{k} \operatorname{Brun}_{k+n}\left(\mathbb{R} P^{2}\right) \longrightarrow \operatorname{Brun}_{n}\left(\mathbb{R} P^{2} \backslash Q_{k}\right) \stackrel{f_{*}}{\rightarrow} \operatorname{Brun}_{n}\left(\mathbb{R} P^{2}\right) \rightarrow \pi_{n-1}\left(\mathbb{S}^{2}\right) \longrightarrow 1 .
$$

Na seguinte proposição faremos uso do homomorfismo $\beta: F_{3}\left(\widehat{z_{0}}, \widehat{z_{1}}, \widehat{z_{2}}\right) \rightarrow P_{4}\left(\mathbb{S}^{2}\right)$, definido no parágrafo anterior à Proposição 6.1 .5 (ver também [9, final da página 313]), bem como sua definição geral para qualquer número de cordas, indicada nas linhas 9-10 da página 314 de [9]. O motivo pelo qual damos o exemplo na Proposição 12, usando a aplicação $\beta$, é de contornar uma imprecisão detectada na Seção 7.4 de [9], a qual é explicada na Seção 6.1.3 deste texto.

Proposição 12 (Proposição 3.4.1). Seja $\beta_{5} \in P_{5}\left(\mathbb{S}^{2}\right)$ o elemento dado por

$$
\beta_{5}=\left[\left[D_{1,2} D_{1,3}, D_{1,4}\right],\left[D_{1,2}, D_{1,3} D_{1,4}\right]\right]
$$

Então, $\beta_{5}=\beta\left(\left[\left[\hat{z}_{1} \hat{z}_{0}, \hat{z}_{0}^{-1} \hat{z}_{1}^{-1} \hat{z}_{2} \hat{z}_{1} \hat{z}_{0}\right],\left[\hat{z}_{0}, \hat{z}_{0}^{-1} \hat{z}_{2} \hat{z}_{1} \hat{z}_{0}\right]\right]\right)$. Além disso, $\beta_{5}$ é uma trança Brunniana com 5 cordas na esfera, $\beta_{5} \in \operatorname{Brun}_{5}\left(\mathbb{S}^{2}\right)$, porém $\beta_{5} \notin f_{*}\left(\operatorname{Brun}_{5}\left(\mathbb{D}^{2}\right)\right)$.

\section{Capítulo 4}

No Capítulo 4 trataremos inicialmente resultados gerais da teoria de homotopia simplicial com o intuito de descrever de diferentes formas os grupos de homotopia de Moore 
de $\Delta$-grupos (ou de grupos simpliciais). Começaremos com a nossa versão do Teorema de Extensão Central em teoria de homotopia simplicial. Uma outra versão com hipóteses diferentes, porém, com uma conclusão similar à apresentada aqui, foi dada no Teorema 3.8 do capítulo intitulado "Simplicial Objects and Homotopy Groups" do livro [8]. Gostaríamos de mencionar que muitas das demonstrações do Capítulo 4 têm idéias inspiradas em [58]. Lembremos que denotamos o centro de um grupo $G$ por $Z(G)$.

Teorema 13 (Teorema de Extensão Central, Teorema 4.1.1). Sejam $\mathcal{G}=\left\{G_{n}\right\}_{n \geq 0}$ uma sequência de grupos qualquer e $\mathcal{H}=\left\{H_{n}\right\}_{n \geq 0}$ um $\Delta$-grupo. Suponhamos que para algum $n \geq 0$ temos que $H_{n} \leq G_{n+1}, H_{n+1} \leq G_{n+2}$ e as seguintes condições são satisfeitas:

i) $\mathcal{H}$ é fibrante.

ii) $\mathcal{B} d_{n} \mathcal{H}$ é um subgrupo normal de $G_{n+1}$.

iii) Se $X$ é um conjunto de geradores de $G_{n+1}$, então para todo elemento $\alpha \in X$, existem $0 \leq i_{0} \leq n$ e uma aplicação $\widehat{d}_{i_{0}}: G_{n+2} \rightarrow G_{n+1}$ tais que $\left.\widehat{d}_{i_{0}}\right|_{H_{n+1}}=d_{i_{0}}$ e

$$
\widehat{d}_{i_{0}}^{-1}(\alpha) \cap \operatorname{Ker}\left(d_{i_{0}+1}: H_{n+1} \rightarrow H_{n}\right)
$$

não é o conjunto vazio, nem o grupo trivial.

Então, o grupo $\pi_{n}(\mathcal{H})$ está contido no centro de $G_{n+1} / \mathcal{B} d_{n} \mathcal{H}$.

Precisaremos do seguinte lema para enunciar o resultado do Teorema 15.

Lema 14 (Lema 4.1.3). Seja $\mathcal{G}=\left\{G_{n} ; \delta_{n+1}\right\}_{n \geq 0}$ uma sequência de grupos e homomorfismos $\delta_{n+1}: G_{n+1} \rightarrow G_{n}$ e denotemos por $H_{n}=\operatorname{Ker}\left(\delta_{n+1}\right)$. Suponhamos que a coleção de subgrupos $\mathcal{H}=\left\{H_{n}\right\}_{n \geq 0}$ é um $\Delta$-grupo e que para algum $n \geq 0$ as seguintes condições são verificadas.

i) Os homomorfismos $\delta_{n+1}: G_{n+1} \rightarrow G_{n}$ e $\delta_{n+1} \mid: Z\left(G_{n+1}\right) \rightarrow Z\left(G_{n}\right)$ são sobrejetores.

ii) $\mathcal{B} d_{n} \mathcal{H}$ é um subgrupo normal de $G_{n+1}$.

iii) $Z\left(H_{n} / \mathcal{B} d_{n} \mathcal{H}\right) \cap A_{n+1}=\{1\}$, onde $A_{n+1}$ é a projeção de $Z\left(G_{n+1}\right)$ em $G_{n+1} / \mathcal{B} d_{n} \mathcal{H}$.

Então, $Z\left(\frac{G_{n+1}}{\mathcal{B} d_{n} \mathcal{H}}\right)$ está contido no produto direto interno de $Z\left(\frac{H_{n}}{\mathcal{B} d_{n} \mathcal{H}}\right)$ com $A_{n+1}$. Ainda mais, se $\mathcal{H}$ é um grupo simplicial e $Z\left(H_{n-1}\right)=\{1\}$, então $Z\left(\frac{G_{n+1}}{\mathcal{B} d_{n} \mathcal{H}}\right)$ está contido no produto direto interno de $\pi_{n}(\mathcal{H})$ com $A_{n+1}$.

Com as hipóteses do Teorema 13 e do Lema 14 obtemos o seguinte resultado. 
Teorema 15 (Teorema 4.1.5). Seja $\mathcal{G}=\left\{G_{n} ; \delta_{n+1}\right\}_{n \geq 0}$ uma sequência de grupos e homomorfismos $\delta_{n+1}: G_{n+1} \rightarrow G_{n}$ e denotemos por $H_{n}=\operatorname{Ker}\left(\delta_{n+1}\right)$. Suponhamos que $\mathcal{H}=\left\{H_{n}\right\}_{n \geq 0}$ é um grupo simplicial tal que, para algum $n \geq 1$, as condições $\left.i\right)$, ii) $e$ iii) do Lema 14 e a condição iii) do Teorema 13 são todas satisfeitas. Vamos supor também que $H_{n-1}$ tem centro trivial, $Z\left(H_{n-1}\right)=\{1\}$. Então,

$$
Z\left(G_{n+1} / \mathcal{B} d_{n} \mathcal{H}\right) \cong \pi_{n}(\mathcal{H}) \times A_{n+1}
$$

onde $A_{n+1}=Z\left(G_{n+1}\right) / \mathcal{B} d_{n} \mathcal{H}$.

A razão pela qual chamamos o teorema abaixo de Teorema de Decomposição vem do fato que o Teorema 3.27 do capítulo intitulado "Simplicial objects and homotopy groups" do livro [8] é um resultado similar ao nosso, e chamado de "Decomposition Theorem".

Teorema 16 (Teorema de Decomposição, Teorema 4.1.7). Seja $\mathcal{H}$ um $\Delta$-grupo fibrante tal que, para algum $n \geq 1$, valem as seguintes afirmações.

1. $\mathcal{B} d_{n-1} \mathcal{H}$ é um grupo livre e $\mathcal{B} d_{n} \mathcal{H}$ é um subgrupo livre e normal em $H_{n}$.

2. Se $X$ é um conjunto de geradores de $H_{n}$, então para todo elemento $\alpha$ de $X$, existe $0 \leq i_{0} \leq n$ tal que $d_{i_{0}}^{-1}(\alpha) \cap \operatorname{Ker}\left(d_{i_{0}+1}: H_{n+1} \rightarrow H_{n}\right)$ não é o conjunto vazio, nem o grupo trivial.

Então,

$$
\mathcal{N}_{n} \mathcal{H} / \mathcal{B} d_{n} \mathcal{H} \cong \pi_{n}(\mathcal{H}) \times \mathcal{B} d_{n-1} \mathcal{H}
$$

Depois de formular o Teorema de Decomposição vamos obter algumas consequências do mesmo, as quais serão aplicadas para alguns grupos simpliciais formados por tranças sobre o disco ou a esfera.

$\mathrm{Na}$ Seção 4.2 vamos construir três grupos simpliciais específicos. O primeiro deles, $\mathcal{K}$, é formado pela sequência de grupos dada pelo grupo fundamental do disco perfurado; o segundo deles, $\mathcal{K}\left(\mathbb{D}^{2}\right)$, é formado por subgrupos de tranças sobre o disco (ver Subseção 4.2.1) e o terceiro, $\mathcal{K}\left(\mathbb{S}^{2}\right)$, por subgrupos de tranças sobre a esfera (ver Subseção 4.2.2). Para esta construção de grupos simpliciais foi usado o Lema 4.2.1.

O seguinte teorema, que é um dos resultados principais deste capítulo, estabelece uma nova conexão entre os grupos de tranças sobre superfícies e os grupos de homotopia da esfera $\mathbb{S}^{2}$. Gostaríamos de observar que o primeiro isomorfismo do Teorema 17 já era conhecido, pois no Teorema 1 de [58] foi obtido o isomorfismo $Z\left(\frac{P_{n+1}}{B d_{n}}\right) \cong \pi_{n+1}\left(\mathbb{S}^{2}\right) \times \mathbb{Z}$ para $n \geq 3$, ver Teorema 1.3.25.

Teorema 17 (Teorema 4.3.4). Seja $n \geq 2$, existem isomorfismos:

1. $Z\left(\frac{P_{n+1}}{\mathcal{B} d_{n} \mathcal{K}\left(\mathbb{D}^{2}\right)}\right) \cong \pi_{n+1}\left(\mathbb{S}^{2}\right) \times \mathbb{Z}$. 
2. $Z\left(\frac{P_{n+2}\left(\mathbb{S}^{2}\right)}{\mathcal{B} d_{n} \mathcal{K}\left(\mathbb{S}^{2}\right)}\right) \cong \pi_{n+1}\left(\mathbb{S}^{2}\right) \times \mathbb{Z}_{2}$

Nos seguintes dois teoremas aplicamos o Teorema de Decomposição (Teorema 16), e seus corolários, aos grupos simpliciais $\mathcal{K}\left(\mathbb{D}^{2}\right)$ e $\mathcal{K}\left(\mathbb{S}^{2}\right)$, respectivamente.

Teorema 18 (Teorema 4.3.5).

1. Seja $n \geq 2$. Existe uma decomposição

$$
\frac{\operatorname{Brun}_{n+1}\left(\mathbb{D}^{2}\right)}{\left[\left\langle A_{0, n+1}\right\rangle^{P_{n+1}}, \ldots,\left\langle A_{n, n+1}\right\rangle^{P_{n+1}}\right]_{S}} \cong \pi_{n+1}\left(\mathbb{S}^{2}\right) \times\left[\left\langle A_{0, n}\right\rangle^{P_{n}}, \ldots,\left\langle A_{n-1, n}\right\rangle^{P_{n}}\right]_{S}
$$

Portanto, para $n \geq 3$,

$$
\operatorname{Tor}\left(\frac{\operatorname{Brun}_{n+1}\left(\mathbb{D}^{2}\right)}{\left[\left\langle A_{0, n+1}\right\rangle^{P_{n+1}}, \ldots,\left\langle A_{n, n+1}\right\rangle^{P_{n+1}}\right]_{S}}\right) \cong \pi_{n+1}\left(\mathbb{S}^{2}\right) .
$$

2. Seja $n \geq 1$. Existe uma decomposição

$$
H_{n}\left(\left(N\left(\mathcal{K}\left(\mathbb{D}^{2}\right)\right)\right)_{a b}\right) \cong \pi_{n+1}\left(\mathbb{S}^{2}\right) \oplus A_{n}
$$

para cada $n$, onde $A_{n}$ é livre de torção.

3. Seja $n \geq 1$. Existe uma decomposição

$$
H_{n}\left(\mathbb{Z}\left(N\left(\mathcal{K}\left(\mathbb{D}^{2}\right)\right)\right)\right) \cong \pi_{n+1}\left(\mathbb{S}^{2}\right) \oplus C_{n}
$$

para algum grupo $C_{n}$.

4. Seja $n \geq 3$. Existe um isomorfismo de grupos

$$
\operatorname{Tor}\left(H_{n}\left(\left(N\left(\mathcal{K}\left(\mathbb{D}^{2}\right)\right)\right)_{a b}\right)\right) \cong \pi_{n+1}\left(\mathbb{S}^{2}\right)
$$

Teorema 19 (Teorema 4.3.6).

1. Seja $n \geq 2$. Existe uma decomposição

$$
\frac{\mathcal{Q}^{1} \operatorname{Brun}_{n+2}\left(\mathbb{S}^{2}\right)}{\left[\left\langle A_{1, n+2}\right\rangle^{P_{n+2}\left(\mathbb{S}^{2}\right)}, \ldots,\left\langle A_{n+1, n+2}\right\rangle^{P_{n+2}\left(\mathbb{S}^{2}\right)}\right]_{S}} \cong \pi_{n+1}\left(\mathbb{S}^{2}\right) \times \mathcal{B} d_{n-1} \mathcal{K}\left(\mathbb{S}^{2}\right)
$$

onde $\mathcal{B} d_{n-1} \mathcal{K}\left(\mathbb{S}^{2}\right)=\left[\left\langle A_{1, n+1}\right\rangle^{P_{n+1}\left(\mathbb{S}^{2}\right)}, \ldots,\left\langle A_{n, n+1}\right\rangle^{P_{n+1}\left(\mathbb{S}^{2}\right)}\right]_{S}$.

Portanto, para $n \geq 3$, temos que

$$
\operatorname{Tor}\left(\frac{\mathcal{Q}^{1} \operatorname{Brun}_{n+2}\left(\mathbb{S}^{2}\right)}{\left[\left\langle A_{1, n+2}\right\rangle^{P_{n+2}\left(\mathbb{S}^{2}\right)}, \ldots,\left\langle A_{n+1, n+2}\right\rangle^{P_{n+2}\left(\mathbb{S}^{2}\right)}\right]_{S}}\right) \cong \pi_{n+1}\left(\mathbb{S}^{2}\right) .
$$


2. Seja $n \geq 1$. Existe uma decomposição

$$
H_{n}\left(\left(N\left(\mathcal{K}\left(\mathbb{S}^{2}\right)\right)\right)_{a b}\right) \cong \pi_{n+1}\left(\mathbb{S}^{2}\right) \oplus A_{n},
$$

para cada $n$, onde $A_{n}$ é livre de torção.

3. Seja $n \geq 1$. Existe uma decomposição

$$
H_{n}\left(\mathbb{Z}\left(N\left(\mathcal{K}\left(\mathbb{S}^{2}\right)\right)\right)\right) \cong \pi_{n+1}\left(\mathbb{S}^{2}\right) \oplus C_{n}
$$

para algum grupo $C_{n}$.

4. Seja $n \geq 3$. Existe um isomorfismo de grupos

$$
\operatorname{Tor}\left(H_{n}\left(\left(N\left(\mathcal{K}\left(\mathbb{S}^{2}\right)\right)\right)_{a b}\right)\right) \cong \pi_{n+1}\left(\mathbb{S}^{2}\right)
$$

No estudo dos grupos Brunnianos, tentando entender sua estrutura, obtivemos a Proposição 20, que foi o ponto de partida para estabelecer a conexão entre grupos de tranças sobre o disco e grupos cristalográficos, que aparece no Capítulo 5.

Proposição 20 (Proposição 4.3.10).

1. O grupo $B_{3} /$ Brun $_{3}\left(\mathbb{D}^{2}\right)$ tem uma apresentação dada pelos geradores $\sigma_{1}, \sigma_{2}$ e as relações: $\sigma_{1} \sigma_{2} \sigma_{1}=\sigma_{2} \sigma_{1} \sigma_{2}$ e $\left(\sigma_{1}^{-1} \sigma_{2}\right)^{3}=1$.

2. $P_{3} / \operatorname{Brun}_{3}\left(\mathbb{D}^{2}\right) \cong \mathbb{Z} \oplus \mathbb{Z} \oplus \mathbb{Z}$.

Para finalizar o Capítulo 4 damos uma prova do Teorema 1 de [58], diferente daquela dada pelos autores em [58]. Notemos que o enunciado proposto a seguir aparenta ser um pouco diferente do original, no entanto, se temos em conta a igualdade de grupos $\mathcal{B} d_{n-1} \mathcal{K}\left(\mathbb{D}^{2}\right)=B d_{n} \mathbb{P}$, vemos que o enunciado original e o enunciado abaixo são iguais.

Teorema 21 (Teorema 1 de [58], Teorema 4.3.12). Seja $n \geq 4$.

1. A seguinte igualdade de grupos é válida $\sqrt{\left(\mathcal{B} d_{n-1} \mathcal{K}\left(\mathbb{D}^{2}\right), P_{n}\right)}=\operatorname{Brun}_{n} \cap \theta\left(\right.$ Brun $\left._{n}\right)$ e existe um isomorfismo de grupos $\sqrt{\left(\mathcal{B} d_{n-1} \mathcal{K}\left(\mathbb{D}^{2}\right), P_{n}\right)} / \mathcal{B} d_{n-1} \mathcal{K}\left(\mathbb{D}^{2}\right) \cong \pi_{n}\left(\mathbb{S}^{2}\right)$.

2. Existem isomorfismos de grupos

(a) $Z\left(P_{n} / \mathcal{B} d_{n-1} \mathcal{K}\left(\mathbb{D}^{2}\right)\right) \cong \pi_{n}\left(\mathbb{S}^{2}\right) \times \mathbb{Z}$,

(b) $Z\left(B_{n} / \mathcal{B} d_{n-1} \mathcal{K}\left(\mathbb{D}^{2}\right)\right) \cong\left\{\alpha \in \pi_{n}\left(\mathbb{S}^{2}\right) \mid 2 \alpha=0\right\} \times \mathbb{Z}$. 


\section{Capítulo 5}

No Capítulo 5 estudaremos a conexão entre os grupos de tranças do disco e os grupos cristalográficos. As principais definições relacionadas aos grupos cristalográficos e de Bieberbach, bem como os Teoremas de Bieberbach, podem ser encontradas na Seção 5.1.

Daremos, no Lema 5.1.12, uma caracterização algébrica dos grupos cristalográficos, a qual será usada no Capítulo 5. Este resultado parece ser conhecido pelos especialistas na área. No entanto, dado que não a encontramos na literatura, decidimos dar uma prova desta caracterização.

Pela Proposição 20 sabemos que $P_{3} / \operatorname{Brun}_{3}\left(\mathbb{D}^{2}\right) \cong \mathbb{Z} \oplus \mathbb{Z} \oplus \mathbb{Z}$. Consideraremos a sequência exata curta

$$
1 \longrightarrow P_{3} / \operatorname{Brun}_{3}\left(\mathbb{D}^{2}\right) \longrightarrow B_{3} / \operatorname{Brun}_{3}\left(\mathbb{D}^{2}\right) \stackrel{\bar{\sigma}}{\longrightarrow} \Sigma_{3} \longrightarrow 1
$$

a qual foi obtida a partir da sequência exata curta

$$
1 \longrightarrow P_{3} \longrightarrow B_{3} \stackrel{\sigma}{\longrightarrow} \Sigma_{3} \longrightarrow 1
$$

Teorema 22 (Teorema 5.2.1). A representação integral $\Theta: \Sigma_{3} \rightarrow \operatorname{Aut}\left(P_{3} / \operatorname{Brun}_{3}\left(\mathbb{D}^{2}\right)\right)$, obtida a partir da sequência exata curta (4), é fiel. Como consequência temos que o grupo quociente $B_{3} /$ Brun $_{3}\left(\mathbb{D}^{2}\right)$ é um grupo cristalográfico de dimensão 3 com grupo de holonomia $\Sigma_{3}$.

Observação 1. Pelo Corolário 2.1.4 temos que $\left[P_{3}, P_{3}\right]=$ Brun $_{3}\left(\mathbb{D}^{2}\right)$. Portanto, o grupo quociente $B_{3} /$ Brun $_{3}\left(\mathbb{D}^{2}\right)=B_{3} /\left[P_{3}, P_{3}\right]$ é um grupo cristalográfico de dimensão 3 , com grupo de holonomia $\Sigma_{3}$.

Um corolário do Teorema 22 garante a existência de grupos cristalográficos de dimensão 3, com grupo de holonomia contido em $\Sigma_{3}$, e obtidos a partir dos grupos de tranças com 3 cordas sobre o disco.

Corolário 23 (Corolário 5.2.4). Seja $H$ um subgrupo qualquer de $\Sigma_{3}$. Então, o grupo

$$
\frac{\sigma^{-1}(H)}{\operatorname{Brun}_{3}\left(\mathbb{D}^{2}\right)}
$$

é um grupo cristalográfico de dimensão 3 e com grupo de holonomia $H$, onde $\sigma$ é o epimorfismo dado na sequência exata curta (5).

Definição 24 (Definição 5.2.5). Seja $H$ um subgrupo de $\Sigma_{3}$, denotemos o grupo dado em (6), do Corolário 23, por $\mathcal{B C G}(3, H)$ e o chamemos de grupo cristalográfico de tranças com 3 cordas e grupo de holonomia H. Pelo Corolário 23, $\mathcal{B C G}(3, H)$ tem dimensão 3.

No seguinte resultado caracterizamos os elementos de ordem 3 no grupo $B_{3} / \operatorname{Brun}_{3}\left(\mathbb{D}^{2}\right)$. 
Teorema 25 (Teorema 5.2.10).

1. Um elemento $\vartheta$ em $B_{3} / \operatorname{Brun}_{3}\left(\mathbb{D}^{2}\right)$ tem ordem 3 se, e somente se, $\vartheta$ é da forma $\delta_{3}^{i} N_{1,2}^{r} N_{2,3}^{s} N_{1,3}^{t}$ com $i=1,2$ e $r+s+t=0$.

2. Sejam $N_{1,2}^{m}, N_{2,3}^{n}, N_{1,3}^{l} \in P_{3} / \operatorname{Brun}_{3}\left(\mathbb{D}^{2}\right)$. Então,

(a) $N_{1,2}^{m} N_{2,3}^{n} N_{1,3}^{l} \tau_{1}\left(\delta_{3}\right) \tau_{1}^{-1} N_{1,3}^{-l} N_{2,3}^{-n} N_{1,2}^{-m}=\delta_{3}^{2}$ se, e somente se, $m=n$ e $l=m+1$.

(b) $N_{1,2}^{m} N_{2,3}^{n} N_{1,3}^{l}\left(\delta_{3} N_{1,2}^{r} N_{2,3}^{s} N_{1,3}^{t}\right) N_{1,3}^{-l} N_{2,3}^{-n} N_{1,2}^{-m}=\delta_{3}$ se, e somente se, $m=n+r$, $n=l+s$ e $l=m+t$.

(c) $N_{1,2}^{m} N_{2,3}^{n} N_{1,3}^{l}\left(\delta_{3}^{2} N_{1,2}^{r} N_{2,3}^{s} N_{1,3}^{t}\right) N_{1,3}^{-l} N_{2,3}^{-n} N_{1,2}^{-m}=\delta_{3}^{2}$ se, e somente se, $m=l+r$, $n=m+s$ el $l=n+t$.

No Teorema 25 caracterizamos os elementos de ordem 3 no grupo cristalográfico de tranças com 3 cordas e grupo de holonomia $\Sigma_{3}, B_{3} / \operatorname{Brun}_{3}\left(\mathbb{D}^{2}\right)$, no seguinte resultado veremos que estes elementos formam uma única classe de conjugação.

Corolário 26 (Corolário 5.2.11). Existe uma única classe de conjugação de elementos de ordem 3 em $B_{3} / \operatorname{Brun}_{3}\left(\mathbb{D}^{2}\right)$.

Até agora estudamos apenas a torção 3 no grupo $B_{3} / \operatorname{Brun}_{3}\left(\mathbb{D}^{2}\right)$, caracterizando os elementos de ordem 3 no Teorema 25 e mostrando que eles formam uma única classe de conjugação no Corolário 26. O seguinte resultado garante que esta é a única possibilidade de torção neste grupo.

Teorema 27 (Teorema 5.2.12). O grupo $B_{3} /$ Brun $_{3}\left(\mathbb{D}^{2}\right)$ não tem elementos de torção 2.

A seguir veremos que a variedade plana $X$ determinada pelo grupo $\mathcal{B C G}\left(3,\left\langle\mu_{1}\right\rangle\right)$ é uma variedade de Seifert não orientável.

Teorema 28 (Teorema 5.2.13). O grupo $\mathcal{B C G}\left(3,\left\langle\mu_{1}\right\rangle\right)$ é um grupo de Bieberbach de dimensão 3, com grupo de holonomia $\mathbb{Z}_{2}$. A variedade plana $X$ unicamente determinada por $\mathcal{B C G}\left(3,\left\langle\mu_{1}\right\rangle\right)$ é não orientável e com primeiro grupo de homologia $H_{1}(X ; \mathbb{Z}) \cong \mathbb{Z} \oplus \mathbb{Z}$. A menos de difeomorfismo afim, $X$ é igual à $\mathscr{B}_{2}$ da classificação das 3-variedades planas dada na Seção 3.5 de [95]. Ainda mais, a variedade plana não orientável $X$ é o espaço total de um fibrado sobre o círculo, $F \rightarrow X \rightarrow \mathbb{S}^{1}$, onde a fibra $F$ pode ser o toro ou a garrafa de Klein.

Uma vez feito o estudo dos grupos de tranças com 3 cordas sobre o disco e sua interação com os grupos cristalográficos, passamos a estudar o caso geral. A partir da sequência exata curta

$$
1 \longrightarrow P_{n}(M) /\left[P_{n}, P_{n}\right] \longrightarrow B_{n}(M) /\left[P_{n}, P_{n}\right] \longrightarrow \Sigma_{n} \longrightarrow 1
$$


obtemos uma representação integral $\Sigma_{n} \rightarrow \operatorname{Aut}\left(P_{n}(M) /\left[P_{n}, P_{n}\right]\right)$, dada por conjugação. O seguinte interessante resultado, que é um dos principais teoremas do Capítulo 5, relacionando os grupos de tranças sobre o disco com a teoria dos grupos cristalográficos será provado na Seção 5.3.

Teorema 29 (Teorema 5.3.1). Sejam $n \geq 3$ e $M=\mathbb{D}^{2}$. A representação obtida a partir da sequência exata curta (7),

$$
\Theta_{n}: \Sigma_{n} \rightarrow \operatorname{Aut}\left(P_{n} /\left[P_{n}, P_{n}\right]\right)
$$

é injetora. Como consequência temos que o grupo quociente $B_{n} /\left[P_{n}, P_{n}\right]$ é um grupo cristalográfico de dimensão $n(n-1) / 2$ e grupo de holonomia $\Sigma_{n}$.

O corolário abaixo garante que qualquer grupo finito é o grupo de holonomia de um grupo cristalográfico, este último obtido a partir dos grupos de tranças do disco.

Corolário 30 (Corolário 5.3.3). Seja $H$ um grupo finito qualquer. Existe $n \geq 3$ tal que $H$ é um subgrupo de $\Sigma_{n}$ e nesse caso o grupo

$$
\frac{\sigma^{-1}(H)}{\left[P_{n}, P_{n}\right]}
$$

é um grupo cristalográfico de dimensão $n(n-1) / 2$ com grupo de holonomia $H$, onde $\sigma e ́$ o epimorfismo dado na sequência exata curta (5.43).

Dado um grupo finito $H$, como no Corolário 30, existe $n \geq 3$ tal que $H$ é um subgrupo de $\Sigma_{n}$. Denotemos o grupo dado em (8) por $\mathcal{B C G}(n, H)$ e o chamemos de grupo cristalográfico de tranças com $n$ cordas e grupo de holonomia $H$.

Observação 2. O Corolário 30 é uma versão similar do Teorema de Auslander e Kuranishi, formulado no Teorema 5.1.8.

Como feito no caso de 3 cordas, queremos estudar os elementos de torção do grupo cristalográfico $B_{n} /\left[P_{n}, P_{n}\right]$. A seguir escrevemos os avanços conseguidos nessa direção.

Teorema 31 (Teorema 5.3.6). Seja $n \geq 3$, então o grupo quociente $B_{n} /\left[P_{n}, P_{n}\right]$ não tem elementos de ordem 2.

Teorema 32 (Teorema 5.3.11). Seja $n \geq 3$ um número ímpar. Então, $B_{n} /\left[P_{n}, P_{n}\right]$ tem elementos de ordem $n$.

Com o Teorema 32 podemos demonstrar que se $m$ é um inteiro maior ou igual que $n$ e $n \geq 3$, então o grupo quociente $B_{m} /\left[P_{m}, P_{m}\right]$ tem elementos de ordem ímpar, para qualquer número ímpar menor ou igual que $m$.

Teorema 33. Sejam $m$ e $n$ dois inteiros quaisquer tais que $1 \leq n \leq m$. 
1. Consideremos o homomorfismo $\iota: B_{n} \rightarrow B_{m}$ definido por $\sigma_{i} \mapsto \sigma_{i}$, para todo $1 \leq i \leq n-1$, e a projeção natural deste homomorfismo no grupo quociente $\bar{\iota}: B_{n} /\left[P_{n}, P_{n}\right] \rightarrow B_{m} /\left[P_{m}, P_{m}\right]$. Então, o homomorfismo $\bar{\iota}$ é injetor.

2. Se $n \geq 3$ e impar, então $B_{m} /\left[P_{m}, P_{m}\right]$ tem elementos de ordem $n$.

Já vimos que, para $n \geq 3$, o grupo quociente $B_{n} /\left[P_{n}, P_{n}\right]$ não tem elementos de torção 2. Agora estamos interessados em subgrupos do grupo cristalográfico $B_{n} /\left[P_{n}, P_{n}\right]$ que são livres de torção, isto é, que são grupos de Bieberbach.

Lema 34 (Lema 5.4.1). Sejam $n \geq 3$ e $H$ um subgrupo de $\Sigma_{n}$. Se $H$ é um 2-grupo finito, então $\mathcal{B C G}(n, H)$ é um grupo de Bieberbach de dimensão $n(n-1) / 2$ que é a dimensão do grupo cristalográfico $B_{n} /\left[P_{n}, P_{n}\right]$.

Veremos na continuação o problema de realização de 2-grupos finitos como grupos de holonomia de grupos de Bieberbach que são subgrupos de $B_{n} /\left[P_{n}, P_{n}\right]$, para algum $n \geq 3$.

Teorema 35 (Teorema 5.4.2). Seja $H$ um 2-grupo finito, então existem $n \geq 3$ e uma variedade plana $M$, de dimensão $n(n-1) / 2$, tal que $H$ é o grupo de holonomia de $M$. Ainda mais, o grupo fundamental de $M$ é um grupo de Bieberbach que é um subgrupo de $B_{n} /\left[P_{n}, P_{n}\right]$.

O Teorema 35 é uma reformulação do Teorema de Auslander e Kuranishi (Teorema 5.1.8) para 2-grupos finitos, via o grupo quociente $B_{n} /\left[P_{n}, P_{n}\right]$.

A seguir aplicamos o Lema 34 tomando $n=4$.

Teorema 36 (Teorema 5.4.3). Sejam $H_{1}=\mathbb{Z}_{4}, H_{2}=\mathbb{Z}_{2} \oplus \mathbb{Z}_{2}$ e $H_{3}$ igual ao grupo diedral (de ordem 8). Para cada $1 \leq i \leq 3$, existe uma variedade plana orientável $\mathscr{Y}_{i}$ de dimensão 6, com grupo de holonomia $H_{i}$, e com grupo fundamental o grupo de Bieberbach $\mathcal{B C G}\left(4, H_{i}\right) \subseteq B_{4} /\left[P_{4}, P_{4}\right]$.

No próximo teorema aplicamos o Lema 34 ao subgrupo cíclico de ordem $2^{k}$ do grupo de permutações $\Sigma_{2^{k}}$.

Teorema 37 (Teorema 5.4.6). Para cada $k \geq 2$ existe uma variedade plana orientável $\mathscr{X}_{k}$, de dimensão $2^{k-1}\left(2^{k}-1\right)$, com grupo de holonomia $\mathbb{Z}_{2^{k}}$, e com grupo fundamental o grupo de Bieberbach $\mathcal{B C G}\left(2^{k},\left\langle\bar{\sigma}\left(\alpha_{0,2^{k}}\right)\right\rangle\right)$. Além disso, o primeiro grupo de homologia é dado por $H_{1}\left(\mathscr{X}_{k} ; \mathbb{Z}\right)=\left(\oplus_{2^{k-1}} \mathbb{Z}\right) \oplus \mathbb{Z}_{2^{k-1}}$.

O Lema 34 será aplicado novamente no seguinte teorema, com $n \geq 4$, para o subgrupo cíclico $\mathbb{Z}_{2}$, de $\Sigma_{n}$, gerado por $\left(\begin{array}{ll}1 & 2\end{array}\right)$.

Teorema 38 (Teorema 5.4.8). Sejam $n \geq 4$ e $\mathscr{M}_{n}$ a variedade plana unicamente determinada pelo grupo de Bieberbach $\mathcal{B C G}\left(n,\left\langle\left(\begin{array}{ll}1 & 2\end{array}\right)\right\rangle\right)$. Então, $\mathscr{M}_{n}$ tem dimensão $n(n-1) / 2$, grupo de holonomia isomorfo a $\mathbb{Z}_{2}$, é uma variedade plana orientável se $n$ é par e não orientável se $n$ é impar. Além disso $H_{1}\left(\mathscr{M}_{n} ; \mathbb{Z}\right)=\mathbb{Z}^{\frac{(n-3) n+4}{2}}$. 
O último resultado do Capítulo 5 é uma aplicação dos resultados obtidos acima à existência de difeomorfismos de Anosov. O resultado abaixo vai ser baseado num teorema de Porteous que dá condições para a existência de difeomorfismos de Anosov quando a holonomia é um grupo cíclico.

Teorema 39 (Teorema 5.4.9). Para todo $n \geq 4$, a variedade plana $\mathscr{M}_{n}$, dada no Teorema 38 (orientável para $n$ par e não orientável para $n$ ímpar), com grupo de holonomia $\mathbb{Z}_{2}$, admite difeomorfismo de Anosov. Para todo $k \geq 3$, a variedade plana orientável $\mathscr{X}_{k}$, de dimensão $2^{k-1}\left(2^{k}-1\right)$, com grupo de holonomia $\mathbb{Z}_{2^{k}}$, do Teorema 37 , admite difeomorfismo de Anosov.

No entanto, a variedade plana orientável $\mathscr{Y}_{1}$, dada no Teorema 36, e a variedade plana orientável $\mathscr{X}_{2}$, do Teorema 37, ambas de dimensão 6 e com grupo de holonomia $\mathbb{Z}_{4}$, não admitem difeomorfismo de Anosov.

\section{Capítulo 6 Apêndice}

No último capítulo destacamos uma análise de dois artigos, sendo que o primeiro deles foi escrito por Berrick, Cohen, Wong e Wu [9] e o segundo deles escrito por Li e Wu [58]. O principal resultado da Seção 6.1 é a seguinte proposição.

Proposição 40 (Proposição 6.1.1). Suponha que $n \geq 2$, então

1. $\partial\left(A_{-1,0}\right) \neq 1$.

2. Como aplicações de $P_{n+2}\left(\mathbb{D}^{2}\right)$ em $P_{n}\left(\mathbb{D}^{2}\right)$, temos $\partial \circ \partial \neq \partial \circ d_{0}$.

Pelo item 2 da Proposição 40 segue que o item 3 do Lema 6.5.2 de [9] está formulado de forma incorreta, pois para $n \geq 2$, os homomorfismos $\partial \circ \partial$ e $\partial \circ d_{0}$ de $P_{n+2}\left(\mathbb{D}^{2}\right)$ em $P_{n}\left(\mathbb{D}^{2}\right)$ não são iguais. A partir deste fato analisamos nas primeiras duas seções do apêndice as consequências deste problema encontrado. 


\section{Capítulo 1}

\section{Preliminares}

Neste capítulo queremos registrar alguns conceitos básicos e resultados necessários para o bom desenvolvimento deste trabalho, no entanto o leitor familiarizado com os tópicos aqui tratados pode omití-los, caso considere conveniente. Na primeira seção, introduziremos alguns conceitos da teoria de grupos, com particular atenção aos ligados à teoria dos comutadores. Na segunda seção faremos uma exposição de aspectos básicos da topologia algébrica e da teoria de tranças, dando atenção aos enlaçamentos e tranças Brunnianas. Na última seção deste capítulo recordaremos as principais definições e propriedades da teoria de homotopia simplicial usadas neste texto. Destacaremos a estrutura simplicial dos grupos de tranças sobre superfícies, donde podemos ver claramente a linguagem usada na conexão entre os grupos de tranças sobre superfícies e os grupos de homotopia da esfera $\mathbb{S}^{2}$, dada inicialmente por Cohen et al. [9].

Gostaríamos de ressaltar que as superfícies consideradas neste texto serão sempre conexas, podendo ser com bordo ou não, assim como orientáveis ou não. Para evitar patologias assumiremos durante todo este texto que dada uma superfície ela será compacta, ou compacta com um conjunto finito de $k$ pontos removidos do interior, onde neste caso dizemos que a superfície é compacta $k$-perfurada.

\section{$1.1 \quad$ Rudimentos da teoria de grupos}

Seja $G$ um grupo qualquer. Ao longo deste texto denotaremos por $\langle a\rangle$ o fecho normal de um elemento a do grupo $G$, isto é, o menor subgrupo normal de $G$ que contém o elemento $a$. Também poderá ser denotado por $\langle a\rangle^{G}$ caso se queira especificar o grupo em questão. Observemos que se $G$ é um grupo simples (isto é, os únicos subgrupos normais de $G$ são os triviais) e $a$ não é o elemento neutro do grupo, então $\langle a\rangle^{G}=G$. Uma apresentação de um grupo $G$ é uma descrição do grupo em termos de geradores e relações, usualmente escrito como $G=\langle X \mid R\rangle$, onde $X$ indica um conjunto de geradores e $R$ um conjunto de relações (que podem ser infinitos). Se $R$ é o conjunto vazio escreveremos $G=\langle X \mid-\rangle$ para representar isto. Quando $X$ é um conjunto finito dizemos que $G$ é finitamente gerado, e no 
caso em que tanto $X$ como $R$ são conjuntos finitos dizemos que $G$ é um grupo finitamente apresentado. Agora, supondo que o grupo $G$ tem um subgrupo normal $N$ tal que $N$ e $G / N$ são grupos finitamente apresentados, então um resultado de Hall garante que o grupo $G$ é finitamente apresentado [81, 2.2.4]. Por exemplo, se um grupo $G$ tem uma cadeia de subgrupos $1 \triangleleft G_{0} \triangleleft G_{1} \triangleleft \cdots \triangleleft G_{i} \triangleleft G_{i+1} \triangleleft \cdots \triangleleft G_{n}=G$ tal que cada quociente $G_{i+1} / G_{i}$ é um grupo cíclico, então $G$ é um grupo finitamente apresentado. Tais grupos são chamados de grupos policíclicos.

Exemplo 1.1.1. Vejamos alguns exemplos de apresentações de grupos.

- O grupo dos inteiros $\mathbb{Z}=\langle a \mid-\rangle$, é o grupo cíclico infinito, ou o grupo livre gerado por um elemento, que é finitamente apresentado.

- Os grupos cíclicos finitos módulo $m, \mathbb{Z}_{m}=\left\langle a \mid a^{m}=1\right\rangle$, são grupos finitamente apresentados.

- Os grupos livres $F_{X}=\langle X \mid-\rangle$, que serão tratados com um pouco de detalhe na próxima subseção. Observamos que se $X$ é finito (resp., infinito), então $F_{X}$ é finitamente apresentado (resp., infinitamente apresentado). No caso em que $X$ é finito $e|X|=n$ podemos denotar o grupo livre $F_{X}$ por $F_{n}$.

- Notemos que o mesmo grupo pode ter diferentes apresentações, ainda podendo ter um número diferente de geradores e relações. Por exemplo,

$$
\left\langle a, b, c, d \mid c^{4}=1, a=b, b^{2}=1, d^{7}=1, c=a b d^{-1}, c a^{-2}=1\right\rangle
$$

é uma outra apresentação do grupo cíclico finito $\mathbb{Z}_{2}$.

- O grupo das permutações em $n$ elementos é finitamente apresentado e tem a seguinte apresentação: $\Sigma_{n}=\left\langle\sigma_{1}, \sigma_{2}, \ldots, \sigma_{n-1} \mid R\right\rangle$, onde $R$ é formado pelas relações $\left\{\sigma_{i}^{2}\right.$, para $\left.1 \leq i \leq n-1\right\},\left\{\sigma_{i} \sigma_{i+1} \sigma_{i}=\sigma_{i+1} \sigma_{i} \sigma_{i+1}\right.$, para $\left.1 \leq i \leq n-2\right\} e$ $\left\{\sigma_{i} \sigma_{j}=\sigma_{j} \sigma_{i}\right.$, quando $\left.|i-j| \geq 2\right\}$.

- O grupo de Heisenberg tem uma apresentação dada por

$$
\left\langle x, y, z \mid z=x y x^{-1} y^{-1}, x z=z x, y z=z y\right\rangle .
$$

- Uma família importante de grupos corresponde aos grupos de Baumslag-Solitar, uma apresentação é dada por $B(m, n)=\left\langle a, b \mid a^{n}=b a^{m} b^{-1}\right\rangle$.

Uma das ferramentas usadas para mostrar que duas apresentações de grupos são isomorfas e portanto correspondem nesse sentido ao mesmo grupo são as transformações de Tietze, como visto no Teorema 1.5 de [60]. Entre os métodos que usaremos com certa 
frequência estão o método de Reidemeister-Schreier ("Reidemeister-Schreier rewriting process") para dar apresentações de subgrupos de um grupo dado, que pode ser encontrado no livro de Magnus, Karras e Solitar [60], no entanto recomendamos também a leitura deste método no livro de Murasugi e Kurpita [75]. Outra técnica que será usada é a apresentação de extensões de grupos $1 \rightarrow L \rightarrow G \rightarrow H \rightarrow 1$, como descrita no livro de Johnson [50].

Nas próximas duas subseções seguiremos o livro de Magnus, Karras e Solitar [60], no entanto uma abordagem do estudo de grupos livres, suas propriedades e assuntos relacionados também podem ser encontrados nos livros de teoria dos grupos de Robinson [81], de Rotman [83] e de Johnson [50].

\subsubsection{Grupos livres}

Consideraremos nesta subseção uma família importante de grupos, os chamados grupos livres, que será bastante usada neste texto. O grupo livre finitamente gerado de posto $n, F_{n}$, nos $n$ geradores livres $x_{1}, x_{2}, \ldots, x_{n}$ é o grupo gerado por $x_{1}, x_{2}, \ldots, x_{n}$ e com nenhuma relação entre eles, usando a bem conhecida notação para apresentações de grupos podemos então escrever $F_{n}=\left\langle x_{1}, x_{2}, \ldots, x_{n} \mid-\right\rangle$. Lembramos que se dois grupos livres de posto finito $F_{n}$ e $F_{m}$ são isomorfos, então $n=m$. Observamos que também existem os grupos livres de posto infinito, que como seu nome indica são grupos gerados por um conjunto infinito de elementos e tais que não existe nenhuma relação entre eles. Uma das propriedades bastante conhecidas e interessantes dos grupos livres é que todo grupo é isomorfo ao grupo quociente de algum grupo livre [50, Proposition 4, p. 7].

O problema da palavra, que consiste em determinar se existe um algoritmo para decidir se dada uma palavra do grupo ela é o elemento identidade ou não, é um de três importantes problemas de decisão em teoria dos grupos que foram inicialmente formulados em 1911 por M. Dehn (ver [81, Seção 2.2], ou [60, Seção 1.3]); os outros são o problema de conjugação, que pergunta se existe um algoritmo para decidir se dados dois elementos de um grupo finitamente apresentado então eles são conjugados, e o problema de isomorfismo: existe algum algoritmo para decidir se dois grupos finitamente apresentados dados são isomorfos? Em geral todos os três problemas tem resposta negativa. No entanto, notamos que o problema da palavra pode ser resolvido para os grupos livres finitamente apresentados, como feito na Seção 1.4 de [60], assim como para os grupos policíclicos.

Entre os grupos livres também encontramos os chamados grupos abelianos livres, que são grupos livres no sentido anterior com a única condição adicional que todos seus geradores comutam entre si. Segue do Teorema Fundamental dos Grupos Abelianos Finitamente Gerados que os grupos abelianos livres finitamente gerados (de posto $n$ ) são isomorfos a uma soma direta de $n$ cópias de $\mathbb{Z}$. No caso de grupos abelianos livres infinitamente gerados temos que eles são isomorfos a um produto direto infinito de cópias de $\mathbb{Z}$. Como é 
de se esperar, todo grupo abeliano é isomorfo a um grupo quociente de um grupo abeliano livre.

Agora, o famoso Teorema de Nielsen-Schreier garante que subgrupos de grupos livres são por sua vez grupos livres [81, 6.1.1]. Tal teorema foi inicialmente provado por Nielsen em 1921 no caso especial de grupos livres finitamente apresentados, isto é, grupos livres de posto finito. Para demonstrar o caso geral é usada a noção de transversal de Schreier, como feito na Seção 6.1 de [81]. Como destacado lá, uma das consequências do Teorema de Nielsen-Schreier é o fato que o subgrupo comutador (que será tratado com mais detalhes na próxima subseção) de um grupo livre com dois ou mais geradores (e portanto não cíclico) é um grupo livre de posto infinito. O Teorema de Nielsen-Schreier tem muitas aplicações, como por exemplo, a técnica usada para dar uma apresentação de um subgrupo de um grupo com uma apresentação dada, chamada de método de Reidemeister-Schreier.

Dizemos que um grupo G é Hopfiano (em homenagem a H. Hopf) se ele não é isomorfo a um grupo quociente próprio, ou de forma equivalente, se todo endomorfismo sobrejetor é um automorfismo. Por exemplo, todos os grupos finitos e todos os grupos simples são Hopfianos, enquanto que os grupos abelianos livres de posto infinito não são Hopfianos.

A questão original levantada por Hopf em 1932 era: Todo grupo finitamente gerado é Hopfiano? É conhecido que a resposta é negativa, o grupo de Baumslag-Solitar $B S(2,3)=\left\langle a, b \mid b a^{2} b^{-1}=a^{3}\right\rangle$ [50, Seção 9.7], é um exemplo simples de um grupo finitamente gerado que não é Hopfiano.

Um resultado interessante provado por Nielsen [81, 6.1.12] é que um grupo livre de posto finito (isto é, finitamente gerado) é Hopfiano e portanto pode-se concluir daí que se $F$ é um grupo livre de posto finito $n$, então ele não pode ser gerado por um conjunto com menos de $n$ elementos. Em particular, do resultado provado por Nielsen, segue que os grupos abelianos livres de posto finito são Hopfianos.

\subsubsection{Subgrupos comutadores em grupos}

Dado um grupo $G$, definimos como subgrupo dos comutadores de $G$ ao menor subgrupo que contém os elementos da forma $[a, b]=a^{-1} b^{-1} a b$ para todos os elementos $a, b \in G$. Este subgrupo é denotado por $[G, G]$ e chamado subgrupo comutador de $G$.

Chamamos a atenção que em [60] é usado a convenção $(a, b)=a^{-1} b^{-1} a b$ e em outros textos é usado que $[a, b]=a b a^{-1} b^{-1}$, porém usaremos sempre a convenção $[a, b]=a^{-1} b^{-1} a b$.

É bem conhecido que o subgrupo comutador $[G, G]$ é um subgrupo normal do grupo $G$ e que $G /[G, G]$ é o grupo abelianizado obtido a partir de $G$. Um método simples para obter informação de um grupo $G$ que é descrito via uma apresentação de grupo em termos de geradores e relações, consiste em abelianizar $G$. Eventualmente é necessário tomar séries centrais descendentes, que serão definidas posteriormente, para obter mais informações do 
grupo. Sejam $a, b$ um par de elementos quaisquer em um grupo $G$. Denotemos a conjugação de elementos por: $a^{b}=b^{-1} a b$ e $a^{-b}=b^{-1} a^{-1} b$.

Teorema 1.1.2 (Teorema 5.1 de [60] - Identidades de Witt-Hall). Para quaisquer três elementos a,b,c de um grupo $G$ valem as seguintes igualdades:

1. $[a, b] \cdot[b, a]=1$.

2. $[a, b \cdot c]=[a, c] \cdot[a, b] \cdot[[a, b], c]$.

3. $[a \cdot b, c]=[a, c] \cdot[[a, c], b] \cdot[b, c]$.

4. $\left[[a, b], c^{a}\right] \cdot\left[[c, a], b^{c}\right] \cdot\left[[b, c], a^{b}\right]=1$.

5. $[[a, b], c] \cdot[[b, c], a] \cdot[[c, a], b]=[b, a] \cdot[c, a] \cdot[c, b]^{a} \cdot[a, b] \cdot[a, c]^{b} \cdot[b, c]^{a} \cdot[a, c] \cdot[c, a]^{b}$.

As primeiras quatro identidades do Teorema 1.1.2 foram obtidas por P. Hall em 1933, enquanto a última foi obtida por Witt em 1937. A última identidade é útil para demonstrar a identidade de Jacobi da Álgebra de Lie construída a partir das séries centrais de um grupo. A teoria das séries centrais descendentes de grupos foi completamente desenvolvida por P. Hall em 1933. A série derivada e central de um grupo $G$ são definidas de forma recursiva como segue. A série derivada de um grupo $G$ é definida como sendo $G^{(0)}=$ $G$, e $G^{(n+1)}=\left[G^{(n)}, G^{(n)}\right]$, para $n=0,1,2,3, \ldots$ Dizemos que um grupo $G$ tal que $G^{(N)}=1$, para algum inteiro positivo $N$, é solúvel. Por exemplo, os grupos cíclicos são grupos solúveis. Um grupo é chamado residualmente solúvel se os elementos da sua série derivada tem como intersecção o elemento identidade. Exemplos de grupos residualmente solúveis são os grupos livres e grupos de tranças de algumas superfícies.

A série central descendente de um grupo $G$ (ou apenas série central de $G$ ) são definidas de forma recursiva como sendo $\Gamma_{1}=G, \Gamma_{n+1}(G)=\left[\Gamma_{n}, G\right]$, para $n=1,2,3, \ldots$ Um grupo $G$ tal que $\Gamma_{N}(G)=1$ para algum inteiro positivo $N$ é chamado de nilpotente. Por exemplo, o grupo dos quatérnios é solúvel. Um grupo é chamado residualmente nilpotente se sua série central descendente se intersecta na identidade. Entre os exemplos de grupos residualmente nilpotentes temos os grupos livres de posto $r \geq 1$, ou ainda os grupos de tranças puras sobre o disco com mais de 2 cordas. Pode ser provado que os grupos residualmente nilpotentes são grupos Hopfianos [60, Teorema 5.5]. Outro resultado interessante relacionado aos grupos livres foi dado por Magnus: Se $F$ é um grupo livre, a intersecção de todos os termos das séries centrais de $F$ é trivial, isto é, $F$ é residualmente nilpotente [81, 6.1.10]. As identidades de Witt-Hall descritas no Teorema 1.1.2 tem a seguinte forma, quando tomarmos módulos pelas séries centrais.

Teorema 1.1.3 (Teorema 5.3 [60]). Sejam a, b, c elementos de um grupo $G$ e $k, m, n$ inteiros positivos tais que $a \in \Gamma_{k}, b \in \Gamma_{m}, c \in \Gamma_{n}(G)$. Então, temos as seguintes equivalências, módulo elementos da série central do grupo: 
1. $a \cdot b \equiv b \cdot a \bmod \Gamma_{k+m}(G)$.

2. $(a, b \cdot c) \equiv(a, b) \cdot(a, c) \bmod \Gamma_{k+m+n}(G)$.

3. $(a \cdot b, c) \equiv(a, c) \cdot(b, c) \bmod \Gamma_{k+m+n}(G)$.

4. $((a, b), c) \cdot((b, c), a) \cdot((c, a), b) \equiv 1 \bmod \Gamma_{k+m+n+1}(G)$.

Os dois teoremas anteriores mostram que a operação produto e o processo de comutatividade num grupo estão quase relacionados com as operações de adição e multiplicação numa Álgebra de Lie. Isto pode ser visto no Teorema 5.12 do Magnus, Karras e Solitar $[60]$.

\subsubsection{O subgrupo comutador simétrico}

Neste trabalho usaremos bastante o subgrupo comutador simétrico, introduzido em [5] e estudado em [59]. Como dito por $\mathrm{Li}$ e Wu em [59, rodapé 1] o subgrupo comutador simétrico foi batizado por Roman Mikhailov em comunicações pessoais com eles. Antes de definirmos tal grupo vamos lembrar algumas definições, como os arranjos de colchetes ("bracket arrangements") e os comutadores amplos ("fat commutators").

Similarmente ao processo feito em [60], mas seguindo o artigo de $\mathrm{Li} \mathrm{e} \mathrm{Wu}$ [59], definimos um arranjo de colchetes de peso n ("bracket arrangements") em um grupo G como sendo uma aplicação $\beta^{n}: G^{n} \rightarrow G$ que é definida indutivamente como segue: $\beta^{1}=\mathrm{id}_{G}$, $\beta^{2}\left(a_{1}, a_{2}\right)=\left[a_{1}, a_{2}\right]$, para quaisquer $a_{1}, a_{2}$ em $G$, onde $\left[a_{1}, a_{2}\right]$ é o comutador dos elementos $a_{1}, a_{2}$. Suponhamos que os arranjos de colchetes de peso $k$ foram definidos para $1 \leq k<n$, com $n \geq 3$. Uma aplicação $\beta^{n}: G^{n} \rightarrow G$ é chamada um arranjo de colchetes de peso $n$ se $\beta^{n}$ é a composta

$$
G^{n}=G^{k} \times G^{n-k} \stackrel{\beta^{k} \times \beta^{n-k}}{\longrightarrow} G \times G \stackrel{\beta^{2}}{\longrightarrow} G
$$

para arranjos de colchetes $\beta^{k}$ e $\beta^{n-k}$ de pesos $k$ e $n-k$, respectivamente, com $1 \leq k<n$. É conhecido que o número de arranjos de colchetes de peso $n$, para $n \geq 2$, é dado por

$$
\frac{1}{n-1}\left(\begin{array}{c}
2 n-2 \\
n
\end{array}\right)
$$

onde o coeficiente binomial é dado por $\left(\begin{array}{c}m \\ k\end{array}\right)=\frac{m !}{k !(m-k) !}($ ver exercício 5 da Seção 5.2 de [60]).

Exemplo 1.1.4. Sejam $a_{1}, a_{2}, a_{3}, a_{4}$ elementos de um grupo $G$.

1. Se $n=3$, existem dois arranjos de colchetes dados por $\left[\left[a_{1}, a_{2}\right], a_{3}\right]$ e $\left[a_{1},\left[a_{2}, a_{3}\right]\right]$. 
2. Para $n=4$, existem cinco arranjos de colchetes dados por $\left[\left[a_{1}, a_{2}\right],\left[a_{3}, a_{4}\right]\right]$, $\left[\left[\left[a_{1}, a_{2}\right], a_{3}\right], a_{4}\right],\left[\left[a_{1},\left[a_{2}, a_{3}\right]\right], a_{4}\right],\left[a_{1},\left[\left[a_{2}, a_{3}\right], a_{4}\right]\right],\left[a_{1},\left[a_{2},\left[a_{3}, a_{4}\right]\right]\right]$.

Consideremos uma coleção de subgrupos (não necessariamente normais) $R_{j}$ de $G$, para $1 \leq j \leq n$. O subgrupo comutador amplo ("fat commutator") $\left[\left[R_{1}, R_{2}, \ldots, R_{n}\right]\right]$ é definido como sendo o subgrupo de $G$ gerado por todos os comutadores da forma

$$
\beta^{t}\left(g_{i_{1}}, \ldots, g_{i_{t}}\right)
$$

onde

1. $1 \leq i_{s} \leq n$;

2. $\left\{i_{1}, \ldots, i_{t}\right\}=\{1, \ldots, n\}$, isto é, cada número inteiro no conjunto $\{1,2, \ldots, n\}$ aparece pelo menos uma vez como um número $i_{s}$;

3. $g_{j} \in R_{j}$

4. $\beta^{t}$ percorre todos os arranjos de colchetes de peso $t(\operatorname{com} t \geq n)$.

Observemos que para $n=1$ temos que $\left[\left[R_{1}\right]\right]=R_{1}$. O subgrupo comutador amplo tem sido usado para aplicações da teoria combinatória de grupo em teoria de homotopia [9], [25], [96]. Uma descrição do subgrupo comutador amplo de subgrupos normais como produto de subgrupos comutadores será dada na sequência.

Dado um grupo $G$ e um conjunto de seus subgrupos $R_{1}, \ldots, R_{n}$, definimos o subgrupo comutador simétrico $\left[R_{1}, R_{2}, \ldots, R_{n}\right]_{S}$ por

$$
\left[R_{1}, R_{2}, \ldots, R_{n}\right]_{S}:=\prod_{\sigma \in \Sigma_{n}}\left[\left[R_{\sigma(1)}, R_{\sigma(2)}\right], \ldots, R_{\sigma(n)}\right]
$$

onde $\Sigma_{n}$ é o grupo de permutações em $n$ elementos e $\left[\left[R_{\sigma(1)}, R_{\sigma(2)}\right], \ldots, R_{\sigma(n)}\right]$ é o subgrupo gerado pelos comutadores iterados à esquerda

$$
\left[\ldots\left[\left[g_{1}, g_{2}\right], g_{3}\right], \ldots, g_{n}\right]
$$

com $g_{i} \in R_{\sigma(i)}$. Observemos que para $n=1$, temos que $\left[R_{1}\right]_{S}=R_{1}$. Segue da própria definição que o subgrupo comutador simétrico é um subgrupo do subgrupo comutador amplo. Ainda mais, foi provado por Li e Wu [59] que esses dois grupos são iguais quando os subgrupos $R_{j}$ são normais em $G$.

Teorema 1.1.5 (Teorema 1.1 [59]). Seja $R_{j}$ qualquer subgrupo normal de um grupo $G$, com $1 \leq j \leq n$. Então,

$$
\left[\left[R_{1}, R_{2}, \ldots, R_{n}\right]\right]=\left[R_{1}, R_{2}, \ldots, R_{n}\right]_{S}
$$


No mesmo artigo Li e Wu também provaram que é possível desconsiderar alguns comutadores da produtória dada em (1.1), como mostra o seguinte teorema.

Teorema 1.1.6 (Teorema 1.2 [59]). Seja $R_{j}$ qualquer subgrupo normal de um grupo $G$, com $1 \leq j \leq n$. Então,

$$
\left[R_{1}, R_{2}, \ldots, R_{n}\right]_{S}=\prod_{\sigma \in \Sigma_{n-1}}\left[\left[R_{1}, R_{\sigma(2)}\right], \ldots, R_{\sigma(n)}\right]
$$

onde $\Sigma_{n-1}$ age sobre $\{2,3, \ldots, n\}$.

Vejamos alguns exemplos de subgrupo comutador simétrico, usando a simplificação dada no Teorema 1.1.6. Vamos supor que os grupos $R_{*}$ na sequência são subgrupos normais de um grupo $G$.

\section{Exemplo 1.1.7.}

1. Se $n=2$, então o subgrupo comutador simétrico é simplesmente o subgrupo comutador dos dois subgrupos normais:

$$
\left[R_{1}, R_{2}\right]_{S}=\left[R_{1}, R_{2}\right] \cdot\left[R_{2}, R_{1}\right]=\left[R_{1}, R_{2}\right]
$$

2. Seguindo a definição de subgrupo comutador simétrico, o caso de $n=3$ fica assim:

$$
\begin{aligned}
{\left[R_{1}, R_{2}, R_{3}\right]_{S}=} & {\left[\left[R_{1}, R_{2}\right], R_{3}\right] \cdot\left[\left[R_{2}, R_{1}\right], R_{3}\right] \cdot\left[\left[R_{1}, R_{3}\right], R_{2}\right] \cdot } \\
& \cdot\left[\left[R_{3}, R_{2}\right], R_{1}\right] \cdot\left[\left[R_{2}, R_{3}\right], R_{1}\right] \cdot\left[\left[R_{3}, R_{1}\right], R_{2}\right]
\end{aligned}
$$

No entanto, usando a simplificação dada por Li e Wu, o subgrupo comutador simétrico de 3 subgrupos é o produto dos seguintes dois subgrupos comutadores:

$$
\left[R_{1}, R_{2}, R_{3}\right]_{S}=\left[\left[R_{1}, R_{2}\right], R_{3}\right] \cdot\left[\left[R_{1}, R_{3}\right], R_{2}\right]
$$

3. No caso $n=4$, pela definição do subgrupo comutador simétrico segue que o grupo $\left[R_{1}, R_{2}, R_{3}, R_{4}\right]_{S}$ é um produto de 24 comutadores. No entanto, com a simplificação do Teorema 1.1.6, podemos escrevê-lo como um produto de apenas comutadores:

$$
\begin{aligned}
{\left[R_{1}, R_{2}, R_{3}, R_{4}\right]_{S}=} & {\left[\left[\left[R_{1}, R_{2}\right], R_{3}\right] R_{4}\right] \cdot\left[\left[\left[R_{1}, R_{3}\right], R_{4}\right] R_{2}\right] \cdot\left[\left[\left[R_{1}, R_{4}\right], R_{2}\right] R_{3}\right] \cdot } \\
& \cdot\left[\left[\left[R_{1}, R_{2}\right], R_{4}\right] R_{3}\right] \cdot\left[\left[\left[R_{1}, R_{3}\right], R_{2}\right] R_{4}\right] \cdot\left[\left[\left[R_{1}, R_{4}\right], R_{3}\right] R_{2}\right]
\end{aligned}
$$

Observação 1.1.8. Pela definição do grupo comutador simétrico segue claramente que os grupos $R_{i}$, para $1 \leq i \leq n$, podem ser escritos em qualquer ordem na descrição de $\left[R_{1}, R_{2}, \ldots, R_{n}\right]_{S}$. Isto é,

$$
\left[R_{1}, R_{2}, \ldots, R_{n}\right]_{S}=\left[R_{i_{1}}, R_{i_{2}}, \ldots, R_{i_{n}}\right]_{S}
$$


onde $\left\{i_{1}, \ldots, i_{n}\right\}=\{1, \ldots, n\}$.

Na próxima seção definiremos os grupos de homotopia de espaços topológicos e os relacionaremos com esta noção de subgrupo comutador simétrico.

\subsection{Topologia Algébrica e Teoria de Tranças}

Na sequência faremos uma revisão sobre aspectos de topologia algébrica e teoria de tranças. Para detalhes básicos sobre topologia algébrica recomendamos ao leitor os livros de Hatcher [46], de Massey [65], [66], e de Munkres [74]; para mais detalhes sobre a teoria de tranças recomendamos os livros de Hansen [45], de Kassel e Turaev [54], de Murasugi [75] e o capítulo de Rolfsen no livro [8]; bem como os textos de Birman e Brendle [11] e de Guaschi e Juan-Pineda [42].

\subsubsection{Grupos de homotopia}

Na sequência definiremos os grupos de homotopia e mencionaremos algumas de suas propriedades, usaremos o livro de Hatcher como referência principal [46]. Para propriedades gerais de teoria de homotopia recomendamos o livro de Whitehead [93] e para assuntos mais específicos o livro de Toda [90].

É bem conchecido que a definição dos grupos de homotopia é fácil de entender, no entanto, calcular estes grupos pode ser bastante difícil. Um dos problemas fundamentais em teoria de homotopia consiste no estudo dos grupos de homotopia das esferas, os quais são desconhecidos, em geral. O cálculo de alguns destes grupos de homotopia das esferas pode ser encontrado em [90], [20], [55]; em particular $\pi_{n}\left(\mathbb{S}^{2}\right)$ é conhecido para $n \leq 64$, ver [20]. Os grupos de homotopia foram introduzidos por W. Hurewicz em 1935 e é bem conhecido que o entendimento destes grupos ajudaria no desenvolvimento de várias áreas da matemática (não somente em topologia algébrica). Na tentativa de calcular tais grupos foram criadas fortes teorias em topologia algébrica como teoria de homotopia simplicial, teoria de bordismo, cobordismo e K-teoria. Também entre alguns métodos específicos para calcular estes grupos citamos, por exemplo, o teorema de suspensão de Freudhental, a fibração de Hopf, as sequências espectrais de Serre e de Adams, bem como os sistemas de Moore-Postnikov. Mais detalhes sobre a história deste problema, entre outros, podem ser encontrados no texto de P. May sobre a história da topologia algébrica estável entre os anos 1945-1966 [68].

Vamos assumir que a noção de homotopia de aplicações já é conhecida. Seja $I^{n}$ o cubo unitário $n$-dimensional, onde $I=[0,1]$ é o intervalo unitário. O bordo $\partial I^{n}$ de $I^{n}$ é o subespaço formado pelos pontos que tem pelo menos uma coordenada igual a 0 ou 1 . Para um espaço $X$ com ponto base $x_{0} \in X$ definamos o n-ésimo grupo de homotopia de $X$ com ponto base $x_{0}$, denotado por $\pi_{n}\left(X, x_{0}\right)$, como sendo o conjunto de classes de homotopia de 
aplicações $f:\left(I^{n}, \partial I^{n}\right) \rightarrow\left(X, x_{0}\right)$, onde as homotopias $f_{t}$ são tais que $f_{t}\left(\partial I^{n}\right)=x_{0}$, para todo $t \in[0,1]$. Esta definição se estende ao caso $n=0$ tomando $I_{0}$ como sendo um ponto e $\partial I_{0}$ como sendo o conjunto vazio, portanto $\pi_{0}\left(X, x_{0}\right)$ é justamente o conjunto formado pelas componentes conexas por caminhos de $X$. O caso $n=1$ é o bem conhecido grupo fundamental de $X$ com ponto base $x_{0}, \pi_{1}\left(X, x_{0}\right)$. A operação de grupo em $\pi_{n}\left(X, x_{0}\right)$ é a concatenação de aplicações

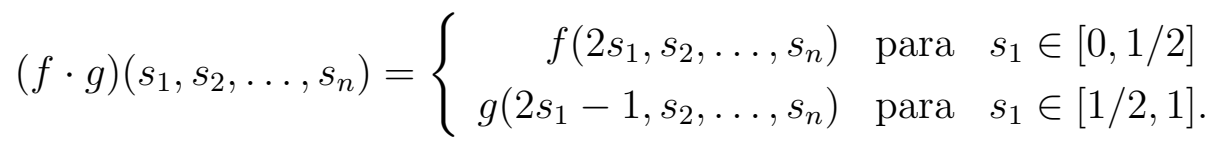

No caso em que $n \geq 2$ o grupo de homotopia do espaço $X$ com ponto base $x_{0}, \pi_{n}\left(X, x_{0}\right)$, é um grupo abeliano e portanto denotaremos a operação do grupo por "+" ao invés de "." como é feito usualmente no grupo fundamental. O elemento neutro nesse grupo é a classe de homotopia da aplicação constante que envia $I^{n}$ em $x_{0}$ e os inversos são dados por $-f\left(s_{1}, s_{2}, \ldots, s_{n}\right)=f\left(1-s_{1}, s_{2}, \ldots, s_{n}\right)$.

Observemos que considerar aplicações $\left(I^{n}, \partial I^{n}\right) \rightarrow\left(X, x_{0}\right)$ é o mesmo que considerar aplicações do quociente $I^{n} / \partial I^{n}=\mathbb{S}^{n}$ em $X$, levando o ponto base $s_{0}=\partial I^{n} / \partial I^{n}$ em $x_{0}$. Isto quer dizer que podemos ver o grupo de homotopia $\pi_{n}\left(X, x_{0}\right)$ como sendo o conjunto formado pelas classes de homotopia de aplicações $\left(\mathbb{S}^{n}, s_{0}\right) \rightarrow\left(X, x_{0}\right)$, onde as homotopias são consideradas por aplicações da mesma forma $\left(\mathbb{S}^{n}, s_{0}\right) \rightarrow\left(X, x_{0}\right)$.

Vamos listar na sequência algumas propriedades dos grupos de homotopia de $X$ com ponto base $x_{0}$.

\section{Propriedades 1.2.1.}

1. Se $X$ é um espaço conexo por caminhos, então o grupo de homotopia de $X$ não depende da escolha do ponto base, isto é, se $x_{0}, x_{1}$ são dois elementos de $X$, então os grupos $\pi_{n}\left(X, x_{0}\right)$ e $\pi_{n}\left(X, x_{1}\right)$ são isomorfos. Nesse caso, podemos escrever o grupo de homotopia de $X$ apenas por $\pi_{n}(X)$.

2. Uma aplicação de revestimento $p:\left(\widetilde{X}, \widetilde{x}_{0}\right) \rightarrow\left(X, x_{0}\right)$ induz um isomorfismo nos grupos de homotopia $p_{*}: \pi_{n}\left(\widetilde{X}, \widetilde{x}_{0}\right) \rightarrow \pi_{n}\left(X, x_{0}\right)$, para todo $n \geq 2$. No caso $n=1$, $p_{*}$ é injetora.

Em particular quando $X$ tem um espaço de revestimento contrátil, tem-se que $\pi_{n}\left(X, x_{0}\right)=0$, para $n \geq 2$. Os espaços $X$ tais que $\pi_{n}(X)=0$, para $n \geq 2$, são chamados de espaços asféricos.

3. Para um produto de uma coleção arbitrária de espaços conexos por caminhos $\prod_{\alpha} X_{\alpha}$ existem isomorfismos $\pi_{n}\left(\prod_{\alpha} X_{\alpha}\right) \cong \prod_{\alpha} \pi_{n}\left(X_{\alpha}\right)$, para todo $n$.

4. Usando o Teorema de Suspensão de Freudhental, pode-se provar que $\pi_{n}\left(\mathbb{S}^{n}\right) \cong \mathbb{Z}$ gerado pela aplicação identidade, para $n \geq 2$, assumindo que $\pi_{1}\left(\mathbb{S}^{1}\right) \cong \mathbb{Z}$. Também 
pode-se provar que a aplicação grau $\pi_{n}\left(\mathbb{S}^{n}\right) \rightarrow \mathbb{Z}$ é um isomorfismo, para todo $n \geq 1$. Como uma consequência do chamado Teorema de Aproximação Celular, pode-se provar que $\pi_{n}\left(\mathbb{S}^{k}\right)=0$ para $n<k$.

5. Um resultado bem conhecido de J. P. Serre afirma que $\pi_{n}\left(\mathbb{S}^{k}\right)$ é um grupo finito exceto para $n=k$ (como vimos no item anterior) e quando $k$ é par e $n=2 k-1$. É bem conhecido que, tomando $k=2 r, \pi_{4 r-1}\left(\mathbb{S}^{2 r}\right)$ é isomorfo a uma soma direta de $\mathbb{Z}$ com um grupo finito. Portanto, toda a dificuldade no cálculo dos grupos de homotopia das esferas está nos grupos abelianos finitos. O problema pode ser assim reduzido a calcular a p-torção em $\pi_{n}\left(\mathbb{S}^{k}\right)$ para todo primo $p$.

Um espaço $X$ tal que o único grupo de homotopia não trivial é o $n$-ésimo, $\pi_{n}(X) \cong G$, é chamado um espaço de Eilenberg-Mac Lane e denotado por $K(G, n)$. As superfícies que são espaços de Eilenberg-Mac Lane são aquelas diferentes da esfera e do plano projetivo e esta distinção entre estes espaços será bastante usada neste texto.

Dizemos que uma aplicação $p: E \rightarrow B$ tem a propriedade de levantamento de homotopias com relação a um espaço $X$ se, dada uma homotopia $g_{t}: X \rightarrow B$ e uma aplicação $\widetilde{g}_{0}: X \rightarrow E$ que é um levantamento de $g_{0}$ tal que $p \widetilde{g_{0}}=g_{0}$, então existe uma homotopia $\widetilde{g}_{t}: X \rightarrow E$ que é um levantamento de $g_{t}: X \rightarrow B$. Uma fibração é uma aplicação $p: E \rightarrow B$ com a propriedade de levantamento de homotopia com relação a todos os espaços $X$. Um exemplo típico e simples é a aplicação projeção na primeira coordenada $B \times F \rightarrow B$. Uma das ferramentas fortes e mais usadas relacionadas às fibrações é a sequência longa em homotopia. Suponhamos que $p: E \rightarrow B$ é uma fibração tal que $B$ é conexo por caminhos, então existe uma sequência exata longa em homotopia

$$
\cdots \rightarrow \pi_{n}\left(F, x_{0}\right) \rightarrow \pi_{n}\left(E, x_{0}\right) \stackrel{p_{*}}{\rightarrow} \pi_{n}\left(B, b_{0}\right) \rightarrow \pi_{n-1}\left(F, x_{0}\right) \rightarrow \cdots \rightarrow \pi_{0}\left(E, x_{0}\right) \rightarrow 0 .
$$

Observemos que esta sequência pode ser obtida no caso mais fraco de uma fibração de Serre, isto é, uma aplicação $p: E \rightarrow B$ que tem a propriedade de levantamento de homotopias para discos $\mathbb{D}^{k}$, com $k \geq 0$.

A seguir veremos que sob algumas hipóteses podemos reduzir o cálculo dos grupos de homotopia à combinatória de grupos, usando intersecções de subgrupos e o subgrupo comutador simétrico. Existe uma noção de conectividade de subgrupos normais de um grupo dado, mencionada no artigo de Ellis e Mikhailov [25], que daremos na sequência. Seja $G$ um grupo com subgrupos normais $N_{1}, N_{2}, \ldots, N_{m}$. Dizemos que uma $m$-upla de subgrupos normais $\left(N_{1}, \ldots, N_{m}\right)$ é conexa se $m \leq 2$ ou $m \geq 3$ e para todos os subconjuntos $I, J \subseteq\{1, \ldots, m\}, \operatorname{com}|I| \geq 2$ e $|J| \geq 1$, a seguinte igualdade é satisfeita:

$$
\left(\bigcap_{i \in I} N_{i}\right)\left(\prod_{j \in J} N_{j}\right)=\bigcap_{i \in I}\left(N_{i}\left(\prod_{j \in J} N_{j}\right)\right) .
$$


Sejam $G$ um grupo com subgrupos normais $N_{1}, \ldots, N_{n}$ e $X$ o colimite dos espaços classificantes $B\left(G / \prod_{i \in I} N_{i}\right)$, onde $I$ percorre todos os subconjuntos estritamente conti$\operatorname{dos} I \subsetneq\{1, \ldots, n\}$. O seguinte resultado estabelece uma importante conexão entre teoria combinatória de grupos e grupos de homotopia, onde tais grupos serão definidos na próxima seção.

Teorema 1.2.2 (Teorema de Ellis-Mikhailov). Se a $(n-1)$-upla $\left(N_{1}, \ldots, \widehat{N}_{i}, \ldots, N_{n}\right)$ é conexa, para todo $1 \leq i \leq n$, então o colimite $X$ é tal que

$$
\begin{gathered}
\pi_{n}(X) \cong \frac{N_{1} \cap \cdots \cap N_{n}}{\prod_{I \cup J=\{1, \ldots, n\}, I \cap J=\emptyset}\left[\cap_{i \in I} N_{i}, \cap_{j \in J} N_{j}\right]}, \\
\pi_{n+1}(X) \cong \operatorname{Ker}\left(\partial: T\left(N_{1}, \ldots, N_{n}\right) \rightarrow G\right) .
\end{gathered}
$$

O Teorema de Ellis-Mikhailov, Teorema 1.2.2, foi usado por Bardakov et al. [5] para dar uma descrição dos grupos de tranças Brunnianas sobre superfícies Eilenberg-Mac Lane como subgrupos comutadores simétricos, bem como para estabelecer uma conexão entre os grupos Brunnianos sobre a esfera e o plano projetivo, com os grupos de homotopia da esfera $\mathbb{S}^{2}$. Usaremos o Teorema 1.2.2 para dar uma descrição dos chamados grupos de tranças quase-Brunnianas sobre superfícies. Com esta descrição estabeleceremos uma conexão entre os grupos quase-Brunnianos sobre a esfera e o plano projetivo, com os grupos de homotopia da esfera.

Enunciaremos aqui um resultado de Jie $\mathrm{Wu}$, que pode ser encontrado em [96, Teorema 1.7], [6, Página 805] ou [25, Página 2108], que relaciona grupos livres e grupos de homotopia da esfera $\mathbb{S}^{2}$.

Teorema 1.2.3 (Teorema de Jie Wu). Seja $F_{n}=F_{n}\left(y_{1}, \ldots, y_{n}\right)$ um grupo livre de posto n. Denotemos por $R_{i}=\left\langle y_{i}\right\rangle^{F_{n}}$, para $1 \leq i \leq n$, e por $R_{n+1}=\left\langle y_{1} \cdots y_{n}\right\rangle^{F_{n}}$. Então, para $n \geq 1$

$$
\frac{R_{1} \cap R_{2} \cap \cdots \cap R_{n} \cap R_{n+1}}{\left[R_{1}, R_{2}, \ldots, R_{n}, R_{n+1}\right]_{S}}=\pi_{n+1}\left(\mathbb{S}^{2}\right) .
$$

Para finalizar, vejamos na Tabela 1.1 os grupos de homotopia da esfera $\pi_{n}\left(\mathbb{S}^{2}\right)$, para $1 \leq n \leq 21$, segundo o livro de H. Toda [90]. A notação usada é a seguinte: $\infty$ representa o grupo abeliano livre $\mathbb{Z}$, o inteiro $m$ indica o grupo cíclico de ordem $m, \mathbb{Z}_{m}$, e o símbolo "+" indica a soma direta dos grupos abelianos.

\begin{tabular}{|c||c|c|c|c|c|c|c|c|c|c|c|c|c|c|}
\hline$n$ & 1 & 2 & 3 & 4 & 5 & 6 & 7 & 8 & 9 & 10 & 11 & 12 & 13 & 14 \\
\hline \hline$\pi_{n}\left(\mathbb{S}^{2}\right)$ & 0 & $\infty$ & $\infty$ & 2 & 2 & 12 & 2 & 2 & 3 & 15 & 2 & $2+2$ & $4+2+3$ & $4+2+2+3+7$ \\
\hline \hline$n$ & 15 & 16 & \multicolumn{1}{|c}{17} & \multicolumn{1}{|c|}{18} & \multicolumn{2}{|c|}{19} & \multicolumn{2}{|c|}{20} & 21 \\
\hline \hline$\pi_{n}\left(\mathbb{S}^{2}\right)$ & $2+2$ & $2+3$ & $2+3+5$ & $2+3+5$ & $2+2+3$ & $4+2+2+3$ & $4+2+2+3$ \\
\hline
\end{tabular}

Tabela 1.1: Grupos de homotopia da esfera $\pi_{n}\left(\mathbb{S}^{2}\right)$, para $1 \leq n \leq 21$. 


\subsubsection{Espaços de configuração}

Os espaços de configuração de espaços topológicos têm sido de interesse em diferentes áreas do conhecimento. Além da matemática tais espaços têm sido estudados em astrofísica, biologia molecular, robótica e criptografia [11], [8]. No livro de E. Fadell e S. Husseini [27] eles expõem os espaços de configuração de variedades de dimensões arbitrárias e seus grupos de homotopia, homologia e cohomologia. Além disso eles usam com frequência as relações de Yang-Baxter no desenvolvimento dos tópicos ali tratados. No capítulo intitulado "Introduction to Configuration Spaces and their Applications", escrito por F.R. Cohen no livro sobre tranças [8], ele aborda em particular os espaços de configuração sobre superfícies e as relações destes com grupos de tranças, "mapping class groups", bem como com a teoria de homotopia simplicial e os grupos de homotopia das esferas.

Nessa subseção, em geral, consideraremos os espaços de configuração sobre superfícies. O motivo disto é que estamos interessados principalmente na relação entre os espaços de configuração e os grupos de tranças sobre superfícies, estabelecida por R. Fox e L. Neuwirth [31] no caso do disco e por E. Fadell e L. Neuwirth para qualquer superfície [28].

Denotemos por $F_{n} M$ o n-ésimo espaço de configuração ordenado de um espaço $M$,

$$
F_{n} M=\left\{\left(x_{1}, \ldots, x_{n}\right) \in M \times \cdots \times M \mid x_{i} \neq x_{j} \text { para } i \neq j\right\} .
$$

Existe uma ação natural de $\Sigma_{n}$ sobre $F_{n} M$, que permuta as coordenadas. Esta ação é livre e portanto $F_{n} M$ é um revestimento de $F_{n} M / \Sigma_{n}$. Denotaremos o espaço de órbitas, chamado de espaço de configuração de pontos não ordenados (ou espaço de n-uplas não ordenadas), por $D_{n} M=F_{n} M / \Sigma_{n}$.

Uma das ferramentas mais usadas quando se estudam grupos de tranças é a fibração de Fadell-Neuwirth e suas generalizações, ver por exemplo [28], [45], [36], [37], [38]. Como observado em [28], se $M$ é uma variedade e $1 \leq n<m$, então a aplicação

$$
\rho: F_{m} M \longrightarrow F_{n} M
$$

definida por $\rho\left(x_{1}, \ldots, x_{m}\right)=\left(x_{1}, \ldots, x_{n}\right)$ é uma fibração (localmente trivial) com fibra $F_{m-n}\left(M \backslash\left\{q_{1}, \ldots, q_{n}\right\}\right)$ e chamada de fibração de Fadell-Neuwirth. Podemos tomar a sequência exata longa de homotopia destes espaços via a fibração de Fadell-Neuwirth. Notemos que a superfície perfurada $M \backslash\left\{q_{1}, \ldots, q_{n}\right\}$, para $n \geq 1$, tem o mesmo tipo de homotopia de um complexo 1-dimensional e portanto, se $M$ é uma superfície de EilenbergMac Lane, então o espaço de configuração $F_{m} M$ também é um espaço de EilenbergMac Lane.

Como casos excepcionais na teoria geral temos a esfera $\mathbb{S}^{2}$ e o plano projetivo $\mathbb{R} P^{2}$, já 
que estas são as únicas superfícies que têm grupos de homotopia superiores não triviais.

\subsubsection{Grupos de Tranças sobre superfícies}

Nesta seção definiremos os grupos de tranças sobre superfícies usando a noção de espaço de configuração sobre superfícies e comentaremos algumas propriedades destes grupos. Para mais detalhes sobre a teoria de tranças recomendamos a leitura dos livros de Kurpita e Murasugi [75], de Kassel e Turaev [54], de Hansen [45], de Birman [13] bem como o texto de Birman e Brendle [11], o artigo de Guaschi e Juan-Pineda [42], onde os grupos de tranças de superfícies são considerados com mais detalhes, também recomendamos a dissertação de mestrado do autor sobre subgrupos geométricos e seus comensuradores em grupos de tranças de superfícies [76].

A relação entre tranças sobre o disco e nós/enlaçamentos pode ser vista na Parte II do livro de Manturov [64]. Observamos que no Capítulo 8 ele mostra a equivalência entre quatro definições diferentes dos grupos de tranças. Existe também uma conexão entre os grupos de tranças e os chamados Mapping Class Groups, que pode ser consultada no Capítulo 9 do livro de Farb e Margalit [30].

O grupo de tranças com $n$ cordas sobre uma superfície $M$, denotado por $B_{n}(M)$, é o conjunto de $n$ tranças geométricas módulo a relação de homotopia e cuja operação é concatenação. O elemento inverso de uma trança $\beta$ é dado pela imagem espelhada de $\beta$ e um representante geométrico do elemento identidade é a trança cujas cordas são todas verticais. Existe um homomorfismo sobrejetor natural do grupo $B_{n}(M)$ no

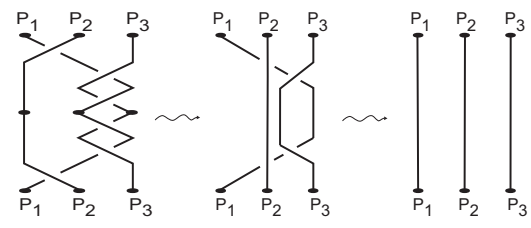

Figura 1.1: Exemplo de concatenação de duas tranças com 3 cordas. grupo simétrico de $n$ elementos $\Sigma_{n}$, que denotaremos por $\sigma: B_{n}(M) \longrightarrow \Sigma_{n}$, e cujo núcleo é chamado de grupo tranças puras com $n$ cordas sobre $M$, denotado por $P_{n}(M)$. Logo, existe a seguinte sequência exata curta de tranças

$$
1 \longrightarrow P_{n}(M) \longrightarrow B_{n}(M) \stackrel{\sigma}{\longrightarrow} \Sigma_{n} \longrightarrow 1 \text {. }
$$

Um exemplo de permutação associada às tranças é dado na Figura 1.2.

No que se segue usaremos a notação dada na seção anterior. R. Fox e L. Neuwirth provaram que podemos ver os grupos de tranças sobre o disco como sendo o grupo fundamental do espaço de configuração do disco e usaram este fato para dar a apresentação de $B_{n}\left(\mathbb{D}^{2}\right)$ originalmente estabelecida por E. Artin em [2]. Seguindo a ideia dada em [31], E. Fadell e L. Neuwirth definiram os grupos de tranças sobre uma variedade $X$ qualquer, com dimensão de $X$ maior ou igual que 2, como sendo o grupo fundamental do espaço de configuração de pontos não ordenados de $X$, [28]. Neste trabalho consideraremos apenas o caso em que $X=M$ é uma superfície. Desta forma, segue dos resultados de R. Fox, 


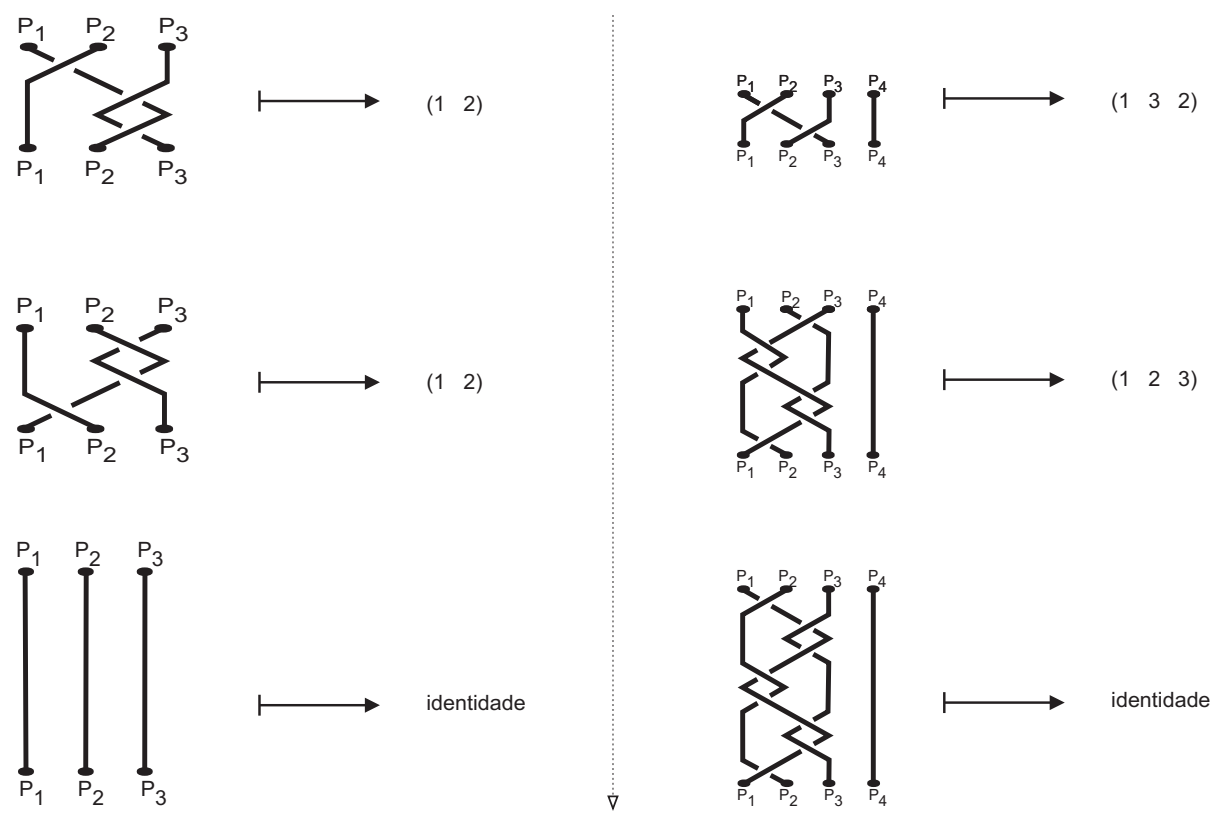

Figura 1.2: Exemplo de permutação associada às tranças.

E. Fadell e L. Neuwirth que o grupo fundamental $\pi_{1}\left(D_{n} M\right)\left(\operatorname{resp} . \pi_{1}\left(F_{n} M\right)\right)$ é o chamado grupo de tranças (resp. tranças puras) com $n$ cordas sobre $M$, denotado por $B_{n}(M)$ (resp. $\left.P_{n}(M)\right)$. Alguns exemplos de tranças sobre superfícies são dados na Figura 1.3. Além disso, se $n=1$, então $\pi_{1}\left(D_{1} M\right)=\pi_{1}\left(F_{1} M\right)=\pi_{1}(M)$.
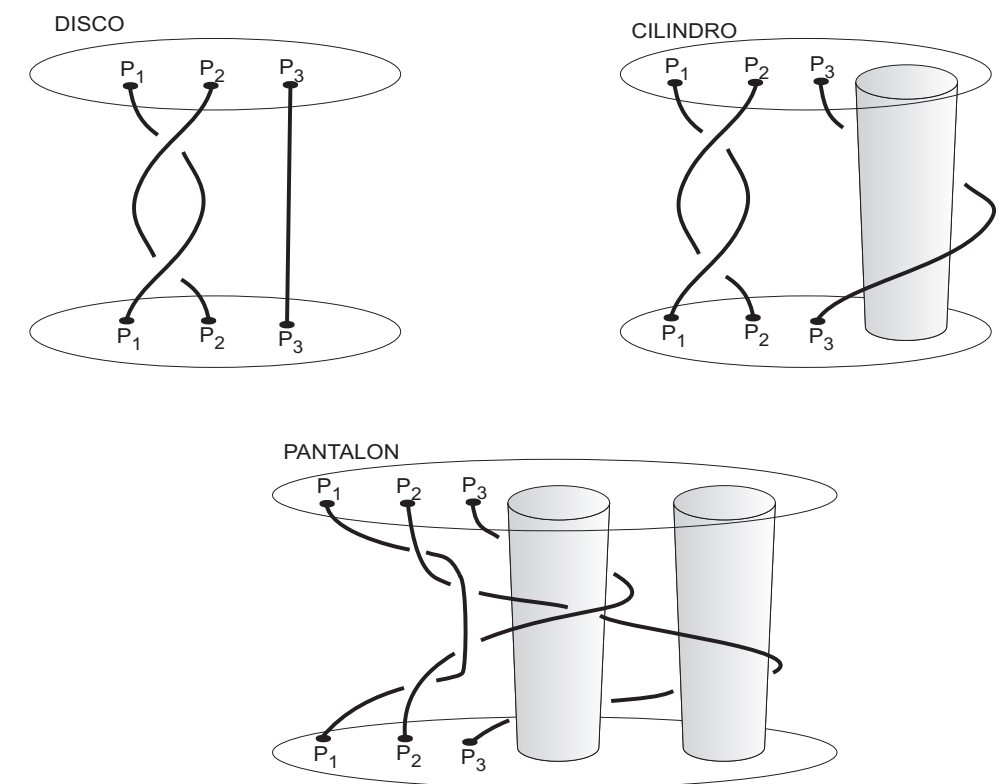

Figura 1.3: Exemplo de tranças com 3 cordas sobre o disco, o anel (cilindro) e a esfera menos 3 pontos (pantalon).

Apresentações dos grupos de tranças sobre superfícies tem sido dadas por vários autores. Inicialmente E. Artin deu a apresentação dos grupos de tranças, e de tranças puras, sobre o disco [2]. Para superfícies as primeiras apresentações destes grupos foram dadas por J. Birman [12] e P. Scott [84] e posteriormente apresentações foram dadas por P. Bel- 
lingeri [7], J. González-Meneses [41], assim como por D. Gonçalves e J. Guaschi em [33] e [38].

O grupo de tranças do disco também é conhecido como o grupo de tranças de Artin, em homenagem a E. Artin que introduziu formalmente esse conceito em [2]. Muitas vezes escreveremos apenas $P_{n}$ e $B_{n}$ no lugar de $P_{n}\left(\mathbb{D}^{2}\right)$ e $B_{n}\left(\mathbb{D}^{2}\right)$, respectivamente. Nas Figuras 1.4 e 1.5 podemos ver os geradores do grupo tranças de Artin $\sigma_{i} \in B_{n}$, para $1 \leq i \leq n-1$, bem como os geradores do grupo tranças puras de Artin

$$
A_{i, j}=\sigma_{j-1} \sigma_{j-2} \cdots \sigma_{i+1} \sigma_{i}^{2} \sigma_{i+1}^{-1} \cdots \sigma_{j-2}^{-1} \sigma_{j-1}^{-1} \in P_{n}
$$

para $1 \leq i<j \leq n$

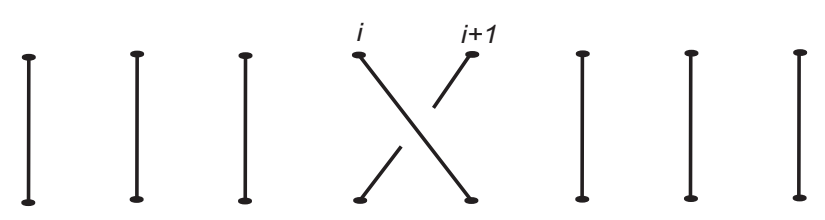

Figura 1.4: Geradores de Artin, $\sigma_{i} \in B_{n}$, para $1 \leq i \leq n-1$.

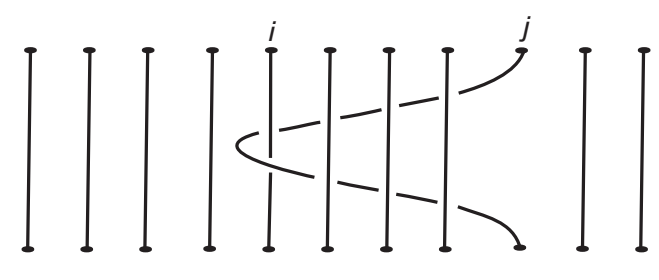

Figura 1.5: Geradores puros de Artin, $A_{i, j}=$ $\sigma_{j-1} \cdots \sigma_{i+1} \sigma_{i}^{2} \sigma_{i+1}^{-1} \cdots \sigma_{j-1}^{-1} \in P_{n}$ para $1 \leq i<j \leq n$.

Alertamos que ao invés da notação usual usada na teoria de tranças em [9] foi usada a notação simplicial, a saber $d_{i}: P_{n+1} \rightarrow P_{n}$ são as aplicações que geometricamente esquecem a $(i+1)$-ésima corda, para $0 \leq i \leq n-1$, e a numeração das cordas de uma trança qualquer sobre uma superfície $M$ começa com o zero. Por exemplo, as trancas $\sigma_{i} \in B_{n+1}$ tem subíndices variando de 0 a $n$ e os elementos $A_{i, j} \in P_{n+1}$ são considerados para valores $0 \leq i<j \leq n$.

Na Seção 6.5 de [9] os autores construíram um homomorfismo do grupo de tranças puras de Artin com $n+1$ cordas no grupo de tranças puras de Artin com $n$ cordas, o qual será usado frequentemente neste texto, e o chamaram de $\partial: P_{n+1}\left(\mathbb{D}^{2}\right) \rightarrow P_{n}\left(\mathbb{D}^{2}\right)$ definido, usando a indexação simplicial para os elementos $A_{i, j}$, por

$$
\partial\left(A_{i, j}\right)=A_{i-1, j-1}, \quad \text { para todo } 0 \leq i<j \leq n
$$

onde

$$
A_{-1, j}=\left(A_{j, j+1} A_{j, j+2} \cdots A_{j, n-1}\right)^{-1}\left(A_{0, j} A_{2, j} \cdots A_{j-1, j}\right)^{-1} \in P_{n}\left(\mathbb{D}^{2}\right),
$$

para $0 \leq j \leq n-1$, ver Figura 1.6 abaixo. Notemos que, em particular, as tranças $A_{-1,0}$ e $A_{-1, n-1}$ de $P_{n}\left(\mathbb{D}^{2}\right)$ são dadas por

$$
A_{-1,0}=\left(A_{0,1} A_{0,2} \cdots A_{0, n-1}\right)^{-1} \quad \text { e } \quad A_{-1, n-1}=\left(A_{0, n-1} A_{1, n-1} \cdots A_{n-2, n-1}\right)^{-1} .
$$


Segue pela própria definição de $\partial$, nos elementos geradores $A_{i, j}$ de $P_{n+1}$, que $\partial$ é um

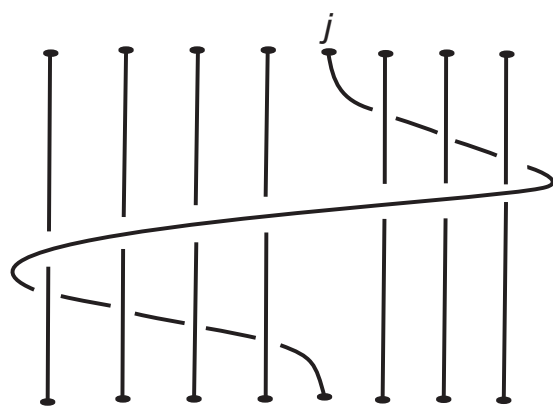

Figura 1.6: Trança $A_{-1, j}$.

homomorfismo sobrejetor.

No enunciado do Lema 6.5.2 de [9], que enunciamos novamente abaixo, não fica claro quem é $\partial\left(A_{-1,0}\right)$. Isto será tratado com detalhes na Subseção 6.1.1. No seguinte resultado é considerada a notação simplicial.

Lema 1.2.4 (Lema 6.5.2 de [9]). Para todo $n$, o homomorfismo $\partial: P_{n+1}\left(\mathbb{D}^{2}\right) \rightarrow P_{n}\left(\mathbb{D}^{2}\right)$ satisfaz as seguintes propriedades:

1. $\partial\left(A_{i, j}\right)=A_{i-1, j-1}$ para qualquer $0 \leq i<j$ e $\partial\left(A_{-1, j}\right)=1$;

2. $d_{i} \circ \partial=\partial \circ d_{i+1}$ para qualquer $0 \leq i \leq n-1$;

3. $\partial \circ \partial=\partial \circ d_{0}$.

Existem algumas imprecisões relacionadas ao Lema 1.2.4, os problemas detectados e algumas de suas consequências serão expostos nas Seções 6.1 e 6.2. Provaremos, no primeiro item da Proposição 6.1.1, que $\partial\left(A_{-1,0}\right) \neq 1$. Uma consequência do item 2 da Proposição 6.1.1, é que o item 3 do Lema 1.2.4 não é correto, ver Observações 6.1.2.

Usando o tipo de homotopia dos espaços de configuração envolvidos e a fibração de Fadell-Neuwirth (1.2) pode-se provar o seguinte resultado (ver [29], [28], [26], [91]).

Proposição 1.2.5. Suponha que $n \geq 3$ se $M=\mathbb{S}^{2}$, que $n \geq 2$ se $M=\mathbb{R} P^{2}$, e que $n \geq 1$ nos outros casos. Para $m>n$, existe uma sequência exata curta

$$
1 \longrightarrow P_{m-n}\left(M \backslash Q_{n}\right) \longrightarrow P_{m}(M) \stackrel{\rho_{*}}{\longrightarrow} P_{n}(M) \longrightarrow 1
$$

onde $Q_{n}=\left\{q_{1}, \ldots, q_{n}\right\}$.

Este resultado é clássico na teoria de tranças e a sequência (1.7) é conhecida como a sequência exata curta de tranças puras. A sequência (1.7) tem sido bastante estudada e pode ser utilizada para determinar apresentações de $P_{m}(M)$, seu centro, bem como estudar elementos de torção nesses grupos ${ }^{1}$. Uma das questões que tem sido de grande

\footnotetext{
1 A aplicação $\rho_{*}$ da Proposição 1.2 .5 pode ser vista como a induzida por uma aplicação geométrica que esquece as últimas $m-n$ cordas da trança.
} 
interesse e estudada por vários autores durante muitos anos, é o problema de cisão da sequência (1.7). A resolução desse problema levou vários estágios, começando desde os anos 60 ([12], [28], [29], [26], [91]), e finalizando com os resultados de D. Gonçalves e J. Guaschi no caso orientável [33], assim como no caso não orientável [38]. Enunciamos na sequência o resultado final do estudo do problema de cisão para os grupos de tranças sobre superfícies.

Teorema 1.2.6 (Teorema 2 [38]). Sejam $m, n \in \mathbb{N}$ e $r \geq 0$. Suponhamos que $N$ é uma superfície compacta conexa podendo ser com bordo, tomemos $\left\{x_{1}, \ldots, x_{r}\right\}$ um subconjunto finito no interior de $N$. Sejam $M=N \backslash\left\{x_{1}, \ldots, x_{r}\right\}$ e $p_{*}: P_{n+m}(M) \rightarrow P_{n}(M)$ a projeção padrão, que geometricamente esquece as últimas $m$ cordas de uma trança em $P_{n+m}(M)$.

1. Se $r>0$ ou se $M$ tem bordo não vazio, então $p_{*}$ admite uma seção para todo $m e$ $n$.

2. Suponhamos que $r=0$ e que $M$ não tem bordo. Então, $p_{*}$ admite uma seção se, e somente se, uma das seguintes condições é verificada:

(a) $M$ é a esfera $\mathbb{S}^{2}$, o toro $\mathbb{T}^{2}$ ou a garrafa de Klein $\mathbb{K}^{2}$ (para todos os valores de $m e n)$.

(b) $M=\mathbb{R} P^{2}, n=2$ e $m=1$.

(c) $M \neq \mathbb{R} P^{2}, \mathbb{S}^{2}, \mathbb{T}^{2}, \mathbb{K}^{2}$ e $n=1$.

Resultados de Fadell e Van Buskirk garantem que, para cada $n \geq 2$, o grupo de tranças de superfície $B_{n}(M)$ tem elementos de ordem finita se, e somente se, $M=\mathbb{S}^{2}$ ou $\mathbb{R} P^{2}$ [29], [75], [91]. Além disso, é provado que os únicos grupos de tranças finitos são $B_{1}\left(\mathbb{R} P^{2}\right)$, $B_{2}\left(\mathbb{R} P^{2}\right), B_{2}\left(\mathbb{S}^{2}\right), B_{3}\left(\mathbb{S}^{2}\right)$, e claramente $B_{1}\left(\mathbb{D}^{2}\right)$ e $B_{1}\left(\mathbb{S}^{2}\right)$ que são triviais. Especificamente, temos que:

- $B_{2}\left(\mathbb{S}^{2}\right)$ é isomorfo a $\mathbb{Z}_{2}$ e $P_{2}\left(\mathbb{S}^{2}\right)=\{1\}$,

- $B_{3}\left(\mathbb{S}^{2}\right)$ tem ordem 12 , é isomorfo ao produto semi-direto $\mathbb{Z}_{3} \rtimes \mathbb{Z}_{4}$, onde a ação é a não trivial, e $P_{3}\left(\mathbb{S}^{2}\right) \cong \mathbb{Z}_{2}$,

- $B_{1}\left(\mathbb{R} P^{2}\right)=P_{1}\left(\mathbb{R} P^{2}\right)$ é isomorfo a $\mathbb{Z}_{2}$,

- $B_{2}\left(\mathbb{R} P^{2}\right)$ é grupo de ordem 16 , isomorfo ao grupo dos quatérnios generalizados $\mathcal{Q}_{16}$ e cujo subgrupo de tranças puras é isomorfo com o grupo dos quatérnios $\{ \pm 1, \pm i, \pm j, \pm k\}$.

Para $n \geq 3$, temos que $B_{n}\left(\mathbb{R} P^{2}\right)$ e $B_{n+1}\left(\mathbb{S}^{2}\right)$ são grupos infinitos com elementos de torção.

Entre alguns objetos importantes dos grupos de tranças temos os chamados "half twist" e "full twist". Este último, denotado por $\Delta_{n}$, gera o centro dos grupos de tranças 
e o centro dos grupos de tranças puras do disco, da esfera, bem como do plano projetivo. Uma descrição para o "full twist" usando alguns dos geradores de $B_{n}(M)$ é dada por $\Delta_{n}=\left(\sigma_{1} \cdots \sigma_{n-1}\right)^{n}$ e um exemplo de uma trança geométrica que o representa, no caso de 5 cordas, é dado na Figura 1.7. No caso do grupo de tranças do disco, o "half twist" também é conhecido como elemento de Garside, pela estrutura de grupo de Garside que este grupo tem.

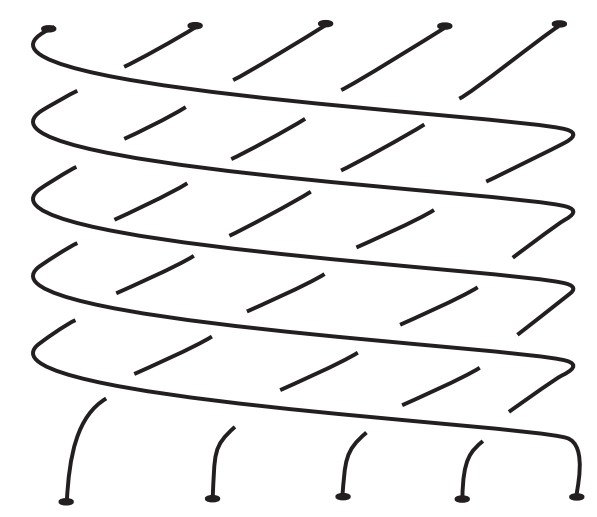

Figura 1.7: "Full twist" com 5 cordas.

Sejam $\Sigma_{n}$ o grupo de permutações dos elementos $\left\{P_{1}, \ldots, P_{n}\right\}$ e $\Sigma_{m-n}$ o grupo de permutações de $\left\{P_{n+1}, \ldots, P_{m}\right\}$. A fibração de Fadell-Neuwirth proporciona uma fibração (localmente trivial)

$$
\widehat{\rho}: F_{m} M /\left(\Sigma_{n} \times \Sigma_{m-n}\right) \longrightarrow F_{n} M / \Sigma_{n}=\widehat{F}_{n} M
$$

com fibra $\left(F_{m-n} M \backslash\left\{P_{1}, \ldots, P_{n}\right\}\right) / \Sigma_{m-n}=\widehat{F}_{m-n} M \backslash\left\{P_{1}, \ldots, P_{n}\right\}$. Portanto, tomando a sequência longa em homotopia para esta fibração podemos deduzir a seguinte proposição.

Proposição 1.2.7. Seja $M$ uma superfície conexa. Suponha que $n \geq 1$ se $M \neq \mathbb{S}^{2}, \mathbb{R} P^{2}$; $n \geq 2$ se $M=\mathbb{R} P^{2}$ e $n \geq 3$ se $M=\mathbb{S}^{2}$, e $m>n$. Então, existe uma sequência exata curta

$$
1 \longrightarrow B_{m-n}\left(M \backslash\left\{P_{1}, \ldots, P_{n}\right\}\right) \longrightarrow \sigma^{-1}\left(\Sigma_{n} \times \Sigma_{m-n}\right) \longrightarrow B_{n} M \longrightarrow 1 .
$$

Observemos que $\Sigma_{n} \times \Sigma_{m-n}$ se identifica a um subgrupo de $\Sigma_{m}$. O grupo $\sigma^{-1}\left(\Sigma_{n} \times \Sigma_{m-n}\right)$ é chamado de grupo misto de tranças com $m$ cordas sobre $M$ e é denotado por $B_{n, m-n}(M)$. Outros detalhes relacionados aos grupos mistos podem ser encontrados, por exemplo, em [35] e [36].

Entre os subgrupos interessantes dos grupos de tranças de superfície destacamos os subgrupos comensuradores estudados por L. Paris e D. Rolfsen [78] e na dissertação de mestrado do autor [76], assim como os subgrupos Brunnianos que definiremos na seguinte subseção e que são grupos com um papel central nesta tese de doutorado. Além da bem 
conhecida aplicação dos grupos de tranças na teoria de ponto fixo, outras aplicações interessantes dos grupos de tranças podem ser citadas, por exemplo, o estudo da propriedade de Borsuk-Ulam para aplicações em superfícies [39], ou o estudo de $K$-teoria de grupos de tranças sobre algumas superfícies [52], [42].

\subsubsection{Enlaçamentos e tranças Brunnianas}

Definimos um nó como sendo a imagem de um mergulho de um círculo $\mathbb{S}^{1}$ no espaço euclidiano $\mathbb{R}^{3}$; de forma mais geral, um $n$-enlaçamento $L$ é uma coleção $\left(l_{1}, \ldots, l_{n}\right)$ de mergulhos $l_{i}: \mathbb{S}^{1} \rightarrow \mathbb{R}^{3}$ tais que as imagens $l_{1}\left(\mathbb{S}^{1}\right), \ldots, l_{n}\left(\mathbb{S}^{1}\right)$ são disjuntas. Para todo $1 \leq i \leq n, l_{i}$ é chamada uma componente do n-enlaçamento $L$. Neste sentido um nó é um 1-enlaçamento. Um subenlaçamento (resp. subenlaçamento próprio) de um $n$ enlaçamento $L=\left(l_{1}, \ldots, l_{n}\right)$ é um subconjunto (resp. subconjunto próprio) de $L$ formado por $t$ (resp. $t<n)$ mergulhos $\left(l_{i_{1}}, \ldots, l_{i_{t}}\right)$, com $i_{1}, \ldots, i_{t} \in\{1, \ldots, n\}$. Ilustramos alguns exemplos nas Figuras 1.8, 1.9, e 1.10. Dizemos que um nó $K$ é trivial se existe um mergulho do disco $\mathbb{D}^{2}$ em $\mathbb{R}^{3}$, digamos $\varphi: \mathbb{D}^{2} \rightarrow \mathbb{R}^{3}$, tal que $K$ é o bordo de $\varphi\left(\mathbb{D}^{2}\right)$. Um nó trivial é tipicamente desenhado como sendo o círculo $\mathbb{S}^{1}$. De forma análoga, dizemos que $L=\left(l_{1}, \ldots, l_{n}\right)$ é um $n$-enlaçamento trivial se para cada $1 \leq i \leq n$, existe um mergulho do disco em $\mathbb{R}^{3}$, digamos $\varphi_{i}: \mathbb{D}^{2} \rightarrow \mathbb{R}^{3}$, de tal forma que $l_{i}\left(\mathbb{S}^{1}\right)$ é o bordo de $\varphi_{i}\left(\mathbb{D}^{2}\right)$, para todo $1 \leq i \leq n$, e $\varphi_{i}\left(\mathbb{D}^{2}\right) \cap \varphi_{j}\left(\mathbb{D}^{2}\right)$ é vazio, para todo $i \neq j$.

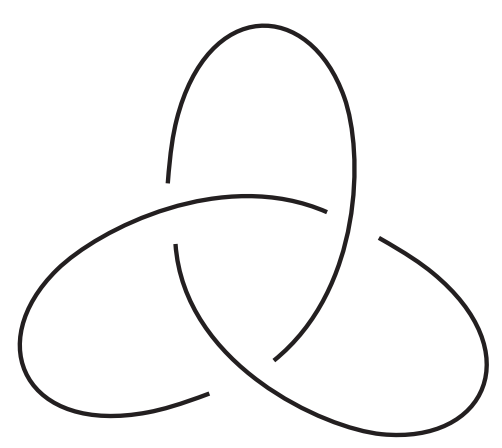

Figura 1.8: Nó Trefoil.

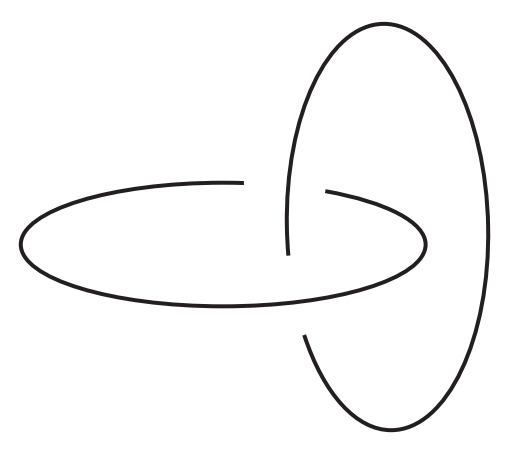

Figura 1.9: Enlaçamento de Hopf.

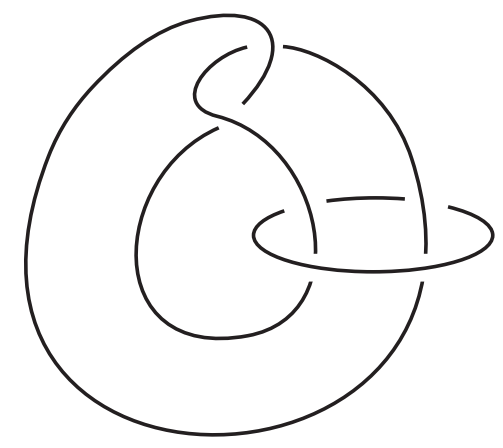

Figura 1.10: Enlaçamento de Whitehead.

Vamos enunciar o bem conhecido Teorema de Alexander que estabelece uma relação entre a teoria de enlaçamentos e a teoria de tranças. Para isto, definamos inicialmente a noção de fecho de uma trança.

Definição 1.2.8. O fecho de uma trança $\beta$ é o enlaçamento $C l(\beta)$ obtido a partir de $\beta$ conectando os pontos iniciais com os pontos finais da trança, como ilustrado na Figura 1.11.

Nas Figuras 1.12, 1.13, e 1.14 veremos tranças cujos fechos correspondem com os enlaçamentos das Figuras 1.8, 1.9, e 1.10, respectivamente. 


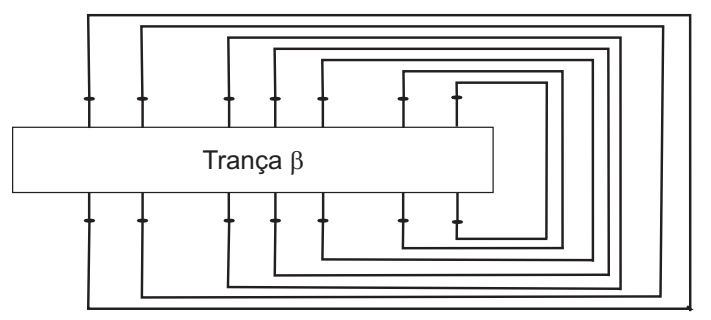

Figura 1.11: Forma geral do fecho de uma trança

Observemos que o fecho de uma trança é considerado orientado: todas as cordas da trança são orientadas da parte superior à parte inferior. O número de componentes do enlaçamento associado a uma trança depende da permutação associada à trança, como diz a seguinte proposição.

Proposição 1.2.9 (Proposição 8.1 de [64]). O número de componentes do enlaçamento dado pelo fecho de uma trança $\beta$ com $n$ cordas, $C l(\beta)$, é igual ao número de órbitas da ação da permutação associada $\sigma(\beta)$ no conjunto $\{1, \ldots, n\}$.

Segue da Proposição 1.2.9 que se uma trança $\beta$ com $n$ cordas sobre o disco é pura, então o fecho desta trança é um enlaçamento com $n$ componentes, enquanto que se $\beta$ é uma trança com permutação associada dada por um cíclo máximo de tamanho $n$, então seu fecho é um nó. Podemos enunciar agora o Teorema de Alexander.

Teorema 1.2.10 (Teorema de Alexander, Teorema 8.6 [64]). Para todo enlaçamento L existe uma trança $\beta$ tal que o fecho de $\beta$ é $L, C l(\beta)=L$.
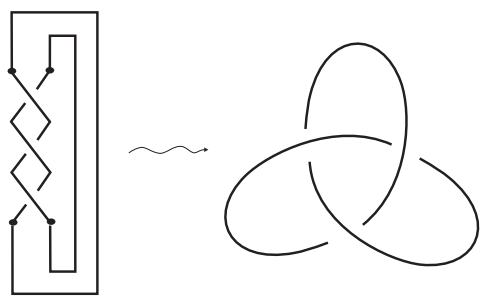

Figura 1.12: Trança $\sigma_{1}^{3} e$ seu fecho, o nó Trefoil.

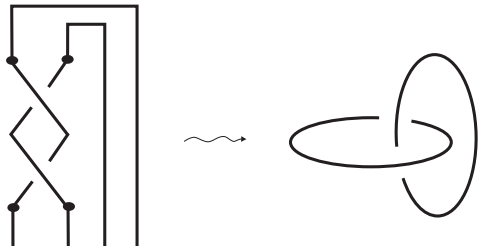

Figura 1.13: Trança $A_{1,2} e$ seu fecho, o enlaçamento de Hopf.

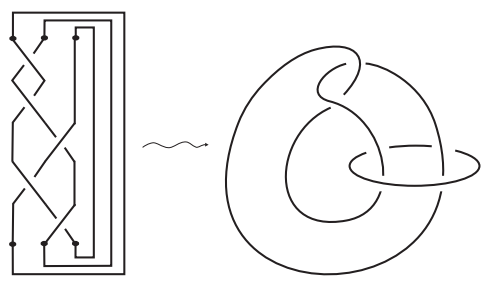

Figura 1.14: Trança $A_{1,2} \sigma_{2}^{-1} \sigma_{1} \sigma_{2}^{-1}$ e seu fecho, o enlaçamento de Whitehead.

O matemático alemão Hermann Brunn (1862-1939) estudou a existência de enlaçamentos com certas propriedades especiais. Em particular, em 1892 H. Brunn construiu nenlaçamentos não triviais $L$, para $n>1$, tais que se retiramos qualquer uma das componentes de $L$ o subenlaçamento próprio resultante é trivial ([15]). Muitos anos depois, em 1954, no artigo intitulado "Link groups" J. Milnor estudou n-enlaçamentos quasetrivias ("almost trivial links"), que ele definiu como sendo $n$-enlaçamentos com a propriedade que qualquer subenlaçamento próprio é trivial [70, Página 189]. Portanto os $n$ enlaçamentos construídos por Brunn, mencionados acima, são enlaçamentos quase-triviais, 
seguindo [70]. Milnor classificou os enlaçamentos quase-triviais, a menos de homotopia de enlaçamentos, usando os invariantes que hoje em dia são chamados de invariantes de Milnor, ver [70]. Estes invariantes de Milnor têm sido muito importantes na teoria dos nós e é bem conhecido que tais invariantes estão relacionados com os produtos de Massey no complemento do enlaçamento.

Posteriormente H. Debrunner, num artigo de 1961 intitulado "Links of Brunnian type", continuou os estudos de H. Brunn [15] e também construiu exemplos de $n$-enlaçamentos da mesma natureza que aqueles obtidos por Brunn, mas também construiu uma certa generalização destes, ver [22]. Na introdução de [22] Debrunner chamou de enlaçamento Brunniano os $n$-enlaçamentos não triviais, para $n>1$, com a propriedade que todo subenlaçamento próprio é trivial quando qualquer uma das componentes for retirada, argumentando que os primeiros exemplos de tais enlaçamentos foram dados por H. Brunn em [15], como mencionamos acima. Em [63], B. Mangum e T. Stanford provaram que os enlaçamentos Brunnianos são determinados por seus complementos. Os Anéis de Borromeo é um dos exemplos mais representativos de um enlaçamento Brunniano, ver Figura 1.15 .

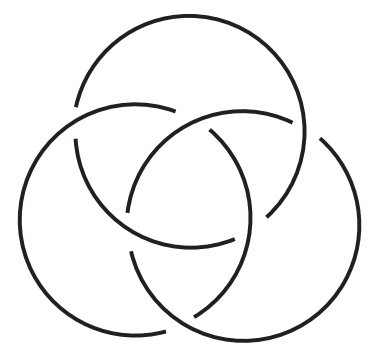

Figura 1.15: Anéis de Borromeo.

Imitando a definição dada em teoria dos nós, chamamos uma trança com $n$ cordas sobre o disco de trança Brunniana se ao removermos qualquer uma de suas cordas obtemos a trança trivial com $n-1$ cordas. Claramente um enlaçamento obtido pelo fecho de uma trança Brunniana é um enlaçamento Brunniano, mas existem enlaçamentos Brunnianos que não são obtidos pelo fecho de uma trança Brunniana, como por exemplo o enlaçamento de Whitehead, ver Figura 1.14.

As tranças Brunnianas foram consideradas inicialmente por D.L. Johnson [49], em 1982, com o nome de tranças suaves (smooth braids), depois H. Levinson [57], em 1973, as chamou de tranças decomponiveis (decomposable braids). De fato H. Levinson considerou um tipo mais geral de tranças, que ele chamou de $k$-decomponiveis. Anos mais tarde, em 1999, T. Stanford usou o termo trança Brunniana no artigo intitulado "Brunnian braids and some of their generalizations" [88].

Essas primeiras considerações de grupos de tranças Brunnianas foram dadas nos grupos de tranças sobre o disco. No entanto, seguindo a mesma ideia pode-se definir o grupo de tranças Brunnianas com $n$ cordas sobre uma superfície $M$, e que denotaremos por 
$\operatorname{Brun}_{n}(M)$. A seguir vamos formalizar a definição de trança Brunniana sobre uma superfície qualquer como sendo uma intersecção de subgrupos de tranças puras de superfície.

Para cada $i=1, \ldots, n$, existe um homomorfismo natural

$$
\rho_{i *}: P_{n}(M) \longrightarrow P_{n-1}(M)
$$

induzido pela aplicação geométrica que "esquece" a $i$-ésima corda, isto é, uma ligeira modificação da fibração de Fadell-Neuwirth (1.2), com $m=n$. Definimos o grupo de tranças Brunnianas com $n$ cordas sobre $M$ como sendo o grupo

$$
\operatorname{Brun}_{n}(M)=\bigcap_{i=1}^{n} \operatorname{Ker}\left(\rho_{i *}\right) .
$$

Ou seja, uma trança $\beta$ pertence a $\operatorname{Brun}_{n}(M)$ se, e somente se, esquecendo qualquer uma das cordas de $\beta$ temos que a trança resultante é a trança identidade em $n-1$ cordas. As tranças Brunnianas têm sido objeto de estudo de vários pesquisadores nos últimos anos, como pode-se ver em [9], [5], [17], [94] e [58]. O seguinte resultado foi provado por Cohen et al. em [9] mas também é parte da Proposição 2.2.7 que será demonstrada no próximo capítulo.

Proposição 1.2.11 (Proposição 4.2.5 de [9]). Seja $M$ uma superfície. Então, $\operatorname{Brun}_{n}(M)$ é um grupo livre para $n \geq 4$ se $M=\mathbb{S}^{2}$ e para $n \geq 3$ nos outros casos. No entanto, $\operatorname{Brun}_{3}\left(\mathbb{S}^{2}\right)=\mathbb{Z}_{2}$.

O seguinte lema, demonstrado em [5] por Bardakov et al., será reformulado e demonstrado no próximo capítulo, ver Lema 2.1.5. Tal resultado será importante no decorrer desse texto.

Lema 1.2.12 (Lema 3.8 de [5]). Sejam $M$ uma superfície e $d_{k}: P_{n}(M) \rightarrow P_{n-1}(M)$ a aplicação que esquece a k-ésima corda.

1. Suponha que $M \neq \mathbb{S}^{2}, \mathbb{R} P^{2}$. Então, para $n \geq 2$, $\operatorname{Ker}\left(d_{n}\right) \cap \operatorname{Ker}\left(d_{k}\right)=\left\langle A_{k, n}\right\rangle^{P}$, para $1 \leq k \leq n-1$ e, portanto $\operatorname{Brun}_{n}(M)=\bigcap_{k=1}^{n-1}\left\langle A_{k, n}\right\rangle^{P}$. Mais ainda,

$$
i_{n *}: \pi_{1}\left(M \backslash\left\{p_{1}, \ldots, p_{n-1}\right\}\right) \rightarrow P_{n}(M)
$$

é um monomorfismo com $i_{n *}\left(\operatorname{Ker}\left(d_{k} \mid\right)\right)=\left\langle A_{k, n}\right\rangle^{P}$, onde

$$
d_{k} \mid: \pi_{1}\left(M \backslash\left\{p_{1}, \ldots, p_{n-1}\right\}\right) \rightarrow \pi_{1}\left(M \backslash\left\{p_{1}, \ldots, p_{k-1}, p_{k+1}, \ldots, p_{n-1}\right\}\right)
$$

é o homomorfismo de grupos induzido pela inclusão que traz de volta o ponto esquecido $p_{k}$.

2. Se $M=\mathbb{S}^{2}$, então o enunciado acima vale para $n \geq 5$. 
3. Se $M=\mathbb{R} P^{2}$, então o enunciado acima vale para $n \geq 4$.

As tranças Brunnianas têm sido de interesse por diferentes motivos, como por exemplo K. Whittlesey [94] atacou um problema em Teoria de aplicações pseudo-Anosov, onde a noção de trança Brunniana foi importante para exibir os primeiros exemplos de subgrupos não triviais de "mapping class groups" que são normais e formados unicamente por elementos pseudo-Anosov. Outra aplicação muito importante corresponde à conexão entre os grupos de tranças e os grupos de homotopia da esfera $\mathbb{S}^{2}$, estudada por exemplo em [5], [9], [58] e [59]. A principal motivação para esta tese é o artigo de 2006 escrito por J. Berrick, F.R. Cohen, Y.L. Wong e J. Wu [9], onde eles exibiram a seguinte conexão:

Teorema 1.2.13 (Teorema 1.2 de [9]). Para $n \geq 5$, a sequência de grupos

$$
1 \longrightarrow \operatorname{Brun}_{n+1}\left(\mathbb{S}^{2}\right) \longrightarrow \operatorname{Brun}_{n}\left(\mathbb{D}^{2}\right) \stackrel{f_{*}}{\longrightarrow} \operatorname{Brun}_{n}\left(\mathbb{S}^{2}\right) \longrightarrow \pi_{n-1}\left(\mathbb{S}^{2}\right) \longrightarrow 1
$$

é exata, onde $f: \mathbb{D}^{2} \hookrightarrow \mathbb{S}^{2}$ é a inclusão natural.

Outros resultados têm sido provados relacionando os grupos de homotopia da esfera $\mathbb{S}^{2}$ e os grupos de tranças sobre a esfera, o plano projetivo e o disco. Um resultado de Li e $\mathrm{Wu}$ em [58], que enunciaremos no Teorema 1.3.25, mostra que o centro de quocientes apropriados dos grupos de tranças puras do disco resulta ser isomorfo com os grupos de homotopia da esfera $\mathbb{S}^{2}$. Posteriormente Bardakov, Mikhailov, Vershinin e Wu, em [5], demonstraram novamente a conexão entre os grupos de tranças Brunnianas sobre a esfera e ainda mais, eles conseguiram uma conexão entre os grupos de tranças Brunnianas sobre o plano projetivo e os grupos de homotopia da esfera $\mathbb{S}^{2}$, ver Teorema 1.2.14 abaixo. Além disso, usando a noção de grupo comutador simétrico, eles deram uma descrição dos grupos de tranças Brunnianas sobre superfícies do tipo Eilenberg-Mac Lane. No próximo teorema, para $1 \leq i<j \leq n$, será usada a notação $A_{i, j}[M]$ para denotar os elementos $A_{i, j}$ usuais de $P_{n}$, mas vistos em $P_{n}(M)$. Em geral não usaremos essa notação, a menos que seja importante distinguir claramente os elementos em cada grupo de tranças.

Teorema 1.2.14 (Teorema 1.1 de [5]). Sejam $M$ uma 2-variedade conexa e $n \geq 2$. Seja

$$
R_{n}(M)=\left[\left\langle A_{1, n}[M]\right\rangle^{P},\left\langle A_{2, n}[M]\right\rangle^{P}, \ldots,\left\langle A_{n-1, n}[M]\right\rangle^{P}\right]_{S}
$$

o subgrupo comutador simétrico.

1. Se $M \neq \mathbb{S}^{2}, \mathbb{R} P^{2}$, então

$$
\operatorname{Brun}_{n}(M)=R_{n}(M)
$$

2. Se $M=\mathbb{S}^{2}$ e $n \geq 5$, então existe uma sequência exata curta

$$
R_{n}(M) \longleftrightarrow \operatorname{Brun}_{n}\left(\mathbb{S}^{2}\right) \longrightarrow \pi_{n-1}\left(\mathbb{S}^{2}\right)
$$


3. Se $M=\mathbb{R} P^{2}$ e $n \geq 4$, então existe uma sequência exata curta

$$
R_{n}(M) \longrightarrow \operatorname{Brun}_{n}\left(\mathbb{R} P^{2}\right) \longrightarrow \pi_{n-1}\left(\mathbb{S}^{2}\right)
$$

Posteriormente mostraremos que de fato existe uma relação entre os grupos de tranças sobre qualquer superfície e os grupos de homotopia da esfera $\mathbb{S}^{2}$. Isto pode ser visto, por exemplo, no Teorema 2.3.3.

Em [97], J. Wu estabeleceu uma conexão entre os grupos de homotopia das esferas como invariantes de enlaçamentos, para enlaçamentos de tipo Brunniano. Isto reafirma a estreita relação entre os grupos de homotopia das esferas e os enlaçamentos ou tranças com a propriedade de virar um enlaçamento ou trança trivial após remover uma componente ou uma corda, respectivamente.

Um tipo de generalização da definição de trança Brunniana (no disco) foi dada por Levinson [56]. Ele considerou a noção de tranças $k$-decomponíveis, sendo que o grupo das tranças Brunnianas é igual ao grupo das tranças 1-decomponíveis. Na sequência estendemos a definição de tranças $k$-decomponíveis a qualquer superfície, dada inicialmente por Levinson para tranças sobre o disco ([56]).

Definição 1.2.15. Seja $1 \leq k \leq n-1$. Uma $n$-trança $\gamma$ sobre uma superfície $M$ será chamada de $k$-decomponível se, e somente se, quando retirarmos qualquer conjunto de $k$ cordas de $\gamma$ obtemos a trança identidade com $n-k$ cordas sobre $M$.

O subconjunto de $P_{n}(M)$ formado pelas tranças $k$-decomponíveis forma um subgrupo, que será denotado nesse texto por $\operatorname{Brun}_{n}^{k}(M)$ e chamado de grupo de tranças $k$-decomponíveis com $n$ cordas sobre $M$. Observamos que em [56], tomando $M=\mathbb{D}^{2}$, este grupo foi denotado por $D_{k, n}$. Retomaremos no próximo capítulo as tranças $k$-decomponíveis, junto com as tranças Brunnianas e um outro tipo de tranças que chamaremos de quase-Brunnianas, as quais nos permitiram estabelecer novas conexões entre os grupos de tranças sobre superfícies e os grupos de homotopia da esfera $\mathbb{S}^{2}$.

\subsection{Teoria de Homotopia Simplicial}

A teoria de homotopia simplicial foi criada praticamente ao mesmo tempo que a Topologia Algébrica. Esta última teve seu começo no início do século XX mas vista como topologia combinatória e em grande parte foi bem sucedida pelas ideias inovadoras de H. Poincaré. Em 1950, S. Eilenberg e J. A. Zilber introduziram a noção de complexo semisimplicial completo (complete semi-simplicial complex) e que posteriormente passou a ser chamado de conjunto simplicial (simplicial set). O estudo dos conjuntos simpliciais evoluiu para uma forte teoria de homotopia simplicial usando métodos combinatórios, sucesso 
este devido a contribuições profundas de forma independente por D. Kan e J. C. Moore nos anos 50 e depois por D. Quillen nos anos 60.

Nesta subseção gostaríamos de introduzir alguns dos objetos principais da teoria de homotopia simplicial, no entanto a exposição feita aqui será apenas de caráter introdutório. O leitor interessado poderá encontrar mais detalhes sobre a teoria de homotopia simplicial nos artigos de Kan [53] e de Moore [73], bem como no texto expositivo de Curtis [19] e no livro de May [67]. Para uma visão da teoria de homotopia simplicial usando teoria de categorias sugerimos o livro de Goerss e Jardine [32], mas alertamos que aqui não usaremos essa abordagem. A conexão entre a teoria de tranças e a teoria de homotopia simplicial pode ser vista no artigo de Cohen et al. [9] e nos capítulos intitulados "Simplicial Objects and Homotopy Groups" e "Introduction to Configuration Spaces and their Applications" do livro [8].

\subsection{1 $\Delta$-grupos}

Um $\Delta$-conjunto é formado por uma sequência de conjuntos $\mathcal{X}=\left\{X_{n}\right\}_{n \geq 0}$ junto com aplicações $d_{i}: X_{n} \rightarrow X_{n-1}$ chamadas de aplicações faces, para todo $0 \leq i \leq n$, tais que

$$
d_{j} d_{i}=d_{i} d_{j+1}, \text { para todo } 0 \leq i \leq j \leq n
$$

Similarmente, um $\Delta$-grupo $\mathcal{G}=\left\{G_{n}\right\}_{n \geq 0}$ é formado por um $\Delta$-conjunto $\mathcal{G}$ no qual cada $G_{n}$ é um grupo e cada face $d_{i}$ é um homomorfismo de grupos. A igualdade (1.8) é chamada algumas vezes de identidade simplicial para os $\Delta$-grupos.

Observação 1.3.1. A rigor um $\Delta$-conjunto deveria ser denotado como uma dupla formada por uma sequência de conjuntos e uma sequência de aplicações

$$
\left\{\mathcal{X} ; d^{\mathcal{X}}\right\}=\left\{X_{n} ;\left(d_{i}^{\mathcal{X}}\right)_{n}: X_{n} \rightarrow X_{n-1}, \text { com } 0 \leq i \leq n\right\}_{n \geq 0}
$$

onde as aplicações $\left(d_{i}^{\mathcal{X}}\right)_{*}$ satisfazem (1.8) e para $n=0$ não consideramos nenhuma aplicação face. É bastante comum na literatura fazer um abuso de linguagem e omitir o exceso de informação. Seguindo essa prática evitaremos nesse trabalho a notação carregada dada em (1.9) para a teoria dos $\Delta$-conjuntos, exceto quando for realmente preciso distinguir os objetos considerados.

Exemplo 1.3.2. A família formada pelos grupos de tranças puras sobre uma superfície $M$ (fixada), $\mathcal{P}(M)=\left\{P_{n+1}(M)\right\}_{n \geq 0}$, tem uma estrutura natural de $\Delta$-grupo que é induzida, em parte, a partir da fibração de Fadell-Neuwirth em cada nivel. Sabemos que o grupo $P_{n+1}(M)=\pi_{1}\left(F_{n+1} M,\left(q_{0}, q_{1}, \ldots, q_{n}\right)\right)$, onde

$$
F_{n+1} M=\left\{\left(x_{0}, x_{1}, \ldots, x_{n}\right) \in M^{n} \mid x_{i} \neq x_{j} \text { para todo } i \neq j\right\}
$$


$e\left(q_{0}, q_{1}, \ldots, q_{n}\right)$ será o ponto base das tranças com $n+1$ cordas sobre $M$. Chamamos a atenção que para o estudo da conexão entre a teoria de tranças e a teoria de homotopia, como feito em [9], é importante especificar o ponto base do grupo fundamental dos espaços de configuração. Como feito em [9, Página 275], definimos para cada $0 \leq i \leq n$ aplicações $d_{i}: F_{n+1} M \rightarrow F_{n} M$ por

$$
d_{i}\left(x_{0}, x_{1}, \ldots, x_{n}\right)=\left(x_{0}, \ldots, x_{i-1}, x_{i+1}, \ldots, x_{n}\right) .
$$

As aplicações induzidas por $d_{i}$, e que por por abuso de notação serão denotadas da mesma forma, são aplicações faces $d_{i}: P_{n+1}(M) \longrightarrow P_{n}(M)$, para $i=0, \ldots, n$, as quais são dadas pelo esquecimento da $(i+1)$-ésima corda seguido de um re-arranjo do ponto base das tranças, conforme ilustrado na Figura 1.18. Na verdade, estas aplicações faces $d_{i}$ são as restrições de umas outras aplicações faces, que por abuso de linguagem também são denotadas por $d_{i}$ porém definidas em um grupo maior, $d_{i}: B_{n+1}(M) \longrightarrow B_{n}(M)$, onde $B_{n+1}(M)=\pi_{1}\left(D_{n+1} M,\left(q_{0}, q_{1}, \ldots, q_{n}\right)\right)$, conforme explicado em detalhes na prova do Teorema 3.2.12 de [9]. A expressão formal da aplicação di é dada na terceira linha da página 283 de [9]. As Figuras 1.16 e 1.17 ilustram a definição das aplicações $d_{i}$ em $B_{n+1}(M)$. Observemos que o $\Delta$-grupo $\mathcal{P}(M)$ acima, formado por grupos de tranças sobre a superfície $M$, foi denotado em [9] por $\mathcal{F}(M)^{\pi_{1}}$.

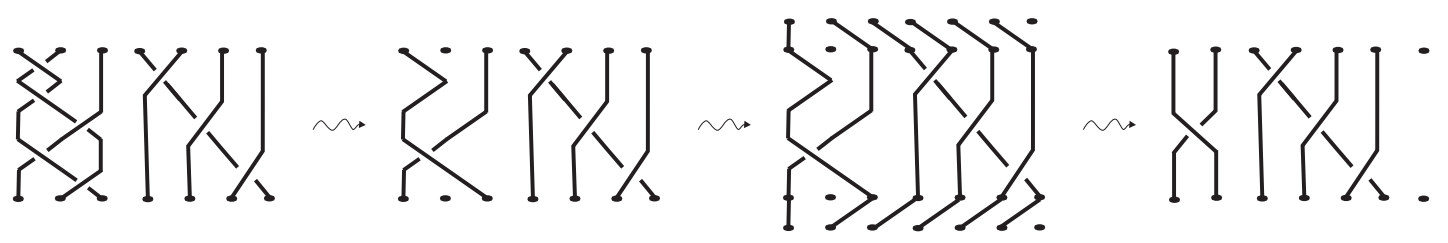

Figura 1.16: Esquecendo a segunda corda de uma trança com 7 cordas, que não é pura, e fazendo um re-arranjo do ponto base.

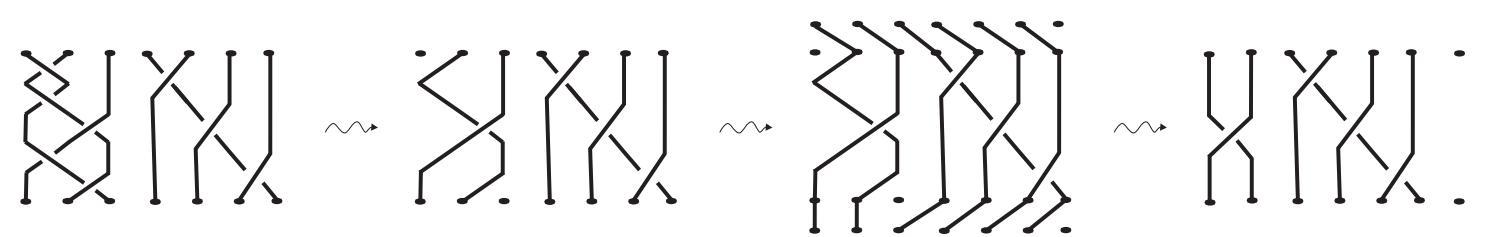

Figura 1.17: Esquecendo a primeira corda de uma trança com 7 cordas, que não é pura, e fazendo um re-arranjo do ponto base.

Uma aplicação $f=\left\{f_{n}\right\}_{n \geq 0}: \mathcal{X} \rightarrow \mathcal{X}^{\prime}$ é chamada uma $\Delta$-aplicação se o seguinte diagrama é comutativo, para todo $0 \leq i \leq n$,

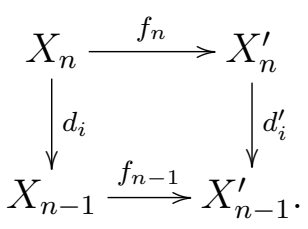



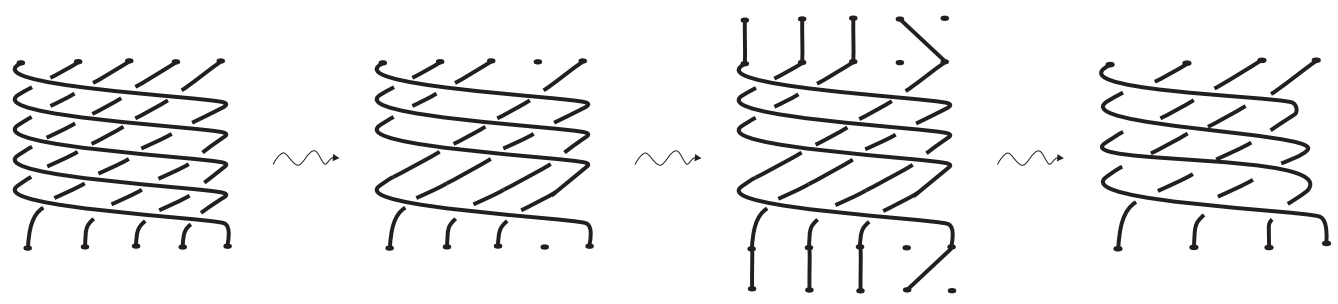

Figura 1.18: Esquecendo a quarta corda do "full twist" com 5 cordas e fazendo um re-arranjo do ponto base.

Em outras palavras, se $d_{i}^{\prime} f_{n}=f_{n-1} d_{i}$, para todo $0 \leq i \leq n$.

Exemplo 1.3.3. Seja $f: \mathbb{D}^{2} \hookrightarrow \mathbb{S}^{2}$ a inclusão natural do disco na esfera e denotemos o homomorfismo induzido nos grupos de tranças puras por $f_{n_{*}}: P_{n}\left(\mathbb{D}^{2}\right) \rightarrow P_{n}\left(\mathbb{S}^{2}\right)$. Então, $\left\{f_{n_{*}}\right\}_{n \geq 0}=f_{*}: \mathcal{P}\left(\mathbb{D}^{2}\right) \rightarrow \mathcal{P}\left(\mathbb{S}^{2}\right)$ é uma $\Delta$-aplicação.

O complexo de Moore $N \mathcal{G}=\left\{\mathcal{N}_{n} \mathcal{G}\right\}_{n \geq 0}$ de um $\Delta$-grupo $\mathcal{G}$ é definido por

$$
\mathcal{N}_{n} \mathcal{G}=\bigcap_{i=1}^{n} \operatorname{Ker}\left(d_{i}: G_{n} \rightarrow G_{n-1}\right)
$$

Lema 1.3.4 ([9], Lema 4.1.1). Seja $\mathcal{G}$ um $\Delta$-grupo. Então, $d_{0}\left(\mathcal{N}_{n} \mathcal{G}\right) \subseteq \mathcal{N}_{n-1} \mathcal{G}$ e $N \mathcal{G}$ com $d_{0}$ formam um complexo de cadeia de grupos.

Segue do lema anterior que dado um $\Delta$-grupo $\mathcal{G}$ os grupos de homologia do complexo de cadeia de grupos $\left(N \mathcal{G}, d_{0}\right)$ é bem definido. Um elemento em $\mathcal{B} d_{n} \mathcal{G}=d_{0}\left(\mathcal{N}_{n+1} \mathcal{G}\right)$ é chamado um bordo de Moore e um elemento em $\mathcal{Z}_{n} \mathcal{G}=\operatorname{Ker}\left(d_{0}: \mathcal{N}_{n} \mathcal{G} \rightarrow \mathcal{N}_{n-1} \mathcal{G}\right)$ é chamado um ciclo de Moore. A n-ésima homotopia de Moore do $\Delta$-grupo $\mathcal{G}, \pi_{n}(\mathcal{G})$, é definida como sendo o conjunto de classes laterais

$$
\pi_{n}(\mathcal{G})=H_{n}\left(N \mathcal{G}, d_{0}\right)=\mathcal{Z}_{n} \mathcal{G} / \mathcal{B} d_{n} \mathcal{G}
$$

Observemos que o conjunto de homotopias $\pi_{n}(\mathcal{G})$ não é necessariamente um grupo, devido a que, em geral, os bordos $\mathcal{B} d_{n} \mathcal{G}$ não necessariamente formam um subgrupo normal dos ciclos $\mathcal{Z}_{n} \mathcal{G}$. Por exemplo, seja $B$ um subgrupo não normal de $A$ e seja $\mathcal{G}$ o $\Delta$-grupo dado por $G_{0}=A, G_{1}=B$ e $G_{n}=\{1\}$, para $n \geq 2$, com a inclusão $d_{0}: G_{1} \hookrightarrow G_{0}$ e a aplicação trivial $d_{1}: G_{1} \rightarrow G_{0}$. Então, $\pi_{0}(\mathcal{G})$ é o conjunto de classes laterais $B / A$. Também é possível que os grupos de homotopia não sejam abelianos. Um exemplo disto pode ser visto na Proposição 7.1.1 de [9] (ver também o Teorema 3.1.6 desse texto), onde é provado que o terceiro grupo de homotopia de Moore do $\Delta$-grupo $\mathcal{P}\left(\mathbb{S}^{2}\right)$ é isomorfo com um subgrupo não abeliano do grupo de Heisenberg.

De forma similar ao feito em Topologia das Variedades, diremos que $\mathcal{G}$ é um $\Delta$-grupo contrátil se todos os seus conjuntos de homotopia de Moore são triviais. É bem conhecido que nenhuma superfície fechada (compacta e sem bordo) é contrátil, ainda mais a única 
superfície contrátil é o disco (com ou sem bordo). Isto não acontece com os $\Delta$-grupos do Exemplo 1.3.2, formados por grupos de tranças puras sobre superfícies e com aplicações faces induzidas pela fibração de Fadell-Neuwirth.

Exemplo 1.3.5. No Capítulo 3 veremos que se $M$ é uma superfície diferente da esfera $\mathbb{S}^{2}$ e do plano projetivo $\mathbb{R} P^{2}$, então os $\Delta$-grupos $\mathcal{P}(M)$ são todos contráteis, ver os Teoremas 3.1.6 e 3.1.8.

Lema 1.3.6 ([9], Lema 4.1.2). Seja $\mathcal{G}$ um $\Delta$-grupo. Então, para todo $n \geq 0$, os bordos de Moore $\mathcal{B} d_{n} \mathcal{G}$ formam um subgrupo normal de $\operatorname{Im}\left(d_{0}: G_{n+1} \rightarrow G_{n}\right)$. Em particular, os conjuntos de homotopia de Moore $\pi_{n}(\mathcal{G})$ tem estrutura de grupo se o grupo formado pelos ciclos de Moore $\mathcal{Z}_{n} \mathcal{G}$ está contido em $\operatorname{Im}\left(d_{0}: G_{n+1} \rightarrow G_{n}\right)$.

Definimos $\pi_{n}(\mathcal{G})$, anteriormente, com referência a $d_{0}$. Em princípio, usando uma aplicação face diferente $\left(d_{i}, \operatorname{com} i \neq 0\right)$, poderiam ser obtidos conjuntos de homotopia distintos, apesar de que os ciclos de Moore sempre são os mesmos e dados por

$$
\mathcal{Z}_{n} \mathcal{G}=\bigcap_{0 \leq j \leq n} \operatorname{Ker}\left(d_{j}: G_{n} \rightarrow G_{n-1}\right)
$$

Pelas relações $d_{k} d_{i}=d_{i-1} d_{k}$, para $k<i$, e $d_{k} d_{i}=d_{i} d_{k+1}$, para $k \geq i$, temos também que, para cada $0 \leq i \leq n+1$,

$$
d_{i}\left(\bigcap_{j \neq i} \operatorname{Ker}\left(d_{j}: G_{n+1} \rightarrow G_{n}\right)\right) \subseteq \mathcal{Z}_{n} \mathcal{G}
$$

De fato, seja $y \in d_{i}\left(\bigcap_{j \neq i} \operatorname{Ker}\left(d_{j}: G_{n+1} \rightarrow G_{n}\right)\right)$. Então, existe

$$
x \in \bigcap_{j \neq i} \operatorname{Ker}\left(d_{j}: G_{n+1} \rightarrow G_{n}\right)
$$

tal que $d_{i}(x)=y$. Queremos provar que $y \in \mathcal{N}_{n} \mathcal{G}=\bigcap_{i=1}^{n} \operatorname{Ker}\left(d_{i}: G_{n} \rightarrow G_{n-1}\right)$ e que $d_{0}(y)=1$. Agora,

$$
d_{k}(y)=d_{k} d_{i}(x)=\left\{\begin{array}{lll}
d_{i-1} d_{k}(x)=1, & \text { para } & k<i \\
d_{i} d_{k+1}(x)=1, & \text { para } & k \geq i
\end{array}\right.
$$

Logo, $y \in \mathcal{N}_{n} \mathcal{G}$. Em particular, para $k=0$ temos que $d_{0}(y)=1$ e portanto $y \in \mathcal{Z}_{n} \mathcal{G}$.

Seja $0 \leq i \leq n+1$, chamemos o grupo $d_{i}\left(\bigcap_{j \neq i} \operatorname{Ker}\left(d_{j}: G_{n+1} \rightarrow G_{n}\right)\right)$ de $i$-ésimo conjunto de bordos do $\Delta$-grupo $\mathcal{G}$, e notemos que o 0 -ésimo conjunto de bordos de $\mathcal{G}$ é igual ao conjunto de bordos de Moore de $\mathcal{G}$ definido anteriormente. Podemos concluir assim que, o grupo $d_{i}\left(\bigcap_{j \neq i} \operatorname{Ker}\left(d_{j}: G_{n+1} \rightarrow G_{n}\right)\right)$ é tal que $d_{i}\left(\bigcap_{j \neq i} \operatorname{Ker}\left(d_{j}: G_{n+1} \rightarrow G_{n}\right)\right) \subseteq \mathcal{Z}_{n} \mathcal{G}$; porém, pode ser diferente de $\mathcal{B} d_{n} \mathcal{G}$. Vamos estudar condições nas quais estes conjuntos de bordos coincidem, para isto introduziremos algumas definições na sequência. Seja 
$\mathcal{X}=\left\{X_{n}\right\}_{n \geq 0}$ um $\Delta$-conjunto. Os elementos $x_{0}, \ldots, x_{i-1}, x_{i+1}, \ldots, x_{n} \in X_{n-1}$ são chamados faces emparelhadas ("matching faces") com relação a $i$ se $d_{j} x_{k}=d_{k} x_{j+1}$, para $0 \leq k \leq j \leq n-1$ e para $k, j+1 \neq i$. Um $\Delta$-conjunto é fibrante se satisfaz a seguinte condição de extensão de homotopia, para todo $0 \leq i \leq n$,

Sejam $x_{0}, \ldots, x_{i-1}, x_{i+1}, \ldots, x_{n} \in X_{n-1}$ elementos quaisquer que são faces emparelhadas com relação a $i$. Então, existe um elemento $w \in X_{n}$ tal que $d_{j} w=x_{j}$ para todo $j \neq i$.

Dizemos que um $\Delta$-grupo é fibrante se ele como $\Delta$-conjunto é fibrante. A propriedade de ser fibrante é muito importante em teoria de homotopia simplicial. A seguinte proposição mostra uma condição, em termos de $\Delta$-grupo fibrante, de tal forma que os conjuntos dos bordos para os vários $d_{i}$ 's coincidem.

Proposição 1.3.7 ([9], Proposição 4.1.3). Seja $\mathcal{G}$ um $\Delta$-grupo fibrante, então

1. $\mathcal{B} d_{n} \mathcal{G}$ é um subgrupo normal de $\mathcal{Z}_{n} \mathcal{G}$, para todo $n \geq 0$;

2. $d_{i}\left(\bigcap_{j \neq i} \operatorname{Ker}\left(d_{j}: G_{n+1} \rightarrow G_{n}\right)\right)=\mathcal{B} d_{n} \mathcal{G}$, para todo $0 \leq i \leq n+1$;

3. $\pi_{n}(\mathcal{G})$ é um grupo abeliano, para todo $n \geq 1$.

Gostaríamos de observar que em algumas referências, como em [19], um $\Delta$-conjunto fibrante que também é um conjunto simplicial (que definiremos na Subeção 1.3.2) é chamado de Complexo de Kan em homenagem a D. Kan.

\subsubsection{Grupos simpliciais}

Um conjunto simplicial $\mathcal{X}$ é uma sequência de conjuntos $\mathcal{X}=\left\{X_{n}\right\}_{n \geq 0}$ junto com aplicações $d_{i}: X_{n} \rightarrow X_{n-1}$, chamadas de aplicações faces, e $s_{i}: X_{n} \rightarrow X_{n+1}$, chamadas de aplicações degenerações, para todo $0 \leq i \leq n$. Estas aplicações devem satisfazer as seguintes identidades simpliciais:

$$
\begin{gathered}
d_{j} d_{i}=d_{i} d_{j+1}, \quad \text { se } i \leq j \\
d_{i} s_{j}=\left\{\begin{array}{lll}
s_{j-1} d_{i}, & \text { se } i<j \\
i d, & \text { se } i=j, j+1 \\
s_{j} d_{i-1}, & \text { se } & i>j+1
\end{array}\right. \\
s_{i} s_{j}=s_{j+1} s_{i}, \quad \text { se } i \leq j .
\end{gathered}
$$

Um grupo simplicial $\mathcal{G}=\left\{G_{n}\right\}_{n \geq 0}$ é um conjunto simplicial em que todo $G_{n}$ é um grupo e as aplicações faces e degenerações são homomorfismos de grupos. Uma aplicação 
simplicial entre conjuntos (grupos) simpliciais $f: \mathcal{K} \rightarrow \mathcal{X}$ é uma família de aplicações $f_{n}: \mathcal{K}_{n} \rightarrow \mathcal{X}_{n}$ que comutam com as aplicações $d_{i}$ e $s_{i}$, ver o diagrama (1.14) abaixo.

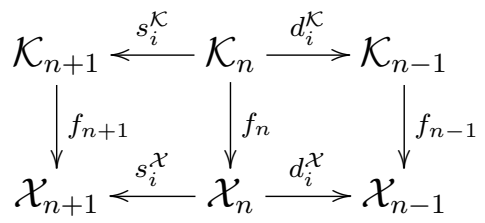

Semelhante à Observação 1.3.1 para $\Delta$-grupos, a rigor, um conjunto simplicial (ou grupo simplicial) deveria ser visto como uma terna $\left\{\mathcal{X} ; d^{\mathcal{X}} ; s^{\mathcal{X}}\right\}$. No entanto, por simplicidade escreveremos apenas $\mathcal{X}$ para denotar tal terna. Observe que um conjunto (resp. grupo) simplicial é um $\Delta$-conjunto (resp. $\Delta$-grupo) onde são adicionadas aplicações degenerações bem como algumas identidades.

Exemplo 1.3.8. Denotemos por $\Sigma_{n+1}$ o grupo simétrico formado pelas bijeções dos símbolos $0,1, \ldots, n$. Seja $\mathcal{S}=\left\{\Sigma_{n+1}\right\}_{n \geq 0}$ a sequência de grupos simétricos em $n+1$ elementos. Como ilustrado no Exemplo 3.1.1 de [9], é possível encontrar aplicações faces $d_{i}$ e degenerações $s_{i}$ de modo que $\mathcal{S}=\left\{\Sigma_{n+1}\right\}_{n \geq 0}$ se torna um conjunto simplicial. Mas estas aplicações não são homomorfismos de grupos e portanto com tais aplicações o conjunto simplicial $\mathcal{S}$ não é um grupo simplicial.

Um grupo simplicial cruzado é um conjunto simplicial $\mathcal{G}=\left\{G_{n}\right\}_{n \geq 0}$ tal que, para todo $n \geq 0, G_{n}$ é um grupo e existe um homomorfismo de grupos $\mu: G_{n} \rightarrow \Sigma_{n+1}$, definido por $g \mapsto \mu_{g}$, satisfazendo as seguintes duas condições:

1. $\mu: \mathcal{G} \rightarrow \mathcal{S}$ é uma aplicação simplicial.

2. Para todo $0 \leq i \leq n$,

$$
\begin{aligned}
& d_{i}\left(g g^{\prime}\right)=d_{i}(g) d_{i \cdot \mu_{g}}\left(g^{\prime}\right), \mathrm{e} \\
& s_{i}\left(g g^{\prime}\right)=s_{i}(g) s_{i \cdot \mu_{g}}\left(g^{\prime}\right) .
\end{aligned}
$$

\section{Exemplo 1.3.9.}

1. O conjunto simplicial $\mathcal{S}=\left\{\Sigma_{n+1}\right\}_{n \geq 0}$ com $\mu$ sendo a aplicação identidade é um grupo simplicial cruzado.

2. Um grupo simplicial $\mathcal{G}$ se torna um grupo simplicial cruzado quando o homomorfismo $\mu: G_{n} \rightarrow \Sigma_{n+1}$ é tomado como o homomorfimo trivial.

Observação 1.3.10. Um grupo simplicial cruzado não é necessariamente um grupo simplicial, pois as aplicações $d_{i}$ e $s_{i}$ não têm que ser homomorfismos de grupos.

Um morfismo $f: \mathcal{H} \rightarrow \mathcal{G}$ de grupos simpliciais cruzados é uma coleção de homomorfismos de grupos $f_{n}: H_{n} \rightarrow G_{n}$ tais que $f=\left\{f_{n}\right\}$ é uma aplicação simplicial e também $\mu_{H}=\mu_{G} \circ f$. Nota-se que $\operatorname{Ker}(\mu: \mathcal{G} \rightarrow \mathcal{S})$ é um grupo simplicial. 
A sequência de grupos $\mathcal{B}(M)=\left\{B_{n+1}(M)\right\}_{n \geq 0}$ junto com as aplicações faces $d_{i}$, que em parte são induzidas pela fibração de Fadell-Neuwirth e definidas na prova do Teorema 3.2 .12 de [9], para $0 \leq i \leq n$, não é um $\Delta$-grupo. No entanto, foi provado no mesmo teorema que $\mathcal{B}(M)$ é um $\Delta$-grupo cruzado, mas em [9] este $\Delta$-grupo cruzado foi denotado por $\mathcal{B}(M)^{\pi_{1}}$.

Exemplo 1.3.11. Existem $\Delta$-grupos que não admitem estrutura de grupo simplicial. No Corolário 3.2.13 de [9] foi provado que o $\Delta$-grupo $\mathcal{P}(M)$, do Exemplo 1.3.2, é um grupo simplicial no caso em que $M$ é o toro, a garrafa de Klein ou uma superfície com bordo não vazio. Isto não acontece para as outras superfícies. Veremos na Proposição 3.1.3 deste trabalho que se $M$ é uma superfície fechada, diferente do toro e da garrafa de Klein, então $\mathcal{P}(M)$ não admite uma estrutura de grupo simplicial com as aplicações faces dadas no Exemplo 1.3.2.

No Capítulo 3 estudaremos com mais detalhes os $\Delta$-grupos formados por grupos de tranças puras sobre superfícies com aplicações faces induzidas pela fibração de FadellNeuwirth.

Lembremos que, na Subseção 1.2.4, definimos uma trança pura em $P_{n+1}(M)$ como sendo Brunniana se ela se torna a trança trivial com $n$ cordas quando qualquer uma de suas cordas é removida e este grupo foi denotado por $\operatorname{Brun}_{n+1}(M)$. Podemos estender esta definição ao grupo de tranças $B_{n+1}(M)$, como veremos a seguir. No contexto de $\Delta$-grupos, definiremos uma trança $\beta \in B_{n+1}(M)$ como sendo uma trança Brunniana simplicial se, e somente se, $d_{i} \beta=1$, para todo $0 \leq i \leq n$, onde $d_{i}: B_{n+1}(M) \rightarrow B_{n}(M)$ é a aplicação que foi definida na prova do Teorema 3.2.12 de [9]. As tranças Brunnianas simpliciais com $n+1$ cordas sobre $M$ formam um grupo que denotaremos por $\mathfrak{B} \mathfrak{r} \mathfrak{u} \mathfrak{n}_{n+1}(M)$.

Observemos que se o número de cordas é maior ou igual que 3, então uma trança Bruniana simplicial é de fato uma trança Brunniana, em vista da seguinte proposição.

Proposição 1.3.12 (Proposição 4.2.2 de [9]). Seja $\beta$ uma trança Brunniana simplicial com $n$ cordas sobre uma superfície $M$. Se $n \geq 3$, então $\beta$ é uma trança pura.

Observação 1.3.13. As tranças Brunnianas simpliciais foram estudadas em [9] e [5], no entanto os autores as chamaram apenas de tranças Brunnianas. Neste trabalho decidimos distinguir as duas definições de forma clara e diferenciar a notação usada para o respectivo grupo formado, com o intuito de não ter confusão entre estes elementos durante este texto.

Segue da Proposição 1.3.12 que as tranças Brunnianas sobre $M$ com mais de 3 cordas são os ciclos de Moore no $\Delta$-grupo $\mathcal{P}(M)$, isto é, para $n \geq 3$

$$
\mathfrak{B r u n}_{n}(M)=\operatorname{Brun}_{n}(M)=\mathcal{Z}_{n-1} \mathcal{P}(M)
$$

Observemos que a conclusão da Proposição 1.3.12 é falsa para $n=2$. Para isto, notemos que o elemento $\sigma_{1}$ do grupo de tranças sobre uma superfície $M$ é uma trança Brunniana 
simplicial, mas tal elemento não é uma trança pura. No entanto, segue da própria definição dos grupos envolvidos que $\operatorname{Brun}_{2}(M)=\mathfrak{B r u n}_{2}(M) \cap P_{2}(M)$. Em [5] foi provado o seguinte resultado sobre os grupos de tranças Brunnianas com duas cordas, que enunciaremos usando a notação acima.

Proposição 1.3.14 (Proposição 3.3 de [5]).

1. $\operatorname{Brun}_{2}(M)=\left\langle A_{1,2}\right\rangle^{B_{2}(M)}$.

2. $\mathfrak{B r u n}_{2}(M)$ é gerado pelos elementos $A_{1,2}$ e $\sigma_{1}$.

Na Proposição 2.4 de [5] os autores demonstraram que para $n \geq 1$ o subgrupo $\operatorname{Brun}_{n}(M)$ é normal em $B_{n}(M)$ e portanto segue da Proposição 1.3.12 a Proposição 1.3.16, que corresponde ao Corolário 2.5 de [5].

Observação 1.3.15. Como vimos acima, na Proposição 3.3 de [5], foi demonstrado que Brun $_{2}(M)=\left\langle A_{1,2}\right\rangle^{B_{2}(M)}$. Porém, para qualquer superfície também vale que

$$
\left\langle A_{1,2}\right\rangle^{B_{2}(M)}=\left\langle A_{1,2}\right\rangle^{P_{2}(M)}
$$

$\operatorname{Logo}$,

$$
\operatorname{Brun}_{2}(M)=\left\langle A_{1,2}\right\rangle^{P_{2}(M)}
$$

Proposição 1.3.16 (Corolário 2.5 de [5]). Seja $n \geq 3$. O grupo $\mathfrak{B r u n}_{n}(M)=\operatorname{Brun}_{n}(M)$ é normal em $B_{n}(M)$.

Retomemos nossas considerações gerais sobre teoria de homotopia simplicial. Uma propriedade importante dos grupos simpliciais, do ponto de vista dos grupos de homotopia, foi provada por Moore e será enunciada abaixo.

Teorema 1.3.17 (Teorema de Moore [8, Teorema 2.48, página 86]). Todo grupo simplicial $\mathcal{G}$ é um conjunto simplicial fibrante.

Em particular, o Teorema de Moore garante que todos os conjuntos de homotopia de um grupo simplicial são grupos abelianos, em vista da Proposição 1.3.7.

Um conjunto simplicial com ponto base é uma dupla $(\mathcal{X}, *)$ formada por um conjunto simplicial $\mathcal{X}$ e com uma escolha de um ponto base $* \in X_{0}$ (ver [53, Definição 2.5]). Observemos que o conjunto $X_{0}$ pode ter mais de um elemento, tendo assim diferentes possíveis escolhas do ponto base. Uma aplicação entre conjuntos simpliciais com ponto base é uma aplicação simplicial que preserva o ponto base.

Enunciamos a seguir o Teorema de Adição em Homotopia ("Homotopy Addition Theorem") que usaremos no Capítulo 4. 
Teorema 1.3.18 (Teorema $2.4[19]$ ). Seja $\mathcal{X}=\left\{X_{n}\right\}_{n \geq 0}$ um conjunto simplicial fibrante com ponto base $*$. Seja $y_{i} \in X_{n}$, com $0 \leq i \leq n+1$, tal que $d_{j} y_{i}=*$, para $0 \leq j \leq n$, com $n \geq 2$. Então, em $\pi_{n}(\mathcal{X})$ temos a igualdade

$$
\left[y_{0}\right]-\left[y_{1}\right]+\left[y_{2}\right]+\cdots+(-1)^{n+1}\left[y_{n+1}\right]=0
$$

se, e somente se, existe $y \in X_{n+1}$ tal que $d_{i}(y)=y_{i}$, para $0 \leq i \leq n+1$.

O Teorema acima é o Teorema 2.67 do capítulo intitulado "Simplicial Objects and Homotopy Groups" do livro [8].

Dizemos que $\mathcal{X}=\left\{X_{n}\right\}_{n \geq 0}$ é um conjunto simplicial reduzido se $X_{0}$ é um conjunto formado por um único elemento, isto é, se $X_{0}=\{*\}$. Para enunciar o seguinte teorema precisaremos de outros elementos da teoria de homotopia simplicial que mencionaremos na continuação e que podem ser encontrados na respectiva seção do capítulo intitulado "Simplicial Objects and Homotopy Groups" do livro [8]. Na Seção 2.1 é construída a n-ésima esfera simplicial $S^{n}$, na Seção 2.2 é definida a realização geométrica para conjuntos simpliciais (denotada por $|\mathcal{X}|$ ) e na Seção 3.3 existe a construção de grupo livre de Milnor. Em particular, o Exercício 2.7 de [8, Página 77] afirma que a realização geométrica da $n$-ésima esfera simplicial $\left|S^{n}\right|$ é homeomorfa com a esfera de dimensão $n, \mathbb{S}^{n}$. No seguinte resultado formulamos um Teorema de Milnor, enunciado também no Teorema 6.1.1 de [9].

Teorema 1.3.19 (Teorema de Milnor). Se $\mathcal{X}$ é um conjunto simplicial reduzido, então a realização geométrica $|F[\mathcal{X}]|$ da construção de grupo livre de Milnor de $\mathcal{X}, F[\mathcal{X}]$, é equivalentemente homotópica a $\Omega \Sigma|\mathcal{X}|$.

No resultado anterior, $\Omega Y$ e $\Sigma Y$ significam, como é usual, o espaço de laços e a suspensão do espaço topológico $Y$, respectivamente. Segue do Teorema de Milnor o seguinte resultado imediato e que será citado posteriormente neste trabalho, onde é usado que para todo $k \geq 1$ a suspensão $\Sigma \mathbb{S}^{k}=\mathbb{S}^{k+1}$ e o fato bem conhecido sobre grupos de homotopia e o espaço de laços de um espaço topológico conexo: $\pi_{n}(\Omega Y)=\pi_{n+1}(Y)$.

Corolário 1.3.20. A construção de grupo livre de Milnor da esfera simplicial $S^{k}$, com $k \geq 1$, é tal que $\pi_{n}\left(\left|F\left[S^{k}\right]\right|\right) \cong \pi_{n+1}\left(\mathbb{S}^{k+1}\right)$, para todo $n \geq 0$.

Observação 1.3.21. Gostaríamos de observar que os grupos de homotopia de grupos simpliciais $\mathcal{G}$ são definidos usando uma certa combinatória por meio das aplicações simpliciais. Porém, na prática, este cálculo é feito usando um teorema de Moore que garante os isomorfismos

$$
H_{n}\left(N \mathcal{G}, d_{0}\right) \cong \pi_{n}(\mathcal{G}) \cong \pi_{n}(|\mathcal{G}|)
$$

para todo $n \geq 0$. Para mais detalhes sobre isto ver a Subseção 2.5.3 e a Seção 3 do capítulo intitulado "Simplicial Objects and Homotopy Groups" do livro [8], onde foi enunciado o Teorema de Moore como sendo o Teorema 3.5, na página 153. 
Antes de finalizar esta subseção veremos dois exemplos de sequências de grupos de tranças construídas em [9] e [58]. Tais sequências de grupos serão mencionadas posteriormente em vários lugares neste trabalho.

Exemplo 1.3.22. Em [9], os autores consideraram a sequência de grupos de tranças puras $\Gamma=\left\{P_{n}\right\}_{n \geq 0}$, onde $P_{0}=\{1\}$, junto com as aplicações faces dadas a seguir. Para $1 \leq i \leq n, d_{i}^{\Gamma}: P_{n} \rightarrow P_{n-1}$ é a aplicação que esquece a i-ésima corda, induzida pela fibração de Fadell-Neuwirth, isto é, $d_{i}^{\mathbb{P}}=d_{i-1}^{\mathcal{P}\left(\mathbb{D}^{2}\right)}$, para $1 \leq i \leq n$, onde $\mathcal{P}\left(\mathbb{D}^{2}\right)$ é o $\Delta$-grupo do Exemplo 1.3.2. A aplicação face $d_{0}$ foi definida como sendo $d_{0}^{\Gamma}=\partial: P_{n} \rightarrow P_{n-1}$, ver equação (1.4).

Veremos na Seção 6.1 que $\Gamma$ não é um $\Delta$-grupo, contrariando o afirmado no Corolário 6.5.3 de [9].

Esta sequência de grupos junto com as aplicações faces foi utilizada em [58], mas denotada por $\mathbb{P}$, ou seja, $\mathbb{P}=\Gamma$. Ainda mais, em [58] foram definidas aplicações degenerações da seguinte forma: $s_{i}^{\mathbb{P}}: P_{n} \rightarrow P_{n+1}$, onde $s_{i}^{\mathbb{P}}=s_{i-1}^{\mathcal{P}\left(\mathbb{D}^{2}\right)}$, para $1 \leq i \leq n$. Isto é, as degenerações $s_{i}^{\mathbb{P}}$, para $1 \leq i \leq n$, são os homomorfismos que dobram a $i$-ésima corda. Foram definidas todas as aplicações degenerações necessárias para termos um quase-grupo simplicial, no entanto, não obtemos um quase-grupo simplicial pelo fato de $\mathbb{P}$ não ser um $\Delta$-grupo. A definição de grupo quase-simplicial pode ser vista em [58, Página 532].

Exemplo 1.3.23. Em [58] é considerada a sequência de subgrupos de tranças puras $\widetilde{\Omega} \mathbb{P}$ dada por $(\widetilde{\Omega} \mathbb{P})_{n}=\operatorname{Ker}\left(d_{n+1}: P_{n+1} \rightarrow P_{n}\right)$, para todo $n \geq 0$, onde $P_{0}=\{1\}$. Para $\widetilde{\Omega} \mathbb{P}$ foram consideradas as seguintes aplicações simpliciais:

- Faces: $d_{i}^{\widetilde{\Omega} \mathbb{P}}:(\widetilde{\Omega} \mathbb{P})_{n} \rightarrow(\widetilde{\Omega} \mathbb{P})_{n-1}$, onde $d_{0}^{\widetilde{\Omega} \mathbb{P}}=\partial$ e $d_{i}^{\widetilde{\Omega} \mathbb{P}}=d_{i-1}^{\mathcal{P}\left(\mathbb{D}^{2}\right)}$, para $1 \leq i \leq n$. Isto é, as aplicações faces $d_{i}^{\widetilde{\Omega}}$, para $1 \leq i \leq n$, são as bem conhecidas aplicações que esquecem a i-ésima corda, induzidas pela fibração de Fadell-Neuwirth.

- Degenerações: $s_{i}^{\widetilde{\Omega} \mathbb{P}}: P_{n} \rightarrow P_{n+1}$, onde $s_{i}^{\widetilde{\Omega} \mathbb{P}}=s_{i-1}^{\mathcal{P}\left(\mathbb{D}^{2}\right)}$, para $1 \leq i \leq n$. Isto é, as degenerações $s_{i}^{\widetilde{\Omega} \mathbb{P}}$ para $1 \leq i \leq n$ são os homomorfismos que dobram a i-ésima $\operatorname{corda}$.

A sequência de grupos $\widetilde{\Omega} \mathbb{P}$ junto com as aplicações faces acima é um $\Delta$-grupo, e se também consideramos as aplicações degenerações acima ele é um grupo quase-simplicial.

Observação 1.3.24. Ao grupo quase-simplicial podemos definir a aplicação degeneração $s_{0}^{\widetilde{\Omega} \mathbb{P}}$ de tal forma que ele se torna um grupo simplicial. Na Subseção 4.2.1 construiremos um grupo simplicial que chamaremos de $\mathcal{K}\left(\mathbb{D}^{2}\right)$ e pode-se ver que, como grupos quasesimpliciais, $\mathcal{K}\left(\mathbb{D}^{2}\right)$ e $\widetilde{\Omega} \mathbb{P}$ são iguais, ver Observação 4.2.6. Desta forma, o grupo quasesimplicial $\widetilde{\Omega} \mathbb{P}$ junto com a aplicação degeneração $s_{0}^{\widetilde{\Omega} \mathbb{P}}$ dada por $s_{0}^{\widetilde{\Omega} \mathbb{P}}=s_{0}^{\mathcal{K}\left(\mathbb{D}^{2}\right)}$ é um grupo simplicial. 
Vamos descrever a notação que será usada no Teorema 1.3.25 que enunciaremos abaixo. Em [58, Página 522] foi definido um automorfismo $\theta: P_{n} \rightarrow P_{n}$ da seguinte forma: $\theta: A_{i, j} \mapsto A_{i, j}$, para $2 \leq i<j \leq n$, e $\theta: A_{1, j} \mapsto A_{1, j}^{-1} A_{0, j} A_{1, j}$. Observemos que estamos usando a notação usual da teoria de tranças para os elementos de $P_{n}$. Este automorfismo tem a propriedade que $d_{1} \theta=\partial: P_{n} \rightarrow P_{n-1}$, onde $d_{1}$ é o homomorfismo que esquece a primeira corda e $\partial$ é o homomorfismo definido em (1.4). Seguindo [58], dado um subgrupo $H$ de um grupo $G$ denotamos o conjunto das raízes de $H$ em $G$ por

$$
\sqrt{(H, G)}=\left\{x \in G \mid x^{q} \in H \text { para algum } q \in \mathbb{Z}, q \neq 0\right\}
$$

Seja

$$
B d_{n}=d_{1} \theta\left(\operatorname{Brun}_{n+1}\left(\mathbb{D}^{2}\right)\right)=\partial\left(\operatorname{Brun}_{n+1}\left(\mathbb{D}^{2}\right)\right) .
$$

Durante todo este trabalho denotaremos o centro de um grupo $G$ por $Z(G)$.

Teorema 1.3.25 (Teorema 1 de [58]). Seja $n \geq 4$.

1. Os seguintes grupos são iguais $\sqrt{\left(B d_{n}, P_{n}\right)}=\operatorname{Brun}_{n} \cap \theta\left(\operatorname{Brun}_{n}\left(\mathbb{D}^{2}\right)\right)$, e existe um isomorfismo de grupos $\frac{\sqrt{\left(B d_{n}, P_{n}\right)}}{B d_{n}} \cong \pi_{n}\left(\mathbb{S}^{2}\right)$.

2. Existem isomorfismos de grupos

(a) $Z\left(P_{n} / B d_{n}\right) \cong \pi_{n}\left(\mathbb{S}^{2}\right) \times \mathbb{Z}$,

(b) $Z\left(B_{n} / B d_{n}\right) \cong\left\{\alpha \in \pi_{n}\left(\mathbb{S}^{2}\right) \mid 2 \alpha=0\right\} \times \mathbb{Z}$.

Gostaríamos de observar que o conjunto das raízes de $H$ em $G$ também pode ser denotado por $\sqrt[G]{H}$ e chamado de isolador de $H$ em $G$. O conjunto $\sqrt[G]{H}$ não é necessariamente um grupo. Dizemos que um grupo $G$ tem a propriedade do isolador se para todo subgrupo $H$ de $G$ tem-se que $\sqrt[G]{H}$ é um grupo. Os grupos nilpotentes têm a propriedade do isolador, ver Teorema 4.5 das notas sobre grupos nilpotentes [44] de P. Hall.

O Teorema 1.3.25, enunciado por Li e Wu em [58], motivou o estudo de descrições dos grupos de homotopia de $\Delta$-grupos, ou de grupos simpliciais, como o centro de grupos quocientes apropriados. Mais detalhes sobre este fato são encontrados no Capítulo 4 desta tese. O Teorema 4.3.12 é o Teorema 1.3.25 acima. No entanto, daremos uma prova alternativa deste resultado e além disso, no início do Capítulo 4, faremos um comentário curto sobre algumas imprecisões encontradas na prova do Teorema 1 de [58]. 


\section{Capítulo 2}

\section{Grupos de tranças Brunnianas, quase-Brunnianas e grupos de homotopia da esfera}

Neste capítulo daremos continuação ao estudo da conexão entre a teoria de tranças sobre superfícies e os grupos de homotopia da esfera $\mathbb{S}^{2}$, motivados principalmente pelos artigos [9], [58] e [5]. A divisão deste capítulo é dada a seguir. Na Seção 2.1 vamos lembrar a definição de tranças Brunnianas sobre superfícies e daremos uma reformulação do Lema 3.8 de [5], que será bastante usada neste texto. Na Seção 2.2 definiremos os grupos de tranças quase-Brunnianas, de ordem $k$, com $n$ cordas, sobre uma superfície $M$, descreveremos algumas de suas propriedades e exibiremos a conexão encontrada destes com os grupos de homotopia de $\mathbb{S}^{2}$. A definição de tranças quase-Brunnianas de ordem $k$, foi motivada pela definição de tranças quase-Brunnianas dada por Cohen e Wu em [17] que por sua vez estende a definição de trança Brunniana. Os resultados obtidos em [9] e [5] relacionam apenas os grupos de tranças sobre a esfera e o plano projetivo com os grupos de homotopia da esfera e isto é confirmado na Seção 2.2. Usando uma outra abordagem, na Seção 2.3, veremos que na realidade os grupos de tranças sobre qualquer superfície estão relacionados com os grupos de homotopia da esfera. Para finalizar o capítulo, na Seção 2.4, provaremos que existe um homomorfismo injetor de $\operatorname{Brun}_{n+1}\left(\mathbb{S}^{2}\right)$ em $\operatorname{Brun}_{n}\left(\mathbb{D}^{2}\right)$. Este homomorfismo injetor junto com o principal teorema da Seção 2.3 nos permitem demonstrar de forma alternativa os Teoremas 1.2.13 e 1.3.25 (1).

\subsection{Grupos de tranças Brunnianas}

Lembremos da definição do grupo de tranças Brunnianas dada na Subseção 1.2.4. Para cada $i=1, \ldots, n$ existe um homomorfismo natural

$$
\rho_{i *}: P_{n}(M) \longrightarrow P_{n-1}(M)
$$


induzido pela aplicação geométrica que "esquece" a $i$-ésima corda, isto é, uma ligeira modificação da fibração de Fadell-Neuwirth (1.2), com $m=n$. Definimos o grupo de tranças Brunnianas com $n$ cordas sobre $M$ como sendo o grupo

$$
\operatorname{Brun}_{n}(M)=\bigcap_{i=1}^{n} \operatorname{Ker}\left(\rho_{i *}\right) \text {. }
$$

Ou seja, uma trança $\beta$ pertence a $\operatorname{Brun}_{n}(M)$ se, e somente se, esquecendo qualquer uma das cordas de $\beta$ temos que a trança resultante é a trança identidade em $n-1$ cordas. Claramente $\operatorname{Brun}_{n}(M)$ é normal em $P_{n}(M)$.

Seja $n \geq 3$, sabemos que o grupo de tranças Brunnianas é um grupo livre, exceto para a esfera no caso de 3 cordas (ver Proposição 1.2.11), e é um subgrupo normal de $B_{n}(M)$ (ver Proposição 1.3.16).

Sem muita dificuldade podemos dar exemplos de tranças Brunnianas, usando expressões algébricas nos geradores de $P_{n}(M)$. Usaremos na continuação os elementos

$$
A_{i, j}=\sigma_{j-1} \sigma_{j-2} \cdots \sigma_{i+1} \sigma_{i}^{2} \sigma_{i+1}^{-1} \cdots \sigma_{j-2}^{-1} \sigma_{j-1}^{-1} \in P_{n}\left(\mathbb{D}^{2}\right)
$$

para $1 \leq i<j \leq n$, correspondentes aos geradores do grupo de tranças puras sobre o disco $P_{n}\left(\mathbb{D}^{2}\right)$, um desenho destes geradores pode ser visto na Figura 1.5.

Observação 2.1.1. Observemos que os geradores de tranças puras $A_{i, j}$ pertencem ao núcleo do homomorfismo $\left(\rho_{i *}\right)$, bem como ao núcleo de $\left(\rho_{j *}\right)$, em outras palavras,

$$
A_{i, j} \in \operatorname{Ker}\left(\rho_{i *}\right) \cap \operatorname{Ker}\left(\rho_{j *}\right) .
$$

\section{Exemplo 2.1.2.}

- A trança $\left[A_{1,4},\left[A_{2,3}, A_{2,5}\right]\right]$ é uma trança pura e Brunniana em $P_{5}\left(\mathbb{D}^{2}\right)$.

- A trança $\left[\left[A_{2,3}, A_{1,6}\right],\left[A_{2,4}, A_{3,5}\right]\right]$ é uma trança pura e Brunniana em $P_{6}\left(\mathbb{D}^{2}\right)$.

- Um exemplo de trança não Brunniana no disco é dado na Figura 2.1, pois se retiramos a última corda, a trança resultante não é a trança identidade. Na Figura 2.2 vemos com um exemplo que se $M$ é simplesmente conexa, então claramente toda trança com 2 cordas é Brunniana, mas isto não acontece no caso em que o grupo fundamental de $M$ não é trivial.

$\mathrm{Na}$ continuação vamos provar que o grupo de tranças Brunnianas com 3 cordas sobre o disco é igual ao subgrupo comutador de um grupo livre em dois geradores.

Proposição 2.1.3. O grupo de tranças Brunnianas com 3 cordas sobre o disco é igual ao subgrupo comutador do grupo livre em dois geradores $F_{2}=\operatorname{Ker}\left(d_{0}: P_{3} \rightarrow P_{2}\right)$, isto é,

$$
\operatorname{Brun}_{3}\left(\mathbb{D}^{2}\right)=\left[F_{2}, F_{2}\right]
$$




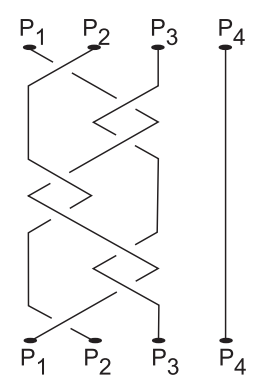

Figura 2.1: Trança com 4 cordas sobre o disco que não é Brunniana.
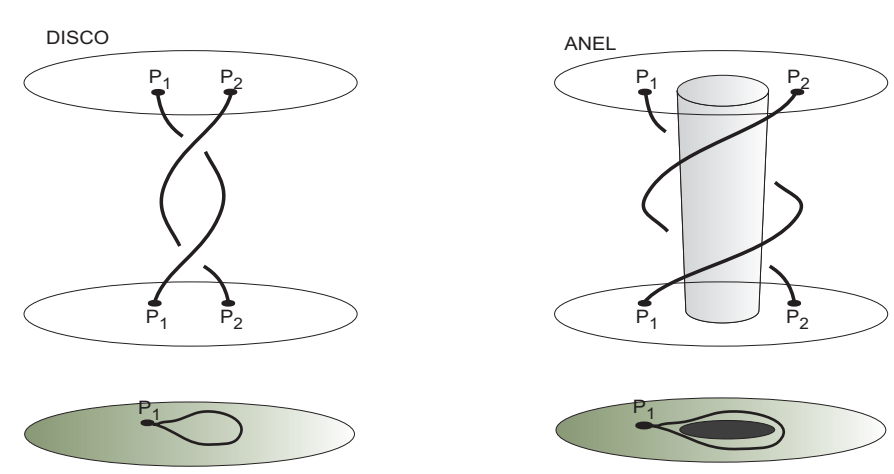

Figura 2.2: Trança com 2 cordas no disco (Brunniana) e no anel (não Brunniana).

Demonstração. Nessa prova usaremos a indexação usual da teoria de homotopia simplicial para as aplicações que esquecem cordas, bem como para os geradores dos grupos de tranças. Claramente tem-se que $\left[F_{2}, F_{2}\right] \subseteq \operatorname{Brun}_{3}\left(\mathbb{D}^{2}\right)$, com $F_{2}=F_{2}\left(A_{0,1}, A_{0,2}\right)$.

Lembremos que $P_{3}\left(\mathbb{D}^{2}\right)=F_{2} \oplus \mathbb{Z}$, onde

$$
F_{2}=F_{2}\left(A_{0,1}, A_{0,2}\right)=\operatorname{Ker}\left(d_{0}: P_{3}\left(\mathbb{D}^{2}\right) \rightarrow P_{2}\left(\mathbb{D}^{2}\right)\right)
$$

Denotemos por $a=A_{0,1}, b=A_{0,2}$. Lembremos também que as aplicações faces aplicam "full twist" em "full twist". As aplicações faces $d_{j}: P_{3}\left(\mathbb{D}^{2}\right) \rightarrow P_{2}\left(\mathbb{D}^{2}\right)$, para $j=0,1,2$, são definidas seguindo a tabela:

\begin{tabular}{|c||c|c|c|}
\hline & $d_{0}$ & $d_{1}$ & $d_{2}$ \\
\hline \hline$a$ & 0 & 0 & $\Delta_{2}$ \\
\hline$b$ & 0 & $\Delta_{2}$ & 0 \\
\hline$\Delta_{3}$ & $\Delta_{2}$ & $\Delta_{2}$ & $\Delta_{2}$ \\
\hline
\end{tabular}

Tabela 2.1: $d_{j}(x)$, para $j=0,1,2$ e $x=a, b, \Delta_{3}$.

Seja $\beta \in P_{3}\left(\mathbb{D}^{2}\right)=F_{2} \oplus \mathbb{Z}$, então a expressão reduzida para $\beta$ é $a^{k_{1}} b^{l_{1}} \cdots a^{k_{t}} b^{l_{t}} \Delta_{3}^{r}$, para $k_{j}, l_{j}, r \in \mathbb{Z}$, com $1 \leq j \leq t$. Lembremos que $P_{n}\left(\mathbb{D}^{2}\right)$ é livre de torção, portanto se $\beta \in \operatorname{Brun}_{3}\left(\mathbb{D}^{2}\right)$, teremos que

$$
\left\{\begin{aligned}
r & =0 \\
\sum k_{j} & =0 \\
\sum l_{j} & =0
\end{aligned}\right.
$$

De $r=0$ segue que $\beta \in F_{2}$, ainda mais, usando a outra informação obtida concluímos que $\beta \in\left[F_{2}, F_{2}\right]$. De fato, considerando a sequência exata curta

$$
1 \longrightarrow\left[F_{2}, F_{2}\right] \longrightarrow F_{2} \stackrel{\rho}{\longrightarrow} F_{2} /\left[F_{2}, F_{2}\right] \longrightarrow 0
$$

temos que para $\beta \in \operatorname{Brun}_{3}\left(\mathbb{D}^{2}\right)$, vale $\rho(\beta)=0=\left(\sum_{j=0}^{t} k_{j}, \sum_{j=0}^{t} l_{j}\right)$. Mostramos assim que $\operatorname{Brun}_{3}\left(\mathbb{D}^{2}\right) \subseteq\left[F_{2}, F_{2}\right]$, terminando a prova. 
Dado que o grupo de tranças $P_{3}$ pode ser descrito como $P_{3}=F_{2} \rtimes \mathbb{Z}$, então segue imediatamente da Proposição 2.1.3 o seguinte resultado.

Corolário 2.1.4. O grupo de tranças Brunnianas com 3 cordas sobre o disco é igual ao subgrupo comutador de $P_{3}$

$$
\operatorname{Brun}_{3}\left(\mathbb{D}^{2}\right)=\left[P_{3}, P_{3}\right]
$$

O seguinte lema é uma reformulação do Lema 3.8 de [5] e sua prova decorre de forma similar àquela dada aí. Notemos também que o Lema 2.1.5 será bastante usado ao longo desse texto.

Lema 2.1.5. Suponhamos que $n \geq 5$ se $M=\mathbb{S}^{2}$, que $n \geq 4$ se $M=\mathbb{R} P^{2}$ e que $n \geq 2$ nos outros casos. Então, a intersecção

$$
\operatorname{Ker}\left(d_{j}: P_{n}(M) \rightarrow P_{n-1}(M)\right) \cap \operatorname{Ker}\left(d_{k}: P_{n}(M) \rightarrow P_{n-1}(M)\right)
$$

é tal que

$$
\begin{aligned}
\operatorname{Ker}\left(d_{j}\right) \cap \operatorname{Ker}\left(d_{k}\right) & =\left\langle A_{k, j}\right\rangle^{i_{j_{*}}\left(\pi_{1}\left(M \backslash\left\{q_{1}, \ldots, \widehat{q}_{j}, \ldots, q_{n}\right\}\right)\right)} \\
& =\left\langle A_{k, j}\right\rangle^{i_{k_{*}}\left(\pi_{1}\left(M \backslash\left\{q_{1}, \ldots, \widehat{q_{k}}, \ldots, q_{n}\right\}\right)\right)}=\left\langle A_{k, j}\right\rangle^{P_{n}(M)}
\end{aligned}
$$

para quaisquer $1 \leq k<j \leq n$.

Fixemos $m$, com $1 \leq m \leq n$. Portanto, para $n \geq 5$ se $M=\mathbb{S}^{2}, n \geq 4$ se $M=\mathbb{R} P^{2}$ e $n \geq 3$ nos outros casos, vale que

$$
\operatorname{Brun}_{n}(M)=\left(\bigcap_{1 \leq l<m}\left\langle A_{l, m}\right\rangle^{P_{n}(M)}\right) \bigcap\left(\bigcap_{m<r \leq n}\left\langle A_{m, r}\right\rangle^{P_{n}(M)}\right) .
$$

Ainda mais, se $n \geq 3$ e $M$ é diferente da esfera $\mathbb{S}^{2}$ e do plano projetivo $\mathbb{R} P^{2}$ então temos a seguinte descrição para o grupo de tranças Brunnianas sobre $M$

$$
\operatorname{Brun}_{n}(M)=\left[\left\langle A_{1, m}\right\rangle^{P_{n}(M)}, \cdots,\left\langle A_{m-1, m}\right\rangle^{P_{n}(M)},\left\langle A_{m, m+1}\right\rangle^{P_{n}(M)}, \ldots,\left\langle A_{m, n}\right\rangle^{P_{n}(M)}\right]_{S} .
$$

Demonstração. Lembremos da identidade simplicial

$$
d_{j-1} d_{k}=d_{k} d_{j} \text {, para } k+1 \leq j \leq n
$$


Consideremos o seguinte diagrama comutativo, para $1 \leq k<j \leq n$ :

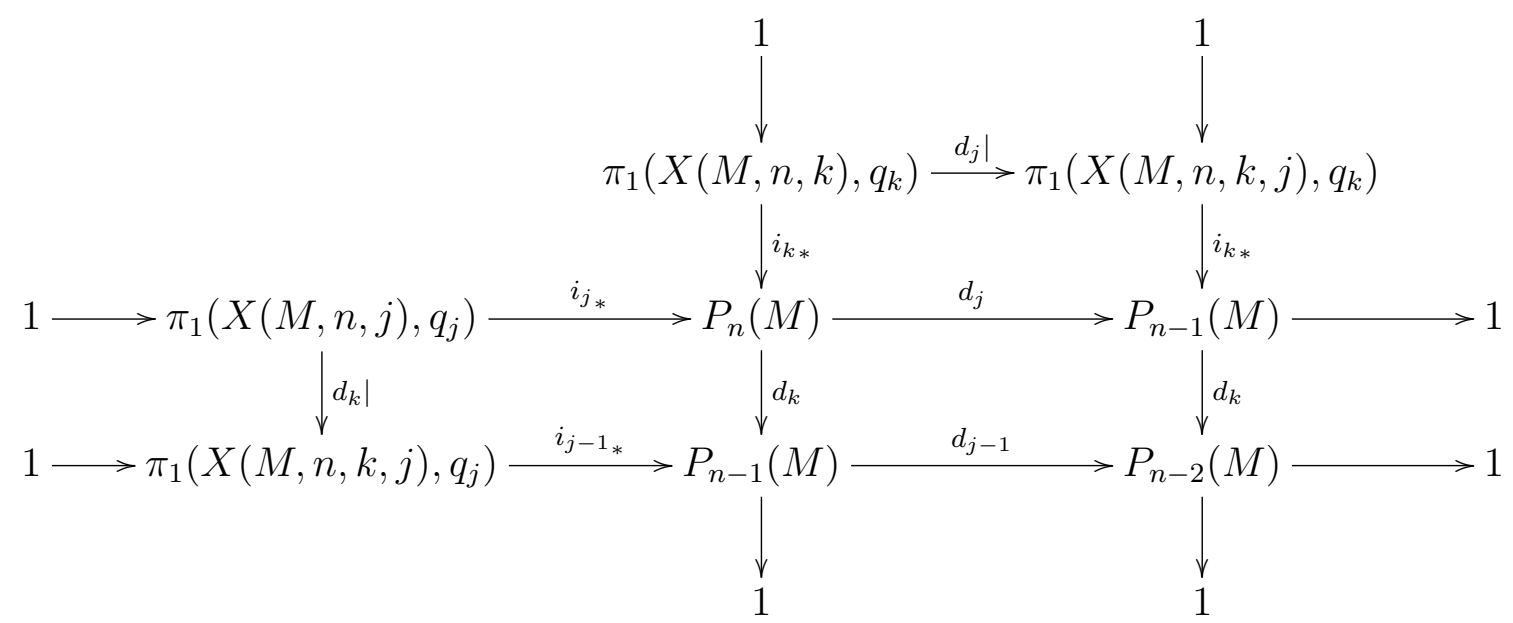

onde os espaços $X(M, n, t)$ e $X(M, n, s, t)$ são dados por $M \backslash\left\{q_{1}, \ldots, \widehat{q_{t}}, \ldots, q_{n}\right\}$ e $M \backslash\left\{q_{1}, \ldots, \widehat{q_{s}}, \ldots, \widehat{q_{t}}, \ldots, q_{n}\right\}$, respectivamente, supondo que $s<t$ e $s, t \in\{1,2, \ldots, n\}$.

Observemos que o quadrado inferior direito no diagrama acima comuta em virtude da identidade simplicial e que, nas hipóteses do lema, a fibração de Fadell-Neuwirth garante que as sequências horizontais e verticais são de fato sequências exatas curtas.

Como consequência, a restrição $i_{j_{*}} \mid: \operatorname{Ker}\left(d_{k} \mid\right) \rightarrow \operatorname{Ker}\left(d_{k}\right) \cap \operatorname{Ker}\left(d_{j}\right)$ é um monomorfismo. Vamos demonstrar que $i_{j_{*}} \mid$ é de fato um isomorfismo, falta provar que tal aplicação é sobrejetora. Seja $\gamma \in \operatorname{Ker}\left(d_{k}\right) \cap \operatorname{Ker}\left(d_{j}\right)$, então existe $\gamma_{1} \in \pi_{1}\left(M \backslash\left\{q_{1}, \ldots, \widehat{q_{j}}, \ldots, q_{n}\right\}, q_{j}\right)$ tal que $i_{j_{*}}\left(\gamma_{1}\right)=\gamma$. Usando a comutatividade do diagrama acima temos que

$$
i_{j-1_{*}} \circ d_{k} \mid\left(\gamma_{1}\right)=d_{k} \circ i_{j_{*}}\left(\gamma_{1}\right)=1
$$

e como $i_{j-1_{*}}$ é um monomorfismo, então segue que $d_{k} \mid\left(\gamma_{1}\right)=1$. Logo, $\gamma_{1} \in \operatorname{Ker}\left(d_{k} \mid\right)$ e $i_{j_{*}}\left(\gamma_{1}\right)=\gamma$. Ou seja, $i_{j_{*}} \mid: \operatorname{Ker}\left(d_{k} \mid\right) \rightarrow \operatorname{Ker}\left(d_{k}\right) \cap \operatorname{Ker}\left(d_{j}\right)$ é sobrejetora. Logo,

$$
i_{j_{*}} \mid: \operatorname{Ker}\left(d_{k} \mid\right) \rightarrow \operatorname{Ker}\left(d_{k}\right) \cap \operatorname{Ker}\left(d_{j}\right)
$$

é um isomorfismo.

De forma completamente similar prova-se que

$$
i_{k *} \mid: \operatorname{Ker}\left(d_{j} \mid\right) \rightarrow \operatorname{Ker}\left(d_{k}\right) \cap \operatorname{Ker}\left(d_{j}\right)
$$

é um isomorfismo.

Similarmente ao feito em [5], notemos que $\operatorname{Ker}\left(d_{k} \mid\right)$ é o fecho normal no grupo fundamental $\pi_{1}\left(M \backslash\left\{q_{1}, \ldots, \widehat{q_{j}}, \ldots, q_{n}\right\}, q_{j}\right)$ gerado pela classe de homotopia $\left[w_{k}\right]$, onde $w_{k}$ é um pequeno círculo em torno de $q_{k}$, isto é, $\operatorname{Ker}\left(d_{k} \mid\right)=\left\langle\left[w_{k}\right]\right\rangle^{\pi_{1}\left(M \backslash\left\{q_{1}, \ldots, \widehat{q}_{j}, \ldots, q_{n}\right\}, q_{j}\right)}$.

Provemos agora que $i_{j_{*}}\left(\left[w_{k}\right]\right)=A_{k, j}^{ \pm 1}$. Para isto consideremos o seguinte diagrama 
comutativo

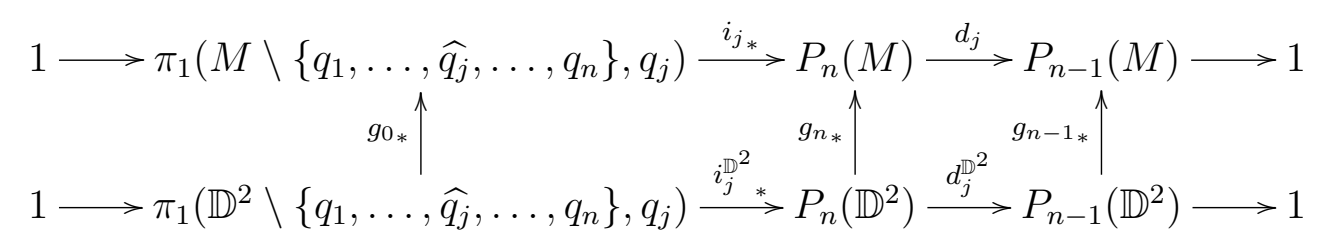

onde $g_{*}$ é induzida geometricamente a partir da inclusão natural $g: \mathbb{D}^{2} \hookrightarrow M$.

Observemos que $w_{k}$ é um pequeno círculo ao redor de $q_{k}$ e portanto podemos dizer que $\left[w_{k}^{\mathbb{D}^{2}}\right] \in \pi_{1}\left(\mathbb{D}^{2} \backslash\left\{q_{1}, \ldots, \widehat{q_{j}}, \ldots, q_{n}\right\}, q_{j}\right)$ é tal que

$$
g_{0 *}\left(\left[w_{k}^{\mathbb{D}^{2}}\right]\right)=\left[w_{k}\right] .
$$

Por outro lado, sabemos que $i_{j}^{\mathbb{D}^{2}}\left(\left[w_{k}^{\mathbb{D}^{2}}\right]\right)=A_{k, j}^{ \pm 1}\left[\mathbb{D}^{2}\right]$. Logo, usando a comutatividade do diagrama anterior e o fato que $g_{n *}\left(A_{k, j}^{ \pm 1}\left[\mathbb{D}^{2}\right]\right)=A_{k, j}^{ \pm 1}[M]$, temos que $i_{j_{*}}\left(\left[w_{k}\right]\right)=A_{k, j}^{ \pm 1}[M]$. $\mathrm{O}$ sinal \pm 1 depende da orientação do pequeno círculo ao redor de $p_{k}$.

Provamos acima que $\operatorname{Ker}\left(d_{k} \mid\right)=\left\langle\left[w_{k}\right]\right\rangle^{\pi_{1}\left(M \backslash\left\{q_{1}, \ldots, \widehat{q}_{j}, \ldots, q_{n}\right\}, q_{j}\right)}$, portanto

$$
i_{j_{*}}\left(\operatorname{Ker}\left(d_{k} \mid\right)\right)=\left\langle A_{k, j}\right\rangle^{i_{j_{*}}\left(\pi_{1}\left(M \backslash\left\{q_{1}, \ldots, \widehat{q}_{j}, \ldots, q_{n}\right\}, q_{j}\right)\right)}=\operatorname{Ker}\left(d_{j}\right) \cap \operatorname{Ker}\left(d_{k}\right)
$$

donde segue que

$$
\operatorname{Ker}\left(d_{j}\right) \cap \operatorname{Ker}\left(d_{k}\right)=i_{j_{*}}\left(\operatorname{Ker}\left(d_{k} \mid\right)\right) \subseteq\left\langle A_{k, j}\right\rangle^{P} .
$$

Além disso, como $A_{k, j}$ pertence ao subgrupo normal $\operatorname{Ker}\left(d_{j}\right) \cap \operatorname{Ker}\left(d_{k}\right)$ de $P_{n}(M)$, então segue claramente que

$$
\left\langle A_{k, j}\right\rangle^{P} \subseteq \operatorname{Ker}\left(d_{j}\right) \cap \operatorname{Ker}\left(d_{k}\right)
$$

Logo, nas hipóteses do lema, para $M$ e $n$ temos que

$$
\left\langle A_{k, j}\right\rangle^{i_{j_{*}}\left(\pi_{1}\left(M \backslash\left\{q_{1}, \ldots, \widehat{q}_{j}, \ldots, q_{n}\right\}, q_{j}\right)\right)}=\operatorname{Ker}\left(d_{j}\right) \cap \operatorname{Ker}\left(d_{k}\right)=\left\langle A_{k, j}\right\rangle^{P}
$$

para qualquer $1 \leq k<j \leq n$.

Similarmente prova-se que

$$
\left\langle A_{k, j}\right\rangle^{i_{k *}\left(\pi_{1}\left(M \backslash\left\{q_{1}, \ldots, \widehat{q_{k}}, \ldots, q_{n}\right\}, q_{k}\right)\right)}=\operatorname{Ker}\left(d_{j}\right) \cap \operatorname{Ker}\left(d_{k}\right)=\left\langle A_{k, j}\right\rangle^{P}
$$

para qualquer $1 \leq k<j \leq n$, completando a demonstração da primeira parte.

Como consequência das igualdades dadas em (2.1) ou (2.2) temos a seguinte descrição para o grupo $\operatorname{Brun}_{n}(M)$. Fixemos um número $m$ arbitrário, $1 \leq m \leq n$. Então, para 
$n \geq 5$ se $M=\mathbb{S}^{2}, n \geq 4$ se $M=\mathbb{R} P^{2}$ e $n \geq 3$ nos outros casos, temos que:

$$
\begin{aligned}
\operatorname{Brun}_{n}(M)= & \operatorname{Ker}\left(d_{1}\right) \cap \operatorname{Ker}\left(d_{2}\right) \cap \cdots \cap \operatorname{Ker}\left(d_{m}\right) \cap \cdots \cap \operatorname{Ker}\left(d_{n}\right) \\
= & \left(\operatorname{Ker}\left(d_{1}\right) \cap \cdots \cap \operatorname{Ker}\left(d_{m}\right)\right) \cap\left(\operatorname{Ker}\left(d_{m}\right) \cap \cdots \cap \operatorname{Ker}\left(d_{n}\right)\right) \\
= & \left(\operatorname{Ker}\left(d_{1}\right) \cap \operatorname{Ker}\left(d_{m}\right)\right) \cap \cdots \cap\left(\operatorname{Ker}\left(d_{m-1}\right) \cap \operatorname{Ker}\left(d_{m}\right)\right) \cap \\
& \left(\operatorname{Ker}\left(d_{m}\right) \cap \operatorname{Ker}\left(d_{m+1}\right)\right) \cap \cdots \cap\left(\operatorname{Ker}\left(d_{m}\right) \cap \operatorname{Ker}\left(d_{n}\right)\right) \\
= & \left(\bigcap_{1 \leq l<m}\left\langle A_{l, m}\right\rangle^{P}\right) \cap\left(\bigcap_{m<r \leq n}\left\langle A_{m, r}\right\rangle^{P}\right)
\end{aligned}
$$

como queríamos provar. Suponhamos que $n \geq 3$ e $M$ é diferente da esfera $\mathbb{S}^{2}$ e do plano projetivo $\mathbb{R} P^{2}$. A demonstração da igualdade

$$
\operatorname{Brun}_{n}(M)=\left[\left\langle A_{1, m}\right\rangle^{P_{n}(M)}, \cdots,\left\langle A_{m-1, m}\right\rangle^{P_{n}(M)},\left\langle A_{m, m+1}\right\rangle^{P_{n}(M)}, \ldots,\left\langle A_{m, n}\right\rangle^{P_{n}(M)}\right]_{S} .
$$

decorre de forma similar com aquela dada para o Teorema 1.1 de [5] (ver Teorema 1.2.14).

Observação 2.1.6. É importante observar que a informação adicional no Lema 2.1.5, comparado com o Lema 3.8 de [5], consiste em permitir na descrição dos grupos Brunnianos a presença de elementos do núcleo $\operatorname{Ker}\left(d_{j}: P_{n}(M) \rightarrow P_{n-1}(M)\right)$, para $1 \leq j \leq n$, e não apenas dos elementos de $\operatorname{Ker}\left(d_{n}: P_{n}(M) \rightarrow P_{n-1}(M)\right)$ como feito em [5]. Isto em alguns casos poderá ser de grande utilidade, como por exemplo, na prova da Proposição 3.4.1.

\subsection{Grupos de tranças quase-Brunnianas e grupos de homotopia da 2-esfera}

Nesta seção introduziremos a noção de grupo de tranças quase-Brunnianas, de ordem $k$, sobre uma superfície $M$ dada. O caso $k=1$, chamado apenas de grupo de tranças quase-Brunnianas sobre $M$ e que será explicado posteriormente, tem sido considerado de forma explícita por Cohen e Wu [17], bem como de forma indireta por Cohen et al. [9] no estudo de conexões entre grupos de tranças sobre a esfera e grupos de homotopia da esfera 2-dimensional. Vale a pena mencionar que os grupos de tranças Brunnianas sobre uma superfície $M$ são subgrupos dos grupos de tranças quase-Brunnianas sobre $M$.

Denotaremos as aplicações que esquecem a $j$-ésima corda por $d_{j}: B_{m}(M) \rightarrow B_{m-1}(M)$ para $j=1,2, \ldots, m$, como em [9], mas não usaremos a notação simplicial que começa indexando as aplicações com o zero. Alertamos que tais aplicações quando definidas no grupo de tranças $B_{m}(M)$ não são homomorfismos, no entanto elas viram homomorfismos quando restritas aos grupos de tranças puras $P_{n}(M)$. Os homomorfismos $d_{j}$ restritos ao grupo de tranças puras $P_{m}(M), d_{j}: P_{m}(M) \rightarrow P_{m-1}(M)$, são exatamente os homomorfismos usuais induzidos pela fibração de Fadell-Neuwirth. 
Seja $\beta \in B_{m}(M)$ tal que $d_{j}(\beta)=1$, para todo $j=k+1, k+2, \ldots, m$. Se $k=0$, então $\beta$ é uma trança Brunniana sobre $M$, e se adicionalmente $m \geq 3, \beta \in P_{m}(M)$, pois $\operatorname{Brun}_{m}(M) \subseteq P_{m}(M)$ para $m \geq 3$. Suponhamos que $1 \leq k \leq m-1$, então esquecendo as últimas $m-k$ cordas de $\beta$ obtemos a trança identidade em $k$ cordas. Logo, $\beta \in B_{1, \ldots, 1, m-k}(M) \subseteq B_{k, m-k}(M)$. Nas Figuras 2.3 e 2.4 pegamos uma trança $\beta \in B_{5}$ e observamos o resultado de tirar as primeiras duas cordas, bem como as três últimas cordas, respectivamente.

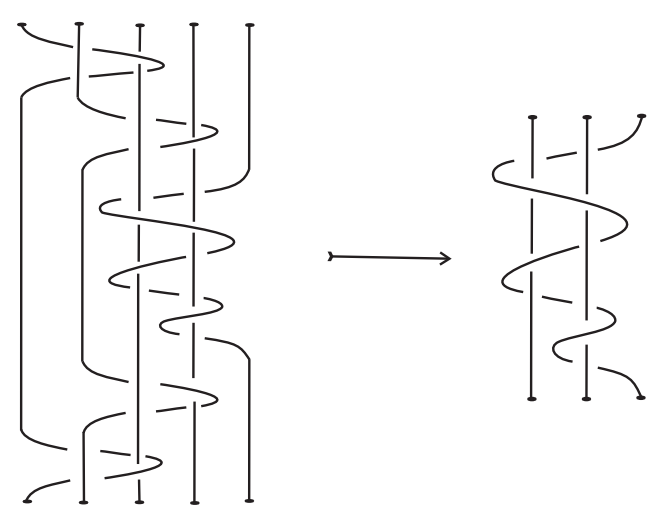

Figura 2.3: Esquecendo as duas primeiras cordas de uma trança $\beta \in B_{5}$

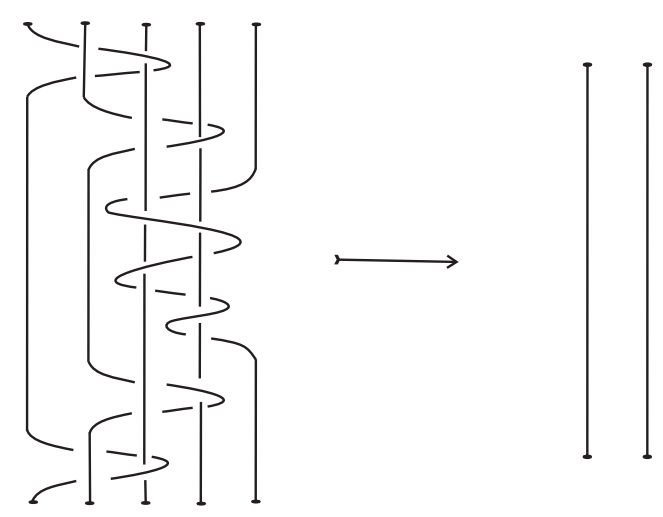

Figura 2.4: Esquecendo as três últimas cordas de uma trança $\beta \in B_{5}$

Dada uma trança $\beta \in B_{n}(M)$ e um número $j \in\{1,2, \ldots, n\}$ definimos $j \cdot \beta=\sigma(\beta)(j)$, onde $\sigma \in \Sigma_{n}$ e $\sigma: B_{n}(M) \rightarrow \Sigma_{n}$ é a projeção canônica. Esta ação foi utilizada na Seção 3 de [9].

Para $0 \leq k \leq m-1$ e $m \geq 3$ o conjunto

$$
\mathcal{Q}_{B}^{k} \operatorname{Brun}_{m}(M)=\left\{\beta \in B_{k, m-k}(M) \mid d_{j}(\beta)=1, \forall j=k+1, \ldots, m\right\}
$$

é um subgrupo do grupo misto $B_{k, m-k}(M)=\sigma^{-1}\left(\Sigma_{k} \times \Sigma_{m-k}\right)$.

De fato, sejam $\beta_{1}, \beta_{2} \in \mathcal{Q}_{B}^{k} \operatorname{Brun}_{m}(M)$. Então, para todo $j=k+1, k+2, \ldots, m$, tem-se que

$$
d_{j}\left(\beta_{1} \beta_{2}^{-1}\right)=d_{j}\left(\beta_{1}\right) d_{j \cdot \beta_{1}}\left(\beta_{2}^{-1}\right)=1 \cdot 1=1
$$

pois $j \cdot \beta_{1} \in\{k+1, \ldots, m\}$ uma vez que $\beta_{1} \in B_{k, m-k}(M)$.

Definição 2.2.1. O grupo $\mathcal{Q}_{B}^{k} \operatorname{Brun}_{m}(M)$ será chamado de grupo misto de tranças quaseBrunnianas de ordem $k$, com $m$ cordas, sobre $M$. Definamos o grupo $\mathcal{Q}_{B}^{k} \operatorname{Brun}_{m}(M)$ restrito ao grupo de tranças puras, $\mathcal{Q}^{k} \operatorname{Brun}_{m}(M)=\mathcal{Q}_{B}^{k} \operatorname{Brun}_{m}(M) \cap P_{m}(M)$, que chamaremos de grupo quase-Brunniano de ordem $k$, com $m$ cordas, sobre $M$.

A trança com 5 cordas que aparece nas Figuras 2.3 e 2.4 é uma trança quase-Brunniana de ordem 2, com 5 cordas, sobre o disco. Um outro exemplo de trança quase-Brunniana é dado na Figura 2.5. 


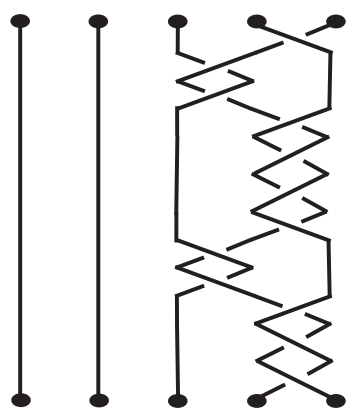

Figura 2.5: Trança quase-Brunniana, de ordem 2, com 5 cordas sobre o disco.

Proposição 2.2.2. Sejam $m \geq 3 e 0 \leq k \leq m-1$.

1. Se $m-k \neq 2$, então $\mathcal{Q}^{k} \operatorname{Brun}_{m}(M)=\mathcal{Q}_{B}^{k} \operatorname{Brun}_{m}(M)$.

2. Se $m-k=2$, então $P_{m}(M) \not \supset \mathcal{Q}_{B}^{k} \operatorname{Brun}_{m}(M) \subset B_{1, \ldots, 1,2}(M)$.

Demonstração. Tomemos uma trança $\gamma$ em $\mathcal{Q}_{B}^{k} \operatorname{Brun}_{m}(M)$. Consideremos os seguintes homomorfismos definidos no grupo misto $B_{k, m-k}(M)$ : o homomorfismo que esquece as primeiras $k$ cordas $\eta_{k}: B_{k, m-k}(M) \rightarrow B_{m-k}(M)$ e aquele que esquece as últimas $m-k$ cordas $\theta_{m-k}: B_{k, m-k}(M) \rightarrow B_{k}(M)$. Observemos que $\eta_{k}$ é a composição da aplicação $d_{1}$ um número $k$ de vezes, isto é, $\eta_{k}=d_{1}^{k}$ e $\theta_{m-k}=d_{k+1} \circ \cdots \circ d_{m}$. Pelas identidades simpliciais válidas em $\Delta$-grupos temos que $\eta_{k} \circ d_{j+k}=d_{j} \circ \eta_{k}$ para $1 \leq j \leq m-k$ e portanto o seguinte diagrama é comutativo, para todo $1 \leq j \leq m-k$ :

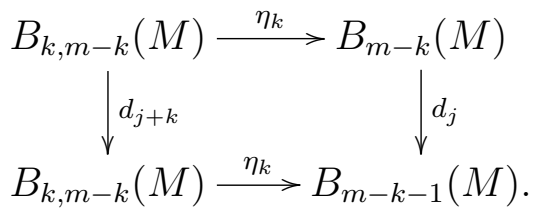

Dado que $\gamma$ pertence a $\mathcal{Q}_{B}^{k} \operatorname{Brun}_{m}(M)$, segue do anterior que $\eta_{k}(\gamma) \in \operatorname{Brun}_{m-k}(M)$. Além disso, como $\theta_{m-k}(\gamma)=1$, então a permutação associada a $\gamma$ pertence ao grupo $\Sigma_{1} \times \cdots \times \Sigma_{1} \times \Sigma_{m-k}$. O caso $m-k=1$ decorre desta última afirmação.

1. Suponhamos que $m-k \geq 3$, então $\eta_{k}(\gamma) \in \operatorname{Brun}_{m-k}(M) \subseteq P_{m-k}(M)$. Portanto, a permutação associada a $\gamma$ pertence ao grupo $\Sigma_{k} \times \Sigma_{1} \times \cdots \times \Sigma_{1}$. Mas também vimos que a permutação associada a $\gamma$ pertence ao grupo $\Sigma_{1} \times \cdots \times \Sigma_{1} \times \Sigma_{m-k}$. Logo, $\gamma \in P_{m}(M)$.

2. Notemos que, no caso em que $m-k=2$, o elemento $\sigma_{m-1}$ pertence ao grupo $\mathcal{Q}_{B}^{m-2} \operatorname{Brun}_{m}(M)$, pois $\sigma_{m-1} \in \operatorname{Ker}\left(d_{m-1}\right) \cap \operatorname{Ker}\left(d_{m}\right)$. Portanto, o grupo misto de tranças quase-Brunnianas $\mathcal{Q}_{B}^{m-2} \operatorname{Brun}_{m}(M)$ de ordem $m-2$, com $m$ cordas sobre $M$ não é um subgrupo de $P_{m}(M)$ e devido a que a permutação associada a $\gamma$ pertence ao grupo $\Sigma_{1} \times \cdots \times \Sigma_{1} \times \Sigma_{m-k}$, temos que $\mathcal{Q}_{B}^{k} \operatorname{Brun}_{m}(M) \subset B_{1, \ldots, 1,2}(M)$. 
Notemos que se $k=0$, então $\mathcal{Q}^{0} \operatorname{Brun}_{m}(M)=\operatorname{Brun}_{m}(M)$. Além disso, se $k=1$, o grupo de tranças quase-Brunnianas de ordem 1 , com $m$ cordas sobre $M$, coincide com o grupo chamado de "quase-Brunniano" por Cohen e Wu [17], isto explica a motivação na escolha do nome para este subgrupo do grupo de tranças sobre $M$.

Já sabemos que $\mathcal{Q}_{B}^{m-2} \operatorname{Brun}_{m}(M) \subset B_{1, \ldots, 1,2}(M)$, mas podemos dizer mais, como veremos na seguinte proposição. Além disso, podemos dar uma descrição dos grupos de tranças quase-Brunnianas como intersecção dos núcleos dos homomorfismos que esquecem cordas $d_{j}: P_{m}(M) \rightarrow P_{m-1}(M)$, bem como intersecção de subgrupos normais de $P_{m}(M)$.

\section{Proposição 2.2.3.}

1. Suponhamos que $m \geq 5$ se $M=\mathbb{S}^{2}$, que $m \geq 4$ se $M=\mathbb{R} P^{2}$ e que $m \geq 3$ nos outros casos. O grupo de tranças quase-Brunnianas pode ser descrito, para $1 \leq k \leq m-1$, assim:

$$
\begin{aligned}
\mathcal{Q}^{k} \operatorname{Brun}_{m}(M) & =\operatorname{Ker}\left(d_{k+1}\right) \cap \operatorname{Ker}\left(d_{k+2}\right) \cap \cdots \cap \operatorname{Ker}\left(d_{m}\right) \\
& =\left(\bigcap_{k+1 \leq l<t}\left\langle A_{l, t}\right\rangle^{P}\right) \cap\left(\bigcap_{t<r \leq m}\left\langle A_{t, r}\right\rangle^{P}\right) .
\end{aligned}
$$

Além disso, para $0 \leq k \leq m$, temos que

$$
\mathcal{Q}^{k} \operatorname{Brun}_{m}(M)=\operatorname{Ker}\left(d_{k+1}: P_{m}(M) \rightarrow P_{m-1}(M)\right) \cap \mathcal{Q}^{k+1} \operatorname{Brun}_{m}(M)
$$

2. Suponhamos que $m \geq 3$. Então, $\mathcal{Q}_{B}^{m-2} \operatorname{Brun}_{m}(M)=\left\langle\mathcal{Q}^{m-2} \operatorname{Brun}_{m}(M), \sigma_{m-1}\right\rangle$.

Demonstração. 1. Seja $\beta \in \mathcal{Q}^{k} \operatorname{Brun}_{m}(M)$, por definição $\beta \in P_{m}(M)$ e $d_{j}(\beta)=1$, para todo $1 \leq k+1 \leq j \leq m$, donde segue que $\beta \in \operatorname{Ker}\left(d_{k+1}\right) \cap \operatorname{Ker}\left(d_{k+2}\right) \cap \cdots \cap \operatorname{Ker}\left(d_{m}\right)$. A outra inclusão é imediata e portanto para todo $0 \leq k \leq m$

$$
\mathcal{Q}^{k} \operatorname{Brun}_{m}(M)=\operatorname{Ker}\left(d_{k+1}\right) \cap \operatorname{Ker}\left(d_{k+2}\right) \cap \cdots \cap \operatorname{Ker}\left(d_{m}\right) .
$$

Em vista do Lema 2.1.5, para $m$ e $M$ como nas hipóteses, segue que

$$
\operatorname{Ker}\left(d_{j}\right) \cap \operatorname{Ker}\left(d_{k}\right)=\left\langle A_{k, j}\right\rangle^{P_{m}(M)},
$$

para quaisquer $1 \leq k<j \leq m$. Logo, para $k+1 \leq t \leq m$, temos que

$$
\begin{aligned}
\mathcal{Q}^{k} \operatorname{Brun}_{m}(M)= & \operatorname{Ker}\left(d_{k+1}\right) \cap \operatorname{Ker}\left(d_{k+2}\right) \cap \cdots \cap \operatorname{Ker}\left(d_{m}\right) \\
= & \left(\operatorname{Ker}\left(d_{k+1}\right) \cap \cdots \cap \operatorname{Ker}\left(d_{t}\right)\right) \cap\left(\operatorname{Ker}\left(d_{t}\right) \cap \cdots \cap \operatorname{Ker}\left(d_{m}\right)\right) \\
= & \left(\operatorname{Ker}\left(d_{k+1}\right) \cap \operatorname{Ker}\left(d_{t}\right)\right) \cap \cdots \cap\left(\operatorname{Ker}\left(d_{t-1}\right) \cap \operatorname{Ker}\left(d_{t}\right)\right) \cap \\
& \left(\operatorname{Ker}\left(d_{t}\right) \cap \operatorname{Ker}\left(d_{t+1}\right)\right) \cap \cdots \cap\left(\operatorname{Ker}\left(d_{t}\right) \cap \operatorname{Ker}\left(d_{m}\right)\right) \\
= & \left(\bigcap_{k+1 \leq l<t}\left\langle A_{l, t}\right\rangle^{P}\right) \cap\left(\bigcap_{t<r \leq m}\left\langle A_{t, r}\right\rangle^{P}\right) .
\end{aligned}
$$


Finalmente, segue da descrição dada em (2.3) que para todo $0 \leq k \leq m$

$$
\begin{aligned}
\mathcal{Q}^{k} \operatorname{Brun}_{m}(M) & =\operatorname{Ker}\left(d_{k+1}\right) \cap\left(\operatorname{Ker}\left(d_{k+2}\right) \cap \cdots \cap \operatorname{Ker}\left(d_{m}\right)\right) \\
& =\operatorname{Ker}\left(d_{k+1}\right) \cap \mathcal{Q}^{k+1} \operatorname{Brun}_{m}(M)
\end{aligned}
$$

2. Lembremos que $\sigma_{m-1} \in \mathcal{Q}_{B}^{m-2} \operatorname{Brun}_{m}(M)$. Da sequência exata curta

$$
1 \longrightarrow P_{m}(M) \longrightarrow B_{1, \ldots, 1,2}(M) \longrightarrow \underbrace{\Sigma_{1} \times \cdots \times \Sigma_{1}}_{(m-2) \text {-vezes }} \times \Sigma_{2} \longrightarrow 1
$$

obtemos o seguinte diagrama comutativo

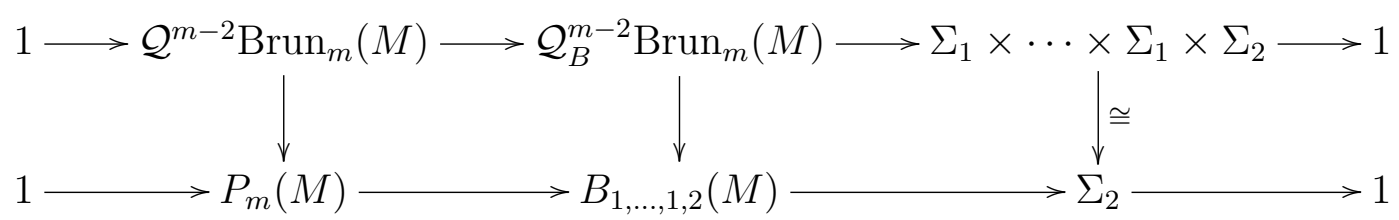

donde segue o resultado.

No item 1 da Proposição 2.2.3 demos uma descrição dos grupos de tranças quaseBrunnianas como intersecção de subgrupos normais dos grupos de tranças puras. Observemos que se tomamos $t=m$ em tal descrição, então

$$
\mathcal{Q}^{k} \operatorname{Brun}_{m}(M)=\bigcap_{l=k+1}^{m}\left\langle A_{l, m}\right\rangle^{P} .
$$

Notemos também que o caso $k=0$ corresponde ao grupo de tranças Brunnianas e um resultado similar foi obtido em [9].

Definição 2.2.4. Sejam $m \geq 3$ e $1 \leq k \leq m-1$. Definimos o grupo semi-Brunniano de ordem $k$, com $m$ cordas, sobre $M$ denotado por $\mathcal{S}^{k} \operatorname{Brun}_{m}(M)$ como sendo a intersecção do núcleo $\operatorname{Ker}\left(\eta_{k}\right)$ com o grupo $\mathcal{Q}_{B}^{k} \operatorname{Brun}_{m}(M)$, onde $\eta_{k}: B_{k, m-k}(M) \rightarrow B_{m-k}(M)$ é o homomorfismo induzido pela fibração de Fadell-Neuwirth generalizada.

Por convenção diremos que $\mathcal{S}^{0} \operatorname{Brun}_{m}(M)=\operatorname{Brun}_{m}(M)$.

Em outras palavras uma trança $\beta \in B_{k, m-k}(M)$ é semi-Brunniana de ordem $k$, com $m$ cordas, sobre $M$ se ela torna-se a trança identidade em $B_{m-k}(M)$ quando removemos o conjunto das primeiras $k$ cordas, assim como vira a trança trivial em $B_{k, m-k-1}(M)$ quando retiramos qualquer uma das últimas $m-k$ cordas.

Observemos que se $k=1$, então

$$
\mathcal{S}^{1} \operatorname{Brun}_{m}(M)=\operatorname{Brun}_{m}(M)
$$




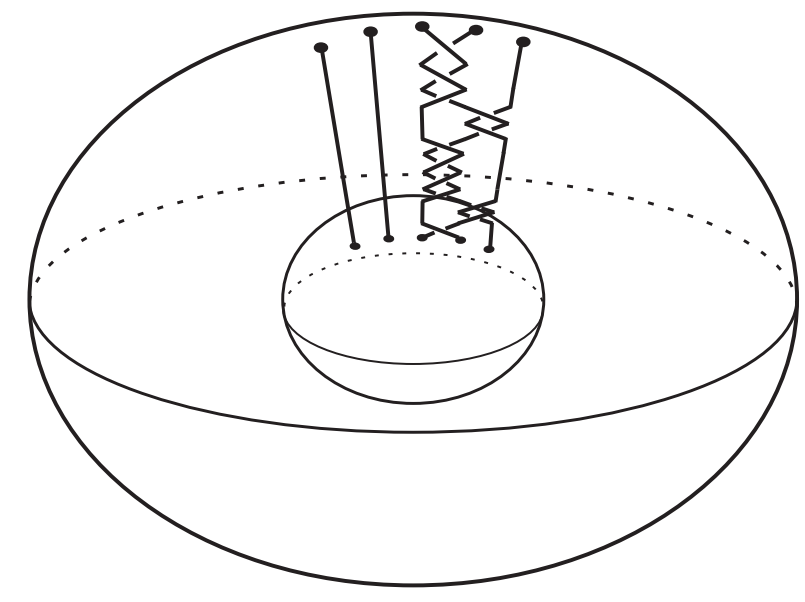

Figura 2.6: Trança semi-Brunniana de ordem 2, com 5 cordas, na esfera.

Contrariamente ao acontecido no caso das tranças quase-Brunnianas de ordem $m-2$, com $m$ cordas, a seguinte proposição mostra que todas as tranças semi-Brunnianas são tranças puras e portanto não precisamos nesse caso de um subíndice $B$.

Proposição 2.2.5. O grupo $\mathcal{S}^{k} \operatorname{Brun}_{m}(M)$ está contido no grupo de tranças puras, para $m \geq 3$ e $0 \leq k \leq m-1$.

Demonstração. Em vista da Proposição 2.2.2 basta apenas verificar o caso $k=m-2$. Por definição,

$$
\mathcal{S}^{m-2} \operatorname{Brun}_{m}(M)=\operatorname{Ker}\left(\eta_{m-2}\right) \cap \mathcal{Q}_{B}^{m-2} \operatorname{Brun}_{m}(M) .
$$

Seja $\beta \in \mathcal{S}^{m-2} \operatorname{Brun}_{m}(M)$. Como $\beta \in \operatorname{Ker}\left(\eta_{m-2}\right)$, então $\sigma(\beta) \in \Sigma_{m-2} \times \Sigma_{1} \times \Sigma_{1}$. Por outro lado, como $\beta \in \mathcal{Q}_{B}^{m-2} \operatorname{Brun}_{m}(M)$, então $\sigma(\beta) \in \underbrace{\Sigma_{1} \times \cdots \times \Sigma_{1}}_{(m-2)-\text { vezes }} \times \Sigma_{2}$. O resultado desejado segue facilmente juntando a informação anterior.

Segue pela definição das tranças semi-Brunnianas que

$$
\mathcal{S}^{k} \operatorname{Brun}_{m}(M)=\operatorname{Ker}\left(\eta_{k}\right) \cap \mathcal{Q}_{B}^{k} \operatorname{Brun}_{m}(M)
$$

No entanto, pela Proposição 2.2.5, temos que

$$
\mathcal{S}^{k} \operatorname{Brun}_{m}(M)=\operatorname{Ker}\left(\eta_{k}\right) \cap\left(\mathcal{Q}_{B}^{k} \operatorname{Brun}_{m}(M) \cap P_{m}(M)\right)=\operatorname{Ker}\left(\eta_{k}\right) \cap \mathcal{Q}^{k} \operatorname{Brun}_{m}(M) .
$$

Observações 2.2.6. Seja $m \geq 3$.

1. O grupo $\mathcal{S}^{k} \operatorname{Brun}_{m}(M)$ é um subgrupo de $\operatorname{Brun}_{m}^{k}(M)$, onde $\operatorname{Brun}_{m}^{k}(M)$ é o grupo de tranças k-decomponíveis sobre $M$ definido na Subseção 1.2.4.

2. F. R. Cohen e J. Wu comentam que o grupo $\mathcal{Q}^{1} \operatorname{Brun}_{m}(M)$ está relacionado com os enlaçamentos do tipo HTB ("Homologically Trivial and Brunnian") [63]. Será que o grupo $\mathcal{Q}^{k} \operatorname{Brun}_{m}(M)$ também se relaciona com os enlaçamentos do tipo HTB? 
3. Observemos também que o grupo $\mathcal{Q}^{1} \operatorname{Brun}_{m}(M)$ é exatamente igual ao conjunto formado pelos complexos de Moore $\mathcal{N}_{m-1} \mathcal{P}(M)$, definidos em [9].

A aplicação $d_{m}: B_{m-1,1}(M) \rightarrow B_{m-1}(M)$ está induzida por uma fibração de FadellNeuwirth generalizada e portanto é um homomorfismo. Notemos que por definição, o grupo de tranças quase-Brunnianas de ordem $m-1$, com $m$ cordas, é simplesmente o núcleo de $d_{m}: B_{m-1,1}(M) \rightarrow B_{m-1}(M)$. Posteriormente daremos uma descrição para os grupos de tranças quase-Brunnianas de ordem $k$, com $m$ cordas, sobre uma superfície $M$, para $1 \leq k \leq m-2$.

Na seguinte proposição destacamos algumas relações (por inclusão) entre os subgrupos definidos acima (inclusive os grupos de tranças $k$-decomponíveis). Como consequência de tais inclusões provaremos que os grupos de tranças quase-Brunnianas e semi-Brunnianas são em geral grupos livres.

Proposição 2.2.7. Suponhamos que $m \geq 3$. Então, as seguintes afirmações são válidas.

1. (a) $\operatorname{Brun}_{m}(M)=\mathcal{Q}^{0} \operatorname{Brun}_{m}(M) \subseteq \mathcal{Q}^{1} \operatorname{Brun}_{m}(M) \subseteq \cdots \subseteq \mathcal{Q}^{k} \operatorname{Brun}_{m}(M) \subseteq$ $\subseteq \mathcal{Q}^{k+1} \operatorname{Brun}_{m}(M) \subseteq \cdots \subseteq \mathcal{Q}^{m-1} \operatorname{Brun}_{m}(M) \subseteq P_{m}(M)$

(b) $\operatorname{Brun}_{m}(M)=\mathcal{S}^{0} \operatorname{Brun}_{m}(M) \subseteq \mathcal{S}^{1} \operatorname{Brun}_{m}(M) \subseteq \cdots \subseteq \mathcal{S}^{k} \operatorname{Brun}_{m}(M) \subseteq$ $\subseteq \mathcal{S}^{k+1} \operatorname{Brun}_{m}(M) \subseteq \cdots \subseteq \mathcal{S}^{m-1} \operatorname{Brun}_{m}(M) \subseteq P_{m}(M)$

(c) $\operatorname{Brun}_{m}(M) \subseteq \mathcal{S}^{r} \operatorname{Brun}_{m}(M) \subseteq \mathcal{Q}^{s} \operatorname{Brun}_{m}(M) \subseteq \operatorname{Brun}_{m}^{t+1}(M) \subseteq P_{m}(M)$, para $1 \leq r \leq s \leq t \leq m-2$.

2. Os grupos de tranças quase-Brunnianas e semi-Brunnianas de ordem $k$, com $m$ cordas, sobre uma superfície $M$ são grupos livres, exceto para $m=3$ e $M=\mathbb{S}^{2}$.

Demonstração. 1. Pela Proposição 2.2.3, temos que $\mathcal{Q}^{k} \operatorname{Brun}_{m}(M) \subseteq \mathcal{Q}^{k+1} \operatorname{Brun}_{m}(M)$ para

$0 \leq k \leq m-1$, obtendo o item $(a)$.

Por definição sabemos que $\mathcal{S}^{k} \operatorname{Brun}_{m}(M)=\operatorname{Ker}\left(\eta_{k}\right) \cap \mathcal{Q}^{k} \operatorname{Brun}_{m}(M)$ e notemos que o núcleo de $\eta_{k}, \operatorname{Ker}\left(\eta_{k}: B_{k, m-k}(M) \rightarrow B_{m-k}(M)\right)$, está contido no grupo

$$
\operatorname{Ker}\left(\eta_{k+1}: B_{k+1, m-k-1}(M) \rightarrow B_{m-k-1}(M)\right)
$$

Isto é, para $0 \leq k \leq m-1$,

$$
\operatorname{Ker}\left(\eta_{k}\right) \subseteq \operatorname{Ker}\left(\eta_{k+1}\right)
$$

Portanto o item (b) segue do item (a) juntamente com as inclusões dadas em (2.4). Agora, pelo item $(b)$, temos que $\mathcal{S}^{r} \operatorname{Brun}_{m}(M) \subseteq \mathcal{S}^{v} \operatorname{Brun}_{m}(M)$ e portanto, para $1 \leq r \leq v \leq m-1$, temos que

$$
\operatorname{Brun}_{m}(M) \subseteq \mathcal{S}^{r} \operatorname{Brun}_{m}(M) \subseteq \mathcal{S}^{v} \operatorname{Brun}_{m}(M)
$$


Notemos que $\mathcal{Q}^{t} \operatorname{Brun}_{m}(M) \subseteq \operatorname{Brun}_{m}^{t+1}(M)$, pois ao retirarmos qualquer conjunto de $t+1$ cordas de uma trança $\beta$ com $m$ cordas em $\mathcal{Q}^{t} \operatorname{Brun}_{m}(M)$, sempre vamos retirar pelo menos uma das cordas no conjunto formado pelas $m-t$ últimas cordas de $\beta$ e portanto obtemos a trança trivial com $m-t-1$ cordas sobre $M$. Como $\operatorname{Brun}_{m}^{t+1}(M) \subseteq P_{m}(M)$ por definição e $\mathcal{Q}^{v} \operatorname{Brun}_{m}(M) \subseteq \mathcal{Q}^{t} \operatorname{Brun}_{m}(M)$, para $1 \leq v \leq t \leq m-2$, pelo item $(a)$ temos que as seguintes inclusões são válidas

$$
\mathcal{Q}^{v} \operatorname{Brun}_{m}(M) \subseteq \operatorname{Brun}_{m}^{t+1}(M) \subseteq P_{m}(M),
$$

completando a demonstração do item 1.

2. Notemos que $P_{3}\left(\mathbb{S}^{2}\right)$ não tem subgrupos livres dado que $P_{3}\left(\mathbb{S}^{2}\right) \cong \mathbb{Z}_{2}$. Suponhamos que $m \geq 4$ se $M=\mathbb{S}^{2}$ e que $m \geq 3$ nos outros casos. Lembremos que $\mathcal{Q}^{m-1} \operatorname{Brun}_{m}(M)=\operatorname{Ker}\left(d_{m}: P_{m}(M) \rightarrow P_{m-1}(M)\right)$ e portanto $\mathcal{Q}^{m-1} \operatorname{Brun}_{m}(M)$ é isomorfo ao grupo livre $\pi_{1}\left(M \backslash Q_{m-1}, q_{m}\right)$, onde $Q_{m-1}$ é um conjunto finito de $m-1$ pontos no interior de $M$. Como consequência, aplicando as inclusões de grupos dadas no item 1 desta proposição, os grupos de tranças quase-Brunnianas e semiBrunnianas de ordem $k$, com $m$ cordas, sobre uma superfície $M$ são subgrupos do grupo livre $\mathcal{Q}^{m-1} \operatorname{Brun}_{m}(M)$, exceto para $m=3$ e $M=\mathbb{S}^{2}$. Segue do Teorema de Nielsen-Schreier que os subgrupos de grupos livres são por sua vez grupos livres, ver Subseção 1.1.1, donde segue o resultado desejado.

Posteriormente provaremos que, como no caso dos grupos Brunnianos, estes grupos são em geral grupos livres de posto infinito, com algumas exceções. Sabemos que o grupo de tranças Brunnianas $\operatorname{Brun}_{m}(M)$ é um subgrupo normal de $B_{m}(M)$. Na continuação vamos demonstrar que os grupos $\mathcal{Q}^{k} \operatorname{Brun}_{m}(M)$ e $\mathcal{S}^{k} \operatorname{Brun}_{m}(M)$ são subgrupos normais de $B_{k, m-k}(M)$.

Proposição 2.2.8. Sejam $M$ uma superfície qualquer, $m \geq 3$ e $0 \leq k \leq m-1$.

1. Os grupos $\mathcal{Q}^{k} \operatorname{Brun}_{m}(M)$ e $\mathcal{S}^{k} \operatorname{Brun}_{m}(M)$ são subgrupos normais de $B_{k, m-k}(M)$. No entanto, $\mathcal{Q}_{B}^{m-2} \operatorname{Brun}_{m}(M)$ não é um subgrupo normal de $B_{m-2,2}(M)$.

2. Existe uma filtração de subgrupos normais em $P_{m}(M)$ :

$$
\mathcal{Q}^{0} \operatorname{Brun}_{m}(M) \unlhd \mathcal{Q}^{1} \operatorname{Brun}_{m}(M) \unlhd \cdots \unlhd \mathcal{Q}^{k} \operatorname{Brun}_{m}(M) \unlhd \cdots \unlhd \mathcal{Q}^{m-1} \operatorname{Brun}_{m}(M) \unlhd P_{m}(M)
$$

Demonstração. 1. Se $k=0$, então $\mathcal{Q}^{0} \operatorname{Brun}_{m}(M)=\operatorname{Brun}_{m}(M)$ e pela Proposição 1.3.16 sabemos que este grupo é normal em $B_{m}(M)$. Para $k=m-1$, temos que $\mathcal{Q}^{k} \operatorname{Brun}_{m}(M)=\operatorname{Ker}\left(d_{m}: B_{m-1,1}(M) \rightarrow B_{m-1}(M)\right)$ e como a aplicação $d_{m}: B_{m-1,1}(M) \rightarrow B_{m-1}(M)$ é um homomorfismo, então seu núcleo é normal em 
$B_{m-1,1}(M)$. Portanto, é claro que $\mathcal{Q}^{k} \operatorname{Brun}_{m}(M)$ é um subgrupo normal de $B_{k, m-k}(M)$ nesses dois casos.

Suponhamos que $1 \leq k \leq m-2$. Tomemos $\beta \in \mathcal{Q}^{k} \operatorname{Brun}_{m}(M)$ e $\gamma \in B_{k, m-k}(M)$. Para todo $i \in\{k+1, k+2, \ldots, m\}$, temos que

$$
d_{i}\left(\gamma \beta \gamma^{-1}\right)=d_{i}(\gamma \beta) d_{i \cdot(\gamma \beta)}\left(\gamma^{-1}\right)=d_{i}(\gamma) d_{i \cdot \gamma}(\beta) d_{i \cdot(\gamma \beta)}\left(\gamma^{-1}\right)=d_{i}(\gamma) d_{i \cdot \gamma}\left(\gamma^{-1}\right)=1
$$

Segue de (2.5) que $\mathcal{Q}^{k} \operatorname{Brun}_{m}(M)$ é um subgrupo normal de $B_{k, m-k}(M)$ para $m \geq 3$ e $1 \leq k \leq m-2$.

Agora, observemos que

$$
\mathcal{S}^{k} \operatorname{Brun}_{m}(M)=\operatorname{Ker}\left(\eta_{k}: B_{k, m-k}(M) \rightarrow B_{m-k}(M)\right) \cap \mathcal{Q}^{k} \operatorname{Brun}_{m}(M)
$$

Dado que $\operatorname{Ker}\left(\eta_{k}\right)$ e $\mathcal{Q}^{k} \operatorname{Brun}_{m}(M)$ são subgrupos normais de $B_{k, m-k}(M)$, para $m \geq 3$ e $0 \leq k \leq m-1$, então $\mathcal{S}^{k} \operatorname{Brun}_{m}(M)$ é um subgrupo normal de $B_{k, m-k}(M)$.

Provemos que $\mathcal{Q}_{B}^{m-2} \operatorname{Brun}_{m}(M)$ não é um subgrupo normal de $B_{m-2,2}(M)$. Sejam $\beta=\sigma_{m-1} \in \mathcal{Q}_{B}^{m-2} \operatorname{Brun}_{m}(M)$ e $\gamma=A_{j, m-1} \in P_{m}(M) \subseteq B_{m-2,2}(M)$, para qualquer $1 \leq j \leq m-2$. Observemos que $(m-1) \cdot \gamma \beta=(m-1) \cdot \beta=m$. Portanto, usando a definição da aplicação simplicial $d_{m-1}: B_{m}(M) \rightarrow B_{m-1}(M)$, temos que

$$
\begin{aligned}
d_{m-1}\left(\gamma \beta \gamma^{-1}\right) & =d_{m-1}(\gamma \beta) d_{(m-1) \cdot \gamma \beta}\left(\gamma^{-1}\right)=d_{m-1}(\gamma) d_{(m-1) \cdot \gamma}(\beta) d_{m}\left(\gamma^{-1}\right) \\
& =A_{j, m-1}^{-1} \in P_{m-1}(M)
\end{aligned}
$$

que é um elemento não trivial no grupo de tranças puras $P_{m-1}(M)$.

Isto é, $d_{m-1}\left(\gamma \beta \gamma^{-1}\right) \neq 1$ e portanto segue, pela definição de $\mathcal{Q}_{B}^{m-2} \operatorname{Brun}_{m}(M)$, que $\gamma \beta \gamma^{-1} \notin \mathcal{Q}_{B}^{m-2} \operatorname{Brun}_{m}(M)$.

2. Para $0 \leq k \leq m-2$

$$
\mathcal{Q}^{k} \operatorname{Brun}_{m}(M)=\operatorname{Ker}\left(d_{k+1} \mid: \mathcal{Q}^{k+1} \operatorname{Brun}_{m}(M) \rightarrow P_{m-1}(M)\right) .
$$

Lembremos que $\mathcal{Q}^{k} \operatorname{Brun}_{m}(M) \unlhd B_{k, m-k}(M)$, portanto temos uma filtração finita de subgrupos normais:

$\mathcal{Q}^{0} \operatorname{Brun}_{m}(M) \unlhd \mathcal{Q}^{1} \operatorname{Brun}_{m}(M) \unlhd \cdots \unlhd \mathcal{Q}^{k} \operatorname{Brun}_{m}(M) \unlhd \cdots \unlhd \mathcal{Q}^{m-1} \operatorname{Brun}_{m}(M) \unlhd P_{m}(M)$.

Como mencionamos acima, os grupos quase-Brunnianos e semi-Brunnianos são em geral livres de posto infinito. Na seguinte proposição, veremos que algumas exceções ocorrem nos casos dos grupos de tranças com poucas cordas sobre a esfera e o plano projetivo. 
Já tínhamos mencionado, na Introdução, que os grupos de tranças sobre a esfera e o plano projetivo são excepcionais na teoria geral, uma razão disto é que os espaços de configuração destas duas superfícies não são espaços de Eilenberg-Mac Lane.

Observação 2.2.9. Sejam $d_{i}, d_{j}: P_{m}(M) \rightarrow P_{m-1}(M)$ homomorfismos que esquecem a $i$-ésima e a j-ésima corda, respectivamente. Notemos que

$$
\operatorname{Ker}\left(d_{i}\right) \cap \operatorname{Ker}\left(d_{j}\right)=\operatorname{Ker}\left(\left.d_{i}\right|_{\operatorname{Ker}\left(d_{j}\right)}\right)=\operatorname{Ker}\left(\left.d_{j}\right|_{\operatorname{Ker}\left(d_{i}\right)}\right) .
$$

Por abuso de notação algumas vezes escreveremos apenas $d_{i}$ no lugar de $d_{i} \mid$.

\section{Proposição 2.2.10.}

1. $\mathcal{Q}^{1} \operatorname{Brun}_{3}\left(\mathbb{S}^{2}\right) \cong \mathbb{Z}_{2}$. Ainda mais, Brun $_{3}\left(\mathbb{S}^{2}\right) \cong \mathbb{Z}_{2}$.

2. $\mathcal{Q}^{1} \operatorname{Brun}_{3}\left(\mathbb{R} P^{2}\right)$ é um grupo livre de posto 5 , livremente gerado por

$$
\left\{\rho_{3}^{4}, A_{2,3},\left[A_{2,3}, \rho_{3}\right],\left[A_{2,3}, \rho_{3}^{2}\right],\left[A_{2,3}, \rho_{3}^{3}\right]\right\}
$$

3. $\mathcal{Q}^{2} \operatorname{Brun}_{4}\left(\mathbb{S}^{2}\right)$ é um grupo livre de posto 3, livremente gerado por

$$
\left\{A_{2,4}^{2}, A_{3,4}, A_{2,4} A_{3,4} A_{2,4}^{-1}\right\}
$$

4. $\mathcal{Q}^{1} \operatorname{Brun}_{4}\left(\mathbb{S}^{2}\right)$ é um grupo livre de posto 5, livremente gerado por

$$
\left\{A_{2,4}^{2},\left[A_{2,4}, A_{3,4}\right], A_{3,4}^{2}, A_{3,4} A_{2,4}^{2} A_{3,4}^{-1}, A_{3,4} A_{2,4} A_{3,4} A_{2,4}^{-1}\right\}
$$

Além disso, verifica-se que

$$
\mathcal{Q}^{1} \operatorname{Brun}_{4}\left(\mathbb{S}^{2}\right)=\operatorname{Brun}_{4}\left(\mathbb{S}^{2}\right)
$$

Demonstração. A afirmação 1. segue imediatamente pelo fato que $P_{2}\left(\mathbb{S}^{2}\right)=\{1\}$ e $P_{3}\left(\mathbb{S}^{2}\right) \cong \mathbb{Z}_{2}$. A afirmação 2. segue da prova da [5, Proposição 4.9], onde o conjunto pedido corresponde ao conjunto $S$ ali descrito.

Para demonstrar as duas últimas afirmações usaremos o método de ReidemeisterSchreier ("Reidemeister-Schreier rewriting process") duas vezes. A notação usada aqui será aquela dada em [75].

Consideremos a seguinte sequência exata curta de Fadell-Neuwirth

$$
1 \longrightarrow \pi_{1}\left(\mathbb{S}^{2} \backslash\left\{q_{1}, q_{2}, q_{3}\right\}, q_{4}\right) \longrightarrow P_{4}\left(\mathbb{S}^{2}\right) \stackrel{d_{4}}{\longrightarrow} P_{3}\left(\mathbb{S}^{2}\right) \longrightarrow 1
$$

Sabemos que $\pi_{1}\left(\mathbb{S}^{2} \backslash\left\{q_{1}, q_{2}, q_{3}\right\}, q_{4}\right)=F_{2}\left(A_{2,4}, A_{3,4}\right)$ e que $P_{3}\left(\mathbb{S}^{2}\right)=\mathbb{Z}_{2}$ é gerado pelo "full 
twist" $\Delta_{3}$. Segue, pela definição, que

$$
\mathcal{Q}^{2} \operatorname{Brun}_{4}\left(\mathbb{S}^{2}\right)=\operatorname{Ker}\left(d_{3} \mid: F_{2}\left(A_{2,4}, A_{3,4}\right) \rightarrow P_{3}\left(\mathbb{S}^{2}\right)\right)
$$

Denotemos por $\Delta$ o "full twist" em $P_{3}\left(\mathbb{S}^{2}\right)$, por 1 o elemento neutro no grupo de tranças $P_{4}\left(\mathbb{S}^{2}\right)$ e por 0 o elemento neutro em $P_{3}\left(\mathbb{S}^{2}\right)=\mathbb{Z}_{2}$. Desta forma notemos que

$$
\begin{aligned}
d_{3} \mid(1) & =d_{3} \mid\left(A_{3,4}\right)=0 \\
d_{3} \mid\left(A_{2,4}\right) & =A_{2,3}
\end{aligned}
$$

e portanto uma transversal de Schreier para $\operatorname{Ker}\left(d_{3} \mid\right)$ pode ser dada por

$$
M=\left\{M_{1}=1, M_{2}=A_{2,4}\right\} .
$$

Em primeiro lugar vamos obter um conjunto de elementos geradores de $\mathcal{Q}^{2} \operatorname{Brun}_{4}\left(\mathbb{S}^{2}\right)$ usando o método já mencionado. Por [75, Proposição 6.2] os elementos $y_{j, k}=\varrho\left(M_{k}, x_{j}\right)$, para $k=1,2$ e $x_{1}=A_{2,4}, x_{2}=A_{3,4}$, são geradores de $\mathcal{Q}^{2} \operatorname{Brun}_{4}\left(\mathbb{S}^{2}\right)$. Escrevamos então tais elementos:

$$
\begin{array}{ll}
y_{1,1}=\varrho\left(M_{1}, x_{1}\right)=A_{2,4} \overline{A_{2,4}}-1 & =1 \\
y_{1,2}=\varrho\left(M_{2}, x_{1}\right)=A_{2,4}^{2} \overline{A_{2,4}^{2}}-1 & =A_{2,4}^{2} \\
y_{2,1}=\varrho\left(M_{1}, x_{2}\right)=A_{3,4} \overline{A_{3,4}}-1 & =A_{3,4} \\
y_{2,2}=\varrho\left(M_{2}, x_{2}\right)=A_{2,4} A_{3,4} \overline{A_{2,4} A_{3,4}}-1 & =A_{2,4} A_{3,4} A_{2,4}^{-1} .
\end{array}
$$

Como subgrupo de um grupo livre é um grupo livre, temos que

$$
\mathcal{Q}^{2} \operatorname{Brun}_{4}\left(\mathbb{S}^{2}\right)=F_{3}\left(A_{2,4}^{2}, A_{3,4}, A_{2,4} A_{3,4} A_{2,4}^{-1}\right),
$$

provando assim a afirmação 3 .

Agora,

$$
\mathcal{Q}^{1} \operatorname{Brun}_{4}\left(\mathbb{S}^{2}\right)=\operatorname{Ker}\left(d_{2} \mid: \mathcal{Q}^{2} \operatorname{Brun}_{4}\left(\mathbb{S}^{2}\right) \rightarrow P_{3}\left(\mathbb{S}^{2}\right)\right)
$$

Repetindo aqui o procedimento feito acima, notemos que

$$
\begin{aligned}
d_{2} \mid(1) & =d_{2} \mid\left(A_{2,4}^{2}\right) & = & 0 \\
d_{2} \mid\left(A_{3,4}\right) & =d_{2} \mid\left(A_{2,4} A_{3,4} A_{2,4}^{-1}\right) & = & A_{2,3}=\Delta .
\end{aligned}
$$

Uma transversal de Schreier para $\operatorname{Ker}\left(d_{2} \mid\right)$ pode ser dada por

$$
M=\left\{M_{1}=1, M_{2}=A_{3,4}\right\} .
$$

Um conjunto de elementos geradores de $\mathcal{Q}^{1} \operatorname{Brun}_{4}\left(\mathbb{S}^{2}\right)$ é dado por $y_{j, k}=\varrho\left(M_{k}, x_{j}\right)$, para 
$k=1,2$ e $x_{1}=A_{2,4}^{2}, x_{2}=A_{3,4}, x_{3}=A_{2,4} A_{3,4} A_{2,4}^{-1}$. Tais elementos são:

$$
\begin{array}{ll}
y_{1,1}=\varrho\left(M_{1}, x_{1}\right)=A_{2,4}^{2} \bar{A}_{2,4}^{2}-1 & =A_{2,4}^{2} \\
y_{2,1}=\varrho\left(M_{1}, x_{2}\right)=A_{3,4} \overline{A_{3,4}}-1 & =1 \\
y_{3,1}=\varrho\left(M_{1}, x_{3}\right)=A_{2,4} A_{3,4} A_{2,4}^{-1} \overline{A_{2,4} A_{3,4} A_{2,4}^{-1}} & =A_{2,4} A_{3,4} A_{2,4}^{-1} A_{3,4}^{-1} \\
y_{1,2}=\varrho\left(M_{2}, x_{1}\right)=A_{3,4} A_{2,4}^{2} \overline{A_{3,4} A_{2,4}^{2}}-1 & =A_{3,4} A_{2,4}^{2} A_{3,4}^{-1} \\
y_{2,2}=\varrho\left(M_{2}, x_{2}\right)=A_{3,4}^{2} \overline{A_{3,4}^{2}}-1 & =A_{3,4}^{2} \\
y_{3,2}=\varrho\left(M_{2}, x_{3}\right)=A_{3,4} A_{2,4} A_{3,4} A_{2,4}^{-1} \overline{A_{3,4} A_{2,4} A_{3,4} A_{2,4}^{-1}}-1 & =A_{3,4} A_{2,4} A_{3,4} A_{2,4}^{-1} .
\end{array}
$$

Logo,

$$
\mathcal{Q}^{1} \operatorname{Brun}_{4}\left(\mathbb{S}^{2}\right)=F_{5}\left(A_{2,4}^{2},\left[A_{2,4}, A_{3,4}\right], A_{3,4}^{2}, A_{3,4} A_{2,4}^{2} A_{3,4}^{-1}, A_{3,4} A_{2,4} A_{3,4} A_{2,4}^{-1}\right)
$$

Por outro lado, $\operatorname{Brun}_{4}\left(\mathbb{S}^{2}\right)=\operatorname{Ker}\left(d_{1} \mid: \mathcal{Q}^{1} \operatorname{Brun}_{4}\left(\mathbb{S}^{2}\right) \rightarrow P_{3}\left(\mathbb{S}^{2}\right)\right)$ e $d_{1} \mid\left(y_{j}\right)=0$, para $y_{j}$ gerador de $\mathcal{Q}^{1} \operatorname{Brun}_{4}\left(\mathbb{S}^{2}\right)$, com $1 \leq j \leq 5$. Isto é,

$$
\operatorname{Brun}_{4}\left(\mathbb{S}^{2}\right)=\operatorname{Ker}\left(d_{1} \mid: \mathcal{Q}^{1} \operatorname{Brun}_{4}\left(\mathbb{S}^{2}\right) \rightarrow P_{3}\left(\mathbb{S}^{2}\right)\right)=\mathcal{Q}^{1} \operatorname{Brun}_{4}\left(\mathbb{S}^{2}\right)
$$

completando a demonstração da última afirmação.

Em [5] foi dada uma descrição dos grupos Brunnianos de tranças sobre superfícies do tipo Eilenberg-Mac Lane usando os subgrupos comutadores simétricos, ver Teorema 1.2.14 no capítulo de preliminares, e na prova foi usado por eles o Teorema 1.2.2. Motivados por isto daremos a seguir uma descrição dos grupos quase-Brunnianos como comutadores simétricos, para isto também usaremos o Teorema 1.2.2 e a noção de conectividade de subgrupos normais de um grupo dado, ver Subseção 1.2.1.

Observação 2.2.11. Sejam $R_{1}, \ldots, R_{m}$ subgrupos normais de um grupo $G$. Notemos que se $\left(R_{1}, \ldots, R_{m}\right)$ é uma m-upla conexa de subgrupos normais em $G$, então segue, pela própria definição, que qualquer $|J|$-upla $\left(R_{i_{1}}, \ldots, R_{i_{|J|}}\right)$ é uma $|J|$-upla conexa de subgrupos normais em $G$, para qualquer subconjunto $J \subsetneq\{1, \ldots, m\}$.

Teorema 2.2.12. Seja $M$ uma superfície qualquer. Vamos supor que $m \geq 3$ se $M$ é diferente de $\mathbb{S}^{2}$ e de $\mathbb{R} P^{2}$, que $m \geq 4$ se $M=\mathbb{R} P^{2}$ e que $m \geq 5$ se $M=\mathbb{S}^{2}$. Então, para $1 \leq k \leq m-2$ vale que

$$
\mathcal{Q}^{k} \operatorname{Brun}_{m}(M)=\left[\left\langle A_{k+1, m}\right\rangle^{P},\left\langle A_{k+2, m}\right\rangle^{P}, \ldots,\left\langle A_{m-1, m}\right\rangle^{P}\right]_{S} .
$$

No caso $k=m-1$, para $m \geq 4$ se $M=\mathbb{S}^{2}$ e para $m \geq 3$ nos outros casos, temos que

$$
\mathcal{Q}^{m-1} \operatorname{Brun}_{m}(M) \cong \pi_{1}\left(M \backslash\left\{q_{1}, \ldots, q_{m-1}\right\}, q_{m}\right)
$$


Demonstração. Provaremos primeiro o caso $m-k=2$. Segue do Lema 2.1.5 que

$$
\operatorname{Ker}\left(d_{m-1}\right) \cap \operatorname{Ker}\left(d_{m}\right)=\left\langle A_{m-1, m}[M]\right\rangle^{P}
$$

para $M$ e $m$ como nas hipóteses do teorema. Logo, pela Proposição 2.2.3, segue que $\mathcal{Q}^{m-2} \operatorname{Brun}_{m}(M)=\left\langle A_{m-1, m}[M]\right\rangle^{P}$.

Consideremos agora o caso $m-k \geq 3$. Usaremos a mesma notação usada no enunciado e a prova do [5, Teorema 3.9]. Observemos que $X=M \backslash\left\{q_{1}, \ldots, q_{k}\right\}$ é um espaço do tipo Eilenberg-Mac Lane que pode ser visto como o colimite dos espaços $X \backslash \coprod_{i \in I} p_{i}$, onde $I=(\{k+1, \ldots, m-1\} \backslash J)$ e $J$ percorre todos os subconjuntos $J \subsetneq\{k+1, k+2, \ldots, m-1\}$.

Denotemos por $G:=\pi_{1}\left(M \backslash\left\{q_{1}, \ldots, q_{m-1}\right\}\right)$ e por $R_{i}:=\operatorname{Ker}\left(\left.d_{i}\right|_{\operatorname{Ker}\left(d_{m}\right)}\right)$ (como em [5]). Como as superfícies perfuradas são espaços do tipo Eilenberg-Mac Lane, então os espaços $X \backslash \coprod_{i \in I} p_{i}$ são espaços classificantes para os grupos $G / \prod_{i \in J} R_{i}$.

Pela Observação 2.2.11 segue que a condição de conectividade é satisfeita para toda $(m-(k+1)-1)$-upla de subgrupos $\left(R_{k+1}, \ldots, \hat{R}_{n}, \ldots, R_{m-1}\right)$, para $k+1 \leq n \leq m-1$. Aqui $\hat{R}_{n}$ significa não considerar o subgrupo mencionado.

Portanto, pelo Teorema 1.2.2, temos que vale a seguinte igualdade:

$$
\bigcap_{j=k+1}^{m-1} \operatorname{Ker}\left(d_{j} \mid\right)=\left[\operatorname{Ker}\left(d_{k+1} \mid\right), \operatorname{Ker}\left(d_{k+2} \mid\right), \ldots, \operatorname{Ker}\left(d_{m-1} \mid\right)\right]_{S} .
$$

Logo,

$$
\begin{aligned}
\mathcal{Q}^{k} \operatorname{Brun}_{m}(M) & =i_{*}\left(\bigcap_{j=k+1}^{m-1} \operatorname{Ker}\left(d_{j} \mid\right)\right) \\
& =i_{*}\left(\left[\operatorname{Ker}\left(d_{k+1} \mid\right), \operatorname{Ker}\left(d_{k+2} \mid\right), \ldots, \operatorname{Ker}\left(d_{m-1} \mid\right)\right]_{S}\right) \\
& =\left[\left\langle A_{k+1, m}\right\rangle^{P},\left\langle A_{k+2, m}\right\rangle^{P}, \ldots,\left\langle A_{m-1, m}\right\rangle^{P}\right]_{S} .
\end{aligned}
$$

A primeira e a última igualdade acima decorrem do Lema 2.1.5.

Finalmente, se $k=m-1$, então

$$
\mathcal{Q}^{m-1} \operatorname{Brun}_{m}(M)=\operatorname{Ker}\left(d_{m}: P_{m}(M) \rightarrow P_{m-1}(M)\right) \cong \pi_{1}\left(M \backslash\left\{q_{1}, \ldots, q_{m-1}\right\}, q_{m}\right)
$$

A descrição dada no Teorema 2.2.12 também vale para $k=0$ se $m \geq 3$ e se $M \neq \mathbb{S}^{2}, \mathbb{R} P^{2}$, pois neste caso temos que $\mathcal{Q}^{0} \operatorname{Brun}_{m}(M)=\operatorname{Brun}_{m}(M)$ e o resultado foi provado por Bardakov et al., ver Teorema 1.2.14.

Na demonstração da Proposição 2.2.2 consideramos a projeção que esquece as primeiras $k$ cordas

$$
\eta_{k}: B_{k, m-k}(M) \rightarrow B_{m-k}(M)
$$

para $1 \leq k \leq m-1$, e vimos que $\eta_{k}\left(\mathcal{Q}^{k} \operatorname{Brun}_{m}(M)\right) \subseteq \operatorname{Brun}_{m-k}(M)$. Portanto, podemos considerar a seguinte restrição de $\eta_{k}$, que por abuso de notação também denotaremos por 
$\eta_{k}: \mathcal{Q}^{k} \operatorname{Brun}_{m}(M) \rightarrow \operatorname{Brun}_{m-k}(M)$. Como uma primeira aplicação do teorema anterior temos o seguinte resultado, envolvendo grupos quase-Brunnianos e grupos de homotopia.

Teorema 2.2.13. Sejam $k \geq 1$ e $m \geq 3$ e suponhamos que $k \leq m-5$ se $M=\mathbb{S}^{2}$, que $k \leq m-4$ se $M=\mathbb{R} P^{2}$ e $k \leq m-3$ nos outros casos.

1. Se $M$ é uma superfície do tipo Eilenberg-Mac Lane, então $\operatorname{coKer}\left(\eta_{k}\right)=\{1\}$.

2. Se $M=\mathbb{R} P^{2}$, então $\operatorname{coKer}\left(\eta_{k}\right) \cong \pi_{m-k-1}\left(\mathbb{S}^{2}\right)$.

3. Se $M=\mathbb{S}^{2}$, então $\operatorname{coKer}\left(\eta_{k}\right) \cong \pi_{m-k-1}\left(\mathbb{S}^{2}\right)$.

Demonstração. Observemos que $\eta_{k}: P_{m}(M) \rightarrow P_{m-k}(M)$ é um epimorfismo e portanto, usando a descrição do grupo de tranças puras quase-Brunnianas, temos que

$$
\begin{aligned}
\eta_{k}\left(\mathcal{Q}^{k} \operatorname{Brun}_{m}(M)\right) & =\eta_{k}\left(\left[\left\langle A_{k+1, m}\right\rangle^{P},\left\langle A_{k+2, m}\right\rangle^{P}, \ldots,\left\langle A_{m-1, m}\right\rangle^{P}\right]_{S}\right) \\
& =\left[\left\langle A_{1, m-k}\right\rangle^{P},\left\langle A_{2, m-k}\right\rangle^{P}, \ldots,\left\langle A_{m-k-1, m-k}\right\rangle^{P}\right]_{S} \\
& =R_{m-k}(M),
\end{aligned}
$$

onde a última igualdade é parte da notação usada no Teorema 1.2.14. Sabemos que $\mathcal{Q}^{k} \operatorname{Brun}_{m}(M) \unlhd B_{k, m-k}(M)$ e, em particular, é normal em $P_{m}(M)$. Usando novamente o fato de $\eta_{k}: P_{m}(M) \rightarrow P_{m-k}(M)$ ser um epimorfismo obtemos que $\eta_{k}\left(\mathcal{Q}^{k} \operatorname{Brun}_{m}(M)\right)$ é normal em $P_{m-k}(M)$, ainda mais, como $\eta_{k}\left(\mathcal{Q}^{k} \operatorname{Brun}_{m}(M)\right) \subseteq \operatorname{Brun}_{m-k}(M)$, então $\eta_{k}\left(\mathcal{Q}^{k} \operatorname{Brun}_{m}(M)\right)$ é normal em $\operatorname{Brun}_{m-k}(M)$.

Consideremos agora o co-núcleo

$$
\operatorname{coKer}\left(\eta_{k}\right)=\frac{\operatorname{Brun}_{m-k}(M)}{\eta_{k}\left(\mathcal{Q}^{k} \operatorname{Brun}_{m}(M)\right)}=\frac{\operatorname{Brun}_{m-k}(M)}{R_{m-k}(M)} .
$$

Logo, aplicando o Teorema 1.2.14 em cada caso obtemos o resultado desejado:

1. Se $M$ é uma superfície do tipo Eilenberg-Mac Lane, então $\operatorname{coKer}\left(\eta_{k}\right)=\{1\}$, pois

$$
\frac{\operatorname{Brun}_{m-k}(M)}{R_{m-k}(M)}=\{1\}
$$

2. Se $M=\mathbb{R} P^{2}$, então $\operatorname{coKer}\left(\eta_{k}\right)=\frac{\operatorname{Brun}_{m-k}\left(\mathbb{R} P^{2}\right)}{R_{m-k}\left(\mathbb{R} P^{2}\right)} \cong \pi_{m-k-1}\left(\mathbb{S}^{2}\right)$.

3. Se $M=\mathbb{S}^{2}$, então $\operatorname{coKer}\left(\eta_{k}\right)=\frac{\operatorname{Brun}_{m-k}\left(\mathbb{S}^{2}\right)}{R_{m-k}\left(\mathbb{S}^{2}\right)} \cong \pi_{m-k-1}\left(\mathbb{S}^{2}\right)$.

Segue imediatamente do teorema anterior, usando as definições dos grupos envolvidos, o seguinte corolário. 
Corolário 2.2.14. Consideremos o homomorfismo $\eta_{k}: B_{k, m-k}(M) \rightarrow B_{m-k}(M)$.

1. Se $1 \leq k \leq m-3$ e $M$ é uma superfície do tipo Eilenberg-Mac Lane, então a seguinte sequência é exata curta

$$
1 \longrightarrow \mathcal{S}^{k} \operatorname{Brun}_{m}(M) \longrightarrow \mathcal{Q}^{k} \operatorname{Brun}_{m}(M) \stackrel{\eta_{k}}{\longrightarrow} \operatorname{Brun}_{m-k}(M) \longrightarrow 1
$$

2. Se $1 \leq k \leq m-4$ e $M=\mathbb{R} P^{2}$, então a seguinte sequência é exata

$$
1 \rightarrow \mathcal{S}^{k} \operatorname{Brun}_{m}\left(\mathbb{R} P^{2}\right) \rightarrow \mathcal{Q}^{k} \operatorname{Brun}_{m}\left(\mathbb{R} P^{2}\right) \stackrel{\eta_{k}}{\rightarrow} \operatorname{Brun}_{m-k}\left(\mathbb{R} P^{2}\right) \rightarrow \pi_{m-k-1}\left(\mathbb{S}^{2}\right) \rightarrow 1
$$

3. Se $1 \leq k \leq m-5$ e $M=\mathbb{S}^{2}$, então a seguinte sequência é exata

$$
1 \longrightarrow \mathcal{S}^{k} \operatorname{Brun}_{m}\left(\mathbb{S}^{2}\right) \longrightarrow \mathcal{Q}^{k} \operatorname{Brun}_{m}\left(\mathbb{S}^{2}\right) \stackrel{\eta_{k}}{\longrightarrow} \operatorname{Brun}_{m-k}\left(\mathbb{S}^{2}\right) \longrightarrow \pi_{m-k-1}\left(\mathbb{S}^{2}\right) \longrightarrow 1
$$

Uma segunda aplicação do Teorema 2.2.12 será dada na seguinte proposição.

Proposição 2.2.15. Suponhamos que $k \leq m-3$, onde $m \geq 6$ e $k \geq 1$ se $M=\mathbb{S}^{2}, m \geq 5$ e $k \geq 1$ se $M=\mathbb{R} P^{2}$ e $m \geq 4$ e $k \geq 0$ nos outros casos. Então, a seguinte sequência é exata curta:

$$
1 \longrightarrow \mathcal{Q}^{k} \operatorname{Brun}_{m}(M) \longrightarrow \mathcal{Q}^{k+1} \operatorname{Brun}_{m}(M) \stackrel{d_{k+1}}{\longrightarrow} \mathcal{Q}^{k} \operatorname{Brun}_{m-1}(M) \longrightarrow 1
$$

Demonstração. Para $k \geq 1$ se $M=\mathbb{S}^{2}, \mathbb{R} P^{2}$ e $k \geq 0$ nos outros casos, temos que

$$
d_{k+1}\left(\mathcal{Q}^{k+1} \operatorname{Brun}_{m}(M)\right)=\mathcal{Q}^{k} \operatorname{Brun}_{m-1}(M) .
$$

De fato, nas hipóteses sobre $M$ e $k$, usando a descrição dos grupos de tranças quaseBrunnianas dada no Teorema 2.2.12, segue que

$$
\begin{aligned}
d_{k+1}\left(\mathcal{Q}^{k+1} \operatorname{Brun}_{m}(M)\right) & =\left[d_{k+1}\left(\left\langle A_{k+2, m}\right\rangle^{P_{m}(M)}\right), \ldots, d_{k+1}\left(\left\langle A_{m-1, m}\right\rangle^{P_{m}(M)}\right)\right]_{S} \\
& =\left[\left\langle A_{k+1, m-1}\right\rangle^{P_{m-1}(M)}, \ldots,\left\langle A_{m-2, m-1}\right\rangle^{P_{m-1}(M)}\right]_{S} \\
& =\mathcal{Q}^{k} \operatorname{Brun}_{m-1}(M)
\end{aligned}
$$

Assim, para $k \geq 1$ se $M=\mathbb{S}^{2}, \mathbb{R} P^{2}$ e $k \geq 0$ nos outros casos a seguinte sequência é exata curta:

$$
1 \longrightarrow \mathcal{Q}^{k} \operatorname{Brun}_{m}(M) \longrightarrow \mathcal{Q}^{k+1} \operatorname{Brun}_{m}(M) \stackrel{d_{k+1}}{\longrightarrow} \mathcal{Q}^{k} \operatorname{Brun}_{m-1}(M) \longrightarrow 1
$$




\subsection{Tranças puras sobre superfícies e grupos de ho- motopia da 2-esfera}

Nesta seção consideraremos $M=M_{g, k}$ uma superfície fechada de genus $g \geq 0$ com $k \geq 0$ pontos removidos. Seja $Q_{n}=q_{1}, \ldots, q_{n}$ uma coleção de $n \geq 1$ pontos no interior de $M$ e suponhamos que

$$
\delta= \begin{cases}1, & \text { se } M \text { é uma superfície não orientável, } \\ 2, & \text { se } M \text { é uma superfície orientável. }\end{cases}
$$

Logo, $\pi_{1}\left(M \backslash Q_{n}, q_{n+1}\right)=F_{n+\delta(g-1)+k+1}\left(z_{1}, \ldots, z_{n+\delta(g-1)+k+1}\right)$. Vamos identificar os geradores do grupo livre $\pi_{1}\left(M \backslash Q_{n}, q_{n+1}\right)$ com tranças em $P_{n+1}(M)$. Para isto usaremos a aplicação $i_{n+1 *}$ da sequência exata curta

$$
1 \longrightarrow \pi_{1}\left(M \backslash\left\{q_{1}, \ldots, q_{n}\right\}, q_{n+1}\right) \stackrel{i_{n+1 *}}{\longrightarrow} P_{n+1}(M) \stackrel{d_{n+1}}{\longrightarrow} P_{n}(M) \longrightarrow 1,
$$

válida para $n \geq 3$ se $M=\mathbb{S}^{2}, n \geq 2$ se $M=\mathbb{R} P^{2}$ e para $n \geq 1$ nos outros casos.

Dada a superfície $M_{g, k}$ definimos

$$
\epsilon=\left\{\begin{array}{lll}
0, & \text { se } \quad k=0 \\
1, & \text { se } \quad k \geq 1
\end{array}\right.
$$

Um primeiro subconjunto de geradores nesta identificação, independe da orientação de $M$

$$
\begin{cases}z_{i} \mapsto A_{i+1-\epsilon, n+1}, & \text { se } \quad 1 \leq i \leq n+\epsilon-1 \\ z_{i} \mapsto w_{i+1-\epsilon, n+1}, & \text { se } \quad n+\epsilon \leq i \leq n-1+k .\end{cases}
$$

Observemos que o número de geradores considerados acima é $n-1+k$.

Já os outros geradores serão enviados por $i_{n+1_{*}}$, dependendo da orientação de $M$, como ilustrado na continuação.

- Para $M$ orientável diferente da esfera:

$$
\begin{cases}z_{i} \mapsto a_{i-n-k+1, n+1}, & \text { se } \quad n+k \leq i \leq n+k+g-1 \\ z_{i} \mapsto b_{i-n-k-g, n+1}, & \text { se } \quad n+k+g \leq i \leq n+k+2 g-1\end{cases}
$$

Observemos que o número de geradores, considerados neste último subconjunto, é $2 g$.

- Para $M$ não orientável:

$$
z_{i} \mapsto \rho_{i-n-k+1, n+1}, \quad \text { se } n+k \leq i \leq n+g+k \text {. }
$$


Observemos que o número de geradores, considerados neste último subconjunto, é $g+1$.

Exemplo 2.3.1. Consideremos o bitoro para ilustrar como foi feita a escolha dos geradores no caso compacto, bem como no caso não compacto.

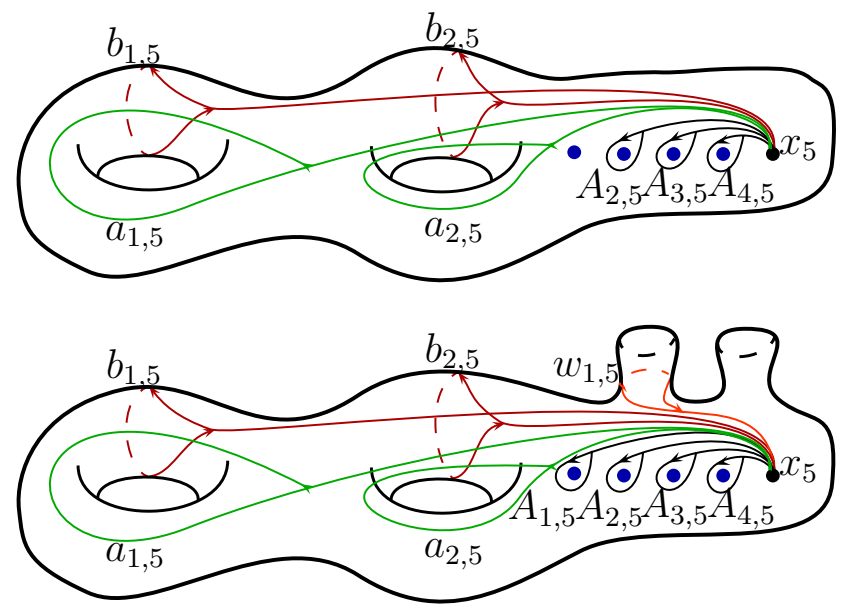

Denotemos por $F=\pi_{1}\left(M \backslash Q_{n}, q_{n+1}\right)=F_{n+\delta(g-1)+k+1}\left(z_{1}, \ldots, z_{n+\delta(g-1)+k+1}\right)$ e por

$$
\left\{\begin{array}{l}
\widehat{G_{n+1}(M)}=\left[\left\langle z_{1}\right\rangle^{F}, \ldots,\left\langle z_{n+\delta(g-1)+k+1}\right\rangle^{F},\left\langle\prod_{i=1}^{n+\delta(g-1)+k+1} z_{i}\right\rangle^{F}\right]_{S} \\
\widehat{E_{n+1}(M)}=\left\langle z_{n+\epsilon}\right\rangle^{F} \cap \cdots \cap\left\langle z_{n+\delta(g-1)+k+1}\right\rangle^{F} \cap\left\langle\prod_{i=1}^{n+\delta(g-1)+k+1} z_{i}\right\rangle^{F} .
\end{array}\right.
$$

Sejam $\left.G_{n+1}(M)=i_{n+1_{*}}\left(\widehat{G_{n+1}(M}\right)\right)$ e $\left.E_{n+1}(M)=i_{n+1 *}\left(\widehat{E_{n+1}(M}\right)\right)$. Pela sequência exata curta (2.6), temos que $i_{n+1_{*}}$ é um monomorfismo tal que $i_{n+1_{*}}(F)=\operatorname{Ker}\left(d_{n+1}\right)$. Portanto,

$$
G_{n+1}(M)=\left[\left\langle i_{n+1 *}\left(z_{1}\right)\right\rangle^{\operatorname{Ker}\left(d_{n+1}\right)}, \ldots,\left\langle i_{n+1 *}\left(z_{r}\right)\right\rangle^{\operatorname{Ker}\left(d_{n+1}\right)},\left\langle\prod_{i=1}^{r} i_{n+1 *}\left(z_{i}\right)\right\rangle^{\operatorname{Ker}\left(d_{n+1}\right)}\right]_{S}
$$

e como um monomorfismo aplicado num número finito de intersecções de grupos é igual à intersecção do monomorfismo aplicado em cada um dos grupos, então

$$
E_{n+1}(M)=\left\langle i_{n+1 *}\left(z_{n+\epsilon}\right)\right\rangle^{\operatorname{Ker}\left(d_{n+1}\right)} \cap \cdots \cap\left\langle i_{n+1 *}\left(z_{r}\right)\right\rangle^{\operatorname{Ker}\left(d_{n+1}\right)} \cap\left\langle\prod_{i=1}^{r} i_{n+1 *}\left(z_{i}\right)\right\rangle^{\operatorname{Ker}\left(d_{n+1}\right),}
$$

onde $r=n+\delta(g-1)+k+1$ nas igualdades (2.7) e (2.8).

Exemplo 2.3.2. Suponhamos que $M$ é o disco, a esfera ou o plano projetivo. Vamos mostrar descrições para os grupos $G_{n+1}(M)$ e $E_{n+1}(M)$ utilizando as igualdades (2.7) e (2.8), respectivamente. Usaremos a notação $D_{i, j}$ para os geradores de tranças puras sobre 
a esfera com o objetivo de distinguí-los daqueles geradores dos grupos de tranças puras do disco. A seguinte relação em $P_{n+1}\left(\mathbb{S}^{2}\right)$ será útil para descrevermos $G_{n+1}\left(\mathbb{S}^{2}\right)$ e $E_{n+1}\left(\mathbb{S}^{2}\right)$ :

$$
D_{1, n+1} D_{2, n+1} \cdots D_{n, n+1}=1 .
$$

Também será usado que o fecho normal de um elemento num grupo $G$ é igual ao fecho normal em $G$ do inverso do mesmo elemento, isto é, se $g \in G$, então $\langle g\rangle^{G}=\left\langle g^{-1}\right\rangle^{G}$. Lembremos que os grupos que fazem parte da notação de um grupo comutador simétrico não são ordenados, ver Observação 1.1.8.

1. Primeiro mostremos o caso dos grupos $E_{n+1}(M)$.

(a) Para $n \geq 1$ e $M=\mathbb{D}^{2}$, temos que

$$
E_{n+1}\left(\mathbb{D}^{2}\right)=\left\langle\prod_{i=1}^{n} i_{n+1_{*}} i_{n+1_{*}}\left(z_{i}\right)\right\rangle^{\operatorname{Ker}\left(d_{n+1}\right)}=\left\langle A_{0, n+1}\right\rangle^{\operatorname{Ker}\left(d_{n+1}\right)},
$$

onde $A_{0, n+1}=\left(A_{1, n+1} A_{2, n+1} \cdots A_{n, n+1}\right)^{-1}$. Ainda mais, como

$$
A_{0,2}=A_{1,2}^{-1} \in P_{2}\left(\mathbb{D}^{2}\right)
$$

então $E_{2}\left(\mathbb{D}^{2}\right)=\left\langle A_{1,2}\right\rangle^{\operatorname{Ker}\left(d_{2}\right)}=P_{2}\left(\mathbb{D}^{2}\right)$, pois $\operatorname{Ker}\left(d_{2}: P_{2}\left(\mathbb{D}^{2}\right) \rightarrow P_{1}\left(\mathbb{D}^{2}\right)\right)=$ $P_{2}\left(\mathbb{D}^{2}\right)$.

(b) Seja $n \geq 2$. O grupo $E_{n+1}\left(\mathbb{R} P^{2}\right)$ tem a seguinte descrição

$$
\begin{aligned}
E_{n+1}\left(\mathbb{R} P^{2}\right) & =\left\langle i_{n+1_{*}}\left(z_{n}\right)\right\rangle^{\operatorname{Ker}\left(d_{n+1}\right)} \cap\left\langle i_{n+1 *}\left(z_{1} z_{2} \cdots z_{n}\right)\right\rangle^{\operatorname{Ker}\left(d_{n+1}\right)} \\
& =\left\langle\rho_{1, n-1}\right\rangle^{\operatorname{Ker}\left(d_{n+1}\right)} \cap\left\langle A_{2, n+1} \cdots A_{n, n+1} \rho_{1, n+1}\right\rangle^{\operatorname{Ker}\left(d_{n+1}\right)} .
\end{aligned}
$$

(c) O grupo $E_{n+1}\left(\mathbb{S}^{2}\right)$, para $n \geq 3$, é dado por

$$
\begin{aligned}
E_{n+1}\left(\mathbb{S}^{2}\right) & =\left\langle i_{n+1 *}\left(z_{1} z_{2} \cdots z_{n-1}\right)\right\rangle^{\operatorname{Ker}\left(\delta_{n+1}\right)} \\
& =\left\langle D_{2, n+1} D_{3, n+1} \cdots D_{n, n+1}\right\rangle^{\operatorname{Ker}\left(\delta_{n+1}\right)}
\end{aligned}
$$

Segue, de (2.9), que $D_{2, n+1} D_{3, n+1} \cdots D_{n, n+1}=D_{1, n+1}^{-1} \in P_{n+1}\left(\mathbb{S}^{2}\right)$ e pelo Lema 2.1.5 sabemos que $\left\langle D_{1, n+1}\right\rangle^{\operatorname{Ker}\left(\delta_{n+1}\right)}=\left\langle D_{1, n+1}\right\rangle^{P_{n+1}\left(\mathbb{S}^{2}\right)}$. Logo,

$$
\begin{aligned}
E_{n+1}\left(\mathbb{S}^{2}\right) & =\left\langle D_{2, n+1} D_{3, n+1} \cdots D_{n, n+1}\right\rangle^{\operatorname{Ker}\left(\delta_{n+1}\right)} \\
& =\left\langle D_{1, n+1}\right\rangle^{\operatorname{Ker}\left(\delta_{n+1}\right)} \\
& =\left\langle D_{1, n+1}\right\rangle^{P_{n+1}\left(\mathbb{S}^{2}\right)}
\end{aligned}
$$

2. Os grupos $G_{n+1}(M)$. 
(a) O grupo $G_{2}\left(\mathbb{D}^{2}\right)=\{1\}$, pois $P_{2}\left(\mathbb{D}^{2}\right)=\mathbb{Z}$. Para $n \geq 2$ temos que $G_{n+1}\left(\mathbb{D}^{2}\right)$ é igual ao grupo

$$
\left[\left\langle i_{n+1 *}\left(z_{1}\right)\right\rangle^{\operatorname{Ker}\left(d_{n+1}\right)}, \ldots,\left\langle i_{n+1 *}\left(z_{n-1}\right)\right\rangle^{\operatorname{Ker}\left(d_{n+1}\right)},\left\langle i_{n+1 *}\left(z_{1} z_{2} \cdots z_{n-1}\right)\right\rangle^{\operatorname{Ker}\left(d_{n+1}\right)}\right]_{S}
$$

e portanto

$$
\begin{aligned}
G_{n+1}\left(\mathbb{D}^{2}\right) & =\left[\left\langle A_{1, n+1}\right\rangle^{\operatorname{Ker}\left(d_{n+1}\right)}, \ldots,\left\langle A_{n, n+1}\right\rangle^{\operatorname{Ker}\left(d_{n+1}\right)}, E_{n+1}\left(\mathbb{D}^{2}\right)\right]_{S} \\
& =\left[\left\langle A_{1, n+1}\right\rangle^{\operatorname{Ker}\left(d_{n+1}\right)}, \ldots,\left\langle A_{n, n+1}\right\rangle^{\operatorname{Ker}\left(d_{n+1}\right)},\left\langle A_{0, n+1}\right\rangle^{\operatorname{Ker}\left(d_{n+1}\right)}\right]_{S} \\
& =\left[\left\langle A_{0, n+1}\right\rangle^{\operatorname{Ker}\left(d_{n+1}\right)},\left\langle A_{1, n+1}\right\rangle^{\operatorname{Ker}\left(d_{n+1}\right)}, \ldots,\left\langle A_{n, n+1}\right\rangle^{\operatorname{Ker}\left(d_{n+1}\right)}\right]_{S} .
\end{aligned}
$$

Pela descrição dos grupos Brunnianos do disco dada no Teorema 1.2.14, temos que

$$
\operatorname{Brun}_{n+2}\left(\mathbb{D}^{2}\right)=\left[\left\langle A_{1, n+2}\right\rangle^{P_{n+2}},\left\langle A_{2, n+2}\right\rangle^{P_{n+2}}, \ldots,\left\langle A_{n+1, n+2}\right\rangle^{P_{n+2}}\right]_{S}
$$

e pelo Lema 2.1.5 sabemos que, para $1 \leq j \leq n+1$, vale a igualdade

$$
\left\langle A_{j, n+2}\right\rangle^{\operatorname{Ker}\left(d_{n+2}\right)}=\left\langle A_{j, n+2}\right\rangle^{P_{n+2}\left(\mathbb{D}^{2}\right)} .
$$

Portanto, usando a definição do homomorfismo d dada em (1.4) e a igualdade (2.11), segue que

$$
\begin{aligned}
G_{n+1}\left(\mathbb{D}^{2}\right) & =\left[\left\langle A_{0, n+1}\right\rangle^{\operatorname{Ker}\left(d_{n+1}\right)},\left\langle A_{1, n+1}\right\rangle^{\operatorname{Ker}\left(d_{n+1}\right)}, \ldots,\left\langle A_{n, n+1}\right\rangle^{\operatorname{Ker}\left(d_{n+1}\right)}\right]_{S} \\
& =\partial\left(\left[\left\langle A_{1, n+2}\right\rangle^{\operatorname{Ker}\left(d_{n+2}\right)}, \ldots,\left\langle A_{n+1, n+2}\right\rangle^{\operatorname{Ker}\left(d_{n+2}\right)}\right]_{S}\right) \\
& =\partial\left(\left[\left\langle A_{1, n+2}\right\rangle^{P_{n+2}},\left\langle A_{2, n+2}\right\rangle^{P_{n+2}}, \ldots,\left\langle A_{n+1, n+2}\right\rangle^{P_{n+2}}\right]_{S}\right) \\
& =\partial\left(\operatorname{Brun}_{n+2}\left(\mathbb{D}^{2}\right)\right) .
\end{aligned}
$$

(b) Para $n \geq 2$ e $M=\mathbb{R} P^{2}$, temos que

$$
\begin{aligned}
& G_{n+1}\left(\mathbb{R} P^{2}\right) \\
& =\left[\left\langle i_{n+1 *}\left(z_{1}\right)\right\rangle^{\operatorname{Ker}\left(d_{n+1}\right)}, \ldots,\left\langle i_{n+1 *}\left(z_{n}\right)\right\rangle^{\operatorname{Ker}\left(d_{n+1}\right)},\left\langle i_{n+1 *}\left(z_{1} z_{2} \cdots z_{n}\right)\right\rangle^{\operatorname{Ker}\left(d_{n+1}\right)}\right]_{S} \\
& =\left[\left\langle A_{2, n+1}\right\rangle^{\operatorname{Ker}\left(d_{n+1}\right)}, \ldots,\left\langle A_{n, n+1}\right\rangle^{\operatorname{Ker}\left(d_{n+1}\right)},\left\langle\rho_{1, n+1}\right\rangle^{\operatorname{Ker}\left(d_{n+1}\right)},\langle\omega\rangle^{\operatorname{Ker}\left(d_{n+1}\right)}\right]_{S},
\end{aligned}
$$

onde $\omega=A_{2, n+1} \cdots A_{n, n+1} \rho_{1, n+1}$.

(c) Sejam $n \geq 3$ e $M=\mathbb{S}^{2}$. Pela igualdade (2.7), temos que $G_{n+1}\left(\mathbb{S}^{2}\right)$ é igual a

$$
\left[\left\langle i_{n+1_{*}}\left(z_{1}\right)\right\rangle^{\operatorname{Ker}\left(\delta_{n+1}\right)}, \ldots,\left\langle i_{n+1_{*}}\left(z_{n-1}\right)\right\rangle^{\operatorname{Ker}\left(\delta_{n+1}\right)},\left\langle i_{n+1_{*}}\left(z_{1} z_{2} \cdots z_{n-1}\right)\right\rangle^{\operatorname{Ker}\left(\delta_{n+1}\right)}\right]_{S}
$$


e pela relação do grupo de tranças $P_{n+1}\left(\mathbb{S}^{2}\right)$, dada em (2.9), temos que

$$
i_{n+1 *}\left(\prod_{i=1}^{n-1}\left(z_{i}\right)\right)=D_{1, n+1}^{-1} .
$$

Lembremos que o homomorfismo $f_{*}: P_{n+1}\left(\mathbb{D}^{2}\right) \rightarrow P_{n+1}\left(\mathbb{S}^{2}\right)$ é definido por $f_{*}\left(A_{i, j}\right)=D_{i, j}$, para todo $1 \leq i<j \leq n+1$. Pelo Lema 2.1.5, para $1 \leq j \leq n$, vale a igualdade $\left\langle A_{j, n+1}\right\rangle^{\operatorname{Ker}\left(d_{n+1}\right)}=\left\langle A_{j, n+1}\right\rangle^{P_{n+1}\left(\mathbb{D}^{2}\right)}$. Vamos usar isto, junto com a descrição dos grupos Brunnianos sobre o disco dada no Teorema 1.2.14, para obtermos a seguinte descrição do grupo $G_{n+1}\left(\mathbb{S}^{2}\right)$.

$$
\begin{aligned}
& G_{n+1}\left(\mathbb{S}^{2}\right)= \\
& =\left[\left\langle D_{2, n+1}\right\rangle^{\operatorname{Ker}\left(\delta_{n+1}\right)}, \ldots,\left\langle D_{n, n+1}\right\rangle^{\operatorname{Ker}\left(\delta_{n+1}\right)},\left\langle D_{2, n+1} \cdots D_{n, n+1}\right\rangle^{\operatorname{Ker}\left(\delta_{n+1}\right)}\right]_{S} \\
& =\left[\left\langle D_{2, n+1}\right\rangle^{\operatorname{Ker}\left(\delta_{n+1}\right)}, \ldots,\left\langle D_{n, n+1}\right\rangle^{\operatorname{Ker}\left(\delta_{n+1}\right)},\left\langle D_{1, n+1}\right\rangle^{\operatorname{Ker}\left(\delta_{n+1}\right)}\right]_{S} \\
& =f_{*}\left(\left[\left\langle A_{2, n+1}\right\rangle^{\operatorname{Ker}\left(d_{n+1}\right)}, \ldots,\left\langle A_{n, n+1}\right\rangle^{\operatorname{Ker}\left(d_{n+1}\right)},\left\langle A_{1, n+1}\right\rangle^{\operatorname{Ker}\left(d_{n+1}\right)}\right]_{S}\right) \\
& =f_{*}\left(\left[\left\langle A_{2, n+1}\right\rangle^{P_{n+1}\left(\mathbb{D}^{2}\right)}, \ldots,\left\langle A_{n, n+1}\right\rangle^{P_{n+1}\left(\mathbb{D}^{2}\right)},\left\langle A_{1, n+1}\right\rangle^{P_{n+1}\left(\mathbb{D}^{2}\right)}\right]_{S}\right) \\
& =f_{*}\left(\operatorname{Brun}_{n+1}\left(\mathbb{D}^{2}\right)\right) .
\end{aligned}
$$

Além disso, para $n \geq 4$, das igualdades dadas em (2.14), segue que $G_{n+1}\left(\mathbb{S}^{2}\right)$ é o grupo $R_{n+1}\left(\mathbb{S}^{2}\right)$ de [5], ver Teorema 1.2.14.

Com a ajuda do Teorema 1.2.3 e a construção feita acima podemos relacionar tranças sobre qualquer superfície e os grupos de homotopia da esfera. Destacamos que, em alguns casos, ainda é possível exibir tranças que se projetam em geradores dos grupos de homotopia baixos da esfera, os quais são livres de torção, ver as Observações 2.3.4.

Teorema 2.3.3. Suponhamos que $n \geq 4$ se $M=\mathbb{S}^{2}, n \geq 3$ se $M=\mathbb{R} P^{2}$ e que $n \geq 1$ nos outros casos. As seguintes sequências são exatas curtas.

1. Para $k=0$ :

$$
1 \longrightarrow G_{n+1}(M) \longrightarrow \mathcal{Q}^{1} \operatorname{Brun}_{n+1}(M) \cap E_{n+1}(M) \longrightarrow \pi_{n+\delta(g-1)+2}\left(\mathbb{S}^{2}\right) \longrightarrow 1
$$

2. Para $k \geq 1$ :

$$
1 \longrightarrow G_{n+1}(M) \longrightarrow \operatorname{Brun}_{n+1}(M) \cap E_{n+1}(M) \longrightarrow \pi_{n+\delta(g-1)+k+2}\left(\mathbb{S}^{2}\right) \longrightarrow 1 .
$$

Demonstração. Suponhamos que $n \geq 4$ se $M=\mathbb{S}^{2}, n \geq 3$ se $M=\mathbb{R} P^{2}$ e que $n \geq 1$ nos outros casos. Pelo Lema 2.1.5, temos que $i_{n+1 *}\left(\left\langle z_{i}\right\rangle^{F}\right)=\left\langle A_{i+1-\epsilon, n+1}\right\rangle^{P}$, para 
$i=1,2, \ldots, n+\epsilon-1$. Além disso, do Lema 2.1.5 e da Proposição 2.2.3, segue que

$$
\begin{aligned}
\mathcal{Q}^{1-\epsilon} \operatorname{Brun}_{n+1}(M) & =\bigcap_{i=1}^{n+\epsilon-1}\left\langle A_{i+1-\epsilon, n+1}\right\rangle^{P} \\
& =\bigcap_{i=1}^{n+\epsilon-1} i_{n+1_{*}}\left(\left\langle z_{i}\right\rangle^{F}\right)
\end{aligned}
$$

O homomorfismo $i_{n+1_{*}}$ da sequência exata curta dada em (2.6) é injetor. Portanto, temos os seguintes isomorfismos

$$
i_{n+1_{*}}: \widehat{G_{n+1}(M)} \rightarrow G_{n+1}(M) \text { e } i_{n+1_{*}}: \widehat{E_{n+1}(M)} \rightarrow E_{n+1}(M) .
$$

Também pelo fato de $i_{n+1 *}$ ser injetora, temos que para qualquer número finito de subgrupos $A_{1}, \ldots, A_{s}$ do grupo fundamental $\pi_{1}\left(M \backslash\left\{q_{1}, \ldots, q_{n}\right\}, q_{n+1}\right)$ vale que

$$
i_{n+1_{*}}\left(A_{1} \cap A_{2} \cap \cdots \cap A_{s}\right)=i_{n+1_{*}}\left(A_{1}\right) \cap i_{n+1_{*}}\left(A_{2}\right) \cap \cdots \cap i_{n+1_{*}}\left(A_{s}\right) .
$$

Portanto,

$$
\left.i_{n+1_{*}}:\left\langle z_{1}\right\rangle^{F} \cap \cdots \cap\left\langle z_{n-1+\epsilon}\right\rangle^{F} \cap \widehat{E_{n+1}(M}\right) \rightarrow \mathcal{Q}^{1-\epsilon} \operatorname{Brun}_{n+1}(M) \cap E_{n+1}(M)
$$

é um isomorfismo.

Como $G_{n+1}(M)$ é um subgrupo comutador simétrico de subgrupos normais em $\operatorname{Ker}\left(d_{n+1}\right)$, então $G_{n+1}(M)$ é um subgrupo normal de $\mathcal{Q}^{1-\epsilon} \operatorname{Brun}_{n+1}(M) \cap E_{n+1}(M)$.

Sabemos que $\pi_{1}\left(M \backslash Q_{n}\right)=F_{n+\delta(g-1)+k+1}\left(z_{1}, \ldots, z_{n+\delta(g-1)+k+1}\right)$ e portanto, pelo Teorema 1.2.3, segue que a seguinte sequência é exata curta:

$$
\left.\left.1 \longrightarrow \widehat{G_{n+1}(M}\right) \longrightarrow\left\langle z_{1}\right\rangle^{F} \cap \cdots \cap\left\langle z_{n-1+\epsilon}\right\rangle^{F} \cap \widehat{E_{n+1}(M}\right) \longrightarrow \pi_{n+\delta(g-1)+k+2}\left(\mathbb{S}^{2}\right) \longrightarrow 1 .
$$

Logo, o seguinte diagrama é comutativo, onde as duas primeiras flechas verticais são isomorfismos e a última é induzida pelas duas primeiras, que também resulta ser um isomorfismo (pelo lema dos cinco):

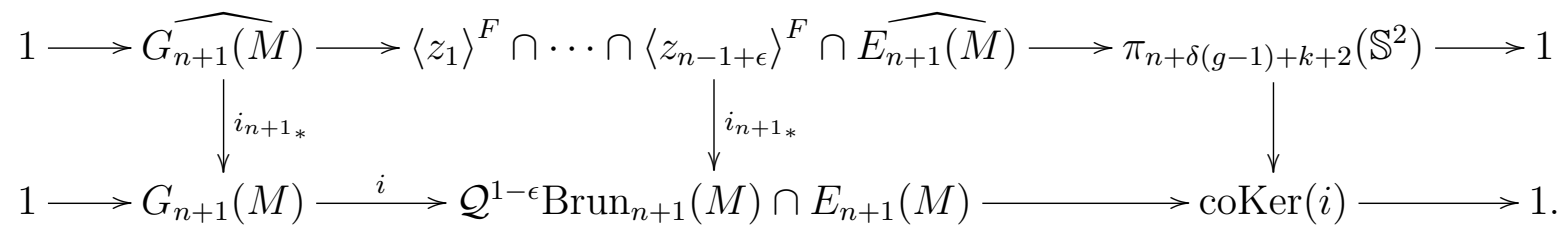

Lembremos que dada a superfície $M=M_{g, k}$

$$
\epsilon=\left\{\begin{array}{lll}
0 & \text { se } & k=0 \\
1 & \text { se } \quad k \geq 1
\end{array}\right.
$$

e lembremos também que $\mathcal{Q}^{0} \operatorname{Brun}_{n+1}(M)=\operatorname{Brun}_{n+1}(M)$. Portanto, o resultado desejado 
segue do diagrama comutativo acima.

O caso $n=1$ e $M \neq \mathbb{S}^{2}, \mathbb{R} P^{2}$ é analisado de forma similar ao feito acima.

Gostaríamos de destacar que o resultado anterior estende a qualquer superfície alguns resultados já conhecidos na literatura sobre a conexão entre os grupos de tranças sobre superfícies e os grupos de homotopia da esfera. Para os grupos de tranças sobre a esfera em [9], sobre o disco em [58] e sobre a esfera e o plano projetivo em [5]. O objetivo da seguinte observação é ilustrar isto.

\section{Observações 2.3.4.}

1. Tomemos $n=3$ se $M=\mathbb{S}^{2}$ e $n=2$ se $M=\mathbb{R} P^{2}$, pois estes casos não foram considerados no Teorema 2.3.3 dado que, pela Proposição 2.2.10, os grupos $\mathcal{Q}^{1} \operatorname{Brun}_{4}\left(\mathbb{S}^{2}\right)$ e $\mathcal{Q}^{1} \operatorname{Brun}_{3}\left(\mathbb{R} P^{2}\right)$ são grupos livres de posto 5. No entanto, seguindo o mesmo raciocínio usado na prova do teorema pode-se provar que as seguintes sequências são exatas curtas:

(a) Para tranças com 4 cordas sobre a esfera.

$$
1 \longrightarrow G_{4}\left(\mathbb{S}^{2}\right) \longrightarrow\left\langle A_{2,4}\right\rangle^{P_{4}\left(\mathbb{S}^{2}\right)} \cap\left\langle A_{3,4}\right\rangle^{P_{4}\left(\mathbb{S}^{2}\right)} \cap E_{4}\left(\mathbb{S}^{2}\right) \longrightarrow \pi_{3}\left(\mathbb{S}^{2}\right) \longrightarrow 1
$$

(b) Para tranças com 3 cordas sobre o plano projetivo.

$$
1 \longrightarrow G_{3}\left(\mathbb{R} P^{2}\right) \longrightarrow\left\langle A_{2,3}\right\rangle^{P_{3}\left(\mathbb{R} P^{2}\right)} \cap E_{3}\left(\mathbb{R} P^{2}\right) \longrightarrow \pi_{3}\left(\mathbb{S}^{2}\right) \longrightarrow 1
$$

2. O resultado acima (sequência exata curta (2.15) do Teorema 2.3.3) no caso particular em que $M=\mathbb{S}^{2}$ coincide com o Teorema 3.10 item (2) de Bardakov et al. [5] (ver Teorema 1.2.14). Vimos, no Exemplo 2.3.2, que $G_{n+1}\left(\mathbb{S}^{2}\right)$ é o grupo $R_{n+1}\left(\mathbb{S}^{2}\right)$ de [5] e que $E_{n+1}\left(\mathbb{S}^{2}\right)=\left\langle A_{1, n+1}\right\rangle^{P_{n+1}\left(\mathbb{S}^{2}\right)}$. Como Brun $n+1\left(\mathbb{S}^{2}\right) \subset \mathcal{Q}^{1}$ Brun $_{n+1}\left(\mathbb{S}^{2}\right)$ pela Proposição 2.2.3 e $\bigcap_{i=1}^{n}\left\langle A_{i, n+1}\right\rangle^{P}=$ Brun $_{n+1}\left(\mathbb{S}^{2}\right)$ pelo Lema 2.1.5, então

$$
\mathcal{Q}^{1} \operatorname{Brun}_{n+1}\left(\mathbb{S}^{2}\right) \cap E_{n+1}\left(\mathbb{S}^{2}\right)=\operatorname{Brun}_{n+1}\left(\mathbb{S}^{2}\right)
$$

3. No caso particular em que $M$ é o disco obtemos o mesmo isomorfismo do item 1 do Teorema 1.3.25, que relaciona grupos de tranças Brunnianas sobre o disco e grupos de homotopia da esfera $\mathbb{S}^{2}$. Com o intuito de verificar a afirmação anterior vamos supor que $n \geq 3$ e lembremos que $B d_{n+1}=\partial\left(\operatorname{Brun}_{n+2}\left(\mathbb{D}^{2}\right)\right)$, ver (1.20), onde d é o homomorfismo que definimos na Equação (1.4) do Capítulo 1.

No Exemplo 2.3.2 vimos que

$$
E_{n+1}\left(\mathbb{D}^{2}\right)=\left\langle A_{0, n+1}\right\rangle^{\operatorname{Ker}\left(d_{n+1}\right)}
$$


onde $A_{0, n+1}=\left(A_{1, n+1} A_{2, n+1} \cdots A_{n, n+1}\right)^{-1} e$

$$
G_{n+1}\left(\mathbb{D}^{2}\right)=\partial\left(\operatorname{Brun}_{n+2}\left(\mathbb{D}^{2}\right)\right)=B d_{n+1}
$$

Lembremos que $f_{*}: P_{n+1}\left(\mathbb{D}^{2}\right) \rightarrow P_{n+1}\left(\mathbb{S}^{2}\right)$ é o homomorfismo induzido pela inclusão natural $f: \mathbb{D}^{2} \hookrightarrow \mathbb{S}^{2}$, com núcleo $\operatorname{Ker}\left(f_{*}\right)=E_{n+1}\left(\mathbb{D}^{2}\right)$. Provaremos no Lema 2.4.2 que $\operatorname{Ker}\left(f_{*}\right) \cap \operatorname{Ker}\left(d_{n+1}\right)=\operatorname{Ker}(\partial) \cap \operatorname{Ker}\left(d_{n+1}\right)$ e assim

$$
\operatorname{Brun}_{n+1}\left(\mathbb{D}^{2}\right) \cap \operatorname{Ker}(\partial)=\operatorname{Brun}_{n+1}\left(\mathbb{D}^{2}\right) \cap \operatorname{Ker}\left(f_{*}\right)=\operatorname{Brun}_{n+1}\left(\mathbb{D}^{2}\right) \cap E_{n+1}\left(\mathbb{D}^{2}\right)
$$

Na prova do Teorema 4.3.12 verificaremos que

$$
\operatorname{Brun}_{n+1}\left(\mathbb{D}^{2}\right) \cap \theta\left(\operatorname{Brun}_{n+1}\left(\mathbb{D}^{2}\right)\right)=\operatorname{Brun}_{n+1}\left(\mathbb{D}^{2}\right) \cap \operatorname{Ker}(\partial) .
$$

$\log o$

$$
\operatorname{Brun}_{n+1}\left(\mathbb{D}^{2}\right) \cap \theta\left(\operatorname{Brun}_{n+1}\left(\mathbb{D}^{2}\right)\right)=\operatorname{Brun}_{n+1}\left(\mathbb{D}^{2}\right) \cap E_{n+1}\left(\mathbb{D}^{2}\right)
$$

Desta forma, a sequência exata curta (2.16) do Teorema 2.3.3, válida para $n \geq 3$, ficaria assim:

$$
1 \longrightarrow B d_{n+1} \longrightarrow \operatorname{Brun}_{n+1}\left(\mathbb{D}^{2}\right) \cap \theta\left(\operatorname{Brun}_{n+1}\left(\mathbb{D}^{2}\right)\right) \longrightarrow \pi_{n+1}\left(\mathbb{S}^{2}\right) \longrightarrow 1
$$

Observemos que $B d_{n+1}$ é normal em $P_{n+1}$, pois $\operatorname{Brun}_{n+2}\left(\mathbb{D}^{2}\right)$ é normal em $P_{n+2}$ e $\partial: P_{n+2} \rightarrow P_{n+1}$ é um homomorfismo sobrejetor (ver o comentário depois da definição de $\partial$ em (1.4)). Logo, existe um isomorfismo de grupos

$$
\frac{\operatorname{Brun}_{n+1}\left(\mathbb{D}^{2}\right) \cap \theta\left(\operatorname{Brun}_{n+1}\left(\mathbb{D}^{2}\right)\right)}{B d_{n+1}} \cong \pi_{n+1}\left(\mathbb{S}^{2}\right)
$$

4. É importante notar que os resultados que aparecem na literatura, inclusive o Teorema 1 de Li e Wu em [58], não mostram a conexão entre tranças e os grupos de homotopia inferiores da esfera.

Por exemplo, podemos exibir tranças com 2 cordas no disco que se projetam no gerador de $\pi_{2}\left(\mathbb{S}^{2}\right)$, similarmente tranças com 3 cordas no disco que se projetam no gerador de $\pi_{3}\left(\mathbb{S}^{2}\right)$. Vejamos alguns exemplos explícitos na continuação, seguindo a construção feita em [25, Página 2109]:

(a) Como $G_{2}\left(\mathbb{D}^{2}\right)=\{1\}$ e $P_{2}\left(\mathbb{D}^{2}\right)=\operatorname{Brun}_{2}\left(\mathbb{D}^{2}\right)=E_{2}\left(\mathbb{D}^{2}\right)=\left\langle A_{1,2}\right\rangle^{P_{2}}$, então segue que o elemento $A_{1,2} \in P_{2}\left(\mathbb{D}^{2}\right)$ está em correspondência com a aplicação grau $\mathbb{S}^{2} \rightarrow \mathbb{S}^{2}$ que gera o grupo de homotopia $\pi_{2}\left(\mathbb{S}^{2}\right)$. Observemos que o fecho da trança $A_{1,2}$ é o enlaçamento de Hopf, ver Figura 1.13. 
(b) O elemento $\left[A_{1,3}, A_{2,3}\right] \in P_{3}\left(\mathbb{D}^{2}\right)$ está em correspondência com a classe de homotopia da fibração de Hopf.

Notemos que o fecho desse elemento corresponde aos anéis de borromeo, ver Figura 2.7.
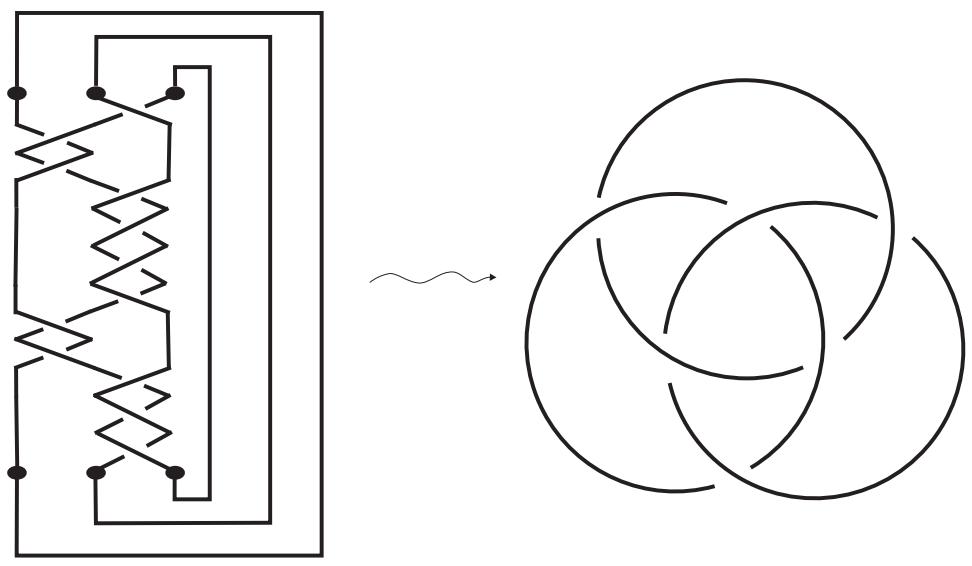

Figura 2.7: Fecho da trança $\left[A_{1,3}, A_{2,3}\right]$ : Anéis de Borromeo.

(c) O elemento $\left[\left[A_{1,4}, A_{2,4}\right],\left[A_{1,4}, A_{2,4} A_{3,4}\right]\right] \in P_{4}\left(\mathbb{D}^{2}\right)$ corresponde à classe de homotopia da composição de aplicações $\mathbb{S}^{4} \rightarrow \mathbb{S}^{3} \rightarrow \mathbb{S}^{2}$.

5. O Teorema 2.3.3, no caso em que a superfície $M$ é o plano projetivo dá lugar a um resultado similar, mas diferente, àquele dado por Bardakov et al. em [5] (ver Teorema 1.2.14). No Teorema 2.3.3 as tranças com $n+1$ cordas sobre o plano projetivo se projetam em elementos de $\pi_{n+1}\left(\mathbb{S}^{2}\right)$, a diferença do resultado de Bardakov et al. em que são as tranças com $n+2$ cordas que se projetam neste grupo de homotopia.

\subsection{Um monomorfismo entre tranças sobre a esfera e tranças sobre o disco}

\subsubsection{Construindo um homomorfismo de grupos de $\operatorname{Brun}_{n+1}\left(\mathbb{S}^{2}\right)$ em $\operatorname{Brun}_{n}\left(\mathbb{D}^{2}\right)$}

Nessa subseção usaremos a seguinte notação:

- Para $1 \leq j \leq n+1$ e $1 \leq i \leq n+1$, as aplicações $\delta_{j}: P_{n+1}\left(\mathbb{S}^{2}\right) \rightarrow P_{n}\left(\mathbb{S}^{2}\right)$ e $d_{i}: P_{n+1}\left(\mathbb{D}^{2}\right) \rightarrow P_{n}\left(\mathbb{D}^{2}\right)$ são homomorfismos induzidos geometricamente por esquecimento da $j$-ésima corda e da $i$-ésima corda, respectivamente.

- $\partial: P_{n+1}\left(\mathbb{D}^{2}\right) \rightarrow P_{n}\left(\mathbb{D}^{2}\right)$ é o homomorfismo definido na Equação (1.4) da Subseção 1.2.3 e o elemento $A_{0, n}=\left(A_{1, n} A_{2, n} \cdots A_{n-1, n}\right)^{-1} \in P_{n}\left(\mathbb{D}^{2}\right)$ foi definido na Equação (1.6). O homomorfismo $\partial$ também será usado no Capítulo 4, bem como nas Seções 6.1 e 6.2 deste texto. 
- $f: \mathbb{D}^{2} \hookrightarrow \mathbb{S}^{2}$ é a inclusão do disco como sendo o hemisfério norte da esfera e $f_{*}: P_{n}\left(\mathbb{D}^{2}\right) \rightarrow P_{n}\left(\mathbb{S}^{2}\right)$ é o homomorfismo induzido.

Lema 2.4.1. Para $n \geq 3$ existe um monomorfismo

$$
\psi_{n+1}: \operatorname{Ker}\left(\delta_{n+1}: P_{n+1}\left(\mathbb{S}^{2}\right) \rightarrow P_{n}\left(\mathbb{S}^{2}\right)\right) \rightarrow P_{n}\left(\mathbb{D}^{2}\right)
$$

que induz isomorfismos

$$
\psi_{n+1}: \operatorname{Ker}\left(\delta_{n+1}\right) \rightarrow \operatorname{Ker}\left(d_{n}\right) \text { e } \psi_{n+1}: \mathcal{Q}^{k} \operatorname{Brun}_{n+1}\left(\mathbb{S}^{2}\right) \rightarrow \mathcal{Q}^{k-1} \operatorname{Brun}_{n}\left(\mathbb{D}^{2}\right)
$$

o segundo sendo para todo $n \geq 4$ e $1 \leq k \leq n$.

Em particular, para $n \geq 4, \psi_{n+1}: \mathcal{Q}^{1}$ Brun $_{n+1}\left(\mathbb{S}^{2}\right) \rightarrow$ Brun $_{n}\left(\mathbb{D}^{2}\right)$ é um isomorfismo.

Demonstração. Seja $n \geq 3$ e consideremos as seguintes sequências exatas, induzidas pela fibração de Fadell-Neuwirth:

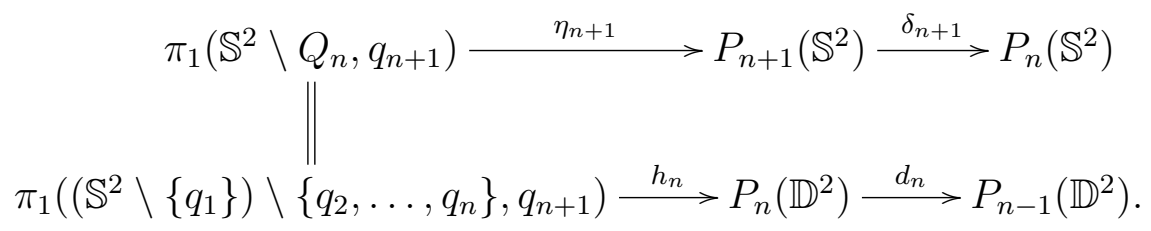

Sabemos que os homomorfismos $h_{n}$ e $\eta_{n+1}$ são de fato monomorfismos. Desta forma podemos definir o homomorfismo $\psi_{n+1}=h_{n} \circ \eta_{n+1}^{-1}$, para $n \geq 3$,

$$
\psi_{n+1}: \operatorname{Ker}\left(\delta_{n+1}\right) \rightarrow P_{n}\left(\mathbb{D}^{2}\right)
$$

Uma apresentação para $\operatorname{Ker}\left(\delta_{n+1}\right)$ e $\operatorname{Ker}\left(d_{n}\right)$ é dada por:

$$
\left\{\begin{array}{l}
\operatorname{Ker}\left(\delta_{n+1}\right)=\left\langle D_{1, n+1}, D_{2, n+1}, \ldots, D_{n, n+1} \mid D_{1, n+1} D_{2, n+1} \cdots D_{n, n+1}=1\right\rangle \\
\operatorname{Ker}\left(d_{n}\right)=\left\langle A_{1, n}, A_{2, n}, \ldots, A_{n-1, n} \mid-\right\rangle
\end{array}\right.
$$

Observemos que $\psi_{n+1}: \operatorname{Ker}\left(\delta_{n+1}\right) \rightarrow \operatorname{Ker}\left(d_{n}\right)$ é um isomorfismo e, para cada $i=1,2, \ldots, n$, temos $\psi_{n+1}\left(D_{i, n+1}\right)=A_{i-1, n}$.

Como $\mathcal{Q}^{k} \operatorname{Brun}_{n+1}\left(\mathbb{S}^{2}\right) \subset \operatorname{Ker}\left(\delta_{n+1}\right)$, o monomorfismo $\psi_{n+1}$ se restringe ao grupo $\mathcal{Q}^{k} \operatorname{Brun}_{n+1}\left(\mathbb{S}^{2}\right)$. Usando as descrições em termos de grupos comutadores simétricos, dadas pelos Teoremas 2.2.12 e 1.2.14, dos grupos quase-Brunnianos e dos grupos Brunnianos respectivamente, temos:

$$
\begin{aligned}
\psi_{n+1}\left(\mathcal{Q}^{k} \operatorname{Brun}_{n+1}\left(\mathbb{S}^{2}\right)\right) & =\psi_{n+1}\left(\left[\left\langle D_{k+1, n+1}\right\rangle^{P_{n+1}\left(\mathbb{S}^{2}\right)}, \ldots,\left\langle D_{n, n+1}\right\rangle^{P_{n+1}\left(\mathbb{S}^{2}\right)}\right]_{S}\right) \\
& =\left[\left\langle A_{k, n}\right\rangle^{P_{n}},\left\langle A_{k+1, n}\right\rangle^{P_{n}}, \ldots,\left\langle A_{n-1, n}\right\rangle^{P_{n}}\right]_{S} \\
& =\mathcal{Q}^{k-1} \operatorname{Brun}_{n}\left(\mathbb{D}^{2}\right)
\end{aligned}
$$


e portanto $\psi_{n+1}: \mathcal{Q}^{k} \operatorname{Brun}_{n+1}\left(\mathbb{S}^{2}\right) \rightarrow \mathcal{Q}^{k-1} \operatorname{Brun}_{n}\left(\mathbb{D}^{2}\right)$ é um isomorfismo.

Observemos que pode-se provar de forma alternativa o isomorfismo mencionado no Lema 2.4.1 $\psi_{n+1}: \mathcal{Q}^{k} \operatorname{Brun}_{n+1}\left(\mathbb{S}^{2}\right) \rightarrow \mathcal{Q}^{k-1} \operatorname{Brun}_{n}\left(\mathbb{D}^{2}\right)$, para $n \geq 4$. Na continuação ilustramos a prova no caso de $k=1$. A demonstração do caso geral é análoga com aquela dada para o caso particular.

Para $2 \leq j \leq n$, o seguinte diagrama é comutativo e induzido por um diagrama geométrico em espaços de configuração, onde

$$
X\left(\mathbb{S}^{2}, n+1, q_{1}\right)=\pi_{1}\left(\mathbb{S}^{2} \backslash Q_{n}, q_{n+1}\right)=\pi_{1}\left(\left(\mathbb{S}^{2} \backslash\left\{q_{1}\right\}\right) \backslash\left\{q_{2}, \ldots, q_{n}\right\}, q_{n+1}\right)
$$

e $\xi$ é a aplicação induzida por colocar de volta o ponto esquecido $q_{j}$ ("filling in the missing point" $\left.q_{j}\right)$ :

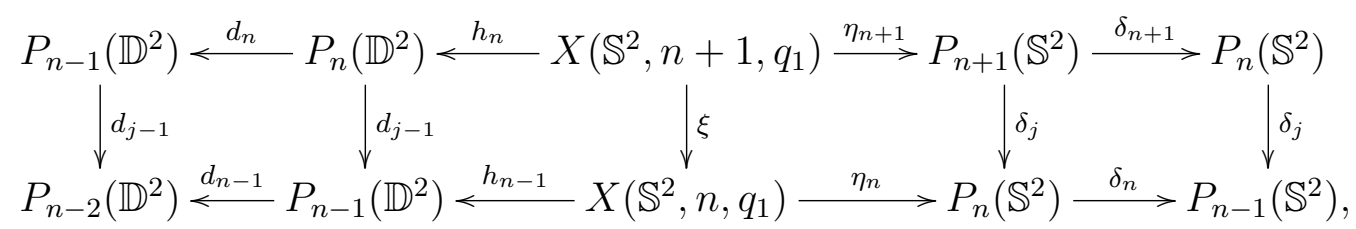

Definimos o homomorfismo $\psi_{n+1}$ como sendo $\psi_{n+1}=h_{n} \circ \eta_{n+1}^{-1}$. Considerando $n \geq 4$ temos que $\psi_{n+1}$ é injetor. Segue, do diagrama comutativo (2.22), que o seguinte quadrado é comutativo, para $n \geq 4$ e $2 \leq j \leq n$,

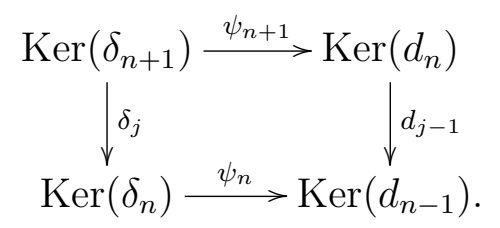

Dada $\beta \in \mathcal{Q}^{1} \operatorname{Brun}_{n+1}\left(\mathbb{S}^{2}\right)$, para $2 \leq j \leq n+1$, do quadrado comutativo (2.23), segue que:

$$
d_{j-1}\left(\psi_{n+1}(\beta)\right)=\psi_{n} \delta_{j}(\beta)=1
$$

e portanto $\psi_{n+1}: \mathcal{Q}^{1} \operatorname{Brun}_{n+1}\left(\mathbb{S}^{2}\right) \rightarrow \operatorname{Brun}_{n}\left(\mathbb{D}^{2}\right)$ está bem definida. Agora, dada uma trança $\gamma \in \operatorname{Brun}_{n}\left(\mathbb{D}^{2}\right)$, chamemos de $\gamma^{\prime}$ a trança $\gamma$ com uma corda vertical adicional à esquerda. Consideremos $\gamma^{\prime} \in P_{n+1}\left(\mathbb{S}^{2}\right)$ e notemos que $\gamma^{\prime} \in \mathcal{Q}^{1} \operatorname{Brun}_{n+1}\left(\mathbb{S}^{2}\right)$, pois $\gamma \in \operatorname{Brun}_{n}\left(\mathbb{D}^{2}\right)$ e ainda mais, seguindo o diagrama $(2.22)$, vemos que $\psi_{n+1}\left(\gamma^{\prime}\right)=\gamma$, isto é $\psi_{n+1}: \mathcal{Q}^{1} \operatorname{Brun}_{n+1}\left(\mathbb{S}^{2}\right) \rightarrow \operatorname{Brun}_{n}\left(\mathbb{D}^{2}\right)$ é sobrejetor e portanto um isomorfismo, para $n \geq 4$.

O seguinte lema estabelece igualdades entre intersecções de núcleos das aplicações $d_{n}: P_{n}\left(\mathbb{D}^{2}\right) \rightarrow P_{n-1}\left(\mathbb{D}^{2}\right), f_{*}: P_{n}\left(\mathbb{D}^{2}\right) \rightarrow P_{n}\left(\mathbb{S}^{2}\right)$ e $\partial: P_{n}\left(\mathbb{D}^{2}\right) \rightarrow P_{n-1}\left(\mathbb{D}^{2}\right)$. Também é provada uma outra igualdade com a imagem do $\operatorname{grupo} \operatorname{Ker}\left(\delta_{n+1}\right) \cap \operatorname{Ker}\left(\delta_{1}\right)$ via o homomorfismo $\psi_{n+1}: \operatorname{Ker}\left(\delta_{n+1}\right) \rightarrow P_{n}\left(\mathbb{D}^{2}\right)$, onde os homomorfismos $\delta_{n+1}$ e $\delta_{1}$ são definidos no grupo de tranças $P_{n+1}\left(\mathbb{S}^{2}\right)$. 
Lema 2.4.2. Para $n \geq 4$ as seguintes igualdades de grupos são válidas no grupo de tranças do disco $P_{n}\left(\mathbb{D}^{2}\right)$ :

1. $\psi_{n+1}\left(\operatorname{Ker}\left(\delta_{n+1}\right) \cap \operatorname{Ker}\left(\delta_{1}\right)\right)=\operatorname{Ker}\left(d_{n}\right) \cap \operatorname{Ker}(\partial)$.

2. $\operatorname{Ker}\left(d_{n}\right) \cap \operatorname{Ker}\left(f_{*}\right)=\operatorname{Ker}\left(d_{n}\right) \cap \operatorname{Ker}(\partial)$.

Demonstração. Seja $n \geq 4$.

1. Usando as apresentações dos grupos $\operatorname{Ker}\left(\delta_{n+1}\right), \operatorname{Ker}\left(\delta_{n}\right), \operatorname{Ker}\left(d_{n}\right)$ e $\operatorname{Ker}\left(d_{n-1}\right)$ (ver Equação (2.21)), junto com a definição das aplicações $\partial$, dada na Equação (1.4), e $\delta_{1}$ temos que

$$
\delta_{1}\left(\operatorname{Ker}\left(\delta_{n+1}\right)\right)=\operatorname{Ker}\left(\delta_{n}\right) \quad \text { e } \quad \partial\left(\operatorname{Ker}\left(d_{n}\right)\right)=\operatorname{Ker}\left(d_{n-1}\right)
$$

Sabemos que $\partial\left(A_{0, j}\right)=1$, para $2 \leq j \leq n$, onde o elemento $A_{0, j}$ aqui é igual ao elemento $A_{-1, j}$ de [9]. Observemos que lá foi usada a indexação simplicial (para mais detalhes sugerimos ver a Seção 6.5 de [9] e a Seção 6.1 desse texto). Agora, notemos que em $P_{n-1}\left(\mathbb{D}^{2}\right)$ valem as seguintes igualdades:

(i)

$$
\partial \circ \psi_{n+1}\left(D_{i, n+1}\right)=\left\{\begin{aligned}
\partial\left(A_{i-1, n}\right)=A_{i-2, n-1}, & \text { se } 2 \leq i \leq n \\
\partial\left(A_{0, n}\right)=1, & \text { se } i=1 .
\end{aligned}\right.
$$

(ii)

$$
\psi_{n} \circ \delta_{1}\left(D_{i, n+1}\right)=\left\{\begin{aligned}
\psi_{n}\left(D_{i-1, n}\right)=A_{i-2, n-1}, & \text { se } 2 \leq i \leq n, \\
1, & \text { se } i=1 .
\end{aligned}\right.
$$

Portanto, por $(i)$ e $(i i)$, o seguinte quadrado é comutativo, para todo $n \geq 4$ :

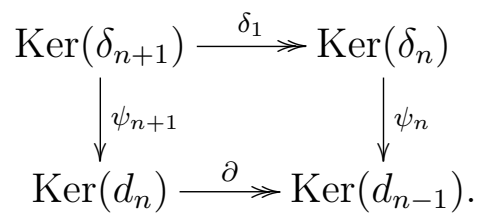

Completando o quadrado (2.24) com os núcleos das aplicações $\delta_{1}$ e $\partial$, temos o seguinte diagrama comutativo:

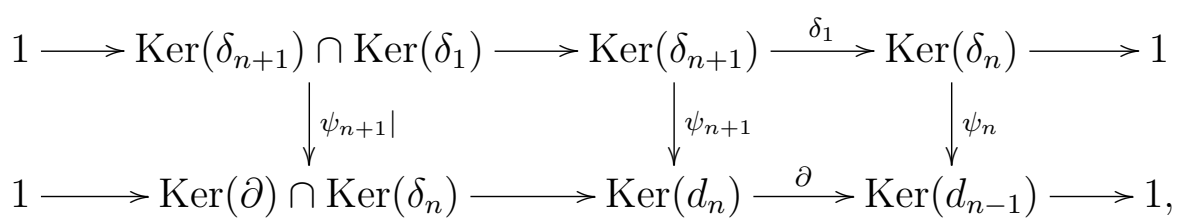

onde as linhas horizontais são sequências exatas curtas e as aplicações $\psi_{n+1}$ e $\psi_{n}$ são isomorfismos pelo Lema 2.4.1. Logo, pelo lema dos cinco, temos que $\psi_{n+1} \mid$ é por 
sua vez um isomorfismo e portanto $\psi_{n+1}\left(\operatorname{Ker}\left(\delta_{n+1}\right) \cap \operatorname{Ker}\left(\delta_{1}\right)\right)=\operatorname{Ker}(\partial) \cap \operatorname{Ker}\left(d_{n}\right)$, provando assim o primeiro item.

2. Notemos que em $P_{n-1}\left(\mathbb{D}^{2}\right)$ vale a seguinte igualdade:

$$
\psi_{n+1} \circ f_{*}\left(A_{i, n}\right)=\left\{\begin{array}{cl}
\psi_{n+1}\left(D_{i, n}\right)=A_{i-1, n-1}, & \text { se } 2 \leq i \leq n \\
\psi_{n+1}\left(D_{1, n}\right)=A_{0, n-1}, & \text { se } i=1
\end{array}\right.
$$

Portanto, segue da igualdade (2.25) e da definição de $\partial$ (dada na Equação (1.4)) que o seguinte quadrado é comutativo, onde $\psi_{n}$ é um isomorfismo para todo $n \geq 4$, pelo Lema 2.4.1:

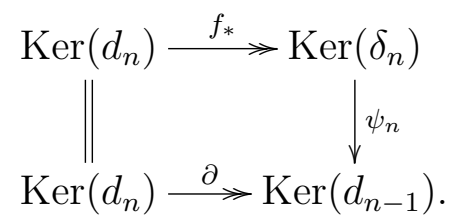

Como no caso anterior temos que $\operatorname{Ker}\left(d_{n}\right) \cap \operatorname{Ker}\left(f_{*}\right)=\operatorname{Ker}\left(d_{n}\right) \cap \operatorname{Ker}(\partial)$.

Observação 2.4.3. O quadrado comutativo (2.26) que aparece na demonstração do item 2 do Lema 2.4.2 é similar àquele dado no Lema 6.6.3 de [9] onde no lugar da $\psi_{n}$ aparece um homomorfismo $\alpha$ (ver o quadrado comutativo (6.3) do Capítulo 3), mas veremos que não pode existir uma tal $\alpha$ com as propriedades mencionadas em [9]. Mais detalhes podem ser encontrados na Seção 6.1.

$\mathrm{Na}$ continuação aplicamos os Lemas 2.4.1 e 2.4.2 e exibimos um isomorfismo do grupo de tranças Brunnianas com $n+1$ cordas sobre a esfera, Brun $_{n+1}\left(\mathbb{S}^{2}\right)$, com o grupo $\operatorname{Brun}_{n}\left(\mathbb{D}^{2}\right) \cap \operatorname{Ker}\left(f_{*}\right)$.

Teorema 2.4.4. Para $n \geq 4$ o homomorfismo

$$
\psi_{n+1}: \operatorname{Brun}_{n+1}\left(\mathbb{S}^{2}\right) \rightarrow \operatorname{Brun}_{n}\left(\mathbb{D}^{2}\right) \cap \operatorname{Ker}\left(f_{*}\right)
$$

é um isomorfismo. Em particular, $\psi_{n+1}: \operatorname{Brun}_{n+1}\left(\mathbb{S}^{2}\right) \rightarrow \operatorname{Brun}_{n}\left(\mathbb{D}^{2}\right)$ é um homomorfismo injetor, para $n \geq 4$.

Demonstração. Seja $n \geq 4$. Pelo Lema 2.4.1 $\psi_{n+1}: \mathcal{Q}^{1} \operatorname{Brun}_{n+1}\left(\mathbb{S}^{2}\right) \rightarrow \operatorname{Brun}_{n}\left(\mathbb{D}^{2}\right)$ é um isomorfismo e pela Proposição 2.2.3 temos que

$$
\operatorname{Brun}_{n+1}\left(\mathbb{S}^{2}\right)=\operatorname{Ker}\left(\delta_{1}\right) \cap \mathcal{Q}^{1} \operatorname{Brun}_{n+1}\left(\mathbb{S}^{2}\right)=\operatorname{Ker}\left(\delta_{1}\right) \cap \mathcal{Q}^{1} \operatorname{Brun}_{n+1}\left(\mathbb{S}^{2}\right) \cap \operatorname{Ker}\left(\delta_{n+1}\right)
$$

Sabemos que um monomorfismo aplicado numa intersecção de grupos é igual à intersecção do monomorfismo aplicado em cada um dos grupos envolvidos. Portanto, usando os Lemas 
2.4.1 e 2.4.2, temos as seguintes igualdades:

$$
\begin{aligned}
\psi_{n+1}\left(\operatorname{Brun}_{n+1}\left(\mathbb{S}^{2}\right)\right) & =\psi_{n+1}\left(\operatorname{Ker}\left(\delta_{1}\right) \cap \operatorname{Ker}\left(\delta_{n+1}\right) \cap \mathcal{Q}^{1} \operatorname{Brun}_{n+1}\left(\mathbb{S}^{2}\right)\right) \\
& =\psi_{n+1}\left(\operatorname{Ker}\left(\delta_{1}\right) \cap \operatorname{Ker}\left(\delta_{n+1}\right)\right) \cap \psi_{n+1}\left(\mathcal{Q}^{1} \operatorname{Brun}_{n+1}\left(\mathbb{S}^{2}\right)\right) \\
& =\left(\operatorname{Ker}(\partial) \cap \operatorname{Ker}\left(d_{n}\right)\right) \cap \operatorname{Brun}_{n}\left(\mathbb{D}^{2}\right) \\
& =\left(\operatorname{Ker}\left(f_{*}\right) \cap \operatorname{Ker}\left(d_{n}\right)\right) \cap \operatorname{Brun}_{n}\left(\mathbb{D}^{2}\right) \\
& =\operatorname{Ker}\left(f_{*}\right) \cap \operatorname{Brun}_{n}\left(\mathbb{D}^{2}\right) .
\end{aligned}
$$

A injetividade de $\psi_{n+1}: \operatorname{Ker}\left(\delta_{n+1}\right) \rightarrow \operatorname{Ker}\left(d_{n}\right)$ implica o resultado.

O Teorema 2.4.4 será usado na Subseção 2.4.2 para dar uma prova alternativa do Teorema 6.6.5 de [9] e também será usado na Subseção 2.4.3 para demonstrar o Teorema 1 item (1) do Li e Wu [58] (ver o Teorema 1.3.25 no Capítulo 1).

\subsubsection{Uma prova diferente do Teorema 1.2 de Cohen et al. [9]}

Vamos dar uma prova diferente do Teorema 6.6.5 de [9], usando o Lema 2.4.2 e os Teoremas 2.3.3, 2.4.4. Melhor ainda, podemos aqui corrigir o problema encontrado na Seção 6.5 de [9] e fornecer uma descrição explícita do homomorfismo que leva tranças Brunnianas sobre a esfera em tranças Brunnianas sobre o disco. Ressaltamos que o Teorema 6.6.5 de [9] é uma versão mais detalhada do Teorema 1.2 do mesmo artigo de Cohen et al.

Teorema 2.4.5 (Teorema 6.6.5 de [9]). Para $n \geq 5$, a sequência

$$
1 \longrightarrow \operatorname{Brun}_{n+1}\left(\mathbb{S}^{2}\right) \stackrel{\psi_{n+1}}{\longrightarrow} \operatorname{Brun}_{n}\left(\mathbb{D}^{2}\right) \stackrel{f_{*}}{\longrightarrow} \operatorname{Brun}_{n}\left(\mathbb{S}^{2}\right) \longrightarrow \pi_{n-1}\left(\mathbb{S}^{2}\right) \longrightarrow 1
$$

é exata e o diferencial algébrico $\psi_{n+1} f_{*}$ coincide com o diferencial geométrico $\partial$.

Demonstração. A comparação dos diferenciais apareceu na prova do item 2 do Lema 2.4.2. Para provar o resultado, inicialmente, vamos detalhar os elementos envolvidos no Teorema 2.3.3 para $M=\mathbb{S}^{2}$ e $k=0$. Pelo Exemplo 2.3.2 sabemos que, para $n \geq 4$, valem as seguintes igualdades:

$$
G_{n+1}\left(\mathbb{S}^{2}\right)=f_{*}\left(\operatorname{Brun}_{n+1}\left(\mathbb{D}^{2}\right)\right) \text {, e } E_{n+1}\left(\mathbb{S}^{2}\right)=\left\langle D_{1, n+1}\right\rangle^{P_{n+1}\left(\mathbb{S}^{2}\right)}
$$

Logo, da Proposição 2.2.3, segue que

$$
\begin{aligned}
E_{n+1}\left(\mathbb{S}^{2}\right) \cap \mathcal{Q}^{1} \operatorname{Brun}_{n+1}\left(\mathbb{S}^{2}\right) & =\left\langle D_{1, n+1}\right\rangle^{P_{n+1}\left(\mathbb{S}^{2}\right)} \cap\left\langle D_{2, n+1}\right\rangle^{P_{n+1}\left(\mathbb{S}^{2}\right)} \cap \cdots \cap\left\langle D_{n, n+1}\right\rangle^{P_{n+1}\left(\mathbb{S}^{2}\right)} \\
& =\operatorname{Brun}_{n+1}\left(\mathbb{S}^{2}\right)
\end{aligned}
$$

e assim pelo Teorema 2.3.3 item (1), no caso em que $M=\mathbb{S}^{2}$ e $k=0$, temos que a seguinte 
sequência é exata curta, para $n \geq 5$,

$$
1 \longrightarrow f_{*}\left(\operatorname{Brun}_{n}\left(\mathbb{D}^{2}\right)\right) \longrightarrow \operatorname{Brun}_{n}\left(\mathbb{S}^{2}\right) \longrightarrow \pi_{n-1}\left(\mathbb{S}^{2}\right) \longrightarrow 1
$$

Por outro lado, pelo Teorema 2.4.4, temos que a seguinte sequência é exata, para $n \geq 4$,

$$
1 \longrightarrow \operatorname{Brun}_{n+1}\left(\mathbb{S}^{2}\right) \stackrel{\psi_{n+1}}{\longrightarrow} \operatorname{Brun}_{n}\left(\mathbb{D}^{2}\right) \stackrel{f_{*}}{\longrightarrow} \operatorname{Brun}_{n}\left(\mathbb{S}^{2}\right)
$$

O resultado desejado segue juntando a informação nas duas sequências exatas (2.27) e $(2.28)$.

\subsubsection{Uma prova diferente do Teorema 1 (1) de Li e Wu em [58]}

O seguinte resultado é uma consequência do Teorema 2.4.4, donde segue, em particular, o isomorfismo descrito no item (1) do Teorema 1 de Li e Wu em [58] (ver Teorema 1.3.25). Como $f_{*}: P_{n+1} \rightarrow P_{n+1}\left(\mathbb{S}^{2}\right)$ é um epimorfismo e $\operatorname{Brun}_{n+1}\left(\mathbb{D}^{2}\right)$ é normal em $P_{n+1}$, então $f_{*}\left(\operatorname{Brun}_{n+1}\left(\mathbb{D}^{2}\right)\right)$ é normal em $P_{n+1}$. Se $n \geq 4$, então $f_{*}\left(\operatorname{Brun}_{n+1}\left(\mathbb{D}^{2}\right)\right)$ está contido em $\operatorname{Brun}_{n+1}\left(\mathbb{S}^{2}\right)$ (ver Teorema 2.4.5) e portanto $f_{*}\left(\operatorname{Brun}_{n+1}\left(\mathbb{D}^{2}\right)\right)$ é normal em $\operatorname{Brun}_{n+1}\left(\mathbb{S}^{2}\right)$.

Teorema 2.4.6. Seja $n \geq 4$. O isomorfismo $\psi_{n+1}: \operatorname{Brun}_{n+1}\left(\mathbb{S}^{2}\right) \rightarrow \operatorname{Brun}_{n}\left(\mathbb{D}^{2}\right) \cap \operatorname{Ker}\left(f_{*}\right)$ induz um isomorfismo de grupos quocientes,

$$
\bar{\psi}_{n+1}: \frac{\operatorname{Brun}_{n+1}\left(\mathbb{S}^{2}\right)}{f_{*}\left(\operatorname{Brun}_{n+1}\left(\mathbb{D}^{2}\right)\right)} \rightarrow \frac{\operatorname{Brun}_{n}\left(\mathbb{D}^{2}\right) \cap \operatorname{Ker}\left(f_{*}\right)}{\left[\left\langle A_{0, n}\right\rangle^{\operatorname{Ker}\left(d_{n}\right)},\left\langle A_{1, n}\right\rangle^{\operatorname{Ker}\left(d_{n}\right)}, \ldots,\left\langle A_{n-1, n}\right\rangle^{\operatorname{Ker}\left(d_{n}\right)}\right]_{S}} .
$$

Demonstração. Seja $n \geq 4$. Pelo Teorema 2.4.4 o homomorfismo

$$
\psi_{n+1}: \operatorname{Brun}_{n+1}\left(\mathbb{S}^{2}\right) \rightarrow \operatorname{Brun}_{n}\left(\mathbb{D}^{2}\right) \cap \operatorname{Ker}\left(f_{*}\right)
$$

é um isomorfismo e pelo Lema 2.4.1, temos que $\psi_{n+1}\left(\operatorname{Ker}\left(\delta_{n+1}\right)\right)=\operatorname{Ker}\left(d_{n}\right)$. Segue da equação (2.14) que

$$
f_{*}\left(\operatorname{Brun}_{n+1}\left(\mathbb{D}^{2}\right)\right)=\left[\left\langle D_{1, n+1}\right\rangle^{\operatorname{Ker}\left(\delta_{n+1}\right)}, \ldots,\left\langle D_{n, n+1}\right\rangle^{\operatorname{Ker}\left(\delta_{n+1}\right)}\right]_{S},
$$

Portanto, das seguintes igualdades de grupos

$$
\begin{aligned}
\psi_{n+1}\left(f_{*}\left(\operatorname{Brun}_{n+1}\left(\mathbb{D}^{2}\right)\right)\right) & =\psi_{n+1}\left(\left[\left\langle D_{1, n+1}\right\rangle^{\operatorname{Ker}\left(\delta_{n+1}\right)}, \ldots,\left\langle D_{n, n+1}\right\rangle^{\operatorname{Ker}\left(\delta_{n+1}\right)}\right]_{S}\right) \\
& =\left[\left\langle A_{0, n}\right\rangle^{\operatorname{Ker}\left(d_{n}\right)},\left\langle A_{1, n}\right\rangle^{\operatorname{Ker}\left(d_{n}\right)}, \ldots,\left\langle A_{n-1, n}\right\rangle^{\operatorname{Ker}\left(d_{n}\right)}\right]_{S},
\end{aligned}
$$

temos que a restrição do homomorfismo $\psi_{n+1}$,

$$
\psi_{n+1} \mid: f_{*}\left(\operatorname{Brun}_{n+1}\left(\mathbb{D}^{2}\right)\right) \rightarrow\left[\left\langle A_{0, n}\right\rangle^{\operatorname{Ker}\left(d_{n}\right)},\left\langle A_{1, n}\right\rangle^{\operatorname{Ker}\left(d_{n}\right)}, \ldots,\left\langle A_{n-1, n}\right\rangle^{\operatorname{Ker}\left(d_{n}\right)}\right]_{S},
$$


é um isomorfismo, para $n \geq 4$.

Como $f_{*}\left(\operatorname{Brun}_{n+1}\left(\mathbb{D}^{2}\right)\right)$ é um subgrupo normal de $\operatorname{Brun}_{n+1}\left(\mathbb{S}^{2}\right)$ e o homomorfismo $\psi_{n+1}: \operatorname{Brun}_{n+1}\left(\mathbb{S}^{2}\right) \rightarrow \operatorname{Brun}_{n}\left(\mathbb{D}^{2}\right) \cap \operatorname{Ker}\left(f_{*}\right)$ é sobrejetor (de fato isomorfismo), então

$$
\psi_{n+1}\left(f_{*}\left(\operatorname{Brun}_{n+1}\left(\mathbb{D}^{2}\right)\right)\right)=\left[\left\langle A_{0, n}\right\rangle^{\operatorname{Ker}\left(d_{n}\right)},\left\langle A_{1, n}\right\rangle^{\operatorname{Ker}\left(d_{n}\right)}, \ldots,\left\langle A_{n-1, n}\right\rangle^{\operatorname{Ker}\left(d_{n}\right)}\right]_{S}
$$

é normal em $\operatorname{Brun}_{n}\left(\mathbb{D}^{2}\right) \cap \operatorname{Ker}\left(f_{*}\right)$. Portanto, a seguinte composição

$$
\operatorname{Brun}_{n+1}\left(\mathbb{S}^{2}\right) \rightarrow \operatorname{Brun}_{n}\left(\mathbb{D}^{2}\right) \cap \operatorname{Ker}\left(f_{*}\right) \rightarrow \frac{\operatorname{Brun}_{n}\left(\mathbb{D}^{2}\right) \cap \operatorname{Ker}\left(f_{*}\right)}{\psi_{n+1}\left(f_{*}\left(\operatorname{Brun}_{n+1}\left(\mathbb{D}^{2}\right)\right)\right)}
$$

é sobrejetora e o núcleo é $\psi_{n+1}^{-1}\left(\psi_{n+1}\left(f_{*}\left(\operatorname{Brun}_{n+1}\left(\mathbb{D}^{2}\right)\right)\right)\right)$, que é igual a $f_{*}\left(\operatorname{Brun}_{n+1}\left(\mathbb{D}^{2}\right)\right)$, donde segue o resultado desejado.

Como consequência do Teorema 2.4.6, obtemos o isomorfismo descrito no Teorema 1 item (1) de Li e Wu [58], que enunciamos no Teorema 1.3.25 do Capítulo 1.

Corolário 2.4.7. Para $n \geq 4$ existe um isomorfismo de grupos

$$
\frac{\operatorname{Brun}_{n}\left(\mathbb{D}^{2}\right) \cap \operatorname{Ker}\left(f_{*}\right)}{\partial\left(\operatorname{Brun}_{n+1}\left(\mathbb{D}^{2}\right)\right)} \cong \pi_{n}\left(\mathbb{S}^{2}\right)
$$

Demonstração. Seja $n \geq 4$. Da sequência exata curta (2.27) que apareceu na prova do Teorema 2.4.5, sabemos que existe o seguinte isomorfismo:

$$
\frac{\operatorname{Brun}_{n+1}\left(\mathbb{S}^{2}\right)}{f_{*}\left(\operatorname{Brun}_{n+1}\left(\mathbb{D}^{2}\right)\right)} \cong \pi_{n}\left(\mathbb{S}^{2}\right)
$$

Agora, pelo Teorema 2.4.6, obtemos o seguinte isomorfismo:

$$
\frac{\operatorname{Brun}_{n+1}\left(\mathbb{S}^{2}\right)}{f_{*}\left(\operatorname{Brun}_{n+1}\left(\mathbb{D}^{2}\right)\right)} \cong \frac{\operatorname{Brun}_{n}\left(\mathbb{D}^{2}\right) \cap \operatorname{Ker}\left(f_{*}\right)}{\left[\left\langle A_{0, n}\right\rangle^{\operatorname{Ker}\left(d_{n}\right)},\left\langle A_{1, n}\right\rangle^{\operatorname{Ker}\left(d_{n}\right)}, \ldots,\left\langle A_{n-1, n}\right\rangle^{\operatorname{Ker}\left(d_{n}\right)}\right]_{S}}
$$

Vimos na sequência de igualdades (2.12), dadas no Exemplo 2.3.2, que

$$
\partial\left(\operatorname{Brun}_{n+1}\left(\mathbb{D}^{2}\right)\right)=\left[\left\langle A_{0, n}\right\rangle^{\operatorname{Ker}\left(d_{n}\right)},\left\langle A_{1, n}\right\rangle^{\operatorname{Ker}\left(d_{n}\right)}, \ldots,\left\langle A_{n-1, n}\right\rangle^{\operatorname{Ker}\left(d_{n}\right)}\right]_{S} .
$$

Portanto, existem isomorfismos de grupos

$$
\frac{\operatorname{Brun}_{n}\left(\mathbb{D}^{2}\right) \cap \operatorname{Ker}\left(f_{*}\right)}{\partial\left(\operatorname{Brun}_{n+1}\left(\mathbb{D}^{2}\right)\right)} \cong \frac{\operatorname{Brun}_{n+1}\left(\mathbb{S}^{2}\right)}{f_{*}\left(\operatorname{Brun}_{n+1}\left(\mathbb{D}^{2}\right)\right)} \cong \pi_{n}\left(\mathbb{S}^{2}\right)
$$

provando assim o resultado desejado.

A demonstração anterior é a segunda prova alternativa e diferente do item (1) do Teorema 1 de Li e Wu [58], sendo que a primeira delas apareceu nas Observações 2.3.4. 
Mais adiante, no Teorema 4.3.12 item 1 do Capítulo 4, daremos uma terceira demonstração alternativa desse resultado, mas aí seguiremos em alguns momentos um roteiro similar com o original proposto pelos autores do artigo [58]. 


\section{Capítulo 3}

\section{$\Delta$-grupos formados por tranças puras sobre superfícies}

Berrick, Cohen, Wong e Wu demonstraram que os grupos de homotopia de Moore dos $\Delta$-grupos formados por grupos de tranças puras sobre a esfera são isomorfos aos grupos de homotopia $\pi_{n}\left(\mathbb{S}^{2}\right)$, para $n \geq 4$ [9, Teorema 1.1]. Além disso, decorre da prova do Teorema 1.2 de [9] que o $\Delta$-grupo $\mathcal{P}\left(\mathbb{D}^{2}\right)$ é um grupo simplicial contrátil. Sendo assim, uma questão natural consiste em calcular os grupos de homotopia de Moore dos $\Delta$-grupos de tranças puras sobre qualquer outra superfície diferente da esfera e o disco. Responderemos esta questão na Seção 3.1. O Teorema 1.2.13 afirma que, para $n \geq 5$, a seguinte sequência de grupos é exata

$$
1 \longrightarrow \operatorname{Brun}_{n+1}\left(\mathbb{S}^{2}\right) \longrightarrow \operatorname{Brun}_{n}\left(\mathbb{D}^{2}\right) \stackrel{f_{*}}{\longrightarrow} \operatorname{Brun}_{n}\left(\mathbb{S}^{2}\right) \longrightarrow \pi_{n-1}\left(\mathbb{S}^{2}\right) \longrightarrow 1
$$

Na Seção 3.3 formularemos uma versão do Teorema 1.2.13 para outras superfícies, envolvendo a noção de grupos Brunnianos, quase-Brunnianos e semi-Brunnianos. Finalmente concluímos este capítulo com um exemplo de uma trança Brunniana com 5 cordas na esfera que se projeta no gerador de $\pi_{4}\left(\mathbb{S}^{2}\right)$.

\subsection{Grupos de homotopia de $\Delta$-grupos formados por tranças puras}

Lembremos que $\mathcal{P}(M)=\left\{P_{n+1}(M)\right\}_{n \geq 0}$ é o $\Delta$-grupo formado por grupos de tranças puras com aplicações faces induzidas pela fibração de Fadell-Neuwirth, fazendo um rearranjo dos pontos base (ver o Exemplo 1.3.2 do Capítulo 1, ou a referência [9]).

Nesta seção mostraremos que se $M$ é uma superfície diferente da esfera e do plano projetivo, então $\pi_{n}(\mathcal{P}(M))=\{1\}$, para todo $n \geq 0$. Já nos casos em que $M$ é a esfera ou o plano projetivo mostraremos que $\pi_{n}(\mathcal{P}(M))=\pi_{n}\left(\mathbb{S}^{2}\right)$, exceto para um número finito 
de casos em que $n$ é pequeno. Provaremos também que o $\Delta$-grupo $\mathcal{P}(M)$ é conexo e simplesmente conexo para qualquer superfície $M$.

Dado que usaremos ferramentas da teoria de homotopia simplicial, mudaremos durante esta seção a indexação das aplicações faces. De forma mais concreta, usaremos as aplicações faces dadas pelo esquecimento da $j$-ésima corda $d_{j}: P_{n}(M) \rightarrow P_{n-1}(M)$ para $j=0,1 \ldots, n-1$. Lembremos que, na Subseção 1.3.1, os conjuntos de homotopia de Moore foram definidos para um $\Delta$-grupo $\mathcal{G}=\left\{G_{n}\right\}_{n \geq 0}$ como sendo $\pi_{n}(\mathcal{G})=\mathcal{Z}_{n} \mathcal{G} / \mathcal{B} d_{n} \mathcal{G}$, onde

- $\mathcal{N}_{n} \mathcal{G}=\bigcap_{j=1}^{n} \operatorname{Ker}\left(d_{j}: G_{n} \rightarrow G_{n-1}\right)$

- $\mathcal{Z}_{n} \mathcal{G}=\operatorname{Ker}\left(d_{0}: \mathcal{N}_{n} \mathcal{G} \rightarrow \mathcal{N}_{n-1} \mathcal{G}\right)=\bigcap_{j=0}^{n} \operatorname{Ker}\left(d_{j}: G_{n} \rightarrow G_{n-1}\right)$

- $\mathcal{B} d_{n} \mathcal{G}=\operatorname{Im}\left(d_{0}: \mathcal{N}_{n+1} \mathcal{G} \rightarrow \mathcal{N}_{n} \mathcal{G}\right)$.

Exemplo 3.1.1. Vamos descrever os complexos, ciclos e bordos de Moore do $\Delta$-grupo $\mathcal{P}(M)$ do Exemplo 1.3.2. Começaremos com o caso $n=0$ para estes três objetos. Observemos que $\mathcal{N}_{0} \mathcal{P}(M)=\mathcal{Z}_{0} \mathcal{P}(M)=P_{1}(M)$ e $\mathcal{B} d_{0} \mathcal{P}(M)=d_{0}\left(\operatorname{Ker}\left(d_{j}: P_{2}(M) \rightarrow P_{1}(M)\right)\right)$.

Seja $n \geq 1$. Os complexos, ciclos e bordos de Moore de $\mathcal{P}(M)$ são dados, respectivamente, por

$$
\begin{gathered}
\mathcal{N}_{n} \mathcal{P}(M)=\bigcap_{j=1}^{n} \operatorname{Ker}\left(d_{j}: P_{n+1}(M) \rightarrow P_{n}(M)\right)=\mathcal{Q}^{1} \operatorname{Brun}_{n+1}(M) . \\
\mathcal{Z}_{n} \mathcal{P}(M)=\bigcap_{j=0}^{n} \operatorname{Ker}\left(d_{j}: P_{n+1}(M) \rightarrow P_{n}(M)\right)=\operatorname{Brun}_{n+1}(M) .
\end{gathered}
$$

$$
\mathcal{B} d_{n} \mathcal{P}(M)=\operatorname{Im}\left(d_{0}: \mathcal{Q}^{1} \operatorname{Brun}_{n+2}(M) \rightarrow \mathcal{Q}^{1} \operatorname{Brun}_{n+1}(M)\right)=d_{0}\left(\mathcal{Q}^{1} \operatorname{Brun}_{n+2}(M)\right) .
$$

Observemos que $\mathcal{Q}^{1}$ Brun $_{n+2}(M)$ é normal em $P_{n+2}(M)$ e como $d_{0}: P_{n+2}(M) \rightarrow P_{n+1}(M)$ é um epimorfismo, então $d_{0}\left(\mathcal{Q}^{1}\right.$ Brun $\left._{n+2}(M)\right)$ é um subgrupo normal de $P_{n+1}(M)$. Portanto, os conjuntos de homotopia de Moore para o $\Delta$-grupo $\mathcal{P}(M)$ são de fato grupos e são dados por

$$
\pi_{n}(\mathcal{P}(M))=\frac{\operatorname{Brun}_{n+1}(M)}{d_{0}\left(\mathcal{Q}^{1} \text { Brun }_{n+2}(M)\right)} .
$$

Pela Proposição 1.3.7 sabemos que se um $\Delta$-grupo é fibrante, então os conjuntos de homotopia de Moore $\pi_{n}$ são de fato grupos. Ainda mais, se $n \geq 1$, são grupos abelianos. Agora, pelo Teorema de Moore (Teorema 1.3.17), temos que todo grupo simplicial $\mathcal{G}$ é fibrante e portanto nesse caso $\pi_{n}(\mathcal{G})$ é um grupo para todo $n$. Sendo assim, gostaríamos de saber para quais superfícies o $\Delta$-grupo $\mathcal{P}(M)$ com aplicações faces dadas por esquecimentos de cordas é fibrante ou admite uma estrutura de grupo simplicial. 
Observação 3.1.2. No caso em que $M$ é o toro, a garrafa de Klein ou uma superfície fechada $k$-perfurada, com $k \geq 1$, o $\Delta$-grupo $\mathcal{P}(M)$ é de fato um grupo simplicial, ver [9, Corolário 3.1].

Proposição 3.1.3. Seja $\mathcal{P}(M)$ o $\Delta$-grupo formado por grupos de tranças puras sobre uma superfície $M$, considerado no Exemplo 1.3.2, cujas aplicações faces são dadas por esquecimento de cordas. Se $M$ é uma superfície fechada, diferente do toro e da garrafa de Klein, então $\mathcal{P}(M)$ não admite uma estrutura de grupo simplicial.

Demonstração. O caso $\mathcal{P}\left(\mathbb{S}^{2}\right)$ foi resolvido por Cohen et al. na Proposição 3.2.14 de [9]. Vamos supor então que $M$ é uma superfície fechada, diferente da esfera, do toro e da garrafa de Klein. Suponhamos por absurdo que $\mathcal{P}(M)$ admite uma estrutura de grupo simplicial. Portanto, pelas identidades simpliciais dadas na Subseção 1.3.2, existe uma seção $s_{i}: P_{n}(M) \rightarrow P_{n+1}(M)$ para a projeção $d_{j}: P_{n+1}(M) \rightarrow P_{n}(M)$, com $j, j+1=i$.

Agora, se $n \geq 3$, a existência de uma seção $s_{i}: P_{n}(M) \rightarrow P_{n+1}(M)$ para a projeção $d_{j}: P_{n+1}(M) \rightarrow P_{n}(M)$, com $j, j+1=i$, contradiz a solução do problema de cisão dado por Gonçalves e Guaschi, como mostra o Teorema 1.2.6.

Logo, se $M$ é uma superfície fechada, diferente do toro e da garrafa de Klein, então $\mathcal{P}(M)$ não admite uma estrutura de grupo simplicial.

Uma demonstração diferente à anterior pode ser dada no caso do $\Delta$-grupo formado por grupos de tranças sobre o plano projetivo, ver a Proposição 6.3.1 no Apêndice.

Observação 3.1.4. Veremos que $\pi_{3}\left(\mathcal{P}\left(\mathbb{S}^{2}\right)\right)$ é um grupo não abeliano (Teorema 3.1.6) e como consequência o $\Delta$-grupo $\mathcal{P}\left(\mathbb{S}^{2}\right)$ não pode ser fibrante, por causa da Proposição 1.3.7. No entanto, desconhecemos se $\mathcal{P}(M)$ é fibrante para $M$ fechada diferente da esfera, do toro e da garrafa de Klein.

O interesse por calcular os grupos de homotopia dos $\Delta$-grupos formados por grupos de tranças sobre qualquer superfície $M$, surge de forma natural na leitura do artigo de Cohen et al. [9], onde eles calculam estes grupos de homotopia no caso em que $M$ é a esfera e o disco.

Observação 3.1.5. Nos Teoremas 3.1 .6 e 3.1 .8 daremos uma descrição dos grupos de homotopia $\pi_{n}(\mathcal{P}(M))$ para qualquer valor de $n$ e qualquer superfície $M$, estendendo assim os resultados obtidos em [9] para o caso da esfera $M=\mathbb{S}^{2}$ (ver o Teorema 1.1 para $n \geq 4$ e a Seção 7.1 para $n \leq 3$ ), e para o disco $M=\mathbb{D}^{2}$ (ver a nota no final da página 303).

Recentemente tivemos conhecimento de um artigo de J. Berrick, L. Hanbury e J. Wu [10] onde são calculados os grupos de homotopia destes $\Delta$-grupos de tranças puras para qualquer superfície $M$. Os resultados exibidos em [10] englobam vários resultados desta seção, no entanto destacamos que a abordagem feita aqui para calcular estes grupos é, em geral, diferente e em algumas ocasiões mais simples que aquela adotada no artigo mencionado. 


\subsubsection{Grupos de homotopia inferiores de $\mathcal{P}(M)$}

No seguinte resultado calculamos os grupos de homotopia inferiores dos $\Delta$-grupos formados por grupos de tranças puras sobre superfícies.

\section{Teorema 3.1.6.}

1. Para qualquer superfície $M$ vale que

$$
\pi_{0}(\mathcal{P}(M))=\{1\}
$$

2. Para qualquer superfície $M$ vale que

$$
\pi_{1}(\mathcal{P}(M))=\{1\}
$$

3. $\pi_{2}\left(\mathcal{P}\left(\mathbb{S}^{2}\right)\right)=\mathbb{Z}_{2}$.

4. $\pi_{2}\left(\mathcal{P}\left(\mathbb{R} P^{2}\right)\right)=\mathbb{Z} \oplus \mathbb{Z} \oplus \mathbb{Z}$.

5. O grupo $\pi_{3}\left(\mathcal{P}\left(\mathbb{S}^{2}\right)\right)$ é um subgrupo do grupo de Heisenberg. É gerado por $w_{0}, w_{1}$ e $\delta$ sujeito às relações $\left[w_{0}, \delta\right]=\left[w_{1}, \delta\right]=1 e\left[w_{0}, w_{1}\right]=\delta^{4}$.

Observação 3.1.7. O item 3 e o item 5 do Teorema 3.1.6 foram provados por Cohen et al., ver Seção \%.1 de [9]. Para o item 3, $\pi_{2}\left(\mathcal{P}\left(\mathbb{S}^{2}\right)\right)=\mathbb{Z}_{2}$, daremos abaixo uma prova curta $e$ diferente da dada em [9].

$O$ conjunto de geradores livres que usaremos para Brun $_{3}\left(\mathbb{R} P^{2}\right)$ na seguinte prova são os geradores livres dados na Proposição 4.9 de [5], com $x_{1}=w$ e $x_{2}=A_{2,3}$. Usaremos na prova a notação usual da teoria de tranças e não a simplicial. Estes elementos aparecem nas apresentações de $P_{3}\left(\mathbb{R} P^{2}\right)$ dadas nos Lemas 4.4 e 4.6 de [5]. O elemento w é definido geometricamente no primeiro parágrafo da página 1631 de [5] e na linha 15 da página 1628 os autores escrevem $w=\sigma_{2} \sigma_{1} \rho \sigma_{1}^{-1} \sigma_{2}^{-1}$. Digamos que $\left(q_{1}, q_{2}, q_{3}\right)$ é ponto base das tranças de $P_{3}\left(\mathbb{R} P^{2}\right)$. Pode-se provar que $w=\rho_{3}$, onde $\rho_{3}$ é representado geometricamente pelo laço que gera o grupo fundamental do plano projetivo com ponto base $q_{3}$ e que "passa por trás" dos pontos $q_{0}$ e $q_{1}$. A descrição das aplicações faces para estes elementos é dada na página 1631 de [5], onde pode ser visto que $d_{3} w=1$. Além disso, na Equação (4-11) da página 1629 de [5] aparece a igualdade $A_{1,3}=w^{2} A_{2,3}^{-1}$ e portanto usando $w=\rho_{3}$ temos que $A_{1,3} A_{2,3}=\rho_{3}^{2}$, sendo esta última uma relação que usaremos na prova do item 4 a seguir.

Demonstração. 1. Notemos que os bordos e ciclos do $\Delta$-grupo $\mathcal{P}(M)$, no nível zero, são $\mathcal{B} d_{0} \mathcal{P}(M)=\operatorname{Im}\left(d_{0} \mid: \operatorname{Ker}\left(d_{1}: P_{2}(M) \rightarrow P_{1}(M)\right) \rightarrow P_{1}(M)\right)$ e $\mathcal{Z}_{0} \mathcal{P}(M)=P_{1}(M)$, respectivamente. Então, basta provar que o homomorfismo $d_{0} \mid: \operatorname{Ker}\left(d_{1}\right) \rightarrow P_{1}(M)$, que corresponde geometricamente a esquecer a primeira corda, é um epimorfismo. 
Seja $\gamma$ um elemento do grupo fundamental $\pi_{1}\left(M, x_{1}\right)$, onde $\left(x_{0}, x_{1}\right)$ é o ponto base das tranças em $P_{2}(M)$. Vamos escolher um laço $c$ baseado em $x_{1}$ que representa $\gamma$ e que evita $x_{0}$, ver Figura 3.1, e peguemos a trança geométrica $\gamma^{\prime}$ que é $\left(x_{0}^{*}, c\right)$, onde $x_{0}^{*}$ é o caminho constante em $x_{0}$. Então, a classe de homotopia de $\gamma^{\prime}$ pertence ao

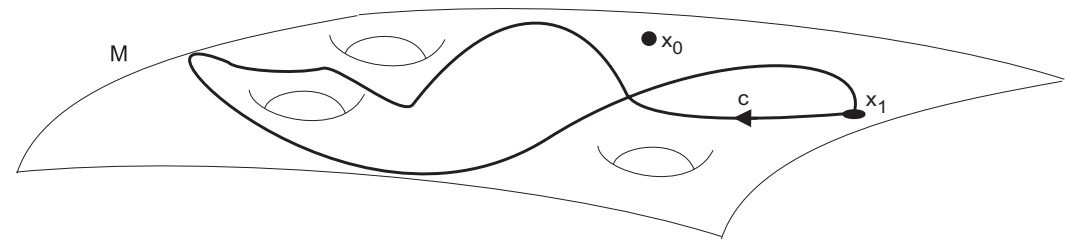

Figura 3.1: Laço c que representa $\gamma$.

grupo de tranças $P_{2}(M)$ e verifica $d_{0}\left(\gamma^{\prime}\right)=\gamma$ e $d_{1}\left(\gamma^{\prime}\right)=1$.

Desta forma provamos que $d_{0}: \operatorname{Ker}\left(d_{1}\right) \rightarrow P_{1}(M)$ é um epimorfismo, como desejado.

2. Pela igualdade (3.4) temos que

$$
\pi_{1}(\mathcal{P}(M))=\operatorname{Brun}_{2}(M) / d_{0}\left(\mathcal{Q}^{1} \operatorname{Brun}_{3}(M)\right)
$$

Vamos renomear as aplicações faces para usar a indexação usual da teoria de tranças. O caso da esfera é imediato, pois $P_{2}\left(\mathbb{S}^{2}\right)=\{1\}$.

Lembremos que, para qualquer superfície $M$, o grupo $\operatorname{Brun}_{2}(M)$ é normalmente gerado por $A_{1,2},\left\langle A_{1,2}\right\rangle^{B_{2}(M)}=\operatorname{Brun}_{2}(M)$, ver Proposição 1.3.14. Vamos analisar agora o grupo $d_{1}\left(\mathcal{Q}^{1} \operatorname{Brun}_{3}(M)\right)$.

Vejamos na continuação o caso do plano projetivo $\mathbb{R} P^{2}$. Pela Proposição 2.2 .10 e o fato que $\rho_{2}^{4}=1$ em $P_{2}\left(\mathbb{R} P^{2}\right)$, segue que $d_{1}\left(\mathcal{Q}^{1} \operatorname{Brun}_{3}\left(\mathbb{R} P^{2}\right)\right)$ é gerado por

$$
\left\{A_{1,2},\left[A_{1,2}, \rho_{2}\right],\left[A_{1,2}, \rho_{2}^{2}\right],\left[A_{1,2}, \rho_{2}^{3}\right]\right\}
$$

Como $A_{1,2}$ é central em $B_{2}\left(\mathbb{R} P^{2}\right)$ (é o "full-twist" em $B_{2}\left(\mathbb{R} P^{2}\right)$ ), uma análise deste conjunto de geradores mostra que $d_{1}\left(\mathcal{Q}^{1} \operatorname{Brun}_{3}\left(\mathbb{R} P^{2}\right)\right)=\left\langle A_{1,2}\right\rangle^{B_{2}\left(\mathbb{R} P^{2}\right)}$ (na verdade este subgrupo é igual a $\left.\left\langle A_{1,2}\right\rangle\right)$.

Vamos supor agora que $M \neq \mathbb{S}^{2}, \mathbb{R} P^{2}$. Então, segue do Teorema 2.2.12, usando a numeração usual (e não a simplicial) para as aplicações faces, que

$$
\begin{aligned}
d_{1}\left(\mathcal{Q}^{1} \operatorname{Brun}_{3}(M)\right) & =d_{1}\left(\left\langle A_{2,3}\right\rangle^{P}\right) \\
& =\left\langle A_{1,2}\right\rangle^{P} .
\end{aligned}
$$

Pela igualdade dada em (1.17), da Observação 1.3.15, temos $\operatorname{Brun}_{2}(M)=\left\langle A_{1,2}\right\rangle^{P_{2}(M)}$. Portanto,

$$
\operatorname{Brun}_{2}(M) /\left\langle A_{1,2}\right\rangle^{P}=\{1\}
$$


3. Pela Proposição 2.2.10 sabemos que $\operatorname{Brun}_{3}\left(\mathbb{S}^{2}\right) \cong \mathbb{Z}_{2}$ e $\mathcal{Q}^{1} \operatorname{Brun}_{4}\left(\mathbb{S}^{2}\right)=\operatorname{Brun}_{4}\left(\mathbb{S}^{2}\right)$. Lembremos que $d_{0}: P_{4}\left(\mathbb{S}^{2}\right) \rightarrow P_{3}\left(\mathbb{S}^{2}\right)$ é a aplicação que esquece a primeira corda, $\operatorname{logo} d_{0}\left(\operatorname{Brun}_{4}\left(\mathbb{S}^{2}\right)\right)=\{1\}$. Desta forma, usando a igualdade (3.4) com $n=2 \mathrm{e}$ $M=\mathbb{S}^{2}$, temos que

$$
\begin{aligned}
\pi_{2}\left(\mathcal{P}\left(\mathbb{S}^{2}\right)\right) & =\operatorname{Brun}_{3}\left(\mathbb{S}^{2}\right) / d_{0}\left(\mathcal{Q}^{1} \operatorname{Brun}_{4}\left(\mathbb{S}^{2}\right)\right) \\
& =\operatorname{Brun}_{3}\left(\mathbb{S}^{2}\right) / d_{0}\left(\operatorname{Brun}_{4}\left(\mathbb{S}^{2}\right)\right) \\
& =\operatorname{Brun}_{3}\left(\mathbb{S}^{2}\right) /\{1\} \\
& \cong \mathbb{Z}_{2} .
\end{aligned}
$$

Nota: Pode ser dada uma terceira prova desta afirmação e diferente daquela dada por Cohen et al. [9], ver Proposição 6.3.2.

4. Usaremos aqui a notação usual da teoria de tranças. Pela Proposição 4.9 de [5], segue que o grupos de tranças Brunnianas com 3 cordas sobre o plano projetivo $\operatorname{Brun}_{3}\left(\mathbb{R} P^{2}\right)$ é um grupo livre de posto 9 com um conjunto de geradores dado por

$$
\begin{array}{lll}
A_{2,3}^{2}, & \rho_{3}^{4}, & {\left[\rho_{3}^{4}, A_{2,3}\right],} \\
{\left[A_{2,3}, \rho_{3}\right],} & {\left[A_{2,3}, \rho_{3}^{2}\right],} & {\left[A_{2,3}, \rho_{3}^{3}\right],} \\
{\left[\left[A_{2,3}, \rho_{3}\right], A_{2,3}\right],} & {\left[\left[A_{2,3}, \rho_{3}^{2}\right], A_{2,3}\right],} & {\left[\left[A_{2,3}, \rho_{3}^{3}\right], A_{2,3}\right] .}
\end{array}
$$

Segue da descrição dada no Teorema 2.2.12 dos subgrupos quase-Brunnianos como subgrupos comutadores simétricos que

$$
\mathcal{Q}^{1} \operatorname{Brun}_{4}\left(\mathbb{R} P^{2}\right)=\left[\left\langle A_{2,4}\right\rangle^{P_{4}\left(\mathbb{R} P^{2}\right)},\left\langle A_{3,4}\right\rangle^{P_{4}\left(\mathbb{R} P^{2}\right)}\right]
$$

A seguir vamos calcular o grupo quociente $\pi_{2}\left(\mathcal{P}\left(\mathbb{R} P^{2}\right)\right)$ dado por:

$$
\begin{aligned}
\pi_{2}\left(\mathcal{P}\left(\mathbb{R} P^{2}\right)\right) & =\operatorname{Brun}_{3}\left(\mathbb{R} P^{2}\right) / d_{1}\left(\mathcal{Q}^{1} \operatorname{Brun}_{4}\left(\mathbb{R} P^{2}\right)\right) \\
& =\operatorname{Brun}_{3}\left(\mathbb{R} P^{2}\right) /\left[\left\langle A_{1,3}\right\rangle^{P_{3}\left(\mathbb{R} P^{2}\right)},\left\langle A_{2,3}\right\rangle^{P_{3}\left(\mathbb{R} P^{2}\right)}\right]
\end{aligned}
$$

Provaremos que $\pi_{2}\left(\mathcal{P}\left(\mathbb{R} P^{2}\right)\right) \cong \mathbb{Z} \oplus \mathbb{Z} \oplus \mathbb{Z}$. Usando as identidades de Witt-Hall no Teorema 1.1 .2 e a identidade $A_{1,3} A_{2,3}=\rho_{3}^{2} \in P_{3}\left(\mathbb{R} P^{2}\right)$, temos que:

- $\left[A_{2,3}, \rho_{3}^{2}\right]=A_{2,3}^{-1} \rho_{3}^{-2} A_{2,3} \rho_{3}^{2}=A_{2,3}^{-1} A_{2,3}^{-1} A_{1,3}^{-1} A_{2,3} A_{1,3} A_{2,3}=A_{2,3}^{-1}\left[A_{2,3}, A_{1,3}\right] A_{2,3}$.

- $\left[\rho_{3}^{4}, A_{2,3}\right]=\left[\rho_{3}^{2}, A_{2,3}\right] \cdot\left[\left[\rho_{3}^{2}, A_{2,3}\right], \rho_{3}^{2}\right] \cdot\left[\rho_{3}^{2}, A_{2,3}\right]$, tomando $\rho_{3}^{4}=\rho_{3}^{2} \rho_{3}^{2}$.

- $\left[A_{2,3}, \rho_{3}^{3}\right]=\left[A_{2,3}, \rho_{3}\right] \cdot\left[A_{2,3}, \rho_{3}^{2}\right] \cdot\left[\left[A_{2,3}, \rho_{3}^{2}\right], \rho_{3}\right]$, tomando $\rho_{3}^{3}=\rho_{3}^{2} \rho_{3}$.

- Lembremos que em $P_{3}\left(\mathbb{R} P^{2}\right)$ vale a seguinte relação $\rho_{3}^{-1} A_{2,3} \rho_{3}=\rho_{3}^{-1} A_{1,3}^{-1} \rho_{3}^{3}$. 
Assim,

$$
\begin{aligned}
{\left[\left[A_{2,3}, \rho_{3}\right], A_{2,3}\right] } & =\left[\rho_{3}^{-1} A_{2,3} \rho_{3}, A_{2,3}\right]=\left[\rho_{3}^{-1} A_{1,3}^{-1} \rho_{3}^{3}, A_{2,3}\right] \\
& =\left[\rho_{3}^{-1} A_{1,3}^{-1} \rho_{3}, A_{2,3}\right] \cdot\left[\left[\rho_{3}^{-1} A_{1,3}^{-1} \rho_{3}, A_{2,3}\right], \rho_{3}^{2}\right] \cdot\left[\rho_{3}^{2}, A_{2,3}\right]
\end{aligned}
$$

Portanto, no grupo quociente $\operatorname{Brun}_{3}\left(\mathbb{R} P^{2}\right) /\left[\left\langle A_{1,3}\right\rangle^{P_{3}\left(\mathbb{R} P^{2}\right)},\left\langle A_{2,3}\right\rangle^{P_{3}\left(\mathbb{R} P^{2}\right)}\right]$, temos que

$$
\begin{gathered}
{\left[A_{2,3}, \rho_{3}^{3}\right]=\left[A_{2,3}, \rho_{3}\right], \text { e }} \\
{\left[\left[A_{2,3}, \rho_{3}^{3}\right], A_{2,3}\right]=\left[\left[A_{2,3}, \rho_{3}^{2}\right], A_{2,3}\right]=\left[\left[A_{2,3}, \rho_{3}\right], A_{2,3}\right]=\left[A_{2,3}, \rho_{3}^{2}\right]=\left[\rho_{3}^{4}, A_{2,3}\right]=1 .}
\end{gathered}
$$

Pelas igualdades (3.5) e (3.6), segue que $\operatorname{Brun}_{3}\left(\mathbb{R} P^{2}\right) /\left[\left\langle A_{1,3}\right\rangle^{P_{3}\left(\mathbb{R} P^{2}\right)},\left\langle A_{2,3}\right\rangle^{P_{3}\left(\mathbb{R} P^{2}\right)}\right]$ é gerado pelos elementos $A_{2,3}^{2}, \rho_{3}^{4},\left[A_{2,3}, \rho_{3}\right]$. Agora, pela Proposição 3.1.3, o $\Delta$-grupo $\mathcal{P}\left(\mathbb{R} P^{2}\right)$ não é um grupo simplicial e portanto não podemos usar o Teorema de Moore e deduzir da teoria simplicial que ele é um grupo abeliano, mas uma verificação direta garante esse fato. Apliquemos as igualdades dadas em (3.6) nas expressões dos comutadores:

- $\left[\left[A_{2,3}, \rho_{3}\right], A_{2,3}^{2}\right]=\left[\left[A_{2,3}, \rho_{3}\right], A_{2,3}\right] \cdot\left[\left[A_{2,3}, \rho_{3}\right], A_{2,3}\right] \cdot\left[\left[\left[A_{2,3}, \rho_{3}\right], A_{2,3}\right], A_{2,3}\right]$

- $\left[\left[A_{2,3}, \rho_{3}\right], \rho_{3}^{4}\right]=\left[A_{2,3}, \rho_{3}\right]^{-1} \cdot\left[A_{2,3}, \rho_{3}^{4}\right]^{-1} \cdot\left[A_{2,3}, \rho_{3}^{5}\right]$

- $\left[A_{2,3}^{2}, \rho_{3}^{4}\right]=\left[A_{2,3}, \rho_{3}^{4}\right] \cdot\left[\left[A_{2,3}, \rho_{3}^{4}\right], A_{2,3}\right] \cdot\left[A_{2,3}, \rho_{3}^{4}\right]$

para ver que as classes de equivalência dos comutadores dos elementos $A_{2,3}^{2}, \rho_{3}^{4}$, $\left[A_{2,3}, \rho_{3}\right]$ no grupo quociente $\operatorname{Brun}_{3}\left(\mathbb{R} P^{2}\right) /\left[\left\langle A_{1,3}\right\rangle^{P_{3}\left(\mathbb{R} P^{2}\right)},\left\langle A_{2,3}\right\rangle^{P_{3}\left(\mathbb{R} P^{2}\right)}\right]$ são triviais. Portanto, o grupo de homotopia de Moore $\pi_{2}\left(\mathcal{P}\left(\mathbb{R} P^{2}\right)\right)$, isomorfo ao grupo quociente $\operatorname{Brun}_{3}\left(\mathbb{R} P^{2}\right) /\left[\left\langle A_{1,3}\right\rangle^{P_{3}\left(\mathbb{R} P^{2}\right)},\left\langle A_{2,3}\right\rangle^{P_{3}\left(\mathbb{R} P^{2}\right)}\right]$ gerado pelos elementos $A_{2,3}^{2}, \rho_{3}^{4},\left[A_{2,3}, \rho_{3}\right]$, é um grupo abeliano gerado por 3 elementos. Vejamos que $\pi_{2}\left(\mathcal{P}\left(\mathbb{R} P^{2}\right)\right)$ é isomorfo à soma direta de 3 cópias de $\mathbb{Z}$.

Consideremos primeiro a abelianização do grupo livre de posto $3, F_{3}$, dado por

$$
F_{3}=\left\langle A_{2,3}, \rho_{3}^{2},\left[A_{2,3}, \rho_{3}\right] \mid-\right\rangle \subseteq \operatorname{Ker}\left(d_{3}\right)
$$

Usando transformações de Tietze elementares e a relação $A_{1,3} A_{2,3}=\rho_{3}^{2}$, podemos verificar a seguinte igualdade de grupos livres

$$
\left\langle A_{2,3}, \rho_{3}^{2},\left[A_{2,3}, \rho_{3}\right] \mid-\right\rangle=\left\langle A_{2,3}, A_{1,3},\left[A_{2,3}, \rho_{3}\right] \mid-\right\rangle \text {. }
$$

Consideremos a abelianização de $F_{3}$ :

$$
1 \longrightarrow \Gamma_{2}\left(F_{3}\right) \longrightarrow\left\langle A_{2,3}, \rho_{3}^{2},\left[A_{2,3}, \rho_{3}\right] \mid-\right\rangle \stackrel{a b}{\longrightarrow} \mathbb{Z} \oplus \mathbb{Z} \oplus \mathbb{Z} \longrightarrow 1
$$


Dado que

$$
\operatorname{Brun}_{3}\left(\mathbb{R} P^{2}\right) \subseteq\left\langle A_{2,3}, A_{1,3},\left[A_{2,3}, \rho_{3}\right] \mid-\right\rangle \text { e }\left[\left\langle A_{1,3}\right\rangle^{P_{3}\left(\mathbb{R} P^{2}\right)},\left\langle A_{2,3}\right\rangle^{P_{3}\left(\mathbb{R} P^{2}\right)}\right] \subseteq \Gamma_{2}\left(F_{3}\right),
$$

então existe $\varphi: \pi_{2}\left(\mathcal{P}\left(\mathbb{R} P^{2}\right)\right) \rightarrow \mathbb{Z} \oplus \mathbb{Z} \oplus \mathbb{Z}$ tal que o seguinte diagrama de sequências exatas curtas é comutativo:

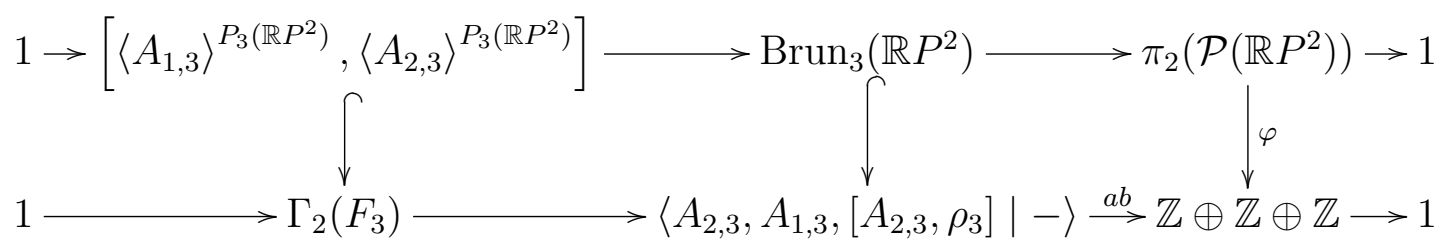

onde $\varphi\left(\overline{A_{2,3}^{2}}\right)=(2,0,0), \varphi\left(\overline{\rho_{3}^{4}}\right)=(0,2,0)$ e $\varphi\left(\left[A_{2,3}, \rho_{3}\right]\right)=(0,0,1)$. Portanto,

$$
\varphi\left(\pi_{2}\left(\mathcal{P}\left(\mathbb{R} P^{2}\right)\right)\right)=2 \mathbb{Z} \oplus 2 \mathbb{Z} \oplus \mathbb{Z} \cong \mathbb{Z} \oplus \mathbb{Z} \oplus \mathbb{Z}
$$

Temos assim um epimorfismo $\varphi: \pi_{2}\left(\mathcal{P}\left(\mathbb{R} P^{2}\right)\right) \rightarrow \mathbb{Z} \oplus \mathbb{Z} \oplus \mathbb{Z}$, que é definido por $\overline{A_{2,3}^{2}} \mapsto(1,0,0), \overline{\rho_{3}^{4}} \mapsto(0,1,0)$ e $\overline{\left[A_{2,3}, \rho_{3}\right]} \mapsto(0,0,1)$.

Definamos agora a seguinte aplicação $\psi: \mathbb{Z} \oplus \mathbb{Z} \oplus \mathbb{Z} \rightarrow\left\langle\overline{A_{2,3}^{2}}, \overline{\rho_{3}^{4}}, \overline{\left[A_{2,3}, \rho_{3}\right]}\right\rangle$, que envia $(1,0,0) \mapsto \overline{A_{2,3}^{2}},(0,1,0) \mapsto \overline{\rho_{3}^{4}}$ e $(0,0,1) \mapsto \overline{\left[A_{2,3}, \rho_{3}\right]}$. Notemos que $\psi$ é um homomorfismo já que $\left\langle\overline{A_{2,3}^{2}}, \overline{\rho_{3}^{4}}, \overline{\left[A_{2,3}, \rho_{3}\right]}\right\rangle$ é abeliano. Ainda mais, observemos que o homomorfismo $\psi$ é sobrejetor pela própria definição.

Consideremos então a seguinte composição:

$$
\mathbb{Z} \oplus \mathbb{Z} \oplus \mathbb{Z} \stackrel{\psi}{\longrightarrow}\left\langle\overline{A_{2,3}^{2}}, \overline{\rho_{3}^{4}}, \overline{\left[A_{2,3}, \rho_{3}\right]}\right\rangle \stackrel{\varphi}{\longrightarrow} \mathbb{Z} \oplus \mathbb{Z} \oplus \mathbb{Z}
$$

definida por

$$
\begin{aligned}
& (1,0,0) \mapsto \overline{A_{2,3}^{2}} \mapsto(1,0,0), \\
& (0,1,0) \mapsto \overline{\rho_{3}^{4}} \mapsto(0,1,0), \\
& (0,0,1) \mapsto \overline{\left[A_{2,3}, \rho_{3}\right]} \mapsto(0,0,1) .
\end{aligned}
$$

Como $\varphi \circ \psi: \mathbb{Z} \oplus \mathbb{Z} \oplus \mathbb{Z} \rightarrow \mathbb{Z} \oplus \mathbb{Z} \oplus \mathbb{Z}$ é um epimorfismo e $\mathbb{Z} \oplus \mathbb{Z} \oplus \mathbb{Z}$ é um grupo Hopfiano, então temos que $\varphi \circ \psi$ é de fato um isomorfismo. Mas isto implica que $\psi$ é monomorfismo e portanto isomorfismo.

$\operatorname{Logo}, \pi_{2}\left(\mathcal{P}\left(\mathbb{R} P^{2}\right)\right) \cong \mathbb{Z} \oplus \mathbb{Z} \oplus \mathbb{Z}$, como queríamos provar.

5. Como mencionado antes, este item foi provado por Cohen et al., ver Proposição 7.1 .1 de $[9]$. 


\subsubsection{Grupos de homotopia superiores de $\mathcal{P}(M)$}

Tomando $k=1$ no Teorema 2.2.13, obtemos o seguinte resultado que descreve os grupos de homotopia altos do $\Delta$-grupo formado por grupos de tranças de superfície. Os Teoremas 3.1.6 e 3.1.8 fornecem uma descrição completa para $\pi_{n}(\mathcal{P}(M)) \operatorname{com} M$ e $n$ quaisquer.

\section{Teorema 3.1.8.}

1. Sejam $n \geq 2$ e $M$ uma superfície diferente da esfera $\mathbb{S}^{2}$ e do plano projetivo $\mathbb{R} P^{2}$. Então,

$$
\pi_{n}(\mathcal{P}(M))=\{1\}
$$

2. Suponhamos que $n \geq 4$ se $M=\mathbb{S}^{2}$ e que $n \geq 3$ se $M=\mathbb{R} P^{2}$. Então,

$$
\pi_{n}(\mathcal{P}(M))=\pi_{n}\left(\mathbb{S}^{2}\right)
$$

Demonstração. Seja $n \geq 2$. Pela igualdade (3.4) sabemos que o grupo de homotopia de Moore $\pi_{n}(\mathcal{P}(M))$ é dado por

$$
\pi_{n}(\mathcal{P}(M))=\operatorname{Brun}_{n+1}(M) / d_{0}\left(\mathcal{Q}^{1} \operatorname{Brun}_{n+2}(M)\right)
$$

Tomando $k=1$ e $m=n+2$ no Teorema 2.2.13, vemos que as restrições de $\eta_{1}$ e $d_{0}$ consideradas como aplicações de $\mathcal{Q}^{1} \operatorname{Brun}_{n+2}(M)$ em $d_{0}\left(\mathcal{Q}^{1} \operatorname{Brun}_{n+2}(M)\right)$ são iguais. Portanto, $\pi_{n}(\mathcal{P}(M))=\operatorname{coKer}\left(\eta_{1}: \mathcal{Q}^{1} \operatorname{Brun}_{n+2}(M) \rightarrow \operatorname{Brun}_{n+1}(M)\right)$ e o resultado segue do Teorema 2.2.13.

A definição de homologia para conjuntos simpliciais pode ser encontrada no livro de Curtis [19]. Enunciaremos, sem prova, o seguinte resultado clássico da teoria de homotopia simplicial [19, Teorema 3.12].

Teorema 3.1.9 (Teorema de Hurewicz simplicial). Se $\mathcal{X}$ é um conjunto simplicial fibrante e conexo com $\pi_{i}(\mathcal{X})=0$, para $i<n(n \geq 2)$, então $\pi_{n}(\mathcal{X})$ é isomorfo a $H_{n}(\mathcal{X})$.

Sabemos que se $M$ é o toro, a garrafa de Klein ou uma superfície fechada $k$-perfurada, com $k>0$, então $\mathcal{P}(M)$ é um grupo simplicial, ver Observação 3.1.2, e portanto pelo Teorema de Moore temos que $\mathcal{P}(M)$ é fibrante. Além disso, pelo Teorema 3.1.6 temos que $\mathcal{P}(M)$ é conexo, para qualquer $M$. Pelo Teorema de Hurewicz simplicial aplicado ao Teorema 3.1.8 obtemos o seguinte resultado sobre os grupos de homologia do $\Delta$-grupo $\mathcal{P}(M)$, para uma superfície fechada $k$-perfurada $M$.

Corolário 3.1.10. Suponhamos que $M$ é o toro, a garrafa de Klein ou uma superfície fechada $k$-perfurada, com $k>0$. Então, para $n \geq 2$,

$$
H_{n}(\mathcal{P}(M))=\pi_{n}(\mathcal{P}(M))=\{1\}
$$




\subsection{O grupo $T_{k, n}(M)$ para uma superfície $M$}

Denotemos por $T_{k, n}(M)$ o subgrupo de tranças Brunnianas sobre uma superfície $M \backslash Q_{k}$ dado por $T_{k, n}(M)=\operatorname{Ker}\left(f_{*}\right) \cap \operatorname{Brun}_{n}\left(M \backslash Q_{k}\right)$, para $k \geq 1$, onde $f: M \backslash Q_{k} \hookrightarrow M$ é a inclusão natural de $M \backslash Q_{k}$ em $M$ e $f_{*}: P_{n}\left(M \backslash Q_{k}\right) \rightarrow P_{n}(M)$ denota a aplicação induzida por $f$ nos grupos de tranças puras.

Teorema 3.2.1. Seja $n \geq 3$. Vamos supor que $k \geq 3$ se $M=\mathbb{S}^{2}$, que $k \geq 2$ se $M=\mathbb{R} P^{2}$ e que $k \geq 1$ nos outros casos. Então,

1. $\operatorname{Brun}_{n}\left(M \backslash Q_{k}\right) \cong \mathcal{Q}^{k} \operatorname{Brun}_{k+n}(M)$.

2. $T_{k, n}(M) \cong \mathcal{S}^{k} \operatorname{Brun}_{k+n}(M)$.

Demonstração. Por hipótese podemos considerar a seguinte sequência exata curta induzida pela fibração de Fadell-Neuwirth

$$
1 \longrightarrow P_{n}\left(M \backslash Q_{k}\right) \stackrel{\eta}{\longrightarrow} P_{k+n}(M) \stackrel{\rho}{\longrightarrow} P_{k}(M) \longrightarrow 1
$$

obtida ao removermos as últimas $n$ componentes na projeção da fibração e portanto $\rho=d_{k} \circ \cdots \circ d_{k+n-1}$. Notemos que $\eta\left(\operatorname{Brun}_{n}\left(M \backslash Q_{k}\right)\right) \subseteq \mathcal{Q}^{k} \operatorname{Brun}_{k+n}(M)$, pois o seguinte diagrama é comutativo para todo $j=0, \ldots, n-1$,

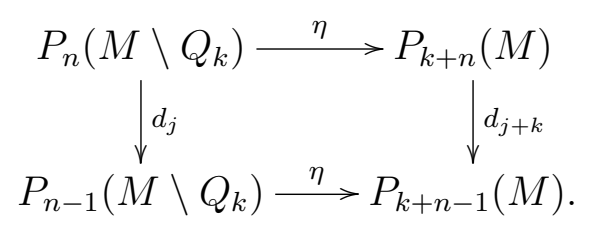

O homomorfismo $\eta: \operatorname{Brun}_{n}\left(M \backslash Q_{k}\right) \rightarrow \mathcal{Q}^{k} \operatorname{Brun}_{k+n}(M)$ é sobrejetor, isto segue da sequência exata curta (3.7) e usando o diagrama comutativo (3.8) (usamos aqui a injetividade de $\eta)$. Demonstramos assim o item 1, pois $\eta: \operatorname{Brun}_{n}\left(M \backslash Q_{k}\right) \rightarrow \mathcal{Q}^{k} \operatorname{Brun}_{k+n}(M)$ é um monomorfismo sobrejetor, isto é, um isomorfismo.

Observemos que o seguinte diagrama também é comutativo, é induzido a partir de um quadrado geométrico comutativo de espaços de configuração.

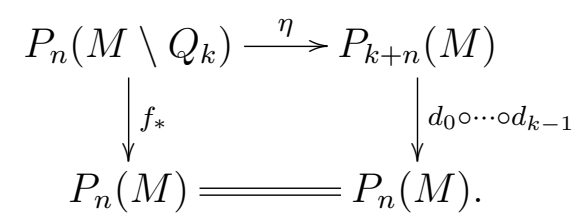

Agora vamos restringir o morfismo $\eta$ ao subgrupo $T_{k, n}(M)$. Veremos na continuação que $\eta\left(T_{k, n}(M)\right)=\mathcal{S}^{k} \operatorname{Brun}_{k+n}(M)$.

Uma das inclusões é imediata usando os diagramas comutativos (3.8) e (3.9), mostraremos apenas que vale a outra. Seja $\beta \in \mathcal{S}^{k} \operatorname{Brun}_{k+n}(M)$, então pela sequência exata curta (3.7) 
existe um elemento $\beta_{1} \in P_{n}\left(M \backslash Q_{k}\right)$ tal que $\eta\left(\beta_{1}\right)=\beta$, ainda mais, segue do diagrama comutativo (3.8) que $\beta_{1} \in \operatorname{Brun}_{n}\left(M \backslash Q_{k}\right)$, pois $\eta$ é monomorfismo. Agora, usando o quadrado (3.9) temos que $\beta_{1} \in \operatorname{Ker}\left(f_{*}\right)$. Logo, $\beta_{1} \in T_{k, n}(M)$ e portanto $\beta \in \eta\left(T_{k, n}(M)\right.$ ).

Como $\eta$ é um monomorfismo, segue que $\eta: T_{k, n}(M) \rightarrow \mathcal{S}^{k} \operatorname{Brun}_{k+n}(M)$ é de fato um isomorfismo mostrando assim o item 2 .

Observação 3.2.2. Seja $0 \leq l \leq n-3$. O item 1 do Teorema 3.2.1 pode ser estendido da seguinte forma

$$
\mathcal{Q}^{l} \operatorname{Brun}_{n}\left(M \backslash Q_{k}\right) \cong \mathcal{Q}^{k+l} \operatorname{Brun}_{k+n}(M),
$$

e a demonstração é análoga à dada anteriormente.

Uma consequência imediata do Teorema 3.2.1, com $k=1$, é descrita na continuação como um corolário.

Corolário 3.2.3. Seja $M$ uma superfície do tipo Eilenberg-Mac Lane. Então, para $n \geq 3$,

1. $\operatorname{Brun}_{n}\left(M \backslash Q_{1}\right) \cong \mathcal{Q}^{1} \operatorname{Brun}_{n+1}(M)=\mathcal{N}_{n} \mathcal{P}(M)$.

2. $\operatorname{Brun}_{n+1}(M) \cong T_{1, n}(M)$.

\subsection{Sequências exatas de grupos de tranças Brunni- anas e grupos de homotopia}

Nesta seção daremos uma formulação da sequência exata do Teorema 1.2.13 para outras superfícies, generalizando assim tal teorema. Para isto precisaremos de alguns resultados prévios.

Seja $L$ uma superfície obtida a partir de $M$ removendo um conjunto finito de pontos (não vazio) de $M$. Portanto, dos Teoremas 3.1.6 e 3.1.8, segue que $\pi_{n}(\mathcal{P}(L))=\{1\}$, para todo $n \geq 0$. Consideremos a inclusão natural de $L$ em $M, f: L \hookrightarrow M$. Denotemos por $f_{*}: P_{n}(L) \rightarrow P_{n}(M)$ a aplicação induzida por $f$ nos grupos de tranças puras. Por um abuso de linguagem também chamaremos por $f_{*}$ sua restrição aos subgrupos de tranças Brunnianas, bem como aos subgrupos dados por complexos, bordos e ciclos de Moore, quando for o caso. Nos próximos resultados faremos uso do funtor Complexo de Moore $N$, ver a Seção 4 de [9] para mais detalhes sobre este funtor.

Lema 3.3.1. Sejam $m \geq 1$ e $n \geq m+1$. Suponhamos que L é uma superfície obtida a partir de $M$ removendo um conjunto finito de pontos (não vazio) de $M$ e tal que $N f_{*}: \mathcal{N}_{n+1} \mathcal{P}(L) \rightarrow \mathcal{N}_{n+1} \mathcal{P}(M)$ é um epimorfismo. Então, a seguinte sequência é exata

$$
1 \longrightarrow \operatorname{Ker}\left(f_{*}\right) \longrightarrow \operatorname{Brun}_{n+1}(L) \stackrel{f_{*}}{\longrightarrow} \operatorname{Brun}_{n+1}(M) \longrightarrow \pi_{n}(\mathcal{P}(M)) \longrightarrow 1
$$


Demonstração. Pelos Teoremas 3.1.6 e 3.1.8 sabemos que, para todo $n \geq 0$, vale que $\pi_{n}(\mathcal{P}(L))=\mathcal{Z}_{n} \mathcal{P}(L) / \mathcal{B} d_{n} \mathcal{P}(L)=\{1\}$. Como consequência $\mathcal{Z}_{n} \mathcal{P}(L)=\mathcal{B} d_{n} \mathcal{P}(L)$.

Sejam $m \geq 1$ e $n \geq m+1$. Observemos que

$$
\mathcal{B} d_{n} \mathcal{P}(M)=d_{0}\left(\mathcal{N}_{n+1} \mathcal{P}(M)\right)=\operatorname{Im}\left(N f_{*}: \mathcal{B} d_{n} \mathcal{P}(L) \rightarrow(\mathcal{P}(M))_{n}\right)
$$

pois o seguinte diagrama é comutativo

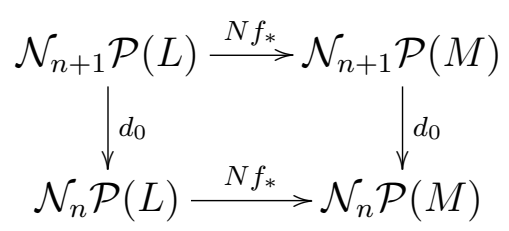

e por hipótese $N f_{*}: \mathcal{N}_{n+1} \mathcal{P}(L) \rightarrow \mathcal{N}_{n+1} \mathcal{P}(M)$ é um epimorfismo.

Pela igualdade dada em (3.2) temos $\mathcal{Z}_{n} \mathcal{P}(L)=\operatorname{Brun}_{n+1}(L)$ e $\mathcal{Z}_{n} \mathcal{P}(M)=\operatorname{Brun}_{n+1}(M)$. Logo,

$$
\begin{aligned}
\mathcal{B} d_{n} \mathcal{P}(M) & =\operatorname{Im}\left(N f_{*}: \mathcal{B} d_{n} \mathcal{P}(L) \rightarrow(\mathcal{P}(M))_{n}\right) \quad(\text { por }(3.11)) \\
& =\operatorname{Im}\left(f_{*}: \operatorname{Brun}_{n+1}(L) \rightarrow P_{n}(M)\right) \quad\left(\text { pois } \mathcal{B} d_{n} \mathcal{P}(L)=\mathcal{Z}_{n} \mathcal{P}(L)\right) \\
& =\operatorname{Im}\left(f_{*}: \operatorname{Brun}_{n+1}(L) \rightarrow \operatorname{Brun}_{n+1}(M)\right) .
\end{aligned}
$$

A última igualdade é garantida pelo fato que $f_{*}\left(\operatorname{Brun}_{n+1}(L)\right) \subseteq \operatorname{Brun}_{n+1}(M)$. Como $\pi_{n}(\mathcal{P}(M))=\frac{\mathcal{Z}_{n} \mathcal{P}(M)}{\mathcal{B} d_{n} \mathcal{P}(M)}$, então temos a seguinte sequência exata,

$$
1 \longrightarrow \operatorname{Ker}\left(f_{*}\right) \longrightarrow \operatorname{Brun}_{n+1}(L) \stackrel{f_{*}}{\longrightarrow} \operatorname{Brun}_{n+1}(M) \longrightarrow \pi_{n}(\mathcal{P}(M)) \longrightarrow 1 .
$$

Denotemos por $X_{n}$ o conjunto de pontos base dos grupos de tranças com $n$ cordas sobre $M$ e sejam $k \geq 1$ e $Q_{k}=\left\{q_{0}, q_{1}, \ldots, q_{k-1}\right\}$ um conjunto finito de pontos (no interior) de $M$ tal que $Q_{k}$ e $X_{n}$ são disjuntos. A seguinte proposição foi demonstrada por Bellingeri no caso em que $M$ é uma superfície orientável de genus maior ou igual que 1 [7, Proposição 1.3]. No caso em que $M$ é uma superfície fechada (compacta sem bordo) e $Q_{k}$ é um conjunto unitário, ou seja, $k=1$, o resultado pode ser encontrado, para as tranças puras, no começo da prova do caso 2 do [5, Teorema 1.2] apenas como uma observação, porém os autores sugerem um roteiro da prova. Não temos conhecimento de uma demonstração da seguinte proposição para os outros casos, diferentes aos que acabamos de mencionar. Por este motivo decidimos dar uma prova aqui, mas observamos que o roteiro da demonstração, no caso das tranças puras e $Q_{k}$ sendo um conjunto com mais de um elemento, é o mesmo daquele sugerido por Bardakov et al. [5]. 
Proposição 3.3.2. Seja $k \geq 1$. Suponhamos que $Q_{k} \cap X_{n}$ é vazio e seja $f: M \backslash Q_{k} \hookrightarrow M$ a inclusão natural. Nesse caso temos que os homomorfismos $f_{*}: P_{n}\left(M \backslash Q_{k}\right) \rightarrow P_{n}(M)$ e $f_{*}: B_{n}\left(M \backslash Q_{k}\right) \rightarrow B_{n}(M)$ são sobrejetores, para todo $n \geq 1$.

Demonstração. Provaremos o resultado inicialmente para as tranças puras, por indução sobre $n$. Seja $\gamma$ um elemento de $\pi_{1}(M)$, podemos escolher um laço $c: \mathbb{S}^{1} \rightarrow M$, representante da classe de $\gamma$, tal que $c$ evita o conjunto $Q_{k}$. Então, se denotamos por $\gamma^{\prime}$ a classe do laço $c$ em $\pi_{1}\left(M \backslash Q_{k}\right)$ temos claramente que $f_{*}\left(\gamma^{\prime}\right)=\gamma$. Provamos assim que $f_{*}: \pi_{1}\left(M \backslash Q_{k}\right) \rightarrow \pi_{1}(M)$ é um epimorfismo e portanto o resultado desta proposição vale para $n=1$.

Notemos que o seguinte diagrama geométrico é comutativo, no qual as linhas horizontais são fibrações de Fadell-Neuwirth.

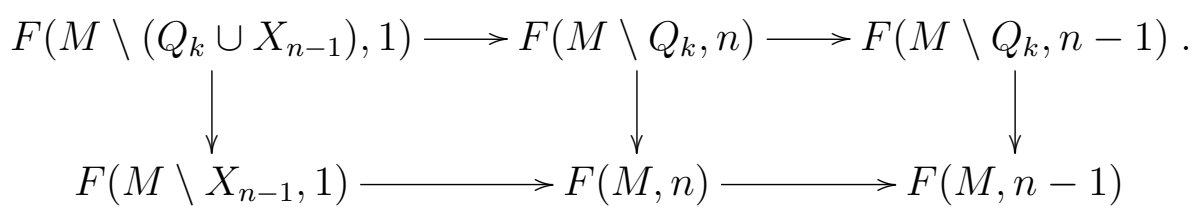

O diagrama (3.12) induz o seguinte diagrama comutativo algébrico, onde as linhas horizontais são sequências exatas curtas de tranças puras (ver a sequência (1.7) da Proposição 1.2.5), e por hipótese de indução vamos assumir que $f_{*}: P_{n-1}\left(M \backslash Q_{k}\right) \rightarrow P_{n-1}(M)$ é um epimorfismo.

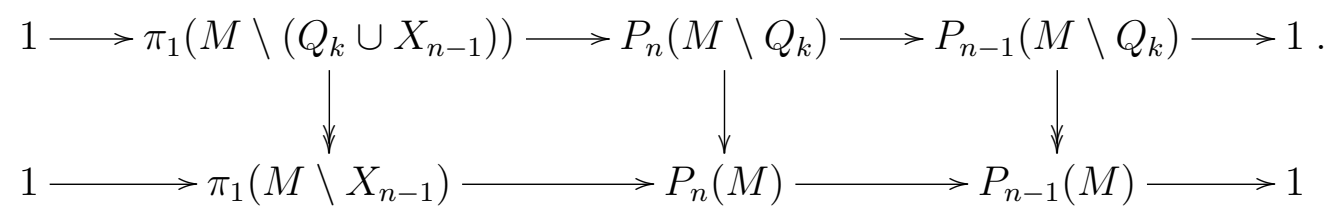

Aplicando o lema dos cinco ao diagrama comutativo (3.13) obtemos o resultado desejado para as tranças puras, isto é, provamos que $f_{*}: P_{n}\left(M \backslash Q_{k}\right) \rightarrow P_{n}(M)$ é um epimorfismo para $n \geq 1$.

Consideremos agora o seguinte diagrama comutativo, onde as linhas horizontais são sequências exatas curtas de tranças dadas em (1.3), no Capítulo 1.

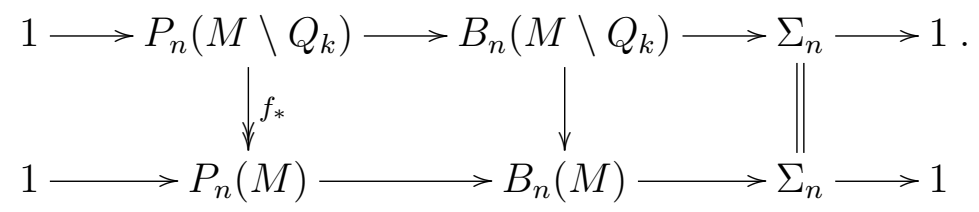

Dado que as flechas verticais nas pontas do diagrama (3.14) são epimorfismos, então podemos aplicar o lema dos cinco e assim demonstramos que $f_{*}: B_{n}\left(M \backslash Q_{k}\right) \rightarrow B_{n}(M)$ é um epimorfismo.

Gostaríamos de mencionar que as técnicas usadas na prova anterior são clássicas e 
bastante usadas na teoria de tranças. A Proposição 3.3.2 será usada para provar o próximo teorema, que estende a outras superfícies a sequência exata de tranças do Teorema 1.2.13. Um resultado similar foi obtido no Corolário 2.2.14 e será usado na prova do Teorema 3.3.3.

Teorema 3.3.3. Vamos supor que $k \geq 3$ se $M=\mathbb{S}^{2}$, que $k \geq 2$ se $M=\mathbb{R} P^{2}$ e que $k \geq 1$ nos outros casos. Seja $Q_{k}$ um conjunto de $k$ pontos no interior de $M$.

1. Para todo $n \geq 3$ e $M \neq \mathbb{S}^{2}, \mathbb{R} P^{2}$ a seguinte sequência é exata curta

$$
1 \longrightarrow \mathcal{S}^{k} \operatorname{Brun}_{k+n}(M) \longrightarrow \operatorname{Brun}_{n}\left(M \backslash Q_{k}\right) \stackrel{f_{*}}{\longrightarrow} \operatorname{Brun}_{n}(M) \longrightarrow 1
$$

2. Para todo $n \geq 5$ a seguinte sequência é exata

$$
1 \longrightarrow \mathcal{S}^{k} \operatorname{Brun}_{k+n}\left(\mathbb{S}^{2}\right) \longrightarrow \operatorname{Brun}_{n}\left(\mathbb{S}^{2} \backslash Q_{k}\right) \stackrel{f_{*}}{\longrightarrow} \operatorname{Brun}_{n}\left(\mathbb{S}^{2}\right) \longrightarrow \pi_{n-1}\left(\mathbb{S}^{2}\right) \longrightarrow 1
$$

3. Para todo $n \geq 4$ a seguinte sequência é exata

$$
1 \longrightarrow \mathcal{S}^{k} \operatorname{Brun}_{k+n}\left(\mathbb{R} P^{2}\right) \longrightarrow \operatorname{Brun}_{n}\left(\mathbb{R} P^{2} \backslash Q_{k}\right) \stackrel{f_{*}}{\longrightarrow} \operatorname{Brun}_{n}\left(\mathbb{R} P^{2}\right) \longrightarrow \pi_{n-1}\left(\mathbb{S}^{2}\right) \longrightarrow 1 .
$$

Observação 3.3.4. O epimorfismo $f_{*}: \operatorname{Brun}_{n}\left(M \backslash Q_{k}\right) \rightarrow \operatorname{Brun}_{n}(M)$ do Teorema 3.3.3 (item 1) está nas entrelinhas do caso 2 da demonstração do Teorema 1.2 de [5].

Demonstração. Vamos supor que $k \geq 3$ se $M=\mathbb{S}^{2}$, que $k \geq 2$ se $M=\mathbb{R} P^{2}$ e que $k \geq 1$ nos outros casos. Pela igualdade (3.1), segue que $\mathcal{N}_{n-1} \mathcal{P}\left(M \backslash Q_{k}\right)=\mathcal{Q}^{1} \operatorname{Brun}_{n}\left(M \backslash Q_{k}\right)$. Consideremos agora $f_{*}: P_{n}\left(M \backslash Q_{k}\right) \rightarrow P_{n}(M)$, dada por $A_{i, j}\left[M \backslash Q_{k}\right] \mapsto A_{i, j}$. Como comentamos no parágrafo anterior ao Teorema 1.2.14 do Capítulo 1 , para $1 \leq i<j \leq n$, os elementos $A_{i, j}\left[M \backslash Q_{k}\right]$ são os elementos $A_{i, j}$ de $P_{n}$, mas vistos em $P_{n}\left(M \backslash Q_{k}\right)$. Inicialmente provaremos que a restrição $f_{*}: \mathcal{Q}^{1} \operatorname{Brun}_{n}\left(M \backslash Q_{k}\right) \rightarrow \mathcal{Q}^{1} \operatorname{Brun}_{n}(M)$ é um epimorfismo, para $n$ e $M$ como nas hipóteses do teorema, em cada item do enunciado. De fato, pela Proposição 3.3.2 temos que $f_{*}: P_{n}\left(M \backslash Q_{k}\right) \rightarrow P_{n}(M)$ é um epimorfismo e portanto

$$
f_{*}\left(\left\langle A_{i, j}\left[M \backslash Q_{k}\right]\right\rangle^{P_{n}\left(M \backslash Q_{k}\right)}\right)=\left\langle A_{i, j}\right\rangle^{P_{n}(M)} .
$$

Assim usando a descrição dos grupos quase-Brunnianos, dada no Teorema 2.2.12 e a Proposição 3.3.2, temos que:

$$
\begin{aligned}
& f_{*}\left(\mathcal{Q}^{1} \operatorname{Brun}_{n}\left(M \backslash Q_{k}\right)\right) \\
& =\left[f_{*}\left(\left\langle A_{2, n}\left[M \backslash Q_{k}\right]\right\rangle^{P_{n}\left(M \backslash Q_{k}\right)}\right), f_{*}\left(\left\langle A_{3, n}\left[M \backslash Q_{k}\right]\right\rangle\right), \ldots, f_{*}\left(\left\langle A_{n-1, n}\left[M \backslash Q_{k}\right]\right\rangle^{P_{n}\left(M \backslash Q_{k}\right)}\right)\right]_{S} \\
& =\left[\left\langle A_{2, n}\right\rangle^{P_{n}(M)},\left\langle A_{3, n}\right\rangle^{P_{n}(M)}, \ldots,\left\langle A_{n-1, n}\right\rangle^{P_{n}(M)}\right]_{S} \\
& =\mathcal{Q}^{1} \operatorname{Brun}_{n}(M) .
\end{aligned}
$$


Portanto, pelo Lema 3.3.1, com $n$ e $M$ como nas hipóteses do teorema, em cada item do enunciado, a seguinte sequência é exata

$$
1 \rightarrow \operatorname{Ker}\left(f_{*}\right) \cap \operatorname{Brun}_{n}\left(M \backslash Q_{k}\right) \rightarrow \operatorname{Brun}_{n}\left(M \backslash Q_{k}\right) \stackrel{f_{*}}{\rightarrow} \operatorname{Brun}_{n}(M) \rightarrow \pi_{n-1}(\mathcal{P}(M)) \rightarrow 1
$$

Lembremos que $T_{k, n}(M)=\operatorname{Ker}\left(f_{*}\right) \cap \operatorname{Brun}_{n}\left(M \backslash Q_{k}\right)$ e portanto a sequência (3.16) é igual a

$$
1 \longrightarrow T_{k, n}(M) \longrightarrow \operatorname{Brun}_{n}\left(M \backslash Q_{k}\right) \stackrel{f_{*}}{\longrightarrow} \operatorname{Brun}_{n}(M) \longrightarrow \pi_{n-1}(\mathcal{P}(M)) \longrightarrow 1
$$

Pelo Teorema 3.2.1 existe um isomorfismo $\eta: \operatorname{Ker}\left(f_{*}\right) \cap \operatorname{Brun}_{n}\left(M \backslash Q_{k}\right) \rightarrow \mathcal{S}^{k} \operatorname{Brun}_{k+n}(M)$. Portanto, a sequência (3.17) fica assim

$$
1 \longrightarrow \mathcal{S}^{k} \operatorname{Brun}_{k+n}(M) \longrightarrow \operatorname{Brun}_{n}\left(M \backslash Q_{k}\right) \stackrel{f_{*}}{\longrightarrow} \operatorname{Brun}_{n}(M) \longrightarrow \pi_{n-1}(\mathcal{P}(M)) \longrightarrow 1
$$

Juntando a sequência exata dada em (3.18) e a descrição dos grupos de homotopia $\pi_{n}(\mathcal{P}(M))$ do Teorema 3.1.8 obtemos o resultado desejado em cada caso.

De forma similar prova-se a versão do resultado anterior no caso de poucas cordas. Nesse caso pode-se usar o cálculo explícito dos grupos de homotopia inferiores dos $\Delta$-grupos formados por grupos de tranças puras sobre superfícies dado no Teorema 3.1.6.

Teorema 3.3.5. Vamos supor que $k \geq 3$ se $M=\mathbb{S}^{2}$, que $k \geq 2$ se $M=\mathbb{R} P^{2}$ e que $k \geq 1$ nos outros casos. Seja $Q_{k}$ um conjunto de $k$ pontos no interior de $M$. As seguintes sequências são exatas.

1. Para $M \neq \mathbb{S}^{2}, \mathbb{R} P^{2}$ :

$$
1 \longrightarrow \mathcal{S}^{k} \operatorname{Brun}_{k+2}(M) \longrightarrow \operatorname{Brun}_{2}\left(M \backslash Q_{k}\right) \stackrel{f_{*}}{\longrightarrow} \operatorname{Brun}_{2}(M) \longrightarrow 1
$$

2. (a) $1 \longrightarrow \mathcal{S}^{k} \operatorname{Brun}_{k+3}\left(\mathbb{S}^{2}\right) \longrightarrow \operatorname{Brun}_{3}\left(\mathbb{S}^{2} \backslash Q_{k}\right) \stackrel{f_{*}}{\rightarrow} \operatorname{Brun}_{3}\left(\mathbb{S}^{2}\right) \longrightarrow \mathbb{Z}_{2} \rightarrow 1$, onde Brun ${ }_{3}\left(\mathbb{S}^{2}\right)=\mathbb{Z}_{2}$ e $\pi_{2}\left(\mathcal{P}\left(\mathbb{S}^{2}\right)\right)=\mathbb{Z}_{2}$. Como consequência, existe um isomorfismo $\mathcal{S}^{k} \operatorname{Brun}_{k+3}\left(\mathbb{S}^{2}\right) \cong \operatorname{Brun}_{3}\left(\mathbb{S}^{2} \backslash Q_{k}\right)$.

(b) $1 \longrightarrow \mathcal{S}^{k} \operatorname{Brun}_{k+4}\left(\mathbb{S}^{2}\right) \longrightarrow \operatorname{Brun}_{4}\left(\mathbb{S}^{2} \backslash Q_{k}\right) \stackrel{f_{*}}{\rightarrow} \operatorname{Brun}_{4}\left(\mathbb{S}^{2}\right) \rightarrow \pi_{3}\left(\mathcal{P}\left(\mathbb{S}^{2}\right)\right) \rightarrow 1$, onde Brun $_{4}\left(\mathbb{S}^{2}\right)=\mathcal{Q}^{1}$ Brun $_{4}\left(\mathbb{S}^{2}\right)=F_{5}$ e $\pi_{3}\left(\mathcal{P}\left(\mathbb{S}^{2}\right)\right)$ é o subgrupo do grupo de Heisenberg dado no item 5 do Teorema 3.1.6.

3. $1 \rightarrow \mathcal{S}^{k} \operatorname{Brun}_{k+3}\left(\mathbb{R} P^{2}\right) \rightarrow \operatorname{Brun}_{3}\left(\mathbb{R} P^{2} \backslash Q_{k}\right) \stackrel{f_{*}}{\rightarrow} \mathbb{Z} \oplus \mathbb{Z} \oplus \mathbb{Z} \stackrel{\varphi}{\rightarrow} \pi_{2}\left(\mathcal{P}\left(\mathbb{R} P^{2}\right)\right) \rightarrow 1$, onde Brun $_{3}\left(\mathbb{R} P^{2}\right)=F_{9}$ e $\pi_{2}\left(\mathcal{P}\left(\mathbb{R} P^{2}\right)\right)=\mathbb{Z} \oplus \mathbb{Z} \oplus \mathbb{Z}$ são os grupos dados no item 4 do Teorema 3.1.6. A projeção $\varphi$ é definida por $A_{2,3}^{2} \mapsto(1,0,0), \rho_{3}^{4} \mapsto(0,1,0)$ 
$e\left[A_{2,3}, \rho_{3}\right] \mapsto(0,0,1)$, os outros geradores livres de Brun $_{3}\left(\mathbb{R} P^{2}\right)$ são enviados em $(0,0,0)$ (ver a prova do item 4 do Teorema 3.1.6).

Demonstração. Usa-se um raciocínio similar ao usado na demonstração do Teorema 3.3.3 para obter as sequências exatas. A descrição dos grupos envolvidos segue dos resultados da Proposição 2.2.10 e do Teorema 3.1.6.

\subsection{Uma trança Brunniana com 5 cordas na esfera que se projeta no gerador de $\pi_{4}$ da esfera}

Nessa subseção queremos dar um exemplo correto, na direção dada inicialmente por Cohen et al. [9], de uma trança Brunniana com 5 cordas na esfera que se projeta no gerador $\eta_{2}^{2}$ de $\pi_{4}\left(\mathbb{S}^{2}\right)$. Desta forma resolvemos o problema encontrado na Subseção 7.4 de [9], o qual será discutido na Subseção 6.1.3. Alertamos que o elemento $\beta_{5}$ construído aqui é diferente daquele construído por Cohen et al., embora seja denotado da mesma forma.

Para começar queremos destacar as seguintes igualdades, válidas em $P_{n}\left(\mathbb{D}^{2}\right)$, para $n \geq 4$ :

$$
\left\{\begin{aligned}
A_{2,3}^{-1} A_{1,3} A_{2,3} & =A_{1,2} A_{1,3} A_{1,2}^{-1} \\
A_{3,4}^{-1} A_{2,4}^{-1} A_{1,4} A_{2,4} A_{3,4} & =A_{1,2} A_{1,3} A_{1,4} A_{1,3}^{-1} A_{1,2}^{-1}
\end{aligned}\right.
$$

De fato,

$$
\begin{aligned}
A_{2,3}^{-1} A_{1,3} A_{2,3} & =\sigma_{2}^{-2} \sigma_{2} \sigma_{1}^{2} \sigma_{2}^{-1} \sigma_{2}^{2}=\sigma_{2}^{-1} \sigma_{1}^{2} \sigma_{2}=\sigma_{1} \sigma_{2}^{2} \sigma_{1}^{-1}=\sigma_{1}^{2} \sigma_{1}^{-1} \sigma_{2}^{2} \sigma_{1} \sigma_{1}^{-2} \\
& =\sigma_{1}^{2} \sigma_{2} \sigma_{1}^{2} \sigma_{2}^{-1} \sigma_{1}^{-2}=A_{1,2} A_{1,3} A_{1,2}^{-1}
\end{aligned}
$$

e

$$
\begin{aligned}
A_{3,4}^{-1} A_{2,4}^{-1} A_{1,4} A_{2,4} A_{3,4} & =\sigma_{3}^{-2} \sigma_{3} \sigma_{2}^{-2} \sigma_{3}^{-1} \sigma_{3} \sigma_{2} \sigma_{1}^{2} \sigma_{2}^{-1} \sigma_{3}^{-1} \sigma_{3} \sigma_{2}^{2} \sigma_{3}^{-1} \sigma_{3}^{2} \\
& =\sigma_{3}^{-1} \sigma_{2}^{-1} \sigma_{1}^{2} \sigma_{2} \sigma_{3}=\sigma_{3}^{-1} \sigma_{1} \sigma_{2}^{2} \sigma_{1}^{-1} \sigma_{3}=\sigma_{1} \sigma_{3}^{-1} \sigma_{2}^{2} \sigma_{3} \sigma_{1}^{-1} \\
& =\sigma_{1} \sigma_{2} \sigma_{3}^{2} \sigma_{2}^{-1} \sigma_{1}^{-1}=\sigma_{1}^{2} \sigma_{1}^{-1} \sigma_{2} \sigma_{3}^{2} \sigma_{2}^{-1} \sigma_{1} \sigma_{1}^{-2} \\
& =\sigma_{1}^{2} \sigma_{1}^{-1} \sigma_{2}^{2} \sigma_{1} \sigma_{1}^{-1} \sigma_{2}^{-1} \sigma_{3}^{2} \sigma_{2} \sigma_{1} \sigma_{1}^{-1} \sigma_{2}^{-2} \sigma_{1} \sigma_{1}^{-2} \\
& =\sigma_{1}^{2} \sigma_{1}^{-1} \sigma_{2}^{2} \sigma_{1} \sigma_{1}^{-1} \sigma_{3} \sigma_{2}^{2} \sigma_{3}^{-1} \sigma_{1} \sigma_{1}^{-1} \sigma_{2}^{-2} \sigma_{1} \sigma_{1}^{-2} \\
& =\sigma_{1}^{2} \sigma_{2} \sigma_{1}^{2} \sigma_{2}^{-1} \sigma_{3} \sigma_{1}^{-1} \sigma_{2}^{2} \sigma_{1} \sigma_{3}^{-1} \sigma_{2} \sigma_{1}^{-2} \sigma_{2}^{-1} \sigma_{1}^{-2} \\
& =\sigma_{1}^{2} \sigma_{2} \sigma_{1}^{2} \sigma_{2}^{-1} \sigma_{3} \sigma_{2} \sigma_{1}^{2} \sigma_{2}^{-1} \sigma_{3}^{-1} \sigma_{2} \sigma_{1}^{-2} \sigma_{2}^{-1} \sigma_{1}^{-2} \\
& =A_{1,2} A_{1,3} A_{1,4} A_{1,3}^{-1} A_{1,2}^{-1} .
\end{aligned}
$$

Retomemos na seguinte proposição o homomorfismo $\beta: F_{3}\left(\widehat{z_{0}}, \widehat{z_{1}}, \widehat{z_{2}}\right) \rightarrow P_{4}\left(\mathbb{S}^{2}\right)$ definido no paragráfo anterior à Proposição 6.1 .5 (ver também [9, final da página 313]), bem como sua definição geral para qualquer número de cordas, indicada nas linhas 9-10 da página 314 de [9]. A rigor o homomorfismo $\beta$ deveria ter um subíndice indicando o 
número de cordas (ou de geradores livres para $F_{n-1}$ ), porém como estamos seguindo [9] não modificaremos a notação já usada para este homomorfismo.

Proposição 3.4.1. Seja $\beta_{5} \in P_{5}\left(\mathbb{S}^{2}\right)$ o elemento dado por

$$
\beta_{5}=\left[\left[D_{1,2} D_{1,3}, D_{1,4}\right],\left[D_{1,2}, D_{1,3} D_{1,4}\right]\right]
$$

Então, $\beta_{5}=\beta\left(\left[\left[\hat{z}_{1} \hat{z}_{0}, \hat{z}_{0}^{-1} \hat{z}_{1}^{-1} \hat{z}_{2} \hat{z}_{1} \hat{z}_{0}\right],\left[\hat{z}_{0}, \hat{z}_{0}^{-1} \hat{z}_{2} \hat{z}_{1} \hat{z}_{0}\right]\right]\right)$. Além disso, $\beta_{5}$ é uma trança Brunniana com 5 cordas na esfera, $\beta_{5} \in \operatorname{Brun}_{5}\left(\mathbb{S}^{2}\right)$, porém $\beta_{5} \notin f_{*}\left(\operatorname{Brun}_{5}\left(\mathbb{D}^{2}\right)\right)$.

Demonstração. Pelas igualdades obtidas em (3.19) e pelo fato que $f_{*}: P_{5}\left(\mathbb{D}^{2}\right) \rightarrow P_{5}\left(\mathbb{S}^{2}\right)$ é o homomorfismo que leva os geradores de tranças puras do grupo de tranças sobre o disco nos geradores do grupo de tranças puras sobre a esfera, $A_{i, j} \mapsto D_{i, j}$, temos que:

$$
\left\{\begin{aligned}
D_{2,3}^{-1} D_{1,3} D_{2,3} & =D_{1,2} D_{1,3} D_{1,2}^{-1} \\
D_{3,4}^{-1} D_{2,4}^{-1} D_{1,4} D_{2,4} D_{3,4} & =D_{1,2} D_{1,3} D_{1,4} D_{1,3}^{-1} D_{1,2}^{-1}
\end{aligned}\right.
$$

Obtemos assim a seguinte igualdade de grupos livres de posto 3

$$
F_{3}\left(D_{1,2}, D_{2,3}^{-1} D_{1,3} D_{2,3}, D_{3,4}^{-1} D_{2,4}^{-1} D_{1,4} D_{2,4} D_{3,4}\right)=F_{3}\left(D_{1,2}, D_{1,3}, D_{1,4}\right) .
$$

Lembremos que todo endomorfismo de um grupo Hopfiano sobrejetor é um automorfismo. Agora, como o homomorfismo $\beta: F_{3}\left(\widehat{z_{0}}, \widehat{z_{1}}, \widehat{z_{2}}\right) \rightarrow P_{5}\left(\mathbb{S}^{2}\right)$ é tal que

$$
\beta\left(F_{3}\left(\widehat{z_{0}}, \widehat{z_{1}}, \widehat{z_{2}}\right)\right)=F_{3}\left(D_{1,2}, D_{1,3}, D_{1,4}\right)
$$

então $\beta$ é um isomorfismo, pois os grupos livres são Hopfianos. Ainda mais, dado que $\operatorname{Ker}\left(d_{1}: P_{5}\left(\mathbb{S}^{2}\right) \rightarrow P_{4}\left(\mathbb{S}^{2}\right)\right)=F_{3}\left(D_{1,2}, D_{1,3}, D_{1,4}\right)$, então a seguinte sequência é exata curta

$$
1 \longrightarrow F_{3}\left(\widehat{z_{0}}, \widehat{z_{1}}, \widehat{z_{2}}\right) \stackrel{\beta}{\longrightarrow} P_{5}\left(\mathbb{S}^{2}\right) \stackrel{d_{1}}{\longrightarrow} P_{4}\left(\mathbb{S}^{2}\right) \longrightarrow 1
$$

Sabemos que a fibração de Fadell-Neuwirth dá lugar à seguinte sequência exata curta

$$
1 \longrightarrow G_{3}\left(w_{0}, w_{1}, w_{2}\right) \stackrel{i_{1 *}}{\longrightarrow} P_{5}\left(\mathbb{S}^{2}\right) \stackrel{d_{1}}{\longrightarrow} P_{4}\left(\mathbb{S}^{2}\right) \longrightarrow 1
$$

onde $G_{3}\left(w_{0}, w_{1}, w_{2}\right)=\pi_{1}\left(\mathbb{S}^{2} \backslash\left\{q_{2}, q_{3}, q_{4}, q_{5}\right\}, q_{1}\right)$. Os geradores livres $w_{0}, w_{1}, w_{2}$, do grupo livre de posto $3, G_{3}\left(w_{0}, w_{1}, w_{2}\right)$, são como na Figura 3.2.

Assim, comparando as duas sequências exatas curtas (3.20) e (3.21), concluímos que $\beta\left(F_{3}\right)=i_{1 *}\left(G_{3}\right)$.

Consideremos na continuação o seguinte isomorfismo de grupos livres, $\theta: G_{3} \rightarrow F_{3}$, 


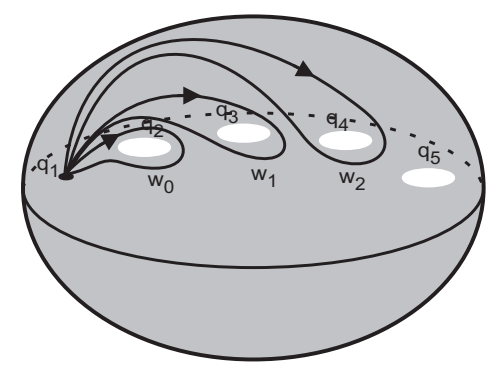

Figura 3.2: Geradores livres $w_{0}, w_{1}, w_{2}$.

definido por

$$
\begin{aligned}
w_{0} & \mapsto \widehat{z_{0}} \\
w_{1} & \mapsto{\widehat{z_{0}}}^{-1} \widehat{z_{1}} \widehat{z_{0}} \\
w_{2} & \mapsto{\widehat{z_{0}}}^{-1}{\widehat{z_{1}}}^{-1} \widehat{z_{2}} \widehat{z_{1}} \widehat{z_{0}} .
\end{aligned}
$$

Tomemos o elemento

$$
\alpha_{5}=\left[\left[w_{0} w_{1}, w_{2}\right],\left[w_{0}, w_{1} w_{2}\right]\right] \in G_{3}\left(w_{0}, w_{1}, w_{2}\right)
$$

Por [25, Página 2109 (item 2)] sabemos que o elemento $\alpha_{5}$ do grupo livre de posto 3 , dado em (3.22), se projeta no gerador $\eta_{2}^{2} \in \pi_{4}\left(\mathbb{S}^{2}\right)$.

Notemos que $\theta\left(\alpha_{5}\right)=\left[\left[\hat{z}_{1} \hat{z}_{0}, \hat{z}_{0}^{-1} \hat{z}_{1}^{-1} \hat{z}_{2} \hat{z}_{1} \hat{z}_{0}\right],\left[\hat{z}_{0}, \hat{z}_{0}^{-1} \hat{z}_{2} \hat{z}_{1} \hat{z}_{0}\right]\right], \beta\left(\theta\left(w_{0}\right)\right)=D_{1,2}$, $\beta\left(\theta\left(w_{1}\right)\right)=D_{1,3}$ e $\beta\left(\theta\left(w_{2}\right)\right)=D_{1,4}$. Portanto, $\beta\left(\theta\left(\alpha_{5}\right)\right)=\beta_{5} \in P_{5}\left(\mathbb{S}^{2}\right)$, verificando a primeira parte da proposição.

Provemos que $\beta_{5}=\left[\left[D_{1,2} D_{1,3}, D_{1,4}\right],\left[D_{1,2}, D_{1,3} D_{1,4}\right]\right] \in \operatorname{Brun}_{5}\left(\mathbb{S}^{2}\right)$. Claramente temos que $d_{j}\left(\beta_{5}\right)=1$, para $j=1,2,3,4$. Assim, resta verificar que $d_{5}\left(\beta_{5}\right)=1$. Usando a relação de superfície $D_{1,2} D_{1,3} D_{1,4}=1$ em $P_{4}\left(\mathbb{S}^{2}\right)$ obtemos a igualdade desejada:

$$
d_{5}\left(\beta_{5}\right)=\left[\left[D_{1,2} D_{1,3}, D_{1,4}\right],\left[D_{1,2}, D_{1,3} D_{1,4}\right]\right]=\left[\left[D_{1,4}^{-1}, D_{1,4}\right],\left[D_{1,2}, D_{1,2}^{-1}\right]\right]=1 \in P_{4}\left(\mathbb{S}^{2}\right) .
$$

Portanto, $\beta_{5} \in \operatorname{Brun}_{5}\left(\mathbb{S}^{2}\right)$.

Pelo Lema 2.1.5, que oferece uma descrição dos grupos de tranças Brunnianas sobre superfícies como uma intersecção de certos subgrupos normais dos grupos de tranças puras, temos que

$$
\left\langle D_{1,5}\right\rangle^{i_{1 *}\left(G_{3}\right)}=\left\langle D_{1,5}\right\rangle^{P_{5}\left(\mathbb{S}^{2}\right)}
$$

e

$$
\left\langle D_{1,2}\right\rangle^{P_{5}\left(\mathbb{S}^{2}\right)} \cap\left\langle D_{1,3}\right\rangle^{P_{5}\left(\mathbb{S}^{2}\right)} \cap\left\langle D_{1,4}\right\rangle^{P_{5}\left(\mathbb{S}^{2}\right)} \cap\left\langle D_{1,5}\right\rangle^{P_{5}\left(\mathbb{S}^{2}\right)}=\operatorname{Brun}_{5}\left(\mathbb{S}^{2}\right) .
$$

Vejamos agora que $\beta_{5} \notin f_{*}\left(\operatorname{Brun}_{5}\left(\mathbb{D}^{2}\right)\right)$. Pelo Teorema 1.2 .3 sabemos que

$$
\pi_{4}\left(\mathbb{S}^{2}\right) \cong \frac{\left\langle w_{0}\right\rangle^{G_{3}} \cap\left\langle w_{1}\right\rangle^{G_{3}} \cap\left\langle w_{2}\right\rangle^{G_{3}} \cap\left\langle w_{0} w_{1} w_{2}\right\rangle^{G_{3}}}{\left[\left\langle w_{0}\right\rangle^{G_{3}},\left\langle w_{1}\right\rangle^{G_{3}},\left\langle w_{2}\right\rangle^{G_{3}},\left\langle w_{0} w_{1} w_{2}\right\rangle^{G_{3}}\right]_{S}}
$$


Lembremos que $D_{1,2} D_{1,3} D_{1,4} D_{1,5}=1$ em $P_{5}\left(\mathbb{S}^{2}\right)$ e $\operatorname{assim} \beta\left(\left\langle\widehat{z_{2}} \widehat{z_{1}} \widehat{z_{0}}\right\rangle^{F_{3}}\right)=\left\langle D_{1,5}\right\rangle^{\beta\left(F_{3}\right)}$. Portanto, usando que $\beta\left(F_{3}\right)=i_{1 *}\left(G_{3}\right)$, temos que

$$
\begin{aligned}
& \pi_{4}\left(\mathbb{S}^{2}\right) \cong \frac{\left\langle w_{0}\right\rangle^{G_{3}} \cap\left\langle w_{1}\right\rangle^{G_{3}} \cap\left\langle w_{2}\right\rangle^{G_{3}} \cap\left\langle w_{0} w_{1} w_{2}\right\rangle^{G_{3}}}{\left[\left\langle w_{0}\right\rangle^{G_{3}},\left\langle w_{1}\right\rangle^{G_{3}},\left\langle w_{2}\right\rangle^{G_{3}},\left\langle w_{0} w_{1} w_{2}\right\rangle^{G_{3}}\right]_{S}} \\
& \cong \frac{\left\langle{\widehat{z_{0}}}^{F_{3}} \cap\left\langle{\widehat{z_{0}}}^{-1} \widehat{z_{1}} \widehat{z_{0}}\right\rangle^{F_{3}} \cap\left\langle{\widehat{z_{0}}}^{-1}{\widehat{z_{1}}}^{-1} \widehat{z_{2}} \widehat{z_{1}} \widehat{z_{0}}\right\rangle^{F_{3}} \cap\left\langle\widehat{z_{2}} \widehat{z_{1}} \widehat{z_{0}}\right\rangle^{F_{3}}\right.}{\left[\left\langle{\widehat{z_{0}}}^{F_{3}},\left\langle{\widehat{z_{0}}}^{-1} \widehat{z_{1}} \widehat{z_{0}}\right\rangle^{F_{3}},\left\langle{\widehat{z_{0}}}^{-1}{\widehat{z_{1}}}^{-1} \widehat{z_{2}} \widehat{z_{1}} \widehat{z_{0}}\right\rangle^{F_{3}},\left\langle\widehat{z_{2}} \widehat{z_{1}} \widehat{z_{0}}\right\rangle^{F_{3}}\right]_{S}\right.} \\
& \cong \frac{\left\langle D_{1,2}\right\rangle^{\beta\left(F_{3}\right)} \cap\left\langle D_{1,3}\right\rangle^{\beta\left(F_{3}\right)} \cap\left\langle D_{1,4}\right\rangle^{\beta\left(F_{3}\right)} \cap\left\langle D_{1,5}\right\rangle^{\beta\left(F_{3}\right)}}{\left[\left\langle D_{1,2}\right\rangle^{\beta\left(F_{3}\right)},\left\langle D_{1,3}\right\rangle^{\beta\left(F_{3}\right)},\left\langle D_{1,4}\right\rangle^{\beta\left(F_{3}\right)},\left\langle D_{1,5}\right\rangle^{\beta\left(F_{3}\right)}\right]_{S}} \\
& =\frac{\left\langle D_{1,2}\right\rangle^{i_{1 *}\left(G_{3}\right)} \cap\left\langle D_{1,3}\right\rangle^{i_{1 *}\left(G_{3}\right)} \cap\left\langle D_{1,4}\right\rangle^{i_{1 *}\left(G_{3}\right)} \cap\left\langle D_{1,5}\right\rangle^{i_{1 *}\left(G_{3}\right)}}{\left[\left\langle D_{1,2}\right\rangle^{i_{1 *}\left(G_{3}\right)},\left\langle D_{1,3}\right\rangle^{i_{1 *}\left(G_{3}\right)},\left\langle D_{1,4}\right\rangle^{i_{1 *}\left(G_{3}\right)},\left\langle D_{1,5}\right\rangle^{i_{1 *}\left(G_{3}\right)}\right]_{S}} \\
& =\frac{\left\langle D_{1,2}\right\rangle^{P_{5}\left(\mathbb{S}^{2}\right)} \cap\left\langle D_{1,3}\right\rangle^{P_{5}\left(\mathbb{S}^{2}\right)} \cap\left\langle D_{1,4}\right\rangle^{P_{5}\left(\mathbb{S}^{2}\right)} \cap\left\langle D_{1,5}\right\rangle^{P_{5}\left(\mathbb{S}^{2}\right)}}{\left[\left\langle D_{1,2}\right\rangle^{P_{5}\left(\mathbb{S}^{2}\right)},\left\langle D_{1,3}\right\rangle^{P_{5}\left(\mathbb{S}^{2}\right)},\left\langle D_{1,4}\right\rangle^{P_{5}\left(\mathbb{S}^{2}\right)},\left\langle D_{1,5}\right\rangle^{P_{5}\left(\mathbb{S}^{2}\right)}\right]_{S}} \\
& =\frac{\operatorname{Brun}_{5}\left(\mathbb{S}^{2}\right)}{f_{*}\left(\operatorname{Brun}_{5}\left(\mathbb{D}^{2}\right)\right)}
\end{aligned}
$$

Como comentado antes, por [25, Página 2109 (item 2)], a classe do elemento $\alpha_{5}$ dado em (3.22) não é trivial no grupo quociente

$$
\frac{\left\langle w_{0}\right\rangle^{G_{3}} \cap\left\langle w_{1}\right\rangle^{G_{3}} \cap\left\langle w_{2}\right\rangle^{G_{3}} \cap\left\langle w_{0} w_{1} w_{2}\right\rangle^{G_{3}}}{\left[\left\langle w_{0}\right\rangle^{G_{3}},\left\langle w_{1}\right\rangle^{G_{3}},\left\langle w_{2}\right\rangle^{G_{3}},\left\langle w_{0} w_{1} w_{2}\right\rangle^{G_{3}}\right]_{S}} \cong \pi_{4}\left(\mathbb{S}^{2}\right) .
$$

Logo, por (3.25), a classe de $\beta_{5}=\beta\left(\theta\left(\alpha_{5}\right)\right)$ não é trivial em $\frac{\operatorname{Brun}_{5}\left(\mathbb{S}^{2}\right)}{f_{*}\left(\operatorname{Brun}_{5}\left(\mathbb{D}^{2}\right)\right)}$, como queríamos provar. 


\section{Capítulo 4}

\section{Centro de $G_{n+1} / \mathcal{B} d_{n} \mathcal{H}$ e os grupos de homotopia $\pi_{n}(\mathcal{H})$}

Em [96] J. Wu estudou os grupos de homotopia de grupos simpliciais, usando fortemente a teoria de homotopia simplicial e, em particular, foi dada uma descrição de tais grupos como sendo o centro de certos quocientes apropriados dos grupos pertencentes ao grupo simplicial. As técnicas usadas na teoria de homotopia simplicial têm se mostrado úteis para dar diferentes descrições dos grupos de homotopia de alguns espaços topológicos. Destacamos a seguir dois trabalhos recentes que evidenciam isto. Em [58], J.Y. Li e J. Wu descreveram os grupos de homotopia da esfera $\mathbb{S}^{2}$ (mais um fator adicional) como sendo o centro de quocientes de grupos de tranças do disco (ver Teorema 1.3.25). Posteriormente, R. Mikhailov e J. Wu em [69], estudaram os grupos de homotopia de alguns espaços topológicos específicos como centros de grupos finitamente apresentados, estes últimos foram construídos a partir da estrutura do espaço topológico considerado.

Nesse capítulo continuaremos estes estudos na teoria de homotopia simplicial e descreveremos os grupos de homotopia de grupos simpliciais $\mathcal{H}$ via o centro de $G_{n+1} / \mathcal{B} d_{n} \mathcal{H}$, onde $G_{n+1}$ é um grupo que contém $\mathcal{H}_{n}$, mas sem nenhuma exigência do ponto de vista simplicial para a sequência de grupos $\left\{G_{n}\right\}_{n \geq 0}$. Como uma aplicação destes conceitos gerais veremos que os grupos de homotopia da esfera $\mathbb{S}^{2}$ podem ser descritos tomando-se $\mathcal{H}$ acima como sendo o grupo simplicial formado por subgrupos de tranças do disco ou da esfera. Transparece que resultados semelhantes devem ser verdadeiros para subgrupos de tranças sobre outras superfícies, porém esse estudo será conduzido futuramente.

Vários dos resultados apresentados nesse capítulo são muito semelhantes aos resultados de [58], no entanto a colocação destes resultados se justifica não só por possíveis variações dos resultados de [58], mas pelas dificuldades e imprecisões existentes em [58]. O estudo do aspecto mencionado acima encontra-se em detalhes no final da Seção 6.2. 


\subsection{O Teorema de Extensão Central e o Teorema de Decomposição}

Nesta seção trataremos resultados gerais da teoria de homotopia simplicial com o intuito de descrever de diferentes formas os grupos de homotopia de $\Delta$-grupos (ou grupos simpliciais). Começaremos esta seção com a nossa versão do Teorema de Extensão Central em teoria de homotopia simplicial. Uma outra versão, com hipóteses diferentes porém, com uma conclusão similar à apresentada aqui, foi dada no Teorema 3.8 do capítulo intitulado "Simplicial Objects and Homotopy Groups" do livro [8]. Gostaríamos de mencionar que muitas das demonstrações desta seção tem ideias inspiradas em [58]. Lembremos que denotamos o centro de um grupo $G$ por $Z(G)$.

Teorema 4.1.1 (Teorema de Extensão Central). Sejam $\mathcal{G}=\left\{G_{n}\right\}_{n \geq 0}$ uma sequência de grupos qualquer e $\mathcal{H}=\left\{H_{n}\right\}_{n \geq 0}$ um $\Delta$-grupo. Suponhamos que para algum $n \geq 0$ temse as inclusões de grupos $H_{n} \subseteq G_{n+1}, H_{n+1} \subseteq G_{n+2}$ e que as seguintes condições são satisfeitas:

i) $\mathcal{H}$ é fibrante.

ii) $\mathcal{B} d_{n} \mathcal{H}$ é um subgrupo normal de $G_{n+1}$.

iii) Se $X$ é um conjunto de geradores de $G_{n+1}$, então para todo elemento $\alpha \in X$, existem $0 \leq i_{0} \leq n$ e uma aplicação $\widehat{d}_{i_{0}}: G_{n+2} \rightarrow G_{n+1}$ tais que $\left.\widehat{d}_{i_{0}}\right|_{H_{n+1}}=d_{i_{0}}$ e

$$
\widehat{d}_{i_{0}}^{-1}(\alpha) \cap \operatorname{Ker}\left(d_{i_{0}+1}: H_{n+1} \rightarrow H_{n}\right)
$$

não é o conjunto vazio, nem o grupo trivial.

Então, o grupo $\pi_{n}(\mathcal{H})$ está contido no centro de $G_{n+1} / \mathcal{B} d_{n} \mathcal{H}$.

Demonstração. Fixemos um número $n \geq 0$. Por definição, $\pi_{n}(\mathcal{H})=\mathcal{Z}_{n} \mathcal{H} / \mathcal{B} d_{n} \mathcal{H}$ e pela Proposição 1.3.7, $\pi_{n}(\mathcal{H})$ é um grupo. Observemos que

$$
\begin{aligned}
\pi_{n}(\mathcal{H}) \subseteq Z\left(G_{n+1} / \mathcal{B} d_{n} \mathcal{H}\right) \Leftrightarrow & {[\bar{\beta}, \bar{\alpha}]=1 \in G_{n+1} / \mathcal{B} d_{n} \mathcal{H}, \text { para todo } \bar{\beta} \in \mathcal{Z}_{n} \mathcal{H} / \mathcal{B} d_{n} \mathcal{H}, } \\
& \text { e para todo } \bar{\alpha} \in G_{n+1} / \mathcal{B} d_{n} \mathcal{H} . \\
\Leftrightarrow & {[\beta, \alpha] \in \mathcal{B} d_{n} \mathcal{H}, \text { para todo } \beta \in \mathcal{Z}_{n} \mathcal{H}, } \\
& \text { e para todo } \alpha \in G_{n+1} .
\end{aligned}
$$

Sejam $\beta \in \mathcal{Z}_{n} \mathcal{H} \subseteq H_{n}$ e $\alpha$ em $G_{n+1}$. Pelas identidades de Witt-Hall formuladas no Teorema 1.1 .2 e pelo fato que $\mathcal{B} d_{n} \mathcal{H}$ é um subgrupo normal de $G_{n+1}$ é suficiente provar que $[\beta, \alpha] \in \mathcal{B} d_{n} \mathcal{H}$ para um conjunto de geradores de $G_{n+1}$. Sendo assim, tomemos o elemento $\alpha$ no conjunto $X$, que é um conjunto de geradores de $G_{n+1}$. Por hipótese existem 
$i_{0} \in\{0,1, \ldots, n\}$ e $\widehat{d}_{i_{0}}: G_{n+2} \rightarrow G_{n+1}$ tais que $\widehat{d}_{i_{0}}^{-1}(\alpha) \cap \operatorname{Ker}\left(d_{i_{0}+1}\right)$ não é o conjunto vazio, nem o grupo trivial. Observemos que os elementos

$$
x_{0}=1, x_{1}=1, \ldots, x_{i_{0}-1}=1, x_{i_{0}}=\beta, x_{i_{0}+2}=1, \ldots, x_{n}=1, x_{n+1}=1
$$

são faces emparelhadas em $H_{n}$, com relação a $i_{0}+1$. Como $\mathcal{H}$ é fibrante, então existe um elemento $\gamma \in H_{n+1}$ tal que $d_{k} \gamma=x_{k}$, para todo $k \neq i_{0}+1$, isto é, $d_{i_{0}} \gamma=\beta \in H_{n}$ e $d_{k} \gamma=1$, para $k \neq i_{0}, i_{0}+1$, com $0 \leq k \leq n+1$. Dado que a intersecção de grupos $\widehat{d}_{i_{0}}^{-1}(\alpha) \cap \operatorname{Ker}\left(d_{i_{0}+1}\right)$ tem pelo menos um elemento diferente da identidade, podemos pegar $\widehat{\alpha} \neq 1$ em $\widehat{d}_{i_{0}}^{-1}(\alpha) \cap \operatorname{Ker}\left(d_{i_{0}+1}\right) \subseteq H_{n+1}$. Seja $\omega=[\gamma, \widehat{\alpha}] \in H_{n+1}$. Então, para $0 \leq k \leq n+1$ temos que:

$$
d_{k} \omega=\left[d_{k} \gamma, d_{k} \widehat{\alpha}\right]= \begin{cases}{\left[1, d_{k} \widehat{\alpha}\right]=1,} & \text { se } k \neq i_{0}, i_{0}+1 \\ {[\beta, \alpha],} & \text { se } k=i_{0} \\ {\left[d_{i_{0}+1} \gamma, 1\right]=1,} & \text { se } k=i_{0}+1\end{cases}
$$

Portanto, provamos que existem $i_{0} \in\{0, \ldots, n\}$ e $\omega \in H_{n+1}$ tais que $d_{i_{0}} \omega=[\beta, \alpha]$ e $d_{k} \omega=1$, para $0 \leq k \leq n+1 \operatorname{com} k \neq i_{0}$. Em outras palavras, $d_{i_{0}} \omega=[\beta, \alpha] \mathrm{e}$

$$
\omega \in \bigcap_{\substack{0 \leq k \leq n+1 \\ k \neq i_{0}}} \operatorname{Ker}\left(d_{k}: H_{n+1} \rightarrow H_{n}\right)
$$

Segue da Proposição 1.3.7 que, para todo $0 \leq i \leq n+1$, o conjunto de bordos de Moore para o $\Delta$-grupo fibrante $\mathcal{H}$ pode ser descrito como

$$
\mathcal{B} d_{n} \mathcal{H}=d_{i}\left(\bigcap_{\substack{0 \leq k \leq n+1 \\ k \neq i}} \operatorname{Ker}\left(d_{k}: H_{n+1} \rightarrow H_{n}\right)\right)
$$

Logo, $d_{i_{0}} \omega=[\beta, \alpha] \in \mathcal{B} d_{n} \mathcal{H}$, para qualquer $\beta \in \mathcal{Z}_{n} \mathcal{H}$ e qualquer elemento $\alpha$ do conjunto de geradores $X$ de $G_{n+1}$, como queríamos provar.

Corolário 4.1.2. Seja $\mathcal{H}=\left\{H_{n}\right\}_{n \geq 0}$ um $\Delta$-grupo fibrante tal que $\mathcal{B} d_{n} \mathcal{H}$ é um subgrupo normal de $H_{n}$. Suponhamos que se $X$ é um conjunto de geradores de $H_{n}$, então para todo elemento $\alpha \in X$, existe $0 \leq i_{0} \leq n$ tal que $d_{i_{0}}^{-1}(\alpha) \cap \operatorname{Ker}\left(d_{i_{0}+1}: H_{n+1} \rightarrow H_{n}\right)$ não é o conjunto vazio, nem o grupo trivial. Então, o grupo $\pi_{n}(\mathcal{H})$ está contido no centro de $H_{n} / \mathcal{B} d_{n} \mathcal{H}$

Demonstração. Seja $\mathcal{G}=\left\{G_{n}\right\}_{n \geq 0}$ uma sequência de grupos qualquer com $G_{n+1}=H_{n}$ e $G_{n+2}=H_{n+1}$. Este resultado decorre facilmente do Teorema de Extensão Central, formulado no Teorema 4.1.1, aplicado a $\mathcal{H}$ e $\mathcal{G}$.

Notemos que se $\varphi: G \rightarrow H$ é um homomorfismo sobrejetor, então a restrição $\varphi \mid: Z(G) \rightarrow Z(H)$ está bem definida. 
Lema 4.1.3. Seja $\mathcal{G}=\left\{G_{n} ; \delta_{n+1}\right\}_{n \geq 0}$ uma sequência de grupos e homomorfismos $\delta_{n+1}: G_{n+1} \rightarrow G_{n}$ e denotemos por $H_{n}$ o grupo Ker $\left(\delta_{n+1}\right)$. Suponhamos que a coleção de subgrupos $\mathcal{H}=\left\{H_{n}\right\}_{n \geq 0}$ é um $\Delta$-grupo e que para algum $n \geq 0$ as seguintes condições são verificadas.

i) Os homomorfismos $\delta_{n+1}: G_{n+1} \rightarrow G_{n}$ e $\delta_{n+1} \mid: Z\left(G_{n+1}\right) \rightarrow Z\left(G_{n}\right)$ são sobrejetores.

ii) $\mathcal{B} d_{n} \mathcal{H}$ é um subgrupo normal de $G_{n+1}$.

iii) $Z\left(H_{n} / \mathcal{B} d_{n} \mathcal{H}\right) \cap A_{n+1}=\{1\}$, onde $A_{n+1}$ é a projeção de $Z\left(G_{n+1}\right)$ em $G_{n+1} / \mathcal{B} d_{n} \mathcal{H}$.

Então, $Z\left(\frac{G_{n+1}}{\mathcal{B} d_{n} \mathcal{H}}\right)$ está contido no produto direto interno de $Z\left(\frac{H_{n}}{\mathcal{B} d_{n} \mathcal{H}}\right)$ com $A_{n+1}$.

Ainda mais, se $\mathcal{H}$ é um grupo simplicial e $Z\left(H_{n-1}\right)=\{1\}$, então $Z\left(\frac{G_{n+1}}{\mathcal{B} d_{n} \mathcal{H}}\right)$ está contido no produto direto interno de $\pi_{n}(\mathcal{H})$ com $A_{n+1}$.

Demonstração. Seja $x \in G_{n+1}$ tal que $\bar{x} \in Z\left(G_{n+1} / \mathcal{B} d_{n} \mathcal{H}\right)$. Portanto, $[x, \beta]$ pertence a $\mathcal{B} d_{n} \mathcal{H} \subseteq H_{n}$, para todo $\beta$ em $G_{n+1}$. Seja $\alpha \in G_{n}$, dado que $\delta_{n+1}: G_{n+1} \rightarrow G_{n}$ é sobrejetora, então existe $\beta \in G_{n+1}$ tal que $\delta_{n+1}(\beta)=\alpha$. Como $[x, \beta] \in \mathcal{B} d_{n} \mathcal{H} \leq H_{n}$ e $H_{n}=\operatorname{Ker}\left(\delta_{n+1}\right)$, então

$$
\left[\delta_{n+1}(x), \alpha\right]=\left[\delta_{n+1}(x), \delta_{n+1}(\beta)\right]=\delta_{n+1}([x, \beta])=1 .
$$

Portanto, $\delta_{n+1}(x) \in Z\left(G_{n}\right)$. Por hipótese, $\delta_{n+1} \mid: Z\left(G_{n+1}\right) \rightarrow Z\left(G_{n}\right)$ é sobrejetora. Assim, podemos tomar $x_{1} \in Z\left(G_{n+1}\right)$ tal que $\delta_{n+1}\left(x_{1}\right)=\delta_{n+1}(x)$. Notemos que $\bar{x}_{1} \in Z\left(G_{n+1}\right) / \mathcal{B} d_{n} \mathcal{H}$ e que vale a seguinte inclusão

$$
Z\left(G_{n+1}\right) / \mathcal{B} d_{n} \mathcal{H} \subseteq Z\left(G_{n+1} / \mathcal{B} d_{n} \mathcal{H}\right)
$$

Seja $y=x x_{1}^{-1} \in G_{n+1}$. Como $\bar{x} \in Z\left(G_{n+1} / \mathcal{B} d_{n} \mathcal{H}\right)$ e $\bar{x}_{1} \in Z\left(G_{n+1}\right) / \mathcal{B} d_{n} \mathcal{H}$, então $\bar{y}=\bar{x} \bar{x}_{1}^{-1} \in Z\left(G_{n+1} / \mathcal{B} d_{n} \mathcal{H}\right)$. Além disso, $y \in H_{n}=\operatorname{Ker}\left(\delta_{n+1}: G_{n+1} \rightarrow G_{n}\right)$ pois

$$
\delta_{n+1}(y)=\delta_{n+1}(x) \delta_{n+1}\left(x_{1}^{-1}\right)=\delta_{n+1}(x) \delta_{n+1}(x)^{-1}=1 .
$$

Como consequência, $\bar{y} \in Z\left(H_{n} / \mathcal{B} d_{n} \mathcal{H}\right)$ e portanto

$$
\bar{x}=\bar{y} \bar{x}_{1} \in Z\left(\frac{H_{n}}{\mathcal{B} d_{n} \mathcal{H}}\right) \cdot \frac{Z\left(G_{n+1}\right)}{\mathcal{B} d_{n} \mathcal{H}} .
$$

Logo, $Z\left(\frac{G_{n+1}}{\mathcal{B} d_{n} \mathcal{H}}\right) \subseteq Z\left(\frac{H_{n}}{\mathcal{B} d_{n} \mathcal{H}}\right) \cdot \frac{Z\left(G_{n+1}\right)}{\mathcal{B} d_{n} \mathcal{H}}$, o que demonstra a primeira parte do lema.

Suponhamos agora que $\mathcal{H}$ é um grupo simplicial e $Z\left(H_{n-1}\right)=\{1\}$. Dado $\bar{x}$ em $Z\left(G_{n+1} / \mathcal{B} d_{n} \mathcal{H}\right)$ vimos em $(4.2)$ que $\bar{x}=\bar{y} \bar{x}_{1} \in Z\left(\frac{H_{n}}{\mathcal{B} d_{n} \mathcal{H}}\right) \cdot \frac{Z\left(G_{n+1}\right)}{\mathcal{B} d_{n} \mathcal{H}}$. Assim, para provar a última afirmação deste lema basta demonstrarmos que o elemento $\bar{y} \in Z\left(H_{n} / \mathcal{B} d_{n} \mathcal{H}\right)$ é 
tal que $\bar{y} \in \pi_{n}(\mathcal{H})=\mathcal{Z}_{n} \mathcal{H} / \mathcal{B} d_{n} \mathcal{H}$. Como $Z\left(H_{n-1}\right)=\{1\}$, então para $0 \leq j \leq n$,

$$
d_{j} y=1 \Leftrightarrow\left[d_{j} y, w\right]=1, \text { para todo } w \in H_{n-1} \subseteq G_{n} .
$$

Usando as identidades simpliciais no grupo simplicial $\mathcal{H}$, dadas em (1.12), vemos que

$$
\left[d_{0} y, w\right]=\left[d_{0} y, d_{0} s_{0} w\right]=d_{0}\left[y, s_{0} w\right]
$$

e para $j \geq 1$

$$
\left[d_{j} y, w\right]=\left[d_{j} y, d_{j} s_{j-1} w\right]=d_{j}\left[y, s_{j-1} w\right] .
$$

Como $\bar{y} \in Z\left(H_{n} / \mathcal{B} d_{n} \mathcal{H}\right)$, então $[y, h] \in \mathcal{B} d_{n} \mathcal{H} \subseteq \mathcal{Z}_{n} \mathcal{H}$, para todo $h \in H_{n}$, e portanto $\left[d_{j} y, w\right]=1$, para todo $w \in H_{n-1}$ e todo $0 \leq j \leq n$. Ou seja, $y \in \mathcal{Z}_{n} \mathcal{H}$ em vista do afirmado em (4.3). Logo, $\bar{y} \in \pi_{n}(\mathcal{H})=\mathcal{Z}_{n} \mathcal{H} / \mathcal{B} d_{n} \mathcal{H}$ como queríamos provar.

Observação 4.1.4. Se na prova da primeira afirmação do Lema 4.1.3 temos que $Z\left(G_{n}\right)=\{1\}$, então $\delta_{n+1}(x)=1$ e portanto $x \in \operatorname{Ker}\left(\delta_{n+1}\right)=H_{n}$. Assim, a demonstração ficaria mais curta pois é claro que $\bar{x} \in Z\left(H_{n} / \mathcal{B} d_{n} \mathcal{H}\right)$.

Juntemos as hipóteses do Teorema 4.1.1 e do Lema 4.1.3 para obter o seguinte resultado.

Teorema 4.1.5. Seja $\mathcal{G}=\left\{G_{n} ; \delta_{n+1}\right\}_{n \geq 0}$ uma sequência de grupos e homomorfismos $\delta_{n+1}: G_{n+1} \rightarrow G_{n}$ e denotemos por $H_{n}$ o grupo $\operatorname{Ker}\left(\delta_{n+1}\right)$. Suponhamos que $\mathcal{H}=\left\{H_{n}\right\}_{n \geq 0}$ é um grupo simplicial tal que, para algum $n \geq 1$, as condições $i$ ), ii) e iii) do Lema 4.1.3 e a condição iii) do Teorema 4.1.1 são todas satisfeitas. Vamos supor também que $H_{n-1}$ tem centro trivial, $Z\left(H_{n-1}\right)=\{1\}$. Então,

$$
Z\left(G_{n+1} / \mathcal{B} d_{n} \mathcal{H}\right) \cong \pi_{n}(\mathcal{H}) \times A_{n+1}
$$

onde $A_{n+1}=Z\left(G_{n+1}\right) / \mathcal{B} d_{n} \mathcal{H}$.

Demonstração. Uma vez que $\mathcal{H}$ é um grupo simplicial segue pelo Teorema de Moore que $\mathcal{H}$ é um $\Delta$-grupo fibrante. Além disso, as condições ii) e iii) do Teorema 4.1.1 são satisfeitas e portanto

$$
\frac{\mathcal{Z}_{n} \mathcal{H}}{\mathcal{B} d_{n} \mathcal{H}} \subseteq Z\left(G_{n+1} / \mathcal{B} d_{n} \mathcal{H}\right)
$$

Observemos que as hipóteses do Corolário 4.1.2 são verificadas e portanto $\pi_{n}(\mathcal{H})=\mathcal{Z}_{n} \mathcal{H} / \mathcal{B} d_{n} \mathcal{H}$ está contido no centro $Z\left(H_{n} / \mathcal{B} d_{n} \mathcal{H}\right)$. Como a intersecção dos grupos $Z\left(H_{n} / \mathcal{B} d_{n} \mathcal{H}\right)$ e $Z\left(G_{n+1}\right) / \mathcal{B} d_{n} \mathcal{H}$ é trivial então a intersecção dos grupos $\mathcal{Z}_{n} \mathcal{H} / \mathcal{B} d_{n} \mathcal{H}$ e $Z\left(G_{n+1}\right) / \mathcal{B} d_{n} \mathcal{H}$ também é trivial. Portanto, podemos tomar o produto direto interno destes grupos. Logo, por (4.4) e (4.1), temos que

$$
\frac{\mathcal{Z}_{n} \mathcal{H}}{\mathcal{B} d_{n} \mathcal{H}} \frac{Z\left(G_{n+1}\right)}{\mathcal{B} d_{n} \mathcal{H}} \subseteq Z\left(G_{n+1} / \mathcal{B} d_{n} \mathcal{H}\right)
$$


Notemos que as hipóteses do Lema 4.1 .3 são satisfeitas e portanto obtemos a seguinte inclusão

$$
Z\left(\frac{G_{n+1}}{\mathcal{B} d_{n} \mathcal{H}}\right) \subseteq \frac{\mathcal{Z}_{n} \mathcal{H}}{\mathcal{B} d_{n} \mathcal{H}} \frac{Z\left(G_{n+1}\right)}{\mathcal{B} d_{n} \mathcal{H}}
$$

Segue das inclusões dadas em (4.5) e (4.6) o resultado desejado

$$
Z\left(\frac{G_{n+1}}{\mathcal{B} d_{n} \mathcal{H}}\right)=\pi_{n}(\mathcal{H}) \frac{Z\left(G_{n+1}\right)}{\mathcal{B} d_{n} \mathcal{H}} \cong \pi_{n}(\mathcal{H}) \times \frac{Z\left(G_{n+1}\right)}{\mathcal{B} d_{n} \mathcal{H}}
$$

Observação 4.1.6. Dados grupos $A \subseteq B \subseteq C$ tais que $A \subseteq Z(C)$, então também é válido que $A \subseteq Z(B)$.

A razão pela qual chamamos o teorema abaixo de Teorema de Decomposição vem do fato que o Teorema 3.27, do capítulo intitulado "Simplicial objects and homotopy groups" do livro [8], é um resultado similar ao nosso e chamado de "Decomposition Theorem".

Teorema 4.1.7 (Teorema de Decomposição). Seja $\mathcal{H}$ um $\Delta$-grupo fibrante tal que, para algum $n \geq 1$, valem as seguintes afirmações.

1. $\mathcal{B} d_{n-1} \mathcal{H}$ é um grupo livre e $\mathcal{B} d_{n} \mathcal{H}$ é um subgrupo livre e normal em $H_{n}$.

2. Se $X$ é um conjunto de geradores de $H_{n}$, então para todo elemento $\alpha$ de $X$, existe $0 \leq i_{0} \leq n$ tal que $d_{i_{0}}^{-1}(\alpha) \cap \operatorname{Ker}\left(d_{i_{0}+1}: H_{n+1} \rightarrow H_{n}\right)$ não é o conjunto vazio, nem o grupo trivial.

Então,

$$
\mathcal{N}_{n} \mathcal{H} / \mathcal{B} d_{n} \mathcal{H} \cong \pi_{n}(\mathcal{H}) \times \mathcal{B} d_{n-1} \mathcal{H}
$$

Demonstração. Segue da definição dos bordos e ciclos de Moore do $\Delta$-grupo $\mathcal{H}$ que existe uma sequência exata curta

$$
1 \longrightarrow \mathcal{Z}_{n} \mathcal{H} \longrightarrow \mathcal{N}_{n} \mathcal{H} \stackrel{d_{0}}{\longrightarrow} \mathcal{B} d_{n-1} \mathcal{H} \longrightarrow 1
$$

Como $\mathcal{B} d_{n} \mathcal{H}$ é um subgrupo normal do grupo $H_{n}$ e, em particular, é normal nos grupos $\mathcal{N}_{n} \mathcal{H}$ e $\mathcal{Z}_{n} \mathcal{H}$, então é possível passar ao quociente a sequência exata curta (4.7)

$$
1 \longrightarrow \mathcal{Z}_{n} \mathcal{H} / \mathcal{B} d_{n} \mathcal{H} \longrightarrow \mathcal{N}_{n} \mathcal{H} / \mathcal{B} d_{n} \mathcal{H} \stackrel{\bar{d}_{0}}{\longrightarrow} \mathcal{B} d_{n-1} \mathcal{H} \longrightarrow 1
$$

Notemos que $\pi_{n}(\mathcal{H})=\mathcal{Z}_{n} \mathcal{H} / \mathcal{B} d_{n} \mathcal{H}$. Como $\mathcal{B} d_{n-1} \mathcal{H}$ é um grupo livre, então a sequência (4.8) cinde. Pelo Corolário 4.1.2, temos que $\pi_{n}(\mathcal{H}) \subseteq Z\left(H_{n} / \mathcal{B} d_{n} \mathcal{H}\right)$. Pela Observação 4.1.6 com $A=\mathcal{Z}_{n} \mathcal{H} / \mathcal{B} d_{n} \mathcal{H}=\pi_{n}(\mathcal{H}), B=\mathcal{N}_{n} \mathcal{H} / \mathcal{B} d_{n} \mathcal{H}$ e $C=H_{n} / \mathcal{B} d_{n} \mathcal{H}$, temos que 
$\pi_{n}(\mathcal{H}) \subseteq Z\left(\mathcal{N}_{n} \mathcal{H} / \mathcal{B} d_{n} \mathcal{H}\right)$ e portanto a sequência exata curta (4.8) é uma extensão central. Logo,

$$
\mathcal{N}_{n} \mathcal{H} / \mathcal{B} d_{n} \mathcal{H} \cong \mathcal{Z}_{n} \mathcal{H} / \mathcal{B} d_{n} \mathcal{H} \times \mathcal{B} d_{n-1} \mathcal{H} \cong \pi_{n}(\mathcal{H}) \times \mathcal{B} d_{n-1} \mathcal{H}
$$

Observação 4.1.8. Se no Teorema 4.1.7 uma das aplicações faces de $\mathcal{H}$ é sobrejetora, digamos $d_{i}: H_{n+1} \rightarrow H_{n}$, então a condição "B $d_{n} \mathcal{H}$ é um subgrupo normal em $H_{n}$ " é garantida. A razão disto é que $\bigcap_{j \neq i} \operatorname{Ker}\left(d_{j}: H_{n+1} \rightarrow H_{n}\right)$ é um subgrupo normal de $H_{n+1}$ e como $\mathcal{H}$ é um $\Delta$-grupo fibrante, então pela Proposição 1.3.7

$$
\mathcal{B} d_{n} \mathcal{H}=d_{i}\left(\bigcap_{j \neq i} \operatorname{Ker}\left(d_{j}: H_{n+1} \rightarrow H_{n}\right)\right) .
$$

Logo, $\mathcal{B} d_{n} \mathcal{H}$ é um subgrupo normal em $H_{n}$.

Lema 4.1.9. Sejam $G, H$ grupos, $\phi: H \rightarrow H$ um endomorfismo e $p: G \rightarrow H$ qualquer homomorfismo sobrejetor tal que $p=\phi \circ p$. Então, $\phi$ é o homomorfismo identidade.

Demonstração. A prova é imediata pois, para todo $h \in H$ existe $g \in G$ tal que $p(g)=h$, e $\operatorname{assim} \phi(h)=\phi \circ p(g)=p(g)=h$.

Seja $\mathcal{H}$ um $\Delta$-grupo tal que o grupo dado pelos bordos de Moore $\mathcal{B} d_{n} \mathcal{H}$ é normal no grupo dado pelos complexos de Moore $\mathcal{N}_{n} \mathcal{H}$. Denotemos a classe de equivalência de $x \in \mathcal{N}_{n} \mathcal{H}$ por:

- $[x] \in \mathcal{N}_{n} \mathcal{H} / \mathcal{B} d_{n} \mathcal{H}$.

- $(x)_{a b} \in\left(\mathcal{N}_{n} \mathcal{H}\right)_{a b}$.

- $([x])_{a b} \in\left(\mathcal{N}_{n} \mathcal{H} / \mathcal{B} d_{n} \mathcal{H}\right)_{a b}$.

- $\left[(x)_{a b}\right]^{I m} \in\left(\mathcal{N}_{n} \mathcal{H}\right)_{a b} / \operatorname{Im}\left(\left(d_{0}\right)_{a b}:\left(\mathcal{N}_{n+1} \mathcal{H}\right)_{a b} \rightarrow\left(\mathcal{N}_{n} \mathcal{H}\right)_{a b}\right)$

Para obter algumas consequências do Teorema de Decomposição (Teorema 4.1.7) precisaremos do seguinte resultado técnico. Com o intuito de evitar diagramas muito grandes, denotaremos $\operatorname{Im}\left(\left(d_{0}\right)_{a b}:\left(\mathcal{N}_{n+1} \mathcal{H}\right)_{a b} \rightarrow\left(\mathcal{N}_{n} \mathcal{H}\right)_{a b}\right)$ por $\operatorname{Im}$.

Proposição 4.1.10. Seja $\mathcal{H}$ um $\Delta$-grupo tal que $\mathcal{B} d_{n} \mathcal{H}$ é um subgrupo normal de $\mathcal{N}_{n} \mathcal{H}$, para algum $n \geq 0$. Então, existe um isomorfismo de grupos abelianos

$$
\phi: \frac{\left(\mathcal{N}_{n} \mathcal{H}\right)_{a b}}{\operatorname{Im}} \rightarrow\left(\mathcal{N}_{n} \mathcal{H} / \mathcal{B} d_{n} \mathcal{H}\right)_{a b}
$$

definido por $\phi\left(\left[(x)_{a b}\right]^{\operatorname{Im}}\right)=([x])_{a b}$. 
Demonstração. Consideremos o seguinte diagrama comutativo de complexos de cadeia de grupos

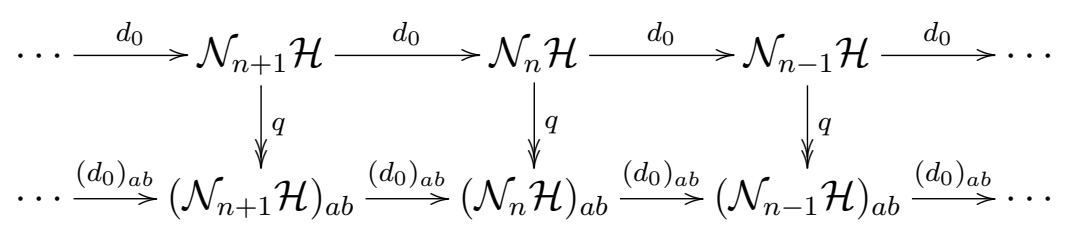

A partir do diagrama (4.9) temos que $q\left(\mathcal{B} d_{n} \mathcal{H}\right)=\operatorname{Im}$. Claramente o seguinte quadrado é comutativo

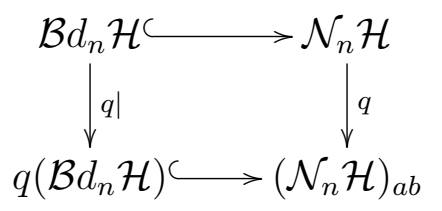

e portanto existe $\psi_{1}$ tal que o seguinte diagrama é comutativo

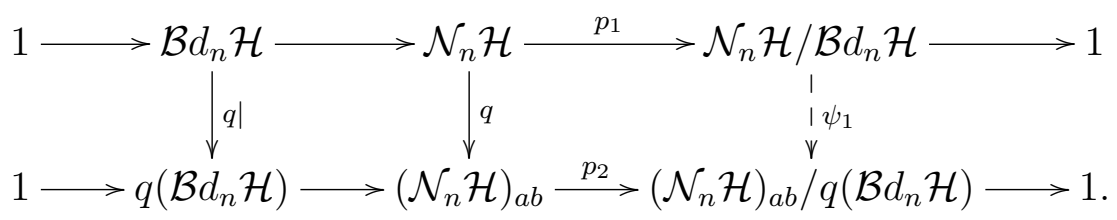

Logo,

$$
\psi_{1} \circ p_{1}=p_{2} \circ q .
$$

Similarmente, existe um homomorfismo $\psi$ tal que o seguinte diagrama é comutativo

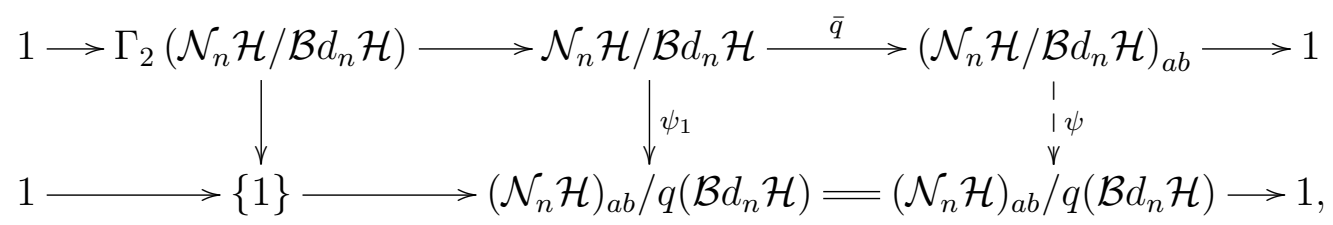

e portanto

$$
\psi_{1}=\psi \circ \bar{q}
$$

Seja $p=\bar{q} \circ p_{1}: \mathcal{N}_{n} \mathcal{H} \rightarrow\left(\mathcal{N}_{n} \mathcal{H} / \mathcal{B} d_{n} \mathcal{H}\right)_{a b}$. Então, pelas igualdades (4.10) e (4.11), temos que

$$
p_{2} \circ q=\psi_{1} \circ p_{1}=\psi \circ \bar{q} \circ p_{1}=\psi \circ p .
$$

Desta forma, provamos que existe $\psi:\left(\mathcal{N}_{n} \mathcal{H} / \mathcal{B} d_{n} \mathcal{H}\right)_{a b} \rightarrow\left(\mathcal{N}_{n} \mathcal{H}\right)_{a b} / \mathrm{Im}$ tal que a composição de homomorfismos

$$
\mathcal{N}_{n} \mathcal{H} \stackrel{q}{\longrightarrow}\left(\mathcal{N}_{n} \mathcal{H}\right)_{a b} \stackrel{p_{2}}{\longrightarrow}\left(\mathcal{N}_{n} \mathcal{H}\right)_{a b} / \operatorname{Im}
$$

se fatora através do grupo quociente $\left(\mathcal{N}_{n} \mathcal{H} / \mathcal{B} d_{n} \mathcal{H}\right)_{a b}$. Notemos que $p=\bar{q} \circ p_{1}$ e $p_{2} \circ q$ são epimorfismos. 
Observemos que existe $\phi_{1}$ tal que o seguinte diagrama é comutativo

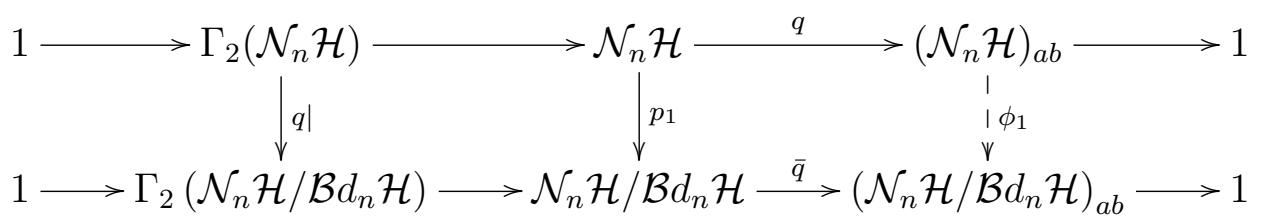

e assim, $p=\bar{q} \circ p_{1}: \mathcal{N}_{n} \mathcal{H} \rightarrow\left(\mathcal{N}_{n} \mathcal{H} / \mathcal{B} d_{n} \mathcal{H}\right)_{a b}$ se fatora através da abelianização de $\mathcal{N}_{n} \mathcal{H}$, $q: \mathcal{N}_{n} \mathcal{H} \rightarrow\left(\mathcal{N}_{n} \mathcal{H}\right)_{a b}$. Notemos que no diagrama (4.13) vale que

$$
\phi_{1}(\operatorname{Im})=\phi_{1}\left(q\left(\mathcal{B} d_{n} \mathcal{H}\right)\right)=p\left(\mathcal{B} d_{n} \mathcal{H}\right)=\{1\}
$$

e como consequência existe $\phi$ tal que o seguinte diagrama é comutativo

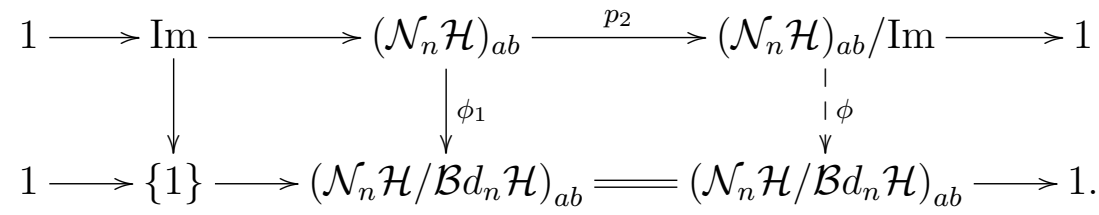

Desta forma, provamos que existe um homomorfismo $\phi:\left(\mathcal{N}_{n} \mathcal{H}\right)_{a b} / \operatorname{Im} \rightarrow\left(\mathcal{N}_{n} \mathcal{H} / \mathcal{B} d_{n} \mathcal{H}\right)_{a b}$ satisfazendo a seguinte igualdade

$$
p=\phi \circ p_{2} \circ q
$$

Portanto, a aplicação quociente $p: \mathcal{N}_{n} \mathcal{H} \rightarrow\left(\mathcal{N}_{n} \mathcal{H} / \mathcal{B} d_{n} \mathcal{H}\right)_{a b}$ se fatora através do grupo $\left(\mathcal{N}_{n} \mathcal{H}\right)_{a b} / \operatorname{Im}$. Pelas igualdades (4.12) e (4.14) temos que $p=\phi \circ \psi \circ p$ e $p_{2} \circ q=\psi \circ \phi \circ p_{2} \circ q$. Como $p$ e $p_{2} \circ q$ são epimorfismos, do Lema 4.1.9, segue que $\phi \circ \psi$ e $\psi \circ \phi$ são homomorfismos identidade definidos nos respectivos grupos. Logo, $\phi:\left(\mathcal{N}_{n} \mathcal{H}\right)_{a b} / \operatorname{Im} \rightarrow\left(\mathcal{N}_{n} \mathcal{H} / \mathcal{B} d_{n} \mathcal{H}\right)_{a b}$ é um isomorfismo, como queríamos provar.

Finalmente, observemos que $p(x)=([x])_{a b}$ e como $p_{2} \circ q$ é sobrejetor e $p=\phi \circ p_{2} \circ q$, então $\phi\left(\left[(x)_{a b}\right]^{I m}\right)=p(x)=([x])_{a b}$.

Observação 4.1.11. Na primeira parte da prova do Teorema 2.11 de [58] existe um isomorfismo similar ao isomorfismo $\phi$ da Proposição 4.1.10, porém, em um caso particular no qual os autores consideraram a sequência de grupos $\mathbb{P}$. A construção dada em [58] nos ajudou a seguir em linhas gerais a ideia da prova da Proposição 4.1.10. Destacamos que nós especificamos de forma explícita a definição da aplicação $\phi$ da Proposição 4.1.10, mas que isso não foi feito em [58] para o caso particular estudado pelos autores.

A seguir, provaremos algumas consequências do Teorema de Decomposição (Teorema 4.1.7), seguindo o roteiro dado em [58] para o caso especial da sequência de grupos $\mathbb{P}$. Consideraremos na continuação a abelianização dos complexos de Moore de um $\Delta$-grupo fibrante $\mathcal{H},(N \mathcal{H})_{a b}=\left\{\left((N \mathcal{H})_{a b}\right)_{n}\right\}_{n \geq 0}$, onde, para cada $n,\left((N \mathcal{H})_{a b}\right)_{n}=\left(\mathcal{N}_{n} \mathcal{H}\right)_{a b}$. Obser- 
vemos que

$$
\mathcal{B} d_{n}(N \mathcal{H})_{a b}=\operatorname{Im}
$$

onde $\operatorname{Im}=\operatorname{Im}\left(\left(d_{0}\right)_{a b}:\left(\mathcal{N}_{n+1} \mathcal{H}\right)_{a b} \rightarrow\left(\mathcal{N}_{n} \mathcal{H}\right)_{a b}\right)$, como fizemos no parágrafo anterior à Proposição 4.1.10.

Corolário 4.1.12. Supondo as hipóteses do Teorema de Decomposição (Teorema 4.1.7) temos que

$$
H_{n}\left((N \mathcal{H})_{a b},\left(d_{0}\right)_{a b}\right) \cong \pi_{n}(\mathcal{H}) \times L_{n}
$$

para qualquer $n \geq 1$, onde $L_{n}$ é um grupo livre de torção.

Demonstração. Como $\mathcal{H}$ é um $\Delta$-grupo fibrante, pela Proposição 1.3.7, temos que $\pi_{n}(\mathcal{H})$ é um grupo abeliano para todo $n \geq 1$. Pelo Teorema de Decomposição (Teorema 4.1.7) segue que

$$
\mathcal{N}_{n} \mathcal{H} / \mathcal{B} d_{n} \mathcal{H} \cong \pi_{n}(\mathcal{H}) \times \mathcal{B} d_{n-1} \mathcal{H}
$$

Portanto, existe uma decomposição de grupos abelianos

$$
\left(\mathcal{N}_{n} \mathcal{H} / \mathcal{B} d_{n} \mathcal{H}\right)_{a b} \cong \pi_{n}(\mathcal{H}) \oplus\left(\mathcal{B} d_{n-1} \mathcal{H}\right)_{a b}
$$

Sejam $q^{\prime}: \mathcal{N}_{n} \mathcal{H} / \mathcal{B} d_{n} \mathcal{H} \rightarrow \pi_{n}(\mathcal{H})$ a projeção da decomposição de grupos dada em (4.16) e

$$
\left(q^{\prime}\right)_{a b}:\left(\mathcal{N}_{n} \mathcal{H} / \mathcal{B} d_{n} \mathcal{H}\right)_{a b} \rightarrow \pi_{n}(\mathcal{H})
$$

a projeção da decomposição dada em (4.17). Tomemos $x \in \mathcal{Z}_{n} \mathcal{H}$. Então, da prova do Teorema de Decomposição (Teorema 4.1.7) segue que $[x] \in \operatorname{Ker}\left(\left.\bar{d}_{0}\right|_{\mathcal{N}_{n} \mathcal{H} / \mathcal{B} d_{n} \mathcal{H}}\right.$ ) e portanto $q^{\prime}([x])=[x]$. Assim, $\left(q^{\prime}\right)_{a b}\left(([x])_{a b}\right)=[x]$. Por definição sabemos que o grupo $H_{n}\left((N \mathcal{H})_{a b}\right)=\mathcal{Z}_{n}(N \mathcal{H})_{a b} / \mathcal{B} d_{n}(N \mathcal{H})_{a b}$ e portanto

$$
H_{n}\left((N \mathcal{H})_{a b}\right)=\frac{\operatorname{Ker}\left(\left(d_{0}\right)_{a b}: \mathcal{N}_{n}(N \mathcal{H})_{a b} \rightarrow \mathcal{N}_{n-1}(N \mathcal{H})_{a b}\right)}{\operatorname{Im}\left(\left(d_{0}\right)_{a b}: \mathcal{N}_{n+1}(N \mathcal{H})_{a b} \rightarrow \mathcal{N}_{n}(N \mathcal{H})_{a b}\right)}
$$

Segue claramente de (4.15), (4.18) e da igualdade $\left((N \mathcal{H})_{a b}\right)_{n}=\left(\mathcal{N}_{n} \mathcal{H}\right)_{a b}$ que existe uma inclusão

$$
i: H_{n}\left((N \mathcal{H})_{a b}\right) \rightarrow\left(\mathcal{N}_{n} \mathcal{H}\right)_{a b} / \mathrm{Im}
$$

Consideremos o homorfismo $q_{*}: H_{n}(N \mathcal{H}) \rightarrow H_{n}\left((N \mathcal{H})_{a b}\right)$, que é induzido pelo homomorfismo $q: \mathcal{N}_{n} \mathcal{H} \rightarrow\left(\mathcal{N}_{n} \mathcal{H}\right)_{n}$ do diagrama comutativo (4.9) e definido, para $x \in \mathcal{Z}_{n} \mathcal{H}$ por

$$
q_{*}([x])=[q(x)]^{\operatorname{Im}}=\left[(x)_{a b}\right]^{\operatorname{Im}} .
$$

Tomemos a seguinte composta:

$$
\pi_{n}(\mathcal{H})=H_{n}(N \mathcal{H}) \stackrel{q_{*}}{\longrightarrow} H_{n}\left((N \mathcal{H})_{a b}\right) \stackrel{i}{\longrightarrow}\left(\mathcal{N}_{n} \mathcal{H}\right)_{a b} / \operatorname{Im} \stackrel{\phi}{\cong}\left(\mathcal{N}_{n} \mathcal{H} / \mathcal{B} d_{n} \mathcal{H}\right)_{a b} \stackrel{\left(q^{\prime}\right)_{a b}}{\longrightarrow} \pi_{n}(\mathcal{H})
$$


onde $\phi$ é o isomorfismo da Proposição 4.1 .10 definido por $\phi\left(\left[(x)_{a b}\right]^{I m}\right)=([x])_{a b}$. Definamos um homomorfismo $g$ como sendo a composição $g:=\phi \circ i$. Como $g \circ q_{*}$ está definido em um grupo quociente de $\mathcal{Z}_{n} \mathcal{H}$, pela equação (4.20), obtemos que $g \circ q_{*}([x])=([x])_{a b}$. Assim,

$$
\left(q^{\prime}\right)_{a b} \circ g \circ q_{*}([x])=\left(q^{\prime}\right)_{a b}\left(([x])_{a b}\right)=[x],
$$

e portanto a composta $\left(q^{\prime}\right)_{a b} \circ g \circ q_{*}$ é o homomorfismo identidade definido em $\pi_{n}(\mathcal{H})$. Logo, a seguinte sequência exata curta de grupos abelianos cinde

$$
1 \longrightarrow \operatorname{Ker}\left(\left(q^{\prime}\right)_{a b} \circ g\right) \longrightarrow H_{n}\left((N \mathcal{H})_{a b}\right) \stackrel{\left(q^{\prime}\right)_{a b} \circ g}{\longrightarrow} \pi_{n}(\mathcal{H}) \longrightarrow 1 .
$$

Como $\phi$ é um isomorfismo e $i$ é injetora, então $g=\phi \circ i$ é injetora. Portanto, pela decomposição (4.17), temos que $L_{n}=g\left(\operatorname{Ker}\left(\left(q^{\prime}\right)_{a b} \circ g\right)\right)$ é isomorfo a um subgrupo do grupo abeliano livre $\left(\mathcal{B} d_{n-1} \mathcal{H}\right)_{a b}$, obtendo o resultado desejado.

Continuemos com as consequências do Teorema de Decomposição (Teorema 4.1.7). Denotemos por $\mathbb{Z} G$ o anel do grupo $G$ sobre os inteiros e por $\mathbb{Z} \mathcal{G}=\left\{\mathbb{Z} G_{n}\right\}_{n \geq 0}$. É bem conhecido que dado um anel $A,(A,+)$ é um grupo abeliano. No seguinte resultado descreveremos os grupos de homologia do grupo simplicial abeliano $\mathbb{Z} \mathcal{G}$.

Corolário 4.1.13. Sob as hipóteses do Teorema de Decomposição (Teorema 4.1.7) temos que

$$
H_{n}(\mathbb{Z}(N \mathcal{H})) \cong \pi_{n}(\mathcal{H}) \times C_{n}
$$

para qualquer $n \geq 1$, e para algum grupo $C_{n}$.

Demonstração. Em geral, dado qualquer grupo $G$, a projeção $q: G \rightarrow(G)_{a b}, g \mapsto(g)_{a b}$, induz um epimorfismo $\theta: \mathbb{Z} G \rightarrow(G)_{a b}$, definido por $\theta: \sum_{i \in I} a_{i} g_{i} \mapsto \sum_{i \in I} a_{i}(g)_{a b}$. Assim, obtemos o quadrado comutativo

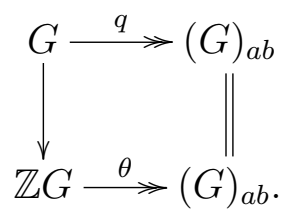

Este diagrama comutativo se estende de forma natural a $\Delta$-grupos e portanto podemos considerar $G=\mathcal{H}$ e $\theta: \mathbb{Z} \mathcal{H} \rightarrow(\mathcal{H})_{a b}$. Seja $\theta_{*}: H_{n}(\mathbb{Z}(N \mathcal{H})) \rightarrow H_{n}\left((N \mathcal{H})_{a b}\right)$ o homomorfismo induzido em homologia. A composição

$$
\pi_{n}(\mathcal{H})=H_{n}(N \mathcal{H}) \longrightarrow H_{n}(\mathbb{Z}(N \mathcal{H})) \stackrel{\theta_{*}}{\longrightarrow} H_{n}\left((N \mathcal{H})_{a b}\right) \stackrel{\left(q^{\prime}\right)_{a b} \circ g}{\longrightarrow} \pi_{n}(\mathcal{H})
$$

é o homomorfismo identidade, onde $\left(q^{\prime}\right)_{a b} \circ g$ é o homomorfismo que apareceu na prova do Corolário 4.1.12. Dessa forma, $H_{n}(\mathbb{Z}(N \mathcal{H})) \cong \pi_{n}(\mathcal{H}) \times C_{n}$, para todo $n \geq 1$, onde o grupo $C_{n}=\operatorname{Ker}\left(\left(q^{\prime}\right)_{a b} \circ g \circ \theta_{*}\right) \subseteq H_{n}(\mathbb{Z}(N \mathcal{H}))$. 
O seguinte resultado é claro e decorre naturalmente do Corolário 4.1.12. Denotemos por $\operatorname{Tor}(G)$ o conjunto dado pelos elementos de torção do grupo $G$.

Corolário 4.1.14. Sob as hipóteses do Teorema de Decomposição (Teorema 4.1.7), se $\pi_{n}(\mathcal{H})$ é um grupo finito, então

$$
\operatorname{Tor}\left(H_{n}\left((N \mathcal{H})_{a b}\right)\right) \cong \pi_{n}(\mathcal{H})
$$

Para finalizar esta seção vamos relacionar o isolador dos bordos de Moore de um $\Delta$-grupo $\mathcal{G}$ com os grupos de homotopia de $\mathcal{G}$. Lembremos que o isolador, ou conjunto de raízes, foi definido no final da Subseção 1.3.2.

Teorema 4.1.15. Suponhamos que $n \geq 1$ e que $\mathcal{G}$ é um $\Delta$-grupo tal que $\mathcal{G}_{n-1}$ é livre de torção e $\pi_{n}(\mathcal{G})$ é um grupo finito. Então, o conjunto de raízes $\sqrt{\left(\mathcal{B} d_{n} \mathcal{G}, \mathcal{G}_{n}\right)}$ é um grupo tal que $\sqrt{\left(\mathcal{B} d_{n} \mathcal{G}, \mathcal{G}_{n}\right)}=\mathcal{Z}_{n} \mathcal{G} e$

$$
\frac{\sqrt{\left(\mathcal{B} d_{n} \mathcal{G}, \mathcal{G}_{n}\right)}}{\mathcal{B} d_{n} \mathcal{G}}=\pi_{n}(\mathcal{G})
$$

Demonstração. Para obtermos as afirmações desejadas basta provarmos a igualdade $\sqrt{\left(\mathcal{B} d_{n} \mathcal{G}, \mathcal{G}_{n}\right)}=\mathcal{Z}_{n} \mathcal{G}$. Seja $x \in \sqrt{\left(\mathcal{B} d_{n} \mathcal{G}, \mathcal{G}_{n}\right)}$. Então, $x \in \mathcal{G}_{n}$ e existe $q \in \mathbb{Z}$, com $q \neq 0$, tal que $x^{q} \in \mathcal{B} d_{n} \mathcal{G}$. Logo, $x^{q}=d_{0} y$ com $y \in \mathcal{N}_{n+1} \mathcal{G}$. Vejamos que $x \in \mathcal{Z}_{n} \mathcal{G}$. Segue pela identidade simplicial (1.11) que

$$
\left(d_{i} x\right)^{q}=d_{i}\left(x^{q}\right)=d_{i} d_{0} y=d_{0} d_{i+1} y=1,
$$

para todo $0 \leq i \leq n$. Provamos assim que para algum $q \in \mathbb{Z}$, com $q \neq 0,\left(d_{i} x\right)^{q}=1$ no grupo livre de torção $\mathcal{G}_{n-1}$. Portanto, $d_{i} x=1$ para todo $0 \leq i \leq n$, isto é, $x \in \mathcal{Z}_{n} \mathcal{G}$. Logo, $\sqrt{\left(\mathcal{B} d_{n} \mathcal{G}, \mathcal{G}_{n}\right)} \subseteq \mathcal{Z}_{n} \mathcal{G}$. Para terminar a demonstração, provemos que vale a outra inclusão. Seja $z \in \mathcal{Z}_{n} \mathcal{G}$, como $\pi_{n}(\mathcal{G})=\mathcal{Z}_{n} \mathcal{G} / \mathcal{B} d_{n} \mathcal{G}$ é um grupo finito, então existe $q \in \mathbb{Z}$, com $q \neq 0$, tal que $z^{q} \in \mathcal{B} d_{n} \mathcal{G}$. Portanto, $\mathcal{Z}_{n} \mathcal{G} \subseteq \sqrt{\left(\mathcal{B} d_{n} \mathcal{G}, \mathcal{G}_{n}\right)}$.

No próximo corolário usaremos a construção de grupo livre de Milnor aplicada na esfera simplicial $S^{r}, F\left[S^{r}\right]$ e o Corolário 1.3.20 que é obtido a partir do Teorema de Milnor (Teorema 1.3.19), ver Subseção 1.3.2.

Corolário 4.1.16. Sejam $n, k \geq 2$. Existe um isomorfismo de grupos

$$
\frac{\sqrt{\left(\mathcal{B} d_{n-1} F\left[S^{k-1}\right], F\left[S^{k-1}\right]_{n-1}\right)}}{\mathcal{B} d_{n-1} F\left[S^{k-1}\right]} \cong \pi_{n}\left(\mathbb{S}^{k}\right),
$$

exceto para $n=k$ e para $n=2 k-1$ quando $k$ é par.

Demonstração. Sejam $n, k \geq 2$. Pela construção do grupo livre de Milnor segue que $F\left[S^{k-1}\right]_{n-1}$ é um grupo livre. Por um Teorema de Moore, ver (1.18) na Observação 
1.3.21, temos que $\pi_{n-1}\left(F\left[S^{k-1}\right]\right) \cong \pi_{n-1}\left(\left|F\left[S^{k-1}\right]\right|\right)$ e assim pelo Corolário 1.3.20 obte$\operatorname{mos} \pi_{n-1}\left(F\left[S^{k-1}\right]\right) \cong \pi_{n}\left(\mathbb{S}^{k}\right)$. Sabemos que $\pi_{n}\left(\mathbb{S}^{k}\right)$ é um grupo finito exceto para $n=k$ e quando $k$ é par e $n=2 k-1$, ver Propriedades 1.2.1 (5). Logo, aplicando o Teorema 4.1.15 ao grupo simplicial (e portanto $\Delta$-grupo) $F\left[S^{k-1}\right]$, temos que

$$
\frac{\sqrt{\left(\mathcal{B} d_{n-1} F\left[S^{k-1}\right], F\left[S^{k-1}\right]_{n-1}\right)}}{\mathcal{B} d_{n-1} F\left[S^{k-1}\right]} \cong \pi_{n}\left(\mathbb{S}^{k}\right)
$$

exceto para $n=k$ e para $n=2 k-1$ quando $k$ é par, como queríamos provar.

\subsection{Construção de grupos simpliciais}

Nessa seção vamos construir três grupos simpliciais específicos. O primeiro deles é formado pela sequência de grupos dada pelo grupo fundamental do disco perfurado, o segundo deles é formado por subgrupos de tranças sobre o disco (ver Subseção 4.2.1) e o terceiro por subgrupos de tranças sobre a esfera (ver Subseção 4.2.2). Para isto, precisaremos do Lema 4.2.1 abaixo. Na Seção 4.3 aplicaremos os resultados obtidos na Seção 4.1 aos grupos simpliciais construídos nesta seção.

Lema 4.2.1. Suponhamos que $\mathcal{H}=\left\{H_{n}\right\}_{n \geq 0}$ é uma sequência de conjuntos (resp. grupos) qualquer, que $\mathcal{G}=\left\{G_{n}\right\}_{n \geq 0}$ é um conjunto (resp. grupo) simplicial com faces $d_{i}^{\mathcal{G}}$ e degenerações $s_{i}^{\mathcal{G}}$ e que $\Phi=\left\{\Phi_{n}\right\}: \mathcal{G} \rightarrow \mathcal{H}$ é uma bijeção (resp. um isomorfismo) entre estes conjuntos (resp. grupos). Então, $\mathcal{H}$ tem uma estrutura de conjunto (resp. grupo) simplicial induzida por $\mathcal{G}$, onde $d_{i}^{\mathcal{H}}=\Phi_{n-1} \circ d_{i}^{\mathcal{G}} \circ \Phi_{n}^{-1}: G_{n} \rightarrow G_{n-1}$ e $s_{i}^{\mathcal{H}}=\Phi_{n+1} \circ s_{i}^{\mathcal{G}} \circ \Phi_{n}^{-1}: G_{n} \rightarrow G_{n+1}$.

Demonstração. Provaremos o resultado apenas para conjuntos simpliciais, pois o caso de grupos simpliciais é completamente análogo. Sejam $\mathcal{G}=\left\{G_{n}\right\}_{n \geq 0}$ um conjunto simplicial com faces $d_{i}^{\mathcal{G}}$ e degenerações $s_{i}^{\mathcal{G}}, \mathcal{H}=\left\{H_{n}\right\}_{n \geq 0}$ é uma sequência de conjuntos qualquer e $\Phi=\left\{\Phi_{n}\right\}: \mathcal{G} \rightarrow \mathcal{H}$ uma bijeção entre estes conjuntos. Como $\Phi_{n}$ é bijeção para todo $n$, podemos tomar o inverso desta aplicação e assim induzir as aplicações faces e degenerações tais que os seguintes quadrados comutam

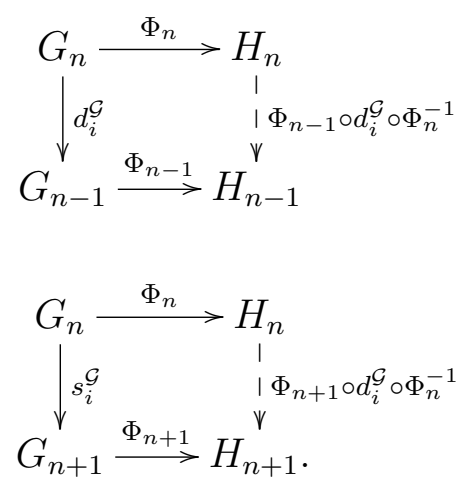

Portanto, dos quadrados comutativos (4.23) e (4.24), podemos definir em $\mathcal{H}$ as aplicações $d_{i}^{\mathcal{H}}=\Phi_{n-1} \circ d_{i}^{\mathcal{G}} \circ \Phi_{n}^{-1}: H_{n} \rightarrow H_{n-1}$ e $s_{i}^{\mathcal{H}}=\Phi_{n+1} \circ s_{i}^{\mathcal{G}} \circ \Phi_{n}^{-1}: H_{n} \rightarrow H_{n+1}$. Claramente, as 
identidades simpliciais

$$
\begin{aligned}
& d_{j}^{\mathcal{H}} d_{i}^{\mathcal{H}}=d_{i}^{\mathcal{H}} d_{j+1}^{\mathcal{H}} \text {, se } i \leq j, \\
& d_{i}^{\mathcal{H}} s_{j}^{\mathcal{H}}= \begin{cases}s_{j-1}^{\mathcal{H}} d_{i}^{\mathcal{H}}, & \text { se } i<j \\
i d^{\mathcal{H}}, & \text { se } i=j, j+1 \\
s_{j}^{\mathcal{H}} d_{i-1}^{\mathcal{H}}, & \text { se } i>j+1,\end{cases} \\
& \text { e } s_{i}^{\mathcal{H}} s_{j}^{\mathcal{H}}=s_{j+1}^{\mathcal{H}} s_{i}^{\mathcal{H}}, \quad \text { se } \quad i \leq j
\end{aligned}
$$

são satisfeitas, pois

$$
\begin{aligned}
d_{j}^{\mathcal{H}} d_{i}^{\mathcal{H}} & =\Phi_{n-2} \circ d_{j}^{\mathcal{G}} \circ \Phi_{n-1}^{-1} \Phi_{n-1} \circ d_{i}^{\mathcal{G}} \circ \Phi_{n}^{-1}=\Phi_{n-2} \circ d_{j}^{\mathcal{G}} \circ d_{i}^{\mathcal{G}} \circ \Phi_{n}^{-1}, \\
d_{i}^{\mathcal{H}} s_{j}^{\mathcal{H}} & =\Phi_{n} \circ d_{i}^{\mathcal{G}} \circ \Phi_{n+1}^{-1} \Phi_{n+1} \circ s_{j}^{\mathcal{G}} \circ \Phi_{n}^{-1}=\Phi_{n} \circ d_{i}^{\mathcal{G}} \circ s_{j}^{\mathcal{G}} \circ \Phi_{n}^{-1}, \\
s_{i}^{\mathcal{H}} s_{j}^{\mathcal{H}} & =\Phi_{n+2} \circ s_{i}^{\mathcal{G}} \circ \Phi_{n+1}^{-1} \Phi_{n+1} \circ s_{j}^{\mathcal{G}} \circ \Phi_{n}^{-1}=\Phi_{n+2} \circ s_{i}^{\mathcal{G}} \circ s_{j}^{\mathcal{G}} \circ \Phi_{n}^{-1},
\end{aligned}
$$

e em $\mathcal{G}$ as identidades simpliciais são válidas.

Seja $Q_{n}$ uma coleção de $n$ pontos no interior de $\mathbb{D}^{2}$, o grupo fundamental $\pi_{1}\left(\mathbb{D}^{2} \backslash Q_{n}\right)$ é um grupo livre de posto $n$, onde $\pi_{1}\left(\mathbb{D}^{2} \backslash Q_{0}\right)=\{1\}$. Para $n \geq 1$ consideremos os geradores $y_{0}, y_{1}, \ldots, y_{n-1}$ de $\pi_{1}\left(\mathbb{D}^{2} \backslash Q_{n}\right)$ como na Figura 4.1.

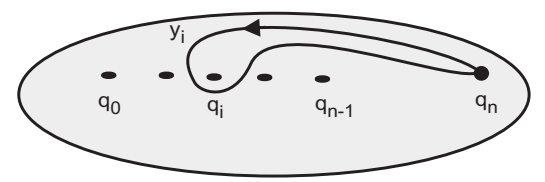

Figura 4.1: Geradores $y_{i}$ de $\pi_{1}\left(\mathbb{D}^{2} \backslash Q_{n}\right)$, com $0 \leq i \leq n-1$, para $n \geq 1$.

Seja a sequência de grupos $\mathcal{K}=\left\{\pi_{1}\left(\mathbb{D}^{2} \backslash Q_{n}\right)\right\}_{n \geq 0}$, para $0 \leq i \leq n$, e consideremos aplicações $d_{i}^{\mathcal{K}}: \pi_{1}\left(\mathbb{D}^{2} \backslash Q_{n}\right) \rightarrow \pi_{1}\left(\mathbb{D}^{2} \backslash Q_{n-1}\right)$ e $s_{i}^{\mathcal{K}}: \pi_{1}\left(\mathbb{D}^{2} \backslash Q_{n}\right) \rightarrow \pi_{1}\left(\mathbb{D}^{2} \backslash Q_{n+1}\right)$, definidas nos elementos geradores $y_{j} \in \pi_{1}\left(\mathbb{D}^{2} \backslash Q_{n}\right)$ por

$$
d_{i}^{\mathcal{K}} y_{j}=\left\{\begin{aligned}
y_{j}, & \text { se } j<i-1, \\
1, & \text { se } j=i-1, \\
y_{j-1}, & \text { se } j \geq i
\end{aligned} \quad \text { e } s_{i}^{\mathcal{K}} y_{j}=\left\{\begin{aligned}
y_{j}, & \text { se } j<i-1 \\
y_{j} y_{j+1}, & \text { se } j=i-1 \\
y_{j+1}, & \text { se } j \geq i
\end{aligned}\right.\right.
$$

para $0 \leq j \leq n-1$, onde $y_{-1}=\left(y_{0} y_{1} \cdots y_{n-1}\right)^{-1}$.

Proposição 4.2.2. A sequência de grupos $\mathcal{K}=\left\{\pi_{1}\left(\mathbb{D}^{2} \backslash Q_{n}\right)\right\}_{n \geq 0}$ é um grupo simplicial com aplicações faces $d_{i}^{\mathcal{K}}$ e degenerações $s_{i}^{\mathcal{K}}$ dadas em $(4.25)$ e $\pi_{n}(\mathcal{K}) \cong \pi_{n+1}\left(\mathbb{S}^{2}\right)$, para todo $n \geq 0$.

Demonstração. Observemos que as aplicações faces e degenerações são as mesmas dadas em [9, Página 295] para o grupo simplicial formado por grupos livres de posto $n$, $F\left[S^{1}\right]=\left\{F\left[S^{1}\right]_{n}\right\}_{n \geq 0}$, onde $F\left[S^{1}\right]$ é a construção de grupo livre de Milnor da esfera simplicial $S^{1}$. Notemos que, como grupos livres, $F\left[S^{1}\right]_{n} \cong \mathcal{K}_{n}=\pi_{1}\left(\mathbb{D}^{2} \backslash Q_{n}\right)$ e portanto 
$F\left[S^{1}\right] \cong \mathcal{K}$ como grupos simpliciais. Por (1.18) na Observação 1.3.21 e pelo Corolário 1.3.20, segue que

$$
\pi_{n}(\mathcal{K}) \cong \pi_{n}\left(F\left[S^{1}\right]\right) \cong \pi_{n+1}\left(\mathbb{S}^{2}\right)
$$

para todo $n \geq 0$.

Corolário 4.2.3. Para o grupo simplicial $\mathcal{K}$ da Proposição 4.2.2, temos que o isolador $\sqrt{\left(\mathcal{B} d_{n} \mathcal{K}, \mathcal{K}_{n}\right)}$ é um grupo e $\sqrt{\left(\mathcal{B} d_{n} \mathcal{K}, \mathcal{K}_{n}\right)}=\mathcal{Z}_{n} \mathcal{K}$. Além disso, para todo $n \geq 3$, existe um isomorfismo de grupos,

$$
\frac{\sqrt{\left(\mathcal{B} d_{n} \mathcal{K}, \mathcal{K}_{n}\right)}}{\mathcal{B} d_{n} \mathcal{K}} \cong \pi_{n+1}\left(\mathbb{S}^{2}\right)
$$

Demonstração. Seja $n \geq 3$. Pela Proposição 4.2.2 sabemos que existe um isomorfismo de grupos $\pi_{n}(\mathcal{K}) \cong \pi_{n+1}\left(\mathbb{S}^{2}\right)$. Além disso, pela construção do grupo simplicial $\mathcal{K}$, temos que $\mathcal{K}_{n}$ é um grupo livre. Como $n \geq 3$, então $\pi_{n+1}\left(\mathbb{S}^{2}\right)$ é um grupo finito, pelas Propriedades 1.2.1 (5). Assim, pelo Teorema 4.1.15 temos que $\sqrt{\left(\mathcal{B} d_{n} \mathcal{K}, \mathcal{K}_{n}\right)}=\mathcal{Z}_{n} \mathcal{K}$ e existe um isomorfismo de grupos

$$
\frac{\sqrt{\left(\mathcal{B} d_{n} \mathcal{K}, \mathcal{K}_{n}\right)}}{\mathcal{B} d_{n} \mathcal{K}} \cong \pi_{n+1}\left(\mathbb{S}^{2}\right)
$$

\subsubsection{O grupo simplicial $\mathcal{K}\left(\mathbb{D}^{2}\right)$}

Consideremos na continuação a numeração usual da teoria de tranças para os subíndices dos homomorfismos e elementos de $P_{n}$. Para $1 \leq i \leq n+1$, denotemos por $d_{i}: P_{n+1} \rightarrow P_{n}$ o homomorfismo que esquece a $i$-ésima corda. Seja $\mathcal{K}\left(\mathbb{D}^{2}\right)=\left\{\left(\mathcal{K}\left(\mathbb{D}^{2}\right)\right)_{n}\right\}_{n \geq 0}$ o conjunto de grupos definidos por

$$
\left(\mathcal{K}\left(\mathbb{D}^{2}\right)\right)_{n}= \begin{cases}\operatorname{Ker}\left(d_{n+1}: P_{n+1} \rightarrow P_{n}\right), & \text { se } n \geq 1 \\ \{1\}, & \text { se } n=0\end{cases}
$$

Aplicando a Proposição 1.2.5 ao disco, com $m=n+1$, temos que a seguinte sequência é exata curta para todo $n \geq 1$

$$
1 \longrightarrow \pi_{1}\left(\mathbb{D}^{2} \backslash Q_{n}\right) \stackrel{i_{n+1}}{\longrightarrow} P_{n+1}\left(\mathbb{D}^{2}\right) \stackrel{d_{n+1}}{\longrightarrow} P_{n}\left(\mathbb{D}^{2}\right) \longrightarrow 1
$$

onde $i_{n+1}: y_{j} \mapsto A_{j+1, n+1}$ para $0 \leq j \leq n-1$. Os geradores $y_{j} \in \pi_{1}\left(\mathbb{D}^{2} \backslash Q_{n}\right)$ são os mesmos dados anteriormente e são representados geometricamente na Figura 4.1. Se $n=0$, considerando $P_{0}=\{1\}$ e $i_{1}=d_{1}$ os homomorfismos triviais, temos que a sequência (4.27) também é exata curta.

Proposição 4.2.4. A sequência de grupos $\mathcal{K}\left(\mathbb{D}^{2}\right)$ tem uma estrutura de grupo simplicial induzida pelo conjunto de isomorfismos $\left\{i_{n+1}\right\}_{n \geq 0}: \mathcal{K} \rightarrow \mathcal{K}\left(\mathbb{D}^{2}\right)$, onde 
$\mathcal{K}=\left\{\pi_{1}\left(\mathbb{D}^{2} \backslash Q_{n}\right)\right\}_{n \geq 0}$ é o grupo simplicial da Proposição 4.2.2. Logo, $\mathcal{K}\left(\mathbb{D}^{2}\right)$ é isomorfo como grupo simplicial a $\mathcal{K}$ e portanto $\pi_{n}\left(\mathcal{K}\left(\mathbb{D}^{2}\right)\right) \cong \pi_{n+1}\left(\mathbb{S}^{2}\right)$, para todo $n \geq 0$.

Demonstração. Do Lema 4.2.1, com $\mathcal{G}=\mathcal{K}, \mathcal{H}=\mathcal{K}\left(\mathbb{D}^{2}\right)$ e $\Phi=\left\{i_{n+1}\right\}_{n \geq 0}$, segue que $\mathcal{K}\left(\mathbb{D}^{2}\right)$ tem uma estrutura de grupo simplicial induzida por $\mathcal{K}$. Como $\mathcal{K}\left(\mathbb{D}^{2}\right) \cong \mathcal{K}$ é um isomorfismo de grupos simpliciais e $\pi_{n}(\mathcal{K}) \cong \pi_{n+1}\left(\mathbb{S}^{2}\right)$, para todo $n \geq 0$, então $\pi_{n}\left(\mathcal{K}\left(\mathbb{D}^{2}\right)\right) \cong \pi_{n+1}\left(\mathbb{S}^{2}\right)$, para todo $n \geq 0$.

Vamos descrever as aplicações simpliciais de $\mathcal{K}\left(\mathbb{D}^{2}\right)$. Chamemos por $d_{i}^{\mathcal{K}}, s_{i}^{\mathcal{K}}$ as aplicações simpliciais de $\mathcal{K}$, ver (4.25). A seguir, vamos chamar apenas por $d_{i}, s_{i}$ as aplicações simpliciais induzidas em $\mathcal{K}\left(\mathbb{D}^{2}\right)$. Pelo Lema 4.2 .1 , para $0 \leq i \leq n$ e para $0 \leq j \leq n-1$, temos que em $\left(\mathcal{K}\left(\mathbb{D}^{2}\right)\right)_{n}$ as aplicações faces são dadas por

$$
d_{i} A_{j+1, n+1}=i_{n} \circ d_{i}^{\mathcal{K}} \circ i_{n+1}^{-1} A_{j+1, n+1}=\left\{\begin{aligned}
A_{j+1, n}, & \text { se } 0 \leq j<i-1 \leq n-1 \\
1, & \text { se } 0 \leq j=i-1 \leq n-1 \\
A_{j, n}, & \text { se } 0 \leq i \leq j \leq n-1
\end{aligned}\right.
$$

onde $A_{0, n}=\left(A_{1, n} A_{2, n} \cdots A_{n-1, n}\right)^{-1} \in P_{n}$, e as aplicações degenerações são dadas por

$s_{i} A_{j+1, n+1}=i_{n+2} \circ s_{i}^{\mathcal{K}} \circ i_{n+1}^{-1} A_{j+1, n+1}=\left\{\begin{aligned} A_{j+1, n+2}, & \text { se } 0 \leq j<i-1 \leq n-1, \\ A_{j+1, n+2} A_{j+2, n+2}, & \text { se } 0 \leq j=i-1 \leq n-1, \\ A_{j+2, n+2}, & \text { se } 0 \leq i \leq j \leq n-1 .\end{aligned}\right.$

Corolário 4.2.5. Para $\mathcal{K}\left(\mathbb{D}^{2}\right)$ temos que $\sqrt{\left(\mathcal{B} d_{n} \mathcal{K}\left(\mathbb{D}^{2}\right),\left(\mathcal{K}\left(\mathbb{D}^{2}\right)\right)_{n}\right)}=\mathcal{Z}_{n} \mathcal{K}\left(\mathbb{D}^{2}\right)$ e existe um isomorfismo de grupos, para todo $n \geq 3$,

$$
\frac{\sqrt{\left(\mathcal{B} d_{n} \mathcal{K}\left(\mathbb{D}^{2}\right),\left(\mathcal{K}\left(\mathbb{D}^{2}\right)\right)_{n}\right)}}{\mathcal{B} d_{n} \mathcal{K}\left(\mathbb{D}^{2}\right)} \cong \pi_{n+1}\left(\mathbb{S}^{2}\right)
$$

Demonstração. Seja $n \geq 3$. Pela Proposição 4.2.4 sabemos que existe um isomorfismo de grupos $\pi_{n}\left(\mathcal{K}\left(\mathbb{D}^{2}\right)\right) \cong \pi_{n+1}\left(\mathbb{S}^{2}\right)$. Além disso, pela construção do grupo simplicial $\mathcal{K}\left(\mathbb{D}^{2}\right)$, temos que $\left(\mathcal{K}\left(\mathbb{D}^{2}\right)\right)_{n}$ é um grupo livre. Como $n \geq 3$, então $\pi_{n+1}\left(\mathbb{S}^{2}\right)$ é um grupo finito e assim, pelo Teorema 4.1 .15 , temos que $\sqrt{\left(\mathcal{B} d_{n} \mathcal{K}\left(\mathbb{D}^{2}\right),\left(\mathcal{K}\left(\mathbb{D}^{2}\right)\right)_{n}\right)}=\mathcal{Z}_{n} \mathcal{K}\left(\mathbb{D}^{2}\right)$ e existe um isomorfismo de grupos

$$
\frac{\sqrt{\left(\mathcal{B} d_{n} \mathcal{K}\left(\mathbb{D}^{2}\right),\left(\mathcal{K}\left(\mathbb{D}^{2}\right)\right)_{n}\right)}}{\mathcal{B} d_{n} \mathcal{K}\left(\mathbb{D}^{2}\right)} \cong \pi_{n+1}\left(\mathbb{S}^{2}\right)
$$

Observação 4.2.6. Consideremos $i=0 \mathrm{em}$ (4.28). Então, para $0 \leq j \leq n-1$, $d_{0} A_{j+1, n+1}=A_{j, n}$, onde $A_{0, n}=\left(A_{1, n} A_{2, n} \cdots A_{n-1, n}\right)^{-1} \in P_{n}$. Portanto, a aplicação face $d_{0}:\left(\mathcal{K}\left(\mathbb{D}^{2}\right)\right)_{n} \rightarrow\left(\mathcal{K}\left(\mathbb{D}^{2}\right)\right)_{n-1}$ é igual a $\partial \mid:\left(\mathcal{K}\left(\mathbb{D}^{2}\right)\right)_{n} \rightarrow\left(\mathcal{K}\left(\mathbb{D}^{2}\right)\right)_{n-1}$. Lembremos que o 
homomorfismo d foi definido na equação (1.4) do Capítulo 1. Sendo assim, podemos estender o homomorfismo face $d_{0}$ a todo o grupo $P_{n+1}$ como sendo $d_{0}=\partial: P_{n+1} \rightarrow P_{n}$. Claramente, para $1 \leq i \leq n$, a restrição do homomorfismo $d_{i}: P_{n+1} \rightarrow P_{n}$ definido em (4.28) corresponde geometricamente a esquecer a i-ésima corda. Além disso, pode-se verificar que as aplicações degenerações definidas em (4.29), para $1 \leq i \leq n$, correspondem a dobrar a i-ésima corda. Para isso, basta comparar estas com as aplicações definidas em [58, Equação (2.5) da página 527].

Logo, como grupo quase-simplicial, $\mathcal{K}\left(\mathbb{D}^{2}\right)$ é igual a $\widetilde{\Omega} \mathbb{P}$. Este último é o grupo quasesimplicial do Exemplo 1.3.23 e que foi estudado em [58]. De forma mais precisa, temos uma igualdade de grupos $\left(\mathcal{K}\left(\mathbb{D}^{2}\right)\right)_{n}=(\widetilde{\Omega} \mathbb{P})_{n}$, para todo $n \geq 0$, e igualdade de homomorfismos $d_{i}^{\mathcal{K}\left(\mathbb{D}^{2}\right)}=d_{i}^{\widetilde{\Omega} \mathbb{P}}$ e $s_{i}^{\mathcal{K}\left(\mathbb{D}^{2}\right)}=s_{i}^{\widetilde{\Omega} \mathbb{P}}$, para todo $i$, exceto para a sequência de degenerações $s_{0}^{\mathcal{K}\left(\mathbb{D}^{2}\right)}:\left(\mathcal{K}\left(\mathbb{D}^{2}\right)\right)_{n} \rightarrow\left(\mathcal{K}\left(\mathbb{D}^{2}\right)\right)_{n+1}$, com $n \geq 0$, pois tanto no Exemplo 1.3.23 como em [58] a aplicação $s_{0}^{\widetilde{\Omega}}$ não foi definida para $\widetilde{\Omega} \mathbb{P}$.

Vimos na Proposição 4.2.4 que $\mathcal{K}\left(\mathbb{D}^{2}\right)$ é um grupo simplicial. Na continuação vamos descrever os complexos, bordos e ciclos de Moore usando os elementos dos grupos de tranças puras sobre o disco.

Proposição 4.2.7. Os complexos, bordos e ciclos de Moore para o grupo simplicial $\mathcal{K}\left(\mathbb{D}^{2}\right)$ são dados, respectivamente, por

1. $\mathcal{N}_{0} \mathcal{K}\left(\mathbb{D}^{2}\right)=\{1\}, \mathcal{N}_{1} \mathcal{K}\left(\mathbb{D}^{2}\right)=P_{2}$, e para $n \geq 2$

$$
\mathcal{N}_{n} \mathcal{K}\left(\mathbb{D}^{2}\right)=\operatorname{Brun}_{n+1}\left(\mathbb{D}^{2}\right)
$$

2. $\mathcal{B} d_{0} \mathcal{K}\left(\mathbb{D}^{2}\right)=\mathcal{B} d_{1} \mathcal{K}\left(\mathbb{D}^{2}\right)=\{1\}$, e para $n \geq 2$

$$
\mathcal{B} d_{n} \mathcal{K}\left(\mathbb{D}^{2}\right)=\left[\left\langle A_{0, n+1}\right\rangle^{P_{n+1}},\left\langle A_{1, n+1}\right\rangle^{P_{n+1}}, \ldots,\left\langle A_{n, n+1}\right\rangle^{P_{n+1}}\right]_{S}
$$

3. $\mathcal{Z}_{0} \mathcal{K}\left(\mathbb{D}^{2}\right)=\{1\}, \mathcal{Z}_{1} \mathcal{K}\left(\mathbb{D}^{2}\right)=P_{2}$, e para $n \geq 2$

$$
\mathcal{Z}_{n} \mathcal{K}\left(\mathbb{D}^{2}\right)=\operatorname{Ker}\left(\partial: P_{n+1} \rightarrow P_{n}\right) \cap \operatorname{Brun}_{n+1}\left(\mathbb{D}^{2}\right)
$$

Demonstração. Como $\left(\mathcal{K}\left(\mathbb{D}^{2}\right)\right)_{0}=\{1\}$, então $\mathcal{N}_{0} \mathcal{K}\left(\mathbb{D}^{2}\right)=\mathcal{B} d_{0} \mathcal{K}\left(\mathbb{D}^{2}\right)=\mathcal{Z}_{0} \mathcal{K}\left(\mathbb{D}^{2}\right)=\{1\}$. Seja $n \geq 1$. Então, $\left(\mathcal{K}\left(\mathbb{D}^{2}\right)\right)_{n}=\operatorname{Ker}\left(d_{n+1}: P_{n+1} \rightarrow P_{n}\right)$ e

$$
\mathcal{N}_{n} \mathcal{K}\left(\mathbb{D}^{2}\right)=\bigcap_{i=1}^{n} \operatorname{Ker}\left(d_{i}:\left(\mathcal{K}\left(\mathbb{D}^{2}\right)\right)_{n} \rightarrow\left(\mathcal{K}\left(\mathbb{D}^{2}\right)\right)_{n-1}\right)=\operatorname{Brun}_{n+1}\left(\mathbb{D}^{2}\right)
$$

onde $\operatorname{Brun}_{2}\left(\mathbb{D}^{2}\right)=P_{2}$.

Com a igualdade dada em (4.30), válida para todo $n \geq 2$, podemos calcular os bordos de Moore para $\mathcal{K}\left(\mathbb{D}^{2}\right)$. Pelo Teorema 1.2.14, sabemos que $\operatorname{Brun}_{3}\left(\mathbb{D}^{2}\right)=\left[\left\langle A_{1,2}\right\rangle^{P_{3}},\left\langle A_{1,3}\right\rangle^{P_{3}}\right]$. 
Logo,

$$
\begin{aligned}
\mathcal{B} d_{1} \mathcal{K}\left(\mathbb{D}^{2}\right) & =d_{0}\left(\mathcal{N}_{2} \mathcal{K}\left(\mathbb{D}^{2}\right)\right)=\partial\left(\left[\left\langle A_{1,2}\right\rangle^{P_{3}},\left\langle A_{1,3}\right\rangle^{P_{3}}\right]\right)=\left[\left\langle A_{0,1}\right\rangle^{P_{2}},\left\langle A_{0,2}\right\rangle^{P_{2}}\right] \\
& =\left[\left\langle A_{1,2}\right\rangle^{P_{2}},\left\langle A_{1,2}\right\rangle^{P_{2}}\right]
\end{aligned}
$$

pois em $P_{2}$ é válido que $A_{0,1}=A_{0,2}=A_{1,2}^{-1}$. Isto é, $\mathcal{B} d_{1} \mathcal{K}\left(\mathbb{D}^{2}\right)$ é um subgrupo comutador do grupo abeliano $P_{2}$ e portanto $\mathcal{B} d_{1} \mathcal{K}\left(\mathbb{D}^{2}\right)=\{1\}$. Provemos agora o resultado para $\mathcal{B} d_{n} \mathcal{K}\left(\mathbb{D}^{2}\right)$, com $n \geq 2$. Para isto, usaremos a descrição dos grupos Brunnianos sobre o disco como grupos comutadores simétricos dada no Teorema 1.2.14. Vimos na Observação 4.2.6 que $d_{0}=\partial \mid$. Assim, usando a definição da aplicação $\partial$, dada na equação (1.4), temos que

$$
\begin{aligned}
\mathcal{B} d_{n} \mathcal{K}\left(\mathbb{D}^{2}\right) & =d_{0}\left(\mathcal{N}_{n+1} \mathcal{K}\left(\mathbb{D}^{2}\right)\right)=\partial\left(\operatorname{Brun}_{n+2}\left(\mathbb{D}^{2}\right)\right) \\
& =\partial\left(\left[\left\langle A_{1, n+2}\right\rangle^{P_{n+2}},\left\langle A_{2, n+2}\right\rangle^{P_{n+2}}, \ldots,\left\langle A_{n+1, n+2}\right\rangle^{P_{n+2}}\right]_{S}\right) \\
& =\left[\left\langle A_{0, n+1}\right\rangle^{P_{n+1}},\left\langle A_{1, n+1}\right\rangle^{P_{n+1}}, \ldots,\left\langle A_{n, n+1}\right\rangle^{P_{n+1}}\right]_{S} .
\end{aligned}
$$

Os ciclos de Moore de $\mathcal{K}\left(\mathbb{D}^{2}\right)$ são dados por

$$
\begin{aligned}
\mathcal{Z}_{n} \mathcal{K}\left(\mathbb{D}^{2}\right) & =\operatorname{Ker}\left(d_{0}: \mathcal{N}_{n} \mathcal{K}\left(\mathbb{D}^{2}\right) \rightarrow \mathcal{N}_{n-1} \mathcal{K}\left(\mathbb{D}^{2}\right)\right) \\
& =\operatorname{Ker}\left(\partial: \operatorname{Brun}_{n+1}\left(\mathbb{D}^{2}\right) \rightarrow \operatorname{Brun}_{n}\left(\mathbb{D}^{2}\right)\right) \\
& =\operatorname{Ker}\left(\partial: P_{n+1}\left(\mathbb{D}^{2}\right) \rightarrow P_{n}\left(\mathbb{D}^{2}\right)\right) \cap \operatorname{Brun}_{n+1}\left(\mathbb{D}^{2}\right) .
\end{aligned}
$$

Como $P_{1}\left(\mathbb{D}^{2}\right)=\{1\}$ e $\operatorname{Brun}_{2}\left(\mathbb{D}^{2}\right)=P_{2}$, então é claro que $\mathcal{Z}_{1} \mathcal{K}\left(\mathbb{D}^{2}\right)=P_{2}$.

No seguinte resultado precisaremos de uma expressão para a conjugação de elementos de $P_{n}$ por elementos de $B_{n}$. Vamos usar a expressão dada no Lema 3.1 de [58] que descreve $\sigma_{k} A_{i, j} \sigma_{k}^{-1}$, para $1 \leq k \leq n-1$ e para $0 \leq i<j \leq n$, onde o elemento $A_{0, j}$ foi aquele definido em (1.5). Dessa forma,

$$
\sigma_{k} A_{i, j} \sigma_{k}^{-1}= \begin{cases}A_{i, j}, & \text { se } k \neq i-1, i, j-1, j, \\ A_{i, k+1}, & \text { se } j=k, \\ A_{i, k+1}^{-1} A_{i, k} A_{i, k+1}, & \text { se } j=k+1 \text { e } i<k, \\ A_{k, k+1}, & \text { se } j=k+1 \text { e } i=k, \\ A_{i+1, j}, & \text { se } i=k<j-1, \\ A_{k+1, j}^{-1} A_{k, j} A_{k+1, j}, & \text { se } i=k+1 .\end{cases}
$$

O caso $j=n$ de (4.31) será bastante usado a seguir. Por este motivo, para efeitos de 
simplificação, vamos destacá-lo abaixo. Para $1 \leq k \leq n-1$ e para $0 \leq i<n$, temos que

$$
\sigma_{k} A_{i, n} \sigma_{k}^{-1}= \begin{cases}A_{i, n}, & \text { se } k \neq i-1, i, n-1, \\ A_{i, n}^{-1} A_{i, n-1} A_{i, n}, & \text { se } k=n-1 \text { e } i<k, \\ A_{n-1, n}, & \text { se } k=i=n-1, \\ A_{i+1, n}, & \text { se } k=i<n-1, \\ A_{i, n}^{-1} A_{i-1, n} A_{i, n}, & \text { se } k=i-1 .\end{cases}
$$

Corolário 4.2.8. Para todo $n \geq 1$, os grupos $\mathcal{N}_{n-1} \mathcal{K}\left(\mathbb{D}^{2}\right)$ e $\mathcal{B} d_{n-1} \mathcal{K}\left(\mathbb{D}^{2}\right)$ são subgrupos normais de $B_{n}$ e $\mathcal{Z}_{n-1} \mathcal{K}\left(\mathbb{D}^{2}\right)$ é um subgrupo normal de $P_{n}$.

Demonstração. Se $n=1,2$ o resultado é claro, ver Proposição 4.2.7. Suponhamos que $n \geq 3$. Pela Proposição 4.2.7, segue que $\mathcal{N}_{n-1} \mathcal{K}\left(\mathbb{D}^{2}\right)=\operatorname{Brun}_{n}\left(\mathbb{D}^{2}\right)$. No Corolário 2.5 de [5] foi provado que $\operatorname{Brun}_{n}\left(\mathbb{D}^{2}\right)$ é normal em $B_{n}$, ver o Corolário 1.3.16. No entanto, podemos dar uma prova diferente aqui. Usando a descrição dos grupos de tranças Brunnianas sobre o disco, como subgrupos comutadores simétricos, dada no Teorema 1.2.14, sabemos que

$$
\operatorname{Brun}_{n}\left(\mathbb{D}^{2}\right)=\left[\left\langle A_{1, n}\right\rangle^{P_{n}},\left\langle A_{2, n}\right\rangle^{P_{n}}, \ldots,\left\langle A_{n-1, n}\right\rangle^{P_{n}}\right]_{S} .
$$

Seja $1 \leq k \leq n-1$. Vejamos a seguir que $\sigma_{k} \operatorname{Brun}_{n}\left(\mathbb{D}^{2}\right) \sigma_{k}^{-1}=\operatorname{Brun}_{n}\left(\mathbb{D}^{2}\right)$, usando a descrição acima dos grupos de tranças Brunnianas e as igualdades dadas em (4.32).

1. Para $k=n-1$,

$$
\begin{aligned}
\sigma_{n-1} \operatorname{Brun}_{n}\left(\mathbb{D}^{2}\right) \sigma_{n-1}^{-1} & =\sigma_{n-1}\left[\left\langle A_{1, n}\right\rangle^{P_{n}},\left\langle A_{2, n}\right\rangle^{P_{n}}, \ldots,\left\langle A_{n-1, n}\right\rangle^{P_{n}}\right]_{S} \sigma_{n-1}^{-1} \\
& =\left[\left\langle A_{1, n-1}\right\rangle^{P_{n}}, \ldots,\left\langle A_{n-2, n-1}\right\rangle^{P_{n}},\left\langle A_{n-1, n}\right\rangle^{P_{n}}\right]_{S}
\end{aligned}
$$

o que é igual a $\operatorname{Brun}_{n}\left(\mathbb{D}^{2}\right)$ pelo Lema 2.1.5.

2. Vamos considerar agora o caso $k \neq n-1$. Nas seguintes igualdades $\left\langle A_{i, n}\right\rangle$ denota $\left\langle A_{i, n}\right\rangle^{P_{n}}$. Assim,

$$
\begin{aligned}
\sigma_{k} \operatorname{Brun}_{n}\left(\mathbb{D}^{2}\right) \sigma_{k}^{-1} & =\sigma_{k}\left[\left\langle A_{1, n}\right\rangle, \ldots,\left\langle A_{k-1, n}\right\rangle,\left\langle A_{k, n}\right\rangle,\left\langle A_{k+1, n}\right\rangle, \ldots,\left\langle A_{n-1, n}\right\rangle\right]_{S} \sigma_{k}^{-1} \\
& =\left[\left\langle A_{1, n}\right\rangle, \ldots,\left\langle A_{k-1, n}\right\rangle,\left\langle A_{k+1, n}\right\rangle,\left\langle A_{k, n}\right\rangle, \ldots,\left\langle A_{n-1, n}\right\rangle\right]_{S} \\
& =\left[\left\langle A_{1, n}\right\rangle, \ldots,\left\langle A_{k-1, n}\right\rangle,\left\langle A_{k, n}\right\rangle,\left\langle A_{k+1, n}\right\rangle, \ldots,\left\langle A_{n-1, n}\right\rangle\right]_{S} \\
& =\operatorname{Brun}_{n}\left(\mathbb{D}^{2}\right) .
\end{aligned}
$$

A penúltima igualdade segue do fato que na expressão do grupo comutador simétrico a ordem dos grupos envolvidos não é importante.

Dessa forma, provamos que o grupo de tranças Brunnianas $\operatorname{Brun}_{n}\left(\mathbb{D}^{2}\right)$ é normal em $B_{n}$, ou equivalentemente, que o grupo $\mathcal{N}_{n-1} \mathcal{K}\left(\mathbb{D}^{2}\right)$ é normal em $B_{n}$. 
Na continuação vamos demonstrar que $\mathcal{B} d_{n-1} \mathcal{K}\left(\mathbb{D}^{2}\right)$ é normal em $B_{n}$. O roteiro desta verificação é similar ao feito acima para $\mathcal{N}_{n-1} \mathcal{K}\left(\mathbb{D}^{2}\right)$. Como $n \geq 3$, da Proposição 4.2.7, segue que

$$
\mathcal{B} d_{n-1} \mathcal{K}\left(\mathbb{D}^{2}\right)=\left[\left\langle A_{0, n}\right\rangle^{P_{n}},\left\langle A_{1, n}\right\rangle^{P_{n}}, \ldots,\left\langle A_{n-1, n}\right\rangle^{P_{n}}\right]_{S} .
$$

1. Vamos supor que $k=n-1$. Pela Proposição 4.2.7 $\mathcal{N}_{n} \mathcal{K}\left(\mathbb{D}^{2}\right)=\operatorname{Brun}_{n+1}\left(\mathbb{D}^{2}\right)$ e pelo Lema 2.1.5 temos que

$$
\operatorname{Brun}_{n+1}\left(\mathbb{D}^{2}\right)=\left[\left\langle A_{1, n}\right\rangle^{P_{n+1}}, \ldots,\left\langle A_{n-1, n}\right\rangle^{P_{n+1}},\left\langle A_{n, n+1}\right\rangle^{P_{n+1}}\right]_{S} .
$$

Logo, usando o fato que o homomorfismo $\partial$ é um epimorfismo, temos as seguintes igualdades

$$
\begin{aligned}
\sigma_{n-1} \mathcal{B} d_{n-1} \mathcal{K}\left(\mathbb{D}^{2}\right) \sigma_{n-1}^{-1} & =\sigma_{n-1}\left[\left\langle A_{0, n}\right\rangle^{P_{n}},\left\langle A_{1, n}\right\rangle^{P_{n}}, \ldots,\left\langle A_{n-1, n}\right\rangle^{P_{n}}\right]_{S} \sigma_{n-1}^{-1} \\
& =\left[\left\langle A_{0, n-1}\right\rangle^{P_{n}}, \ldots,\left\langle A_{n-2, n-1}\right\rangle^{P_{n}},\left\langle A_{n-1, n}\right\rangle^{P_{n}}\right]_{S} \\
& =\partial\left(\left[\left\langle A_{1, n}\right\rangle^{P_{n+1}}, \ldots,\left\langle A_{n-1, n}\right\rangle^{P_{n+1}},\left\langle A_{n, n+1}\right\rangle^{P_{n+1}}\right]_{S}\right) \\
& =\partial\left(\operatorname{Brun}_{n+1}\left(\mathbb{D}^{2}\right)\right)=\partial\left(\mathcal{N}_{n} \mathcal{K}\left(\mathbb{D}^{2}\right)\right)=\mathcal{B} d_{n-1} \mathcal{K}\left(\mathbb{D}^{2}\right) .
\end{aligned}
$$

Na última igualdade usamos a igualdade $d_{0}=\partial: P_{n+1} \rightarrow P_{n}$, ver Observação 4.2.6.

2. Vamos considerar agora o caso $k \neq n-1$. Nas seguintes igualdades $\left\langle A_{i, n}\right\rangle$ denota $\left\langle A_{i, n}\right\rangle^{P_{n}}$. Assim,

$$
\begin{aligned}
\sigma_{k} \mathcal{B} d_{n-1} \mathcal{K}\left(\mathbb{D}^{2}\right) \sigma_{k}^{-1} & =\sigma_{k}\left[\left\langle A_{0, n}\right\rangle^{P_{n}},\left\langle A_{1, n}\right\rangle^{P_{n}}, \ldots,\left\langle A_{n-1, n}\right\rangle^{P_{n}}\right]_{S} \sigma_{k}^{-1} \\
& =\left[\left\langle A_{0, n}\right\rangle, \ldots,\left\langle A_{k-1, n}\right\rangle,\left\langle A_{k+1, n}\right\rangle,\left\langle A_{k, n}\right\rangle, \ldots,\left\langle A_{n-1, n}\right\rangle\right]_{S} \\
& =\left[\left\langle A_{0, n}\right\rangle, \ldots,\left\langle A_{k-1, n}\right\rangle,\left\langle A_{k, n}\right\rangle,\left\langle A_{k+1, n}\right\rangle, \ldots,\left\langle A_{n-1, n}\right\rangle\right]_{S} \\
& =\mathcal{B} d_{n-1} \mathcal{K}\left(\mathbb{D}^{2}\right) .
\end{aligned}
$$

A penúltima igualdade segue do fato que na expressão do grupo comutador simétrico a ordem dos grupos envolvidos não é importante.

Portanto, acabamos de provar que $\mathcal{B} d_{n-1} \mathcal{K}\left(\mathbb{D}^{2}\right)$ é um subgrupo normal de $B_{n}$.

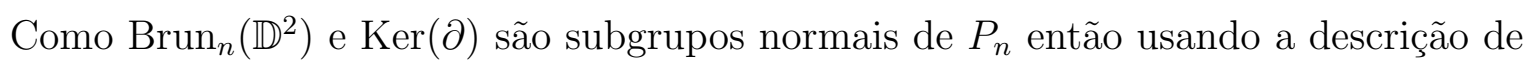
$\mathcal{Z}_{n-1} \mathcal{K}\left(\mathbb{D}^{2}\right)$ dada na Proposição 4.2 .7 é claro que $\mathcal{Z}_{n-1} \mathcal{K}\left(\mathbb{D}^{2}\right)$ é um subgrupo normal de $P_{n}$.

Corolário 4.2.9. Para $n \geq 2$ existe um isomorfismo

$$
\frac{\operatorname{Ker}\left(\partial: P_{n+1} \rightarrow P_{n}\right) \cap \operatorname{Brun}_{n+1}\left(\mathbb{D}^{2}\right)}{\left[\left\langle A_{0, n+1}\right\rangle^{P_{n+1}},\left\langle A_{1, n+1}\right\rangle^{P_{n+1}}, \ldots,\left\langle A_{n, n+1}\right\rangle^{P_{n+1}}\right]_{S}} \cong \pi_{n+1}\left(\mathbb{S}^{2}\right)
$$

Demonstração. Seja $n \geq 2$. Pela Proposição 4.2.7 sabemos que os ciclos de Moore do grupo simplicial $\mathcal{K}\left(\mathbb{D}^{2}\right)$ são dados por $\mathcal{Z}_{n} \mathcal{K}\left(\mathbb{D}^{2}\right)=\operatorname{Ker}\left(\partial: P_{n+1} \rightarrow P_{n}\right) \cap \operatorname{Brun}_{n+1}\left(\mathbb{D}^{2}\right)$ e 
os bordos de Moore por $\mathcal{B} d_{n} \mathcal{K}\left(\mathbb{D}^{2}\right)=\left[\left\langle A_{0, n+1}\right\rangle^{P_{n+1}},\left\langle A_{1, n+1}\right\rangle^{P_{n+1}}, \ldots,\left\langle A_{n, n+1}\right\rangle^{P_{n+1}}\right]_{S}$. Por outro lado, da Proposição 4.2.4, segue que $\frac{\mathcal{Z}_{n} \mathcal{K}\left(\mathbb{D}^{2}\right)}{\mathcal{B} d_{n} \mathcal{K}\left(\mathbb{D}^{2}\right)} \cong \pi_{n+1}\left(\mathbb{S}^{2}\right)$. Logo,

$$
\frac{\operatorname{Ker}\left(\partial: P_{n+1} \rightarrow P_{n}\right) \cap \operatorname{Brun}_{n+1}\left(\mathbb{D}^{2}\right)}{\left[\left\langle A_{0, n+1}\right\rangle^{P_{n+1}},\left\langle A_{1, n+1}\right\rangle^{P_{n+1}}, \ldots,\left\langle A_{n, n+1}\right\rangle^{P_{n+1}}\right]_{S}} \cong \pi_{n+1}\left(\mathbb{S}^{2}\right)
$$

Seja $\chi: B_{n+1} \rightarrow B_{n+1}$ a reflexão espelho definida por $\sigma_{i} \mapsto \sigma_{i}^{-1}$, para $1 \leq i \leq n$. Notemos que o Lema 4.3 de [58] afirma que o grupo $\operatorname{Brun}_{n+1}\left(\mathbb{D}^{2}\right)$ é invariante sob $\chi$. Na seguinte proposição além de fornecer uma prova alternativa também estendemos esse lema, dado que $\mathcal{N}_{n} \mathcal{K}\left(\mathbb{D}^{2}\right)=\operatorname{Brun}_{n+1}\left(\mathbb{D}^{2}\right)$, como vimos na Proposição 4.2.7.

Proposição 4.2.10. As seguintes igualdades são verificadas em $P_{n+1}$ :
1. $\chi\left(\mathcal{N}_{n} \mathcal{K}\left(\mathbb{D}^{2}\right)\right)=\mathcal{N}_{n} \mathcal{K}\left(\mathbb{D}^{2}\right)$.
2. $\chi\left(\mathcal{B} d_{n} \mathcal{K}\left(\mathbb{D}^{2}\right)\right)=\mathcal{B} d_{n} \mathcal{K}\left(\mathbb{D}^{2}\right)$.
3. $\chi\left(\mathcal{Z}_{n} \mathcal{K}\left(\mathbb{D}^{2}\right)\right)=\mathcal{Z}_{n} \mathcal{K}\left(\mathbb{D}^{2}\right)$.

Demonstração. Inicialmente observemos que, para $1 \leq i \leq n$,

$$
\chi\left(A_{i, n+1}\right)=A_{n, n+1}^{-1} \cdots A_{i+1, n+1}^{-1} A_{i, n+1}^{-1} A_{i+1, n+1} \cdots A_{n, n+1}
$$

e também

$$
\chi\left(A_{0, n+1}\right)=A_{0, n+1}^{-1} .
$$

Verifiquemos apenas alguns casos particulares para ilustrar o caso geral:

- $\chi\left(A_{n, n+1}\right)=\chi\left(\sigma_{n}^{2}\right)=\sigma_{n}^{-2}=A_{n, n+1}^{-1}$,

- $\chi\left(A_{n-1, n+1}\right)=\chi\left(\sigma_{n} \sigma_{n-1}^{2} \sigma_{n}^{-1}\right)=\sigma_{n}^{-1} \sigma_{n-1}^{-2} \sigma_{n}=A_{n, n+1}^{-1} A_{n-1, n+1}^{-1} A_{n, n+1}, \mathrm{e}$

- $\chi\left(A_{0, n+1}\right)=\chi\left(\sigma_{n}^{-1} \cdots \sigma_{2}^{-1} \sigma_{1}^{-2} \sigma_{2}^{-1} \cdots \sigma_{n}^{-1}\right)=\sigma_{n} \cdots \sigma_{2} \sigma_{1}^{2} \sigma_{2} \cdots \sigma_{n}=A_{0, n+1}^{-1}$.

Além disso, como $\chi$ é um automorfismo, da igualdade (4.33), segue que

$$
\chi\left(\left\langle A_{i, n+1}\right\rangle^{P_{n+1}}\right)=\left\langle A_{i, n+1}^{-1}\right\rangle^{P_{n+1}}=\left\langle A_{i, n+1}\right\rangle^{P_{n+1}},
$$

para todo $0 \leq i \leq n$.

1. Verifiquemos a primeira igualdade desta proposição. Usaremos a equação (4.34).

$$
\begin{aligned}
\chi\left(\mathcal{N}_{n} \mathcal{K}\left(\mathbb{D}^{2}\right)\right) & =\chi\left(\operatorname{Brun}_{n+1}\left(\mathbb{D}^{2}\right)\right)=\chi\left(\left[\left\langle A_{1, n+1}\right\rangle^{P_{n+1}}, \ldots,\left\langle A_{n, n+1}\right\rangle^{P_{n+1}}\right]_{S}\right) \\
& =\left[\left\langle A_{1, n+1}\right\rangle^{P_{n+1}}, \ldots,\left\langle A_{n, n+1}\right\rangle^{P_{n+1}}\right]_{S}=\operatorname{Brun}_{n+1}\left(\mathbb{D}^{2}\right) .
\end{aligned}
$$


2. Usando a descrição do grupo $\mathcal{B} d_{n} \mathcal{K}\left(\mathbb{D}^{2}\right)$ como subgrupo comutador simétrico dada na Proposição 4.2 .7 e a igualdade dada em (4.34), temos que

$$
\begin{aligned}
\chi\left(\mathcal{B} d_{n} \mathcal{K}\left(\mathbb{D}^{2}\right)\right) & =\chi\left(\left[\left\langle A_{0, n+1}\right\rangle^{P_{n+1}},\left\langle A_{1, n+1}\right\rangle^{P_{n+1}}, \ldots,\left\langle A_{n, n+1}\right\rangle^{P_{n+1}}\right]_{S}\right) \\
& =\left[\left\langle A_{0, n+1}^{-1}\right\rangle^{P_{n+1}},\left\langle A_{1, n+1}^{-1}\right\rangle^{P_{n+1}}, \ldots,\left\langle A_{n, n+1}^{-1}\right\rangle^{P_{n+1}}\right]_{S} \\
& =\mathcal{B} d_{n} \mathcal{K}\left(\mathbb{D}^{2}\right) .
\end{aligned}
$$

3. A igualdade $d_{i} \chi=\chi d_{i}$ é válida para todo $1 \leq i \leq n$, em vista da Proposição 2.1 de [58], e $\partial \chi=\chi \partial$ pelo item 4 do Lema 2.3 de [58]. Seja $\beta \in \chi\left(\mathcal{Z}_{n} \mathcal{K}\left(\mathbb{D}^{2}\right)\right)$. Como

$$
\chi\left(\mathcal{Z}_{n} \mathcal{K}\left(\mathbb{D}^{2}\right)\right)=\chi\left(\operatorname{Ker}\left(\partial: P_{n+1} \rightarrow P_{n}\right) \cap \operatorname{Brun}_{n+1}\left(\mathbb{D}^{2}\right)\right),
$$

então existe $\beta_{1} \in \operatorname{Ker}(\partial) \cap \operatorname{Brun}_{n+1}\left(\mathbb{D}^{2}\right)$ tal que $\chi\left(\beta_{1}\right)=\beta$. Assim, para $1 \leq j \leq n+1$,

$$
d_{j}(\beta)=d_{j} \chi\left(\beta_{1}\right)=\chi d_{j}\left(\beta_{1}\right)=\chi(1)=1 .
$$

Portanto, $\beta \in \operatorname{Brun}_{n+1}\left(\mathbb{D}^{2}\right)$. Além disso,

$$
\partial(\beta)=\partial \chi\left(\beta_{1}\right)=\chi \partial\left(\beta_{1}\right)=\chi(1)=1
$$

Com isto provamos que $\chi\left(\mathcal{Z}_{n} \mathcal{K}\left(\mathbb{D}^{2}\right)\right) \subseteq \mathcal{Z}_{n} \mathcal{K}\left(\mathbb{D}^{2}\right)$. Para demonstrar a outra inclusão tomemos $\gamma \in \operatorname{Brun}_{n+1}\left(\mathbb{D}^{2}\right) \cap \operatorname{Ker}(\partial)$. Então, pelo item 1 desta proposição, existe $\gamma_{1} \in \operatorname{Brun}_{n+1}\left(\mathbb{D}^{2}\right)$ tal que $\chi\left(\gamma_{1}\right)=\gamma$. Como $\partial(\gamma)=1$, então $\partial \chi\left(\gamma_{1}\right)=$ $\chi\left(\partial\left(\gamma_{1}\right)\right)=1$ e como $\chi$ é um automorfismo podemos concluir que $\partial\left(\gamma_{1}\right)=1$. Portanto, existe $\gamma_{1} \in \operatorname{Ker}(\partial) \cap \operatorname{Brun}_{n+1}\left(\mathbb{D}^{2}\right)$ tal que $\chi\left(\gamma_{1}\right)=\gamma$ e assim provamos que $\mathcal{Z}_{n} \mathcal{K}\left(\mathbb{D}^{2}\right) \subseteq \chi\left(\mathcal{Z}_{n} \mathcal{K}\left(\mathbb{D}^{2}\right)\right)$. Logo, $\chi\left(\mathcal{Z}_{n} \mathcal{K}\left(\mathbb{D}^{2}\right)\right)=\mathcal{Z}_{n} \mathcal{K}\left(\mathbb{D}^{2}\right)$.

Na Seção 4.3 vamos aplicar os resultados da Seção 4.1 com $\mathcal{H}=\mathcal{K}\left(\mathbb{D}^{2}\right)$. Para isto vamos destacar algumas propriedades do grupo de tranças $P_{n+1}$ e do grupo simplicial $\mathcal{K}\left(\mathbb{D}^{2}\right)$.

\section{Propriedades 4.2.11.}

1. Sejam $n \geq 1$ e $A_{i, j} \in P_{n+1}$. Então, $A_{i+1, j+1} \in d_{i}^{-1}\left(A_{i, j}\right) \cap \operatorname{Ker}\left(d_{i+1}: P_{n+2} \rightarrow P_{n+1}\right)$, para $1 \leq i<j \leq n+1$.

2. Notemos que, para $n \geq 1, A_{n+1}=Z\left(P_{n+1}\right) / \mathcal{B} d_{n} \mathcal{K}\left(\mathbb{D}^{2}\right) \cong \mathbb{Z}$ é gerado pelo "full twist" $\Delta_{n+1}$ e que $\left(\mathcal{K}\left(\mathbb{D}^{2}\right)\right)_{1} / \mathcal{B} d_{1} \mathcal{K}\left(\mathbb{D}^{2}\right)=\mathbb{Z}$ é gerado pelo "full twist" $\Delta_{2}$. Além disso, para $n \geq 2, \Delta_{n+1}$ não pertence ao grupo livre $\left(\mathcal{K}\left(\mathbb{D}^{2}\right)\right)_{n}$. Seja $x \in P_{n+1}$ tal que

$$
\bar{x} \in \frac{\left(\mathcal{K}\left(\mathbb{D}^{2}\right)\right)_{n}}{\mathcal{B} d_{n} \mathcal{K}\left(\mathbb{D}^{2}\right)} \bigcap \frac{Z\left(P_{n+1}\right)}{\mathcal{B} d_{n} \mathcal{K}\left(\mathbb{D}^{2}\right)}
$$


Então, $\bar{x}=\bar{y}$, com $y \in\left(\mathcal{K}\left(\mathbb{D}^{2}\right)\right)_{n}$ e $\bar{x}=\overline{\Delta_{n+1}^{r}}$, para algum $r \in \mathbb{Z}$. Como $y^{-1} \Delta_{n+1}^{r} \in \mathcal{B} d_{n} \mathcal{K}\left(\mathbb{D}^{2}\right)$ e $\mathcal{B} d_{n} \mathcal{K}\left(\mathbb{D}^{2}\right)=d_{0}\left(\mathcal{N}_{n+1} \mathcal{K}\left(\mathbb{D}^{2}\right)\right)=\partial\left(\right.$ Brun $\left._{n+2}\left(\mathbb{D}^{2}\right)\right)$, então $y^{-1} \Delta_{n+1}^{r}=\partial(z)$, com $z \in$ Brun $_{n+2}\left(\mathbb{D}^{2}\right)$. Portanto,

$$
d_{n+1}\left(y^{-1} \Delta_{n+1}^{r}\right)=\Delta_{n}^{r}
$$

$e$

$$
d_{n+1} \partial(z)=\partial d_{n+2}(z)=\partial(1)=1 .
$$

Como as equações dadas em (4.35) e (4.36) são iguais e $Z\left(P_{n}\right)=\mathbb{Z}=\left\langle\Delta_{n}\right\rangle$, então $\Delta_{n}^{r}=1$ implica que $r=0$. Desta forma provamos que $\bar{x}=\overline{\Delta_{n+1}^{r}}=1$.

Logo,

$$
\frac{\left(\mathcal{K}\left(\mathbb{D}^{2}\right)\right)_{n}}{\mathcal{B} d_{n} \mathcal{K}\left(\mathbb{D}^{2}\right)} \bigcap \frac{Z\left(P_{n+1}\right)}{\mathcal{B} d_{n} \mathcal{K}\left(\mathbb{D}^{2}\right)}=\{1\}
$$

3. Sabemos que $d_{n+1}: P_{n+1} \rightarrow P_{n}$ é o epimorfismo que corresponde a esquecer a $(n+1)$-ésima corda de tranças em $P_{n+1}$. O centro de $P_{n+1}$ é isomorfo a $\mathbb{Z}$ e gerado pelo "full twist" $\Delta_{n+1}$, ainda mais, $d_{n+1}\left(\Delta_{n+1}\right)=\Delta_{n}$. Logo $d_{n+1}: Z\left(P_{n+1}\right) \rightarrow Z\left(P_{n}\right)$ é um isomorfismo para todo $n \geq 2$ e um epimorfismo para $n=1$.

\subsubsection{O grupo simplicial $\mathcal{K}\left(\mathbb{S}^{2}\right)$}

Vamos agora construir um grupo simplicial no qual a maior parte dos grupos são formados por subgrupos de tranças sobre a esfera, usaremos também grupos do grupo simplicial $\mathcal{K}$ (ver Proposição 4.2.2).

Consideremos a numeração usual para os subíndices dos homomorfismos e elementos de $P_{n}\left(\mathbb{S}^{2}\right)$. Para $1 \leq i \leq n+1$, seja $d_{i}: P_{n+1}\left(\mathbb{S}^{2}\right) \rightarrow P_{n}\left(\mathbb{S}^{2}\right)$ o homomorfismo que esquece a $i$-ésima corda. Seja $\mathcal{K}\left(\mathbb{S}^{2}\right)=\left\{\left(\mathcal{K}\left(\mathbb{S}^{2}\right)\right)_{n}\right\}_{n \geq 0}$ o conjunto de grupos definido por

$$
\left(\mathcal{K}\left(\mathbb{S}^{2}\right)\right)_{n}= \begin{cases}\operatorname{Ker}\left(d_{n+2}: P_{n+2}\left(\mathbb{S}^{2}\right) \rightarrow P_{n+1}\left(\mathbb{S}^{2}\right)\right), & \text { se } n \geq 2 \\ \mathcal{K}_{n}, & \text { se } n=0,1\end{cases}
$$

Pela fibração de Fadell-Neuwirth para a esfera temos que a seguinte sequência é exata curta, para todo $n \geq 2$,

$$
1 \longrightarrow \pi_{1}\left(\mathbb{S}^{2} \backslash Q_{n+1}\right) \stackrel{i_{n+2}}{\longrightarrow} P_{n+2}\left(\mathbb{S}^{2}\right) \stackrel{d_{n+2}}{\longrightarrow} P_{n+1}\left(\mathbb{S}^{2}\right) \longrightarrow 1
$$

onde $\pi_{1}\left(\mathbb{S}^{2} \backslash Q_{n+1}\right)=\left\langle z_{0}, z_{1}, \ldots, z_{n} \mid z_{0} z_{1} \cdots z_{n}=1\right\rangle$ e o homomorfismo $i_{n+2}$ é definido por

$$
i_{n+2}: z_{j} \mapsto A_{j+1, n+2}, \text { para } 0 \leq j \leq n
$$

Notemos que, para todo $n \geq 2, i_{n+2}\left(\pi_{1}\left(\mathbb{S}^{2} \backslash Q_{n+1}\right)\right)=\left(\mathcal{K}\left(\mathbb{S}^{2}\right)\right)_{n}$, pois a sequência (4.39) é 
exata curta e assim

$$
i_{n+2}: \pi_{1}\left(\mathbb{S}^{2} \backslash Q_{n+1}\right) \rightarrow\left(\mathcal{K}\left(\mathbb{S}^{2}\right)\right)_{n}
$$

é um isomorfismo, para todo $n \geq 2$.

Para $n=0,1$, por convenção, diremos que $i_{2}: \mathcal{K}_{0} \rightarrow \pi_{1}\left(\mathbb{S}^{2} \backslash Q_{1}\right), i_{3}: \mathcal{K}_{1} \rightarrow \pi_{1}\left(\mathbb{S}^{2} \backslash Q_{2}\right)$ são homomorfismos identidade, dado que $\pi_{1}\left(\mathbb{S}^{2} \backslash Q_{n+1}\right) \cong \pi_{1}\left(\mathbb{D}^{2} \backslash Q_{n}\right)=\mathcal{K}_{n}$. O grupo simplicial $\mathcal{K}=\left\{\mathcal{K}_{n}\right\}_{n \geq 0}$ foi construído antes da Proposição 4.2.2.

Seguindo a construção feita em [9, Página 296], seja $\hat{F}_{n+1}$ o grupo quociente obtido a partir do grupo livre $F\left(z_{0}, z_{1}, \ldots, z_{n}\right)$ restrito à relação $z_{0} z_{1} \cdots z_{n}=1$ e $\hat{z}_{j}$ a imagem de $z_{j}$ em $\hat{F}_{n+1}$. Claramente

$$
\hat{F}_{n+1} \cong F\left(\hat{z}_{0}, \hat{z}_{1}, \ldots, \hat{z}_{n-1}\right)
$$

é um grupo livre de posto $n$ e é um grupo simplicial. As faces $d_{i}$ e degenerações $s_{i}$ sobre $\hat{F}=\left\{\hat{F}_{n+1}\right\}_{n \geq 0}$ são definidas como segue

$$
d_{i} \hat{z}_{j}=\left\{\begin{aligned}
\hat{z}_{j}, & \text { se } j<i, \\
1, & \text { se } j=i, \\
\hat{z}_{j-1}, & \text { se } j>i,
\end{aligned} \quad \text { e } s_{i} \hat{z}_{j}=\left\{\begin{aligned}
\hat{z}_{j}, & \text { se } j<i, \\
\hat{z}_{i} \hat{z}_{i+1}, & \text { se } j=i, \\
\hat{z}_{j+1}, & \text { se } j>i
\end{aligned}\right.\right.
$$

Consideremos a sequência de isomorfismos naturais $j_{n+2}: \hat{F}_{n+1} \rightarrow \pi_{1}\left(\mathbb{S}^{2} \backslash Q_{n+1}\right)$, para $n \geq 0, j_{2}: \hat{F}_{0} \rightarrow \mathcal{K}_{0}$ e $j_{3}: \hat{F}_{1} \rightarrow \mathcal{K}_{1}$. Para todo $n \geq 0$ definamos o seguinte isomorfismo $\Phi_{n}=i_{n+2} \circ j_{n+2}(\operatorname{ver}(4.40))$.

Proposição 4.2.12. A sequência de grupos $\mathcal{K}\left(\mathbb{S}^{2}\right)$ tem uma estrutura de grupo simplicial induzida pelo conjunto de isomorfismos $\left\{\Phi_{n}\right\}_{n \geq 0}: \hat{F} \rightarrow \mathcal{K}\left(\mathbb{S}^{2}\right)$. Logo, $\mathcal{K}\left(\mathbb{S}^{2}\right)$ é isomorfo, como grupo simplicial, a $\hat{F}$ e portanto $\pi_{n}\left(\mathcal{K}\left(\mathbb{S}^{2}\right)\right) \cong \pi_{n+1}\left(\mathbb{S}^{2}\right)$, para todo $n \geq 0$.

Demonstração. A prova deste resultado é análoga a dada para a Proposição 4.2.4. O resultado segue do Lema 4.2.1 com $\mathcal{G}=\hat{F}, \mathcal{H}=\mathcal{K}\left(\mathbb{S}^{2}\right)$ e $\Phi=\left\{\Phi_{n}\right\}_{n \geq 0}$. Pela Proposição 6.1.2 de [9] temos que $\hat{F} \cong F\left[S^{1}\right]$ como grupos simpliciais e portanto, para todo $n \geq 0$, $\pi_{n}\left(\mathcal{K}\left(\mathbb{S}^{2}\right)\right) \cong \pi_{n}(\hat{F}) \cong \pi_{n}\left(F\left[S^{1}\right]\right) \cong \pi_{n+1}\left(\mathbb{S}^{2}\right)$.

Sejam $n \geq 2$ e $0 \leq i \leq n$. Pelo Lema 4.2.1 e pelas aplicações dadas em (4.41), as aplicações faces e degenerações para $\mathcal{K}\left(\mathbb{S}^{2}\right)$ são dadas por:

$$
\begin{aligned}
& d_{i}^{\mathcal{K}\left(\mathbb{S}^{2}\right)} A_{j+1, n+2}=\left\{\begin{aligned}
A_{j+1, n+1}, & \text { se } 0 \leq j<i \leq n \\
1, & \text { se } 0 \leq j=i \leq n \\
A_{j, n+1}, & \text { se } 0 \leq i<j \leq n
\end{aligned}\right. \\
& s_{i}^{\mathcal{K}\left(\mathbb{S}^{2}\right)} A_{j+1, n+2}=\left\{\begin{aligned}
A_{j+1, n+3}, & \text { se } 0 \leq j<i \leq n, \\
A_{j+1, n+3} A_{j+2, n+3}, & \text { se } 0 \leq j=i \leq n, \\
A_{j+2, n+3}, & \text { se } 0 \leq i<j \leq n .
\end{aligned}\right.
\end{aligned}
$$


Observação 4.2.13. Notemos que as aplicações faces definidas em (4.42) para $n \geq 2$ coincidem com as aplicações usuais de esquecimento de cordas $d_{i}: P_{n+2}\left(\mathbb{S}^{2}\right) \rightarrow P_{n+1}\left(\mathbb{S}^{2}\right)$, fazendo um ajuste no subíndice: $d_{i}^{\mathcal{K}\left(\mathbb{S}^{2}\right)}=d_{i+1}$. As aplicações faces $d_{i}^{\mathcal{K}\left(\mathbb{S}^{2}\right)}$ foram usadas em [9] para dar uma estrutura de $\Delta$-grupo para $\mathcal{P}\left(\mathbb{S}^{2}\right)$.

Vamos descrever, no seguinte resultado, os complexos, bordos e ciclos de Moore para o grupo simplicial $\mathcal{K}\left(\mathbb{S}^{2}\right)$ usando grupos já conhecidos.

Proposição 4.2.14. Os complexos, bordos e ciclos de Moore para o grupo simplicial $\mathcal{K}\left(\mathbb{S}^{2}\right)$ são dados, respectivamente, por

1. $\mathcal{N}_{0} \mathcal{K}\left(\mathbb{S}^{2}\right)=\{1\}, \mathcal{N}_{1} \mathcal{K}\left(\mathbb{S}^{2}\right) \cong \mathbb{Z}$ e para $n \geq 2$

$$
\mathcal{N}_{n} \mathcal{K}\left(\mathbb{S}^{2}\right)=\mathcal{Q}^{1} \operatorname{Brun}_{n+2}\left(\mathbb{S}^{2}\right)
$$

2. $\mathcal{B} d_{0} \mathcal{K}\left(\mathbb{S}^{2}\right)=\mathcal{B} d_{1} \mathcal{K}\left(\mathbb{D}^{2}\right)=\{1\}$ e para $n \geq 2$

$$
\mathcal{B} d_{n} \mathcal{K}\left(\mathbb{S}^{2}\right)=\left[\left\langle A_{1, n+2}\right\rangle^{P_{n+2}\left(\mathbb{S}^{2}\right)},\left\langle A_{2, n+2}\right\rangle^{P_{n+2}\left(\mathbb{S}^{2}\right)}, \ldots,\left\langle A_{n+1, n+2}\right\rangle^{P_{n+2}\left(\mathbb{S}^{2}\right)}\right]_{S}
$$

3. $\mathcal{Z}_{0} \mathcal{K}\left(\mathbb{S}^{2}\right)=\{1\}, \mathcal{Z}_{1} \mathcal{K}\left(\mathbb{S}^{2}\right) \cong \mathbb{Z}$ e para $n \geq 2$

$$
\mathcal{Z}_{n} \mathcal{K}\left(\mathbb{S}^{2}\right)=\operatorname{Brun}_{n+2}\left(\mathbb{S}^{2}\right)
$$

Demonstração. A ideia usada aqui é similar com a dada na prova da Proposição 4.2.7. Como $\left(\mathcal{K}\left(\mathbb{S}^{2}\right)\right)_{0}=\{1\}$, então $\mathcal{N}_{0} \mathcal{K}\left(\mathbb{S}^{2}\right)=\mathcal{B} d_{0} \mathcal{K}\left(\mathbb{S}^{2}\right)=\mathcal{Z} \mathcal{Z}_{0} \mathcal{K}\left(\mathbb{S}^{2}\right)=\{1\}$ e $\mathcal{N}_{1} \mathcal{K}\left(\mathbb{S}^{2}\right)=\mathcal{Z}_{1} \mathcal{K}\left(\mathbb{S}^{2}\right)=\left(\mathcal{K}\left(\mathbb{S}^{2}\right)\right)_{1} \cong \mathbb{Z}$

Seja $n \geq 2$. Então, $\left(\mathcal{K}\left(\mathbb{S}^{2}\right)\right)_{n}=\operatorname{Ker}\left(d_{n+2}: P_{n+2}\left(\mathbb{S}^{2}\right) \rightarrow P_{n+1}\left(\mathbb{S}^{2}\right)\right)$ e os complexos de Moore do grupo simplicial $\mathcal{K}\left(\mathbb{S}^{2}\right)$ são dados por

$$
\begin{aligned}
\mathcal{N}_{n} \mathcal{K}\left(\mathbb{S}^{2}\right) & =\bigcap_{i=1}^{n} \operatorname{Ker}\left(d_{i}^{\mathcal{K}\left(\mathbb{S}^{2}\right)}:\left(\mathcal{K}\left(\mathbb{S}^{2}\right)\right)_{n} \rightarrow\left(\mathcal{K}\left(\mathbb{S}^{2}\right)\right)_{n-1}\right) \\
& =\bigcap_{i=1}^{n} \operatorname{Ker}\left(d_{i+1}: \operatorname{Ker}\left(d_{n+2}\right) \rightarrow \operatorname{Ker}\left(d_{n+1}\right)\right. \\
& =\bigcap_{i=1}^{n+1} \operatorname{Ker}\left(d_{i+1}: P_{n+2}\left(\mathbb{S}^{2}\right) \rightarrow P_{n+1}\left(\mathbb{S}^{2}\right)\right. \\
& =\mathcal{Q}^{1} \operatorname{Brun}_{n+2}\left(\mathbb{S}^{2}\right) .
\end{aligned}
$$

Vejamos que os ciclos de Moore de $\mathcal{K}\left(\mathbb{S}^{2}\right)$ são dados por

$$
\begin{aligned}
\mathcal{Z}_{n} \mathcal{K}\left(\mathbb{S}^{2}\right) & =\operatorname{Ker}\left(d_{0}^{\mathcal{K}\left(\mathbb{S}^{2}\right)}: \mathcal{N}_{n} \mathcal{K}\left(\mathbb{S}^{2}\right) \rightarrow \mathcal{N}_{n-1} \mathcal{K}\left(\mathbb{S}^{2}\right)\right) \\
& =\operatorname{Ker}\left(d_{1}: \mathcal{Q}^{1} \operatorname{Brun}_{n+2}\left(\mathbb{S}^{2}\right) \rightarrow \mathcal{Q}^{1} \operatorname{Brun}_{n+1}\left(\mathbb{S}^{2}\right)\right) \\
& =\operatorname{Brun}_{n+2}\left(\mathbb{S}^{2}\right)
\end{aligned}
$$

A última igualdade decorre da Proposição 2.2.3. 
Para finalizar, vamos calcular os bordos de Moore para $\mathcal{K}\left(\mathbb{S}^{2}\right)$. Pela Proposição 2.2.10 sabemos que vale a igualdade de grupos $\mathcal{Q}^{1} \operatorname{Brun}_{4}\left(\mathbb{S}^{2}\right)=\operatorname{Brun}_{4}\left(\mathbb{S}^{2}\right)$. Como

$$
d_{0}^{\mathcal{K}\left(\mathbb{S}^{2}\right)}=d_{1}: \operatorname{Ker}\left(d_{4}: P_{4}\left(\mathbb{S}^{2}\right) \rightarrow P_{3}\left(\mathbb{S}^{2}\right)\right) \rightarrow P_{3}\left(\mathbb{S}^{2}\right),
$$

então o bordo de Moore com $n=1$ é dado por

$$
\begin{aligned}
\mathcal{B} d_{1} \mathcal{K}\left(\mathbb{S}^{2}\right) & =d_{0}^{\mathcal{K}\left(\mathbb{S}^{2}\right)}\left(\mathcal{N}_{2} \mathcal{K}\left(\mathbb{S}^{2}\right)\right)=d_{0}^{\mathcal{K}\left(\mathbb{S}^{2}\right)}\left(\mathcal{Q}^{1} \operatorname{Brun}_{4}\left(\mathbb{S}^{2}\right)\right) \\
& =d_{1}\left(\operatorname{Brun}_{4}\left(\mathbb{S}^{2}\right)\right)=\{1\}
\end{aligned}
$$

Para isto usaremos a descrição dada no Teorema 2.2.12 dos grupos quase-Brunnianos sobre a esfera como grupos comutadores simétricos e a definição da aplicação que esquece a primeira corda, $d_{1}: P_{n+3}\left(\mathbb{S}^{2}\right) \rightarrow P_{n+2}\left(\mathbb{S}^{2}\right)$. Assim,

$$
\begin{aligned}
\mathcal{B} d_{n} \mathcal{K}\left(\mathbb{S}^{2}\right) & =d_{0}^{\mathcal{K}\left(\mathbb{S}^{2}\right)}\left(\mathcal{N}_{n+1} \mathcal{K}\left(\mathbb{S}^{2}\right)\right) \\
& =d_{1}\left(\mathcal{Q}^{1} \operatorname{Brun}_{n+3}\left(\mathbb{S}^{2}\right)\right) \\
& =d_{1}\left(\left[\left\langle A_{2, n+3}\right\rangle^{P_{n+3}\left(\mathbb{S}^{2}\right)},\left\langle A_{3, n+3}\right\rangle^{P_{n+3}\left(\mathbb{S}^{2}\right)}, \ldots,\left\langle A_{n+2, n+3}\right\rangle^{P_{n+3}\left(\mathbb{S}^{2}\right)}\right]_{S}\right) \\
& =\left[\left\langle A_{1, n+2}\right\rangle^{P_{n+2}\left(\mathbb{S}^{2}\right)},\left\langle A_{2, n+2}\right\rangle^{P_{n+2}\left(\mathbb{S}^{2}\right)}, \ldots,\left\langle A_{n+1, n+2}\right\rangle^{P_{n+2}\left(\mathbb{S}^{2}\right)}\right]_{S} .
\end{aligned}
$$

Corolário 4.2.15. Seja $n \geq 2$. O grupo $\mathcal{B} d_{n} \mathcal{K}\left(\mathbb{S}^{2}\right)$ é normal em $P_{n+2}\left(\mathbb{S}^{2}\right), \mathcal{N}_{n} \mathcal{K}\left(\mathbb{S}^{2}\right)$ é normal no grupo misto $B_{1, n+1}\left(\mathbb{S}^{2}\right)$ e $\mathcal{Z}_{n} \mathcal{K}\left(\mathbb{S}^{2}\right)$ é normal em $B_{n+2}\left(\mathbb{S}^{2}\right)$.

Demonstração. Seja $n \geq 2$. O resultado segue da descrição dada na Proposição 4.2.14 para os grupos $\mathcal{B} d_{n} \mathcal{K}\left(\mathbb{S}^{2}\right), \mathcal{N}{ }_{n} \mathcal{K}\left(\mathbb{S}^{2}\right)$ e $\mathcal{Z}_{n} \mathcal{K}\left(\mathbb{S}^{2}\right)$, do fato que subgrupos simétricos de um grupo são normais no grupo e da Proposição 2.2.8.

Corolário 4.2.16. Para $\mathcal{K}\left(\mathbb{S}^{2}\right)$ temos que $\sqrt{\left(\mathcal{B} d_{n} \mathcal{K}\left(\mathbb{S}^{2}\right),\left(\mathcal{K}\left(\mathbb{S}^{2}\right)\right)_{n}\right)}=\mathcal{Z}_{n} \mathcal{K}\left(\mathbb{S}^{2}\right)$ e existe um isomorfismo de grupos, para todo $n \geq 3$,

$$
\frac{\sqrt{\left(\mathcal{B} d_{n} \mathcal{K}\left(\mathbb{S}^{2}\right),\left(\mathcal{K}\left(\mathbb{S}^{2}\right)\right)_{n}\right)}}{\mathcal{B} d_{n} \mathcal{K}\left(\mathbb{S}^{2}\right)} \cong \pi_{n+1}\left(\mathbb{S}^{2}\right)
$$

Demonstração. Seja $n \geq 3$. Pela Proposição 4.2.12 sabemos que existe um isomorfismo de grupos $\pi_{n}\left(\mathcal{K}\left(\mathbb{S}^{2}\right)\right) \cong \pi_{n+1}\left(\mathbb{S}^{2}\right)$. Além disso, pela construção do grupo simplicial $\mathcal{K}\left(\mathbb{S}^{2}\right)$, temos que $\left(\mathcal{K}\left(\mathbb{S}^{2}\right)\right)_{n}$ é um grupo livre. Como $n \geq 3$, então $\pi_{n+1}\left(\mathbb{S}^{2}\right)$ é um grupo finito e assim, pelo Teorema 4.1 .15 , temos que $\sqrt{\left(\mathcal{B} d_{n} \mathcal{K}\left(\mathbb{S}^{2}\right),\left(\mathcal{K}\left(\mathbb{S}^{2}\right)\right)_{n}\right)}=\mathcal{Z}_{n} \mathcal{K}\left(\mathbb{S}^{2}\right)$ e existe um isomorfismo de grupos

$$
\frac{\sqrt{\left(\mathcal{B} d_{n} \mathcal{K}\left(\mathbb{S}^{2}\right),\left(\mathcal{K}\left(\mathbb{S}^{2}\right)\right)_{n}\right)}}{\mathcal{B} d_{n} \mathcal{K}\left(\mathbb{S}^{2}\right)} \cong \pi_{n+1}\left(\mathbb{S}^{2}\right)
$$


Corolário 4.2.17. Para $n \geq 2$ existem isomorfismos

$$
\frac{\operatorname{Brun}_{n+2}\left(\mathbb{S}^{2}\right)}{\left[\left\langle A_{1, n+2}\right\rangle^{P_{n+2}\left(\mathbb{S}^{2}\right)},\left\langle A_{2, n+2}\right\rangle^{P_{n+2}\left(\mathbb{S}^{2}\right)}, \ldots,\left\langle A_{n+1, n+2}\right\rangle^{P_{n+2}\left(\mathbb{S}^{2}\right)}\right]_{S}} \cong \pi_{n+1}\left(\mathbb{S}^{2}\right) .
$$

Demonstração. Pela Proposição temos que $4.2 .12 \pi_{n}\left(\mathcal{K}\left(\mathbb{S}^{2}\right)\right)=\frac{\mathcal{Z}_{n} \mathcal{K}\left(\mathbb{S}^{2}\right)}{\mathcal{B} d_{n} \mathcal{K}\left(\mathbb{S}^{2}\right)} \cong \pi_{n+1}\left(\mathbb{S}^{2}\right)$, para todo $n \geq 0$. O resultado segue substituindo os elementos obtidos na Proposição 4.2.14.

Propriedades 4.2.18. Seja $n \geq 2$.

1. Seja $A_{i, j} \in P_{n+1}\left(\mathbb{S}^{2}\right)$. Então, $A_{i+1, j+1} \in d_{i}^{-1}\left(A_{i, j}\right) \cap \operatorname{Ker}\left(d_{i+1}: P_{n+2}\left(\mathbb{S}^{2}\right) \rightarrow P_{n+1}\left(\mathbb{S}^{2}\right)\right)$.

2. Sabemos que $d_{n+2}: P_{n+2}\left(\mathbb{S}^{2}\right) \rightarrow P_{n+1}\left(\mathbb{S}^{2}\right)$ é o epimorfismo que corresponde a esquecer a $(n+2)$-ésima corda de tranças em $P_{n+2}\left(\mathbb{S}^{2}\right)$. O centro de $P_{n+2}\left(\mathbb{S}^{2}\right)$ é isomorfo a $\mathbb{Z}_{2}$ e gerado pelo "full twist" $\Delta_{n+2}$, ainda mais $d_{n+2}\left(\Delta_{n+2}\right)=\Delta_{n+1}$. Logo $d_{n+2}: Z\left(P_{n+2}\left(\mathbb{S}^{2}\right)\right) \rightarrow Z\left(P_{n+1}\left(\mathbb{S}^{2}\right)\right)$ é um isomorfismo.

3. Notemos que $A_{n+1}=Z\left(P_{n+2}\left(\mathbb{S}^{2}\right)\right) / \mathcal{B} d_{n} \mathcal{K}\left(\mathbb{S}^{2}\right) \cong \mathbb{Z}_{2}$ gerado pela classe do "full twist" $\Delta_{n+2}$. Além disso $\Delta_{n+2}$ não pertence ao grupo livre $\left(\mathcal{K}\left(\mathbb{S}^{2}\right)\right)_{n}$. De forma similar a como foi feito no caso do disco, nas Propriedades 4.2.11, pode-se provar a seguinte igualdade

$$
\frac{\left(\mathcal{K}\left(\mathbb{S}^{2}\right)\right)_{n}}{\mathcal{B} d_{n} \mathcal{K}\left(\mathbb{S}^{2}\right)} \bigcap \frac{Z\left(P_{n+2}\left(\mathbb{S}^{2}\right)\right)}{\mathcal{B} d_{n} \mathcal{K}\left(\mathbb{S}^{2}\right)}=\{1\}
$$

\subsection{Aplicações}

Pela Proposição 4.2.4 sabemos que $\mathcal{H}=\mathcal{K}\left(\mathbb{D}^{2}\right)$ é um grupo simplicial e portanto pelo Teorema de Moore $\mathcal{K}\left(\mathbb{D}^{2}\right)$ é um $\Delta$-grupo fibrante. Pelo Teorema de Nielsen-Schreier, como $\left(\mathcal{K}\left(\mathbb{D}^{2}\right)\right)_{n}$ é um grupo livre, para todo $n \geq 0$, então $\mathcal{B} d_{n} \mathcal{H}$ é um grupo livre. Além disso, dado $A_{i, n+1} \in \operatorname{Ker}\left(d_{n+1}: P_{n+1} \rightarrow P_{n}\right)$ o elemento $A_{i+1, n+2} \in P_{n+2}$ é tal que $A_{i+1, n+2} \in d_{i}^{-1}\left(A_{i, n+1}\right) \cap \operatorname{Ker}\left(d_{i+1}\right)$. Logo, o $\Delta$-grupo fibrante $\mathcal{K}\left(\mathbb{D}^{2}\right)$ satizfaz as hipóteses do Teorema de Decomposição (Teorema 4.1.7).

A seguir, vamos aplicar os resultados gerais de teoria de homotopia simplicial, obtidos na Seção 4.1, aos grupos simpliciais construídos nas Subseções 4.2.1 e 4.2.2. Desta forma, podemos obter novas conexões entre os grupos de homotopia da esfera e os grupos de tranças sobre superfícies.

\subsubsection{Os conjuntos de grupos de tranças $\mathbb{P}$ e $\mathcal{P}\left(\mathbb{S}^{2}\right)$}

Nesta subseção aplicaremos os resultados obtidos na Seção 4.1 nos seguintes casos: 
- À sequência de grupos de tranças sobre o disco $\mathcal{G}=\mathbb{P}=\left\{P_{n}\right\}_{n \geq 0}$ do Exemplo 1.3.22 com $P_{0}=\{1\}$ e ao grupo simplicial $\mathcal{H}=\mathcal{K}\left(\mathbb{D}^{2}\right)$ da Proposição 4.2.4.

- À sequência de grupos de tranças sobre a esfera $\mathcal{G}=\mathcal{P}\left(\mathbb{S}^{2}\right)=\left\{P_{n+1}\left(\mathbb{S}^{2}\right)\right\}_{n \geq 0}$ do Exemplo 1.3.2 e ao grupo simplicial $\mathcal{H}=\mathcal{K}\left(\mathbb{S}^{2}\right)$ da Proposição 4.2.12.

Proposição 4.3.1. Para todo $n \geq 2$, o grupo de homotopia $\pi_{n}\left(\mathcal{K}\left(\mathbb{D}^{2}\right)\right.$ ) está contido no centro de $P_{n+1} / \mathcal{B} d_{n} \mathcal{K}\left(\mathbb{D}^{2}\right)$.

Demonstração. Seja $n \geq 2$. Segue das Propriedades 4.2 .11 que $\mathcal{G}=\mathbb{P}$ e $\mathcal{H}=\mathcal{K}\left(\mathbb{D}^{2}\right)$ satisfazem as hipóteses do Teorema de Extensão Central (Teorema 4.1.1) e portanto

$$
\pi_{n}\left(\mathcal{K}\left(\mathbb{D}^{2}\right)\right) \subseteq Z\left(P_{n+1} / \mathcal{B} d_{n} \mathcal{K}\left(\mathbb{D}^{2}\right)\right)
$$

Proposição 4.3.2. Seja $n \geq 2$. O centro do grupo quociente $P_{n+1} / \mathcal{B} d_{n} \mathcal{K}\left(\mathbb{D}^{2}\right)$ está contido em $Z\left(\frac{\left(\mathcal{K}\left(\mathbb{D}^{2}\right)\right)_{n}}{\mathcal{B} d_{n} \mathcal{K}\left(\mathbb{D}^{2}\right)}\right) \times \mathbb{Z}$

Demonstração. Seja $n \geq 2$. Segue das Propriedades 4.2 .11 que $\mathcal{G}=\mathbb{P}, \mathcal{H}=\mathcal{K}\left(\mathbb{D}^{2}\right)$ e $\delta_{n+1}=d_{n+1}$ satisfazem as hipóteses do Lema 4.1 .3 e portanto

$$
Z\left(\frac{P_{n}}{\mathcal{B} d_{n-1} \mathcal{K}\left(\mathbb{D}^{2}\right)}\right) \subseteq Z\left(\frac{\left(\mathcal{K}\left(\mathbb{D}^{2}\right)\right)_{n}}{\mathcal{B} d_{n} \mathcal{K}\left(\mathbb{D}^{2}\right)}\right) \times \mathbb{Z}
$$

Proposição 4.3.3. Para $n \geq 2$ existe um isomorfismo

$$
Z\left(\frac{\left(\mathcal{K}\left(\mathbb{D}^{2}\right)\right)_{n}}{\mathcal{B} d_{n} \mathcal{K}\left(\mathbb{D}^{2}\right)}\right) \cong \pi_{n+1}\left(\mathbb{S}^{2}\right)
$$

Demonstração. Seja $n \geq 2$. Tomemos $\mathcal{H}=\mathcal{K}\left(\mathbb{D}^{2}\right)$ e consideremos neste caso $\mathcal{G}=\left\{G_{n}\right\}_{n \geq 0}$ dado por $G_{n+1}=H_{n}=\left(\mathcal{K}\left(\mathbb{D}^{2}\right)\right)_{n}$, para todo $n \geq 0$, e os homomorfismos $\delta_{n+1}=d_{n+1}:\left(\mathcal{K}\left(\mathbb{D}^{2}\right)\right)_{n} \rightarrow\left(\mathcal{K}\left(\mathbb{D}^{2}\right)\right)_{n-1}$. Pelas Propriedades 4.2.11 segue que as hipóteses do Teorema de Extensão Central (Teorema 4.1.1) e do Lema 4.1.3 são verificadas. Além disso, os grupos livres $\left(\mathcal{K}\left(\mathbb{D}^{2}\right)\right)_{n}$ têm centro trivial e portanto nesse caso $A_{n+1}=\{1\}$. Logo, pelo Teorema 4.1 .5 , temos que o centro de $\frac{\left(\mathcal{K}\left(\mathbb{D}^{2}\right)\right)_{n}}{\mathcal{B} d_{n} \mathcal{K}\left(\mathbb{D}^{2}\right)}$ é igual ao grupo $\pi_{n}\left(\mathcal{K}\left(\mathbb{D}^{2}\right)\right)$. O resultado segue pela Proposição 4.2.4 que garante o isomorfismo $\pi_{n}\left(\mathcal{K}\left(\mathbb{D}^{2}\right)\right) \cong \pi_{n+1}\left(\mathbb{S}^{2}\right)$.

O seguinte resultado estabelece uma nova conexão entre os grupos de tranças sobre superfícies e os grupos de homotopia da esfera $\mathbb{S}^{2}$. Gostaríamos de observar que o primeiro isomorfismo do Teorema 4.3.4 já era conhecido, pois no Teorema 1 de [58] foi obtido um isomorfismo (usando a notação de [58]) $Z\left(\frac{P_{n+1}}{B d_{n+1}}\right) \cong \pi_{n+1}\left(\mathbb{S}^{2}\right) \times \mathbb{Z}$ para $n \geq 3$, ver Teorema 1.3.25. 
Teorema 4.3.4. Seja $n \geq 2$, existem isomorfismos:
1. $Z\left(\frac{P_{n+1}}{\mathcal{B} d_{n} \mathcal{K}\left(\mathbb{D}^{2}\right)}\right) \cong \pi_{n+1}\left(\mathbb{S}^{2}\right) \times \mathbb{Z}$.
2. $Z\left(\frac{P_{n+2}\left(\mathbb{S}^{2}\right)}{\mathcal{B} d_{n} \mathcal{K}\left(\mathbb{S}^{2}\right)}\right) \cong \pi_{n+1}\left(\mathbb{S}^{2}\right) \times \mathbb{Z}_{2}$.

Demonstração. Seja $n \geq 2$.

1. Em vista das Propriedades 4.2.11 segue que a sequência de grupos $\mathcal{G}=\mathbb{P}$, o grupo simplicial conexo $\mathcal{H}=\mathcal{K}\left(\mathbb{D}^{2}\right)$ e os homomorfismos $\delta_{n+1}=d_{n+1}$ satisfazem as hipóteses do Teorema de Extensão Central (Teorema 4.1.1) e do Lema 4.1.3. Nas Propriedades 4.2 .11 vimos que $A_{n+1}=Z\left(P_{n+1}\right) / \mathcal{B} d_{n} \mathcal{K}\left(\mathbb{D}^{2}\right) \cong \mathbb{Z}$ gerado pela classe do "full twist" $\Delta_{n+1}$. Além disso, os grupos livres $\left(\mathcal{K}\left(\mathbb{D}^{2}\right)\right)_{n}$ têm centro trivial. Logo, pelo Teorema 4.1.5, temos que

$$
Z\left(\frac{P_{n+1}}{\mathcal{B} d_{n} \mathcal{K}\left(\mathbb{D}^{2}\right)}\right) \cong \pi_{n}\left(\mathcal{K}\left(\mathbb{D}^{2}\right)\right) \times \mathbb{Z}
$$

Pela Proposição 4.2 .4 temos $\pi_{n}\left(\mathcal{K}\left(\mathbb{D}^{2}\right)\right) \cong \pi_{n+1}\left(\mathbb{S}^{2}\right)$ e assim, por $(4.45)$, existe um isomorfismo

$$
Z\left(\frac{P_{n+1}}{\mathcal{B} d_{n} \mathcal{K}\left(\mathbb{D}^{2}\right)}\right) \cong \pi_{n+1}\left(\mathbb{S}^{2}\right) \times \mathbb{Z}
$$

2. A demonstração dada a seguir é similar com aquela do item anterior. Segue das Propriedades 4.2.18 que a sequência de grupos $\mathcal{G}=\mathcal{P}\left(\mathbb{S}^{2}\right)$, o grupo simplicial conexo $\mathcal{H}=\mathcal{K}\left(\mathbb{S}^{2}\right)$ e os homomorfismos $\delta_{n+1}=d_{n+2}$ satisfazem as hipóteses do Teorema de Extensão Central (Teorema 4.1.1) e do Lema 4.1.3. Nas Propriedades 4.2.18 vimos que $A_{n+1}=Z\left(P_{n+2}\left(\mathbb{S}^{2}\right)\right) / \mathcal{B} d_{n} \mathcal{K}\left(\mathbb{S}^{2}\right) \cong \mathbb{Z}_{2}$ gerado pela classe do "full twist" $\Delta_{n+2}$. Além disso, os grupos livres $\left(\mathcal{K}\left(\mathbb{S}^{2}\right)\right)_{n}$ têm centro trivial. Logo, pelo Teorema 4.1.5, temos que

$$
Z\left(\frac{P_{n+2}\left(\mathbb{S}^{2}\right)}{\mathcal{B} d_{n} \mathcal{K}\left(\mathbb{S}^{2}\right)}\right) \cong \pi_{n}\left(\mathcal{K}\left(\mathbb{S}^{2}\right)\right) \times \mathbb{Z}_{2}
$$

Pela Proposição 4.2.12 existe um isomorfismo $\pi_{n}\left(\mathcal{K}\left(\mathbb{S}^{2}\right)\right) \cong \pi_{n+1}\left(\mathbb{S}^{2}\right)$ e portanto, por (4.46), existe um isomorfismo

$$
Z\left(\frac{P_{n+2}\left(\mathbb{S}^{2}\right)}{\mathcal{B} d_{n} \mathcal{K}\left(\mathbb{S}^{2}\right)}\right) \cong \pi_{n+1}\left(\mathbb{S}^{2}\right) \times \mathbb{Z}_{2}
$$

Como comentamos no início desta seção, o $\Delta$-grupo fibrante $\mathcal{K}\left(\mathbb{D}^{2}\right)$ satizfaz as hipóteses do Teorema de Decomposição (Teorema 4.1.7) e portanto podemos deduzir o seguinte resultado. 


\section{Teorema 4.3.5.}

1. Seja $n \geq 2$. Existe uma decomposição

$$
\frac{\operatorname{Brun}_{n+1}\left(\mathbb{D}^{2}\right)}{\left[\left\langle A_{0, n+1}\right\rangle^{P_{n+1}}, \ldots,\left\langle A_{n, n+1}\right\rangle^{P_{n+1}}\right]_{S}} \cong \pi_{n+1}\left(\mathbb{S}^{2}\right) \times\left[\left\langle A_{0, n}\right\rangle^{P_{n}}, \ldots,\left\langle A_{n-1, n}\right\rangle^{P_{n}}\right]_{S} .
$$

Portanto, para $n \geq 3$,

$$
\operatorname{Tor}\left(\frac{\operatorname{Brun}_{n+1}\left(\mathbb{D}^{2}\right)}{\left[\left\langle A_{0, n+1}\right\rangle^{P_{n+1}}, \ldots,\left\langle A_{n, n+1}\right\rangle^{P_{n+1}}\right]_{S}}\right) \cong \pi_{n+1}\left(\mathbb{S}^{2}\right) .
$$

2. Seja $n \geq 1$. Existe uma decomposição

$$
H_{n}\left(\left(N\left(\mathcal{K}\left(\mathbb{D}^{2}\right)\right)\right)_{a b}\right) \cong \pi_{n+1}\left(\mathbb{S}^{2}\right) \oplus A_{n}
$$

para cada $n$, onde $A_{n}$ é livre de torção.

3. Seja $n \geq 1$. Existe uma decomposição

$$
H_{n}\left(\mathbb{Z}\left(N\left(\mathcal{K}\left(\mathbb{D}^{2}\right)\right)\right)\right) \cong \pi_{n+1}\left(\mathbb{S}^{2}\right) \oplus C_{n}
$$

para algum grupo $C_{n}$.

4. Seja $n \geq 3$. Existe um isomorfismo de grupos

$$
\operatorname{Tor}\left(H_{n}\left(\left(N\left(\mathcal{K}\left(\mathbb{D}^{2}\right)\right)\right)_{a b}\right)\right) \cong \pi_{n+1}\left(\mathbb{S}^{2}\right)
$$

Demonstração. Segue das Propriedades 1.2.1 que os grupos de homotopia $\pi_{n+1}\left(\mathbb{S}^{2}\right)$ são grupos abelianos finitos, para $n \geq 3$. Seja $n \geq 2$. Pela Proposição 4.2 .4 temos que

$$
\pi_{n}\left(\mathcal{K}\left(\mathbb{D}^{2}\right)\right) \cong \pi_{n+1}\left(\mathbb{S}^{2}\right)
$$

O primeiro item segue do Teorema de Decomposição (Teorema 4.1.7) junto com a descrição dada na Proposição 4.2.7 para os complexos e bordos de Moore de $\mathcal{K}\left(\mathbb{D}^{2}\right)$. Observemos que o grupo $\mathcal{B} d_{n} \mathcal{K}\left(\mathbb{D}^{2}\right)$ está contido no grupo livre $\left(\mathcal{K}\left(\mathbb{D}^{2}\right)\right)_{n}$ e portanto é por sua vez um grupo livre (aplicando o Teorema de Nielsen-Schreier).

O segundo e terceiro item decorrem claramente dos Corolários 4.1.12 e 4.1.13. Pelo Corolário 4.1.14 aplicado no $\Delta$-grupo $\mathcal{K}\left(\mathbb{D}^{2}\right)$, obtemos o último item.

No seguinte resultado aplicamos o Teorema de Decomposição (Teorema 4.1.7) ao grupo simplicial $\mathcal{K}\left(\mathbb{S}^{2}\right)$. A prova segue da mesma forma que fizemos acima com o grupo simplicial $\mathcal{K}\left(\mathbb{D}^{2}\right)$. 


\section{Teorema 4.3.6.}

1. Seja $n \geq 2$. Existe uma decomposição

$$
\frac{\mathcal{Q}^{1} \operatorname{Brun}_{n+2}\left(\mathbb{S}^{2}\right)}{\left[\left\langle A_{1, n+2}\right\rangle^{P_{n+2}\left(\mathbb{S}^{2}\right)}, \ldots,\left\langle A_{n+1, n+2}\right\rangle^{P_{n+2}\left(\mathbb{S}^{2}\right)}\right]_{S}} \cong \pi_{n+1}\left(\mathbb{S}^{2}\right) \times \mathcal{B} d_{n-1} \mathcal{K}\left(\mathbb{S}^{2}\right)
$$

onde $\mathcal{B} d_{n-1} \mathcal{K}\left(\mathbb{S}^{2}\right)=\left[\left\langle A_{1, n+1}\right\rangle^{P_{n+1}\left(\mathbb{S}^{2}\right)}, \ldots,\left\langle A_{n, n+1}\right\rangle^{P_{n+1}\left(\mathbb{S}^{2}\right)}\right]_{S}$.

Portanto, para $n \geq 3$, temos que

$$
\operatorname{Tor}\left(\frac{\mathcal{Q}^{1} \operatorname{Brun}_{n+2}\left(\mathbb{S}^{2}\right)}{\left[\left\langle A_{1, n+2}\right\rangle^{P_{n+2}\left(\mathbb{S}^{2}\right)}, \ldots,\left\langle A_{n+1, n+2}\right\rangle^{P_{n+2}\left(\mathbb{S}^{2}\right)}\right]_{S}}\right) \cong \pi_{n+1}\left(\mathbb{S}^{2}\right) .
$$

2. Seja $n \geq 1$. Existe uma decomposição

$$
H_{n}\left(\left(N\left(\mathcal{K}\left(\mathbb{S}^{2}\right)\right)\right)_{a b}\right) \cong \pi_{n+1}\left(\mathbb{S}^{2}\right) \oplus A_{n}
$$

para cada $n$, onde $A_{n}$ é livre de torção.

3. Seja $n \geq 1$. Existe uma decomposição

$$
H_{n}\left(\mathbb{Z}\left(N\left(\mathcal{K}\left(\mathbb{S}^{2}\right)\right)\right)\right) \cong \pi_{n+1}\left(\mathbb{S}^{2}\right) \oplus C_{n}
$$

para algum grupo $C_{n}$.

4. Seja $n \geq 3$. Existe um isomorfismo de grupos

$$
\operatorname{Tor}\left(H_{n}\left(\left(N\left(\mathcal{K}\left(\mathbb{S}^{2}\right)\right)\right)_{a b}\right)\right) \cong \pi_{n+1}\left(\mathbb{S}^{2}\right)
$$

Demonstração. Seja $n \geq 2$. Pela Proposição 4.2 .12 temos que $\pi_{n}\left(\mathcal{K}\left(\mathbb{S}^{2}\right)\right) \cong \pi_{n+1}\left(\mathbb{S}^{2}\right)$. O primeiro item segue do Teorema de Decomposição (Teorema 4.1.7) junto com a descrição dada na Proposição 4.2.14 para os complexos e bordos de Moore de $\mathcal{K}\left(\mathbb{S}^{2}\right)$.

O segundo e terceiro item decorrem claramente dos Corolários 4.1.12 e 4.1.13.

Para $n \geq 3$ o grupo $\pi_{n+1}\left(\mathbb{S}^{2}\right)$ é um grupo abeliano finito, ver Propriedades 1.2.1. Portanto, pelo Corolário 4.1.14 aplicado no $\Delta$-grupo $\mathcal{K}\left(\mathbb{S}^{2}\right)$, obtemos o último item.

\subsubsection{Quocientes finitamente apresentados e $\pi_{*}\left(\mathbb{S}^{2}\right)$}

Seja $G$ um grupo finitamente apresentado e suponhamos que $H$ é um subgrupo normal de $G$. Em geral, o grupo quociente $G / H$ não precisa ser finitamente apresentado, mas condições para que isto aconteça podem ser dadas. Se $H \triangleleft G$ e existe um conjunto finito de elementos de $H$ que geram normalmente (em $G$ ) o grupo $H$, então, nesse caso, não é 
difícil de provar que o grupo quociente $G / H$ é um grupo finitamente apresentado. Sabemos que os grupos de tranças de Artin $B_{n}$ e $P_{n}$ são grupos finitamente apresentados. Aplicando o Teorema 4.3 de [69] aos grupos $\operatorname{Brun}_{n}\left(\mathbb{D}^{2}\right)$ e $\mathcal{B} d_{n-1} \mathcal{K}\left(\mathbb{D}^{2}\right)$ (junto com o Teorema 1.2.14 e a Proposição 4.2.7) temos que estes grupos são normalmente gerados em $B_{n}$ e $P_{n}$. Desta forma temos os seguintes dois resultados.

Corolário 4.3.7 (Corolário 3.8 de [58]). Para $n \geq 2$, os grupos quocientes $P_{n} /$ Brun $_{n}\left(\mathbb{D}^{2}\right)$ e $B_{n} /$ Brun $_{n}\left(\mathbb{D}^{2}\right)$ são finitamente apresentados.

Corolário 4.3.8 (Corolário 3.16 de [58]). Para todo $n \geq 1$, os grupos quocientes $P_{n} / \mathcal{B} d_{n-1} \mathcal{K}\left(\mathbb{D}^{2}\right)$ e $B_{n} / \mathcal{B} d_{n-1} \mathcal{K}\left(\mathbb{D}^{2}\right)$ são finitamente apresentados.

A seguinte descrição de $\operatorname{Brun}_{3}\left(\mathbb{D}^{2}\right)$ será de utilidade para provar a Proposição 4.3 .10 abaixo.

Lema 4.3.9. O grupo $\operatorname{Brun}_{3}\left(\mathbb{D}^{2}\right)$ pode ser descrito como sendo o fecho normal de elementos de $P_{3}$,

$$
\operatorname{Brun}_{3}\left(\mathbb{D}^{2}\right)=\left\langle\left\{\left[A_{1,2}, A_{1,3}\right],\left[A_{1,2}, A_{2,3}\right]\right\}\right\rangle^{P_{3}}
$$

Ou também, como sendo o fecho normal de um elemento de $B_{3}$,

$$
\operatorname{Brun}_{3}\left(\mathbb{D}^{2}\right)=\left\langle\left[A_{1,2}, A_{2,3}\right]\right\rangle^{B_{3}}
$$

Demonstração. Sabemos, pelo Lema 2.1.5, que $\operatorname{Brun}_{3}\left(\mathbb{D}^{2}\right)=\left[\left\langle A_{1,2}\right\rangle^{P_{3}},\left\langle A_{1,3}\right\rangle^{P_{3}}\right]$. Provemos inicialmente que $\operatorname{Brun}_{3}\left(\mathbb{D}^{2}\right)=\left[\left\langle A_{1,2}\right\rangle^{P_{3}}, \operatorname{Ker}\left(d_{3}: P_{3} \rightarrow P_{2}\right)\right]$. Como $\operatorname{Brun}_{3}\left(\mathbb{D}^{2}\right)=\left[\left\langle A_{1,2}\right\rangle^{P_{3}},\left\langle A_{1,3}\right\rangle^{P_{3}}\right]$ e $\left\langle A_{1,3}\right\rangle^{P_{3}} \subseteq \operatorname{Ker}\left(d_{3}: P_{3} \rightarrow P_{2}\right)$, então é claro que

$$
\operatorname{Brun}_{3}\left(\mathbb{D}^{2}\right) \subseteq\left[\left\langle A_{1,2}\right\rangle^{P_{3}}, \operatorname{Ker}\left(d_{3}: P_{3} \rightarrow P_{2}\right)\right]
$$

Agora, dado um elemento $\beta$ em $\left[\left\langle A_{1,2}\right\rangle^{P_{3}}, \operatorname{Ker}\left(d_{3}: P_{3} \rightarrow P_{2}\right)\right]$, notemos que

$$
d_{1}(\beta)=d_{2}(\beta)=d_{3}(\beta)=1
$$

Logo, $\beta \in \operatorname{Brun}_{3}\left(\mathbb{D}^{2}\right)$. Com isto provamos que $\operatorname{Brun}_{3}\left(\mathbb{D}^{2}\right)=\left[\left\langle A_{1,2}\right\rangle^{P_{3}}, \operatorname{Ker}\left(d_{3}: P_{3} \rightarrow P_{2}\right)\right]$.

Pela Afirmação 4.4 de Mikhailov \& Wu [69] (ou [5, Lema 5.2]), temos que

$$
\operatorname{Brun}_{3}\left(\mathbb{D}^{2}\right)=\left\langle\left\{\left[A_{1,2}, A_{1,3}\right],\left[A_{1,2}, A_{2,3}\right]\right\}\right\rangle^{P_{3}}
$$

Por outro lado,

- $\sigma_{1}\left(\left[A_{1,2}, A_{1,3}\right]\right) \sigma_{1}^{-1}=\left[A_{1,2}, A_{2,3}\right] \in \operatorname{Brun}_{3}\left(\mathbb{D}^{2}\right)$.

- $\sigma_{1}\left(\left[A_{1,2}, A_{2,3}\right]\right) \sigma_{1}^{-1}=\left[A_{1,2}, A_{2,3}^{-1} A_{1,3} A_{2,3}\right] \in \operatorname{Brun}_{3}\left(\mathbb{D}^{2}\right)$.

- $\sigma_{2}\left(\left[A_{1,2}, A_{1,3}\right]\right) \sigma_{2}^{-1}=\left[A_{1,3}, A_{2,3}^{-1} A_{1,2} A_{2,3}\right] \in \operatorname{Brun}_{3}\left(\mathbb{D}^{2}\right)$. 
- $\sigma_{2}\left(\left[A_{1,2}, A_{2,3}\right]\right) \sigma_{2}^{-1}=\left[A_{1,3}, A_{2,3}\right] \in \operatorname{Brun}_{3}\left(\mathbb{D}^{2}\right)$.

Portanto, $\left\langle\left\{\left[A_{1,2}, A_{1,3}\right],\left[A_{1,2}, A_{2,3}\right]\right\}\right\rangle^{B_{3}} \subseteq \operatorname{Brun}_{3}\left(\mathbb{D}^{2}\right)$ e é claro que a outra inclusão é válida, isto é,

$$
\operatorname{Brun}_{3}\left(\mathbb{D}^{2}\right)=\left\langle\left\{\left[A_{1,2}, A_{1,3}\right],\left[A_{1,2}, A_{2,3}\right]\right\}\right\rangle^{P_{3}} \subseteq\left\langle\left\{\left[A_{1,2}, A_{1,3}\right],\left[A_{1,2}, A_{2,3}\right]\right\}\right\rangle^{B_{3}} .
$$

Finalmente, como $\sigma_{1}\left(\left[A_{1,2}, A_{1,3}\right]\right) \sigma_{1}^{-1}=\left[A_{1,2}, A_{2,3}\right] \in \operatorname{Brun}_{3}\left(\mathbb{D}^{2}\right)$, então

$$
\operatorname{Brun}_{3}\left(\mathbb{D}^{2}\right)=\left\langle\left\{\left[A_{1,2}, A_{1,3}\right],\left[A_{1,2}, A_{2,3}\right]\right\}\right\rangle^{B_{3}}=\left\langle\left[A_{1,2}, A_{1,3}\right]\right\rangle^{B_{3}} .
$$

Usando o Lema 4.3.9 podemos verificar diretamente que os grupos quocientes $B_{3} / \operatorname{Brun}_{3}\left(\mathbb{D}^{2}\right)$ e $P_{3} / \operatorname{Brun}_{3}\left(\mathbb{D}^{2}\right)$ são grupos finitamente apresentados, ainda melhor, podemos dar uma descrição mais precisa para estes grupos, como veremos a seguir.

\section{Proposição 4.3.10.}

1. O grupo $B_{3} /$ Brun $_{3}\left(\mathbb{D}^{2}\right)$ tem uma apresentação dada pelos geradores $\sigma_{1}, \sigma_{2}$ e as relações: $\sigma_{1} \sigma_{2} \sigma_{1}=\sigma_{2} \sigma_{1} \sigma_{2} e\left(\sigma_{1}^{-1} \sigma_{2}\right)^{3}=1$.

2. $P_{3} / \operatorname{Brun}_{3}\left(\mathbb{D}^{2}\right) \cong \mathbb{Z} \oplus \mathbb{Z} \oplus \mathbb{Z}$.

Demonstração. 1. Pelo Lema 4.3.9 segue que $\operatorname{Brun}_{3}\left(\mathbb{D}^{2}\right)=\left\langle\left[A_{1,2}, A_{2,3}\right]\right\rangle^{B_{3}}$. Portanto,

$$
\left.B_{3} / \operatorname{Brun}_{3}\left(\mathbb{D}^{2}\right)=\left\langle\sigma_{1}, \sigma_{2}\right| \sigma_{1} \sigma_{2} \sigma_{1}=\sigma_{2} \sigma_{1} \sigma_{2} \text { e }\left[A_{1,2}, A_{2,3}\right]=1\right\rangle
$$

Logo, da igualdade $\left[A_{1,2}, A_{2,3}\right]=\sigma_{1}^{-2} \sigma_{2}^{-2} \sigma_{1}^{2} \sigma_{2}^{2}=\left(\sigma_{1}^{-1} \sigma_{2}\right)^{3}$, obtemos o resultado desejado para esse item.

2. Usando novamente o Lema 4.3 .9 e uma apresentação de $P_{3}$ temos

$$
\begin{array}{cc}
P_{3} / \operatorname{Brun}_{3}\left(\mathbb{D}^{2}\right)=\left\langle A_{1,2}, A_{1,3}, A_{2,3}\right|\left[A_{1,2}, A_{1,3}\right]=\left[A_{2,3}^{-1}, A_{1,3}\right], \\
{\left[A_{1,2}, A_{2,3}\right]=\left[A_{2,3}^{-1}, A_{1,3}^{-1}\right],} \\
& \left.\left[A_{1,2}, A_{1,3}\right]=1,\left[A_{1,2}, A_{2,3}\right]=1\right\rangle \\
=\left\langle A_{1,2}, A_{1,3}, A_{2,3}\right|\left[A_{1,2}, A_{1,3}\right]=1, & \left.\left[A_{1,2}, A_{2,3}\right]=1,\left[A_{1,2}, A_{2,3}\right]=1\right\rangle \\
\cong \mathbb{Z} \oplus \mathbb{Z} \oplus \mathbb{Z} . &
\end{array}
$$

Notemos que outra forma de provar o segundo item da última proposição é usando o fato que o grupo de tranças Brunnianas com 3 cordas sobre o disco é exatamente igual ao grupo comutador do grupo de tranças puras, $\operatorname{Brun}_{3}\left(\mathbb{D}^{2}\right)=\left[P_{3}, P_{3}\right]$, ver o Corolário 2.1.4. 
Observação 4.3.11. O estudo do grupo quociente $B_{3} /$ Brun ${ }_{3}\left(\mathbb{D}^{2}\right)$ nos levou a descobrir que os grupos de tranças sobre o disco estão relacionados com os grupos cristalográficos, como veremos no Capítulo 5. De forma mais precisa, provaremos que o grupo quociente $B_{3} /$ Brun $_{3}\left(\mathbb{D}^{2}\right)$ é um grupo cristalográfico com grupo de holonomia $\Sigma_{3}$ e dimensão 3. Este resultado pode ser generalizado a um número de cordas arbitrário.

Para finalizar esta subseção vamos dar uma prova do Teorema 1 de [58], formulado no Teorema 1.3.25. A igualdade de grupos $\mathcal{B} d_{n-1} \mathcal{K}\left(\mathbb{D}^{2}\right)=B d_{n} \mathbb{P}$ é válida para todo $n$. Basta apenas verificar a expressão dos grupos envolvidos, para $\mathcal{B} d_{n-1} \mathcal{K}\left(\mathbb{D}^{2}\right)$ na Proposição 4.2 .7 e para $B d_{n} \mathbb{P}$ em [58].

Notemos que o enunciado proposto a seguir aparenta ser um pouco diferente do original, no entanto, se temos em conta a igualdade de grupos $\mathcal{B} d_{n-1} \mathcal{K}\left(\mathbb{D}^{2}\right)=B d_{n} \mathbb{P}$, vemos que o enunciado original e o enunciado abaixo são iguais. Ressaltamos que nossa demonstração é ligeiramente diferente daquela dada por Li \& Wu. Usaremos na seguinte prova o homomorfismo $\chi_{\sigma_{k}}: P_{n} \rightarrow P_{n}$, definido por $\beta \mapsto \sigma_{k} \beta \sigma_{k}^{-1}$. Este homomorfismo não é mais do que a ação por conjugação dos $\sigma_{i}$ sobre $P_{n}$, foi definido inicialmente na página 539, Seção 3, do artigo de Li e Wu [58]. Também usaremos o automorfismo $\theta: P_{n} \rightarrow P_{n}$, definido antes do Teorema 1.3.25.

Teorema 4.3.12 (Teorema 1 de [58]). Seja $n \geq 4$.

1. A seguinte igualdade é válida $\sqrt{\left(\mathcal{B} d_{n-1} \mathcal{K}\left(\mathbb{D}^{2}\right), P_{n}\right)}=\operatorname{Brun}_{n}\left(\mathbb{D}^{2}\right) \cap \theta\left(\operatorname{Brun}_{n}\left(\mathbb{D}^{2}\right)\right)$ e existe um isomorfismo de grupos $\sqrt{\left(\mathcal{B} d_{n-1} \mathcal{K}\left(\mathbb{D}^{2}\right), P_{n}\right)} / \mathcal{B} d_{n-1} \mathcal{K}\left(\mathbb{D}^{2}\right) \cong \pi_{n}\left(\mathbb{S}^{2}\right)$.

2. Existem isomorfismos de grupos

(a) $Z\left(P_{n} / \mathcal{B} d_{n-1} \mathcal{K}\left(\mathbb{D}^{2}\right)\right) \cong \pi_{n}\left(\mathbb{S}^{2}\right) \times \mathbb{Z}$,

(b) $Z\left(B_{n} / \mathcal{B} d_{n-1} \mathcal{K}\left(\mathbb{D}^{2}\right)\right) \cong\left\{\alpha \in \pi_{n}\left(\mathbb{S}^{2}\right) \mid 2 \alpha=0\right\} \times \mathbb{Z}$.

Demonstração. Suponhamos que $n \geq 4$.

1. Pelo Corolário 4.2.5 temos a seguinte igualdade de grupos

$$
\mathcal{Z}_{n-1} \mathcal{K}\left(\mathbb{D}^{2}\right)=\sqrt{\left(\mathcal{B} d_{n-1} \mathcal{K}\left(\mathbb{D}^{2}\right), P_{n}\right)}
$$

$\mathrm{e}$

$$
\pi_{n}\left(\mathbb{S}^{2}\right) \cong \mathcal{Z}_{n-1} \mathcal{K}\left(\mathbb{D}^{2}\right) / \mathcal{B} d_{n-1} \mathcal{K}\left(\mathbb{D}^{2}\right)=\sqrt{\left(\mathcal{B} d_{n-1} \mathcal{K}\left(\mathbb{D}^{2}\right), P_{n}\right)} / \mathcal{B} d_{n-1} \mathcal{K}\left(\mathbb{D}^{2}\right)
$$

A seguir, provaremos que

$$
\mathcal{Z}_{n-1} \mathcal{K}\left(\mathbb{D}^{2}\right)=\operatorname{Brun}_{n}\left(\mathbb{D}^{2}\right) \cap \theta\left(\operatorname{Brun}_{n}\left(\mathbb{D}^{2}\right)\right)
$$

Lembremos que, pela Proposição 4.2.7, $\mathcal{Z}_{n-1} \mathcal{K}\left(\mathbb{D}^{2}\right)=\operatorname{Brun}_{n}\left(\mathbb{D}^{2}\right) \cap \operatorname{Ker}(\partial)$. 
Como

$$
\begin{aligned}
\theta\left(\operatorname{Brun}_{n}\left(\mathbb{D}^{2}\right)\right) & =\theta\left(\left[\left\langle A_{1, n}\right\rangle^{P_{n}},\left\langle A_{2, n}\right\rangle^{P_{n}}, \ldots,\left\langle A_{n-1, n}\right\rangle^{P_{n}}\right]_{S}\right) \\
& =\left[\left\langle A_{0, n}\right\rangle^{P_{n}},\left\langle A_{2, n}\right\rangle^{P_{n}}, \ldots,\left\langle A_{n-1, n}\right\rangle^{P_{n}}\right]_{S},
\end{aligned}
$$

então é claro que $\theta\left(\operatorname{Brun}_{n}\left(\mathbb{D}^{2}\right)\right) \subseteq \operatorname{Ker}(\partial)$, pois $\partial\left(A_{0, n}\right)=1$, para $n \geq 2(\operatorname{ver}(1.4))$. Logo,

$$
\operatorname{Brun}_{n}\left(\mathbb{D}^{2}\right) \cap \theta\left(\operatorname{Brun}_{n}\left(\mathbb{D}^{2}\right)\right) \subseteq \operatorname{Brun}_{n}\left(\mathbb{D}^{2}\right) \cap \operatorname{Ker}(\partial)=\mathcal{Z}_{n-1} \mathcal{K}\left(\mathbb{D}^{2}\right)
$$

Veremos que $\left.\partial\right|_{\operatorname{Ker}\left(d_{n}\right)}=\left.d_{1} \theta\right|_{\operatorname{Ker}\left(d_{n}\right)}$ avaliando no conjunto de geradores livres de $\operatorname{Ker}\left(d_{n}\right)$.

- $\partial\left(A_{j, n}\right)=A_{j-1, n-1}$, para $1 \leq j \leq n-1$.

- Como $\theta$ é um automorfismo tal que

$$
\theta^{-1}\left(A_{j, n}\right)= \begin{cases}A_{0, n}, & \text { se } j=1 \\ A_{j, n}, & \text { se } j=2, \ldots, n-1\end{cases}
$$

então $\theta^{2}$ é igual ao homomorfismo identidade e portanto

$$
d_{1} \theta^{-1}\left(A_{j, n}\right)=d_{1} \theta\left(A_{j, n}\right)=A_{j-1, n-1},
$$

para $1 \leq j \leq n-1$, pois $d_{1} \theta=\partial$ (ver o parágrafo que antecede o Teorema 1.3.25).

Sejam $x \in \operatorname{Brun}_{n}\left(\mathbb{D}^{2}\right) \cap \operatorname{Ker}(\partial)$ e $y=\theta^{-1}(x)$. Observemos que $x \in \operatorname{Ker}\left(d_{n}\right)$ e portanto

$$
d_{1} y=d_{1} \theta^{-1}(x)=\partial(x)=1 .
$$

Como $x \in \operatorname{Brun}_{n}\left(\mathbb{D}^{2}\right)=\left[\left\langle A_{1, n}\right\rangle^{P_{n}}, \ldots,\left\langle A_{n-1, n}\right\rangle^{P_{n}}\right]_{S}$, então

$$
x=\left[\alpha_{1} A_{1, n} \alpha_{1}^{-1}, \alpha_{2} A_{2, n} \alpha_{2}^{-1}, \ldots, \alpha_{n-1} A_{n-1, n} \alpha_{n-1}^{-1}\right]_{S}, \text { com } \alpha_{i} \in P_{n}
$$

e $\operatorname{assim} y=\theta^{-1}(x)=\left[\beta_{1} A_{0, n} \beta_{1}^{-1}, \beta_{2} A_{2, n} \beta_{2}^{-1}, \ldots, \beta_{n-1} A_{n-1, n} \beta_{n-1}^{-1}\right]_{S}$, onde $\beta_{j}=\theta^{-1}\left(\alpha_{j}\right)$, para $j=1,2, \ldots, n-1$.

Desta forma, é claro que

$$
d_{j}(y)=d_{j} \theta^{-1}(x)=1,
$$

para $j=2,3, \ldots, n$. Portanto, $d_{j}(y)=1$, para $1 \leq j \leq n$. Em outras palavras, $y \in \operatorname{Brun}_{n}\left(\mathbb{D}^{2}\right)$. Logo, $x \in \theta\left(\operatorname{Brun}_{n}\left(\mathbb{D}^{2}\right)\right)$ e por hipótese $x \in \operatorname{Brun}_{n}\left(\mathbb{D}^{2}\right)$, obtendo o resultado desejado. 
2. O primeiro isomorfismo que aparece nesse item, o subitem (a), corresponde ao item 1 do Teorema 4.3.4.

A seguir vamos provar o segundo isomorfismo, correspondente ao subitem (b):

$$
Z\left(B_{n} / \mathcal{B} d_{n-1} \mathcal{K}\left(\mathbb{D}^{2}\right)\right) \cong\left\{\alpha \in \pi_{n}\left(\mathbb{S}^{2}\right) \mid 2 \alpha=0\right\} \times \mathbb{Z}
$$

Lembremos que $\pi_{n}\left(\mathbb{S}^{2}\right) \cong \frac{\mathcal{Z}_{n-1} \mathcal{K}\left(\mathbb{D}^{2}\right)}{\mathcal{B} d_{n-1} \mathcal{K}\left(\mathbb{D}^{2}\right)}$. Primeiro demonstraremos que

$$
\left\{\alpha \in \frac{\mathcal{Z}_{n-1} \mathcal{K}\left(\mathbb{D}^{2}\right)}{\mathcal{B} d_{n-1} \mathcal{K}\left(\mathbb{D}^{2}\right)} \mid 2 \alpha=0\right\} \subseteq Z\left(B_{n} / \mathcal{B} d_{n-1} \mathcal{K}\left(\mathbb{D}^{2}\right)\right)
$$

Seja $x \in \mathcal{Z}_{n-1} \mathcal{K}\left(\mathbb{D}^{2}\right)$ tal que $x^{2} \in \mathcal{B} d_{n-1} \mathcal{K}\left(\mathbb{D}^{2}\right)$. Vamos provar que $x$ e $\sigma_{i} x \sigma_{i}^{-1}$ representam o mesmo elemento em $\mathcal{Z}_{n-1} \mathcal{K}\left(\mathbb{D}^{2}\right) / \mathcal{B} d_{n-1} \mathcal{K}\left(\mathbb{D}^{2}\right)$, para $1 \leq i \leq n-1$. Observemos que em $\pi_{n-1}\left(\mathcal{K}\left(\mathbb{D}^{2}\right)\right)$,

$$
(-1)^{i-1}[x]+(-1)^{i+1}[x]= \pm 2[x]=0 .
$$

Pela Proposição 4.2.4 sabemos que $\mathcal{K}\left(\mathbb{D}^{2}\right) \cong F\left[\mathbb{S}^{1}\right]$ é um grupo simplicial. Pelo Teorema de Adição em Homotopia (Teorema 1.3 .18 com $y_{i-1}=x, y_{i+1}=x$ e $y_{j}=1$ para $j \neq i-1$ e $j \neq i+1)$, dado que $x \in\left(\mathcal{K}\left(\mathbb{D}^{2}\right)\right)_{n-1}$, existe $y \in\left(\mathcal{K}\left(\mathbb{D}^{2}\right)\right)_{n}$ tal que

$$
d_{i-1}(y)=x, \quad d_{i+1}(y)=x
$$

e $d_{j}(y)=1$ para $0 \leq j \leq n \operatorname{com} j \neq i-1, i+1$.

Notemos que os geradores $\sigma_{i}$ estão indexados usando a notação usual de tranças, ao invés da notação adotada na teoria de homotopia simplicial.

Seja $w=\sigma_{i+1} y \sigma_{i+1}^{-1}$. Então,

- $\operatorname{para} j=0: d_{0} w=d_{0} \chi_{\sigma_{i+1}}(y)=\chi_{\sigma_{i}} d_{0}(y)= \begin{cases}1, & \text { se } i \geq 2 \\ \sigma_{1} x \sigma_{1}^{-1}, & \text { se } i=1\end{cases}$

- $\operatorname{Para} 1 \leq j \leq i$ :

$$
d_{j} w=d_{j} \chi_{\sigma_{i+1}}(y)=\chi_{d_{j-1}^{B} \sigma_{i+1}} d_{j \cdot \sigma_{i+1}}(y)=\chi_{\sigma_{i}} d_{j}(y)= \begin{cases}1, & \text { se } j \neq i-1 \\ \sigma_{i} x \sigma_{i}^{-1}, & \text { se } j=i-1\end{cases}
$$

- $\operatorname{para} j=i+1: d_{i+1} w=d_{i+1} \chi_{\sigma_{i+1}}(y)=d_{i} y=1$;

- para $j=i+2: d_{i+2} w=d_{i+2} \chi_{\sigma_{i+1}}(y)=\chi_{d_{i+1}^{B} \sigma_{i+1}} d_{i+2 \cdot \sigma_{i+1}}(y)=i d \circ d_{i+1}(y)=x$;

- $\operatorname{para} j>i+2: d_{j} w=d_{j} \chi_{\sigma_{i+1}}(y)=\chi_{d_{j-1}^{B} \sigma_{i+1}} d_{j \cdot \sigma_{i+1}}(y)=\chi_{\sigma_{i+1}} d_{j}(y)=1$.

Como $\mathcal{Z}_{n-1} \mathcal{K}\left(\mathbb{D}^{2}\right) \unlhd B_{n}$, então $\sigma_{i} x \sigma_{i}^{-1} \in \mathcal{Z}_{n-1} \mathcal{K}\left(\mathbb{D}^{2}\right)$. Aplicando mais uma vez o 
Teorema de Adição em Homotopia (Teorema 1.3.18) temos que, em $\pi_{n-1}\left(\mathcal{K}\left(\mathbb{D}^{2}\right)\right.$ ),

$$
(-1)^{i-1}\left[\sigma_{i} x \sigma_{i}^{-1}\right]+(-1)^{i+2}[x]=0
$$

e portanto $\left[\sigma_{i} x \sigma_{i}^{-1}\right]=[x] \in \pi_{n-1}\left(\mathcal{K}\left(\mathbb{D}^{2}\right)\right)$. Logo, $x \sigma_{i} x^{-1} \sigma_{i}^{-1} \in \mathcal{B} d_{n-1} \mathcal{K}\left(\mathbb{D}^{2}\right)$ e assim

$$
\left\{\alpha \in \frac{\mathcal{Z}_{n-1} \mathcal{K}\left(\mathbb{D}^{2}\right)}{\mathcal{B} d_{n-1} \mathcal{K}\left(\mathbb{D}^{2}\right)} \mid 2 \alpha=0\right\} \subseteq Z\left(B_{n} / \mathcal{B} d_{n-1} \mathcal{K}\left(\mathbb{D}^{2}\right)\right)
$$

É claro que $\mathbb{Z} \cong Z\left(B_{n}\right) \subseteq Z\left(B_{n} / \mathcal{B} d_{n-1} \mathcal{K}\left(\mathbb{D}^{2}\right)\right)$. Então, usando a última inclusão, temos que

$$
\left\{\alpha \in \frac{\mathcal{Z}_{n-1} \mathcal{K}\left(\mathbb{D}^{2}\right)}{\mathcal{B} d_{n-1} \mathcal{K}\left(\mathbb{D}^{2}\right)} \mid 2 \alpha=0\right\} \times \mathbb{Z} \subseteq Z\left(B_{n} / \mathcal{B} d_{n-1} \mathcal{K}\left(\mathbb{D}^{2}\right)\right)
$$

Provaremos agora que a outra inclusão é válida. Dado que a seguinte sequência é exata curta

$$
1 \longrightarrow P_{n} / \mathcal{B} d_{n-1} \mathcal{K}\left(\mathbb{D}^{2}\right) \longrightarrow B_{n} / \mathcal{B} d_{n-1} \mathcal{K}\left(\mathbb{D}^{2}\right) \stackrel{\bar{\pi}}{\longrightarrow} \Sigma_{n} \longrightarrow 1
$$

e $Z\left(\Sigma_{n}\right)=\{1\}$, para $n \geq 4$, então

$$
Z\left(B_{n} / \mathcal{B} d_{n-1} \mathcal{K}\left(\mathbb{D}^{2}\right)\right) \subseteq Z\left(P_{n} / \mathcal{B} d_{n-1} \mathcal{K}\left(\mathbb{D}^{2}\right)\right)=\mathcal{Z}_{n-1} \mathcal{K}\left(\mathbb{D}^{2}\right) / \mathcal{B} d_{n-1} \mathcal{K}\left(\mathbb{D}^{2}\right) \times \mathbb{Z}
$$

Como consequência, é suficiente demonstrar que

$$
\operatorname{Tor}\left(Z\left(B_{n} / \mathcal{B} d_{n-1} \mathcal{K}\left(\mathbb{D}^{2}\right)\right)\right) \subseteq\left\{\alpha \in \frac{\mathcal{Z}_{n-1} \mathcal{K}\left(\mathbb{D}^{2}\right)}{\mathcal{B} d_{n-1} \mathcal{K}\left(\mathbb{D}^{2}\right)} \mid 2 \alpha=0\right\}
$$

Seja $x \in B_{n}$ tal que $[x] \in \operatorname{Tor}\left(Z\left(B_{n} / \mathcal{B} d_{n-1} \mathcal{K}\left(\mathbb{D}^{2}\right)\right)\right)$. Então, $[x] \in Z\left(B_{n} / \mathcal{B} d_{n-1} \mathcal{K}\left(\mathbb{D}^{2}\right)\right)$ e existe $q \in \mathbb{Z}$ tal que $q$ é o menor número tal que $x^{q} \in \mathcal{B} d_{n-1} \mathcal{K}\left(\mathbb{D}^{2}\right)$, donde $x^{q}=d_{0} y$, para algum $y \in \mathcal{N}_{n} \mathcal{K}\left(\mathbb{D}^{2}\right)$. Pela inclusão dada em (4.47) segue que $[x] \in P_{n} / \mathcal{B} d_{n-1} \mathcal{K}\left(\mathbb{D}^{2}\right)$ e portanto $x \in P_{n}$. Agora, por (1.8), para todo $0 \leq i \leq n-1$, valem as seguintes igualdades

$$
\left(d_{i} x\right)^{q}=d_{i}\left(x^{q}\right)=d_{i} d_{0} y=d_{0} d_{i+1} y=d_{0}(1)=1 .
$$

Logo, como $P_{n-1}$ é livre de torção, $d_{i} x=1$, para todo $0 \leq i \leq n-1$, isto é, $x \in \mathcal{Z}_{n-1} \mathcal{K}\left(\mathbb{D}^{2}\right)$. 
Seja $w=\sigma_{n-1} s_{n-2} x \sigma_{n-1}^{-1}$. Portanto,

$$
\begin{aligned}
d_{n+1} w & =d_{n+1} \chi_{\sigma_{n-1}}\left(s_{n-2} x\right)=\chi_{d_{n}^{B} \sigma_{n-1}} d_{(n+1) \cdot \sigma_{n-1}}\left(s_{n-2} x\right) \\
& =d_{n+1} s_{n-2} x=s_{n-2} d_{n} x=1 .
\end{aligned}
$$

Logo, $w \in\left(\mathcal{K}\left(\mathbb{D}^{2}\right)\right)_{n}$.

Agora, calculemos as outras faces aplicadas a $w$. Lembremos que o subíndice das faces são da forma simplicial, isto quer dizer que começam com o zero, enquanto o subíndice dos geradores de $B_{n}$ são os usuais, isto é, começando com o um.

- $d_{0} w=d_{0} \chi_{\sigma_{n-1}} s_{n-2} x=\chi_{\sigma_{n-2}} d_{0} s_{n-2} x=\chi_{\sigma_{n-2}} s_{n-2} d_{0} x=1$.

- Para $1 \leq j \leq n-3$ :

$$
\begin{aligned}
d_{j} w & =d_{j} \chi_{\sigma_{n-1}} s_{n-2} x=\chi_{d_{j-1}^{B} \sigma_{n-1}} d_{j \cdot \sigma_{n-1}} s_{n-2} x \\
& =\chi_{\sigma_{n-2}} d_{j} s_{n-2} x=\chi_{\sigma_{n-2}} s_{n-3} d_{j} x=1 .
\end{aligned}
$$

- Para $j=n-2$ :

$$
\begin{aligned}
d_{n-2} w & =d_{n-2} \chi_{\sigma_{n-1}} s_{n-2} x=\chi_{d_{n-3}^{B} \sigma_{n-1}} d_{(n-2) \cdot \sigma_{n-1}} s_{n-2} x \\
& =\chi_{\sigma_{n-2}} d_{n-2} s_{n-2} x=\chi_{\sigma_{n-2}}(x) .
\end{aligned}
$$

- Para $j=n-1$ :

$$
\begin{aligned}
d_{n-1} w & =d_{n-1} \chi_{\sigma_{n-1}} s_{n-2} x=\chi_{d_{n-2}^{B} \sigma_{n-1}} d_{(n-1) \cdot \sigma_{n-1}} s_{n-2} x \\
& =\chi_{\sigma_{n-1}} d_{n} s_{n-2} x=\chi_{\sigma_{n-1}} s_{n-2} d_{n-1} x=1 .
\end{aligned}
$$

- Para $j=n$ :

$$
\begin{aligned}
d_{n} w & =d_{n} \chi_{\sigma_{n-1}} s_{n-2} x=\chi_{d_{n-1}^{B} \sigma_{n-1}} d_{(n) \cdot \sigma_{n-1}} s_{n-2} x \\
& =d_{n-1} s_{n-2} x=x,
\end{aligned}
$$

pois $d_{n-1}^{B} \sigma_{n-1}=1$.

Resumindo: os elementos $1, x, \chi_{\sigma_{n-2}}(x)$ são "spherical" em $\left(\mathcal{K}\left(\mathbb{D}^{2}\right)\right)_{n-1}$; e existe um elemento $w \in\left(\mathcal{K}\left(\mathbb{D}^{2}\right)\right)_{n}$ que satisfaz as igualdades $d_{n-2}(w)=\chi_{\sigma_{n-2}}(x), d_{n}(w)=x$ e $d_{j}(w)=1$, para $j \neq n, n-2$.

Portanto, pelo Teorema de Adição em Homotopia (Teorema 1.3.18), temos que

$$
(-1)^{n-2}\left[\chi_{\sigma_{n-2}}(x)\right]+(-1)^{n}[x]=0 .
$$

Como $[x] \in Z\left(B_{n} / \mathcal{B} d_{n-1} \mathcal{K}\left(\mathbb{D}^{2}\right)\right)$, então $[x]=\left[\chi_{\sigma_{n-2}}(x)\right]$ e pela igualdade dada em 
(4.48), temos que

$$
2[x]=0,
$$

obtendo assim o resultado desejado.

Gostaríamos de fazer uma última observação. Em particular, na prova dada acima para o segundo item do Teorema 4.3.12, não fizemos uso do Lema 4.1 de [58], como feito originalmente. 


\section{Capítulo 5}

\section{Grupos de tranças e Grupos cristalográficos}

No estudo dos grupos de tranças Brunnianas sobre superfícies e sua conexão com os grupos de homotopia da esfera $\mathbb{S}^{2}$ nos deparamos com o grupo quociente $B_{3} / \operatorname{Brun}_{3}\left(\mathbb{D}^{2}\right)$ e descobrimos que este quociente é um grupo cristalográfico de dimensão 3. Ainda mais, descobrimos que, para $n \geq 3$, o grupo quociente $B_{n} /\left[P_{n}, P_{n}\right]$ é um grupo cristalográfico de dimensão $n(n-1) / 2$. Até onde conhecemos, esta ponte entre a teoria de tranças e os grupos cristalográficos é completamente nova na literatura.

Os grupos cristalográficos aparecem em muitos contextos dentro e fora da matemática. Os de dimensão 2 também são chamados grupos cristalográficos planos, ou grupos de simetrias do plano (ou ainda "wallpaper groups"). Inicialmente E. Fedorov (1891) demonstrou que existem somente 17 grupos cristalográficos planos, a menos de isomorfismo, e posteriormente uma segunda prova foi dada por G. Pólya (1924). Para mais detalhes sobre a história destes grupos ver [92]. É bem conhecido que os grupos cristalográficos planos estão relacionados com todas as possíveis tesselações ("tessellations") do plano, uma clara exposição disto pode ser encontrada em [47]. Estes grupos também foram muito usados por M.C. Escher em seu magnífico trabalho artístico (ver [72]). Em dimensão 3 os grupos cristalográficos foram enumerados por E. Fedorov (1891), e independentemente por A.M. Schönflies (1891) e W. Barlow (1894) [92]. É possível também encontrar na literatura os nomes "space group" para os grupos cristalográficos de dimensão 3 e "point group" para os respectivos grupos de holonomia.

Eventualmente denotaremos por $g \cdot x$ a ação por conjugação de um elemento $g$ de um grupo $G$ sobre um elemento $x$ do subgrupo normal $X \unlhd G$, isto é, $g \cdot x=g x g^{-1}$.

Dividiremos este capítulo como segue. Na primeira seção definiremos formalmente os grupos cristalográficos seguindo as definições tradicionais geométrica e algébrica. Mostraremos uma outra definição algébrica de grupo cristalográfico, a qual aparentemente já é conhecida, na literatura, pelos especialistas da teoria dos grupos cristalográficos. Esta última definição equivalente será usada aqui. Também na primeira seção, daremos outras 
definições que serão usadas neste capítulo, especialmente a de grupo de Bieberbach. Na segunda seção estudaremos o caso motivador deste capítulo $B_{3} / \operatorname{Brun}_{3}\left(\mathbb{D}^{2}\right)$, onde obtemos resultados detalhados deste grupo. Inicialmente provaremos que este quociente é um grupo cristalográfico e que os elementos de torção deste grupo só tem ordem 3. Ainda mais, os elementos de ordem 3 formam uma única classe de conjugação. Na terceira seção estenderemos para $n \geq 3$ alguns dos resultados da seção anterior. Investigaremos a ação (por conjugação) de $\Sigma_{n}$ em $P_{n} /\left[P_{n}, P_{n}\right]$ e mostraremos que o grupo quociente $B_{n} /\left[P_{n}, P_{n}\right]$, para

$n \geq 3$, é um grupo cristalográfico sem elementos de torção 2. Na última seção relacionaremos vários dos resultados obtidos nas seções anteriores com variedades Riemannianas compactas planas e discutiremos a existência de difeomorfismos de Anosov em alguns destes casos.

\subsection{Grupos cristalográficos e de Bieberbach}

Iniciaremos esta seção com algumas definições, seguindo a Seção 2.1 de [23] e a Seção 1.1 de [16]. Denotemos por Aff $\left(\mathbb{R}^{n}\right)$ o grupo afim de $\mathbb{R}^{n}$, Aff $\left(\mathbb{R}^{n}\right)=\mathbb{R}^{n} \rtimes G L(n ; \mathbb{R})$, onde $G L(n ; \mathbb{R})$ é o grupo de Lie formado por matrizes invertíveis de tamanho $n \times n$ sobre os reais. Observemos que o grupo $G L(n ; \mathbb{R})$ é igual, como grupo de Lie, ao grupo de automorfismos de $\mathbb{R}^{n}$, Aut $\left(\mathbb{R}^{n}\right)$. Diremos que um subgrupo $\Pi$ de $G L(n ; \mathbb{R})$ é uniforme se $\Pi \backslash \mathbb{R}^{n}$ é compacto onde o espaço de órbitas, $\Pi \backslash \mathbb{R}^{n}$, é o conjunto de órbitas com a topologia quociente. Se a ação de $\Pi$ sobre $\mathbb{R}^{n}$ é livre (e portanto, $\Pi$ é livre de torção), então o espaço quociente $\Pi \backslash \mathbb{R}^{n}$ é uma variedade. Denotaremos por $O(n ; \mathbb{R})$ o subgrupo das matrizes ortogonais de $G L(n ; \mathbb{R})$. É bem conhecido que $O(n ; \mathbb{R})$ é um subgrupo compacto maximal de $G L(n ; \mathbb{R})$.

Definição 5.1.1. Um subgrupo discreto uniforme $\Pi$ de $\mathbb{R}^{n} \rtimes O(n ; \mathbb{R}) \subseteq A f f\left(\mathbb{R}^{n}\right)$ é chamado um grupo cristalográfico de dimensão $n$. Se П é livre de torção, então П é um grupo de Bieberbach de dimensão $n$.

Entre algumas referências bibliográficas sobre os grupos cristalográficos e que são bem conhecidas citamos os livros de L. Charlap [16], K. Dekimpe [23] e J. Wolf [95]. Assim como no livro de Wolf, nos livros de W. Thurston [89] e J. Ratcliffe [80] também são estudados os grupos cristalográficos e de Bieberbach por sua grande importância geométrica. Não é o propósito desta introdução explicar a origem da palavra "cristalográfico" que aparece na Definição 5.1.1. No entanto, remetemos o leitor ao livro de H. Brown, R. Bülow, J. Neubüser, H. Wondratschek e H. Zassenhaus intitulado "Crystallographic groups of four-dimensional space" [14] onde é explicado com detalhes este e outros assuntos relacionados a tais grupos.

Os grupos cristalográficos são bem conhecidos pelo trabalho de L. Bieberbach, que formulou e demonstrou três famosos teoremas sobre grupos cristalográficos (um deles também é conhecido como Teorema de Fröbenius), os quais foram publicados entre 1911 e 1912 e 
hoje em dia são conhecidos como os três Teoremas de Bieberbach. Estes teoremas estão relacionados com aspectos da Geometria Riemanniana, por este motivo introduziremos a seguinte definição. Assumiremos que o conceito de variedade Riemanniana é familiar.

Definição 5.1.2. Uma variedade Riemanniana $M$ é chamada plana se sua curvatura é zero em cada ponto ("flat Riemannian manifold").

A seguir, enunciaremos e comentaremos os Teoremas de Bieberbach sobre grupos cristalográficos da forma como aparece no livro de Dekimpe [23]. Como mencionado em [23], a ordem dos teoremas abaixo não corresponde com a ordem histórica em que eles apareceram.

Teorema 5.1.3 (Primeiro Teorema de Bieberbach). Seja $\Pi$ um grupo cristalográfico de dimensão n. Então $\Gamma=\Pi \cap \mathbb{R}^{n}$ é um reticulado de $\mathbb{R}^{n}$ e $\Pi / \Gamma$ é finito.

Este teorema diz que para um grupo cristalográfico $\Pi$ de dimensão $n$, a parte das translações de $\mathbb{R}^{n}, \Gamma=\Pi \cap \mathbb{R}^{n}$, é um grupo abeliano livre isomorfo com $\mathbb{Z}^{n}$ e tal que o espaço vetorial sobre os reais gerado por $\Gamma$ é o próprio espaço $\mathbb{R}^{n}$. Se $\Pi=\Gamma$, então $\Gamma$ é livre de torção e a variedade $\Pi \backslash \mathbb{R}^{n}$ é um toro de dimensão $n$. Se $\Pi$ é um grupo livre de torção, temos que a variedade $M={ }_{\Pi} \backslash \mathbb{R}^{n}$ herda a estrutura Riemanniana plana de $\mathbb{R}^{n}$ e portanto $M$ é uma variedade Riemanniana compacta plana ("compact flat Riemannian manifold"). Ainda mais, todas as variedades Riemannianas compactas planas são obtidas desta forma. Assim, existe uma correspondência um-a-um entre os grupos de Bieberbach e os grupos fundamentais das variedades Riemannianas compactas planas.

Observação 5.1.4. No que segue nesse trabalho, por abuso de linguagem, diremos de forma curta que $M$ é uma variedade plana, assumindo que $M$ é uma variedade Riemanniana compacta plana.

Como comentado em [16, Observação i) na página 17], porém usando a notação do Teorema 5.1.3, se П é um grupo cristalográfico, então existe uma sequência exata curta

$$
0 \longrightarrow \mathbb{Z}^{n} \longrightarrow \Pi \longrightarrow \Phi \longrightarrow 1
$$

onde $\mathbb{Z}^{n}=\Gamma$ é um reticulado de posto $n$ e $\Phi=\Pi / \Gamma$ é um grupo finito. Chamaremos o grupo $\Phi$ de grupo de holonomia de $\Pi$.

Teorema 5.1.5 (Segundo Teorema de Bieberbach). Sejam $\Pi$ e $\Pi^{\prime}$ dois grupos cristalográficos de dimensão $n$. Se $\psi: \Pi \rightarrow \Pi^{\prime}$ é um isomorfismo, então existe um elemento $\alpha \in \operatorname{Aff}\left(\mathbb{R}^{n}\right)$ tal que, para todo $\gamma \in \Pi, \psi(\gamma)=\alpha \gamma \alpha^{-1}$.

Do segundo Teorema de Bieberbach podemos deduzir que as variedades planas são, a menos de um difeomorfismo apropriado, completamente determinadas por seu grupo fundamental. 
Teorema 5.1.6 (Terceiro Teorema de Bieberbach ou Teorema de Fröbenius). A menos de conjugação em $\operatorname{Aff}\left(\mathbb{R}^{n}\right)$, existe unicamente um número finito de grupos cristalográficos de dimensão $n$.

Este último teorema implica que, a menos de um difeomorfismo afim, existem finitas variedades planas de dimensão $n$.

Observação 5.1.7. Os três teoremas de Bieberbach respondem parcialmente ao Problema 18, dos 23, presentados por D. Hilbert durante o Congresso Internacional de Matemáticas, realizado em Paris em 1900 [71].

É natural perguntar quais grupos finitos são realizados como o grupo de holonomia de uma variedade plana. Uma surpreendente resposta a esta pergunta foi dada por L. Auslander e M. Kuranishi em 1957.

Teorema 5.1.8 (Teorema de Auslander e Kuranishi, Teorema 3.4.8 de [95]). Seja G um grupo finito qualquer. Então $G$ é o grupo de holonomia de alguma variedade plana.

A estrutura algébrica dos grupos cristalográficos é bem conhecida e será enunciada na continuação como um teorema, uma parte segue do Primeiro Teorema de Bieberbach e a recíproca é atribuída a $\mathrm{H}$. Zassenhaus.

Teorema 5.1.9 (Caracterização algébrica, Teorema 2.1.4 de [23]). Seja П um grupo cristalográfico de dimensão $n$, então $\Gamma=\Pi \cap \mathbb{R}^{n}$ é o único subgrupo normal, abeliano maximal de $\Pi$.

Reciprocamente, seja $Q$ um grupo abstrato tal que $Q$ contém um subgrupo normal, abeliano maximal $\Gamma$ de indice finito, com $\Gamma \cong \mathbb{Z}^{n}$, então existe um homomorfismo injetor $\phi: Q \rightarrow \operatorname{Aff}\left(\mathbb{R}^{n}\right)$ tal que $\phi(Q)$ é um grupo cristalográfico de dimensão $n$.

Dado um grupo $Q$ satisfazendo as condições do Teorema 5.1 .9 o chamaremos de grupo cristalográfico (ou de Bieberbach, quando for o caso), mas entendendo que ele é isomorfo a um grupo cristalográfico da Definição 5.1.1.

Para atingir um dos nossos objetivos neste capítulo, precisaremos de uma definição algébrica equivalente a dada no Teorema 5.1.9. Para isto, inicialmente teremos que introduzir a definição de representação integral de grupos finitos, ver a Seção 6 do Capítulo I de [16]. Uma matriz unimodular $M$ é uma matriz quadrada $n \times n$ com entradas inteiras e determinante +1 ou -1 . O conjunto $J_{n}$ formado pelas matrizes unimodulares de tamanho $n \times n$ é um subgrupo de $G L(n ; \mathbb{Z})$.

Definição 5.1.10. Seja $\Phi$ um grupo. Uma representação integral de $\Phi$ de posto $n$ é um homomorfismo $\Theta: \Phi \rightarrow J_{n}$. Diremos que duas tais representações são equivalentes se suas imagens são conjugadas em $J_{n}$. Se o homomorfismo $\Theta: \Phi \rightarrow J_{n}$ é injetor diremos que $\Theta$ é uma representação fiel. 
Proposição 5.1.11. Seja П um grupo cristalográfico de dimensão n com grupo de holonomia $\Phi$. Então, existe uma sequência exata curta, como em (5.1), tal que a representação integral $\Theta: \Phi \rightarrow J_{n}$ é fiel.

Demonstração. A conclusão deste resultado é garantida pelo parágrafo anterior à Definição 6.2 de [16], pois a partir da sequência exata curta (5.1) temos uma representação integral $\Theta: \Phi \rightarrow J_{n}$ e pela Proposição 6.1 de [16] a representação $\Theta$ é fiel.

Nosso objetivo na continuação é demonstrar a recíproca da Proposição 5.1.11, com isto teremos uma definição equivalente de grupo cristalográfico. Esta segunda caracterização algébrica será usada neste capítulo.

Lema 5.1.12. Sejam $\Phi$ um grupo finito, П um grupo qualquer e $n \geq 1$ um número inteiro. $\Pi$ é um grupo cristalográfico de dimensão n, com grupo de holonomia $\Phi$ se, e somente se, existe uma sequência exata curta

$$
0 \longrightarrow \mathbb{Z}^{n} \longrightarrow \Pi \longrightarrow \Phi \longrightarrow 1
$$

tal que a representação integral, induzida pela ação por conjugação, $\Theta: \Phi \rightarrow J_{n}$ é fiel.

Observação 5.1.13. Aparentemente a equivalência dada no Lema 5.1.12 já é conhecida. Por exemplo, tal equivalência foi usada em [1], porém os autores citam a página 84 do livro de Charlap [16] e em tal página apenas encontramos a caracterização dada no Teorema 5.1.9. Contudo, já que nós não encontramos uma demonstração do Lema 5.1.12, decidimos dar uma prova aqui.

Demonstração. Sejam $\Phi$ um grupo finito, $\Pi$ um grupo e $n \geq 1$ um número inteiro. Suponhamos que existe uma sequência exata curta

$$
0 \longrightarrow \mathbb{Z}^{n} \longrightarrow \Pi \stackrel{\zeta}{\longrightarrow} \Phi \longrightarrow 1
$$

tal que a representação integral, induzida pela ação por conjugação, $\Theta: \Phi \rightarrow J_{n}$ é fiel. Vamos supor por contradição que П não é um grupo cristalográfico, então pela caracterização algébrica dada no Teorema 5.1.9 temos que $\mathbb{Z}^{n}$ não pode ser um grupo abeliano maximal em П. Portanto, existe um grupo abeliano $A$ tal que $\mathbb{Z}^{n} \subsetneq A \subseteq \Pi$. Seja $a \in A \backslash \mathbb{Z}^{n}$. Como $\mathbb{Z}^{n}$ é um subgrupo estritamente contido no grupo abeliano $A$, então $\zeta(a) \neq 1$ e todo elemento de $\mathbb{Z}^{n}$ é fixo pela ação por conjugação por $a$. Isto é, provamos que existe um elemento $a \in \Pi$ tal que $\zeta(a) \neq 1$ e $\Theta(a)$ é igual à matriz identidade de $J_{n}$. Logo, $\Theta$ não é injetora, mas isto é uma contradição e portanto $\Pi$ é um grupo cristalográfico de dimensão $n$ com grupo de holonomia $\Phi$.

A recíproca é o enunciado da Proposição 5.1.11, que já foi demonstrada. 
Definição 5.1.14. Seja П um grupo cristalográfico de dimensão n com grupo de holonomia $\Phi$. A representação integral $\Theta: \Phi \rightarrow J_{n}$, do Lema 5.1.12, é chamada de representação de holonomia de $\Pi$.

Lembramos que dada uma propriedade $P$, o grupo $G$ é chamado virtualmente $P$ se existe um subgrupo de índice finito $H \leq G$ tal que $H$ tem a propriedade $P$. Observamos que, pelo Lema 5.1.12, os grupos cristalográficos são grupos virtualmente abelianos e portanto virtualmente nilpotentes, bem como grupos virtualmente policíclicos ("polycyclicby-finite groups").

Como mencionado na introdução de [48], diremos que a variedade plana determinada pelo grupo de Bieberbach $\Pi$ é uma variedade plana orientável se, e somente se, a representação integral $\Theta: \Phi \rightarrow J_{n}$ é tal que $\Theta(\Phi) \subseteq S O(n ; \mathbb{Z})$ e neste caso diremos que $\Pi$ é um grupo de Bieberbach orientável.

Diremos que uma representação integral $\Psi$ é decomponivel se existem representações integrais $\Psi_{1}$ e $\Psi_{2}$ tais que $\Psi$ é equivalente à soma $\Psi_{1} \oplus \Psi_{2}$. Se $\Psi$ não é decomponível diremos então que $\Psi$ é indecomponível, ver [82, Página 151]. No livro intitulado "Linear representations of finite groups" [85], de J. P. Serre, as representações (in)decomponíveis são chamadas de (ir)redutíveis. O interesse em estudar representações decomponíveis provém da teoria de representações, no entanto no caso dos grupos cristalográficos pode ter outras aplicações, como por exemplo, a informação obtida sobre a decomposição de representações integrais de grupos finitos, associados a grupos de Bieberbach, foi utilizada por Hiss e Szczepański [48] para determinar a existência de estruturas spin em variedades planas orientáveis. Porém, isto não será tratado aqui.

\subsection{Tranças com 3 cordas sobre o disco e grupos cris- talográficos}

Nesta seção investigaremos o grupo quociente $B_{3} / \operatorname{Brun}_{3}\left(\mathbb{D}^{2}\right)$. Mostraremos que este grupo é cristalográfico e estudaremos seus elementos de torção. Para isto utilizaremos a forma matricial da representação integral $\Theta: \Sigma_{3} \rightarrow \operatorname{Aut}\left(P_{3} / \operatorname{Brun}_{3}\left(\mathbb{D}^{2}\right)\right)$.

\subsubsection{A representação integral $\Theta: \Sigma_{3} \rightarrow \operatorname{Aut}\left(P_{3} / \operatorname{Brun}_{3}\left(\mathbb{D}^{2}\right)\right)$}

Pela Proposição 4.3.10 sabemos que $P_{3} / \operatorname{Brun}_{3}\left(\mathbb{D}^{2}\right) \cong \mathbb{Z} \oplus \mathbb{Z} \oplus \mathbb{Z}$. Vamos provar nesta subseção que o grupo $B_{3} / \operatorname{Brun}_{3}\left(\mathbb{D}^{2}\right)$ é um grupo cristalográfico e para isto estudaremos a representação $\Theta: \Sigma_{3} \rightarrow \operatorname{Aut}\left(P_{3} / \operatorname{Brun}_{3}\left(\mathbb{D}^{2}\right)\right)$, onde $P_{3} / \operatorname{Brun}_{3}\left(\mathbb{D}^{2}\right) \cong \mathbb{Z} \oplus \mathbb{Z} \oplus \mathbb{Z}$. Consideraremos nesse caso a sequência exata curta

$$
1 \longrightarrow P_{3} / \operatorname{Brun}_{3}\left(\mathbb{D}^{2}\right) \longrightarrow B_{3} / \operatorname{Brun}_{3}\left(\mathbb{D}^{2}\right) \stackrel{\bar{\sigma}}{\longrightarrow} \Sigma_{3} \longrightarrow 1
$$


a qual foi obtida a partir da sequência exata curta

$$
1 \longrightarrow P_{3} \longrightarrow B_{3} \stackrel{\sigma}{\longrightarrow} \Sigma_{3} \longrightarrow 1
$$

Seja $q: B_{3} \rightarrow B_{3} / \operatorname{Brun}_{3}\left(\mathbb{D}^{2}\right)$ o homomorfismo quociente. A seguinte notação será usada nesta subseção, $\tau_{i}=q\left(\sigma_{i}\right)$, para $i=1,2$, e $N_{i, j}=q\left(A_{i, j}\right)$, para $1 \leq i<j \leq 3$. Além disso, denotaremos os elementos $\bar{\sigma}\left(\tau_{i}\right)=\sigma\left(\sigma_{i}\right)$, para $i=1,2$, por $\mu_{i}$.

Teorema 5.2.1. A representação integral $\Theta: \Sigma_{3} \rightarrow \operatorname{Aut}\left(P_{3} / \operatorname{Brun}_{3}\left(\mathbb{D}^{2}\right)\right)$, obtida a partir da sequência exata curta (5.4) é fiel. Como consequência temos que o grupo quociente $B_{3} /$ Brun $_{3}\left(\mathbb{D}^{2}\right)$ é um grupo cristalográfico de dimensão 3 com grupo de holonomia $\Sigma_{3}$.

Demonstração. Sabemos que o grupo $P_{3}\left(\mathbb{D}^{2}\right) / \operatorname{Brun}_{3}\left(\mathbb{D}^{2}\right)=\mathbb{Z} \oplus \mathbb{Z} \oplus \mathbb{Z}$ é gerado pelas classes de equivalência de $N_{1,2}, N_{1,3}, N_{2,3}$ [40]. Denotemos por $e_{1}=(1,0,0)=N_{1,2}$, $e_{2}=(0,1,0)=N_{2,3}$ e $e_{3}=(0,0,1)=N_{1,3}$ os geradores deste quociente.

A conjugação $\sigma_{k} A_{i, j} \sigma_{k}^{-1}$, para $1 \leq k \leq n-1$ e para $1 \leq i<j \leq n$, foi descrita em (4.31). Logo, a ação por conjugação dos elementos $\tau_{1}, \tau_{2}$ de $B_{3} / \operatorname{Brun}_{3}\left(\mathbb{D}^{2}\right)$ sobre os elementos $e_{1}, e_{2}, e_{3}$ é dada por

$$
\begin{array}{lll}
\tau_{1} e_{1} \tau_{1}^{-1}=e_{1}, & \tau_{1} e_{2} \tau_{1}^{-1}=e_{3}, & \tau_{1} e_{3} \tau_{1}^{-1}=e_{2}, \\
\tau_{2} e_{1} \tau_{2}^{-1}=e_{3}, & \tau_{2} e_{2} \tau_{2}^{-1}=e_{2}, & \tau_{2} e_{3} \tau_{2}^{-1}=e_{1} .
\end{array}
$$

Portanto, a representação

$$
\Theta: \Sigma_{3} \rightarrow \operatorname{Aut}\left(P_{3} / \operatorname{Brun}_{3}\left(\mathbb{D}^{2}\right)\right)
$$

é definida por:

$$
\begin{array}{rlrl}
(1) & \mapsto\left(\begin{array}{ccc}
1 & 0 & 0 \\
0 & 1 & 0 \\
0 & 0 & 1
\end{array}\right), & \tau_{2} \tau_{1} \mapsto\left(\begin{array}{ccc}
0 & 1 & 0 \\
0 & 0 & 1 \\
1 & 0 & 0
\end{array}\right), \\
\tau_{1} \mapsto\left(\begin{array}{ccc}
1 & 0 & 0 \\
0 & 0 & 1 \\
0 & 1 & 0
\end{array}\right), & \tau_{1} \tau_{2} \mapsto\left(\begin{array}{ccc}
0 & 0 & 1 \\
1 & 0 & 0 \\
0 & 1 & 0
\end{array}\right), \\
\tau_{2} \mapsto\left(\begin{array}{ccc}
0 & 0 & 1 \\
0 & 1 & 0 \\
1 & 0 & 0
\end{array}\right), & \tau_{1} \tau_{2} \tau_{1} \mapsto\left(\begin{array}{ccc}
0 & 1 & 0 \\
1 & 0 & 0 \\
0 & 0 & 1
\end{array}\right) .
\end{array}
$$

Pode-se ver claramente que as matrizes dadas em (5.7) são matrizes de permutação, portanto ortogonais, e desta forma $\Theta\left(\Sigma_{3}\right) \subseteq O(3 ; \mathbb{Z})$. Podemos considerar assim a representação integral

$$
\Theta: \Sigma_{3} \rightarrow O(3 ; \mathbb{Z}) .
$$


Além disso, as matrizes de (5.7) são diferentes entre si. Logo, o homomorfismo

$$
\Theta: \Sigma_{3} \rightarrow O(3 ; \mathbb{Z})
$$

é uma representação integral fiel. Portanto, pelo Lema 5.1.12, $B_{3} / \operatorname{Brun}_{3}\left(\mathbb{D}^{2}\right)$ é um grupo cristalográfico de dimensão 3 com grupo de holonomia $\Sigma_{3}$.

Observação 5.2.2. Pelo Corolário 2.1.4, temos que $\left[P_{3}, P_{3}\right]=$ Brun $_{3}\left(\mathbb{D}^{2}\right)$ e portanto o grupo quociente $B_{3} /$ Brun $_{3}\left(\mathbb{D}^{2}\right)=B_{3} /\left[P_{3}, P_{3}\right]$ é um grupo cristalográfico de dimensão 3 , com grupo de holonomia $\Sigma_{3}$.

Corolário 5.2.3. Seja $\Theta: \Sigma_{3} \rightarrow O(3 ; \mathbb{Z})$ a representação integral dada em (5.8). As matrizes $\Theta\left(\tau_{1}\right), \Theta\left(\tau_{2}\right)$ e $\Theta\left(\tau_{1} \tau_{2} \tau_{1}\right)$ têm determinante -1 . As matrizes $\Theta\left(\tau_{1} \tau_{2}\right)$ e $\Theta\left(\tau_{2} \tau_{1}\right)$ têm determinante 1 .

Como consequência, a representação integral $\Theta: \Sigma_{3} \rightarrow O(3 ; \mathbb{Z})$ é tal que

$$
\Theta(H) \nsubseteq S O(3 ; \mathbb{Z})
$$

onde $H \subseteq \Sigma_{3}$ é um subgrupo de ordem 2. Porém, a restrição de $\Theta$ ao subgrupo alternado $\mathcal{A}_{3}$ de $\Sigma_{3}$ é tal que

$$
\Theta \mid: \mathcal{A}_{3} \rightarrow S O(3 ; \mathbb{Z})
$$

Demonstração. A prova deste resultado segue claramente analisando cada uma das matrizes dadas em (5.7).

Um segundo corolário do Teorema 5.2.1 garante a existência de grupos cristalográficos de dimensão 3 , com grupo de holonomia contido em $\Sigma_{3}$ e obtidos a partir dos grupos de tranças com 3 cordas sobre o disco.

Corolário 5.2.4. Seja $H$ um subgrupo qualquer de $\Sigma_{3}$. Então, o grupo

$$
\frac{\sigma^{-1}(H)}{\operatorname{Brun}_{3}\left(\mathbb{D}^{2}\right)}
$$

é um grupo cristalográfico de dimensão 3 e com grupo de holonomia $H$, onde $\sigma$ é o epimorfismo dado na sequência exata curta (5.5).

Demonstração. Seja $H$ um subgrupo de $\Sigma_{3}$. Consideremos a sequência exata curta

$$
1 \longrightarrow P_{3} / \operatorname{Brun}_{3}\left(\mathbb{D}^{2}\right) \longrightarrow \frac{\sigma^{-1}(H)}{\operatorname{Brun}_{3}\left(\mathbb{D}^{2}\right)} \stackrel{\bar{\sigma}}{\longrightarrow} H \longrightarrow 1
$$

Pelo Teorema 5.2.1 a representação de holonomia $\Theta: \Sigma_{3} \rightarrow \operatorname{Aut}\left(P_{3} / \operatorname{Brun}_{3}\left(\mathbb{D}^{2}\right)\right)$ é fiel e portanto sua restrição ao grupo $H, \Theta \mid: H \rightarrow \operatorname{Aut}\left(P_{3} / \operatorname{Brun}_{3}\left(\mathbb{D}^{2}\right)\right)$, é por sua vez injetora. 
Logo, pelo Lema 5.1 .12 e pelo fato que $P_{3} / \operatorname{Brun}_{3}\left(\mathbb{D}^{2}\right) \cong \mathbb{Z} \oplus \mathbb{Z} \oplus \mathbb{Z}$, temos que $\frac{\sigma^{-1}(H)}{\operatorname{Brun}_{3}\left(\mathbb{D}^{2}\right)}$ é um grupo cristalográfico de dimensão 3 e com grupo de holonomia $H$.

Definição 5.2.5. Seja $H$ um subgrupo de $\Sigma_{3}$, denotaremos o grupo dado em (5.10) do Corolário 5.2.4 por $\mathcal{B C G}(3, H)$ e o chamaremos de grupo cristalográfico de tranças com 3 cordas com grupo de holonomia $H$. Pelo Corolário 5.2.4, $\mathcal{B C G}(3, H)$ tem dimensão 3.

Observemos que $\mathcal{B C G}\left(3, \Sigma_{3}\right)=B_{3} / \operatorname{Brun}_{3}\left(\mathbb{D}^{2}\right)$ e $\mathcal{B C G}(3,\{1\})=\mathbb{Z} \oplus \mathbb{Z} \oplus \mathbb{Z}$ é um grupo de Bieberbach que é o grupo fundamental do toro de dimensão $3, \mathbb{S}^{1} \times \mathbb{S}^{1} \times \mathbb{S}^{1}$.

Lembremos que $\Sigma_{3}=\mathbb{Z}_{3} \rtimes \mathbb{Z}_{2}$, onde $\mathbb{Z}_{3}=\mathcal{A}_{3}$ o subgrupo alternado e $\mathbb{Z}_{2}=\left\langle\sigma_{1} \mid \sigma_{1}^{2}=1\right\rangle$. A seguir, vamos dar atenção especial aos grupos cristalográficos $\mathcal{B C} \mathcal{G}\left(3, \mathcal{A}_{3}\right)$ e $\mathcal{B C G}\left(3,\left\langle\sigma_{1}\right\rangle\right)$. Observemos que $\sigma^{-1}\left(\left\langle\sigma_{1} \mid \sigma_{1}^{2}=1\right\rangle\right)=B_{2,1}$, um subgrupo misto de $B_{3}$, e portanto

$$
\mathcal{B C G}\left(3,\left\langle\sigma_{1}\right\rangle\right)=\frac{B_{2,1}}{\operatorname{Brun}_{3}\left(\mathbb{D}^{2}\right)}
$$

Na seguinte proposição usaremos a seguinte notação: $\alpha_{0,3}=\tau_{1} \tau_{2}$ e $\overline{\Delta_{3}}=q\left(\left(\sigma_{1} \sigma_{2}\right)^{3}\right)$, onde $\Delta_{3}$ é o "full twist" em $B_{3}$ e $q: B_{3} \rightarrow B_{3} / \operatorname{Brun}_{3}\left(\mathbb{D}^{2}\right)$ é o homomorfismo quociente.

\section{Proposição 5.2.6.}

1. O grupo cristalográfico de dimensão 3 com grupo de holonomia $\mathbb{Z}_{3}, \mathcal{B C G}\left(3, \mathcal{A}_{3}\right)$, é normal em $\mathcal{B C G}\left(3, \Sigma_{3}\right)$. Este grupo tem uma apresentação dada por

- Geradores: $N_{1,2}, N_{2,3}, N_{1,3}, \alpha_{0,3}$.

- Relações:

(a) $\left[N_{1,2}, N_{1,3}\right]=1,\left[N_{1,2}, N_{2,3}\right]=1,\left[N_{1,3}, N_{1,3}\right]=1$.

(b) $\alpha_{0,3}^{3}=\overline{\Delta_{3}}$.

(c) $\alpha_{0,3} N_{1,2} \alpha_{0,3}^{-1}=N_{2,3}, \alpha_{0,3} N_{1,3} \alpha_{0,3}^{-1}=N_{1,2}, \alpha_{0,3} N_{2,3} \alpha_{0,3}^{-1}=N_{1,3}$.

Segue desta apresentação dada para o grupo $\mathcal{B C G}\left(3, \mathcal{A}_{3}\right)$ que

$$
\left(\mathcal{B C G}\left(3, \mathcal{A}_{3}\right)\right)_{a b}=\left\langle N_{1,2}, \alpha_{0,3} \mid\left[N_{1,2}, \alpha_{0,3}\right]=1, \alpha_{0,3}^{3}=N_{1,2}^{3}\right\rangle,
$$

e portanto $\left(\mathcal{B C G}\left(3, \mathcal{A}_{3}\right)\right)_{a b} \cong \mathbb{Z} \oplus \mathbb{Z}_{3}$, gerado por $N_{1,2}$ e $N_{1,2} \alpha_{0,3}^{-1}$.

2. O grupo cristalográfico de dimensão 3 com grupo de holonomia $\mathbb{Z}_{2}, \mathcal{B C G}\left(3,\left\langle\mu_{1}\right\rangle\right)$, não é normal em $\mathcal{B C G}\left(3, \Sigma_{3}\right)$. Uma apresentação para $\mathcal{B C G}\left(3,\left\langle\mu_{1}\right\rangle\right)$ é dada por

- Geradores: $N_{1,2}, N_{2,3}, N_{1,3}, \tau_{1}$.

- Relações:

(a) $\left[N_{1,2}, N_{1,3}\right]=1,\left[N_{1,2}, N_{2,3}\right]=1,\left[N_{1,3}, N_{1,3}\right]=1$. 


$$
\begin{aligned}
& \text { (b) } \tau_{1}^{2}=N_{1,2} . \\
& \text { (c) } \tau_{1} N_{1,2} \tau_{1}^{-1}=N_{1,2}, \tau_{1} N_{1,3} \tau_{1}^{-1}=N_{2,3}, \tau_{1} N_{2,3} \tau_{1}^{-1}=N_{1,3}
\end{aligned}
$$

Segue desta apresentação dada para o grupo $\mathcal{B C G}\left(3,\left\langle\mu_{1}\right\rangle\right)$ que

$$
\left(\mathcal{B C G}\left(3,\left\langle\mu_{1}\right\rangle\right)\right)_{a b}=\left\langle N_{1,2}, N_{1,3}, \tau_{1} \mid\left[N_{1,2}, \tau_{1}\right]=1,\left[N_{1,3}, \tau_{1}\right]=1, \tau_{1}^{2}=N_{1,2}\right\rangle
$$

e portanto $\left(\mathcal{B C G}\left(3,\left\langle\mu_{1}\right\rangle\right)\right)_{a b} \cong \mathbb{Z} \oplus \mathbb{Z}$

Demonstração. Como $\mathcal{B C G}(3, H)=\frac{\sigma^{-1}(H)}{\operatorname{Brun}_{3}\left(\mathbb{D}^{2}\right)}$, então o resultado sobre normalidade desta proposição é uma consequência do resultado sobre normalidade em $\Sigma_{3}=\mathbb{Z}_{3} \rtimes \mathbb{Z}_{2}$.

As apresentações dadas para $\mathcal{B C G}(3, H)$, onde $H$ é um dos grupos $\mathcal{A}_{3}$ ou $\left\langle\mu_{1}\right\rangle$, foram obtidas usando o método de apresentações de extensões de grupos dado na Seção 10.2 (página 138) do livro do Johnson [50]. A expressão para o grupo abelianizado, em cada caso, foi obtida a partir da apresentação dada para o grupo $\mathcal{B C G}(3, H)$.

\subsubsection{Elementos de torção em $B_{3} / \operatorname{Brun}_{3}\left(\mathbb{D}^{2}\right)$}

Nesta seção provaremos que o grupo quociente $B_{3} / \operatorname{Brun}_{3}\left(\mathbb{D}^{2}\right)$ não tem elementos de torção 2. Também provaremos que todo elemento de torção não trivial tem ordem 3 e pertence a uma única classe de conjugação.

Lema 5.2.7. Se $\omega$ em $B_{3} /$ Brun $_{3}\left(\mathbb{D}^{2}\right)$ é um elemento de torção, então ele tem ordem 2 ou 3.

Demonstração. Como um elemento de torção de $\Sigma_{3}$ tem ordem 2 ou 3, então estas são as únicas possibilidades para a ordem dos elementos de torção em $B_{3} / \operatorname{Brun}_{3}\left(\mathbb{D}^{2}\right)$, pois $\bar{\sigma}: B_{3} / \operatorname{Brun}_{3}\left(\mathbb{D}^{2}\right) \rightarrow \Sigma_{3}$ é um homomorfismo e o núcleo é livre de torção.

Destacaremos um par de exemplos de elementos de ordem 3 em $B_{3} / \operatorname{Brun}_{3}\left(\mathbb{D}^{2}\right)$.

\section{Exemplo 5.2.8.}

- O elemento $A_{2,3}^{-1} \sigma_{2} \sigma_{1}=\sigma_{2}^{-1} \sigma_{1}$ é tal que

$$
\begin{aligned}
\left(A_{2,3}^{-1} \sigma_{2} \sigma_{1}\right)^{3} & =\left(\sigma_{2}^{-1} \sigma_{1}\right)^{3} \\
& =\sigma_{2}^{-2} \sigma_{1}^{-2} \sigma_{2}^{2} \sigma_{1}^{2} \\
& =\left[A_{2,3}, A_{1,2}\right] .
\end{aligned}
$$

Portanto, $A_{2,3}^{-1} \sigma_{2} \sigma_{1}=\sigma_{2}^{-1} \sigma_{1} \in B_{3}$ é um representante da classe de um elemento de ordem 3 no quociente $B_{3} /\left[P_{3}, P_{3}\right]$. 
- De forma similar $A_{1,2}^{-1} \sigma_{1} \sigma_{2}=\sigma_{1}^{-1} \sigma_{2} \in B_{3}$ é um representante da classe de um elemento de ordem 3 no quociente $B_{3} /\left[P_{3}, P_{3}\right]$, pois $\left(A_{1,2}^{-1} \sigma_{1} \sigma_{2}\right)^{3}=\left[A_{1,2}, A_{2,3}\right]$. Observemos que o fecho da trança $\left(A_{1,2}^{-1} \sigma_{1} \sigma_{2}\right)^{3}$ é o enlaçamento chamado de anéis de Borromeo, ver Figuras 1.15 e 2.7.

Lembremos que $P_{3} / \operatorname{Brun}_{3}\left(\mathbb{D}^{2}\right) \cong \mathbb{Z} \oplus \mathbb{Z} \oplus \mathbb{Z}$ e gerado por $e_{1}=N_{1,2}, e_{2}=N_{2,3}$ e $e_{3}=N_{1,3}$. Seja $\delta_{3}=\tau_{1}^{-1} \tau_{2}=q\left(\sigma_{1}^{-1} \sigma_{2}\right) \in B_{3} / \operatorname{Brun}_{3}\left(\mathbb{D}^{2}\right)$ e como antes, consideremos $\alpha_{0,3}=\tau_{1} \tau_{2} \in B_{3} / \operatorname{Brun}_{3}\left(\mathbb{D}^{2}\right)$. Segue de $(5.7)$ que a ação por conjugação de $\delta_{3}$ e $\alpha_{0,3}$ nos elementos $e_{1}, e_{2}, e_{3}$ é dada por:

$$
\begin{array}{ll}
\delta_{3} \cdot e_{1}=e_{2}, & \alpha_{0,3} \cdot e_{1}=e_{2}, \\
\delta_{3} \cdot e_{2}=e_{3}, & \alpha_{0,3} \cdot e_{2}=e_{3}, \\
\delta_{3} \cdot e_{3}=e_{1}, & \alpha_{0,3} \cdot e_{3}=e_{1} .
\end{array}
$$

Observemos que $\left(\sigma_{1} \sigma_{2}\right)^{3}=\Delta_{3}$ é o "full twist" em $P_{3}$ e portanto

$$
\overline{\Delta_{3}}=\alpha_{0,3}^{3}=(1,1,1) \in \mathbb{Z} \oplus \mathbb{Z} \oplus \mathbb{Z}=P_{3} / \operatorname{Brun}_{3}\left(\mathbb{D}^{2}\right) .
$$

Além disso, pelo Exemplo 5.2.8, temos que $\delta_{3}^{3}=1$ em $B_{3} / \operatorname{Brun}_{3}\left(\mathbb{D}^{2}\right)$.

Lema 5.2.9. Sejam $\omega=(r, s, t) \in P_{3} / \operatorname{Brun}_{3}\left(\mathbb{D}^{2}\right)$.

1. Existe uma decomposição de $B_{3} /$ Brun $_{3}\left(\mathbb{D}^{2}\right)$ em conjuntos disjuntos

$$
B_{3} / \operatorname{Brun}_{3}\left(\mathbb{D}^{2}\right)=\mathcal{B C G}\left(3,\left\langle\mu_{1}\right\rangle\right) \bigsqcup \delta_{3} \mathcal{B C G}\left(3,\left\langle\mu_{1}\right\rangle\right) \bigsqcup \delta_{3}^{2} \mathcal{B C G}\left(3,\left\langle\mu_{1}\right\rangle\right) .
$$

2. A sequência exata curta

$$
1 \longrightarrow \mathbb{Z} \oplus \mathbb{Z} \oplus \mathbb{Z} \longrightarrow \mathcal{B C G}\left(3, \mathcal{A}_{3}\right) \stackrel{\bar{\sigma}}{\longrightarrow} \mathcal{A}_{3} \longrightarrow 1
$$

cinde, onde $\mathcal{A}_{3}=\left\langle\left(\begin{array}{lll}1 & 3 & 2\end{array}\right) \mid\left(\begin{array}{lll}1 & 3 & 2\end{array}\right)^{3}=(1)(2)(3)\right\rangle=\mathbb{Z}_{3}$ é o grupo alternado. Logo,

$$
\mathcal{B C G}\left(3, \mathcal{A}_{3}\right) \cong \mathbb{Z}^{3} \rtimes \mathbb{Z}_{3}
$$

3. $\left(\alpha_{0,3} \omega\right)^{3}=(1,1,1) \in \mathbb{Z} \oplus \mathbb{Z} \oplus \mathbb{Z}=P_{3} /$ Brun $_{3}\left(\mathbb{D}^{2}\right)$ se, e somente se, $\omega$ satisfaz a equação linear $r+s+t=0$.

4. $\left(\delta_{3} \omega\right)^{3}=\left(\delta_{3}^{2} \omega\right)^{3}$. Como consequência, $\delta_{3} \omega$ tem ordem 3 se, se somente se, $\delta_{3}^{2} \omega$ tem ordem 3 .

Demonstração. Suponhamos que $\omega=(r, s, t) \in \mathbb{Z} \oplus \mathbb{Z} \oplus \mathbb{Z}=P_{3} / \operatorname{Brun}_{3}\left(\mathbb{D}^{2}\right)$.

1. O grupo cristalográfico de tranças com 3 cordas com grupo de holonomia $\left\langle\mu_{1}\right\rangle$, $\mathcal{B C G}\left(3,\left\langle\mu_{1}\right\rangle\right)$, não tem elementos de ordem 3 , pois $\bar{\sigma}\left(\mathcal{B C G}\left(3,\left\langle\mu_{1}\right\rangle\right)\right)=\mathbb{Z}_{2}$ e o núcleo 
$\operatorname{Ker}(\bar{\sigma})$ é livre de torção. Pelo Exemplo 5.2.8 temos que $\delta_{3}^{3}=1 \in B_{3} / \operatorname{Brun}_{3}\left(\mathbb{D}^{2}\right)$ e portanto existe uma decomposição de $B_{3} / \operatorname{Brun}_{3}\left(\mathbb{D}^{2}\right)$ em conjuntos disjuntos dada por

$$
B_{3} / \operatorname{Brun}_{3}\left(\mathbb{D}^{2}\right)=\mathcal{B C G}\left(3,\left\langle\mu_{1}\right\rangle\right) \bigsqcup \delta_{3} \mathcal{B C G}\left(3,\left\langle\mu_{1}\right\rangle\right) \bigsqcup \delta_{3}^{2} \mathcal{B C G}\left(3,\left\langle\mu_{1}\right\rangle\right)
$$

2. Consideremos a sequência exata curta

$$
1 \longrightarrow \frac{P_{3}}{\operatorname{Brun}_{3}\left(\mathbb{D}^{2}\right)} \longrightarrow \mathcal{B C G}\left(3, \mathcal{A}_{3}\right) \stackrel{\bar{\sigma}}{\longrightarrow} \mathcal{A}_{3} \longrightarrow 1
$$

Uma seção para $\bar{\sigma}: \mathcal{B C G}\left(3, \mathcal{A}_{3}\right) \rightarrow \mathbb{Z}_{3}$ é dada por

$$
\mu_{1}^{-1} \mu_{2} \mapsto \delta_{3} \in \mathcal{B C G}\left(3, \mathcal{A}_{3}\right)
$$

onde $\mu_{1}^{-1} \mu_{2} \in \mathbb{Z}_{3}=\mathcal{A}_{3}=\left\langle\left(\begin{array}{lll}1 & 3 & 2\end{array}\right) \mid\left(\begin{array}{lll}1 & 3 & 2\end{array}\right)^{3}=(1)(2)(3)\right\rangle \leq \Sigma_{3}$. Logo, a sequência exata curta (5.14) cinde e portanto

$$
\mathcal{B C G}\left(3, \mathcal{A}_{3}\right) \cong \mathbb{Z}^{3} \rtimes \mathbb{Z}_{3}
$$

3. Observemos que

$$
\begin{aligned}
\left(\alpha_{0,3} \omega\right)^{3} & =\alpha_{0,3} \omega \alpha_{0,3} \omega \alpha_{0,3} \omega \\
& =\left(\alpha_{0,3} \omega \alpha_{0,3}^{-1}\right)\left(\alpha_{0,3}^{2} \omega \alpha_{0,3}^{-2}\right) \alpha_{0,3}^{3} \omega \\
& =(t, r, s)+(s, t, r)+(1,1,1)+(r, s, t),
\end{aligned}
$$

e portanto

$$
\left(\alpha_{0,3} \omega\right)^{3}=(r+s+t+1, r+s+t+1, r+s+t+1)
$$

Logo, da equação (5.16), segue que $\left(\alpha_{0,3} \omega\right)^{3}=(1,1,1) \in P_{3} / \operatorname{Brun}_{3}\left(\mathbb{D}^{2}\right)$ se, e somente se, $\omega$ satisfaz a equação linear $r+s+t=0$.

4. Vejamos que $\left(\delta_{3} \omega\right)^{3}=\left(\delta_{3}^{2} \omega\right)^{3}$ examinando por separado cada uma das expressões que aí aparecem e usando o fato que $\delta_{3}^{3}=1$.

$$
\begin{aligned}
\left(\delta_{3} \omega\right)^{3} & =\delta_{3} \omega \delta_{3} \omega \delta_{3} \omega \\
& =\delta_{3} \omega \delta_{3}^{-1} \delta_{3}^{2} \omega \delta_{3}^{-2} \delta_{3}^{3} \omega \\
& =\delta_{3} \omega \delta_{3}^{-1} \delta_{3}^{2} \omega \delta_{3}^{-2} \omega .
\end{aligned}
$$




$$
\begin{aligned}
\left(\delta_{3}^{2} \omega\right)^{3} & =\delta_{3}^{2} \omega \delta_{3}^{2} \omega \delta_{3}^{2} \omega \\
& =\delta_{3}^{2} \omega \delta_{3}^{-2} \delta_{3}^{4} \omega \delta_{3}^{-4} \delta_{3}^{6} \omega \\
& =\delta_{3}^{2} \omega \delta_{3}^{-2} \delta_{3}^{1} \omega \delta_{3}^{-1} \omega \\
& =\delta_{3}^{1} \omega \delta_{3}^{-1} \delta_{3}^{2} \omega \delta_{3}^{-2} \omega
\end{aligned}
$$

Na última igualdade que aparece em (5.18) usamos a comutatividade do grupo abeliano $P_{3} / \operatorname{Brun}_{3}\left(\mathbb{D}^{2}\right)$. Então, por (5.17) e (5.18), obtemos a igualdade

$$
\left(\delta_{3} \omega\right)^{3}=\left(\delta_{3}^{2} \omega\right)^{3} .
$$

Pelo Lema 5.2.7 sabemos que se um elemento tem torção $3 \mathrm{em} B_{3} / \operatorname{Brun}_{3}\left(\mathbb{D}^{2}\right)$ então tal elemento é de ordem 3. O Lema 5.2.9 será usado a seguir para caracterizar estes elementos de ordem 3 .

Teorema 5.2.10.

1. Um elemento $\vartheta$ em $B_{3} / \operatorname{Brun}_{3}\left(\mathbb{D}^{2}\right)$ tem ordem 3 se, e somente se, $\vartheta$ é da forma $\delta_{3}^{i} N_{1,2}^{r} N_{2,3}^{s} N_{1,3}^{t}$, com $i=1,2$ e $r+s+t=0$.

2. Sejam $N_{1,2}^{m}, N_{2,3}^{n}, N_{1,3}^{l} \in P_{3} / \operatorname{Brun}_{3}\left(\mathbb{D}^{2}\right)$. Então,

(a) $N_{1,2}^{m} N_{2,3}^{n} N_{1,3}^{l} \tau_{1}\left(\delta_{3}\right) \tau_{1}^{-1} N_{1,3}^{-l} N_{2,3}^{-n} N_{1,2}^{-m}=\delta_{3}^{2}$ se, e somente se, $m=n$ e $l=m+1$.

(b) $N_{1,2}^{m} N_{2,3}^{n} N_{1,3}^{l}\left(\delta_{3} N_{1,2}^{r} N_{2,3}^{s} N_{1,3}^{t}\right) N_{1,3}^{-l} N_{2,3}^{-n} N_{1,2}^{-m}=\delta_{3}$ se, e somente se, $m=n+r$, $n=l+s$ e $l=m+t$.

(c) $N_{1,2}^{m} N_{2,3}^{n} N_{1,3}^{l}\left(\delta_{3}^{2} N_{1,2}^{r} N_{2,3}^{s} N_{1,3}^{t}\right) N_{1,3}^{-l} N_{2,3}^{-n} N_{1,2}^{-m}=\delta_{3}^{2}$ se, e somente se, $m=l+r$, $n=m+s$ e $l=n+t$.

Demonstração. 1. Seja $\omega \in \mathcal{B C G}\left(3,\left\langle\mu_{1}\right\rangle\right)$, então $\omega$ tem permutação associada trivial ou $\mu_{1}=\left(\begin{array}{ll}1 & 2\end{array}\right)$. Provemos inicialmente que se a permutação associada de $\omega$ é $\left(\begin{array}{ll}1 & 2\end{array}\right), \bar{\sigma}(\omega)=\left(\begin{array}{ll}1 & 2\end{array}\right)$, então $\delta_{3} \omega \neq 0$ e $\delta_{3}^{2} \omega \neq 0$. Como $\bar{\sigma}\left(\delta_{3}\right)=\left(\begin{array}{lll}1 & 3 & 2\end{array}\right)$, então $\bar{\sigma}\left(\delta_{3} \omega\right)=\left(\begin{array}{lll}1 & 3 & 2\end{array}\right)\left(\begin{array}{ll}1 & 2\end{array}\right)=\left(\begin{array}{ll}1 & 3\end{array}\right)$ e portanto $\bar{\sigma}\left(\left(\delta_{3} \omega\right)^{3}\right)=\left(\begin{array}{ll}1 & 3\end{array}\right)$, que claramente não é a permutação trivial $(1)(2)(3)$ e portanto $\left(\delta_{3} \omega\right)^{3} \neq 0$. Para provar que $\delta_{3}^{2} \omega \neq 0$ seguimos o mesmo roteiro anterior, apenas tendo em conta que $\bar{\sigma}\left(\left(\delta_{3}^{2} \omega\right)^{3}\right)=\left(\begin{array}{ll}2 & 3\end{array}\right)$. Consideremos agora $\bar{\sigma}(\omega)=(1)(2)(3)$. A seguir usaremos o Lema 5.2.9, para isto tomemos $\omega=(r, s, t) \in P_{3} / \operatorname{Brun}_{3}\left(\mathbb{D}^{2}\right) \cong \mathbb{Z} \oplus \mathbb{Z} \oplus \mathbb{Z}$ e observemos que $\delta_{3}=N_{1,2}^{-1} \alpha_{0,3}$. Vejamos que $\left(\delta_{3} \omega\right)^{3}=(0,0,0)$ em $\mathbb{Z} \oplus \mathbb{Z} \oplus \mathbb{Z}$ se, e somente se, $r+s+t=0$.

$$
\begin{aligned}
\left(\delta_{3} \omega\right)^{3} & =\delta_{3} \omega \delta_{3} \omega \delta_{3} \omega \\
& =N_{1,2}^{-1} \alpha_{0,3} \omega N_{1,2}^{-1} \alpha_{0,3} \omega N_{1,2}^{-1} \alpha_{0,3} \omega \\
& =N_{1,2}^{-1}\left(\alpha_{0,3} \omega N_{1,2}^{-1} \omega^{-1} \alpha_{0,3}^{-1}\right)\left(\left(\alpha_{0,3} \omega\right)^{2} N_{1,2}^{-1}\left(\alpha_{0,3} \omega\right)^{-2}\right)\left(\alpha_{0,3} \omega\right)^{3} \\
& =N_{1,2}^{-1} N_{2,3}^{-1} N_{1,3}^{-1}\left(\alpha_{0,3} \omega\right)^{3} .
\end{aligned}
$$


Logo, temos que

$$
\left(\delta_{3} \omega\right)^{3}=(-1,-1,-1)+\left(\alpha_{0,3} \omega\right)^{3} .
$$

Pelo item 3 do Lema 5.2.9 sabemos que $\left(\alpha_{0,3} \omega\right)^{3}=(1,1,1)$ se, e somente se, $r+s+t=0$. Portanto, por (5.19), $\left(\delta_{3} \omega\right)^{3}=(0,0,0)$ se, e somente se, $r+s+t=0$. Pelo item 4 do Lema 5.2.9 sabemos que $\left(\delta_{3} \omega\right)^{3}=\left(\delta_{3}^{2} \omega\right)^{3}$ e portanto $\left(\delta_{3} \omega\right)^{3}=(0,0,0)$ se, e somente se, $\left(\delta_{3}^{2} \omega\right)^{3}=(0,0,0)$.

Provamos assim que, um elemento em $B_{3} / \operatorname{Brun}_{3}\left(\mathbb{D}^{2}\right)$ tem ordem 3 se, e somente se, é da forma $\delta_{3}^{i} N_{1,2}^{r} N_{2,3}^{s} N_{1,3}^{t}$, com $i=1,2$ e $r+s+t=0$.

2. Tomemos $N_{1,2}^{m}, N_{2,3}^{n}, N_{1,3}^{l} \in P_{3} / \operatorname{Brun}_{3}\left(\mathbb{D}^{2}\right)$.

(a) Pelo Exemplo 5.2.8, o elemento $\delta_{3}$ de $B_{3} / \operatorname{Brun}_{3}\left(\mathbb{D}^{2}\right)$ é tal que $\delta_{3}^{3}=1$ e

$$
\begin{aligned}
\tau_{1} \delta_{3} \tau_{1}^{-1} & =\tau_{1} \tau_{1}^{-1} \tau_{2} \tau_{1}^{-1} \\
& =\tau_{2} \tau_{1}^{-1} \delta_{3} \delta_{3}^{2} \\
& =\tau_{2} \tau_{1}^{-1} \tau_{2} \delta_{3}^{2} \\
& =N_{1,3}^{-1} N_{2,3} \delta_{3}^{2} .
\end{aligned}
$$

Agora, usaremos a igualdade obtida em (5.20) e a ação de $\delta_{3}$ por conjugação nos elementos de $P_{3} / \operatorname{Brun}_{3}\left(\mathbb{D}^{2}\right)$ na seguinte conta

$$
\begin{aligned}
N_{1,2}^{m} N_{2,3}^{n} N_{1,3}^{l} \tau_{1} \delta_{3} \tau_{1}^{-1} N_{1,3}^{-l} N_{2,3}^{-n} N_{1,2}^{-m} & =N_{1,2}^{m} N_{2,3}^{n} N_{1,3}^{l} N_{1,3}^{-1} N_{2,3} \delta_{3}^{2} N_{1,3}^{-l} N_{2,3}^{-n} N_{1,2}^{-m} \\
& =N_{1,2}^{m} N_{2,3}^{n+1} N_{1,3}^{l-1} \delta_{3}^{2} N_{1,3}^{-l} N_{2,3}^{-n} N_{1,2}^{-m} \delta_{3}^{-2} \delta_{3}^{2} \\
& =N_{1,2}^{m} N_{2,3}^{n+1} N_{1,3}^{l-1} N_{2,3}^{-l} N_{1,2}^{-n} N_{1,3}^{-m} \delta_{3}^{2} \\
& =N_{1,2}^{m-n} N_{2,3}^{n-l+1} N_{1,3}^{t-m-1} \delta_{3}^{2} .
\end{aligned}
$$

De (5.21) segue que

$$
N_{1,2}^{m} N_{2,3}^{n} N_{1,3}^{l} \tau_{1} \delta_{3} \tau_{1}^{-1} N_{1,3}^{-l} N_{2,3}^{-n} N_{1,2}^{-m}=N_{1,2}^{m-n} N_{2,3}^{n-l+1} N_{1,3}^{t-m-1} \delta_{3}^{2}
$$

Logo, por (5.22), temos que $N_{1,2}^{m} N_{2,3}^{n} N_{1,3}^{l} \tau_{1} \delta_{3} \tau_{1}^{-1} N_{1,3}^{-l} N_{2,3}^{-n} N_{1,2}^{-m}=\delta_{3}^{2}$ se, e somente se, $m=n$ e $l=m+1$.

(b) Usando a ação de $\delta_{3}$ por conjugação nos elementos de $P_{3} / \operatorname{Brun}_{3}\left(\mathbb{D}^{2}\right)$ obtemos

$$
\begin{aligned}
N_{1,2}^{m} N_{2,3}^{n} N_{1,3}^{l} \delta_{3} N_{1,2}^{r-m} N_{2,3}^{s-n} N_{1,3}^{t-l} & =N_{1,2}^{m} N_{2,3}^{n} N_{1,3}^{l} \delta_{3} N_{1,2}^{r-m} N_{2,3}^{s-n} N_{1,3}^{t-l} \delta_{3}^{-1} \delta_{3} \\
& =N_{1,2}^{m} N_{2,3}^{n} N_{1,3}^{l} N_{2,3}^{r-m} N_{1,3}^{s-n} N_{1,2}^{t-l} \delta_{3} \\
& =N_{1,2}^{m+t-l} N_{2,3}^{n+r-m} N_{1,3}^{l+s-n} \delta_{3} .
\end{aligned}
$$


Pela igualdade

$$
N_{1,2}^{m} N_{2,3}^{n} N_{1,3}^{l} \delta_{3} N_{1,2}^{r} N_{2,3}^{s} N_{1,3}^{t} N_{1,3}^{-l} N_{2,3}^{-n} N_{1,2}^{-m}=N_{1,2}^{m} N_{2,3}^{n} N_{1,3}^{l} \delta_{3} N_{1,2}^{r-m} N_{2,3}^{s-n} N_{1,3}^{t-l}
$$

e por (5.23), temos que

$$
N_{1,2}^{m} N_{2,3}^{n} N_{1,3}^{l} \delta_{3} N_{1,2}^{r} N_{2,3}^{s} N_{1,3}^{t} N_{1,3}^{-l} N_{2,3}^{-n} N_{1,2}^{-m}=N_{1,2}^{m+t-l} N_{2,3}^{n+r-m} N_{1,3}^{l+s-n} \delta_{3} .
$$

Logo, $N_{1,2}^{m} N_{2,3}^{n} N_{1,3}^{l} \delta_{3} N_{1,2}^{r} N_{2,3}^{s} N_{1,3}^{t} N_{1,3}^{-l} N_{2,3}^{-n} N_{1,2}^{-m}=\delta_{3}$ se, e somente se, $m=n+r$, $n=l+s$ e $l=m+t$.

(c) Seguindo a mesma ideia dos itens anteriores, temos que

$$
\begin{aligned}
N_{1,2}^{m} N_{2,3}^{n} N_{1,3}^{l} \delta_{3}^{2} N_{1,2}^{r-m} N_{2,3}^{s-n} N_{1,3}^{t-l} & =N_{1,2}^{m} N_{2,3}^{n} N_{1,3}^{l} \delta_{3}^{2} N_{1,2}^{r-m} N_{2,3}^{s-n} N_{1,3}^{t-l} \delta_{3}^{-2} \delta_{3}^{2} \\
& =N_{1,2}^{m} N_{2,3}^{n} N_{1,3}^{l} N_{1,3}^{r-m} N_{1,2}^{s-n} N_{2,3}^{t-l} \delta_{3}^{2} \\
& =N_{1,2}^{m+s-n} N_{2,3}^{n+t-l} N_{1,3}^{l+r-m} \delta_{3}^{2} .
\end{aligned}
$$

De (5.25) segue que

$$
N_{1,2}^{m} N_{2,3}^{n} N_{1,3}^{l} \delta_{3}^{2} N_{1,2}^{r} N_{2,3}^{s} N_{1,3}^{t} N_{1,3}^{-l} N_{2,3}^{-n} N_{1,2}^{-m}=N_{1,2}^{m+s-n} N_{2,3}^{n+t-l} N_{1,3}^{l+r-m} \delta_{3}^{2} .
$$

Logo, $\quad N_{1,2}^{m} N_{2,3}^{n} N_{1,3}^{l} \delta_{3}^{2} N_{1,2}^{r} N_{2,3}^{s} N_{1,3}^{t} \tau_{1}^{-1} N_{1,3}^{-l} N_{2,3}^{-n} N_{1,2}^{-m}=\delta_{3}^{2}$ se, e somente se, $m=l+r, n=m+s$ e $l=n+t$.

No Teorema 5.2.10 caracterizamos os elementos de ordem 3 no grupo cristalográfico de tranças com 3 cordas com grupo de holonomia $\Sigma_{3}, B_{3} / \operatorname{Brun}_{3}\left(\mathbb{D}^{2}\right)$, no seguinte resultado veremos que estes elementos formam uma única classe de conjugação.

Corolário 5.2.11. Existe uma única classe de conjugação de elementos de ordem 3 no grupo quociente $B_{3} /$ Brun $_{3}\left(\mathbb{D}^{2}\right)$.

Demonstração. Vimos no item 1 do Teorema 5.2.10 que os elementos de ordem 3 de $B_{3} / \operatorname{Brun}_{3}\left(\mathbb{D}^{2}\right)$ são da forma $\delta_{3}^{i} N_{1,2}^{r} N_{2,3}^{s} N_{1,3}^{t}$, com $i=1,2$ e $r+s+t=0$, e pelo item 2 do Teorema 5.2.10 temos que qualquer um destes elementos é conjugado a $\delta_{3}$. Consideremos alguns valores específicos de $m, n, l$ para ilustrar a última frase.

Se tomamos $l=1$ e $m=n=0$ no item 2(a) do Teorema 5.2.10, vemos que existe um elemento de $B_{3} / \operatorname{Brun}_{3}\left(\mathbb{D}^{2}\right)$ tal que $\delta_{3}$ e $\delta_{3}^{2}$ são conjugados:

$$
\delta_{3}^{2}=N_{1,3} \tau_{1} \delta_{3} \tau_{1}^{-1} N_{1,3}^{-1}
$$


No item 2(b) tomemos $n=0$ e portanto $m=r$ e $l=-s$. Logo,

$$
\delta_{3}=N_{1,2}^{r} N_{1,3}^{-s} \delta_{3} N_{1,2}^{r} N_{2,3}^{s} N_{1,3}^{t} N_{1,3}^{s} N_{1,2}^{-r}
$$

Para o caso do item 2(c) consideremos $n=0$ e portanto $m=-s$ e $l=t$, donde

$$
\delta_{3}^{2}=N_{1,2}^{-s} N_{1,3}^{t} \delta_{3}^{2} N_{1,2}^{r} N_{2,3}^{s} N_{1,3}^{t} N_{1,3}^{-t} N_{1,2}^{s}
$$

Desta forma, juntando a informação das igualdades (5.27) e (5.29), temos que

$$
\delta_{3}=\left(N_{1,3} \tau_{1}\right)^{-1} N_{1,2}^{-s} N_{1,3}^{t} \delta_{3}^{2} N_{1,2}^{r} N_{2,3}^{s} N_{1,3}^{t} N_{1,3}^{-t} N_{1,2}^{s} N_{1,3} \tau_{1}
$$

Logo, das equações (5.28) e (5.30) podemos concluir que os elementos de torção 3 do grupo $B_{3} / \operatorname{Brun}_{3}\left(\mathbb{D}^{2}\right), \delta_{3}^{i} N_{1,2}^{r} N_{2,3}^{s} N_{1,3}^{t}$, com $i=1,2$ e $r+s+t=0$, são todos conjugados a $\delta_{3}$.

Até agora estudamos apenas a torção 3 no grupo $B_{3} / \operatorname{Brun}_{3}\left(\mathbb{D}^{2}\right)$, caracterizando os elementos de ordem 3 no Teorema 5.2.10 e mostrando que eles formam uma única classe de conjugação no Corolário 5.2.11. A seguir provaremos que esta é a única possibilidade de torção neste grupo.

Teorema 5.2.12. O grupo $B_{3} /$ Brun $_{3}\left(\mathbb{D}^{2}\right)$ não tem elementos de torção 2.

Demonstração. É suficiente provar que não tem elementos de ordem 2, em vista do Lema 5.2.7. Vamos supor por absurdo que existe um elemento $\gamma$ de ordem 2 em $B_{3} / \operatorname{Brun}_{3}\left(\mathbb{D}^{2}\right)$. Observemos que tal $\gamma$ não pode ter permutação associada trivial, isto é, $\gamma$ não é uma trança pura. Lembremos da decomposição (5.13),

$$
B_{3} / \operatorname{Brun}_{3}\left(\mathbb{D}^{2}\right)=\mathcal{B C G}\left(3,\left\langle\mu_{1}\right\rangle\right) \bigsqcup \delta_{3} \mathcal{B C G}\left(3,\left\langle\mu_{1}\right\rangle\right) \bigsqcup \delta_{3}^{2} \mathcal{B C G}\left(3,\left\langle\mu_{1}\right\rangle\right)
$$

dada no item 1 do Lema 5.2.9.

Como $\gamma \in B_{3} / \operatorname{Brun}_{3}\left(\mathbb{D}^{2}\right)$, então $\gamma$ deve pertencer a um, e somente um, dos conjuntos do lado direito na igualdade (5.31). Vamos analisar cada um destes casos e chegar numa contradição, concluindo assim a prova deste teorema.

1. Vamos supor que $\gamma \in \mathcal{B C G}\left(3,\left\langle\mu_{1}\right\rangle\right)$. Como $\gamma$ não é pura, então $\gamma=N \tau_{1}$, com

$$
N=\left(N_{1}, N_{2}, N_{3}\right) \in P_{3} / \operatorname{Brun}_{3}\left(\mathbb{D}^{2}\right) \cong \mathbb{Z} \oplus \mathbb{Z} \oplus \mathbb{Z}
$$

Portanto, considerando que $\tau_{1}^{2}=N_{1,2}$ e aplicando a ação por conjugação de $\tau_{1}$ nos 
elementos de $P_{3} / \operatorname{Brun}_{3}\left(\mathbb{D}^{2}\right)$, dada em (5.12), temos que

$$
\begin{aligned}
\left(N \tau_{1}\right)^{2} & =N \tau_{1} N \tau_{1} \\
& =\left(N_{1}, N_{2}, N_{3}\right) \tau_{1}\left(N_{1}, N_{2}, N_{3}\right) \tau_{1}^{-1} \tau_{1}^{2} \\
& =\left(N_{1}, N_{2}, N_{3}\right)+\left(N_{1}, N_{3}, N_{2}\right)+(1,0,0) \\
& =\left(2 N_{1}+1, N_{2}+N_{3}, N_{2}+N_{3}\right) .
\end{aligned}
$$

Como estamos supondo que $\gamma^{2}=(0,0,0)$, então de (5.32) segue que

$$
\left(2 N_{1}+1, N_{2}+N_{3}, N_{2}+N_{3}\right)=(0,0,0)
$$

e portanto $2 N_{1}+1=0$ em $\mathbb{Z}$ o que é uma contradição. Logo, $\mathcal{B C G}\left(3,\left\langle\mu_{1}\right\rangle\right)$ não contém elementos de ordem 2.

2. Tomemos agora $\gamma \in \delta_{3} \mathcal{B C} \mathcal{G}\left(3,\left\langle\mu_{1}\right\rangle\right)$. Seguiremos a ideia anterior. Consideremos uma expressão para $\gamma$, como sendo $\gamma=\delta_{3} N \tau_{1}$, com $N=\left(N_{1}, N_{2}, N_{3}\right) \in P_{3} / \operatorname{Brun}_{3}\left(\mathbb{D}^{2}\right)$. Lembremos que $P_{3} / \operatorname{Brun}_{3}\left(\mathbb{D}^{2}\right) \cong \mathbb{Z} \oplus \mathbb{Z} \oplus \mathbb{Z}$.

$$
\begin{aligned}
\left(\delta_{3} N \tau_{1}\right)^{2} & =\delta_{3} N \tau_{1} \delta_{3} N \tau_{1} \\
& =\delta_{3}\left(N_{1}, N_{2}, N_{3}\right) \delta_{3}^{-1} \delta_{3}\left(\tau_{1} \delta_{3}\left(N_{1}, N_{2}, N_{3}\right)\left(\tau_{1} \delta_{3}\right)^{-1}\right) \delta_{3}^{-1} \delta_{3} \tau_{1} \delta_{3} \tau_{1} \\
& =\delta_{3}\left(N_{1}, N_{2}, N_{3}\right) \delta_{3}^{-1} \delta_{3}\left(\sigma_{2}\left(N_{1}, N_{2}, N_{3}\right)\left(\sigma_{2}\right)^{-1}\right) \delta_{3}^{-1} \tau_{1}^{-1} \sigma_{2} \tau_{1} \\
& =\left(N_{3}, N_{1}, N_{2}\right)+\left(N_{3}, N_{2}, N_{1}\right)+(0,1,0) \\
& =\left(2 N_{3}, N_{1}+N_{2}+1, N_{1}+N_{2}\right) .
\end{aligned}
$$

Como estamos supondo que $\gamma^{2}=(0,0,0)$, então de (5.33) segue que

$$
\left(2 N_{3}, N_{1}+N_{2}+1, N_{1}+N_{2}\right)=(0,0,0)
$$

e portanto $N_{1}+N_{2}+1=N_{1}+N_{2}$ o que é uma contradição. Logo, $\delta_{3} \mathcal{B C G}\left(3,\left\langle\mu_{1}\right\rangle\right)$ não contém elementos de ordem 2 .

3. Finalmente, se $\gamma \in \delta_{3}^{2} \mathcal{B C G}\left(3,\left\langle\mu_{1}\right\rangle\right)$ fazemos uma análise como nos dois itens anteriores. Lembremos que $\delta_{3}^{2}=\delta_{3}^{-1}$.

$$
\begin{aligned}
\left(\delta_{3}^{2} N \tau_{1}\right)^{2} & =\delta_{3}^{2} N \tau_{1} \delta_{3}^{2} N \tau_{1} \\
& =\delta_{3}^{2}\left(N_{1}, N_{2}, N_{3}\right) \delta_{3}^{-2} \delta_{3}^{2}\left(\tau_{1} \delta_{3}^{2}\left(N_{1}, N_{2}, N_{3}\right)\left(\tau_{1} \delta_{3}^{2}\right)^{-1}\right) \delta_{3}^{-2} \delta_{3}^{2} \tau_{1} \delta_{3}^{2} \tau_{1} \\
& =\delta_{3}^{-1}\left(N_{1}, N_{2}, N_{3}\right) \delta_{3} \delta_{3}^{-1}\left(\tau_{1} \delta_{3}^{-1}\left(N_{1}, N_{2}, N_{3}\right)\left(\tau_{1} \delta_{3}^{-1}\right)^{-1}\right) \delta_{3} \delta_{3}^{-1} \tau_{1} \delta_{3}^{-1} \tau_{1} \\
& =\left(N_{2}, N_{3}, N_{1}\right)+\left(N_{1}, N_{3}, N_{2}\right)+(1,1,-1) \\
& =\left(N_{1}+N_{2}+1,2 N_{3}+1, N_{1}+N_{2}-1\right) .
\end{aligned}
$$


Como estamos supondo que $\gamma^{2}=(0,0,0)$, então de (5.34) segue que

$$
\left(N_{1}+N_{2}+1,2 N_{3}+1, N_{1}+N_{2}-1\right)=(0,0,0)
$$

e portanto $N_{1}+N_{2}+1=N_{1}+N_{2}-1$ (e $\left.2 N_{3}+1=0\right)$ o que é uma contradição. Logo, $\delta_{3}^{2} \mathcal{B C G}\left(3,\left\langle\mu_{1}\right\rangle\right)$ não contém elementos de ordem 2 .

Para finalizar esta subseção, vejamos que a variedade plana $X$ determinada pelo grupo $\mathcal{B C G}\left(3,\left\langle\mu_{1}\right\rangle\right)$ é uma variedade de Seifert não orientável. A definição de variedade de Seifert pode ser encontrada no livro de Orlik [77].

Teorema 5.2.13. O grupo $\mathcal{B C G}\left(3,\left\langle\mu_{1}\right\rangle\right)$ é um grupo de Bieberbach de dimensão 3, com grupo de holonomia $\mathbb{Z}_{2}$. A variedade plana $X$ unicamente determinada por $\mathcal{B C G}\left(3,\left\langle\mu_{1}\right\rangle\right)$ é não orientável e com primeiro grupo de homologia $H_{1}(X ; \mathbb{Z}) \cong \mathbb{Z} \oplus \mathbb{Z}$. A menos de difeomorfismo afim, $X$ é igual à variedade $\mathscr{B}_{2}$ da classificação das 3-variedades planas dada na Seção 3.5 de [95]. Ainda mais, a variedade plana não orientável $X$ é o espaço total de um fibrado sobre o círculo, $F \rightarrow X \rightarrow \mathbb{S}^{1}$, onde a fibra $F$ pode ser o toro ou a garrafa de Klein.

Demonstração. Pelo Teorema 5.2.12 sabemos que $B_{3} / \operatorname{Brun}_{3}\left(\mathbb{D}^{2}\right)$ não tem elementos de torção 2 e como o subgrupo $\left\langle\mu_{1}\right\rangle$ de $\mu_{3}$ tem ordem 2 , então $\mathcal{B C G}\left(3,\left\langle\mu_{1}\right\rangle\right)$ é um grupo cristalográfico livre de torção. Isto é, $\mathcal{B C G}\left(3,\left\langle\mu_{1}\right\rangle\right)$ é um grupo de Bieberbach de dimensão 3 com grupo de holonomia $\left\langle\mu_{1}\right\rangle=\mathbb{Z}_{2}$. Seja $X$ a variedade plana unicamente determinada por $\mathcal{B C G}\left(3,\left\langle\mu_{1}\right\rangle\right)$, ou seja $\pi_{1}(X)=\mathcal{B C G}\left(3,\left\langle\mu_{1}\right\rangle\right)$. Pelo Corolário 5.2.3 temos que $\operatorname{det}\left(\Theta\left(\mu_{1}\right)\right)=-1$ e portanto $X$ é não orientável. Como $X$ é uma variedade plana de dimensão 3 , não orientável e com grupo de holonomia $\mathbb{Z}_{2}$, então, da classificação dada no Teorema 3.5.9 de [95], segue que, a menos de difeomorfismo afim, $X$ pode ser $\mathscr{B}_{1}$ ou $\mathscr{B}_{2}$ (usando a notação de [95]). Agora, pela Proposição 5.2.6 temos que $H_{1}(X ; \mathbb{Z}) \cong \mathbb{Z} \oplus \mathbb{Z}$ e portanto, pela classificação dada na tabela da página 122 de [95], temos que $X$ é, a menos de difeomorfismo afim, a variedade $\mathscr{B}_{2}$.

No que segue consideraremos a apresentação de $\mathcal{B C G}\left(3,\left\langle\mu_{1}\right\rangle\right)$ dada na Proposição 5.2.6. Provemos inicialmente que $X$ é o espaço total de um fibrado sobre o círculo, $F \rightarrow X \rightarrow \mathbb{S}^{1}$, onde a fibra $F$ é o toro $\mathbb{T}^{2}$. Consideremos o produto semidireto $\mathbb{Z} \oplus \mathbb{Z} \rtimes \mathbb{Z}$ tal que o subgrupo normal de $\mathbb{Z} \oplus \mathbb{Z} \rtimes \mathbb{Z}$ é gerado por $\left(d_{1}, 0\right)$ e $\left(0, d_{2}\right)$, enquanto o subgrupo que não é normal é gerado por $t$. Daí existe uma sequência exata curta

$$
1 \longrightarrow \mathbb{Z} \oplus \mathbb{Z} \longrightarrow \mathbb{Z} \oplus \mathbb{Z} \rtimes \mathbb{Z} \longrightarrow \mathbb{Z} \longrightarrow 1
$$

Vamos demonstrar que $\mathcal{B C G}\left(3,\left\langle\mu_{1}\right\rangle\right) \cong \mathbb{Z} \oplus \mathbb{Z} \rtimes \mathbb{Z}$. Seja $\varphi: \mathcal{B C G}\left(3,\left\langle\mu_{1}\right\rangle\right) \rightarrow \mathbb{Z}$ a aplicação definida por $\varphi\left(N_{1,3}\right)=\varphi\left(N_{2,3}\right)=0, \varphi\left(N_{1,2}\right)=2$ e $\varphi\left(\tau_{1}\right)=1$. Notemos que $\varphi$ é homomor- 
fismo pois as relações do grupo são preservadas. Portanto, temos uma sequência exata curta

$$
1 \longrightarrow \operatorname{Ker}(\varphi) \longrightarrow \mathcal{B C G}\left(3,\left\langle\mu_{1}\right\rangle\right) \stackrel{\varphi}{\longrightarrow} \mathbb{Z} \longrightarrow 1
$$

Seja $\eta_{1}: \mathbb{Z} \oplus \mathbb{Z} \rtimes \mathbb{Z} \rightarrow \mathcal{B C G}\left(3,\left\langle\mu_{1}\right\rangle\right)$ a aplicação definida por $\eta_{1}\left(d_{1}\right)=N_{1,3}, \eta_{1}\left(d_{2}\right)=N_{2,3}$ e $\eta_{1}(t)=\sigma_{1}$. Usando a apresentação do grupo $\mathcal{B C G}\left(3,\left\langle\mu_{1}\right\rangle\right)$ dada na Proposição 5.2.6 verifica-se que $\eta_{1}$ é um homomorfismo. Além disso, como $N_{1,3}, N_{2,3}$ pertencem ao núcleo de $\varphi$, então $\eta_{1}(\mathbb{Z} \oplus \mathbb{Z}) \subseteq \operatorname{Ker}(\varphi)$. Definamos $\eta_{2}: \mathcal{B C G}\left(3,\left\langle\mu_{1}\right\rangle\right) \rightarrow \mathbb{Z} \oplus \mathbb{Z} \rtimes \mathbb{Z}$ por $\eta_{2}\left(N_{1,3}\right)=d_{1}$, $\eta_{2}\left(N_{2,3}\right)=d_{2}$ e $\eta_{2}\left(\tau_{1}\right)=t$. Como $\eta_{2}$ preserva as relações do grupo $\mathcal{B C} \mathcal{G}\left(3,\left\langle\mu_{1}\right\rangle\right)$, então $\eta_{2}$ é um homomorfismo. Ainda mais, pela construção, segue que $\eta_{1} \circ \eta_{2}$ é o homomorfismo identidade definido no grupo $\mathcal{B C G}\left(3,\left\langle\mu_{1}\right\rangle\right)$ e $\eta_{2} \circ \eta_{1}$ é o homomorfismo identidade de $\mathbb{Z} \oplus \mathbb{Z} \rtimes \mathbb{Z}$. Notemos que os elementos de $\mathcal{B C G}\left(3,\left\langle\mu_{1}\right\rangle\right)$ são da forma $\omega=N_{1,3}^{n_{1}} N_{2,3}^{n_{2}} \tau_{1}^{r}$ e se $\omega \in \operatorname{Ker}(\varphi)$, então $r=0$ e $\omega=N_{1,3}^{n_{1}} N_{2,3}^{n_{2}}$. Portanto, $\eta_{2}(\operatorname{Ker}(\varphi)) \subseteq \mathbb{Z} \oplus \mathbb{Z}$. Juntando a informação anterior, temos um quadrado comutativo

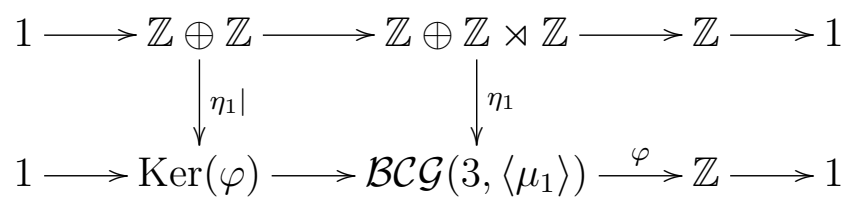

onde $\eta_{1}$ e $\eta_{1} \mid$ são isomorfismos. Logo, as sequências exatas curtas (5.35) e (5.36) são isomorfas, pois existe um isomorfismo $\mathbb{Z} \rightarrow \mathbb{Z}$ (por (5.37)) tal que o seguinte diagrama é comutativo e as flechas verticais são isomorfismos

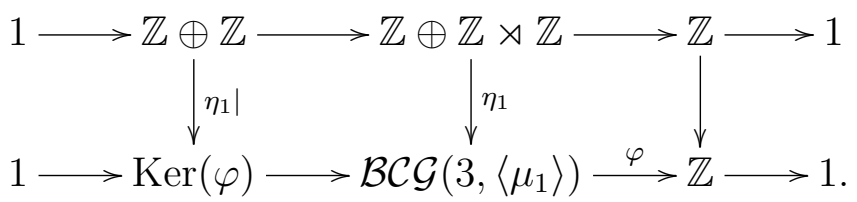

Agora, vamos demonstrar que $X$ é o espaço total de um fibrado sobre o círculo, $F \rightarrow X \rightarrow \mathbb{S}^{1}$, onde a fibra $F$ é a garrafa de Klein. O roteiro a seguir é similar com o anterior, em que provamos que a fibra $F$ é o toro.

Denotemos por Kb a garrafa de Klein. Consideremos o produto semidireto $\pi_{1}(\mathrm{~Kb}) \rtimes \mathbb{Z}$ tal que o subgrupo normal $\pi_{1}(\mathrm{~Kb})$ é gerado por $u$ e $\epsilon$, sujeito à única relação $\epsilon u \epsilon^{-1}=u^{-1}$, enquanto o subgrupo $\mathbb{Z}$ (que não é normal) é gerado por $t$, satisfazendo as igualdades $t u t^{-1}=u$ e $t \epsilon t^{-1}=u \epsilon$. Daí, existe uma sequência exata curta

$$
1 \longrightarrow \pi_{1}(\mathrm{~Kb}) \longrightarrow \pi_{1}(\mathrm{~Kb}) \rtimes \mathbb{Z} \longrightarrow \mathbb{Z} \longrightarrow 1
$$

Vamos demonstrar que $\mathcal{B C G}\left(3,\left\langle\mu_{1}\right\rangle\right) \cong \pi_{1}(\mathrm{~Kb}) \rtimes \mathbb{Z}$. Seja $\xi: \mathcal{B C G}\left(3,\left\langle\mu_{1}\right\rangle\right) \rightarrow \mathbb{Z}$ a aplicação definida por $\xi\left(N_{1,3}\right)=\xi\left(N_{2,3}\right)=1$ e $\xi\left(N_{1,2}\right)=\xi\left(\tau_{1}\right)=0$. Notemos que $\xi$ é homomorfismo, 
pois as relações do grupo são preservadas. Portanto, temos uma sequência exata curta

$$
1 \longrightarrow \operatorname{Ker}(\xi) \longrightarrow \mathcal{B C G}\left(3,\left\langle\mu_{1}\right\rangle\right) \stackrel{\xi}{\longrightarrow} \mathbb{Z} \longrightarrow 1
$$

Seja $\lambda_{1}: \pi_{1}(\mathrm{~Kb}) \rtimes \mathbb{Z} \rightarrow \mathcal{B C G}\left(3,\left\langle\mu_{1}\right\rangle\right)$ a aplicação definida por $\lambda_{1}(u)=N_{1,3} N_{2,3}^{-1}, \lambda_{1}(\epsilon)=\tau_{1}$ e $\lambda_{1}(t)=N_{1,3}$. Usando a apresentação do grupo $\mathcal{B C G}\left(3,\left\langle\mu_{1}\right\rangle\right)$, dada na Proposição 5.2.6, verifica-se que $\lambda_{1}$ é um homomorfismo. Além disso, como $N_{1,3} N_{2,3}^{-1}$ e $\tau_{1}$ pertencem ao núcleo de $\xi$, então $\lambda_{1}\left(\pi_{1}(\mathrm{~Kb})\right) \subseteq \operatorname{Ker}(\xi)$. Definamos $\lambda_{2}: \mathcal{B C G}\left(3,\left\langle\mu_{1}\right\rangle\right) \rightarrow \pi_{1}(\mathrm{~Kb}) \rtimes \mathbb{Z}$ por $\lambda_{2}\left(N_{1,3}\right)=t, \lambda_{2}\left(N_{2,3}\right)=t u^{-1}$ e $\lambda_{2}\left(\tau_{1}\right)=\epsilon$. Como $\lambda_{2}$ preserva as relações do grupo $\mathcal{B C G}\left(3,\left\langle\mu_{1}\right\rangle\right)$, então $\lambda_{2}$ é um homomorfismo. Ainda mais, segue pela construção que $\lambda_{1} \circ \lambda_{2}$ é o homomorfismo identidade definido no grupo $\mathcal{B C} \mathcal{G}\left(3,\left\langle\mu_{1}\right\rangle\right)$ e $\lambda_{2} \circ \lambda_{1}$ é o homomorfismo identidade de $\pi_{1}(\mathrm{~Kb}) \rtimes \mathbb{Z}$. Notemos que os elementos de $\mathcal{B C G}\left(3,\left\langle\mu_{1}\right\rangle\right)$ são da forma $\omega=N_{1,3}^{n_{1}} N_{2,3}^{n_{2}} \tau_{1}^{r}$ e se $\omega \in \operatorname{Ker}(\xi)$, então $n_{1}=-n_{2}$ e $\omega=\left(N_{1,3} N_{2,3}^{-1}\right)^{n_{1}} \tau_{1}^{r}$. Portanto, $\lambda_{2}(\operatorname{Ker}(\xi)) \subseteq \pi_{1}(\mathrm{~Kb})$. Juntando a informação anterior, temos um quadrado comutativo

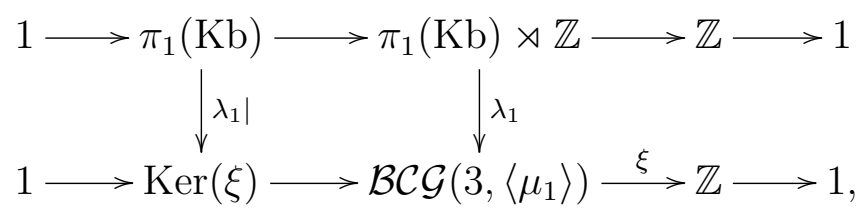

onde $\lambda_{1}$ e $\lambda_{1} \mid$ são isomorfismos. Logo, as sequências exatas curtas (5.39) e (5.40) são isomorfas, pois existe um isomorfismo $\mathbb{Z} \rightarrow \mathbb{Z}$ (por (5.41)) tal que o seguinte diagrama é comutativo e as flechas verticais são isomorfismos

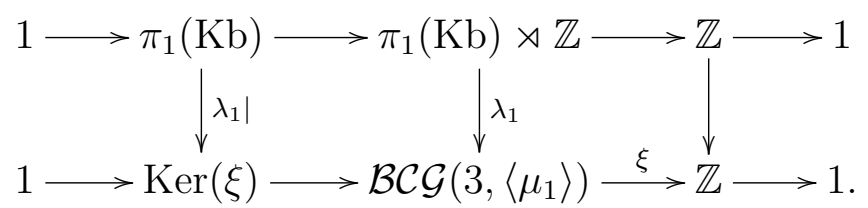

Observação 5.2.14. A variedade $X$ do Teorema 5.2.13 já se apresenta nesse formato como um fibrado sobre o círculo com fibra a garrafa de klein, segundo a apresentação de Orlik [7r]. X é a variedade de Seifert $N_{2}$ do Teorema 4, na página 140, de [7r7].

\subsection{Tranças com $n$ cordas sobre o disco e grupos cris- talográficos}

Seja $M$ uma superfície qualquer, podendo ser não orientável, com bordo, ou compacta sem um número finito de pontos no interior de $M$. Como antes, denotaremos por $\Sigma_{n}$ o grupo simétrico em $n$ elementos, para $n \geq 1$. Sabemos que existe uma sequência exata 
curta associada aos grupos de tranças

$$
1 \longrightarrow P_{n}(M) \longrightarrow B_{n}(M) \stackrel{\sigma}{\longrightarrow} \Sigma_{n} \longrightarrow 1
$$

Como $\Gamma_{2}\left(P_{n}(M)\right)=\left[P_{n}(M), P_{n}(M)\right]$ é um subgrupo normal de $B_{n}(M)$, então podemos passar ao quociente a sequência (5.43), obtendo a seguinte sequência exata curta

$$
1 \longrightarrow P_{n}(M) / \Gamma_{2}\left(P_{n}(M)\right) \longrightarrow B_{n}(M) / \Gamma_{2}\left(P_{n}(M)\right) \stackrel{\bar{\sigma}}{\longrightarrow} \Sigma_{n} \longrightarrow 1
$$

De (5.44) obtemos uma representação $\Sigma_{n} \rightarrow \operatorname{Aut}\left(P_{n}(M) / \Gamma_{2}\left(P_{n}(M)\right)\right)$.

Nessa seção tomaremos $M$ como sendo o disco e provaremos que o grupo quociente $B_{n} /\left[P_{n}, P_{n}\right]$, com $n \geq 3$, também é um grupo cristalográfico, onde, neste caso, o grupo de holonomia é $\Sigma_{n}$ e a dimensão $n(n-1) / 2$. Além disso, como feito no caso de 3 cordas, estudaremos os elementos de torção no quociente $B_{n} /\left[P_{n}, P_{n}\right]$.

\subsubsection{O grupo quociente $B_{n} /\left[P_{n}, P_{n}\right]$ é um grupo cristalográfico}

Lembremos que um conjunto de geradores para $B_{n}$ (resp. $P_{n}$ ) é dado pelos elementos $\sigma_{i}$, para $1 \leq i \leq n-1$ (resp. $A_{i, j}$ para $1 \leq i<j \leq n$ ). Vamos especificar parte da notação a ser usada durante esta seção. Sejam $q_{n}: B_{n} \rightarrow B_{n} /\left[B_{n}, B_{n}\right]$ o homomorfismo quociente e $\tau_{i}=q_{n}\left(\sigma_{i}\right)$, para $1 \leq i \leq n-1$, e $N_{i, j}=q_{n}\left(A_{i, j}\right)$ para $1 \leq i<j \leq n$, ver as Figuras 1.4 e 1.5. Seja $\mu_{i}=\sigma\left(\sigma_{i}\right)=\bar{\sigma}\left(\tau_{i}\right)$.

Teorema 5.3.1. Sejam $n \geq 3$ e $M=\mathbb{D}^{2}$. A representação obtida a partir da sequência exata curta (5.44)

$$
\Theta_{n}: \Sigma_{n} \rightarrow \operatorname{Aut}\left(P_{n} /\left[P_{n}, P_{n}\right]\right)
$$

é injetora. Como consequência temos que o grupo quociente $B_{n} /\left[P_{n}, P_{n}\right]$ é um grupo cristalográfico de dimensão $n(n-1) / 2$ com grupo de holonomia $\Sigma_{n}$.

Demonstração. Suponhamos que $n \geq 3$, vejamos que a representação

$$
\Theta_{n}: \Sigma_{n} \rightarrow \operatorname{Aut}\left(P_{n} /\left[P_{n}, P_{n}\right]\right)
$$

é injetora. O caso $n=4$ será tratado separado no final da prova, portanto vamos supor agora que $n \neq 4$. O resultado desejado para $n=3$ já foi estudado acima, no entanto a demonstração dada aqui, neste caso especial, é diferente daquela dada para o Teorema 5.2.1. Observemos que se $n=3$, ou $n \geq 5$, o único subgrupo normal do grupo de permutações $\Sigma_{n}$ é o subgrupo alternado $\mathcal{A}_{n}$, além disso $\Sigma_{n} / \mathcal{A}_{n}=\mathbb{Z}_{2}$, o subgrupo cíclico de ordem 2 . O núcleo de um homomorfismo é um subgrupo normal do grupo de saída do homomorfismo e portanto $\operatorname{Ker}\left(\Theta_{n}\right) \unlhd \Sigma_{n}$. Dessa forma, nossa estratégia para provar que $\operatorname{Ker}\left(\Theta_{n}\right)=\{1\}$ é provar que $\operatorname{Ker}\left(\Theta_{n}\right) \neq \mathcal{A}_{n}$, ou, de forma equivalente, que $\operatorname{Im}\left(\Theta_{n}\right)$ tem elementos (não 
triviais) de ordem diferente a 2 .

Sejam $\alpha=\mu_{2} \mu_{1}=\sigma\left(\tau_{2} \tau_{1}\right) \in \Sigma_{n}$ e $N_{i, j} \in P_{n} /\left[P_{n}, P_{n}\right]$, para $1 \leq i<j \leq n$. Então, pelas igualdades em (4.31), a ação de $\alpha$ nos elementos de $P_{n} /\left[P_{n}, P_{n}\right]$ é dada por

$$
\begin{aligned}
\alpha \cdot N_{i, j},= & \begin{cases}\mu_{2} \cdot N_{i, j}, & \text { se } i \neq 1,2 ; j \neq 2 \\
\mu_{2} \cdot N_{1,2}, & \text { se } i=1 ; j=2 \\
\mu_{2} \cdot N_{2, j}, & \text { se } i=1<j-1 \\
\mu_{2} \cdot N_{1, j}, & \text { se } i=2<j\end{cases} \\
= & \begin{array}{lll}
N_{i, j}, & \text { se } i \neq 1,2,3 ; j \neq 2,3 \\
N_{2, j}, & \text { se } i=3 \\
N_{1,3}, & \text { se } i=1 ; j=2 \\
N_{2,3}, & \text { se } i=1 ; j=3 \\
N_{3, j}, & \text { se } i=1<j-2 \\
N_{1,2}, & \text { se } i=2 ; j=3 \\
N_{1, j}, & \text { se } i=2<j-1 .
\end{array}
\end{aligned}
$$

Portanto, o automorfismo

$$
\Theta_{n}(\alpha): P_{n} /\left[P_{n}, P_{n}\right] \rightarrow P_{n} /\left[P_{n}, P_{n}\right]
$$

é definido por

$$
N_{1,2} \stackrel{\Theta_{n}(\alpha)}{\longmapsto} N_{1,3} \stackrel{\Theta_{n}(\alpha)}{\longmapsto} N_{2,3} \stackrel{\Theta_{n}(\alpha)}{\longmapsto} N_{1,2}
$$

e para $j \geq 4$

$$
N_{1, j} \stackrel{\Theta_{n}(\alpha)}{\longmapsto} N_{3, j} \stackrel{\Theta_{n}(\alpha)}{\longmapsto} N_{2, j} \stackrel{\Theta_{n}(\alpha)}{\longmapsto} N_{1, j}
$$

Das equações (5.45) e (5.46) é claro que $\Theta_{n}(\alpha)$ é um elemento de ordem 3 no grupo $\Theta_{n}\left(\Sigma_{n}\right)$, para $n \geq 3$. Portanto, $\operatorname{Im}\left(\Theta_{n}\right)$ tem elementos de ordem 3 e assim o grupo quociente $\Sigma_{n} / \Theta_{n}\left(\Sigma_{n}\right)$ é diferente de $\mathbb{Z}_{2}$. Logo, $\operatorname{Ker}\left(\Theta_{n}\right)=\{1\}$, como queríamos provar.

A seguir consideramos o caso dos grupos de tranças de Artin com 4 cordas, para isto descreveremos as 24 matrizes da representação $\Theta_{4}: \Sigma_{4} \rightarrow \operatorname{Aut}\left(P_{4} / \Gamma_{2}\left(P_{4}\right)\right)$. Lembremos que existe uma sequência exata curta

$$
1 \longrightarrow P_{4} / \Gamma_{2}\left(P_{4}\right) \longrightarrow B_{4} / \Gamma_{2}\left(P_{4}\right) \stackrel{\rho_{4}}{\longrightarrow} \Sigma_{4} \longrightarrow 1
$$

O grupo $P_{4} / \Gamma_{2}\left(P_{4}\right)=\mathbb{Z} \oplus \mathbb{Z} \oplus \mathbb{Z} \oplus \mathbb{Z} \oplus \mathbb{Z} \oplus \mathbb{Z}$ é gerado pelas classes de equivalência de $N_{1,2}, N_{1,3}, N_{1,4}, N_{2,3}, N_{2,4}, N_{3,4}[40]$. Denotemos por

$$
\begin{array}{ll}
e_{1}=N_{1,2}=(1,0,0,0,0,0), & e_{4}=N_{2,3}=(0,0,0,1,0,0), \\
e_{2}=N_{1,3}=(0,1,0,0,0,0), & e_{5}=N_{2,4}=(0,0,0,0,1,0), \\
e_{3}=N_{1,4}=(0,0,1,0,0,0), & e_{6}=N_{3,4}=(0,0,0,0,0,1),
\end{array}
$$


os geradores deste quociente. A ação por conjugação de $B_{4} /\left[P_{4}, P_{4}\right]$ sobre estes elementos é dada por

$$
\begin{array}{lll}
\tau_{1} e_{1} \tau_{1}^{-1}=e_{1}, & \tau_{2} e_{1} \tau_{2}^{-1}=e_{2}, & \tau_{3} e_{1} \tau_{3}^{-1}=e_{1}, \\
\tau_{1} e_{2} \tau_{1}^{-1}=e_{4}, & \tau_{2} e_{2} \tau_{2}^{-1}=e_{1}, & \tau_{3} e_{2} \tau_{3}^{-1}=e_{3}, \\
\tau_{1} e_{3} \tau_{1}^{-1}=e_{5}, & \tau_{2} e_{3} \tau_{2}^{-1}=e_{3}, & \tau_{3} e_{3} \tau_{3}^{-1}=e_{2}, \\
\tau_{1} e_{4} \tau_{1}^{-1}=e_{2}, & \tau_{2} e_{4} \tau_{2}^{-1}=e_{4}, & \tau_{3} e_{4} \tau_{3}^{-1}=e_{5}, \\
\tau_{1} e_{5} \tau_{1}^{-1}=e_{3}, & \tau_{2} e_{5} \tau_{2}^{-1}=e_{6}, & \tau_{3} e_{5} \tau_{3}^{-1}=e_{4}, \\
\tau_{1} e_{6} \tau_{1}^{-1}=e_{6}, & \tau_{2} e_{6} \tau_{2}^{-1}=e_{5}, & \tau_{3} e_{6} \tau_{3}^{-1}=e_{6} .
\end{array}
$$

Para $1 \leq i \leq 6$, denotemos por $v_{i}$ o vetor coluna de uma matriz de ordem $6 \times 6$ que tem 0 em todas as entradas, exceto a $i$-ésima, na qual tem o número 1 . Por exemplo, a matriz identidade é escrita usando os $v_{i}$ como $\left(\begin{array}{llllll}v_{1} & v_{2} & v_{3} & v_{4} & v_{5} & v_{6}\end{array}\right)$. Denotemos os 24 elementos de $\Sigma_{4}$ por

$$
\begin{array}{llll}
\text { (1) ident. } & \kappa_{1}=\tau_{1} & \kappa_{2}=\tau_{2} & \kappa_{3}=\tau_{3} \\
\kappa_{4}=\tau_{1} \tau_{2} \tau_{3} \tau_{2} \tau_{1} & \kappa_{5}=\tau_{1} \tau_{2} \tau_{1} & \kappa_{6}=\tau_{2} \tau_{3} \tau_{2} & \kappa_{7}=\tau_{1} \tau_{3} \\
\kappa_{8}=\kappa_{2} \kappa_{4} & \kappa_{9}=\kappa_{5} \kappa_{6} & \kappa_{10}=\tau_{1} \tau_{2} & \kappa_{11}=\tau_{2} \tau_{3} \\
\kappa_{12}=\tau_{2} \tau_{1} & \kappa_{13}=\tau_{3} \tau_{2} & \kappa_{14}=\kappa_{6} \kappa_{1} & \kappa_{15}=\kappa_{1} \kappa_{6} \\
\kappa_{16}=\kappa_{3} \kappa_{4} & \kappa_{17}=\kappa_{4} \kappa_{3} & \kappa_{18}=\kappa_{3} \kappa_{2} \kappa_{1} & \kappa_{19}=\kappa_{2} \kappa_{3} \kappa_{1} \\
\kappa_{20}=\kappa_{1} \kappa_{2} \kappa_{4} & \kappa_{21}=\kappa_{1} \kappa_{3} \kappa_{2} & \kappa_{22}=\kappa_{4} \kappa_{2} \kappa_{1} & \kappa_{23}=\kappa_{1} \kappa_{2} \kappa_{3} .
\end{array}
$$

Portanto, a representação

$$
\Theta_{4}: \Sigma_{4} \rightarrow \operatorname{Aut}\left(P_{4} /\left[P_{4}, P_{4}\right]\right)
$$

induzida pela ação por conjugação de $B_{4} /\left[P_{4}, P_{4}\right]$ sobre $P_{4} /\left[P_{4}, P_{4}\right]$, descrita em $(5.48)$, é definida nos elementos de $\Sigma_{4}$ dados em (5.49) por

$$
\begin{aligned}
& (1) \mapsto\left(\begin{array}{cccccc}
v_{1} & v_{2} & v_{3} & v_{4} & v_{5} & v_{6}
\end{array}\right), \quad \kappa_{12} \mapsto\left(\begin{array}{cccccc}
v_{2} & v_{4} & v_{6} & v_{1} & v_{3} & v_{5}
\end{array}\right), \\
& \kappa_{1} \mapsto\left(\begin{array}{cccccc}
v_{1} & v_{4} & v_{5} & v_{2} & v_{3} & v_{6}
\end{array}\right), \quad \kappa_{13} \mapsto\left(\begin{array}{llllll}
v_{3} & v_{1} & v_{2} & v_{5} & v_{6} & v_{4}
\end{array}\right), \\
& \kappa_{2} \mapsto\left(\begin{array}{cccccc}
v_{2} & v_{1} & v_{3} & v_{4} & v_{6} & v_{5}
\end{array}\right), \quad \kappa_{14} \mapsto\left(\begin{array}{cccccc}
v_{3} & v_{6} & v_{5} & v_{2} & v_{1} & v_{4}
\end{array}\right), \\
& \kappa_{3} \mapsto\left(\begin{array}{cccccc}
v_{1} & v_{3} & v_{2} & v_{5} & v_{4} & v_{6}
\end{array}\right), \quad \kappa_{15} \mapsto\left(\begin{array}{cccccc}
v_{5} & v_{4} & v_{1} & v_{6} & v_{3} & v_{2}
\end{array}\right), \\
& \kappa_{4} \mapsto\left(\begin{array}{cccccc}
v_{5} & v_{6} & v_{3} & v_{4} & v_{1} & v_{2}
\end{array}\right), \quad \kappa_{16} \mapsto\left(\begin{array}{llllll}
v_{4} & v_{6} & v_{2} & v_{5} & v_{1} & v_{3}
\end{array}\right), \\
& \kappa_{5} \mapsto\left(\begin{array}{cccccc}
v_{4} & v_{2} & v_{6} & v_{1} & v_{5} & v_{3}
\end{array}\right), \quad \kappa_{17} \mapsto\left(\begin{array}{cccccc}
v_{5} & v_{3} & v_{6} & v_{1} & v_{4} & v_{2}
\end{array}\right), \\
& \kappa_{6} \mapsto\left(\begin{array}{cccccc}
v_{3} & v_{2} & v_{1} & v_{6} & v_{5} & v_{4}
\end{array}\right), \quad \kappa_{18} \mapsto\left(\begin{array}{cccccc}
v_{3} & v_{5} & v_{6} & v_{1} & v_{2} & v_{4}
\end{array}\right), \\
& \kappa_{7} \mapsto\left(\begin{array}{cccccc}
v_{1} & v_{5} & v_{4} & v_{3} & v_{2} & v_{6}
\end{array}\right), \quad \kappa_{19} \mapsto\left(\begin{array}{cccccc}
v_{2} & v_{6} & v_{4} & v_{3} & v_{1} & v_{5}
\end{array}\right), \\
& \kappa_{8} \mapsto\left(\begin{array}{cccccc}
v_{6} & v_{5} & v_{3} & v_{4} & v_{2} & v_{1}
\end{array}\right), \quad \kappa_{20} \mapsto\left(\begin{array}{llllll}
v_{6} & v_{3} & v_{5} & v_{2} & v_{4} & v_{1}
\end{array}\right), \\
& \kappa_{9} \mapsto\left(\begin{array}{cccccc}
v_{6} & v_{2} & v_{4} & v_{3} & v_{5} & v_{1}
\end{array}\right), \quad \kappa_{21} \mapsto\left(\begin{array}{llllll}
v_{5} & v_{1} & v_{4} & v_{3} & v_{6} & v_{2}
\end{array}\right), \\
& \kappa_{10} \mapsto\left(\begin{array}{cccccc}
v_{4} & v_{1} & v_{5} & v_{2} & v_{6} & v_{3}
\end{array}\right), \quad \kappa_{22} \mapsto\left(\begin{array}{llllll}
v_{6} & v_{4} & v_{2} & v_{5} & v_{3} & v_{1}
\end{array}\right), \\
& \kappa_{11} \mapsto\left(\begin{array}{cccccc}
v_{2} & v_{3} & v_{1} & v_{6} & v_{4} & v_{5}
\end{array}\right), \quad \kappa_{23} \mapsto\left(\begin{array}{llllll}
v_{4} & v_{5} & v_{1} & v_{6} & v_{2} & v_{3}
\end{array}\right) .
\end{aligned}
$$


De (5.50) podemos ver que todas as matrizes são diferentes duas a duas e portanto a representação $\Theta_{4}: \Sigma_{4} \rightarrow \operatorname{Aut}\left(P_{4} /\left[P_{4}, P_{4}\right]\right)$ é injetora, como queríamos provar.

Observação 5.3.2. Gostaríamos de fazer alguns comentários relacionados ao caso de 4 cordas na prova do Teorema 5.3.1. Lembramos que $\Sigma_{4}$ tem dois subgrupos normais não triviais: o grupo alternado $A_{4}$ em cujo caso $\Sigma_{4} / A_{4}=\mathbb{Z}_{2}$ e o 4-subgrupo de Klein $V_{4}$, donde $\Sigma_{4} / V_{4}=\Sigma_{3}$. Observamos que $\operatorname{Ker}\left(\Theta_{n}\right) \neq A_{4}$, usando o mesmo argumento acima, para $n=3$ ou $n \geq 5$. Portanto, o núcleo de $\Theta_{n}$ poderia ser $V_{4}$ ou trivial $\{1\}$. Nesse momento não podemos distinguir qual dos dois grupos corresponde ao núcleo, a não ser o estudo da definição explícita de $\Theta_{4}: \Sigma_{4} \rightarrow \operatorname{Aut}\left(P_{4} /\left[P_{4}, P_{4}\right]\right)$ como feito acima. No entanto, veremos na seguinte seção que $B_{n} /\left[P_{n}, P_{n}\right]$ não tem elementos de torção 2, para $n \geq 3$. Logo, necessariamente $\operatorname{Ker}\left(\Theta_{4}\right) \neq V_{4}$.

Porém, a representação $\Theta_{4}: \Sigma_{4} \rightarrow A u t\left(P_{4} /\left[P_{4}, P_{4}\right]\right)$, dada em (5.50), será bastante útil na última seção, quando relacionarmos os grupos cristalográficos de tranças com variedades planas, bem como no estudo de existência de difeomorfismos de Anosov para tais variedades.

O seguinte corolário garante que qualquer grupo finito é o grupo de holonomia de um grupo cristalográfico, este último obtido a partir dos grupos de tranças do disco.

Corolário 5.3.3. Seja $H$ um grupo finito qualquer. Existe $n \geq 3$ tal que $H$ é um subgrupo de $\Sigma_{n}$ e nesse caso o grupo

$$
\frac{\sigma^{-1}(H)}{\left[P_{n}, P_{n}\right]}
$$

é um grupo cristalográfico de dimensão $n(n-1) / 2$ com grupo de holonomia $H$, onde $\sigma e ́$ o epimorfismo dado na sequência exata curta (5.43).

Demonstração. Pelo Teorema de Cayley todo grupo finito $H$ é isomorfo a um subgrupo de um grupo de permutações $\Sigma_{n}$, para algum $n$. Sem perda de generalidade suponhamos que de fato $H$ é um subgrupo de $\Sigma_{n}$ e que $n \geq 3$.

Consideremos a sequência exata curta

$$
1 \longrightarrow P_{n} /\left[P_{n}, P_{n}\right] \longrightarrow \frac{\sigma^{-1}(H)}{\left[P_{n}, P_{n}\right]} \stackrel{\bar{\sigma}}{\longrightarrow} H \longrightarrow 1
$$

Pela demonstração do Teorema 5.3.1, a representação $\Theta_{n}: \Sigma_{n} \rightarrow \operatorname{Aut}\left(P_{n} /\left[P_{n}, P_{n}\right]\right)$ é fiel, onde $P_{n} /\left[P_{n}, P_{n}\right] \cong \mathbb{Z}^{n(n-1) / 2}$. Portanto, a restrição $\Theta_{n} \mid: H \rightarrow \operatorname{Aut}\left(P_{n} /\left[P_{n}, P_{n}\right]\right)$ é por sua vez uma representação fiel. Logo, $\frac{\sigma^{-1}(H)}{\left[P_{n}, P_{n}\right]}$ é um grupo cristalográfico de dimensão $n(n-1) / 2$ e com grupo de holonomia $H$.

Dado um grupo finito $H$ como no Corolário 5.3.3, temos que existe $n \geq 3$ tal que $H$ é um subgrupo de $\Sigma_{n}$. Denotemos o grupo dado em (5.51) por $\mathcal{B C} \mathcal{G}(n, H)$ e o chamemos de 
grupo cristalográfico de tranças com $n$ cordas com grupo de holonomia H. Sabemos pelo Corolário 5.3.3 que o grupo cristalográfico de tranças $\mathcal{B C} \mathcal{G}(n, H)$ tem dimensão $n(n-1) / 2$. Observemos que $\mathcal{B C G}\left(n, \Sigma_{n}\right)=B_{n} /\left[P_{n}, P_{n}\right]$ e $\mathcal{B C G}(n,\{1\})=\mathbb{Z}^{n(n-1) / 2}$, este último é o grupo fundamental do toro $\mathbb{T}^{n(n-1) / 2}$.

Observação 5.3.4. O Corolário 5.3.3 é uma versão similar do Teorema de Auslander e Kuranishi, formulado no Teorema 5.1.8.

\subsubsection{Sobre a torção 2 em $B_{n} /\left[P_{n}, P_{n}\right]$}

O objetivo desta subseção é provar que, para $n \geq 3$, o grupo quociente $B_{n} /\left[P_{n}, P_{n}\right]$ não tem elementos de ordem 2. Inicialmente, no seguinte lema, estudaremos a conjugação de elementos de $P_{n}$ por elementos de $B_{n}$.

Lema 5.3.5. Sejam $n \geq 3,1 \leq i \leq k \leq n-1$, e $\gamma_{i, k}=\sigma_{i} \sigma_{i+1} \cdots \sigma_{k-1} \sigma_{k} \sigma_{k-1} \cdots \sigma_{i} \in B_{n}$, onde $\gamma_{k, k}=\sigma_{k}$. Então,

1. A permutação associada ao elemento $\gamma_{i, k}$ é a transposição $(i \quad(k+1))$, mesmo quando $i=k$.

2. Suponhamos que $i \neq k$. Então, $\sigma_{i+1} \sigma_{i+2} \cdots \sigma_{k-1} \sigma_{k} \cdot A_{i, k+1}=\omega_{i+1} A_{i, i+1} \omega_{i+1}^{-1}$, para algum $\omega_{i+1}$ em $P_{n}$. Além disso, $\sigma_{k} \sigma_{k-1} \cdots \sigma_{i+2} \sigma_{i+1} \cdot A_{i, i+1}=A_{i, k+1}$.

3. $\gamma_{i, k} A_{i, k+1} \gamma_{i, k}^{-1}=\alpha A_{i, k+1} \alpha^{-1}$, para algum $\alpha$ em $P_{n}$.

4. $\left(\gamma_{i, k}\right)^{2}=A_{i, k+1} A_{i+1, k+1} \cdots A_{k, k+1} A_{i, i+1} A_{i, i+2} \cdots A_{i, k}$.

Demonstração. 1. É claro pela própria definição do elemento $\gamma_{i, k}$. Um desenho da trança $\gamma_{i, k}$ é dado na Figura 5.1 abaixo.

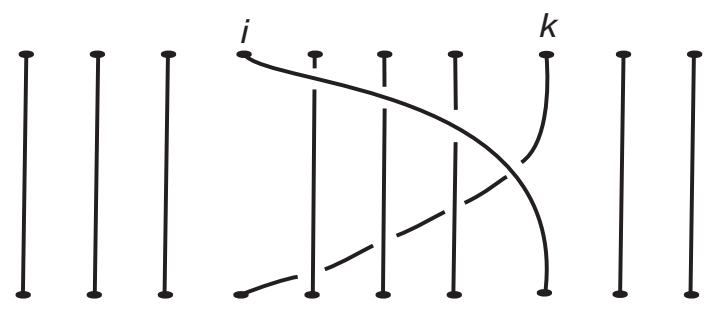

Figura 5.1: Desenho da trança $\gamma_{i, k}$.

2. Suponhamos que $i \neq j$. Observamos que, por (4.31), $\sigma_{j} \cdot A_{i, j+1}=A_{i, j+1}^{-1} A_{i, j} A_{i, j+1}$, para $i<j$. Logo,

$$
\begin{aligned}
\sigma_{i+1} \sigma_{i+2} \cdots \sigma_{k-1} \sigma_{k} \cdot A_{i, k+1} & =\sigma_{i+1} \sigma_{i+2} \cdots \sigma_{k-1} \cdot\left(\omega_{j} A_{i, k+1} \omega_{j}^{-1}\right) \\
& =\sigma_{i+1} \sigma_{i+2} \cdots \sigma_{k-2} \cdot\left(\omega_{j-1} A_{i, k+1} \omega_{j-1}^{-1}\right) \\
& \vdots \\
& =\omega_{i+1} A_{i, i+1} \omega_{i+1}^{-1},
\end{aligned}
$$


para algum $\omega_{i+1}$ em $P_{n}$. Usando que $\sigma_{j} \cdot A_{i, j}=A_{i, j+1}$ (por (4.31)), temos claramente, para $i \neq k$, que

$$
\sigma_{k} \sigma_{k-1} \cdots \sigma_{i+2} \sigma_{i+1} \cdot A_{i, i+1}=A_{i, k+1}
$$

3. Suponhamos inicialmente que $i=k$. Assim, $\gamma_{i, k}=\sigma_{k}$, e portanto

$$
\sigma_{k} A_{k, k+1} \sigma_{k}^{-1}=\sigma_{k}^{2}=A_{k, k+1}
$$

Vamos supor agora que $i \neq k$. Usando o item anterior temos que

$$
\begin{aligned}
\gamma_{i, k} A_{i, k+1} \gamma_{i, k}^{-1} & =\left(\sigma_{k} \sigma_{k-1} \cdots \sigma_{i+2} \sigma_{i+1}\right)\left(\sigma_{i}\right)\left(\sigma_{i+1} \sigma_{i+2} \cdots \sigma_{k-1} \sigma_{k}\right) \cdot A_{i, k+1} \\
& =\left(\sigma_{k} \sigma_{k-1} \cdots \sigma_{i+2} \sigma_{i+1}\right)\left(\sigma_{i}\right) \cdot\left(\omega_{i+1} A_{i, i+1} \omega_{i+1}^{-1}\right) \\
& =\left(\sigma_{k} \sigma_{k-1} \cdots \sigma_{i+2} \sigma_{i+1}\right) \cdot\left(\alpha_{1} A_{i, i+1} \alpha_{1}^{-1}\right) \\
& =\alpha A_{i, k+1} \alpha^{-1}
\end{aligned}
$$

onde $\alpha_{1}=\sigma_{i} \cdot \omega_{i+1} \in P_{n}$ e $\alpha=\left(\sigma_{k} \sigma_{k-1} \cdots \sigma_{i+2} \sigma_{i+1}\right)\left(\alpha_{1}\right) \in P_{n}$.

4. Se $i=k$ o resultado é claro. Suponhamos que $i \neq k(i<k)$. Provaremos inicialmente a seguinte igualdade, por indução em $n=k-i$ :

$$
\sigma_{k} \sigma_{k-1} \cdots \sigma_{i+1}\left(\sigma_{i} \sigma_{i+1} \cdots \sigma_{k-1} \sigma_{k}\right)^{2}=A_{i, k+1} A_{i+1, k+1} \cdots A_{k, k+1} \sigma_{i} \sigma_{i+1} \cdots \sigma_{k-1} .
$$

Verifiquemos o passo base, $n=1$

$$
\sigma_{i+1} \sigma_{i} \sigma_{i+1} \sigma_{i} \sigma_{i+1}=\sigma_{i+1} \sigma_{i}^{2} \sigma_{i+1} \sigma_{i}=A_{i, i+2} A_{i+1, i+2} \sigma_{i}
$$

Suponhamos que a afirmação é valida para $n-1$, vamos provar que também é válida para $n$. Usaremos aqui o fato que $\sigma_{k} \cdot A_{j, k}=A_{j, k+1}(\operatorname{ver}(4.31)$ ).

$$
\begin{aligned}
\sigma_{k} \cdots \sigma_{i+1}\left(\sigma_{i} \cdots \sigma_{k}\right)^{2} & =\sigma_{k} \sigma_{k-1} \cdots \sigma_{i+1}\left(\sigma_{i} \cdots \sigma_{k-1}\right)\left(\sigma_{k}\right)\left(\sigma_{i} \cdots \sigma_{k-2}\right) \sigma_{k-1} \sigma_{k} \\
& =\sigma_{k} \sigma_{k-1} \cdots \sigma_{i+1}\left(\sigma_{i} \cdots \sigma_{k-1}\right)\left(\sigma_{i} \cdots \sigma_{k-2}\right) \sigma_{k} \sigma_{k-1} \sigma_{k} \\
& =\sigma_{k}\left(\sigma_{k-1} \cdots \sigma_{i+1}\right)\left(\sigma_{i} \cdots \sigma_{k-1}\right)\left(\sigma_{i} \cdots \sigma_{k-2}\right) \sigma_{k} \sigma_{k-1} \sigma_{k} \\
& =\sigma_{k}\left(\sigma_{k-1} \cdots \sigma_{i+1}\right)\left(\sigma_{i} \cdots \sigma_{k-1}\right)^{2} \sigma_{k-1}^{-1} \sigma_{k} \sigma_{k-1} \sigma_{k} \\
& \overbrace{}^{\text {H.I. }} \sigma_{k}\left(A_{i, k} A_{i+1, k} \cdots A_{k-1, k} \sigma_{i} \sigma_{i+1} \cdots \sigma_{k-2}\right) \sigma_{k-1}^{-1} \sigma_{k} \sigma_{k-1} \sigma_{k} \\
& =\sigma_{k}\left(A_{i, k} A_{i+1, k} \cdots A_{k-1, k} \sigma_{i} \sigma_{i+1} \cdots \sigma_{k-2}\right) \sigma_{k} \sigma_{k-1} \\
& =\sigma_{k}\left(A_{i, k} A_{i+1, k} \cdots A_{k-1, k}\right) \sigma_{k}^{-1} \sigma_{k}^{2} \sigma_{i} \sigma_{i+1} \cdots \sigma_{k-2} \sigma_{k-1} \\
& =\sigma_{k}\left(A_{i, k} A_{i+1, k} \cdots A_{k-1, k}\right) \sigma_{k}^{-1} A_{k, k+1} \sigma_{i} \sigma_{i+1} \cdots \sigma_{k-2} \sigma_{k-1} \\
& =\left(A_{i, k+1} A_{i+1, k+1} \cdots A_{k, k+1}\right) A_{k, k+1} \sigma_{i} \sigma_{i+1} \cdots \sigma_{k-2} \sigma_{k-1} \\
& =A_{i, k+1} A_{i+1, k+1} \cdots A_{k, k+1} \sigma_{i} \sigma_{i+1} \cdots \sigma_{k-1} \cdot
\end{aligned}
$$


Sabemos que

$$
\begin{aligned}
\gamma_{i, k} & =\sigma_{i} \sigma_{i+1} \cdots \sigma_{k-1} \sigma_{k} \sigma_{k-1} \cdots \sigma_{i} \\
& =\sigma_{k} \sigma_{k-1} \cdots \sigma_{i+1} \sigma_{i} \sigma_{i+1} \cdots \sigma_{k}
\end{aligned}
$$

onde $\gamma_{k, k}=\sigma_{k}$. Portanto, para $i \neq k$, usando a afirmação anterior, temos que

$$
\begin{aligned}
\left(\gamma_{i, k}\right)^{2} & =\left(\sigma_{k} \sigma_{k-1} \cdots \sigma_{i+1} \sigma_{i} \sigma_{i+1} \cdots \sigma_{k}\right)\left(\sigma_{i} \sigma_{i+1} \cdots \sigma_{k-1} \sigma_{k} \sigma_{k-1} \cdots \sigma_{i}\right) \\
& =\sigma_{k} \cdots \sigma_{i+1}\left(\sigma_{i} \cdots \sigma_{k}\right)^{2}\left(\sigma_{k-1} \cdots \sigma_{i}\right) \\
& =A_{i, k+1} A_{i+1, k+1} \cdots A_{k, k+1} \sigma_{i} \sigma_{i+1} \cdots \sigma_{k-1}\left(\sigma_{k-1} \cdots \sigma_{i}\right) \\
& =A_{i, k+1} A_{i+1, k+1} \cdots A_{k, k+1}\left(\sigma_{i} \sigma_{i+1} \cdots \sigma_{k-2} \sigma_{k-1}^{2} \sigma_{k-2} \cdots \sigma_{i}\right) .
\end{aligned}
$$

Finalmente, usando as relações do grupo de tranças do disco, temos que

$$
\sigma_{i} \sigma_{i+1} \cdots \sigma_{k-2} \sigma_{k-1}^{2} \sigma_{k-2} \cdots \sigma_{i}=A_{i, i+1} A_{i, i+2} \cdots A_{i, k}
$$

e portanto

$$
\left(\gamma_{i, k}\right)^{2}=A_{i, k+1} A_{i+1, k+1} \cdots A_{k, k+1} A_{i, i+1} A_{i, i+2} \cdots A_{i, k}
$$

Teorema 5.3.6. Seja $n \geq 3$, então o grupo quociente $B_{n} /\left[P_{n}, P_{n}\right]$ não tem elementos de ordem 2.

Demonstração. Seja $n \geq 3$. Observemos que se um elemento em $B_{n} /\left[P_{n}, P_{n}\right]$ tem ordem 2 , então ele se projeta por $\bar{\sigma}$ numa transposição em $\Sigma_{n}$. Em vista desta observação, basta provarmos o resultado para os elementos na pré-imagem das transposições de $\Sigma_{n}$. Suponhamos por absurdo que existe um elemento $\beta \in B_{n} /\left[P_{n}, P_{n}\right]$ de ordem 2. Então, $\beta$ tem permutação associada $(i(k+1))$ e é da forma $\beta=N \lambda_{i, k}, \operatorname{com} N \in P_{n} /\left[P_{n}, P_{n}\right]$ e $\lambda_{i, k}=q_{n}\left(\gamma_{i, k}\right) \in B_{n} /\left[P_{n}, P_{n}\right]$, com $1 \leq i \leq k \leq n-1$ e $\gamma_{i, k}$ como no lema anterior. Portanto, $\beta^{2}=N \lambda_{i, k} N \lambda_{i, k}$ e assim

$$
N \lambda_{i, k} N \lambda_{i, k}^{-1} \lambda_{i, k}^{2}=(0, \ldots, 0) \in P_{n} /\left[P_{n}, P_{n}\right]=\mathbb{Z}^{n(n-1) / 2} .
$$

Pelo item 3 do Lema 5.3.5

$$
\lambda_{i, k} \cdot N_{i, k+1}=N_{i, k+1}
$$

e pelo item 4 do mesmo lema, o elemento $N_{i, k+1}$ faz parte da expressão de $\lambda_{i, k}^{2}$. Se $N_{i, k+1}$ não faz parte da expressão para $N$ então teremos que $1=0$, por (5.52), mas isto é uma contradição. Agora, se $N_{i, k+1}^{v}$ faz parte da expressão para $N$ então teremos que $2 v+1=0$, por (5.52) e (5.53), encontrando mais uma vez uma contradição.

Provamos que não existem elementos de ordem 2 em $B_{n} /\left[P_{n}, P_{n}\right]$, para $n \geq 3$, e com isto concluimos a prova do teorema. 
Observação 5.3.7. No caso de duas cordas temos que

$$
B_{2} /\left[P_{2}, P_{2}\right]=B_{2} / P_{2} \cong \mathbb{Z}_{2}
$$

\subsubsection{Elementos de ordem ímpar em $B_{n} /\left[P_{n}, P_{n}\right]$}

Vamos agora provar que, em geral, o grupo quociente $B_{n} /\left[P_{n}, P_{n}\right]$ tem elementos de torção ímpar, para $n \geq 3$.

Proposição 5.3.8. Seja $n \geq 3$ e definamos $\alpha_{0, n}=\tau_{1} \tau_{2} \ldots \tau_{n-1} \in B_{n} /\left[P_{n}, P_{n}\right]$. As seguintes igualdades são válidas em $P_{n} /\left[P_{n}, P_{n}\right]$.

1. $\alpha_{0, n} N_{i, j} \alpha_{0, n}^{-1}=N_{i+1, j+1}$, para $1 \leq i<j \leq n-1$.

2. $\alpha_{0, n} N_{i, n} \alpha_{0, n}^{-1}=N_{1, i+1}$, para $1 \leq i<j=n$.

Demonstração. Provaremos inicialmente que para $1 \leq i<j \leq n-1$ vale

$$
\alpha_{0, n} N_{i, j} \alpha_{0, n}^{-1}=N_{i+1, j+1}
$$

Observemos que, para $n=3$,

$$
\alpha_{0,3} N_{1,2} \alpha_{0,3}^{-1}=\tau_{1} \tau_{2} \tau_{1}^{2} \tau_{2}^{-1} \tau_{1}^{-1}=\tau_{1} \tau_{2} \tau_{1} \tau_{1} \tau_{2}^{-1} \tau_{1}^{-1}=\tau_{2}^{2}=N_{2,3}
$$

Suponhamos que a igualdade (5.54) é válida para $n-1$, provaremos que ela é válida para $n$.

$$
\alpha_{0, n} \cdot N_{i, j}=\left(\alpha_{0, n-1} \tau_{n-1}\right) \cdot N_{i, j}= \begin{cases}\alpha_{0, n-1} \cdot N_{i, j}, & \text { para } j \leq n-2 \\ \alpha_{0, n-1} \cdot N_{i, n}, & \text { para } i<j=n-1 .\end{cases}
$$

Pela hipótese de indução $\alpha_{0, n-1} N_{i, j}=N_{i+1, j+1}$, para $j \leq n-2$. Para o caso $j=n-1$ temos, por (4.31), que

$$
\begin{aligned}
\alpha_{0, n-1} N_{i, n} & =\tau_{1} \cdots \tau_{i} \tau_{i+1} \cdots \tau_{n-2} \cdot N_{i, n} \\
& =\tau_{1} \cdots \tau_{i} \cdot N_{i, n} \\
& =\tau_{1} \cdots \tau_{i-1} \cdot N_{i+1, n} \\
& =N_{i+1, n} \\
& =N_{i+1, j+1}
\end{aligned}
$$

Dessa forma completamos o resultado desejado para o item 1. Agora, verifiquemos o item 2 dessa proposição. De (4.31) segue que $\tau_{k} N_{k+1, k+2} \tau_{k}^{-1}=N_{k, k+2}$ e portanto para $i \neq 1$ temos

$$
\tau_{1} \tau_{2} \cdots \tau_{i-1} N_{i, i+1} \tau_{i-1}^{-1} \cdots \tau_{2}-1 \tau_{1}^{-1}=N_{1, i+1}
$$


Claramente $\tau_{i} N_{i, i+1} \tau_{i}^{-1}=N_{i, i+1}$ e, pelo Lema 5.3.5, temos

$$
\tau_{i+1} \cdots \tau_{n-1} N_{i, n} \tau_{n-1}^{-1} \cdots \tau_{i+1}^{-1}=N_{i, i+1}
$$

Logo,

$$
\begin{aligned}
\alpha_{0, n} N_{i, n} \alpha_{0, n}^{-1} & =\left(\tau_{1} \tau_{2} \cdots \tau_{i-1}\right)\left(\tau_{i}\right)\left(\tau_{i+1} \cdots \tau_{n-1}\right) \cdot N_{i, n} \\
& =\left(\tau_{1} \tau_{2} \cdots \tau_{i-1}\right)\left(\tau_{i}\right) \cdot N_{i, i+1} \\
& =\tau_{1} \tau_{2} \cdots \tau_{i-1} \cdot N_{i, i+1} \\
& =N_{1, i+1} .
\end{aligned}
$$

Com isto terminamos a prova do segundo item, assim como desta proposição.

A Proposição 5.3 .8 é útil para estudarmos ciclos em $P_{n} /\left[P_{n}, P_{n}\right]$ induzidos pela ação por conjugação do elemento $\alpha_{0, n} \in P_{n} /\left[P_{n}, P_{n}\right]$. Vamos enunciar por separado o caso de um número par de cordas $n$ do caso ímpar. Por um abuso de linguagem escrevemos $\alpha_{0, n}=\Theta_{n}\left(\alpha_{0, n}\right): P_{n} /\left[P_{n}, P_{n}\right] \rightarrow P_{n} /\left[P_{n}, P_{n}\right]$ nos seguintes dois resultados.

Corolário 5.3.9. Seja $n \geq 4$ um número par. Em $P_{n} /\left[P_{n}, P_{n}\right]$ existem $(n-2) / 2$ ciclos de tamanho $n$ e um ciclo de tamanho $n / 2$ induzidos pela ação de $\alpha_{0, n}$ nos geradores de $P_{n} /\left[P_{n}, P_{n}\right]$. Os $n / 2$ ciclos são dados por:

- $N_{1,2} \stackrel{\alpha_{0, n}}{\longmapsto} N_{2,3} \stackrel{\alpha_{0, n}}{\longmapsto} \cdots \stackrel{\alpha_{0, n}}{\longmapsto} N_{n-2, n-1} \stackrel{\alpha_{0, n}}{\longmapsto} N_{n-1, n} \stackrel{\alpha_{0, n}}{\longmapsto} N_{1, n} \stackrel{\alpha_{0, n}}{\longmapsto} N_{1,2}$.

- $N_{1,3} \stackrel{\alpha_{0, n}}{\longmapsto} N_{2,4} \stackrel{\alpha_{0, n}}{\longmapsto} \cdots \stackrel{\alpha_{0, n}}{\longmapsto} N_{n-2, n} \stackrel{\alpha_{0, n}}{\longmapsto} N_{1, n-1} \stackrel{\alpha_{0, n}}{\longmapsto} N_{2, n} \stackrel{\alpha_{0, n}}{\longmapsto} N_{1,3}$.

- $N_{1, \frac{n}{2}} \stackrel{\alpha_{0, n}}{\longmapsto} N_{2, \frac{n+2}{2}} \stackrel{\alpha_{0, n}}{\longmapsto} \cdots \stackrel{\alpha_{0, n}}{\longmapsto} N_{\frac{n+2}{2}, n} \stackrel{\alpha_{0, n}}{\longmapsto} N_{1, \frac{n+4}{2}} \stackrel{\alpha_{0, n}}{\longmapsto} \cdots \stackrel{\alpha_{0, n}}{\longmapsto} N_{\frac{n-2}{2}, n} \stackrel{\alpha_{0, n}}{\longmapsto} N_{1, \frac{n}{2}}$.

- $N_{1, \frac{n+2}{2}} \stackrel{\alpha_{0, n}}{\longmapsto} N_{2, \frac{n+4}{2}} \stackrel{\alpha_{0, n}}{\longmapsto} \cdots \stackrel{\alpha_{0, n}}{\longmapsto} N_{\frac{n}{2}, n} \stackrel{\alpha_{0, n}}{\longmapsto} N_{1, \frac{n+2}{2}}$.

Na Seção 5.4 estudaremos grupos de Bieberbach de tranças com grupo de holonomia de ordem uma potência de 2 e para esse estudo o Corolário 5.3.9 será de grande utilidade, em particular, será aplicado para determinar a existência de difeomorfismos de Anosov de algumas variedades planas.

Corolário 5.3.10. Seja $n$ um número ímpar. Em $P_{n} /\left[P_{n}, P_{n}\right]$ existem $(n-1) / 2$ ciclos de ordem $n$ induzidos pela ação de $\alpha_{0, n}$ nos geradores de $P_{n} /\left[P_{n}, P_{n}\right]$. Por um abuso de linguagem escrevemos $\alpha_{0, n}=\Theta_{n}\left(\alpha_{0, n}\right): P_{n} /\left[P_{n}, P_{n}\right] \rightarrow P_{n} /\left[P_{n}, P_{n}\right]$ e assim os $(n-1) / 2$ ciclos são dados por:

- $N_{1,2} \stackrel{\alpha_{0, n}}{\longmapsto} N_{2,3} \stackrel{\alpha_{0, n}}{\longmapsto} \cdots \stackrel{\alpha_{0, n}}{\longmapsto} N_{n-2, n-1} \stackrel{\alpha_{0, n}}{\longmapsto} N_{n-1, n} \stackrel{\alpha_{0, n}}{\longmapsto} N_{1, n} \stackrel{\alpha_{0, n}}{\longmapsto} N_{1,2}$.

- $N_{1,3} \stackrel{\alpha_{0, n}}{\longmapsto} N_{2,4} \stackrel{\alpha_{0, n}}{\longmapsto} \cdots \stackrel{\alpha_{0, n}}{\longmapsto} N_{n-2, n} \stackrel{\alpha_{0, n}}{\longmapsto} N_{1, n-1} \stackrel{\alpha_{0, n}}{\longmapsto} N_{2, n} \stackrel{\alpha_{0, n}}{\longmapsto} N_{1,3}$. 
- $N_{1,4} \stackrel{\alpha_{0, n}}{\longmapsto} N_{2,5} \stackrel{\alpha_{0, n}}{\longmapsto} \cdots \stackrel{\alpha_{0, n}}{\longmapsto} N_{n-3, n} \stackrel{\alpha_{0, n}}{\longmapsto} N_{1, n-2} \stackrel{\alpha_{0, n}}{\longmapsto} N_{2, n-1} \stackrel{\alpha_{0, n}}{\longmapsto} N_{3, n} \stackrel{\alpha_{0, n}}{\longmapsto} N_{1,4}$.

- $N_{1, \frac{n+1}{2}} \stackrel{\alpha_{0, n}}{\longmapsto} N_{2, \frac{n+3}{2}} \stackrel{\alpha_{0, n}}{\longmapsto} \cdots \stackrel{\alpha_{0, n}}{\longmapsto} N_{\frac{n+1}{2}, n} \stackrel{\alpha_{0, n}}{\longmapsto} N_{1, \frac{n+3}{2}} \stackrel{\alpha_{0, n}}{\longmapsto} \cdots \stackrel{\alpha_{0, n}}{\longmapsto} N_{\frac{n-1}{2}, n} \stackrel{\alpha_{0, n}}{\longmapsto} N_{1, \frac{n+1}{2}}$.

O Corolário 5.3.10 será fundamental para provar que o grupo cristalográfico de tranças $B_{n} /\left[P_{n}, P_{n}\right]$, com $n \geq 3$ ímpar, tem elementos de ordem $n$.

Teorema 5.3.11. Seja $n \geq 3$ um número impar. Então, $B_{n} /\left[P_{n}, P_{n}\right]$ tem elementos de ordem $n$.

Demonstração. Notemos que $P_{n} /\left[P_{n}, P_{n}\right] \cong \mathbb{Z}^{\frac{n(n-1)}{2}}$. Consideremos a seguinte base para $P_{n} /\left[P_{n}, P_{n}\right]$, de acordo com o Corolário 5.3.10:

$$
\begin{array}{ccccc}
e_{1,1}=N_{1,2}, & e_{1,2}=N_{2,3}, & \ldots & e_{1, n-1}=N_{n-1, n}, & e_{1, n}=N_{1, n}, \\
e_{2,1}=N_{1,3}, & e_{2,2}=N_{2,4}, & \ldots & e_{2, n-1}=N_{1, n-1}, & e_{2, n}=N_{2, n}, \\
\vdots & \vdots & & \vdots & \vdots \\
e_{\frac{n-1}{2}, 1}=N_{1, \frac{n+1}{2}}, & e_{\frac{n-1}{2}, 2}=N_{2, \frac{n+3}{2}}, & \ldots & e_{\frac{n-1}{2}, n-1}=N_{\frac{n-3}{2}, n-1}, & e_{\frac{n-1}{2}, n}=N_{\frac{n-1}{2}, n},
\end{array}
$$

Desta forma, pelo Corolário 5.3.10, segue que

$$
\begin{gathered}
e_{1,1} \stackrel{\alpha_{0, n}}{\longmapsto} e_{1,2} \stackrel{\alpha_{0, n}}{\longmapsto} \cdots \stackrel{\alpha_{0, n}}{\longmapsto} e_{1, n-1} \stackrel{\alpha_{0, n}}{\longmapsto} e_{1, n} \stackrel{\alpha_{0, n}}{\longmapsto} e_{1,1} \\
\vdots \\
e_{\frac{n-1}{2}, 1} \stackrel{\alpha_{0, n}}{\longmapsto} e_{\frac{n-1}{2}, 2} \stackrel{\alpha_{0, n}}{\longmapsto} \cdots \stackrel{\alpha_{0, n}}{\longmapsto} e_{\frac{n-1}{2}, n-1} \stackrel{\alpha_{0, n}}{\longmapsto} e_{\frac{n-1}{2}, n} \stackrel{\alpha_{0, n}}{\longmapsto} e_{\frac{n-1}{2}, 1} .
\end{gathered}
$$

Seja $N \in P_{n} /\left[P_{n}, P_{n}\right]$, podemos supor que

$$
N=\left(a_{1,1}, \ldots, a_{1, n} ; a_{2,1}, \ldots, a_{2, n} ; \cdots ; a_{\frac{n-1}{2}, 1}, \ldots, a_{\frac{n-1}{2}, n}\right)
$$

Portanto, usando a informação anterior, temos que

$$
\left(N \cdot \alpha_{0, n}\right)^{n}=N+\alpha_{0, n} N \alpha_{0, n}^{-1}+\alpha_{0, n}^{2} N \alpha_{0, n}^{-2}+\cdots+\alpha_{0, n}^{n-1} N \alpha_{0, n}^{1-n}+\alpha_{0, n}^{n}
$$

Como $\alpha_{0, n}=q_{n}\left(\Delta_{n}\right)$ (a classe do "full twist" em $P_{n} /\left[P_{n}, P_{n}\right]$ ), então $\alpha_{0, n}=(1,1, \ldots, 1)$, o elemento que tem 1 em todas as coordenadas. Notemos que

$$
\alpha_{0, n} N \alpha_{0, n}^{-1}=\left(a_{1, n}, a_{1,1}, \ldots, a_{1, n-1} ; a_{2, n}, a_{2,1}, \ldots, a_{2, n-1} ; \cdots ; a_{\frac{n-1}{2}, n}, a_{\frac{n-1}{2}, 1}, \ldots, a_{\frac{n-1}{2}, n-1}\right)
$$

e assim por diante permutamos ciclicamente as entradas de $N$, para $\alpha_{0, n}^{r} N \alpha_{0, n}^{-r}$, com 
$2 \leq r \leq n-1$. Portanto, podemos deduzir que a igualdade

$$
\left(N \cdot \alpha_{0, n}\right)^{n}=(0, \ldots, 0) \in P_{n} /\left[P_{n}, P_{n}\right]
$$

é equivalente ao seguinte sistema de equações

$$
\left\{\begin{array}{c}
a_{1,1}+a_{1,2}+a_{1, n}+1=0 \\
a_{2,1}+a_{2,2}+a_{2, n}+1=0 \\
\vdots \\
a_{\frac{n-1}{2}, 1}+a_{\frac{n-1}{2}, 2}+a_{\frac{n-1}{2}, n}+1=0 .
\end{array}\right.
$$

Como o sistema de equações lineares (5.55) tem infinitas soluções, então o resultado desejado é obtido.

Acabamos de provar que se $n \geq 3$ é ímpar, então o grupo quociente $B_{n} /\left[P_{n}, P_{n}\right]$ tem elementos de ordem $n$ e com isto podemos demonstrar que se $m$ é um inteiro maior ou igual que $n \geq 3$, então o grupo quociente $B_{m} /\left[P_{m}, P_{m}\right]$ tem elementos de ordem ímpar, para qualquer ímpar menor ou igual que $m$.

Teorema 5.3.12. Sejam $m$ e $n$ dois inteiros quaisquer tais que $1 \leq n \leq m$.

1. Consideremos o homomorfismo $\iota: B_{n} \rightarrow B_{m}$ definido por $\sigma_{i} \mapsto \sigma_{i}$, para todo $1 \leq i \leq n-1$, e a projeção natural deste homomorfismo no grupo quociente $\bar{\iota}: B_{n} /\left[P_{n}, P_{n}\right] \rightarrow B_{m} /\left[P_{m}, P_{m}\right]$. Então, o homomorfismo $\bar{\iota}$ é injetor.

2. Se $n \geq 3$ e impar, então $B_{m} /\left[P_{m}, P_{m}\right]$ tem elementos de ordem $n$.

Demonstração. Sejam $m$ e $n$ dois inteiros quaisquer tais que $1 \leq n \leq m$.

1. Vamos começar provando que o homomorfismo $\bar{\iota} \mid: P_{n} /\left[P_{n}, P_{n}\right] \rightarrow P_{m} /\left[P_{m}, P_{m}\right]$ é injetor, onde $\iota \mid: P_{n} \rightarrow P_{m}$ é definida por $A_{i, j} \mapsto A_{i, j}$, para $1 \leq i<j \leq n$. Lembremos que $P_{n} /\left[P_{n}, P_{n}\right] \cong \mathbb{Z}^{n(n-1) / 2}$ é um grupo livre abeliano de posto $n(n-1) / 2$ gerado pelas classes de equivalência $\overline{A_{i, j}}$, para $1 \leq i<j \leq n$. O mesmo acontece com o grupo livre abeliano $P_{m} /\left[P_{m}, P_{m}\right] \cong \mathbb{Z}^{m(m-1) / 2}$. Portanto, usando a notação aditiva para os grupos $P_{n} /\left[P_{n}, P_{n}\right]$ e $P_{m} /\left[P_{m}, P_{m}\right]$, temos que $\bar{\iota} \mid: P_{n} /\left[P_{n}, P_{n}\right] \rightarrow P_{m} /\left[P_{m}, P_{m}\right]$ é definida por

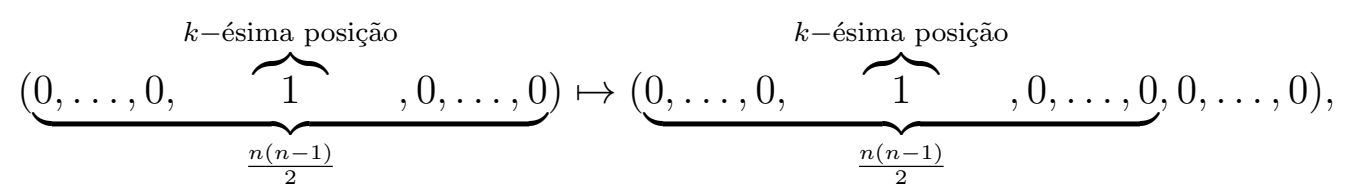

donde claramente vemos que $\bar{\iota} \mid: P_{n} /\left[P_{n}, P_{n}\right] \rightarrow P_{m} /\left[P_{m}, P_{m}\right]$ é injetor. Observemos que o grupo quociente $B_{n} /\left[P_{n}, P_{n}\right]$ se projeta no grupo de permutações $\Sigma_{n}$ e este 
último está contido no grupo de permutações $\Sigma_{m}$. Logo, o seguinte diagrama é comutativo

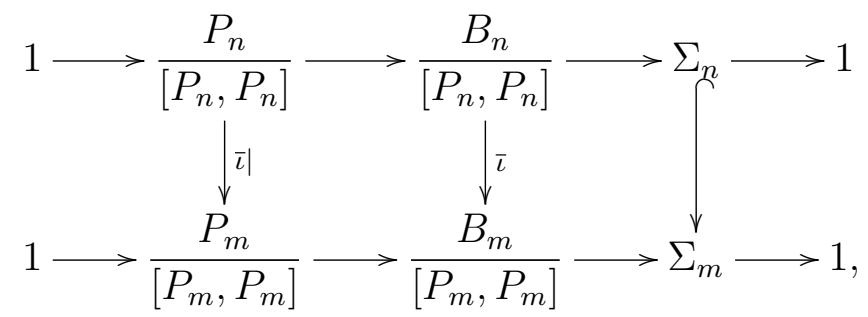

onde as flechas verticais nas pontas são injetoras. Desta forma, usando a comutatividade do diagrama temos que $\bar{\iota}: B_{n} /\left[P_{n}, P_{n}\right] \rightarrow B_{m} /\left[P_{m}, P_{m}\right]$ é injetor.

2. Suponhamos que $n \geq 3$ e ímpar. Como $n$ é ímpar, do Teorema 5.3.11, segue que o grupo $B_{n} /\left[P_{n}, P_{n}\right]$ tem elementos de ordem $n$ e como, pelo item 1 deste resultado, o homomorfismo $\bar{\iota}: B_{n} /\left[P_{n}, P_{n}\right] \rightarrow B_{m} /\left[P_{m}, P_{m}\right]$ é injetor, então o grupo quociente $B_{m} /\left[P_{m}, P_{m}\right]$ tem elementos de ordem $n$ ímpar com $3 \leq n \leq m$.

\subsection{Tranças, variedades planas e existência de difeo- morfismos de Anosov}

Nesta seção estamos interessados nos grupos cristalográficos de tranças livres de torção, isto é, nos grupos de Bieberbach obtidos a partir do quociente $B_{n} /\left[P_{n}, P_{n}\right]$. Mais especificamente os subgrupos deste grupo que são de Bieberbach e que têm a mesma dimensão do grupo cristalográfico $B_{n} /\left[P_{n}, P_{n}\right]$. Na primeira subseção nós descreveremos como obter tais subgrupos e daremos uma nova prova do Teorema de Auslander e Kuranishi (Teorema 5.1.8) para holonomia dada por 2-grupos finitos. Nas demais subseções estudaremos com mais detalhes alguns casos especiais, a saber, o caso $n=4$ e o caso onde a holonomia é cíclica $\mathbb{Z}_{2^{k}}$. Finalmente, na última subseção para vários desses grupos estudaremos o problema de existência de difeomorfismos de Anosov.

\subsubsection{Os grupos de Bieberbach e o Teorema de Auslander e Kuranishi}

Começaremos esta subseção com o seguinte lema.

Lema 5.4.1. Sejam $n \geq 3$ e $H$ um subgrupo de $\Sigma_{n}$. Se $H$ é um 2-grupo finito, então $\mathcal{B C G}(n, H)$ é um grupo de Bieberbach de dimensão $n(n-1) / 2$ que é a dimensão do grupo cristalográfico $B_{n} /\left[P_{n}, P_{n}\right]$. 
Demonstração. Sejam $n \geq 3$ e $H$ um 2-grupo finito contido em $\Sigma_{n}$. Pelo Corolário 5.3.3 sabemos que

$$
\mathcal{B C G}(n, H)=\frac{\sigma^{-1}(H)}{\left[P_{n}, P_{n}\right]}
$$

é um grupo cristalográfico de dimensão $n(n-1) / 2$ com grupo de holonomia $H$, onde $\sigma$ é o epimorfismo dado na sequência exata curta (5.43). Como $H$ é um 2-grupo finito, então só tem torção 2 e portanto a única possibilidade de torção para o grupo $\mathcal{B C} \mathcal{G}(n, H)$ é 2 . Mas, pelo Teorema 5.3.6, o grupo $B_{n} /\left[P_{n}, P_{n}\right]$ não tem torção 2 e $\operatorname{assim} \mathcal{B C G}(n, H)$ também não tem torção 2, donde segue que $\mathcal{B C G}(n, H)$ é um grupo critalográfico livre de torção, ou seja, é um grupo de Bieberbach.

Não é claro no presente se, a menos de isomorfismo, essa família representa todos os subgrupos de $B_{n} /\left[P_{n}, P_{n}\right]$ que são grupos de Bieberbach de dimensão $n(n-1) / 2$.

A seguir, vamos dar uma formulação do Teorema de Auslander e Kuranishi (Teorema $5.1 .8)$, via o grupo quociente $B_{n} /\left[P_{n}, P_{n}\right]$, para 2-grupos finitos. Isto dá uma solução ao problema da realização de 2-grupos finitos como grupos de holonomia de variedades planas.

Teorema 5.4.2. Seja $H$ um 2-grupo finito. Então, existem $n \geq 3$ e uma variedade plana $M$, de dimensão $n(n-1) / 2$, tal que $H$ é o grupo de holonomia de $M$. Ainda mais, o grupo fundamental de $M$ é um grupo de Bieberbach que é um subgrupo de $B_{n} /\left[P_{n}, P_{n}\right]$.

Demonstração. Seja $H$ um 2-grupo finito. Pelo Teorema de Cayley existe um inteiro $n \geq 3$ tal que $H$ é um subgrupo de $\Sigma_{n}$. Pelo Lema 5.4 .1 sabemos que $\mathcal{B C G}(n, H)$ é um grupo de Bieberbach de dimensão $n(n-1) / 2$ com grupo de holonomia $H$ e contido em $B_{n} /\left[P_{n}, P_{n}\right]$. Logo, pelos Teoremas de Bieberbach, existe uma variedade plana $M$, de dimensão $n(n-1) / 2$, com grupo de holonomia $H$, e tal que

$$
\pi_{1}(M)=\mathcal{B C} \mathcal{G}(n, H)
$$

O Teorema de Auslander e Kuranishi, Teorema 5.1.8, põe a questão natural: dado um grupo finito $H$, qual é a menor dimensão da variedade plana que tem grupo de holonomia $H$ ? Em vista da prova do Teorema 5.4.2, o que obtemos aqui é uma realização da holonomia que depende do menor grupo de permutações que contém o 2-grupo finito $H$. Em geral, isso não vai coincidir com a menor dimensão. 


\subsubsection{Grupos de Bieberbach $\mathcal{B C} G(4, H)$ e com grupo de holono- $\operatorname{mia} \mathbb{Z}_{2^{k}}$}

Pelo Lema 5.4.1 se $H$ é um 2-grupo finito contido em $\Sigma_{n}$, para $n \geq 3$, então $\mathcal{B C G}(n, H)$ é um grupo de Bieberbach $\mathcal{B C G}(n, H)$ de dimensão $n(n-1) / 2$ com grupo de holonomia $H$. O objetivo desta subseção é estudar, para $n \geq 4$, todos os possíveis grupos $\mathcal{B C} \mathcal{G}(4, H)$, e para $n$ arbitrário estudar os grupos $\mathcal{B C G}(n, H)$, com $H$ um 2-grupo cíclico.

Teorema 5.4.3. Sejam $H_{1}=\mathbb{Z}_{4}, H_{2}=\mathbb{Z}_{2} \oplus \mathbb{Z}_{2}$ e $H_{3}$ igual ao grupo diedral (de ordem 8). Para cada $1 \leq i \leq 3$, existe uma variedade plana orientável $\mathscr{Y}_{i}$ de dimensão 6 , com grupo de holonomia $H_{i}$, e com grupo fundamental o grupo de Bieberbach $\mathcal{B C G}\left(4, H_{i}\right) \subseteq B_{4} /\left[P_{4}, P_{4}\right]$

Demonstração. Para $1 \leq i \leq 3$, consideremos os grupos $H_{i}$ como subgrupos de $\Sigma_{4}$. Assim,

- O grupo cíclico $H_{1}=\mathbb{Z}_{4}$, é o grupo $\left\langle\left(\begin{array}{llll}1 & 2 & 3 & 4\end{array}\right)\right\rangle$.

- Lembremos que $\Sigma_{4}$ tem dois subgrupos de Klein, isomorfos a $\mathbb{Z}_{2} \oplus \mathbb{Z}_{2}$, um deles normal e o outro não. Seja $H_{2}=\left\{(1),\left(\begin{array}{ll}1 & 2\end{array}\right)\left(\begin{array}{ll}3 & 4\end{array}\right),\left(\begin{array}{lll}1 & 3\end{array}\right)\left(\begin{array}{ll}2 & 4\end{array}\right),\left(\begin{array}{ll}1 & 4\end{array}\right)\left(\begin{array}{ll}2 & 3\end{array}\right)\right\}$ o grupo de Klein normal em $\Sigma_{4}$.

- O grupo diedral (de ordem 8) é igual a $H_{3}=\left\langle\left(\begin{array}{llll}1 & 2 & 3 & 4\end{array}\right),\left(\begin{array}{ll}1 & 3\end{array}\right)\right\rangle$.

Para cada $1 \leq i \leq 3$, pelo Lema 5.4.1 com $n=4, \mathcal{B C G}\left(4, H_{i}\right)$ é um grupo de Bieberbach de dimensão 6 com grupo de holonomia $H_{i}$. Pelo Teorema 5.4.2 existe uma variedade plana $\mathscr{Y}_{i}$ de dimensão 6 , com grupo de holonomia $H_{i}$, e com grupo fundamental o grupo de Bieberbach $\mathcal{B C G}\left(4, H_{i}\right)$. Vejamos que as variedades planas $\mathscr{Y}_{i}$ são orientáveis. Usando a notação do Teorema 5.3.1, temos que $H_{1}=\left\langle\kappa_{18}\right\rangle, H_{2}=\left\{(1), \kappa_{7}, \kappa_{9}, \kappa_{8}\right\}$ e $H_{3}=\left\langle\kappa_{18}, \kappa_{5}\right\rangle$. Por simples inspeção, nas matrizes

$$
\Theta_{4}\left(\kappa_{7}\right), \Theta_{4}\left(\kappa_{18}\right), \Theta_{4}\left(\kappa_{9}\right), \Theta_{4}\left(\kappa_{8}\right), \Theta_{4}\left(\kappa_{5}\right)
$$

dadas em (5.50), vemos que trocando as colunas um número par de vezes obtemos a matriz identidade e portanto as matrizes que aparecem em (5.57) tem determinante 1. Isto é, para $1 \leq i \leq 3$, a imagem da representação $\Theta_{4}: H_{i} \rightarrow \operatorname{Aut}\left(P_{4} /\left[P_{4}, P_{4}\right]\right)$ está contida no grupo $S O(6 ; \mathbb{Z})$. Logo, para $1 \leq i \leq 3$, a representação de holonomia é $\Theta_{4}: H_{i} \rightarrow S O(6 ; \mathbb{Z})$ e as variedades planas $\mathscr{Y}_{i}$ de dimensão 6 com grupo de holonomia $H_{i}$ são orientáveis.

Observação 5.4.4. O caso $\mathbb{Z}_{2} \subset \Sigma_{4}$ será considerado mais na frente. $O$ caso $\mathbb{Z}_{4} \subset \Sigma_{4}$ também será considerado num caso mais geral, mas com ciclo máximo distinto. Os 2grupos de ordem 8 são, a menos de isomorfismo, $\mathbb{Z}_{2} \oplus \mathbb{Z}_{2} \oplus \mathbb{Z}_{2}, \mathbb{Z}_{4} \oplus \mathbb{Z}_{2}, \mathbb{Z}_{8}$, o grupo dos quatérnios e o grupo diedral (ordem 8). É bem conhecido que, entre os 2-grupos de ordem 8, apenas o grupo diedral aparece como subgrupo de $\Sigma_{4}$, na verdade os únicos 2-grupos que são isomorfos a 2-grupos de $\Sigma_{4}$ são $\mathbb{Z}_{2}$ e os grupos $H_{1}, H_{2}, H_{3}$ dados acima. 
Na continuação vamos considerar os 2-grupos cíclicos $\mathbb{Z}_{2^{k}}$, com $k \geq 2$, gerados pelo ciclo máximo

$$
\bar{\sigma}\left(\alpha_{0,2^{k}}\right)=\left(\begin{array}{llllll}
1 & 2^{k} & 2^{k}-1 & \cdots & 3 & 2
\end{array}\right)
$$

de $\Sigma_{2^{k}}$, onde o elemento $\alpha_{0,2^{k}}$ é o elemento dado na Proposição 5.3 .8 e o homomorfismo $\bar{\sigma}$ é aquele da sequência exata curta (5.44).

Teorema 5.4.5. Seja $k \geq 2$. O grupo cristalográfico de tranças $\mathcal{B C G}\left(2^{k},\left\langle\bar{\sigma}\left(\alpha_{0,2^{k}}\right)\right\rangle\right)$ é um grupo de Bieberbach com uma apresentação dada por

- Geradores: $\alpha_{0,2^{k}}$ e $N_{i, j}$, para $1 \leq i<j \leq 2^{k}$.

- Relações:

1. Todos os $N_{i, j}$, para $1 \leq i<j \leq 2^{k}$, comutam.

2. $\alpha_{0,2^{k}}^{2^{k}}=\prod_{1 \leq i<j \leq 2^{k}} N_{i, j}$.

3. $\alpha_{0,2^{k}} N_{i, j} \alpha_{0,2^{k}}^{-1}=N_{i+1, j+1}$, para $1 \leq i<j \leq 2^{k}-1$.

4. $\alpha_{0,2^{k}} N_{i, 2^{k}} \alpha_{0,2^{k}}^{-1}=N_{1, i+1}$, para $1 \leq i \leq 2^{k}-1$.

Como consequência o grupo abelianizado de $\mathcal{B C G}\left(2^{k},\left\langle\bar{\sigma}\left(\alpha_{0,2^{k}}\right)\right\rangle\right)$ é $\left(\oplus_{2^{k-1}} \mathbb{Z}\right) \oplus \mathbb{Z}_{2^{k-1}}$.

Demonstração. Pelo Lema 5.4.1 sabemos que $\mathcal{B C G}\left(2^{k},\left\langle\bar{\sigma}\left(\alpha_{0,2^{k}}\right)\right\rangle\right)$ é um grupo cristalográfico. Observemos que $\alpha_{0,2^{k}}^{2^{k}}=\left(\tau_{1} \cdots \tau_{2^{k}-1}\right)^{2^{k}}=\overline{\Delta_{2^{k}}}$, este último é a classe do "full twist" em $B_{2^{k}} /\left[P_{2^{k}}, P_{2^{k}}\right]$, e pela Proposição 5.3.8 conhecemos a ação por conjugação do elemento $\alpha_{0,2^{k}}$ nos elementos de $P_{n} /\left[P_{n}, P_{n}\right]=\mathbb{Z}^{2^{k-1}\left(2^{k}-1\right)}$. Logo, utilizando a técnica do livro do Johnson, [50, Seção 10.2], para calcular apresentações de extensões de grupos, aplicada na sequência exata curta

$$
1 \longrightarrow \mathbb{Z}^{2^{k-1}\left(2^{k}-1\right)} \longrightarrow \mathcal{B C G}\left(2^{k},\left\langle\bar{\sigma}\left(\alpha_{0,2^{k}}\right)\right\rangle\right) \stackrel{\bar{\sigma}}{\longrightarrow} \mathbb{Z}_{2^{k}} \longrightarrow 1
$$

obtemos a primeira afirmação deste resultado, que se refere à apresentação do grupo de Bieberbach $\mathcal{B C G}\left(2^{k},\left\langle\bar{\sigma}\left(\alpha_{0,2^{k}}\right)\right\rangle\right)$.

Com a apresentação obtida para $\mathcal{B C G}\left(2^{k},\left\langle\bar{\sigma}\left(\alpha_{0,2^{k}}\right)\right\rangle\right)$ vamos dar uma apresentação do grupo abelianizado e assim mostrar que

$$
\left(\mathcal{B C G}\left(2^{k},\left\langle\bar{\sigma}\left(\alpha_{0,2^{k}}\right)\right\rangle\right)\right)_{a b}
$$

é da forma $\left(\oplus_{2^{k-1}} \mathbb{Z}\right) \oplus \mathbb{Z}_{2^{k-1}}$. Supondo que o elemento $\alpha_{0,2^{k}}$ comuta com todos os elementos $N_{i, j}$ de $P_{2^{k}} /\left[P_{2^{k}}, P_{2^{k}}\right]$, para $1 \leq i<j \leq 2^{k}$, segue que

1. $N_{i, j}=N_{i+1, j+1}$, para $1 \leq i<j \leq 2^{k}-1$.

2. $N_{i, 2^{k}}=N_{1, i+1}$, para $1 \leq i \leq 2^{k}-1$. 
Logo, o grupo $\left(\mathcal{B C G}\left(2^{k},\left\langle\bar{\sigma}\left(\alpha_{0,2^{k}}\right)\right\rangle\right)\right)_{a b}$ tem uma apresentação dada por

- Geradores: $\alpha_{0,2^{k}}$ e $N_{1, j}$, para $2 \leq j \leq 2^{k-1}+1$.

- Relações:

1. Os elementos $\alpha_{0,2^{k}}$ e $N_{1, j}$, para $2 \leq j \leq 2^{k-1}+1$, comutam todos dois a dois.

2. $\alpha_{0,2^{k}}^{2^{k}}=\left(\prod_{j=1}^{2^{k-1}} N_{1, j}^{2^{k}}\right) N_{1,2^{k-1}+1}^{2^{k-1}}$.

Para esclarecer a igualdade $\alpha_{0,2^{k}}^{2^{k}}=\left(\prod_{j=1}^{2^{k-1}} N_{1, j}^{2^{k}}\right) N_{1,2^{k-1}+1}^{2^{k-1}}$ sugerimos ver novamente o Corolário 5.3.9. Reescrevendo a relação $\alpha_{0,2^{k}}^{2^{k}}=\left(\prod_{j=1}^{2^{k-1}} N_{1, j}^{2^{k}}\right) N_{1,2^{k-1}+1}^{2^{k-1}}$ da forma

$$
1=\left(\left(\alpha_{0,2^{k}}^{-1} \prod_{j=1}^{2^{k-1}} N_{1, j}\right)^{2} N_{1,2^{k-1}+1}\right)^{2^{k-1}}
$$

segue que o grupo $\left(\mathcal{B C G}\left(2^{k},\left\langle\bar{\sigma}\left(\alpha_{0,2^{k}}\right)\right\rangle\right)\right)_{a b}$ é isomorfo ao grupo abeliano $\left(\oplus_{2^{k-1}} \mathbb{Z}\right) \oplus \mathbb{Z}_{2^{k-1}}$, onde cada um dos elementos $\alpha_{0,2^{k}}$ e $N_{1, j}$, para $2 \leq j \leq 2^{k-1}$, gera uma cópia de $\mathbb{Z}$ e $\mathbb{Z}_{2^{k-1}}$ é gerado por $t=\left(\alpha_{0,2^{k}}^{-1} \prod_{j=1}^{2^{k-1}} N_{1, j}\right)^{2} N_{1,2^{k-1}+1}$.

No seguinte resultado vamos estudar os grupos cíclicos de ordem $2^{k}$, para $k \geq 2$, dados por $\left\langle\bar{\sigma}\left(\alpha_{0,2^{k}}\right)\right\rangle=\mathbb{Z}_{2^{k}} \subseteq \Sigma_{2^{k}}$. Observemos que o menor grupo simétrico que contém o grupo cíclico $\mathbb{Z}_{2^{k}}$ é justamente $\Sigma_{2^{k}}$. O caso de holonomia cíclica de ordem 2 será tratado no Teorema 5.4.8. Para demonstrar o seguinte resultado faremos uso das matrizes circulantes, ver Seção 6.4 .

Teorema 5.4.6. Seja $k \geq 2$. Para todo $k$ existe uma variedade plana orientável $\mathscr{X}_{k}$, de dimensão $2^{k-1}\left(2^{k}-1\right)$, com grupo de holonomia $\mathbb{Z}_{2^{k}}$, e com grupo fundamental o grupo de Bieberbach $\mathcal{B C G}\left(2^{k},\left\langle\bar{\sigma}\left(\alpha_{0,2^{k}}\right)\right\rangle\right)$. Além disso o primeiro grupo de homologia é dado por $H_{1}\left(\mathscr{X}_{k} ; \mathbb{Z}\right)=\left(\oplus_{2^{k-1}} \mathbb{Z}\right) \oplus \mathbb{Z}_{2^{k-1}}$.

Demonstração. Seja $k \geq 2$. Pelo Teorema 5.4.5 sabemos que $\mathcal{B C G}\left(2^{k},\left\langle\bar{\sigma}\left(\alpha_{0,2^{k}}\right)\right\rangle\right)$ é um grupo de Bieberbach de dimensão $2^{k-1}\left(2^{k}-1\right)$ e com grupo de holonomia $\mathbb{Z}_{2^{k}}$. Como na prova do Teorema 5.4.2, existe uma variedade plana $\mathscr{X}_{k}$, de dimensão $2^{k-1}\left(2^{k}-1\right)$, com grupo de holonomia $\mathbb{Z}_{2^{k}}$, e grupo fundamental o grupo de Bieberbach $\mathcal{B C G}\left(n,\left\langle\alpha_{0,2^{k}}\right\rangle\right)$. 
Como o primeiro grupo de homologia de $\mathscr{X}_{k}$ é igual ao grupo abelianizado do seu grupo fundamental $\mathcal{B C G}\left(n,\left\langle\alpha_{0,2^{k}}\right\rangle\right)$, então pelo Teorema 5.4.5 segue que

$$
H_{1}\left(\mathscr{X}_{k} ; \mathbb{Z}\right)=\left(\oplus_{2^{k-1}} \mathbb{Z}\right) \oplus \mathbb{Z}_{2^{k-1}}
$$

Falta por provar que a variedade plana $\mathscr{X}_{k}$ é orientável, para isto vamos demonstrar que a representação de holonomia $\Theta_{k}:\left\langle\bar{\sigma}\left(\alpha_{0,2^{k}}\right)\right\rangle=\mathbb{Z}_{2^{k}} \rightarrow O\left(2^{k-1}\left(2^{k}-1\right) ; \mathbb{Z}\right)$ é tal que $\Theta_{k}\left(\left\langle\bar{\sigma}\left(\alpha_{0,2^{k}}\right)\right\rangle\right) \subseteq S O\left(2^{k-1}\left(2^{k}-1\right) ; \mathbb{Z}\right)$, ou de forma equivalente, que a matriz $\Theta_{k}\left(\bar{\sigma}\left(\alpha_{0,2^{k}}\right)\right)$ tem determinante igual a 1 . Tomemos uma base para o grupo $P_{2^{k}} /\left[P_{2^{k}}, P_{2^{k}}\right]=\mathbb{Z}^{2^{k-1}\left(2^{k}-1\right)}$ usando a ação por conjugação de $\alpha_{0,2^{k}}$ nos elementos $N_{i, j}$, para todo $1 \leq i<j \leq 2^{k}$, dada no Corolário 5.3.9. Desta forma, uma base para $P_{2^{k}} /\left[P_{2^{k}}, P_{2^{k}}\right]$ pode ser dada por:

- Para $1 \leq i \leq 2^{k-1}, e_{i, 1}=N_{1, i+1}$.

- Para $1 \leq i \leq 2^{k-1}-1$ e $1 \leq j \leq 2^{k}-1, e_{i, j+1}=\alpha_{0,2^{k}}^{j}\left(e_{i, 1}\right)$.

- Para $1 \leq j \leq 2^{k-1}-1, e_{2^{k-1}, j+1}=\alpha_{0,2^{k}}^{j}\left(e_{2^{k-1}, 1}\right)$.

Fixemos um valor $s$ entre 1 e $2^{k-1}-1$. Então, segundo o Corolário 5.3.9, o ciclo gerado pela ação de $\alpha_{0,2^{k}}^{j}$ no elemento $e_{s, 1}$ é dado por

$$
e_{s, 1} \mapsto e_{s, 2} \mapsto e_{s, 3} \cdots \mapsto e_{s, 2^{k}-1} \mapsto e_{s, 1}
$$

A matriz associada a este ciclo é de tamanho $2^{k} \times 2^{k}$ e da forma

$$
M_{k, s}=\left(\begin{array}{cccccccc}
0 & 0 & 0 & \cdots & 0 & 0 & 0 & 1 \\
1 & 0 & 0 & \cdots & 0 & 0 & 0 & 0 \\
0 & 1 & 0 & \cdots & 0 & 0 & 0 & 0 \\
0 & 0 & 1 & \cdots & 0 & 0 & 0 & 0 \\
0 & 0 & 0 & \ddots & 0 & 0 & 0 & 0 \\
0 & 0 & 0 & \cdots & 1 & 0 & 0 & 0 \\
0 & 0 & 0 & \cdots & 0 & 1 & 0 & 0 \\
0 & 0 & 0 & \cdots & 0 & 0 & 1 & 0
\end{array}\right) .
$$

De forma similar, a matriz associada ao ciclo de tamanho $2^{k-1}$ gerada pela ação de $\alpha_{0,2^{k}}^{j}$ no elemento $e_{2^{k-1}, 1}$ é de tamanho $2^{k-1} \times 2^{k-1}$ e da forma

$$
M_{k, 2^{k-1}}=\left(\begin{array}{cccccc}
0 & 0 & \cdots & 0 & 0 & 1 \\
1 & 0 & \cdots & 0 & 0 & 0 \\
0 & 1 & \cdots & 0 & 0 & 0 \\
0 & 0 & \ddots & 0 & 0 & 0 \\
0 & 0 & \cdots & 1 & 0 & 0 \\
0 & 0 & \cdots & 0 & 1 & 0
\end{array}\right) .
$$


Logo, a matriz dada pela representação de holonomia

$$
\Theta_{k}:\left\langle\bar{\sigma}\left(\alpha_{0,2^{k}}\right)\right\rangle=\mathbb{Z}_{2^{k}} \rightarrow O\left(2^{k-1}\left(2^{k}-1\right) ; \mathbb{Z}\right)
$$

é formada por blocos, da seguinte forma

$$
\Theta_{k}\left(\bar{\sigma}\left(\alpha_{0,2^{k}}\right)\right)=M_{k}=\left(\begin{array}{ccccc}
M_{k, 1} & 0 & 0 & 0 & 0 \\
0 & M_{k, 2} & 0 & 0 & 0 \\
0 & 0 & \ddots & 0 & 0 \\
0 & 0 & 0 & M_{k, 2^{k-1}-1} & 0 \\
0 & 0 & 0 & 0 & M_{k, 2^{k-1}}
\end{array}\right)
$$

onde $M_{k, s}$, para $1 \leq s \leq 2^{k-1}-1$, são matrizes circulantes principais, com entrada principal 1, de tamanho $2^{k} \times 2^{k}, \operatorname{PCM}\left(2^{k}, 1\right)$; a matriz $M_{k, 2^{k-1}}$ é uma matriz circulante principal, com entrada principal 1 , de tamanho $2^{k-1} \times 2^{k-1}, \operatorname{PCM}\left(2^{k-1}, 1\right)$ e os $0^{\prime}$ s indicam matrizes (do tamanho apropriado) com todas as entradas iguais a zero. Dado que as matrizes circulantes principais $M_{k, s}$, para $1 \leq s \leq 2^{k-1}$, são de tamanho par, então cada uma destas matrizes tem determinante igual a -1 , ver Seção 6.4. Logo, o determinante de $M_{k}$ é igual a 1, pois o número de blocos de matrizes $M_{k, s}$ é par e $\operatorname{det}\left(M_{k}\right)=\prod_{s=1}^{2^{k-1}} \operatorname{det}\left(M_{k, s}\right)$.

Vamos estudar agora o 2-grupo $\mathbb{Z}_{2}$ visto como o grupo gerado pela transposição $\left(\begin{array}{ll}1 & 2\end{array}\right)$, daí $\mathbb{Z}_{2}=\left\langle\left(\begin{array}{ll}1 & 2\end{array}\right)\right\rangle$. Seja $n \geq 3$. Uma apresentação de $\mathcal{B C G}\left(n,\left\langle\left(\begin{array}{ll}1 & 2\end{array}\right)\right\rangle\right)$ será dada no seguinte teorema e com esta apresentação veremos que o abelianizado deste grupo é um grupo abeliano livre.

Teorema 5.4.7. Seja $n \geq 3$. O grupo cristalográfico de tranças $\mathcal{B C G}(n,\langle(12)\rangle)$ é um grupo de Bieberbach com uma apresentação dada por

- Geradores: $\tau_{1}$ e $N_{i, j}$, para $1 \leq i<j \leq n$.

- Relações:

1. Todos os $N_{i, j}$, para $1 \leq i<j \leq n$, comutam.

2. $\tau_{1}^{2}=N_{1,2}$.

3. $\tau_{1} N_{1,2} \tau_{1}^{-1}=N_{1,2}$ e $\tau_{1} N_{i, j} \tau_{1}^{-1}=N_{i, j}$, para $3 \leq i<j \leq n$.

4. $\tau_{1} N_{1, j} \tau_{1}^{-1}=N_{2, j}$, para $3 \leq j \leq n$.

5. $\tau_{1} N_{2, j} \tau_{1}^{-1}=N_{1, j}$, para $3 \leq j \leq n$.

Como consequência o grupo abelianizado de $\mathcal{B C G}\left(n,\left\langle\left(\begin{array}{ll}1 & 2\end{array}\right)\right\rangle\right)$ é um grupo abeliano livre de posto $\frac{(n-3) n+4}{2}$. 
Demonstração. Seja $n \geq 3$. Pelo Corolário 5.3.3, o grupo $\mathcal{B C G}(n,\langle(12)\rangle) \subseteq B_{n} /\left[P_{n}, P_{n}\right]$ é um grupo cristalográfico de dimensão $n(n-1) / 2$, com grupo de holonomia $\left\langle\left(\begin{array}{ll}1 & 2\end{array}\right)\right\rangle=\mathbb{Z}_{2}$. Pelo Teorema 5.3.6 $B_{n} /\left[P_{n}, P_{n}\right]$ não tem elementos de torção 2 . $\operatorname{Logo}, \mathcal{B C G}(n,\langle(12)\rangle)$ é livre de torção e portanto um grupo de Bieberbach.

Para mostrar a apresentação dada no enunciado para o grupo $\mathcal{B C} \mathcal{G}\left(n,\left\langle\left(\begin{array}{ll}1 & 2\end{array}\right)\right\rangle\right)$, aplicamos a técnica do livro do Johnson (para calcular apresentações de extensões de grupos), [50, Seção 10.2], aplicada na extensão de grupos

$$
1 \longrightarrow \mathbb{Z}^{\frac{n(n-1)}{2}} \longrightarrow \mathcal{B C G}(n,\langle(1 \quad 2)\rangle) \longrightarrow \mathbb{Z}_{2} \longrightarrow 1
$$

Com a apresentação de grupo podemos dar uma apresentação do grupo abelianizado e assim determinar que é um grupo abeliano livre de posto finito

$$
\frac{n(n-1)}{2}-(n-2)=\frac{(n-3) n+4}{2},
$$

onde cada cópia de $\mathbb{Z}$ é gerada por um, e somente um, dos elementos $\tau_{1}, N_{i, j}$, para $3 \leq i<j \leq n$, e $N_{1, j}$, para $3 \leq j \leq n$.

O Teorema 5.4.7 será usado no seguinte resultado, para determinar o primeiro grupo de homologia de uma certa variedade plana dada.

Teorema 5.4.8. Sejam $n \geq 4$ e $\mathscr{M}_{n}$ a variedade plana unicamente determinada pelo grupo de Bieberbach $\mathcal{B C G}\left(n,\left\langle\left(\begin{array}{ll}1 & 2\end{array}\right)\right\rangle\right)$. Então, $\mathscr{M}_{n}$ tem dimensão $n(n-1) / 2$, grupo de holonomia isomorfo a $\mathbb{Z}_{2}$, e é uma variedade plana orientável se $n$ é par e não orientável se $n$ é ímpar. Além disso, $H_{1}\left(\mathscr{M}_{n} ; \mathbb{Z}\right)=\mathbb{Z} \frac{(n-3) n+4}{2}$.

Demonstração. Seja $n \geq 4$. Pelo Teorema 5.4.7 temos que $\mathcal{B C G}\left(n,\left\langle\left(\begin{array}{ll}1 & 2\end{array}\right)\right\rangle\right)$ é um grupo de Bieberbach. Pelos Teoremas de Bieberbach sabemos que existe uma correspondência um a um entre os grupos de Bieberbach e as variedades planas. Sejam $n \geq 4$ e $\mathscr{M}_{n}$ a variedade plana unicamente determinada pelo grupo de Bieberbach $\mathcal{B C G}\left(n,\left\langle\left(\begin{array}{ll}1 & 2\end{array}\right)\right\rangle\right)$. Pelo Teorema 5.4 .7 sabemos que o abelianizado do grupo $\mathcal{B C G}\left(n,\left\langle\left(\begin{array}{ll}1 & 2\end{array}\right)\right\rangle\right)$ é um grupo livre de posto $\frac{(n-3) n+4}{2}$ e portanto o primeiro grupo de homologia de $\mathscr{M}_{n}$ é isomorfo a $\mathbb{Z}^{\frac{(n-3) n+4}{2}}$.

Vamos renomear os elementos $N_{i, j}$ do subgrupo $\mathbb{Z}^{\frac{n(n-1)}{2}}$ de $\mathcal{B C G}(n,\langle(1 \quad 2)\rangle$ ) (dados no Teorema 5.4.7) da seguinte forma:

- $e_{2 j-5}=N_{1, j}$, para $3 \leq j \leq n$,

- $e_{2 j-4}=N_{2, j}$, para $3 \leq j \leq n$,

- e por $e_{k}$ os outros geradores que são fixos pela ação por conjugação. Observemos que $\mathrm{s}$ ao $\frac{n(n-1)}{2}-2(n-2)=\frac{(n-5) n+8}{2}$ no total. A ordem em que estão enumerados estes últimos elementos $e_{k}$ não é relevante para o propósito a seguir. 
Usando a representação de holonomia induzida pela sequência exata curta

$$
1 \longrightarrow \mathbb{Z}^{\frac{n(n-1)}{2}} \longrightarrow \mathcal{B C G}(n,\langle(1 \quad 2)\rangle) \longrightarrow \mathbb{Z}_{2} \longrightarrow 1
$$

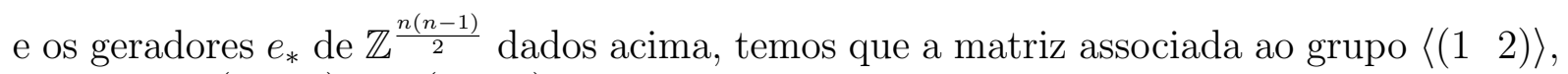
de ordem $\frac{n(n-1)}{2} \times \frac{n(n-1)}{2}$, é dada por

$$
\left(\begin{array}{cccccccccccccc}
0 & 1 & 0 & 0 & 0 & 0 & 0 & 0 & 0 & 0 & 0 & \ldots & 0 & 0 \\
1 & 0 & 0 & 0 & 0 & 0 & 0 & 0 & 0 & 0 & 0 & \ldots & 0 & 0 \\
0 & 0 & 0 & 1 & 0 & 0 & 0 & 0 & 0 & 0 & 0 & \ldots & 0 & 0 \\
0 & 0 & 1 & 0 & 0 & 0 & 0 & 0 & 0 & 0 & 0 & \ldots & 0 & 0 \\
0 & 0 & 0 & 0 & \ddots & 0 & 0 & 0 & 0 & 0 & 0 & \ldots & 0 & 0 \\
0 & 0 & 0 & 0 & 0 & 0 & 1 & 0 & 0 & 0 & 0 & \ldots & 0 & 0 \\
0 & 0 & 0 & 0 & 0 & 1 & 0 & 0 & 0 & 0 & 0 & \ldots & 0 & 0 \\
0 & 0 & 0 & 0 & 0 & 0 & 0 & 1 & 0 & 0 & 0 & \ldots & 0 & 0 \\
0 & 0 & 0 & 0 & 0 & 0 & 0 & 0 & \ddots & 0 & 0 & \ldots & 0 & 0 \\
\vdots & \vdots & \vdots & \vdots & \vdots & \vdots & \vdots & \vdots & \vdots & \ddots & \vdots & & \vdots & \vdots \\
0 & 0 & 0 & 0 & 0 & 0 & 0 & 0 & 0 & 0 & 1 & \ldots & 0 & 0 \\
0 & 0 & 0 & 0 & 0 & 0 & 0 & 0 & 0 & 0 & 0 & \ddots & 0 & 0 \\
0 & 0 & 0 & 0 & 0 & 0 & 0 & 0 & 0 & 0 & 0 & \ldots & 1 & 0 \\
0 & 0 & 0 & 0 & 0 & 0 & 0 & 0 & 0 & 0 & 0 & \ldots & 0 & 1
\end{array}\right)
$$

Observemos que, em (5.66), o número de blocos de submatrizes de ordem $2 \times 2$ da forma $\left(\begin{array}{ll}0 & 1 \\ 1 & 0\end{array}\right)$ é $n-2$ e o número de submatrizes de ordem $1 \times 1$ com entrada 1 é $\frac{(n-5) n+8}{2}$. Portanto, o determinante da matriz que aparece em (5.66) é \pm 1 e depende apenas do número de blocos de ordem $2 \times 2$. Isto é, o determinante é 1 se temos um número par de blocos (em cujo caso $n-2$ é par) e o determinante é -1 se o número de blocos é ímpar (em cujo caso $n-2$ é ímpar).

\subsubsection{Existência de difeomorfismos de Anosov para algumas va- riedades planas}

Vimos no Teorema 5.3.1 que, para $n \geq 3$, o grupo quociente $B_{n} /\left[P_{n}, P_{n}\right]$ é um grupo cristalográfico e no Teorema 5.3.6 verificamos que este grupo não tem torção 2. Com isto construímos nos Teoremas 5.4.3, 5.4.6 e 5.4.8, variedades planas com grupo de holonomia um 2-grupo finito e grupo fundamental um grupo de Bieberbach que é subgrupo de um quociente do grupo de tranças de Artin. Estando agora no ambiente da Geometria podemos fazer muitas perguntas sobre a variedade plana $M$, que é unicamente determi- 
nada por $\mathcal{B C G}(n, H)$ e em alguns casos será possível dar uma resposta a estas perguntas usando a estrutura do grupo $\mathcal{B C G}(n, H)$, bem como sua representação de holonomia $\Theta: H \rightarrow \operatorname{Aut}\left(P_{n} /\left[P_{n}, P_{n}\right]\right)$. Uma dessas questões consiste no problema de existência de difeomorfismos de Anosov. Para uma grande parte dessas variedades encontradas vamos estudar essa questão.

Observemos que um exemplo da interação entre as propriedades algébricas e geométricas de variedades planas $M$ foi dado nos Teoremas 5.2.13, 5.4.3, 5.4.6 e 5.4.8, onde foi possível decidir a orientação das variedades e seu primeiro grupo de homologia; ainda mais, no Teorema 5.2.13 conseguimos decidir que a variedade correspondente é um fibrado sobre o círculo com fibra a garrafa de Klein.

A questão da existência de difeomorfismos de Anosov é um problema importante em Sistemas Dinâmicos, a definição precisa de difeomorfismo de Anosov e mais detalhes sobre este problema podem ser vistos na Seção 6.4. No resultado abaixo utilizaremos um Teorema de Porteous que dá condições para a existência de difeomorfismos de Anosov para variedades planas com grupo de holonomia um grupo cíclico. Ver a formulação completa do Teorema de Porteous no Teorema 6.4.1 da Seção 6.4.

Teorema 5.4.9. Para todo $n \geq 4$, a variedade plana $\mathscr{M}_{n}$ dada no Teorema 5.4.8 (orientável para $n$ par e não orientável para $n$ impar), com grupo de holonomia $\mathbb{Z}_{2}$, admite difeomorfismo de Anosov. Para todo $k \geq 3$ a variedade plana orientável $\mathscr{X}_{k}$ do Teorema 5.4.6, de dimensão $2^{k-1}\left(2^{k}-1\right)$, com grupo de holonomia $\mathbb{Z}_{2^{k}}$, admite difeomorfismo de Anosov.

No entanto, a variedade plana orientável $\mathscr{Y}_{1}$ dada no Teorema 5.4 .3 e a variedade plana orientável $\mathscr{X}_{2}$ do Teorema 5.4.6, ambas de dimensão 6 e com grupo de holonomia $\mathbb{Z}_{4}$, não admitem difeomorfismo de Anosov.

Demonstração. Seja $n \geq 4$, vamos provar inicialmente que a variedade plana $\mathscr{M}_{n}$ com grupo de holonomia $\mathbb{Z}_{2}$, do Teorema 5.4.8, admite difeomorfismo de Anosov. A matriz que representa o gerador de $\mathbb{Z}_{2}$ usando a representação de holonomia $\mathbb{Z}_{2} \rightarrow \operatorname{Aut}\left(P_{n} /\left[P_{n}, P_{n}\right]\right)$ é dada em (5.66). Observemos que os autovalores da matriz (5.66) são 1 ou -1 e cada um destes autovalores aparece com multiplicidade maior que 1 , pois $n \geq 4$ e o número de blocos de submatrizes de ordem $2 \times 2$ é igual a $n-2$. Logo, aplicando o Teorema de Porteous, formulado no Teorema 6.4.1, podemos concluir que a variedade plana $\mathscr{M}_{n}$ admite difeomorfismo de Anosov.

Fixemos um valor para $k$, com $k \geq 3$, e tomemos a variedade plana orientável $\mathscr{X}_{k}$ do Teorema 5.4.6, que tem dimensão $2^{k-1}\left(2^{k}-1\right)$ com grupo de holonomia $\mathbb{Z}_{2^{k}}$. Vamos demonstrar que $\mathscr{X}_{k}$ admite difeomorfismo de Anosov, aplicando o Teorema de Porteous (Teorema 6.4.1). Para isto, precisamos garantir que os autovalores da matriz $\Theta_{k}\left(\bar{\sigma}\left(\alpha_{0,2^{k}}\right)\right)=M_{k}$, dada em (5.63), não tem os seguintes autovalores

$$
1,-1, i,-i, \omega, \omega^{2},-\omega,-\omega^{2}
$$


com multiplicidade algébrica 1 (onde $\omega^{3}=1$ ). Observemos que o polinômio característico $p_{M_{k}}(x)$ de $M_{k}$ é igual ao produto dos polinômios característicos $p_{M_{k, s}}(x)$ das matrizes $M_{k, s}$, para $1 \leq s \leq 2^{k-1}, p_{M_{k}}(x)=\prod_{s=1}^{2^{k-1}} p_{M_{k, s}}(x)$. Vamos analisar os autovalores das matrizes $M_{k, s}$, para $1 \leq s \leq 2^{k-1}$. Os autovalores de uma matriz circulante principal de tamanho $n \times n$, com entrada principal 1 , são dados por $\lambda_{j}=\omega_{j}^{n-1}$, para $j=0, \ldots, n-1$ com

$$
\omega_{j}=\exp \left(\frac{2 \pi i j}{n}\right)
$$

uma $n$-ésima raiz da unidade e $i=\sqrt{-1}$, ver (6.14) na Seção 6.4. Observemos que as matrizes $M_{k, s}$, para $1 \leq s \leq 2^{k-1}$, dadas em (5.61) e (5.62), são todas matrizes circulantes principais com entrada principal 1. Logo, seus autovalores são dados por $\lambda_{(k, s), j}=\omega_{j}^{n-1}$, para $j=0,1, \ldots, n-1$, com $\omega_{j}$ dado em (5.68) e onde $n=2^{k}$ se $1 \leq s \leq 2^{k}-1$ e $n=2^{k-1}$ se $s=2^{k-1}$. Examinando estes autovalores de $M_{k, s}$, para $1 \leq s \leq 2^{k-1}$, podemos concluir que

$$
\{1,-1, i,-i\}
$$

são autovalores para todas as matrizes $M_{k, s}$, e que nenhum $\lambda_{(k, s), j}$ (diferente de 1) é igual a um número $\omega$ ou $\omega^{2}$ satisfazendo a igualdade $\omega^{3}=1$. Além disso, como o número de matrizes $M_{k, s}$ é maior ou igual que 2, pois tomamos $k \geq 3$, então a multiplicidade algébrica dos 4 autovalores $\{1,-1, i,-i\}$ de $M_{k}$, dados em (5.69), é maior ou igual que 2. Provamos que os autovalores da matriz $\Theta_{k}\left(\bar{\sigma}\left(\alpha_{0,2^{k}}\right)\right)=M_{k}$, dada em (5.63), não tem os seguintes autovalores $1,-1, i,-i, \omega, \omega^{2},-\omega,-\omega^{2}$ com multiplicidade algébrica 1 (onde $\omega^{3}=1$ ) e portanto pelo Teorema de Porteous (Teorema 6.4.1) a variedade plana $\mathscr{X}_{k}$ admite difeomorfismo de Anosov.

Agora vamos analisar o caso da variedade plana $\mathscr{Y}_{1}$ do Teorema 5.4.3. A matriz $\Theta_{4}\left(\kappa_{18}\right)$ dada em (5.50) é a matriz que representa o gerador de $\mathbb{Z}_{4}$, usando a representação de holonomia $\mathbb{Z}_{4} \rightarrow \operatorname{Aut}\left(P_{4} /\left[P_{4}, P_{4}\right]\right)$. Notemos que a matriz (5.50) tem polinômio característico $(x-1)^{2}(x+1)^{2}\left(x^{2}+1\right)$ e autovalores $-1,-1,1,1, i,-i$. Similarmente, para $\mathscr{X}_{2}$, observamos que a matriz $M_{2}$, dada em (5.63) no caso de $k=2$, tem autovalores $-1,-1,1,1, i,-i$. Logo, pelo Teorema de Porteous (Teorema 6.4.1) aplicado no grupo de holonomia cíclico $\mathbb{Z}_{4}$, podemos concluir que as variedades planas $\mathscr{Y}_{1}$ e $\mathscr{X}_{2}$ não admitem difeomorfismo de Anosov. 


\section{Capítulo 6}

\section{Apêndice}

Este apêndice tem por objetivo analisar alguns problemas encontrados em artigos relacionados à conexão entre os grupos de tranças e os grupos de homotopia da esfera, e apresentar algumas provas distintas de resultados já obtidos anteriormente neste texto, porém, de forma mais elementar. Dividimos este apêndice como segue. Na Seção 6.1 vamos detalhar algumas imprecisões encontradas no artigo de Cohen et al. [9], explicando em cada caso os problemas encontrados. Abordaremos na Seção 6.2 uma análise detalhada das imprecisões encontradas em [58], onde algumas delas são decorrentes dos problemas encontrados no Lema 6.5.2 de [9] e outras de outra natureza. Na Seção 6.3, faremos uma exposição de algumas ideias iniciais do estudo dos grupos Brunnianos, quase-Brunnianos e sua relação com teoria de homotopia, além de apresentar provas mais simples de alguns resultados obtidos anteriormente. Na última seção, a Seção 6.4, definiremos os difeomorfismos de Anosov sobre variedades e as matrizes circulantes, objetos estes que são usados na Subseção 5.4.3 desta tese.

\subsection{O artigo de J. Berrick, F. Cohen, Y.L. Wong e J. $\mathrm{Wu}$}

Nesta seção vamos expor algumas imprecisões detectadas nas Subseções 6.5, 6.6 e 7.4 do artigo de Berrick, Cohen, Wong e Wu [9]. Em particular, destacamos que o item 3 do Lema 6.5.2 de [9] não é válido para todos os elementos do grupo de tranças do disco. Isto tem consequências em outros resultados de [9] que mencionaremos nesta seção, bem como repercussões significativas num subsequente artigo de Li e Wu de 2009 [58], que serão tratadas na Seção 6.2. Além disso, na Seção 7.4 de [9] os autores ilustram com exemplos algumas tranças que estão em correspondência com elementos dos grupos de homotopia baixos da esfera, no entanto veremos que tais tranças não são tranças Brunnianas sobre a esfera, contrariando o afirmado por eles. 


\subsubsection{Algumas imprecisões detectadas nas Subseções 6.5 e 6.6 de $[9]$}

Observamos que, nesta seção, continuamos usando a notação e indexação usual da teoria de homotopia simplicial para os grupos de tranças. Consideremos o homomorfismo $\partial: P_{n+1} \rightarrow P_{n}$ definido na Equação (1.4) do Capítulo 1 e como antes seja $d_{0}: P_{n+1} \rightarrow P_{n}$ o homomorfismo que geometricamente esquece a primeira corda.

Proposição 6.1.1. Suponha que $n \geq 2$. Então,

1. $\partial\left(A_{-1,0}\right) \neq 1$.

2. Como aplicações de $P_{n+2}\left(\mathbb{D}^{2}\right)$ em $P_{n}\left(\mathbb{D}^{2}\right)$, temos $\partial \circ \partial \neq \partial \circ d_{0}$.

Demonstração. 1. Lembramos que o elemento $A_{-1,0}$ do grupo de tranças do disco foi definido na Equação (1.6), usando a notação usual de teoria de tranças, como sendo $A_{-1,0}=\left(A_{0,1} A_{0,2} \cdots A_{0, n}\right)^{-1} \in P_{n+1}\left(\mathbb{D}^{2}\right)$. Consideremos o homomorfismo $\theta_{n-2}: P_{n}\left(\mathbb{D}^{2}\right) \rightarrow P_{2}\left(\mathbb{D}^{2}\right)$, que geometricamente esquece as últimas $(n-2)$ cordas. Observemos que $\theta_{n-2}$ é induzido geometricamente pela fibração de Fadell-Neuwirth, além disso podemos pensar que $\theta_{n-2}=d_{2} \circ d_{3} \circ \cdots \circ d_{n-1}$. Dessa forma, segue claramente que

$$
\theta_{n-2}\left(A_{i, j}\right)=\left\{\begin{array}{rll}
1, & \text { para } & 2 \leq j \leq n-1 \\
A_{0,1}, & \text { para } & j=1
\end{array}\right.
$$

Pelas igualdades dadas em (6.1) obtemos

$$
\begin{cases}\theta_{n-2}\left(A_{-1, j}\right)=\theta_{n-2}\left(\left(A_{j, j+1} \cdots A_{j, n-1}\right)^{-1}\left(A_{0, j} A_{1, j} \cdots A_{j-1, j}\right)^{-1}\right) & =1 . \\ \theta_{n-2}\left(A_{-1,0}\right)=\theta_{n-2}\left(\left(A_{0,1} A_{0,2} \cdots A_{0, n-1}\right)^{-1}\right) & =\left(A_{0,1}\right)^{-1} \\ \theta_{n-2}\left(A_{-1,1}\right)=\theta_{n-2}\left(\left(A_{1,2} A_{1,3} \cdots A_{1, n-1}\right)^{-1}\left(A_{0,1}\right)^{-1}\right) & =\left(A_{0,1}\right)^{-1}\end{cases}
$$

com $2 \leq j \leq n-1$.

Concluímos assim que,

$$
\theta_{n-2}\left(A_{-1, j}\right)=\left\{\begin{array}{rll}
1, & \text { para } & 2 \leq j \leq n-1 \\
A_{0,1}^{-1}, & \text { para } & j=0,1
\end{array}\right.
$$

Logo, pelas igualdades dadas em (1.4), (6.1) e (6.2), temos que

$$
\begin{aligned}
\theta_{n-2}\left(\partial\left(A_{-1,0}\right)\right) & =\theta_{n-2} \circ \partial\left(\left(A_{0,1} A_{0,2} \cdots A_{0, n}\right)^{-1}\right) \\
& =\theta_{n-2}\left(\left(A_{-1,0} A_{-1,1} A_{-1,2} \cdots A_{-1, n-1}\right)^{-1}\right) \\
& =\theta_{n-2}\left(\left(A_{-1,0} A_{-1,1}\right)^{-1}\right)=\left(A_{0,1}^{-1} A_{0,1}^{-1}\right)^{-1}=A_{0,1}^{2}
\end{aligned}
$$


Como o elemento $A_{0,1}^{2}$ é um elemento não trivial de $P_{2}\left(\mathbb{D}^{2}\right)$, então $\theta_{n-2}\left(\partial\left(A_{-1,0}\right)\right) \neq 1$, e dado que $\theta_{n-2}$ é homomorfismo, temos $\partial\left(A_{-1,0}\right) \neq 1$, demonstrando assim o primeiro item.

2. Consideremos o elemento $b=A_{0,1} \in P_{n+2}\left(\mathbb{D}^{2}\right)$. Observemos que $A_{0,1}$ é um gerador de $P_{n+2}\left(\mathbb{D}^{2}\right)$, usando a apresentação clássica dos grupos de tranças sobre o disco. Então, $\partial \circ d_{0}\left(A_{0,1}\right)=1$, pois $d_{0}\left(A_{0,1}\right)=1$. Pela definição do homomorfismo $\partial$ temos que $\partial\left(A_{0,1}\right)=A_{-1,0} \in P_{n+1}\left(\mathbb{D}^{2}\right)$ e pelo item 1 sabemos que $\partial \circ \partial\left(A_{0,1}\right)=\partial\left(A_{-1,0}\right) \neq 1$. Logo, com $b=A_{0,1} \in P_{n+2}\left(\mathbb{D}^{2}\right)$, para $n \geq 2$, temos que

$$
\partial \circ \partial(b) \neq \partial \circ d_{0}(b)
$$

Observações 6.1.2. Vamos fazer alguns comentários sobre o Lema 6.5.2 de [9], que foi enunciado no Lema 1.2.4.

1. Pelo item 2 da Proposição 6.1.1 segue que o item 3 do Lema 6.5.2 de [9] está formulado de forma incorreta, pois para $n \geq 2$, os homomorfismos $\partial \circ \partial$ e $\partial \circ d_{0} d e$ $P_{n+2}\left(\mathbb{D}^{2}\right)$ em $P_{n}\left(\mathbb{D}^{2}\right)$ não são iguais.

2. Seja $d_{n+1}: P_{n+2}\left(\mathbb{D}^{2}\right) \rightarrow P_{n+1}\left(\mathbb{D}^{2}\right)$ o homomorfismo que geometricamente esquece a última corda. Então, $\operatorname{Ker}\left(d_{n+1}\right)$ é o grupo livre, de posto $n+1$, gerado por

$$
\left\{A_{0, n+1}, A_{1, n+1}, A_{2, n+1}, \ldots, A_{n, n+1}\right\} \text {. }
$$

Vejamos que o item 3 do Lema 1.2.4 é válido quando nos restringirmos a este subgrupo, avaliando nos geradores de $\operatorname{Ker}\left(d_{n+1}\right)$ e usando a definição do homomorfismo ə: $P_{n+2}\left(\mathbb{D}^{2}\right) \rightarrow P_{n+1}\left(\mathbb{D}^{2}\right)$ dada na Equação (1.4) do Capítulo 1:

$$
\begin{aligned}
& \partial \partial\left(A_{i, n+1}\right)=\partial\left(A_{i-1, n}\right)= \begin{cases}1, & \text { se } i=0 \\
A_{i-2, n-1}, & \text { se } 1 \leq i \leq n\end{cases} \\
& \partial d_{0}\left(A_{i, n+1}\right)=\left\{\begin{array}{ll}
\partial(1), & \text { se } i=0 \\
\partial\left(A_{i-1, n}\right), & \text { se } 1 \leq i \leq n
\end{array}= \begin{cases}1, & \text { se } i=0 \\
A_{i-2, n-1}, & \text { se } 1 \leq i \leq n .\end{cases} \right.
\end{aligned}
$$

Logo, ӘӘ $=\partial d_{0}$ no subgrupo $\operatorname{Ker}\left(d_{n+1}\right) \leq P_{n+2}\left(\mathbb{D}^{2}\right)$.

\subsubsection{Sobre o item 3 do Lema 6.5.2 de [9]}

Destacamos na continuação algumas consequências da não validade do item 3 do Lema 1.2.4 para todos os elementos de $P_{n+1}\left(\mathbb{D}^{2}\right)$.

\section{Observações 6.1.3.}


1. No Corolário 6.5.3 de [9] foi considerada uma sequência de conjuntos chamada de $\Gamma$ e que pode ser vista no Exemplo 1.3.22. Vejamos que $\Gamma$ não é um $\Delta$-grupo, como afirmado pelos autores de [9].

De fato, se $\left(\Gamma, d_{j}^{\Gamma}\right)$ é um $\Delta$-grupo, então tem que valer a identidade simplicial $d_{j}^{\Gamma} d_{i}^{\Gamma}=d_{i}^{\Gamma} d_{j+1}^{\Gamma}$, para $0 \leq i \leq j$. Em particular, tem que valer a igualdade $d_{0}^{\Gamma} d_{0}^{\Gamma}=d_{0}^{\Gamma} d_{1}^{\Gamma}$ para todos os elementos de $P_{n+2}\left(\mathbb{D}^{2}\right)$, com $n \geq 0$.

As seguintes igualdades de aplicações são válidas, $d_{0}^{\Gamma}=\partial$ e $d_{1}^{\Gamma}=d_{0}$. Notemos que $d_{0}$ é a aplicação que geometricamente esquece a primeira corda de tranças no disco. Vimos no item 2 da Proposição 6.1.1 que para $A_{0,1}$ em $\Gamma_{n+2}=P_{n+2}\left(\mathbb{D}^{2}\right)$ tem-se que $\partial \partial\left(A_{0,1}\right) \neq \partial d_{0}\left(A_{0,1}\right)$, se $n \geq 2$. Logo, a identidade simplicial $d_{0}^{\Gamma} d_{0}^{\Gamma}=d_{0}^{\Gamma} d_{1}^{\Gamma}$ não é válida para todos os elementos de $P_{n+2}\left(\mathbb{D}^{2}\right)$, com $n \geq 2$.

2. O Corolário 6.5.4 de [9] como enunciado no artigo não é totalmente correto, pois $\Gamma$ não é um $\Delta$-grupo.

3. A demonstração do Lema 6.6.3 de [9] tem um erro. De fato, a seguinte igualdade que aparece na quarta linha da demonstração do Lema 6.6.3 de [9]:

$$
\partial\left(A_{-1,0}^{-1}\right)=1
$$

é falsa, em virtude do item 1 da Proposição 6.1.1.

Além disso, dado que o homomorfismo $\partial: P_{n+1} \rightarrow P_{n}$ é sobrejetor, pode-se verificar que não pode existir um tal homomorfismo $\alpha: P_{n+1}\left(\mathbb{S}^{2}\right) \rightarrow P_{n}$ tal que o seguinte diagrama comuta:

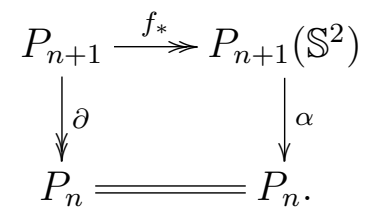

Vamos supor que existe um homomorfismo $\alpha: P_{n+1}\left(\mathbb{S}^{2}\right) \rightarrow P_{n}$ tal que o quadrado (6.3) é comutativo. Como $f_{*}$ e d são sobrejetoras, então pela comutatividade do quadrado (6.3) temos que a também é sobrejetora. Consideremos agora a abelianização do homomorfismo $\alpha,(\alpha)_{a b}:\left(P_{n+1}\left(\mathbb{S}^{2}\right)\right)_{a b} \rightarrow\left(P_{n}\right)_{a b}$, que é por sua vez sobrejetora. Como afirmado na prova do item (b) da Proposição 4 de [40], $\left(P_{n}\right)_{a b}$ é um grupo abeliano livre de posto $n(n-1) / 2$. Além disso, na prova do item (e) do Teorema 5 de [40], os autores deduzem que o grupo abelianizado de tranças puras sobre a esfera, $\left(P_{n+1}\left(\mathbb{S}^{2}\right)\right)_{a b}$, é da forma $\left(P_{n+1}\left(\mathbb{S}^{2}\right)\right)_{a b}=\mathbb{Z}^{(n+1)(n-2) / 2} \oplus \mathbb{Z}_{2}$. Observemos que $(\alpha)_{a b}$ manda o fator $\mathbb{Z}_{2}$ no zero e portanto se restringe a um homomorfismo sobrejetor de $\mathbb{Z}^{(n+1)(n-2) / 2}$ em $\mathbb{Z}^{n(n-1) / 2}$.

Por outro lado, sabemos que o posto do grupo abeliano livre $\left(P_{n}\right)_{a b}$ é $\frac{n(n-1)}{2} e$ 
que o posto do grupo abelianizado $\left(P_{n+1}\left(\mathbb{S}^{2}\right)\right)_{a b}$ é $\frac{(n+1)(n-2)}{2}=\frac{n(n-1)}{2}-1$. É claro que não pode existir um homomorfismo sobrejetor de grupos abelianos livres $H \rightarrow G$ com posto de $H$ menor que o posto de $G$. Desta forma temos encontrado uma contradição e portanto não pode existir uma aplicação $\alpha: P_{n+1}\left(\mathbb{S}^{2}\right) \rightarrow P_{n}$ tal que o diagrama (6.3) comuta.

4. Como o Lema 6.6.4 e o Teorema 6.6.5 de [9] fazem uso do homomorfismo $\alpha: P_{n+1}\left(\mathbb{S}^{2}\right) \rightarrow P_{n}$ construído no Lema 6.6.3 de [9], então esses resultados da forma que foram enunciados não fazem mais sentido. No entanto, como vimos na Seção 2.4, existe um outro homomorfismo chamado de $\psi_{n+1}$ para o qual tais enunciados tomam sentido; mas este novo homomorfismo não foi definido em todo $P_{n+1}\left(\mathbb{S}^{2}\right)$, apenas no subgrupo $\operatorname{Ker}\left(d_{n}: P_{n+1}\left(\mathbb{S}^{2}\right) \rightarrow P_{n}\left(\mathbb{S}^{2}\right)\right)$, onde $d_{n}$ é o homomorfismo que esquece a última corda.

Observação 6.1.4. Faremos, na continuação, um par de comentários sobre o problema encontrado no Corolário 6.5.3 de [9]. Gostaríamos de obter uma estrutura de $\Delta$-grupo para a sequência $\Gamma=\left\{\Gamma_{n}\right\}_{n \geq 0}$ definida por $\Gamma_{0}=1$ e, para $n \geq 1, \Gamma_{n}=P_{n}$.

1. Se as aplicações faces $d_{i}^{\Gamma}=d_{i}: \Gamma_{n} \rightarrow \Gamma_{n-1}$, para $1 \leq i \leq n$ (usando a notação simplicial seria $d_{i}^{\Gamma}=d_{i-1}: \Gamma_{n} \rightarrow \Gamma_{n-1}$, para $1 \leq i \leq n$ ), são as canônicas dadas pelo esquecimento da i-ésima corda, então os ciclos $\mathcal{Z}_{n} \Gamma$ estarão contidos em $\operatorname{Brun}_{n}\left(\mathbb{D}^{2}\right)$. No entanto, teríamos que mudar a aplicação $d_{0}^{\Gamma}=\partial$ pois vimos que não dá certo com essa escolha. Mas mudando $d_{0}^{\Gamma}$ não podemos garantir a afirmação do Corolário 6.5.4 de [9], que é muito interessante, nem nenhuma outra afirmação relacionada com $\partial$. Em outras palavras, teríamos que escolher uma boa aplicação $d_{0}^{\Gamma}$ e demonstrar, se possivel, tudo de novo com essa nova aplicação.

2. Se mantemos a aplicação face $d_{0}^{\Gamma}=\partial$, que é muito boa, possivelmente não teremos que os ciclos de Moore $\mathcal{Z}_{n} \Gamma$ estejam contidos em Brun ${ }_{n}\left(\mathbb{D}^{2}\right)$. O motivo disto é que teremos que escolher as aplicações $d_{i}$, para $i \geq 1$, tal que não sejam exatamente as aplicações que esquecem cordas.

\subsubsection{Sobre o exemplo da Subseção 7.4 de [9]}

Lembramos que em [9] foi dado um exemplo de uma trança com 5 cordas na esfera que se projeta no gerador de $\pi_{4}\left(\mathbb{S}^{2}\right)$, no entanto esta trança não é uma trança Brunniana, embora isto seja o afirmado em [9]. As contas feitas na segunda e terceira linha da página 318 de [9] estão certas, mas depois são substituidos os elementos $\hat{z}_{i}$ pelos elementos de $P_{5}\left(\mathbb{S}^{2}\right)$ usando uma aplicação $\beta$ definida antes da Proposição 7.2.2 de [9] (no final da página 313).

Seja $f_{*}: B_{n}\left(\mathbb{D}^{2}\right) \rightarrow B_{n}\left(\mathbb{S}^{2}\right)$ o homomorfismo induzido pela inclusão natural do disco na esfera. Denotemos por $\delta_{i}$ a imagem dos geradores de Artin $\sigma_{i} \in B_{n}, f_{*}\left(\sigma_{i}\right)=\delta_{i} \in B_{n}\left(\mathbb{S}^{2}\right)$, 
para $i=1, \cdots, n-1$, e por $D_{i, j}$ a imagem dos geradores de tranças puras de Artin, $f_{*}\left(A_{i, j}\right)=D_{i, j} \in B_{n}\left(\mathbb{S}^{2}\right)$, para $1 \leq i<j \leq n$. Notemos que o conjunto de índices aqui começa com 1 e não com o zero, como feito em [9]. Lembremos que o homomorfismo $f_{*}$ é um epimorfismo, ainda quando restrito às tranças puras.

Consideremos o disco menos 3 pontos, $\mathbb{D}^{2} \backslash Q_{3}$ com $Q_{3}=\left\{q_{1}, q_{2}, q_{3}\right\}$ um conjunto de pontos no interior do disco, e tomemos o grupo fundamental deste espaço com ponto base no interior, obtendo um grupo livre em 3 elementos que denotaremos por $F\left(\hat{z}_{0}, \hat{z}_{1}, \hat{z}_{2}\right)$. Podemos associar a cada elemento deste grupo livre uma trança em $P_{4}\left(\mathbb{S}^{2}\right)$, tal aplicação é chamada de $\beta$ em [9, página 313]. Nos geradores do grupo livre $F\left(\hat{z}_{0}, \hat{z}_{1}, \hat{z}_{2}\right)$ tal associação é dada por

- $\beta\left(\hat{z}_{0}\right)=\delta_{1}^{2}=D_{1,2}$

- $\beta\left(\hat{z}_{1}\right)=\delta_{1} \delta_{2}^{2} \delta_{1}^{-1}=D_{2,3}^{-1} D_{1,3} D_{2,3} \mathrm{e}$

- $\beta\left(\hat{z}_{2}\right)=\delta_{1} \delta_{2} \delta_{3}^{2} \delta_{2}^{-1} \delta_{1}^{-1}=D_{3,4}^{-1} D_{2,4}^{-1} D_{1,4} D_{2,4} D_{3,4}$.

Como antes, $\Delta_{n}$ denota o "full twist" em $P_{n}\left(\mathbb{S}^{2}\right)$.

Proposição 6.1.5. O grupo de tranças puras com 4 cordas sobre a esfera, $P_{4}\left(\mathbb{S}^{2}\right)$, é gerado pelos elementos $D_{1,4}, D_{2,4}$ e pelo "full twist" $\Delta_{4}$.

Demonstração. Notemos que, pela fibração de Fadell-Neuwirth, o núcleo da aplicação $d_{4}: P_{4}\left(\mathbb{S}^{2}\right) \rightarrow P_{3}\left(\mathbb{S}^{2}\right)$ (que geometricamente esquece a última corda da trança) é o grupo livre em dois elementos $\pi_{1}\left(\mathbb{S}^{2} \backslash Q_{3}\right)$. Além disso, os elementos $D_{1,4}, D_{2,4}, D_{3,4}$ estão no núcleo de $d_{4}$. Usando a relação $D_{1,4} D_{2,4} D_{3,4}=1$ em $P_{4}\left(\mathbb{S}^{2}\right)$, temos que $D_{3,4}=D_{2,4}^{-1} D_{1,4}^{-1}$.

O "full twist" em $P_{4}\left(\mathbb{S}^{2}\right)$ é dado por $\Delta_{4}=D_{1,2} D_{1,3} D_{2,3}$. Observemos que

$$
d_{4}\left(D_{1,3} \Delta_{4}\right)=d_{4}\left(D_{1,2} \Delta_{4}\right)=d_{4}\left(D_{2,3} \Delta_{4}\right)=1
$$

e portanto estes elementos pertencem ao núcleo de $d_{4}$. Usando as relações de superfície $D_{1,2} D_{1,3} D_{1,4}=1, D_{1,2} D_{2,3} D_{2,4}=1$ podemos expressar os geradores usuais faltantes $\left(D_{1,2}, D_{1,3}, D_{2,3}\right)$ em termos do "full twist" e os geradores $D_{1,4}, D_{2,4}$ :

$$
\left\{\begin{aligned}
D_{1,3} \Delta_{4} & =D_{1,3} D_{1,2} D_{1,3} D_{2,3}=D_{1,3} D_{1,4}^{-1} D_{2,3}=D_{1,3} D_{2,3} D_{1,4}^{-1}=D_{3,4}^{-1} D_{1,4}^{-1} \\
& =D_{1,4} D_{2,4} D_{1,4}^{-1} \\
D_{1,2} & =D_{1,4}^{-1} D_{1,3}^{-1}=\Delta_{4} D_{3,4}=\Delta_{4} D_{2,4}^{-1} D_{1,4}^{-1} \\
D_{2,3} & =D_{1,2}^{-1} D_{2,4}^{-1}=\Delta_{4}^{-1} D_{3,4}^{-1} D_{2,4}^{-1}=\Delta_{4}^{-1} D_{1,4} .
\end{aligned}\right.
$$

A expressão de $D_{1,2}$ em (6.4) foi obtida da expressão anterior para $D_{1,3}$ e a expressão de $D_{2,3}$ em (6.4) foi obtida da expressão anterior para $D_{1,2}$. Logo, temos a seguinte descrição 
para os elementos de $P_{4}\left(\mathbb{S}^{2}\right)$ em termos de $D_{1,4}, D_{2,4}$ e o "full twist" $\Delta_{4}$ :

$$
\left\{\begin{aligned}
D_{1,2} & =\Delta_{4} D_{2,4}^{-1} D_{1,4}^{-1} ; \\
D_{1,3} & =D_{1,4} D_{2,4} D_{1,4}^{-1} \Delta_{4}^{-1} ; \\
D_{2,3} & =\Delta_{4}^{-1} D_{1,4} ; \\
D_{3,4} & =D_{2,4}^{-1} D_{1,4}^{-1} .
\end{aligned}\right.
$$

Sejam $G$ um grupo e $a, b \in G$. Como dito na Subseção 1.1.2, o comutador de $a$ com $b$ corresponde ao elemento $[a, b]=a^{-1} b^{-1} a b$.

Proposição 6.1.6. Seja $\gamma=\left[\left[D_{1,2} D_{2,3}^{-1} D_{3,4}^{-1}, D_{1,4}\right],\left[D_{1,2}, D_{2,3}^{-1} D_{3,4}^{-1} D_{1,4}\right]\right]$ em $P_{4}\left(\mathbb{S}^{2}\right)$. Então, $\gamma$ não representa o elemento neutro em $P_{4}\left(\mathbb{S}^{2}\right)$, isto é, $\gamma \neq 1$ em $P_{4}\left(\mathbb{S}^{2}\right)$.

Demonstração. Observemos que $\gamma \in \operatorname{Ker}\left(d_{4}: P_{4}\left(\mathbb{S}^{2}\right) \rightarrow P_{3}\left(\mathbb{S}^{2}\right)\right)$. Vamos reescrever $\gamma$ usando a descrição dos elementos de $P_{4}\left(\mathbb{S}^{2}\right)$ dada na Proposição 6.1.5. Lembremos que o "full twist" é um elemento central e que em $P_{4}\left(\mathbb{S}^{2}\right)$ vale que $D_{1,4} D_{2,4} D_{3,4}=1$. Portanto, $\gamma$ pode ser escrito assim:

$$
\begin{aligned}
\gamma & =\left[\left[\Delta_{4} D_{3,4} \Delta_{4} D_{1,4}^{-1} D_{3,4}^{-1}, D_{1,4}\right],\left[\Delta_{4} D_{3,4}, \Delta_{4} D_{1,4}^{-1} D_{3,4}^{-1} D_{1,4}\right]\right] \\
& =\left[D_{1,4}, D_{2,4}^{-1} D_{1,4}^{-1} D_{2,4}\right]\left[D_{2,4} D_{1,4}, D_{2,4}^{-1} D_{1,4}^{-1}\right]\left[D_{2,4}^{-1} D_{1,4}^{-1} D_{2,4}, D_{1,4}\right]\left[D_{2,4}^{-1} D_{1,4}^{-1}, D_{2,4} D_{1,4}\right] .
\end{aligned}
$$

Como cada um dos comutadores envolvidos na expressão para $\gamma$ é não nulo, nem o produto destes tem cancelamentos elementares no grupo livre $\operatorname{Ker}\left(d_{4}\right)$. Então, provamos que $\gamma \neq 1$ em $P_{4}\left(\mathbb{S}^{2}\right)$.

Em [9, Pagina 318] é construído o elemento $\alpha_{5}=\left[\left[\hat{z}_{0} \hat{z}_{1}, \hat{z}_{2}\right],\left[\hat{z}_{0}, \hat{z}_{1} \hat{z}_{2}\right]\right]$ que está em correspondência com o gerador $\eta_{2}^{2}$ de $\pi_{4}\left(\mathbb{S}^{2}\right)$. Portanto, a trança $\beta\left(\alpha_{5}\right)=\beta_{5}$, dada por

$$
\beta_{5}=\left[\left[D_{1,2} D_{2,3}^{-1} D_{1,3} D_{2,3}, D_{3,4}^{-1} D_{2,4}^{-1} D_{1,4} D_{2,4} D_{3,4}\right],\left[D_{1,2}, D_{2,3}^{-1} D_{1,3} D_{2,3} D_{3,4}^{-1} D_{2,4}^{-1} D_{1,4} D_{2,4} D_{3,4}\right]\right]
$$

deve ser uma trança não trivial em $\operatorname{Brun}_{5}\left(\mathbb{S}^{2}\right) / f_{*}\left(\operatorname{Brun}_{5}\left(\mathbb{D}^{2}\right)\right)$.

Portanto, $\beta_{5}$ deve satisfazer inicialmente que $d_{j}\left(\beta_{5}\right)=1$ em $P_{4}\left(\mathbb{S}^{2}\right)$, para $j=1,2,3,4,5$. Claramente $d_{j}\left(\beta_{5}\right)=1$ em $P_{4}\left(\mathbb{S}^{2}\right)$, para $j=1,2,3,4$. Falta analisar o caso da aplicação face $d_{5}$, que esquece a última corda.

Notemos que, para $1 \leq i \leq 4$, a expressão de $\beta_{5}$ acima não envolve geradores do tipo $D_{i, 5}$ e portanto $d_{5}\left(\beta_{5}\right) \in P_{4}\left(\mathbb{S}^{2}\right)$ tem a mesma expressão que $\beta_{5}$. Usando a relação de superfície $D_{1,4} D_{2,4} D_{3,4}=1$ em $P_{4}\left(\mathbb{S}^{2}\right)$ aplicada à trança $d_{5}\left(\beta_{5}\right)$, cuja expressão foi dada em $(6.5)$, vemos que em $P_{4}\left(\mathbb{S}^{2}\right)$ tem-se que $d_{5}\left(\beta_{5}\right)=\gamma$, para $\gamma$ como na Proposição 6.1.6. Portanto, $d_{5}\left(\beta_{5}\right) \neq 1$ em $P_{4}\left(\mathbb{S}^{2}\right)$ o que demonstra que $\beta_{5} \in P_{5}\left(\mathbb{S}^{2}\right)$ não é uma trança Brunniana.

Concluímos assim que a trança $\beta_{5} \in P_{5}\left(\mathbb{S}^{2}\right)$ construída em [9] está em correspondência com o elemento gerador $\eta_{2}^{2}$ de $\pi_{4}\left(\mathbb{S}^{2}\right)$, mas não é uma trança Brunniana como foi afirmado 
em [9]. Não é muito difícil achar exemplos de tranças Brunnianas em $P_{5}\left(\mathbb{S}^{2}\right)$ que se projetam em $\eta_{2}^{2}$, ver Seção 2.3. Por outro lado, encontrar um exemplo usando a aplicação $\beta$ construída em [9] já não foi um processo tão simples, ver Seção 3.4.

Observações 6.1.7. Algumas observações finais relacionadas com estas contas são dadas a seguir.

1. Mesmo usando a convenção $[a, b]=a b a^{-1} b^{-1}$, para o comutador de $a, b \in G$, provase que $d_{5}\left(\beta_{5}\right) \neq 1$, com o mesmo raciocínio feito acima. Ou seja, o problema não está nas contas feitas pela escolha do comutador.

2. No artigo aparece uma trança diferente à trança $\beta_{5}$ citada aqui, mas acreditamos que foi um problema de digitação por parte dos autores do artigo. Mesmo assim fizemos a verificação com a trança de $P_{5}\left(\mathbb{S}^{2}\right)$,

$$
\beta_{5}^{*}=\left[\left[\delta_{0}^{3} \delta_{1}, \delta_{0} \delta_{1} \delta_{2}^{2} \delta_{1}^{-1} \delta_{0}^{-1}\right],\left[\delta_{0}^{2}, \delta_{0}^{2} \delta_{1}^{3} \delta_{2}^{2} \delta_{1}^{-1} \delta_{0}^{-1}\right]\right]
$$

obtendo que $d_{5}\left(\beta_{5}^{*}\right) \neq 1$ em $P_{4}\left(\mathbb{S}^{2}\right)$.

3. Além disso, no artigo é afirmado que existe um outro elemento $\alpha_{5}^{\prime}$, que também representa uma trança Brunniana não trivial com 5 cordas na esfera módulo tranças Brunnianas sobre o disco. Digamos que $\beta\left(\alpha_{5}^{\prime}\right)=\beta_{5}^{\prime}$. Usando o método anterior provamos que $d_{5}\left(\beta_{5}^{\prime}\right) \neq 1$ em $P_{4}\left(\mathbb{S}^{2}\right)$.

\subsection{O artigo de J.Y. Li e J. Wu}

No artigo de Li e Wu intitulado "Artin braid groups and homotopy groups" [58] os autores estudam uma nova conexão entre grupos de tranças de Artin e os grupos de homotopia da esfera. No entanto, ao longo do artigo existem várias imprecisões de diferente natureza, não somente nos resultados principais como também em vários resultados intermediários. Nesta seção faremos uma exposição detalhada dos problemas existentes em [58] justificando em cada caso o problema detectado. Para evitar repetição não reescreveremos os enunciados, apenas os comentários respectivos. Também gostaríamos de mencionar que o Capítulo 4 contém as reformulações adequadas de resultados e problemas do artigo de Li e Wu. Em particular, destacamos que uma prova alternativa do principal teorema de [58] foi dada, ver o Teorema 4.3.12 na Seção 4.3.

\subsubsection{Sobre a Seção 2}

Sobre o Lema 2.3 
O Lema 2.3 de [58] contém 6 itens, sendo que os 3 primeiros são os mesmos enunciados no Lema 6.5.2 de Cohen et al. [9] (ver também o Lema 1.2.4). Vimos na Seção 6.1 que o item (3) do Lema 6.5.2 de [9] não é válido para todos os elementos do grupo de tranças do disco.

Analisamos as 3 últimas afirmações do Lema 2.3 de [58] e concluímos que o item (5) deste lema tem um erro, pois a igualdade não é válida para todos os elementos do grupo de tranças $P_{n}$. Para isto, temos que o item (5) afirma que em $P_{n}$ a igualdade

$$
\partial s_{k}=s_{k-1} \partial
$$

é válida, para $k \geq 1$. A igualdade (6.6) é verificada pelos autores de [58] de forma clara para $k \geq 1$ no subconjunto de geradores $\left\{A_{i, j} \mid 2 \leq i<j \leq n\right\}$. Também foi calculado no artigo $\partial s_{k} A_{i, j}$ para $k \geq 1$ e $i=1$, obtendo:

$$
\partial s_{k} A_{1, j}= \begin{cases}A_{0, j}, & \text { se } 1<k+1<j \\ A_{0, j-1} A_{0, j}, & \text { se } k+1=j \\ A_{0, j-1}, & \text { se } k+1>j\end{cases}
$$

Em [58] é proposto o uso de desenhos de tranças geométricas para finalizar a verificação da igualdade (6.6) nos geradores $A_{1, j}, \operatorname{com} 2 \leq j \leq n$. Nós provaremos na seguinte proposição que existem elementos de $P_{n}$ para os quais não vale a igualdade (6.6).

Proposição 6.2.1. Seja $1 \leq k \leq n$. A igualdade $\partial s_{k}=s_{k-1} \partial$ dada em (6.6) é válida em $P_{n}$ exceto para os elementos $A_{1, j} \in P_{n}$, com $k=j-1$ e $2 \leq j \leq n$.

Nota: A notação simplicial é usada para as aplicações degenerações $s_{k}$ mas não para os geradores $A_{i, j}$ de $P_{n}$.

Demonstração. Vamos utilizar a definição explícita da aplicação simplicial $s_{k}$, dada em [58, Página 527], donde obtemos:

$$
s_{k-1} \partial A_{1, j}=s_{k-1} A_{0, j-1}= \begin{cases}A_{0, j-1}, & \text { se } k-1 \geq j-1 \\ A_{0, j}, & \text { se } k-1 \leq j-3 \\ s_{j-2} A_{0, j-1}, & \text { se } k-1=j-2,\end{cases}
$$

com $s_{j-2} A_{0, j-1}$ dado por

$$
\begin{aligned}
s_{j-2} A_{0, j-1}= & \left(A_{j-1, j+1} A_{j, j+1} A_{j-1, j+2} A_{j, j+2} \cdots A_{j-1, n-1} A_{j, n-1} A_{j-1, n} A_{j, n}\right)^{-1} \\
& \left(A_{1, j-1} A_{1, j} A_{2, j-1} A_{2, j} \cdots A_{j-2, j-1} A_{j-2, j}\right)^{-1} .
\end{aligned}
$$

Pelas equações dadas em (6.7) e (6.8) é claro que a igualdade (6.6) é satisfeita para os valores de $k$ e $j$ tais que $k-1 \neq j-2$. Observemos que, para $2 \leq j \leq n$, o produto 
$A_{0, j-1} A_{0, j}$ tem a seguinte expressão:

$$
\begin{aligned}
A_{0, j-1} A_{0, j}= & \left(A_{j-1, j} \cdots A_{j-1, n-2} A_{j-1, n-1}\right)^{-1}\left(A_{1, j-1} A_{2, j-1} \cdots A_{j-2, j-1}\right)^{-1} \\
& \left(A_{j, j+1} A_{j, j+2} \cdots A_{j, n}\right)^{-1}\left(A_{1, j} A_{2, j} \cdots A_{j-2, j} A_{j-1, j}\right)^{-1}
\end{aligned}
$$

Agora, consideremos o homomorfismo de $P_{n}$ em $P_{2}$ dado pelo esquecimento das $n-2$ cordas de uma trança em $P_{n}$, que são diferentes das cordas $(j-1)$-ésima e $j$-ésima. Denotemos tal homomorfismo por

$$
\theta_{n-2,\{j-1, j\}}: P_{n} \rightarrow P_{2}
$$

Então, aplicando $\theta_{n-2,\{j-1, j\}}$ nas igualdades (6.9) e (6.10) temos que

$$
\theta_{n-2,\{j-1, j\}}\left(s_{j-2} \partial A_{1, j}\right)=1 \text { e } \theta_{n-2,\{j-1, j\}}\left(\partial s_{j-1} A_{1, j}\right)=A_{1,2}^{-1},
$$

respectivamente. Observemos que o elemento $\theta_{n-2,\{j-1, j\}}\left(\partial s_{j-1} A_{1, j}\right)$ é diferente de 1 , pois $A_{1,2}^{-1} \neq 1$ em $P_{2}$.

Como $\theta_{n-2,\{j-1, j\}}$ é um homomorfismo, concluímos que $s_{j-2} \partial A_{1, j} \neq \partial s_{j-1} A_{1, j}$ no grupo de tranças puras $P_{n}$.

\section{Sobre a Proposição 2.4}

Na continuação do artigo é construída a sequência $\mathbb{P}=\left\{P_{n}\right\}_{n \geq 0}$ formada por grupos de tranças puras sobre o disco, onde $P_{0}=\{1\}$, dada no Exemplo 1.3.22. A Proposição 2.4 afirma o seguinte:

$\mathbb{P}$ é um quase-grupo simplicial.

Dado que a igualdade (6.6) correspondente ao item (5) do Lema 2.3 de [58] não é válida para todos os elementos de $P_{n}$, como vimos na Proposição 6.2.1, então podemos concluir que $\mathbb{P}$ não é um quase-grupo simplicial. Ainda mais, já tínhamos visto na Seção 6.1 que $\mathbb{P}$ não é nem $\Delta$-grupo, pois $\mathbb{P}$ é igual à sequência de conjuntos $\Gamma$ do Corolário 6.6 .3 de [9].

\section{Sobre a Proposição 2.5}

Observemos que os conjuntos de homotopia de Moore $\pi_{n}(\mathcal{G})$ foram definidos para $\Delta$-grupos $\mathcal{G}$, mas $\mathbb{P}$ não é $\Delta$-grupo e portanto $\pi_{n}(\mathbb{P})$ não foi definido.

\section{Sobre a Proposição 2.6}

Sabemos que $\mathbb{P}$ não é um quase-grupo simplicial, ainda mais, nem $\Delta$-grupo e sendo assim claramente $\mathbb{P}$ não pode ser um conjunto simplicial. No entanto, a demonstração 
escrita em [58] para a Proposição 2.6 pode ser facilmente adaptada para o enunciado da proposição abaixo.

Seja $\mathcal{A}=\left\{P_{n}, d_{i}, s_{j}\right\}_{n \geq 0}$ a sequência formada pelos grupos de tranças $P_{n}$, com $P_{0}=\{1\}$, junto com aplicações $d_{i}: P_{n} \rightarrow P_{n-1}$, para $1 \leq i \leq n$, e $s_{j}: P_{n} \rightarrow P_{n+1}$, para $1 \leq j \leq n$, como no Exemplo 1.3.22.

Proposição 6.2.2. Suponhamos que existe uma sequência de aplicações sobrejetoras $\left\{d_{0}: P_{n+1} \rightarrow P_{n}\right\}_{n \geq 0}$ tais que $\mathcal{A}$ é um $\Delta$-grupo. Então, não existe uma sequência de aplicações $\left\{s_{0}: P_{n} \rightarrow P_{n+1}\right\}_{n \geq 0}$ tal que $\mathcal{A}=\left\{P_{n}\right\}$ é um conjunto simplicial com $d_{0}, s_{0} e$ as aplicações faces e degenerações já existentes para $\left\{P_{n}, d_{i}, s_{j}\right\}_{n \geq 0}$.

\section{Sobre a Proposição 2.9}

A Proposição 2.9 afirma que $\mathbb{P}$ é um $\Delta$-grupo fibrante, mas já sabemos que $\mathbb{P}$ não é nem $\Delta$-grupo, ver Proposições 6.1.1 e 6.2.1. Além disso, independentemente deste fato, a prova deste resultado tem um erro, que explicaremos na continuação.

Caso I $(i<n)$ :

Tomemos $x_{0}, \ldots, x_{i-1}, x_{i+1}, \ldots, x_{n}$ elementos em $P_{n-1}$ que são faces emparelhadas com relação a $i$. Seja $y_{j}=x_{j}\left(d_{j} s_{n-1} x_{n}\right)^{-1}$, para $0 \leq j \leq n$ e $j \neq i$. Seguindo a prova, precisamos verificar que $y_{0}, \ldots, y_{i-1}, y_{i+1}, \ldots, y_{n}$ são faces emparelhadas com relação a $i$ (ver linha 3 da prova da Proposição 2.9 de [58]). Isto é, tem que valer a seguinte igualdade

$$
d_{j} y_{k}=d_{k} y_{j+1}
$$

para $k \leq j$ e $k, j+1 \neq 1$. Sabemos que $d_{j} x_{k}=d_{k} x_{j+1}$, para $k \leq j$ e $k, j+1 \neq 1$. Observemos que

- $d_{j} y_{k}=d_{j}\left(x_{k}\left(d_{k} s_{n-1} x_{n}\right)^{-1}\right)=d_{j} x_{k}\left(d_{j} d_{k} s_{n-1} x_{n}\right)^{-1}$.

- $d_{k} y_{j+1}=d_{k}\left(x_{j+1}\left(d_{j+1} s_{n-1} x_{n}\right)^{-1}\right)=d_{k} x_{j+1}\left(d_{k} d_{j+1} s_{n-1} x_{n}\right)^{-1}$.

Se as identidades simpliciais dos $\Delta$-grupos são satisfeitas, então não existe problema algum, mas este não é o caso. Portanto, podemos escolher candidatos naturais com o intuito de provar que

em geral $d_{j} y_{k} \neq d_{k} y_{j+1}$, para $k \leq j$ e $k, j+1 \neq 1$.

Vamos escolher $x_{n}=A_{1,2} \in P_{n-1}, j=0, k=0$, e $i \neq 0,1$. Assim, supondo que $n \geq 4$, $s_{n-1} A_{1,2}=A_{1,2} \in P_{n}$ e portanto

- $d_{0}^{\mathbb{P}} y_{0}=d_{0}^{\mathbb{P}} d_{0}^{\mathbb{P}} s_{n-1}^{\mathbb{P}} A_{1,2}=d_{0}^{\mathbb{P}} d_{0}^{\mathbb{P}} A_{1,2}=d_{0}^{\mathbb{P}} A_{0,1} \neq 1$.

- $d_{0}^{\mathbb{P}} y_{1}=d_{0}^{\mathbb{P}} d_{1}^{\mathbb{P}} s_{n-1}^{\mathbb{P}} A_{1,2}=d_{0}^{\mathbb{P}} d_{1}^{\mathbb{P}} A_{1,2}=d_{0}^{\mathbb{P}} 1=1$. 
Portanto, em geral, os elementos $y_{0}, \ldots, y_{i-1}, y_{i+1}, \ldots, y_{n}$ não são faces emparelhadas com relação a $i$, como queríamos provar. Logo, existe um erro na demonstração da Proposição 4.9 de [58].

Chamamos a atenção no fato que o caso II da prova da Proposição 4.9 usa o caso I, que tem um erro como acabamos de ver.

\section{Sobre a Proposição 2.10, Teorema 2.11, Corolário 2.13, Corolário 2.14 e Proposição 2.16}

Estes resultados da Seção 2 de [58] têm alguns elementos que não foram definidos dado que $\mathbb{P}$ não é um $\Delta$-grupo, no entanto podem ser reformulados considerarando o $\Delta$-grupo $\mathcal{K}\left(\mathbb{D}^{2}\right)$ ao invés da sequência de conjuntos $\mathbb{P}$. Os enunciados alternativos são, respectivamente, a Proposição 4.3.1, Teorema 4.3.5 item 2, Teorema 4.3.5 item 3, Teorema 4.3.5 item 4 e Proposição 4.3.2.

\subsubsection{Sobre a Seção 3}

\section{As Proposições 3.2 e 3.3}

Mostraremos abaixo que as igualdades do item (2) da Proposição 3.2 e do item (1) da Proposição 3.3 não são válidas para o elemento gerador $A_{1,2}$ de $P_{n}\left(\mathbb{D}^{2}\right)$. Observemos que para os outros geradores tais igualdade são válidas. Lembremos que em $\mathbb{P}$ a aplicação face $d_{0}$ é dada por $d_{0}=\partial$.

Proposição 6.2.3. $d_{0} \chi_{\sigma_{1}}\left(A_{1,2}\right) \neq \theta d_{0}\left(A_{1,2}\right)$.

Demonstração. Notemos que

- $d_{0} \chi_{\sigma_{1}}\left(A_{1,2}\right)=d_{0}\left(A_{1,2}\right)=A_{0,1}, \mathrm{e}$

- $\theta d_{0}\left(A_{1,2}\right)=\theta\left(A_{0,1}\right)=\left(A_{1,2}^{-1} A_{0,2} A_{1,2} \ldots A_{1, n-1}^{-1} A_{0, n-1} A_{1, n-1}\right)$.

Portanto, pela descrição dada acima, a igualdade $d_{0}\left(\theta d_{0}\left(A_{1,2}\right)\right)=1$ é clara, enquanto que, pela Proposição 6.1.1, temos $d_{0}\left(d_{0} \chi_{\sigma_{1}}\left(A_{1,2}\right)\right) \neq 1$. Logo, $d_{0} \chi_{\sigma_{1}}\left(A_{1,2}\right) \neq \theta d_{0}\left(A_{1,2}\right)$.

Proposição 6.2.4. $\theta \chi_{\sigma_{1}} \theta\left(A_{1,2}\right) \neq \chi_{\sigma_{1}} \theta \chi_{\sigma_{1}}\left(A_{1,2}\right)$.

Demonstração. Observemos que:

- $\theta \chi_{\sigma_{1}} \theta\left(A_{1,2}\right)=\theta \chi_{\sigma_{1}}\left(A_{1,2}^{-1} A_{0,2} A_{1,2}\right)=\theta\left(A_{1,2}^{-1} A_{1,2} A_{0,1} A_{1,2}^{-1} A_{1,2}\right)=\theta\left(A_{0,1}\right)$.

- $\chi_{\sigma_{1}} \theta \chi_{\sigma_{1}}\left(A_{1,2}\right)=\chi_{\sigma_{1}} \theta\left(A_{1,2}\right)=\chi_{\sigma_{1}}\left(A_{1,2}^{-1} A_{0,1} A_{1,2}\right)=A_{1,2}^{-1} A_{1,2} A_{0,1} A_{1,2}^{-1} A_{1,2}=A_{0,1}$.

Na prova da Proposição 6.2.3 vimos que $\theta\left(A_{0,1}\right) \neq A_{0,1}$ e portanto obtemos o resultado desejado. 


\section{Sobre o Corolário 3.4}

Como consequência das Proposições 6.2.3 e 6.2.4 temos que $\Phi$ não é um homomorfismo e portanto não pode ser uma representação do grupo de tranças $B_{n+1}$ como afirmado pelos autores. Especificamente, temos que

$$
\Phi\left(\sigma_{1} \sigma_{2} \sigma_{1}\right) \neq \Phi\left(\sigma_{2} \sigma_{1} \sigma_{2}\right)
$$

pois $\Phi\left(\sigma_{1} \sigma_{2} \sigma_{1}\right)=\theta \chi_{\sigma_{1}} \theta$ e $\Phi\left(\sigma_{2} \sigma_{1} \sigma_{2}\right)=\chi_{\sigma_{1}} \theta \chi_{\sigma_{1}}$.

Além disso, para $\beta=\sigma_{1}$ o seguinte quadrado, mencionado no enunciado do Corolário 3.4 de [58], não é comutativo para todos os elementos de $P_{n+1}$.

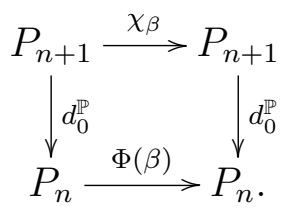

O motivo disto é que

$$
d_{0} \chi_{\sigma_{1}}\left(A_{1,2}\right) \neq \theta d_{0}\left(A_{1,2}\right)
$$

como vimos na Proposição 6.2.3, e $\theta d_{0}\left(A_{1,2}\right)=\Phi\left(\sigma_{1}\right) d_{0}\left(A_{1,2}\right)$.

\section{Observações feitas por Mikhailov e Wu}

R. Mikhailov e J. Wu afirmaram no seu artigo intitulado "Homotopy groups as centers of finitely presented groups" [69] que existem alguns erros no artigo de Li e Wu [58]. Eles afirmam que os seguintes resultados não são verdadeiros:

Lema 3.6, Teorema 3.7, Lema 3.11, Corolário 3.12, Corolário 3.13, Corolário 3.14 e Proposição 3.15 .

\section{Sobre a Proposição 3.9}

O enunciado é correto, mas a prova usa o Teorema 3.7 que não é verdadeiro como mencionado por Mikhailov \& Wu [69]. Uma demonstração deste resultado foi dada na Proposição 4.3.10 item (1).

\section{Sobre o Lema 3.10 e o Corolário 3.16}

Os enunciados e as respectivas provas destes resultados devem ser modificados dado que $\mathbb{P}$ não é um $\Delta$-grupo. Enunciados alternativos foram dados no Corolário 4.2 .8 e Corolário 4.3.8, respectivamente. 


\subsubsection{Sobre a Seção 4}

\section{Sobre o Lema 4.1}

O Lema 4.1 de [58] não é válido para $n=3$. O caso geral $(n \geq 4)$ deste resultado é válido, mas para isso é necessário fazer as seguintes adaptações: ao invés de $\mathcal{Z}_{n-1} \mathbb{P}$ tomar $\mathcal{Z}_{n-2} \mathcal{K}\left(\mathbb{D}^{2}\right)$ e no lugar de $\pi_{n-1}(\mathbb{P})$ tomar $\pi_{n-2}\left(\mathcal{K}\left(\mathbb{D}^{2}\right)\right)$.

Para entender o problema encontrado no caso específico $n=3$ precisaremos da definição de elemento "spherical", dada a seguir, que pode ser encontrada em [8]. Seja $\mathcal{X}=\left\{X_{n}\right\}_{n \geq 0}$ qualquer conjunto simplicial com ponto base. Dizemos que um elemento $x \in X_{n}$ é "spherical" se $d_{i} x=*$, para todo $0 \leq i \leq n$. Notemos que se $\mathcal{X}$ é um $\Delta$-grupo, então dizer que $x \in X_{n}$ é "spherical" equivale a dizer que $x$ é um ciclo de Moore, isto é, $x \in \mathcal{Z}_{n} \mathcal{X}$.

Lebremos que o grupo simplicial $\mathcal{K}\left(\mathbb{D}^{2}\right)$ tem ponto base 1 . Para $0 \leq j \leq 3$ e $0 \leq i \leq 2$ a composta $P_{3} \stackrel{d_{j}}{\rightarrow} P_{2} \stackrel{d_{i}}{\rightarrow} P_{1}$ é trivial, pois $P_{1}=\{1\}$. Portanto, para todo $y \in P_{3}$ e $0 \leq j \leq 3$ temos que $d_{j}(y)$ é "spherical". Ainda mais:

- $\mathcal{B} d_{1}\left(\mathcal{K}\left(\mathbb{D}^{2}\right)\right)=\partial\left(\operatorname{Brun}_{3}\left(\mathbb{D}^{2}\right)\right)=\{1\}$, pois $P_{2}$ é um grupo abeliano.

- $\operatorname{Brun}_{2}\left(\mathbb{D}^{2}\right)=P_{2}$ e $\mathcal{Z}_{1} \mathcal{K}\left(\mathbb{D}^{2}\right)=P_{2}$.

- $\pi_{2}\left(\mathbb{S}^{2}\right)=\pi_{1}\left(\mathcal{K}\left(\mathbb{D}^{2}\right)\right)=\mathcal{Z}_{1} \mathcal{K}\left(\mathbb{D}^{2}\right) / \mathcal{B} d_{1} \mathcal{K}\left(\mathbb{D}^{2}\right)=P_{2} \cong \mathbb{Z}$.

Então, para $y=A_{1,2} \in P_{3}$, temos que

- $d_{0}(y)=A_{0,1}=A_{1,2}^{-1} \in P_{2}$,

- $d_{1}(y)=d_{2}(y)=1$,

- $d_{3}(y)=A_{1,2}$,

e portanto, em $P_{2}=\pi_{1}\left(\mathcal{K}\left(\mathbb{D}^{2}\right)\right)$

$$
\left[d_{0}(y)\right]-\left[d_{1}(y)\right]+\left[d_{2}(y)\right]-\left[d_{3}(y)\right]=-2\left[A_{1,2}\right] \neq 0 \in \pi_{1}\left(\mathcal{K}\left(\mathbb{D}^{2}\right)\right) .
$$

Logo, o Lema 4.1 não é verdadeiro para $n=3$.

\section{Sobre o Lema 4.2}

Notamos que no enunciado deste lema é necessário escrever $\mathcal{Z}_{n-1} \mathcal{K}\left(\mathbb{D}^{2}\right)$ ao invés de $\mathcal{Z}_{n} \mathbb{P}$. Com esta modificação foi dada uma prova alternativa para este resultado, no caso de normalidade em $P_{n}$, ver Corolário 4.2.8. 
É preciso considerar o $\Delta$-grupo $\mathcal{K}\left(\mathbb{D}^{2}\right)$ no lugar da sequência de grupos $\mathbb{P}$, quando necessário. Exceto isso, os enunciados e demonstrações estão corretos. Uma prova alternativa para o enunciado proposto para o Lema 4.3 é fornecida numa parte da demonstração da Proposição 4.2.10.

O Teorema 3 de [58] e sua prova são corretos uma vez esta mudança é feita e sendo assim, para efeitos de ilustração vamos enunciar sem prova este importante resultado. Seja $\chi: B_{n} \rightarrow B_{n}$, definida por $\sigma_{i} \mapsto \sigma_{i}^{-1}$, a reflexão espelho do grupo de tranças. Denotemos por $\operatorname{Fix}^{\phi}(G)$ o subgrupo formado pelos pontos fixos de uma ação $\phi$ sobre um grupo $G$.

Teorema 6.2.5 (Teorema 3 de [58]). O subgrupo $\mathcal{B} d_{n-1}\left(\mathcal{K}\left(\mathbb{D}^{2}\right)\right)$ é invariante pela reflexão espelho $\chi$. Ainda mais, existe um isomorfismo de grupos

$$
F i x^{\chi}\left(B_{n} / \mathcal{B} d_{n-1}\left(\mathcal{K}\left(\mathbb{D}^{2}\right)\right)\right) \cong \pi_{n}\left(\mathbb{S}^{2}\right)
$$

para $n \geq 3$.

\subsection{Algumas provas alternativas}

Nesta seção queremos destacar algumas provas alternativas de resultados obtidos nos Capítulos 2 e 3 . O intuito com isto é não deixar de lado algumas ideias iniciais no estudo dos problemas que relacionam grupos de tranças Brunnianas e grupos de homotopia da esfera. Eventualmente as primeiras tentativas podem ter ideias que sejam úteis quando estudarmos/atacarmos outros problemas relacionados aos grupos de tranças Brunnianas sobre uma superfície $M$ qualquer.

O seguinte resultado está contido na Proposição 3.1.3, no entanto podemos dar uma prova mais elementar usando uma ideia similar à dada na prova da Proposição 3.2.14 de $[9]$.

Proposição 6.3.1. O $\Delta$-grupo $\mathcal{P}\left(\mathbb{R} P^{2}\right)$ não admite uma estrutura de grupo simplicial, com as aplicações faces como no Exemplo 1.3.2.

Demonstração. Sejam $n \geq 2$ e $\Delta_{n}=\left(\sigma_{1} \cdots \sigma_{n-1}\right)^{n}$ o "full twist" em $P_{n}\left(\mathbb{R} P^{2}\right)$. Pela Proposição 15 de [34] sabemos que $\Delta_{n}$ é o único elemento de ordem 2 em $P_{n}\left(\mathbb{R} P^{2}\right)$, para $n \geq 2$.

Vamos supor por absurdo que $\mathcal{P}\left(\mathbb{R} P^{2}\right)$ admite uma estrutura de grupo simplicial. Então, para $i=0,1$, vale que

$$
\left(s_{i}\left(\Delta_{2}\right)\right)^{2}=s_{i}\left(\Delta^{2}\right)=s_{i}(1)=1
$$

e pela unicidade dos elementos de ordem 2 em $P_{n}\left(\mathbb{R} P^{2}\right)$, segue que $s_{0}\left(\Delta_{2}\right)=s_{1}\left(\Delta_{2}\right)$. 
Portanto, usando as identidades simpliciais, temos que

$$
\Delta_{2}=d_{0} s_{0}\left(\Delta_{2}\right)=d_{0} s_{1}\left(\Delta_{2}\right)=s_{0} d_{0}\left(\Delta_{2}\right)=s_{0}(1)=1
$$

o que é claramente uma contradição.

Logo, o $\Delta$-grupo $\mathcal{P}\left(\mathbb{R} P^{2}\right)$ não admite uma estrutura de grupo simplicial, com as aplicações faces como no Exemplo 1.3.2.

Uma prova mais curta da seguinte proposição foi dada anteriormente, no item 3 do Teorema 3.1.6. No entanto, a prova dada na continuação usa ferramentas mais simples.

Proposição 6.3.2. $\pi_{2}\left(\mathcal{P}\left(\mathbb{S}^{2}\right)\right)=\mathbb{Z}_{2}$.

Demonstração. Lembremos que $\pi_{2}\left(\mathcal{P}\left(\mathbb{S}^{2}\right)\right)=\mathcal{Z}_{2} \mathcal{P}\left(\mathbb{S}^{2}\right) / \mathcal{B} d_{2} \mathcal{P}\left(\mathbb{S}^{2}\right)$. Calculemos então cada um dos grupos envolvidos. Para isto é útil ter em conta que $P_{1}\left(\mathbb{S}^{2}\right)=P_{2}\left(\mathbb{S}^{2}\right)=\{1\}$, $P_{3}\left(\mathbb{S}^{2}\right)=\mathbb{Z}_{2}$ é gerado pelo "full twist" $\Delta_{3}$ e que $P_{4}\left(\mathbb{S}^{2}\right)=F_{2} \oplus \mathbb{Z}_{2}$, onde $F_{2}$ é o grupo livre em dois geradores dado por $F_{2}=\operatorname{Ker}\left(d_{0}: P_{4}\left(\mathbb{S}^{2}\right) \rightarrow P_{3}\left(\mathbb{S}^{2}\right)\right)$.

Assim,

$$
\begin{aligned}
\mathcal{Z}_{2} \mathcal{P}\left(\mathbb{S}^{2}\right) & =\bigcap_{j=0}^{2} \operatorname{Ker}\left(d_{j}: P_{3}\left(\mathbb{S}^{2}\right) \rightarrow P_{2}\left(\mathbb{S}^{2}\right)\right) \\
& =\bigcap_{j=0}^{2} \operatorname{Ker}\left(d_{j}: \mathbb{Z}_{2} \rightarrow\{1\}\right) \\
& =\mathbb{Z}_{2}
\end{aligned}
$$

Para calcular $\mathcal{B} d_{2} \mathcal{P}\left(\mathbb{S}^{2}\right)=\operatorname{Im}\left(d_{0}: \mathcal{N}_{3} \mathcal{P}\left(\mathbb{S}^{2}\right) \rightarrow \mathcal{N}_{2} \mathcal{P}\left(\mathbb{S}^{2}\right)\right)$, devemos calcular primeiro os complexos de Moore envolvidos:

$$
\begin{gathered}
\mathcal{N}_{2} \mathcal{P}\left(\mathbb{S}^{2}\right)=\bigcap_{j=1}^{2} \operatorname{Ker}\left(d_{j}: P_{3}\left(\mathbb{S}^{2}\right) \rightarrow P_{2}\left(\mathbb{S}^{2}\right)\right)=P_{3}\left(\mathbb{S}^{2}\right), \\
\begin{aligned}
\mathcal{N}_{3} \mathcal{P}\left(\mathbb{S}^{2}\right) & =\bigcap_{j=1}^{3} \operatorname{Ker}\left(d_{j}: P_{4}\left(\mathbb{S}^{2}\right) \rightarrow P_{3}\left(\mathbb{S}^{2}\right)\right) \\
& =\bigcap_{j=1}^{3} \operatorname{Ker}\left(d_{j}: F_{2} \oplus \mathbb{Z}_{2} \rightarrow \mathbb{Z}_{2}\right) .
\end{aligned}
\end{gathered}
$$

O grupo $F_{2}$ é gerado livremente pelos elementos $a=A_{0,1}, b=A_{0,2}$, onde $A_{i, j}$ representa um gerador de tranças puras. Observemos que as aplicações faces aplicam o "full twist" no "full twist". As aplicações faces $d_{j}: P_{4}\left(\mathbb{S}^{2}\right) \rightarrow P_{3}\left(\mathbb{S}^{2}\right)$, para $j=0,1,2,3$, são definidas conforme a seguinte tabela:

\begin{tabular}{|c|c|c|c|}
\hline & $a$ & $b$ & $\Delta_{4}$ \\
\hline \hline$d_{0}$ & 0 & 0 & $\Delta_{3}$ \\
\hline$d_{1}$ & 0 & $\Delta_{3}$ & $\Delta_{3}$ \\
\hline$d_{2}$ & $\Delta_{3}$ & 0 & $\Delta_{3}$ \\
\hline$d_{3}$ & $\Delta_{3}$ & $\Delta_{3}$ & $\Delta_{3}$ \\
\hline
\end{tabular}

Tabela 6.1: $d_{j}(x)$, para $j=0,1,2,3$ e $x=a, b, \Delta_{4}$. 
Seja $w \in P_{4}\left(\mathbb{S}^{2}\right)=F_{2} \oplus \mathbb{Z}_{2}$, então a expressão reduzida para o elemento $w$ é dada por $w=a^{k_{1}} b^{l_{1}} \cdots a^{k_{t}} b^{l_{t}} \Delta_{4}^{r}$, para $k, l, r \in \mathbb{Z}$. Logo,

$$
\begin{aligned}
w \in \operatorname{Ker}\left(d_{1}\right) & \Leftrightarrow r+l_{1}+\cdots+l_{t} \equiv 0(\bmod 2) \\
w \in \operatorname{Ker}\left(d_{2}\right) & \Leftrightarrow r+k_{1}+\cdots+k_{t} \equiv 0(\bmod 2) \\
w \in \operatorname{Ker}\left(d_{3}\right) & \Leftrightarrow r+l_{1}+\cdots+l_{t}+k_{1}+\cdots+k_{t} \equiv 0(\bmod 2) .
\end{aligned}
$$

Daí, $w \in \mathcal{N}_{3} \mathcal{P}\left(\mathbb{S}^{2}\right)$ implica que $r \equiv 0(\bmod 2)$ e portanto $w \in F_{2}$. Ou seja, $\Delta_{4}$ não aparece na expressão de $w$. Como consequência disto teremos que, em $P_{3}\left(\mathbb{S}^{2}\right)$,

$$
d_{0}(w)=d_{0}\left(a^{k_{1}} b^{l_{1}} \cdots a^{k_{t}} b^{l_{t}}\right)=0
$$

$\operatorname{Logo}, d_{0}\left(\mathcal{N}_{3} \mathcal{P}\left(\mathbb{S}^{2}\right)\right)=\mathcal{B} d_{2} \mathcal{P}\left(\mathbb{S}^{2}\right)=\{0\}$ e assim $\pi_{2}\left(\mathcal{P}\left(\mathbb{S}^{2}\right)\right)=\mathbb{Z}_{2}$

A prova da seguinte proposição é interessante no sentido que explicamos na continuação. Demonstramos inicialmente o resultado para as superfícies perfuradas e depois, usando isto, passamos das superfícies perfuradas para as fechadas. Aqui não é considerado o caso da esfera nem do plano projetivo.

Proposição 6.3.3. Sejam $n \geq 2$ e $M$ uma superfície (orientável ou não) diferente da esfera e do plano projetivo, podendo ser com bordo não vazio. Então, a seguinte sequência é exata

$$
1 \longrightarrow T_{n+1} \longrightarrow \operatorname{Brun}_{n+1}\left(M \backslash Q_{k}\right) \stackrel{f_{*}}{\longrightarrow} \operatorname{Brun}_{n+1}(M) \longrightarrow \pi_{n}(\mathcal{P}(M)) \longrightarrow 1 .
$$

Demonstração. Suponhamos que $n \geq 2$. Primeiro provaremos o resultado no caso em que $M$ é uma superfície com bordo não vazio, ou um toro ou a garrafa de Klein. Pelo Corolário 3.2.13 de [9], para estas superfícies, o $\Delta$-grupo $\mathcal{P}(M)$ é um grupo simplicial e portanto, pelo Teorema de Moore, segue que $\mathcal{P}(M)$ é um $\Delta$-grupo fibrante. Além disso, pela Proposição 3.3.2, segue que $f_{*}: \mathcal{P}\left(M \backslash Q_{k}\right) \rightarrow \mathcal{P}(M)$ é um epimorfismo. Portanto, observemos que

$$
1 \longrightarrow \operatorname{Ker}\left(f_{*}\right) \longrightarrow \mathcal{P}\left(M \backslash Q_{k}\right) \stackrel{f_{*}}{\longrightarrow} \mathcal{P}(M) \longrightarrow 1
$$

é uma sequência exata curta de $\Delta$-grupos fibrantes. Assim, pela Proposição 4.1.4 de [9], segue que

$$
N f_{*}: \mathcal{N}_{n} \mathcal{P}\left(M \backslash Q_{k}\right) \rightarrow \mathcal{N}_{n} \mathcal{P}(M)
$$

é um epimorfismo para todo $n \geq 2$. Logo, aplicando o Lema 3.3.1 $\operatorname{com} L=M \backslash Q_{k}$, a sequência dada em (6.11) é exata no caso em que $M$ é uma superfície com bordo não vazio, ou um toro ou a garrafa de Klein.

Além disso, se $M$ é uma superfície com bordo não vazio, segue pelos Teoremas 3.1.6 e 
3.1.8 que $\pi_{n}(\mathcal{P}(M))=\{1\}$, para todo $n \geq 0$. Aplicando isto na sequência (6.11) para superfícies com bordo não vazio temos que a seguinte sequência é exata curta para $n \geq 2$,

$$
1 \longrightarrow T_{n+1} \longrightarrow \operatorname{Brun}_{n+1}\left(M \backslash Q_{k}\right) \stackrel{f_{*}}{\longrightarrow} \operatorname{Brun}_{n+1}(M) \longrightarrow 1
$$

Na continuação vamos demonstrar o resultado no caso em que $M$ é uma superfície fechada diferente da esfera e do plano projetivo, ou seja, as superfícies fechadas do tipo Eilenberg-Mac Lane. Para isto, reduzimos o problema a uma situação de superfícies perfuradas e usamos a sequência exata curta (6.12). Observamos que a seguir usaremos a notação simplicial.

Seja $h=f \mid: M \backslash\left\{Q_{k} \cup\left\{x_{0}\right\}\right\} \rightarrow M \backslash\left\{x_{0}\right\}$, onde $x_{0}$ é o ponto base correspondente à primeira corda de uma trança em $P_{n+1}(M)$. Consideremos as seguintes composições de homomorfismos

$\theta_{1}=d_{1} \circ \cdots \circ d_{n}: P_{n+1}(M) \rightarrow P_{1}(M)$ e $\theta_{2}=d_{1} \circ \cdots \circ d_{n}: P_{n+1}\left(M \backslash Q_{k}\right) \rightarrow P_{1}\left(M \backslash Q_{k}\right)$,

que esquecem $n$ cordas de uma trança dada. Desta forma temos o seguinte diagrama comutativo de sequências exatas curtas,

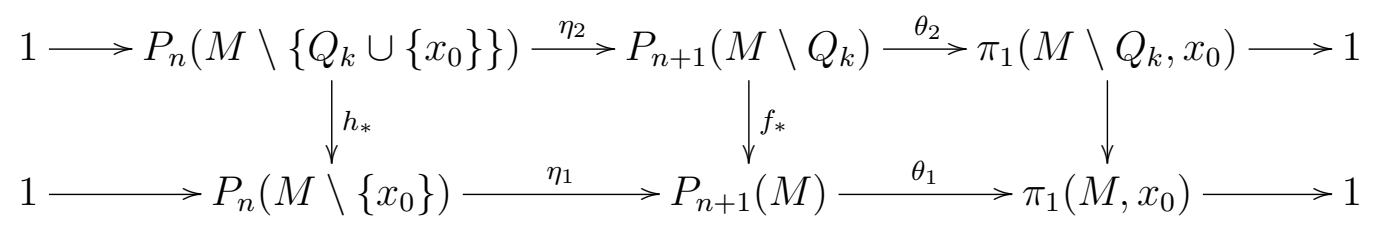

Notemos que o primeiro quadrado é comutativo, pelo fato que geometricamente $h$ é a restrição da inclusão natural $f$ e $\eta_{1}, \eta_{2}$ são os monomorfismos canônicos, induzidos geometricamente pela aplicação que coloca de volta uma corda vertical no ponto base $x_{0}$.

Seja $\beta \in \mathcal{N}_{n} \mathcal{P}(M)$. Então, $\theta_{1}(\beta)=1$ e assim $\beta \in \operatorname{Ker}\left(\theta_{1}\right)=\operatorname{Im}\left(\eta_{1}\right)$. Logo, existe um único elemento $\beta_{1} \in P_{n}\left(M \backslash\left\{x_{0}\right\}\right)$ tal que $\eta_{1}\left(\beta_{1}\right)=\beta$. Como $\eta_{1} \circ d_{k}=d_{k+1} \circ \eta_{1}$,

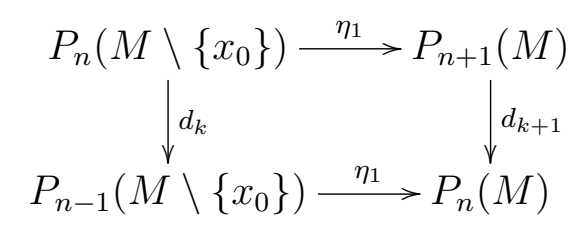

para $k=0, \ldots, n-1$ e $\eta_{1}$ é monomorfismo (aqui usa o fato que $M \neq \mathbb{S}^{2}, \mathbb{R} P^{2}$ ), então $\beta_{1} \in \operatorname{Brun}_{n}\left(M \backslash\left\{x_{0}\right\}\right)$.

Pela sequência exata curta dada em (6.12), entre grupos Brunnianos sobre superfícies perfuradas, temos que $h_{*} \mid: \operatorname{Brun}_{n}\left(M \backslash\left\{Q_{k} \cup\left\{x_{0}\right\}\right\}\right) \rightarrow \operatorname{Brun}_{n}\left(M \backslash\left\{x_{0}\right\}\right)$ é um epimorfismo. Logo, existe $\beta_{2} \in \operatorname{Brun}_{n}\left(M \backslash\left\{Q_{k} \cup\left\{x_{0}\right\}\right\}\right)$ tal que $h_{*}\left(\beta_{2}\right)=\beta_{1}$. Se $\beta_{3}=\eta_{2}\left(\beta_{2}\right)$, então $\beta_{3} \in \mathcal{N}_{n} \mathcal{P}\left(M \backslash Q_{k}\right)$, já que para $\eta_{2}$ vale também a igualdade $\eta_{2} \circ d_{k}=d_{k+1} \circ \eta_{2}$, para 
$k=0, \ldots, n-1$ (não precisa neste caso da injetividade de $\eta_{2}$ ). Logo,

$$
N f_{*} \mid: \mathcal{N}_{n} \mathcal{P}\left(M \backslash Q_{k}\right) \rightarrow \mathcal{N}_{n} \mathcal{P}(M)
$$

é um epimorfismo e como consequência do Lema 3.3.1 temos a seguinte sequência exata para $n \geq 2$, como queríamos provar,

$$
1 \longrightarrow T_{n+1} \longrightarrow \operatorname{Brun}_{n+1}\left(M \backslash Q_{k}\right) \stackrel{f_{*}}{\longrightarrow} \operatorname{Brun}_{n+1}(M) \longrightarrow \pi_{n}(\mathcal{P}(M)) \longrightarrow 1 .
$$

\subsection{Sobre difeomorfismos de Anosov}

Durante o que se segue desta seção assumiremos que o leitor está familiarizado com alguns conceitos básicos de Geometria Riemanniana. Seja $M$ uma variedade Riemanniana, compacta plana. Um difeomorfismo $f: M \rightarrow M$ é chamado um difeomorfismo de Anosov se para qualquer (e portanto para toda) métrica sobre $M$ existem constantes $c>0$ e $0<\lambda<1$ tais que em qualquer ponto $m$ de $M$ o espaço tangente $T M_{m}$ se decompõe como uma soma direta de uma parte que se contrai e uma outra parte que se expande, isto é, $T M_{m}=E^{s} \oplus E^{u}$, onde $\left\|T f^{r} v\right\| \leq c \lambda^{r}\|v\|$, para todo $v \in E^{s}$ e todo inteiro $r>0$ e $\left\|T f^{-r} w\right\| \leq c \lambda^{r}\|w\|$, para todo $w \in E^{u}$ e todo inteiro $r>0$, (ver [79]), onde a norma provem da métrica Riemanniana. As letras s e $u$ indicam as palavras (em inglês) "estável" e "instável". É bem conhecido, por exemplo, que o toro admite um difeomorfismo de Anosov mas a garrafa de Klein não, ver o exemplo na página 307 de [79].

O problema de caracterizar as variedades que admitem difemomorfismos de Anosov é um problema de interesse na área de Sistemas Dinâmicos. Este problema foi proposto por D. Anosov em 1966, durante sua conferência no Congresso Internacional de Matemáticos em Moscou [87]. Ele questionou sobre a existência de exemplos de variedades, distintas do toro, que admitem difeomorfismo de Anosov [87]. Os primeiros exemplos foram dados por S. Smale em 1967 [87] e por M. Shub em 1969 [86]. Até agora, os únicos exemplos conhecidos de variedades compactas que admitem difeomorfismo de Anosov são de natureza algébrica, por exemplo, os toros, as variedades planas, assim como as chamadas nilvariedades e infra-nilvariedades. Para mais detalhes sobre este problema e alguns exemplos citamos [87], [86], [62] e [24].

H. Porteous, em 1971, deu uma importante caracterização algébrica das variedades planas que admitem difeomorfismo de Anosov, ver Seção 4 e Teorema 6.1 de [79]. Tal caracterização depende da representação de holonomia e um caso particular ocorre quando o grupo de holonomia $H$, da variedade plana $M$, é um grupo cíclico finito. Enunciaremos abaixo o resultado neste caso específico, que é de grande utilidade para mostrar que exis- 
tem variedades planas, determinadas por quocientes de grupos de tranças, que admitem difeomorfismo de Anosov.

Teorema 6.4.1 (Teorema de Porteous, Teorema 7.1 de [79]). Seja M uma variedade plana cujo grupo de holonomia $F$ é cíclico e $T: F \rightarrow G L(n ; \mathbb{Z})$ é a representação de holonomia. Se $N=T(g)$, onde $g$ é um gerador de $F$, então $M$ admite um difeomorfismo de Anosov se, e somente se, $N$ não tem os seguintes autovalores $1,-1, i,-i, \omega, \omega^{2},-\omega,-\omega^{2}$ com multiplicidade algébrica 1 (onde $\omega^{3}=1$ ).

A seguir faremos uma exposição curta sobre matrizes circulantes, as quais são de utilidade no estudo da existência de difeomorfismos de Anosov para as variedades planas obtidas a partir dos grupos de Bieberbach de tranças. Um estudo mais completo destas matrizes pode ser visto no livro de P. Davis [21].

Uma matriz circulante de tamanho $n \times n$ é uma matriz da forma

$$
C=\left(\begin{array}{ccccc}
c_{0} & c_{n-1} & \ldots & c_{2} & c_{1} \\
c_{1} & c_{0} & c_{n-1} & & c_{2} \\
\ldots & c_{1} & c_{0} & \ddots & \vdots \\
c_{n-2} & & \ddots & \ddots & c_{n-1} \\
c_{n-1} & c_{n-2} & \ldots & c_{1} & c_{0}
\end{array}\right)
$$

Os autovalores da matriz $C$, dada em (6.13), são da forma

$$
\lambda_{j}=c_{0}+c_{n-1} \omega_{j}+c_{n-2} \omega_{j}^{2}+\cdots+c_{1} \omega_{j}^{n-1}, \quad 0 \leq j \leq n-1
$$

onde

$$
\omega_{j}=\exp \left(\frac{2 \pi i j}{n}\right)
$$

uma $n$-ésima raiz da unidade e $i=\sqrt{-1}$.

Diremos que uma matriz circulante é uma matriz circulante principal se $c_{1} \neq 0$ e para os outros valores de $i$ tem-se que $c_{i}=0$. Esta matriz é da forma

$$
\left(\begin{array}{ccccc}
0 & 0 & \ldots & 0 & c_{1} \\
c_{1} & 0 & 0 & & 0 \\
\vdots & c_{1} & 0 & \ddots & \vdots \\
0 & & \ddots & \ddots & 0 \\
0 & 0 & \ldots & c_{1} & 0
\end{array}\right)
$$

e a entrada $c_{1}$ é chamado de entrada principal da matriz circulante principal. Denotaremos as matrizes circulantes principais por $\operatorname{PCM}(n, c)$, onde a matriz tem ordem $n \times n$ e $c$ indica 
a entrada principal. A matriz circulante principal com entrada principal 1 dada por

$$
\operatorname{PCM}(n, 1)=\left(\begin{array}{ccccc}
0 & 0 & \ldots & 0 & 1 \\
1 & 0 & 0 & & 0 \\
\vdots & 1 & 0 & \ddots & \vdots \\
0 & & \ddots & \ddots & 0 \\
0 & 0 & \ldots & 1 & 0
\end{array}\right)
$$

tem determinante 1 se $n$ é ímpar e -1 se $n$ é par. Além disso, dada qualquer matriz circulante $C$ como em (6.13), a matriz $C$ pode ser escrita em função de $\operatorname{PCM}(n, 1)$ da seguinte forma

$$
C=c_{0} I+c_{1} \operatorname{PCM}(n, 1)+c_{2} \operatorname{PCM}(n, 1)^{2}+\cdots+c_{n-1} \operatorname{PCM}(n, 1)^{n-1},
$$

onde $\operatorname{PCM}(n, 1)^{k}$ quer dizer que tomamos o produto da matriz $\operatorname{PCM}(n, 1)$ um número $k$ de vezes. 


\section{Referências Bibliográficas}

[1] Adem, A., Ge, J., Pan, J., And Petrosyan, N. Compatible actions and cohomology of crystallographic groups. Journal of Algebra 320, 1 (2008), 341 - 353. 139

[2] Artin, E. Theorie der Zöpfe. Abh. Math. Sem. Univ. Hamburg 4 (1925), 47-72. xxi, $14,15,16$

[3] Artin, E. Braids and permutations. Ann. Math. 48 (1947), 643-649. xxi

[4] Artin, E. Theory of Braids. Ann. Math. 48 (1947), 101-126. xxi

[5] Bardakov, V. G., Mikhailov, R., Vershinin, V., and Wu, J. Brunnian braids on surfaces. Algebr. Geom. Topol. 12, 3 (2012), 1607-1648. xxiii, xxv, xxviii, $6,12,23,24,32,33,37,40,41,43,52,54,55,62,64,66,78,80,86,88,113,126$

[6] Baues, H., And Mikhailov, R. Intersection of subgroups in free groups and homotopy groups. Internat. J. Algebra Comput. 18, 5 (2008), 803-823. 12

[7] Bellingeri, P. On presentations of surface braid groups. Journal of Algebra 274, 2 (2004), $543-563.16,86$

[8] Berrick, A. J., Cohen, F., Hanbury, E., Wong, Y.-L., And Wu, J., Eds. Braids - Introductory lectures on braids, configurations and their applications, vol. 19 of IMS Lecture Notes. World Scientific, Singapore, 2009. xxi, xxii, xxxiii, xxxiv, 9, $13,26,33,34,96,100,190$

[9] Berrick, A. J., Cohen, F. R., Wong, Y. L., And Wu, J. Configurations, braids and homotopy groups. J. Amer. Math. Soc. 19 (2006), 265-326. xi, xii, xxiii, xxv, xxvi, xxxi, xxxii, xli, 1, 7, 16, 17, 23, 24, 26, 27, 28, 29, 30, 31, 32, 34, 35, 37, $43,44,47,49,64,69,70,71,75,77,78,80,82,85,90,91,108,118,119,177,178$, $179,180,181,182,183,184,185,186,191,193,208$

[10] Berrick, A. J., Hanbury, E., And Wu, J. Brunnian subgroups of mapping class groups and braid groups. Proc. London Math. Soc. (to appear). xxiii, xxxii, 77 
[11] Birman, J., And Brendle, T. Braids: a survey. In Handbook of knot theory, W. Menasco and M. Thistlethwaite, Eds. Elsevier, Amsterdam, 2005, pp. 19-104. xxii, $9,13,14$

[12] Birman, J. S. On braid groups. Comm. Pure Appl. Math. 22 (1969), 41-72. 15, 18

[13] Birman, J. S. Braids, links, and mapping class groups, vol. 82 of Annals of Math. Studies. Princeton Univ. Press, 1975. xxi, 14

[14] Brown, H., Bülow, R., Neubüser, J., Wondratschek, H., And ZasseNHAUs, H. Crystallographic groups of four-dimensional space. Wiley Monographs in Crystallography. Wiley-Interscience, 1978. 136

[15] Brunn, H. Ueber Verkettung. S.-B. Math.-Phys. Cl. Akad. Wiss. München 22 (1892), 77-99. xxii, 21, 22

[16] Charlap, L. Bieberbach groups and flat manifolds. Springer-Verlag, New York, 1986. $136,137,138,139$

[17] Cohen, F., And Wu, J. On braids groups and homotopy groups. Geometry and Topology Monographs 13 (2008), 169-193. xxiii, 23, 37, 43, 46

[18] Cohen, F. R. Artin's braid groups and classical homotopy theory. In Contemporary Mathematics, Combinatorial methods in topology and algebraic geometry, vol. 44. American Mathematical Society, Providence, RI, 1985, pp. 207-220. xxi

[19] Curtis, E. B. Simplicial homotopy theory. Advances in Math. 6 (1971), 107-209. $26,30,34,83$

[20] Curtis, E. B., And Mahowald, M. The unstable Adams spectral sequence for $S^{3}$. In Contemporary Mathematics, Algebraic Topology (Evanston, IL, 1988), vol. 96. American Mathematical Society, 1989, pp. 125-162. 9

[21] Davis, P. J. Circulant matrices. Pure and Applied Mathematics (John Wiley). John Wiley, New York, 1979. 196

[22] Debrunner, H. Links of Brunnian type. Duke Math. J. 28 (1961), 17-23. xxii, 22

[23] Dekimpe, K. Almost-Bieberbach groups: affine and polynomial structures, vol. 1639 of Lecture Notes in Mathematics. Springer, Berlin, 1996. 136, 137, 138

[24] Dekimpe, K., De Rock, B., And Malfait, W. The Nielsen numbers of Anosov diffeomorphisms on flat Riemannian manifolds. Forum Math. 17, 2 (2005), 325-341. 195 
[25] Ellis, G., And Mikhailov, R. A colimit of classifying spaces. Advances in Math. 223 (2010), 2097-2113. 7, 11, 12, 65, 92, 93

[26] FADELL, E. Homotopy groups of configuration spaces and the string problem of Dirac. Duke Math. Journal 29 (1962), 231-242. 17, 18

[27] Fadell, E., And Husseini, S. Geometry and Topology of Configuration Spaces. Springer Monographs in Mathematics, Springer, 2001. 13

[28] Fadell, E., And Neuwirth, L. Configuration spaces. Math. Scand. 10 (1962), 111-118. 13, 14, 17, 18

[29] Fadell, E., And Van Buskirk, J. The braid groups of $E^{2}$ and $S^{2}$. Duke Math. J. 29 (1962), 243-258. 17, 18

[30] Farb, B., AND Margalit, D. A primer on mapping class groups. Princeton University Press, Princeton, NJ, 2011. xxi, 14

[31] Fox, R. H., And Neuwirth, L. The braid groups. Math. Scand. 10 (1962), 119-126. xxi, 13, 14

[32] Goerss, P. G., And Jardine, J. F. Simplicial homotopy theory. Birkhäuser, 1999. 26

[33] Gonçalves, D. L., And Guaschi, J. On the structure of surface pure braid groups. J. Pure Appl. Algebra 182, 1 (2003), 33-64. 16, 18

[34] Gonçalves, D. L., And Guaschi, J. The braid groups of the projective plane. Algebraic and Geometric Topology 4 (2004), 757-780. 191

[35] Gonçalves, D. L., And Guaschi, J. The roots of the full twist for surface braid groups. Math. Proc. Camb. Phil. Soc. 137 (2004), 307-320. 19

[36] Gonçalves, D. L., And Guaschi, J. The braid group $B_{n, m}\left(S^{2}\right)$ and a generalisation of the Fadell-Neuwirth short exact sequence. J. Knot Theory and its Ramifications 14, 3 (2005), 375-403. 13, 19

[37] Gonçalves, D. L., And Guaschi, J. The braid groups of the projective plane and the Fadell-Neuwirth short exact sequence. Geom. Dedicata 130 (2007), 93-107. 13

[38] Gonçalves, D. L., And Guaschi, J. Braid groups of non-orientable surfaces and the Fadell-Neuwirth short exact sequence. J. Pure Appl. Algebra 214 (2010), 667-677. 13, 16, 18 
[39] Gonçalves, D. L., And Guaschi, J. The Borsuk-Ulam theorem for maps into a surface. Topology Appl. 15\%, 10-11 (2010), 1742-1759. xxi, 20

[40] Gonçalves, D. L., And Guaschi, J. Minimal generating and normally generating sets for the braid and mapping class groups of $\mathbb{D}^{2}, \mathbb{S}^{2}$ and $\mathbb{R} P^{2}$. Math. Z. 274, 1-2 (2013), 667-683. 141, 156, 180

[41] González-Meneses, J. New presentations of surface braid groups. J. Knot Theory Ramifications 10, 3 (2001), 431-451. 16

[42] Guaschi, J., And Juan-Pineda, D. A survey of surface braid groups and the lower algebraic K-theory of their group rings. Handbook of Group Actions, Kunming Conference Proceedings (to appear). xxi, xxii, 9, 14, 20

[43] Gurzo, G. G. The group of smooth braids. In 16th All-Union Algebra Conference, Abstract II (1981), p. 3940. xxiii

[44] Hall, P. The Edmonton notes on Nilpotent groups. Queen Mary College Mathematics Notes, 1969. 36

[45] Hansen, V. L. Braids and Coverings: selected topics. Cambridge University Press, 1989. xxii, $9,13,14$

[46] Hatcher, A. Algebraic topology. Cambridge University Press, Cambridge, 2002. 9

[47] Hilden, H. M., Montesinos, J. M., Tejada, D. M., and Toro, M. M. Artifacts for stamping symmetric designs. Am. Math. Mon. 118, 4 (2011), 327-343. 135

[48] Hiss, G., ANd Szczepański, A. Spin structures on flat manifolds with cyclic holonomy. Commun. Algebra 36, 1 (2008), 11-22. 140

[49] Johnson, D. L. Towards a characterization of smooth braids. Math. Proc. Camb. Philos. Soc. 92 (1982), 425-427. xxii, xxiii, 22

[50] Johnson, D. L. Presentations of groups, 2 ed. Cambridge University Press, Cambridge, 1997. 3, 4, 144, 169, 173

[51] Jones, V. F. R. Hecke algebra representation of braid groups and link polynomials. Ann. Math. 126 (1987), 335-388. xxi

[52] Juan-Pineda, D., And Millán-López, S. The Whitehead group and the lower algebraic $K$-theory of braid groups on $\mathbb{S}^{2}$ and $\mathbb{R} P^{2}$. Algebr. Geom. Topol. 10, 4 (2010), 1887-1903. xxi, 20 
[53] KAn, D. A combinatorial definition of homotopy groups. Ann. Math. 67 (1958), 288-312. 26, 33

[54] Kassel, C., And Turaev, V. Braid groups. With the graphical assistance of Olivier Dodane. Springer-Verlag, New York, 2008. xxii, 9, 14

[55] Kochman, S., And Mahowald, M. On the computation of stable stems. In Contemporary Mathematics, The Čech centennial (Boston, MA, 1993), vol. 181. American Mathematical Society, Providence, RI, 1995, pp. 299-316. 9

[56] Levinson, H. Decomposable braids and linkages. Trans. Amer. Math. Soc. 178 (1973), 111-126. 25

[57] Levinson, H. Decomposable braids as subgroups of braid groups. Trans. Am. Math. Soc. 202 (1975), 51-55. xxii, 22

[58] Li, J. Y., AND Wu, J. Artin braid groups and homotopy groups. Proc. London Math. Soc. 99, 3 (2009), 521-556. xi, xxiii, xxv, xxvi, xxxi, xxxiii, xxxiv, xxxvi, xli, $23,24,35,36,37,64,65,71,72,73,74,95,96,103,111,112,115,116,122,126,128$, $133,177,184,185,186,187,188,189,190,191$

[59] LI, J. Y., AND Wu, J. On symmetric commutator subgroups, braids, links and homotopy groups. Trans. Amer. Math. Soc. 363, 7 (2011), 3829-3852. 6, 7, 8, 24

[60] Magnus, W., Karrass, A., And Solitar, D. Combinatorial Group Theory, 2 ed. Dover, New York, 1976. xxiii, 2, 3, 4, 5, 6

[61] MaKanin, G. S. The Kourovka notebook (Unsolved Questions in group theory), 7 ed. Novosibirsk, 1980. xxii

[62] Malfait, W. Flat manifolds with prescribed first Betti number admitting Anosov diffeomorphisms. Monatsh. Math. 133, 2 (2001), 157-162. 195

[63] Mangum, B., And Stanford, T. Brunnian links are determined by their complements. Algebr. Geom. Topol. 1 (2001), 143-152. 22, 48

[64] Manturov, V. Knot theory. Chapman \& Hall, 2004. xxi, 14, 21

[65] Massey, W. S. Algebraic topology: An introduction. Springer-Verlag, New York, 1967. 9

[66] Massey, W. S. A basic course in algebraic topology. Springer-Verlag, New York, 1991. 9

[67] May, J. P. Simplicial objects in algebraic topology. Van Nostrand Mathematical Studies. Van Nostrand, Princeton, 1968. 26 
[68] May, J. P. Stable algebraic topology, 1945-1966. James, I. M. (ed.), History of topology. Amsterdam: Elsevier. 665-723, 1999. 9

[69] Mikhailov, R., And Wu, J. Homotopy groups as centers of finitely presented groups. Izv. Math. (to appear). 95, 126, 189

[70] Milnor, J. Link groups. Annals of Math. 59, 2 (1954), 177-195. xxii, 21, 22

[71] Milnor, J. W. Hilbert's problem 18: On crystallographic groups, fundamental domains, and on sphere packing. Math. Dev. Hilbert Probl., Proc. Symp. Pure Math. 28, De Kalb 1974, 491-506, 1976. 138

[72] Montesinos, J. M. Crystallographic groups and topology of Escher. Rev. R. Acad. Cienc. Exactas Fís. Nat. (Esp.) 104, 1 (2010), 27-47. 135

[73] Moore, J. C. Homotopie des complexes Monöideaux. Seminaire Henri Cartan (1954-55). 26

[74] Munkres, J. R. Elements of algebraic topology. Perseus Books, Cambridge, 1984. 9

[75] Murasugi, K., And Kurpita, B. A study of braids, vol. 484 of Mathematics and its Applications. Kluwer Academic Publishers, Dordrecht, 1999. xxii, 3, 9, 14, 18, 52, 53

[76] Ocampo, O. E. Subgrupos geométricos e seus comensuradores em grupos de tranças de superfície. Master's thesis, Universidade de São Paulo, Brasil, 2009. 14, 19

[77] OrLik, P. Seifert manifolds, vol. 291 of Lecture Notes in Mathematics. SpringerVerlag, 1972. 152, 154

[78] Paris, L., And Rolfsen, D. Geometric subgroups of surface braid groups. Ann. Inst. Fourier 49 (1999), 417-472. 19

[79] Porteous, H. L. Anosov diffeomorphisms of flat manifolds. Topology 11 (1972), 307-315. 195, 196

[80] Ratcliffe, J. G. Foundations of hyperbolic manifolds, 2 ed. Springer-Verlag, New York, 2006. 136

[81] Robinson, D. A course in the theory of groups, 2 ed. Springer-Verlag, New York, 1995. $2,3,4,5$

[82] Rossetti, J., And Tirao, P. Five-dimensional Bieberbach groups with holonomy group $\mathbb{Z}_{2} \oplus \mathbb{Z}_{2}$. Geom. Dedicata 7\%, 2 (1999), 149-172. 140 
[83] Rotman, J. J. An introduction to the theory of groups, 4 ed. Springer-Verlag, New York, 1995. 3

[84] Scott, G. P. Braid groups and the group of homeomorphisms of a surface. Proc. Camb. Phil. Soc. 68 (1970), 605-617. 15

[85] Serre, J. P. Linear representations of finite groups. Springer-Verlag, New York, 1977. 140

[86] Shub, M. Endomorphisms of compact differentiable manifolds. Am. J. Math. 91 (1969), 175-199. 195

[87] Smale, S. Differentiable dynamical systems. Bull. Am. Math. Soc. 73, 6 (1967), 747-817. 195

[88] Stanford, T. Brunnian braids and some of their generalizations. arXiv:math/9907072 [math.GT] (1999). xxiii, 22

[89] Thurston, W. Three-dimensional geometry and topology. Princeton University Press, Princeton, NJ, 1997. 136

[90] TodA, H. Composition methods in homotopy groups of spheres, vol. 49 of Annals of Mathematics Studies. Princeton University Press, 1962. 9, 12

[91] VAn Buskirk, J. Braid groups of compact 2-manifolds with elements of finite order. Trans. Amer. Math. Soc. 122 (1966), 81-97. 17, 18

[92] Weyl, H. Symmetry. Princeton University Press, Princeton, 1952. 135

[93] Whitehead, G. W. Elements of homotopy theory. Springer-Verlag, New York, 1978. 9

[94] Whittlesey, K. Normal all pseudo-Anosov subgroups of mapping class groups. Geom. Topol. \& (2000), 293-307. xxi, xxiii, 23, 24

[95] Wolf, J. A. Spaces of constant curvature, 6 ed. AMS Chelsea Publishing, Providence, RI, 2011. xxxviii, 136, 138, 152

[96] Wu, J. Combinatorial descriptions of the homotopy groups of certain spaces. Proc. Camb. Phil. Soc. 130 (2001), 489-513. 7, 12, 95

[97] Wu, J. On brunnian-type links and the link invariants given by homotopy groups of spheres. arXiv:0909.4973 [math.AT] (2009). 25

[98] Zariski, O. On the poincaré group of rational plane curves. Amer. J. Math. 58 (1936), 607-619. xxi 
[99] ZARIski, O. The topological discriminant group of a riemann surface of genus $p$. Amer. J. Math. 59 (1937), 335-358. xxi 


\section{Índice Remissivo}

$\Delta$-conjunto, 26

fibrante, 30, 33, 83, 193

$\Delta$-grupo, 26

Bordo de Moore, 28

Ciclo de Moore, 28

contrátil, 28

fibrante, 76

Homotopia de Moore do, 28, 76

$\Delta$-grupo formado por grupos de tranças puras, 75

Homotopia do, 75

$\pi_{n}\left(\mathbb{S}^{2}\right)$, para $1 \leq n \leq 21,12$

Aplicações

degenerações, 30

faces, 26

Arranjo de colchetes, 6

Caracterização algébrica dos grupos cristalográficos, 138

Complexo de Kan, 30

Complexo de Moore, 28

Funtor, 85

Comutador de dois elementos, 4

Conectividade de subgrupos normais, 11

Conjunto das raízes de $H$ em $G$ (isolador), 36

Conjunto simplicial, 30

com ponto base, 33

reduzido, 34

Difeomorfismo de Anosov, 195

Espaço

de configuração, 13 de Eilenberg-Mac Lane, 11

Espaço asférico, 10

Faces emparelhadas, 30

Fecho normal de um elemento, 1

Fibração, 11

de Fadell-Neuwirth, 13

Fibração de Fadell-Neuwirth, 19

Geradores

$\sigma_{i}, 16$

$A_{i, j}, 16$

Grupo

abelianizado, 4

abeliano livre, 3

cíclico, 2

de Baumslag-Solitar, 2

de Heisenberg, 2

de homotopia, 9

de permutações, 2

finitamente apresentado, 2

fundamental, 10

Hopfiano, 4, 82

livre, 3

policíclico, 2

residualmente nilpotente, 5

solúvel, 5

virtualmente $P, 140$

Grupo cristalográfico, 136

de tranças, 143, 159

Grupo de Bieberbach, 136

Grupo de Holonomia, 137

Grupo de tranças, 14

de Artin, 16 
puras, 14

Grupo simplicial, 30

cruzado, 31

Homomorfismo $\partial, 16$

Identidades

de Witt-Hall, 5, 80

simpliciais, 30

Isolador de $H$ em $G, 36$

Lema 6.5.2 de [9], 17

Método de Reidemeister-Schreier, 3

Matriz circulante, 196

Matriz circulante principal, 196

Entrada principal de uma, 196

Reflexão espelho de tranças, 191

Representação

de holonomia, 140

fiel, 138

integral, 138

Série

central, 5

derivada, 5

Segunda caracterização algébrica dos grupos cristalográficos, 139

Sequência exata curta

de tranças, 14

de tranças puras, 17

Subgrupo comutador, 4

amplo, 7

simétrico, 7

Subgrupo comutador simétrico, 6, 8

Superfície $k$-perfurada, 1

\section{Teorema}

de adição em homotopia, 34, 131, 132

de Auslander e Kuranishi, 138

de Decomposição, 100 de Ellis-Mikhailov, 12

de Extensão Central, 96

de Jie Wu, 12, 63

de Milnor, 34

de Moore, 33, 193

de Nielsen-Schreier, 4

de Porteous, 196

Teorema 1.2 de [9], 24, 71, 75

Teoremas de Bieberbach, 137, 138

Trança Brunniana, 22

Trança Brunniana simplicial, 32

Tranças $k$-decomponíveis, 25, 48, 49

Variedade plana, 137

orientável, 140 